
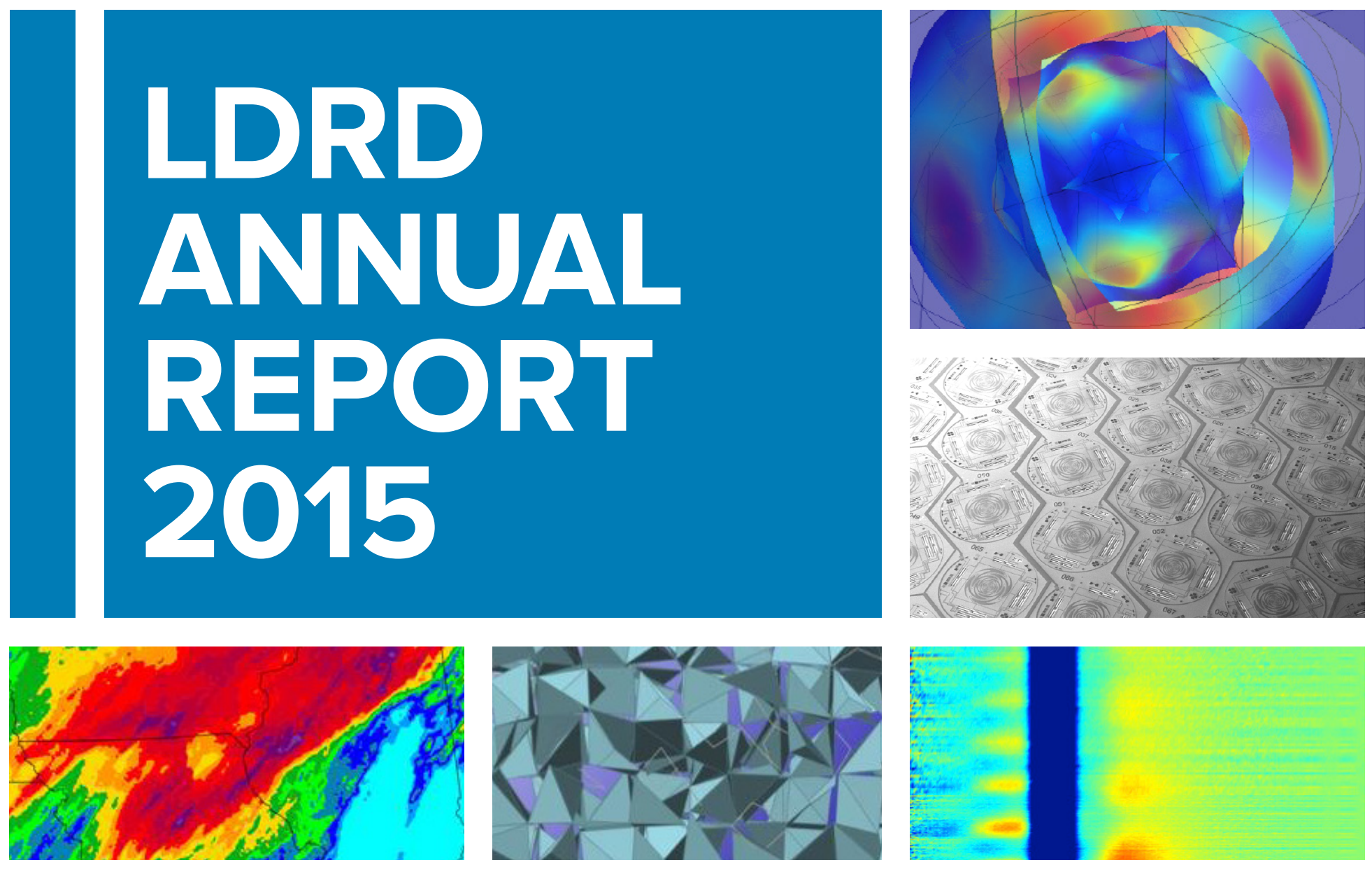

\title{
Laboratory Directed Research and Development Program Activities
}




\section{Every day, scientists and engineers at Argonne National Laboratory make important discoveries that translate into a real difference in the world, working toward the Lab's mission to develop energy solutions, preserve the environment, protect the nation, and help the economy thrive.}

\section{ON THE COVER}

1 A computer simulation of mechanical deformations of a $20-\mathrm{nm}$ gold core inside a 50 -nm silver shell as part of a study of nanoscale high frequency oscillators. [Related to, but not shown in, Extreme Optomechanics (2013-035-R2, page 6).]

2 A scanning electron micrograph of some of the pixels in a superconducting transition edge sensor (TES) detector array. Each approximately seven-millimeter pixel houses six TES bolometers. [See Transition Edge Sensors for Fundamental Physics (2013-219-R2, page 152).]

3 Observed total precipitation throughout one full mid-April day during the Chicago 2013 spring floods. [See Large-Scale Modeling and Simulation for an Adaptive and Resilient Power Grid (2015-159-NO, page 88).]

4 A computer simulation "snapshot" of the structure of uranium dioxide at high temperature, just below the melting point, showing its $\mathrm{UO}_{8}$ cubic polyhedra. [See Understanding Atomic-Scale Uranium Interactions under Severe Accident Conditions (2015-096-NO, page 41).]

5 After an initial pulse starts a gold-silver core-shell nanoparticle vibrating, the ring-down of its two vibrational frequencies at 24 and 40 gigahertz are observed in the transient absorption of a subsequently applied optical probe pulse shown here. [See Extreme Optomechanics (2013-035-R2, page 6).]
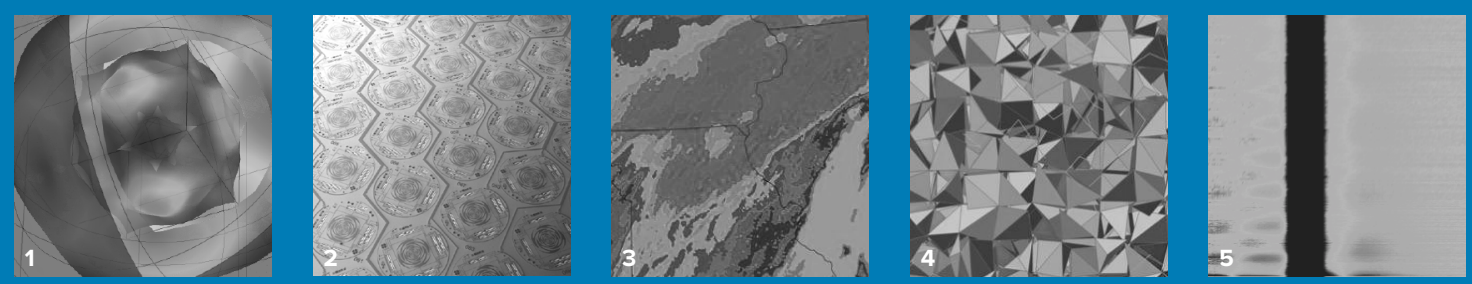


\section{DIRECTOR'S MESSAGE}

With great pleasure I present to you the Annual Report of Laboratory-Directed Research and Development (LDRD) activities at Argonne National Laboratory for fiscal year 2015. In these project reports you will learn about leading-edge projects overseen by scientists at the forefront of their fields.

Our LDRD projects deliver on their promise to advance the Laboratory's major initiatives-evidence is found in the follow-on sponsorship garnered in many cases, as well as the numerous publications, invited presentations, inventions, and new staff hires that can be traced directly to LDRD results. LDRD takes our programs in new directions, enabling us to adapt to new opportunities in a changing environment and enhance the capabilities of our staff and R\&D facilities.

As Argonne aims to make its LDRD program invaluable to DOE as a resource to meet the future science and technology needs of the nation, we also reap the rewards of advancing our leadership in science and technology, exploring new ideas, building new capabilities, and pursuing emerging research opportunities. LDRD has enabled virtually every major Argonne initiative, including: the original Advanced Photon Source and its upgrade; the Argonne Leadership Computing Facility; the Joint Center for Energy Storage Research; four Energy Frontier Research Centers; advanced nuclear fuel cycle and reactor modeling/simulation programs; and computational combustion and materials scale-up.

I attest that in managing the LDRD program, Argonne has adhered without exception to the requirements of DOE Order $413.2 \mathrm{~b}$ and associated guidelines. Our program management operates with a strategy of continuous quality improvement-in addition to meeting all reporting requirements, throughout FY 2015 the LDRD Office continued to implement process enhancements that better serve all LDRD stakeholders, from our researchers and managers to our DOE Site Office colleagues.

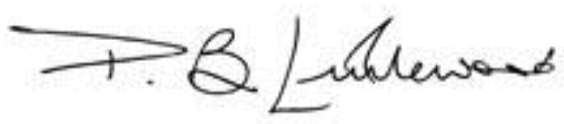

Peter B. Littlewood

Laboratory Director 


\section{LABORATORY OVERVIEW}

Argonne National Laboratory, as a U.S. Department of Energy (DOE) national laboratory, is dedicated to advancing the energy frontier through science and technology. Argonne works to accelerate innovation in science and technology for its primary sponsor, the DOE Office of Science (SC), as well as for DOE's Office of Energy Efficiency and Renewable Energy (EERE), for its National Nuclear Security Administration (NNSA), and for a variety of other federal agencies.

As a national laboratory, Argonne concentrates on scientific and technological challenges that can be addressed only through a sustained, interdisciplinary focus at a national scale. Argonne's seven major initiatives, as enumerated in its laboratory plan, are Biological and Environmental Systems, Energy Storage, Hard X-ray Sciences, Leadership Computing and Computational Science, Materials for Energy, Nuclear Energy and Security, and Sustainable Transportation.

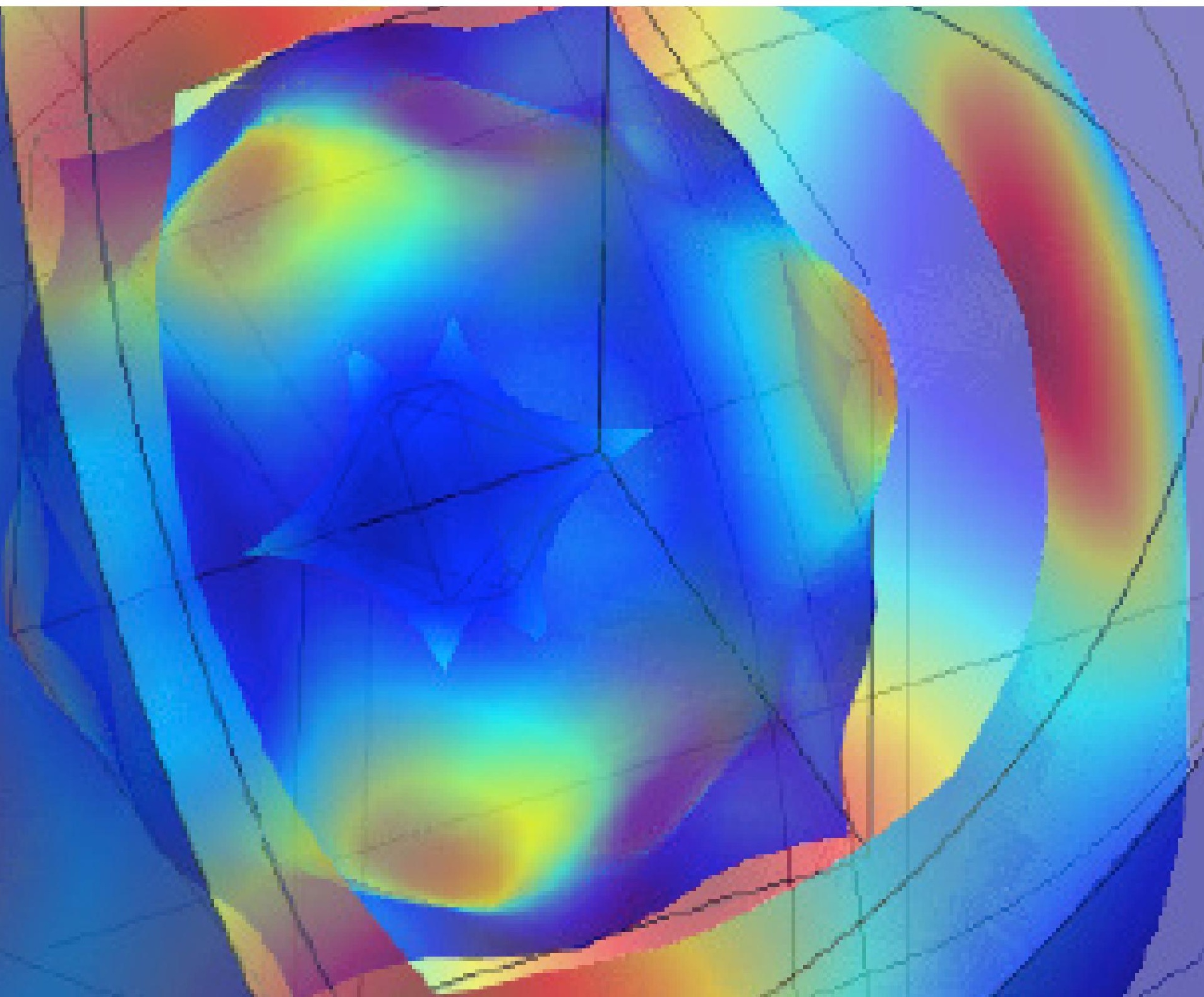




\section{TABLE OF CONTENTS}

PROGRAM OVERVIEW vii

FISCAL YEAR 2015 LDRD PROGRAM COMPONENTS ix

Strategic Initiatives ix

Director's Grand Challenge ix

Director's Competitive Grants (DCGs) x

RESEARCH REPORTS BY COMPONENT Xi

Director's Competitive Grants $\quad 1$

Director's Grand Challenge $\quad 45$

Advanced Computing 51

Applied Energy and Sustainable Transportation 75

Biology and Environment 92

Hard X-ray Sciences $\quad 121$

Materials for Energy 145

National Security 180

Nuclear Energy and Security 189

Other Novel R\&D 200

PROGRAM ASSESSMENT 208

Performance Metrics 209

LDRD Project Characteristics 211

FY 2016 LDRD Program Update $\quad 212$

LDRD Projects Begun in FY 2016

APPENDICES 214

Intellectual Property Events Occuring in FY 2015

Publications and Presentations I 223

Publications and Presentations II 280

Author Index $\quad 292$ 


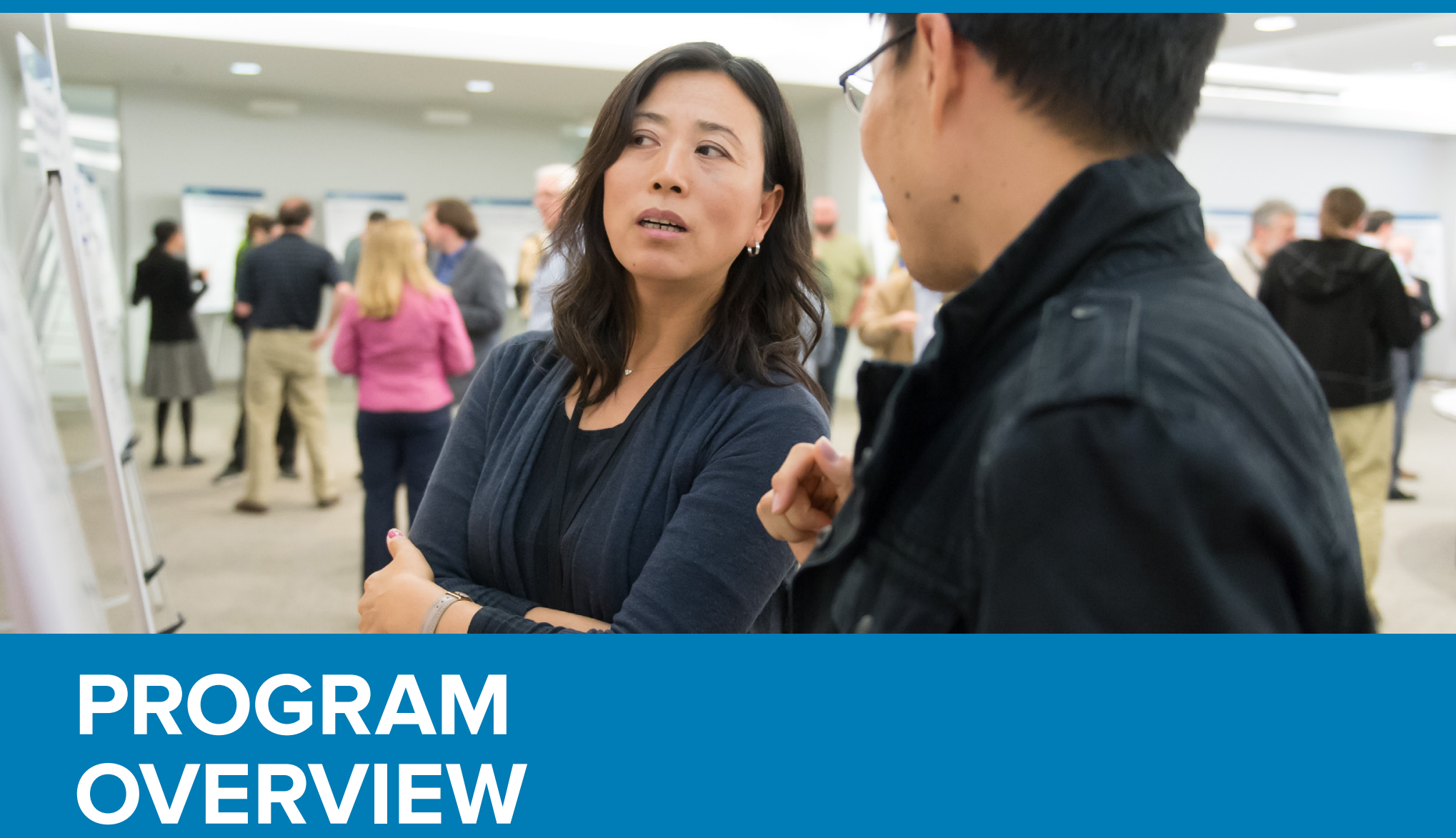




\section{PROGRAM OVERVIEW}

Argonne's Laboratory-Directed Research and Development (LDRD) program strives to encourage the development of novel technical concepts, to enhance the Laboratory's research and development (R\&D) capabilities, and to enable pursuit of strategic laboratory goals.

Argonne's LDRD projects are proposal based and peer reviewed, supporting ideas that require advanced exploration so they can be sufficiently developed to pursue support through normal programmatic channels. Among the aims of the projects supported by the LDRD program are the establishment of engineering proofs of principle, assessment of design feasibility for prospective facilities, development of instrumentation or computational methods or systems, and discoveries in fundamental science and exploratory development.

The projects supported by the LDRD program are distributed across the major mission areas at Argonne, as indicated in the Laboratory's LDRD Plan for fiscal year (FY) 2015. All LDRD projects have demonstrable ties to one or more of the science, energy, environment, and national security missions of the U.S. Department of Energy (DOE), and many are also relevant to the missions of other federal agencies that sponsor work at Argonne. A natural consequence of the more "applied" type projects is their concurrent relevance to industry.
In addition to their relevance to strategic and programmatic activities, LDRD projects naturally fall under one or more areas of science and technology corresponding to the disciplines and fields in which Argonne's technical staff are trained and excel in supporting existing programs. The project reports in this document demonstrate that more often than not, expertise is drawn from several technical areas to pursue the goals of any given project. This multidisciplinary approach is a hallmark of Argonne as a multi-program national laboratory and reflects the synergistic manner in which both LDRD and programmatic work are pursued.

The FY 2015 DOE-approved funding cap for LDRD was $\$ 32.0$ million, or about 4.1\% of Argonne's estimated FY 2015 operating plus capital budgets. Actual expenditures amounted to $\$ 31.0$ million, which includes an overhead levy of approximately $33 \%$.

Individual brief project reports for FY 2015 make up the bulk of this document. 


\section{LDRD MANAGEMENT PROCESS}

Argonne's R\&D activities are organized under four associate laboratory directors (ALDs), who oversee the research conducted in some 16 programmatic divisions. Responsibility for management and oversight lies with the Laboratory Director, who has delegated the management task to the Deputy Laboratory Director for Operations and the Director for Strategy and Innovation. This senior management group makes final recommendations concerning the LDRD program investment and management.

Argonne's LDRD program consists of four components: the Strategic Initiatives, the Director's Grand Challenge, the Director's Competitive Grants (DCGs), and the Director's Strategic Reserve (a portion of the total allowed expenditure temporarily reserved to handle mid-year opportunities, strategic hires for LDRD projects, and LDRD collaborative arrangements). The Strategic Initiatives, Director's Grand Challenge, and Director's Strategic Reserve components fund R\&D projects that are aligned with initiatives within Argonne's Strategic Plan, while the DCG component supports exploratory research aligned with DOE mission areas and core competencies of the Laboratory. Annually, the Laboratory Director determines the appropriate balance of funding among the four components. All final decisions concerning Argonne's LDRD program reside with the Laboratory Director. Certain responsibilities regarding funding, oversight, proposal evaluation, and project direction are delegated.

The LDRD Program Office reviews all proposals to ensure their adherence to DOE Order 413.2b and associated guidelines, as well as to Laboratory administrative procedures.

The LDRD program is funded lab-wide through the Laboratory's indirect budget, which derives from a levy against all program operating and equipment budgets. Although two- or three-year durations are proposed for many projects, funding levels and project selection are determined annually and based on technical progress and the Laboratory's strategic goals and resources.
LDRD funds may be used in collaborative arrangements with third parties to enhance the application or scope of the research, with attendant beneficial interactions. To effect these arrangements, the Laboratory has in place DOE-approved procedures and instruments, such as Cooperative Research and Development Agreements (CRADAs), subcontracts, and site access Guest Agreements. These instruments and procedures enable the application of LDRD funds while ensuring that appropriate terms and conditions are applied thereto, including those related to intellectual property protection.

Line managers in Argonne's programmatic divisions are responsible for monitoring the progress and performance of LDRD research during execution. Strategic Initiative contacts, line managers, and subject matter experts confirm that mid-year progress reviews have occurred and indicate whether any remedial action or advice is needed. The responsibility for the actual conduct of all LDRD projects, including associated environmental safety and health requirements, resides with the ALDs and their line managers.

During FY 2015, all program management requirements were satisfied. Reports required by DOE, including the Annual Report for FY 2014, the LDRD Program Plan for FY 2016, and project data uploaded to the DOE/CFO (Office of the Chief Financial Officer) database, were submitted completely and in a timely fashion.

Concurrence request forms (also referred to as "data sheets" in relevant guidance documents) were submitted electronically for each FY 2015 LDRD project to DOE's Argonne Site Office (ASO) to obtain the DOE Site Manager's concurrence before work on the project proceeded. 


\section{FISCAL YEAR 2015 LDRD PROGRAM COMPONENTS}

\section{STRATEGIC INITIATIVES}

This larger component of LDRD emphasizes R\&D explicitly aligned with Laboratory and Department missions as reflected in strategic initiatives proposed by senior management and consistent with Argonne's Strategic Plan. Strategic goals are revised periodically and reevaluated as necessary. The choice of initiative areas reflects an evaluation of the state of development of the relevant technical fields, the potential value to the Department and the nation of advancing those fields, and, of course, the compatibility and synergy that these fields have with existing facilities, capabilities, and staff expertise at Argonne.

In fiscal year (FY) 2015, leadership of the strategic initiative areas and of the Laboratory's major initiative areas as delineated in Argonne's Strategic Plan were focused under respective Associate Laboratory Director-led teams. Under this arrangement, there were six strategic initiative areas in which proposals could be submitted. The FY 2015 review procedures continued to ensure that all individual proposals identified as fitting under a strategic initiative were reviewed for their scientific quality, innovativeness, and value to each respective mission-related initiative.

The review of strategic initiative proposals relies on a combination of peer and management reviews within each initiative area. ALDs appoint Strategic Initiative points of contact for each respective Strategic Initiative. The points of contact select internal and external subject matter experts (SMEs) to serve on review panels that assist in reviewing proposals. Results are reported to the Laboratory Director, who makes final selections and approves the content and aggregate budgets.

The six FY 2015 Strategic Initiative areas are as follows:

\section{ADVANCED COMPUTING}

The key elements of this initiative fall under the following general areas: next-generation architectures, programming models and software stacks; data-intensive computing infrastructure and systems; computational science application code development; and novel computing applications and over-the-horizon technologies.

\begin{abstract}
APPLIED ENERGY AND SUSTAINABLE TRANSPORTATION The key elements of this initiative fall under the following areas: sustainable transportation, advanced manufacturing, and renewable energy.
\end{abstract}

\section{BIOLOGY AND ENVIRONMENT}

The key elements of this initiative fall under the following general areas: miniaturizing and automating high-throughput laboratory and field sampling and monitoring processes; studying microbial ecosystems and community processes; and accelerating the understanding of protein/DNA sequence-structurefunction relationships.

\section{HARD X-RAY SCIENCES}

Three key focus areas for projects under this initiative area are research and development in support of the Advanced Photon Source upgrade project, science enablers, and future hard $\mathrm{x}$-ray sources.

\section{MATERIALS FOR ENERGY}

Three research focus themes were selected for this initiative, viz., molecular materials design and discovery (M2D2), computational chemistry and materials (CCM), and the integrated imaging initiative (I3).

\section{NUCLEAR ENERGY AND SECURITY}

The key elements of this initiative fall under the following areas: nuclear energy science, engineering and technology demonstration, nuclear energy modeling and simulation, and geospatial analytics for national security.

\section{DIRECTOR'S GRAND CHALLENGE}

The Director's Grand Challenge component of the LDRD portfolio may be included in the LDRD program of any given fiscal year by the Laboratory Director. Also at the discretion of the Laboratory Director is the schedule for the call for proposals and for the review and selection of projects for this component, which does not need to match the schedules for the Strategic Initiatives or Director's Competitive Grants components. For a Grand Challenge project, sufficient talent and resources are devoted to a specific technical topic within an area that is of substantial strategic value to the Laboratory and to DOE, in order to allow major strides to be made toward achieving its R\&D goals. The emphasis is on cross-cutting initiatives that synergistically draw on Argonne's core competencies. 
As a precursor to formal LDRD proposal submissions, brief "white papers" are solicited and subsequently evaluated by a Grand Challenge Review and Oversight Panel composed of four to ten internal and external SMEs. The panel members recommend which white paper authors should be invited to submit more extensive formal proposals. The latter are reviewed on the basis of their expected impact, creativity, degree of cross-cutting utilization of Argonne core capabilities and staff, and potential for success. The awarded project is reviewed by the Grand Challenge Review and Oversight Panel semiannually to evaluate progress.

In FY 2015, there were no new Director's Grand Challenge topics funded. However, the data-driven science-related project from FY 2013 was continued. The project was awarded \$1.6M in FY 2015. The project will conclude in the first quarter of FY 2016, with funding expected to be $\$ 500 \mathrm{~K}$.

\section{DIRECTOR'S COMPETITIVE GRANTS (DCGS)}

The DCG component invests in a full spectrum of investigator-initiated proposals across the Laboratory in DOE-mission-related science and engineering areas. This component provides an avenue for R\&D staff to propose highly innovative projects directly to the Laboratory Director. Proposals are subjected to peer review by a Director's Review Committee to assess their scientific and technical quality. The Committee is composed of nonmanagerial scientists and engineers selected by the Laboratory Director. The Committee currently comprises 23 members who have substantial expertise in their technical fields, are familiar with the peer review process, and represent a broad cross section of the Laboratory. The Director's Review Committee conducts an initial peer review that results in a smaller number of proposals being retained for further review by the Committee. The products of the review procedure are a rank-ordered list of proposals given to the Laboratory Director and brief critiques of proposals sent to the proposing investigators.

The DCG review process is augmented by the recruitment of members of the Laboratory's research community who serve as volunteer SMEs to help the Director's Review Committee review proposals. Approximately 275 researchers volunteered, and their assistance made it possible for the Director's Review Committee to recommend the highest-quality proposals for funding in FY 2015. 


\title{
RESEARCH REPORTS BY COMPONENT
}

\author{
Director's Competitive Grants \\ 2013-013-R2 Improving the Stability of Rubisco Activase, the Weak-Link in the Biological $\mathrm{CO}_{2}$

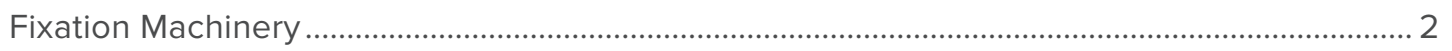

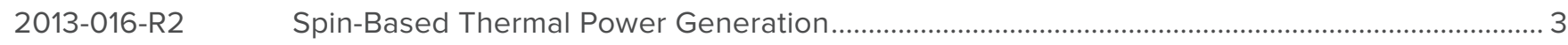 \\ 2013-022-R2 Superlubricious Carbon Films Derived from Natural Gas for Home-Refueling Applications.... 4

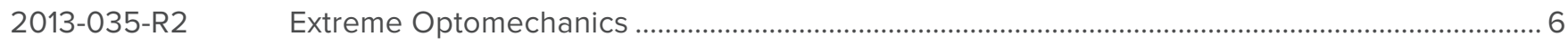 \\ 2013-036-R2 Development of Near-Field Enhanced Terahertz Pump X-ray Probe Techniques \\ for Ultrafast Control of Strongly Correlated Materials ......................................................................... \\ 2013-063-R2 Visualization of Stress-Induced Polarization Switching in Electromechanically Coupled

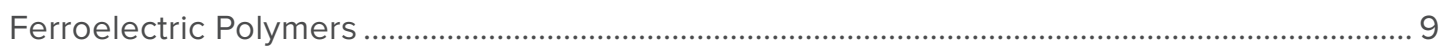 \\ 2013-070-R2 Imaging Ecological Engineers: A Novel Quantum Dots Approach to Map Microbes \\ in Complex Soil Structures with X-rays..................................................................................... \\ 2013-080-R2 Ultra-Low-Loss Superconducting Microstrip for Multi-Chroic Cosmic Microwave

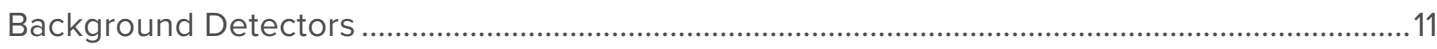 \\ 2013-100-R2 3-D Compositional Control of Intermediate-Band Solar Cells .....................................................13 \\ 2013-116-R2 Nanolaminate Coatings for Improved Nuclear Fuel Cladding Performance ..............................15 \\ 2014-004-R1 Magnetic Separation of Rare Earth Elements ……….......................................................................... 17

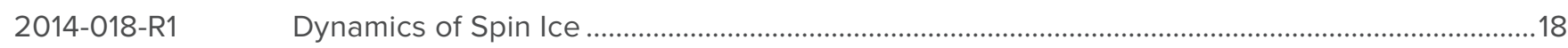 \\ 2014-019-R1 Exploring the Universe with Full-Sky Simulations of the Cosmic Microwave Background .....20 \\ 2014-023-R1 Development of Large-Area Vacuum Ultraviolet Microchannel Plate Photodetectors \\ for Use in Large Liquid Argon and Xenon Time Projection Chambers..........................................21 \\ 2014-025-R1 New Paradigms for High $T_{\mathrm{c}}$ Superconductivity in Acene-Based Materials .................................22 \\ 2014-046-R1 Plastic Artificial Leaves for Water Splitting ...................................................................................23 \\ 2014-051-R1 Carbon Nano-Network as Next-Generation Support for Catalysis and Electrocatalysis ..........25

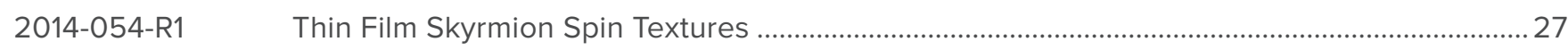 \\ 2014-077-R1 Directly Probing Nanoscale Dynamics in Shear-Thickening Complex Fluids ............................28 \\ 2014-081-R1 Pb-Assisted Corrosion/Cracking Mechanisms at the Interface between Pb-Containing

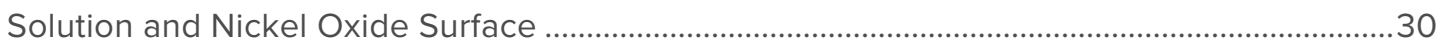 \\ 2014-084-R1 Probing the Chemistry of Atmospheric Dust Particles Using X-ray Spectromicroscopy:

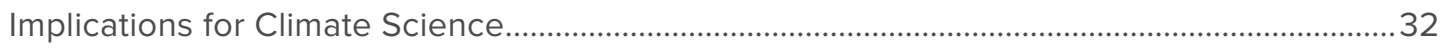 \\ 2014-095-R1 Tuning the Transport Properties of Coupled Majorana …………….............................................34
}

\footnotetext{
* Some projects topically associated with a strategic initiative area, as well as other innovative projects, were reviewed and selected by the Laboratory Director outside the normal annual LDRD call for proposals and review cycle.
} 
2014-108-R1 Single-Cell Structural Genomics of Uncultured Sediment Archaea: On the Trail

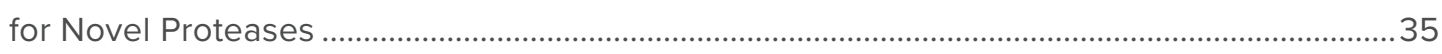

2015-015-NO Detection of Dark Matter Directionality by Means of Columnar Recombination........................ 37

2015-078-NO Josephson Plasma Wave-Based Ultra-High-Frequency Electronics ............................................38

2015-091-NO Next-Generation Natural Gas Adsorbent through Rational Design and Modeling ...................40

2015-096-NO Understanding Atomic-Scale Uranium Interactions under Severe Accident Conditions ..........41

2015-121-NO Development of Advanced $\mathrm{VO}_{2}$ Nano-Composite Thermochromic Materials

for High-Performance Smart Windows .......................................................................................... 43

Director's Grand Challenge

2013-165-R2 Integrating Simulation and Observation: Discovery Engines for Big Data. 46

Advanced Computing

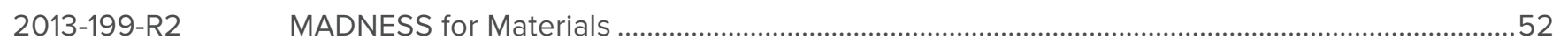

2013-206-R2 Mathematical Techniques to Model Urban Data ……………........................................................53

2013-208-R2 General-Purpose Technical Cloud Platforms ................................................................................54

2013-212-R2 Multiscale Materials Modeling Using Accurate ab-initio Approaches $\left(\mathrm{M}^{3} \mathrm{~A}^{3}\right)$..........................56

2013-213-R2 X-PECT: Performance Framework to Characterize and Transform Applications and Architectures at Extreme Scales...........................................................................................58

2014-160-R1 Developing an Integrated Sensor Network for Science..............................................................59

2014-163-R1 Emerging Compute and Data Infrastructure ................................................................................60

2014-166-R1 Scalable Stochastic Algorithms for Exascale Computational Mesoscience ................................62

2014-167-R1 Improving and Validating Models of the Urban-Climate Connection with Dense

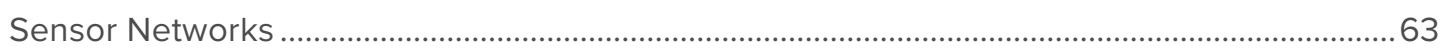

2014-174-R1 Advanced Pipeline for High-Throughput Digitization of Large-Scale Collections ....................64

2014-181-R1* PARIS: Data Knowledge-Based Extreme-Scale Resilience ...........................................................66

2014-182-R1 Dynamic Data Mirroring for Data-Intensive Science .........................................................................67

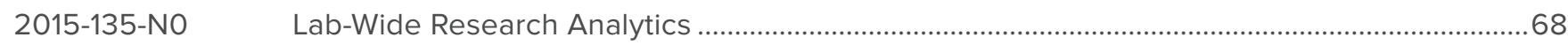

2015-139-NO Implementing a New Extreme-Scale Parallel Programming Model with a Full

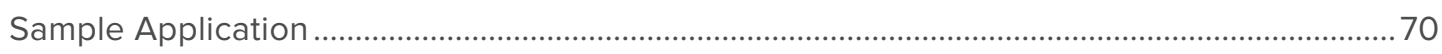

2015-169-NO* Agent-Based Behavioral Modeling of Ebola Spread in Chicago and Other Large

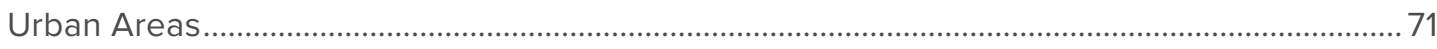

2015-172-NO* Determining Mechanical Properties of Material Systems Using Parameter-Free

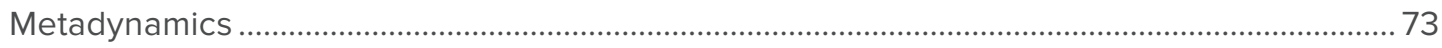

\footnotetext{
* Some projects topically associated with a strategic initiative area, as well as other innovative projects, were reviewed and selected by the Laboratory Director outside the normal annual LDRD call for proposals and review cycle.
} 
Biology and Environment

2012-205-R3* Modeling the Interactions of Biophysical, Biogeochemical, and Microbial Dynamics in Permafrost-Affected Soils: From Pore Scale to Regional Scale ................................................93

2012-206-R3* Developing, Improving, and Testing Methods for Predicting Spatial and Vertical Distributions of Soil Organic Carbon at Regional Scales ..............................................................94

2013-177-R2 Identifying Novel Pathways for Anaerobic Microbial Oxidation of Methane .............................96

2013-194-R2 Annotating, Modeling, and Exploring Enzyme Promiscuity ...........................................................98

2014-132-R1 Identifying Patterns and Associations among Hyperspectral Data and Meteorological and Biological Measurements for Investigating Near-Surface Atmosphere-Biosphere

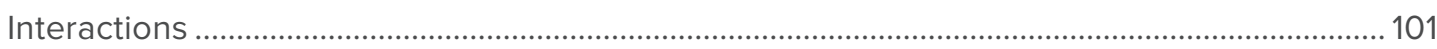

2014-141-R1 Minimizing Environmental Microbial Community Complexity at the Bench: Isolating and Characterizing Minimal Stable Communities (MSCs) Over Time........................................... 102

2014-145-R1 Developing Remote Automated Sensors to Direct Sampling of Aerobic-Anaerobic Switching in Floodplain Ecosystems to Characterize the Response of Microbial Carbon Metabolism at High Temporal Resolution ...................................................................................... 104

2014-157-R1 Biology@Speed: D-Factory, A Novel Experimental Framework .................................................. 105

2014-183-R1 Impact of Radiation and Surface Turbulent Fluxes on the Transition from Stratocumulus to Cumulus Cloud Regime... .107

2014-187-R1* Self-Assembled Functional Membranes for Filtration and Photocatalytic Water Treatment 108

2014-188-R1* Timescale of Groundwater Transport: A Prerequisite for Developing and Maintaining Groundwater Reservoirs.

\footnotetext{
* Some projects topically associated with a strategic initiative area, as well as other innovative projects, were reviewed and selected by the Laboratory Director outside the normal annual LDRD call for proposals and review cycle.
} 


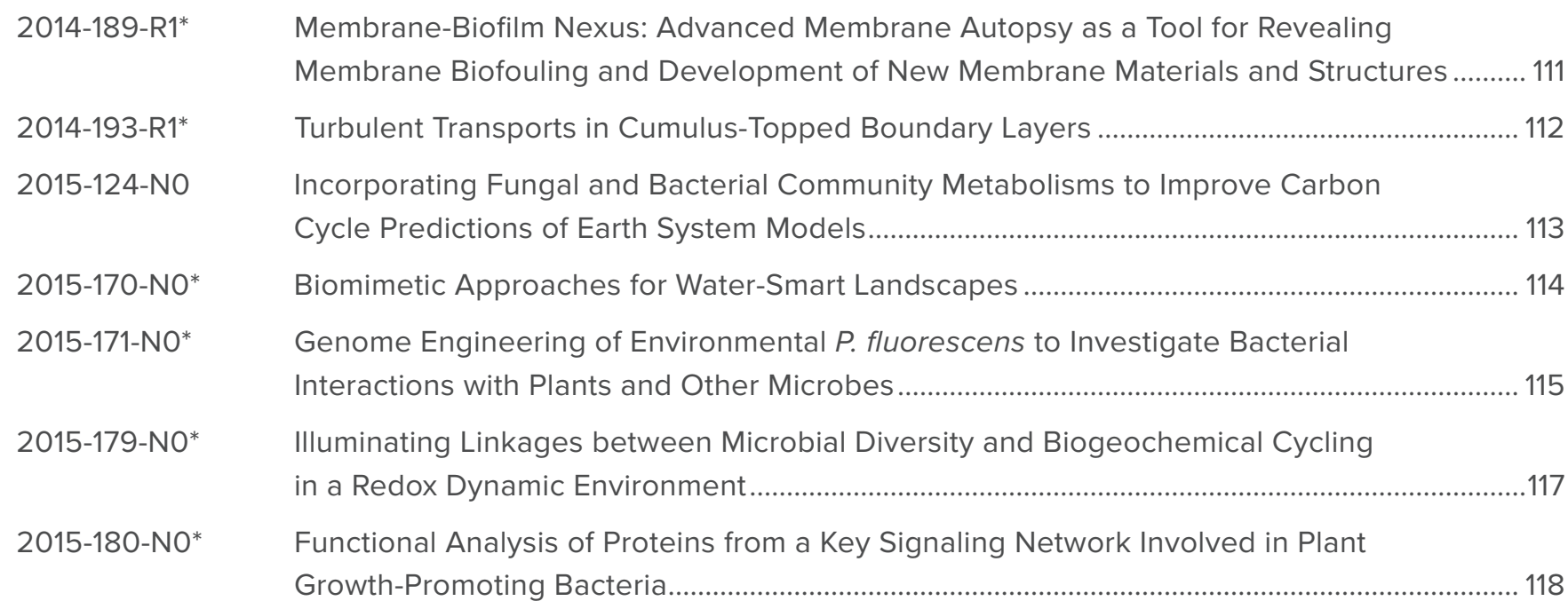

Hard X-ray Sciences

2013-111-R2 Feasibility Study of Applying Thin-Film High-Temperature Superconducting Films

on Copper or Niobium-Sputtered-on-Copper for the Purpose of Achieving Helium-Free

Operation with Cryocoolers ........................................................................................................ 122

2013-168-R2* The Tao of Fusion: Pathways for Big-Data Analysis of Energy Materials at Work ................... 123

2014-127-R1 Development of a Novel Analyzer System for Resonant Inelastic X-ray Scattering

with Better Than 10-meV Resolution ........................................................................................... 124

2014-134-R1 Three-Dimensional Coherent Diffraction Imaging Using Polychromatic Hard X-rays ............. 126

2014-137-R1 $\quad \mathrm{YBa}_{2} \mathrm{Cu}_{3} \mathrm{O}_{7}$ High-Temperature Superconducting Prototype Undulator .....................................127

2014-175-R1 Automation of In Situ Crystallization Plate Screening and Data Collection

at Room Temperature ............................................................................................................... 129

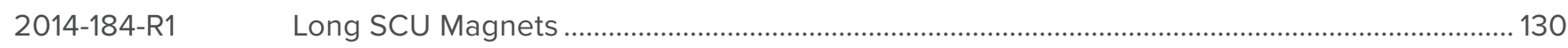

2015-141-NO Using Hard X-rays to Accelerate the Synthesis of Materials....................................................... 131

2015-147-NO Development of a Compact 352-MHz/150-kW CW Solid State RF Power Amplifier

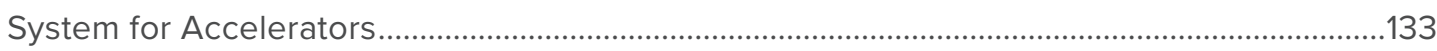

2015-150-NO Unraveling Mesoscale Spatial-Temporal Correlations in Materials Using Coherent

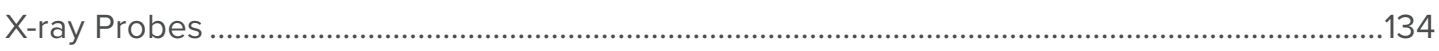

2015-153-NO The VelociProbe: Ultra-High-Resolution Ptychographic Hard X-ray Nanoprobe .................... 136

2015-161-NO Ion Beam Figuring with In-situ Metrology: Diffraction Limited X-ray Optics and Dynamic Aperture for Three-Dimensional Control of Thin-Film Deposition and Ion-Beam Erosion .....137

2015-164-NO Next-Generation Mössbauer Spectroscopy ……………............................................................. 139

2015-173-NO* Isotope Geochemistry via Sn Isotope Fractionation Using Inelastic X-ray Scattering of Synchrotron Radiation .......................................................................................................... 140

2015-182-NO* Developing New Schemes for Nuclear Resonant Scattering Measurements at an Upgraded APS.

\footnotetext{
* Some projects topically associated with a strategic initiative area, as well as other innovative projects, were reviewed and selected by the Laboratory Director outside the normal annual LDRD call for proposals and review cycle.
} 
Materials for Energy

2012-209-R3 Atomistically Informed Mesoscale Modeling for Advanced Electrical Energy

Storage Systems

2013-184-R2

Hierarchical Modeling of Self Assembly in Nanostructured Soft Materials at Equilibrium

and Far from Equilibrium..

2013-202-R2

Computational Molecular Science.

149

2013-216-R2*

Directed Self-Assembly and Three-Dimensional Characterization of Block Copolymers

in Semi-Thick Films 151

2013-219-R2*

Transition Edge Sensors for Fundamental Physics 152

2014-121-R1

Integration of Scalable Microwave Reactor with High-Energy X-ray Beamline for High-Throughput Screening of Energetic Nanomaterial Synthesis.

2014-128-R1

Length-Scale Bridging Computational Scheme for Structure and Transport 156

2014-129-R1

The Design and Synthesis of Novel Oxides: Coupling Materials Informatics with a Next-Generation Deposition System Employing In-situ X-ray Scattering and Photoemission Spectroscopy. 158

2014-133-R1

In-situ X-ray Characterization of Doped Materials Synthesized by Atomic Layer

Deposition for Energy Applications 159

2014-139-R1

Fast Electronic Structure Methods for Rapid Reaction Screening for Inorganic Materials Synthesis and Particle Formation. 160

2014-151-R1 Developing Predictive Models of Wide Bandgap Semiconductor Synthesis and Processing

2014-161-R1

Bridging the Electronic and Atomistic Scales: Force Field Development for Reactive Interfaces from First Principles. 163

2014-191-R1*

Defect-Localized Spins in Semiconductors for Quantum Optoelectronics. 164

2014-192-R1*

Computational Spectroscopy of Heterogeneous Interfaces. 166

2015-144-NO

Framework for Integrating Multi-Modal Imaging of Materials for Energy Storage 168

2015-149-NO Integrated Imaging, Modeling, and Analysis of Ultrafast Energy Transport in Nanomaterials. 169

2015-152-NO

2015-154-NO In-situ Co-Analysis of Atomic and Electronic Structural Evolution for Materials Synthesis ....170 2015-167-NO* Integrated Imaging to Understand and Advance Photocatalysis. .172 Coherent X-ray Studies of Materials Synthesis and Dynamics .174

2015-168-NO*

The Computational Design of New Functional Materials from Complex Transition Metal Oxides.

\footnotetext{
* Some projects topically associated with a strategic initiative area, as well as other innovative projects, were reviewed and selected by the Laboratory Director outside the normal annual LDRD call for proposals and review cycle.
} 
2015-175-NO* Magnetic Phases in Highly Oxidized, Low-Dimensional Oxides 176

2015-178-NO* Towards Ionotronics: First-Principles Strategies for Coupling Electronic and Ionic Properties in Complex Oxides.

2015-185-NO*

Development of a Cryogenic Correlative Confocal Light Microscope ( $\left.C^{3} L M\right)$ for Integrated Imaging.

National Security

2013-171-R2

Unconventional Signatures for Characterizing Culture Conditions

2013-173-R2

Ratiometric Semiconductor Nanocrystal-Based Sensors for Threat

Reduction Applications ..... 183

2013-178-R2

Uranium and Plutonium Detection by Plasmonic Graphene-Based Nanosensors 184

2014-194-R1* Crime on the Urban Edge: Simulating the Interface between Transnational and Local Crime. 186

\section{Nuclear Energy and Security}

2013-152-R2

2014-177-R1

2015-129-NO

2015-136-NO

2015-145-NO
Nanolaminate Materials for Extreme Environments: A Demonstration of Argonne Capabilities for Design, Synthesis, and Accelerated Testing of Radiation-Tolerant Nuclear Engineering Materials 190

Development of a Computational Fluid Dynamics Multiphase Boiling Capability to Predict the Critical Heat Flux in Nuclear Reactor Fuel Assemblies

Economic and Technical Aspects of Nuclear Energy Competitiveness in the Current U.S. Deregulated Electricity Markets 194

Nuclear Materials under Extreme Conditions 196

Understanding Embrittlement in Cast Austenitic Stainless Steels and Stainless

Steel Welds

Other Novel R\&D

2014-185-R1*

Enabling Sodium-Ion Batteries for Grid Storage

201

2015-174-NO*

Conversion of $\mathrm{C}^{2}$ and $\mathrm{C}^{3}$ Paraffins into Liquid-Phase Products 203

2015-177-NO* Integration of Multiple Infrastructure Dependencies and Interdependencies into Infrastructure Hazard Analysis 204

2015-181-NO*

\footnotetext{
* Some projects topically associated with a strategic initiative area, as well as other innovative projects, were reviewed and selected by the Laboratory Director outside the normal annual LDRD call for proposals and review cycle.
} 


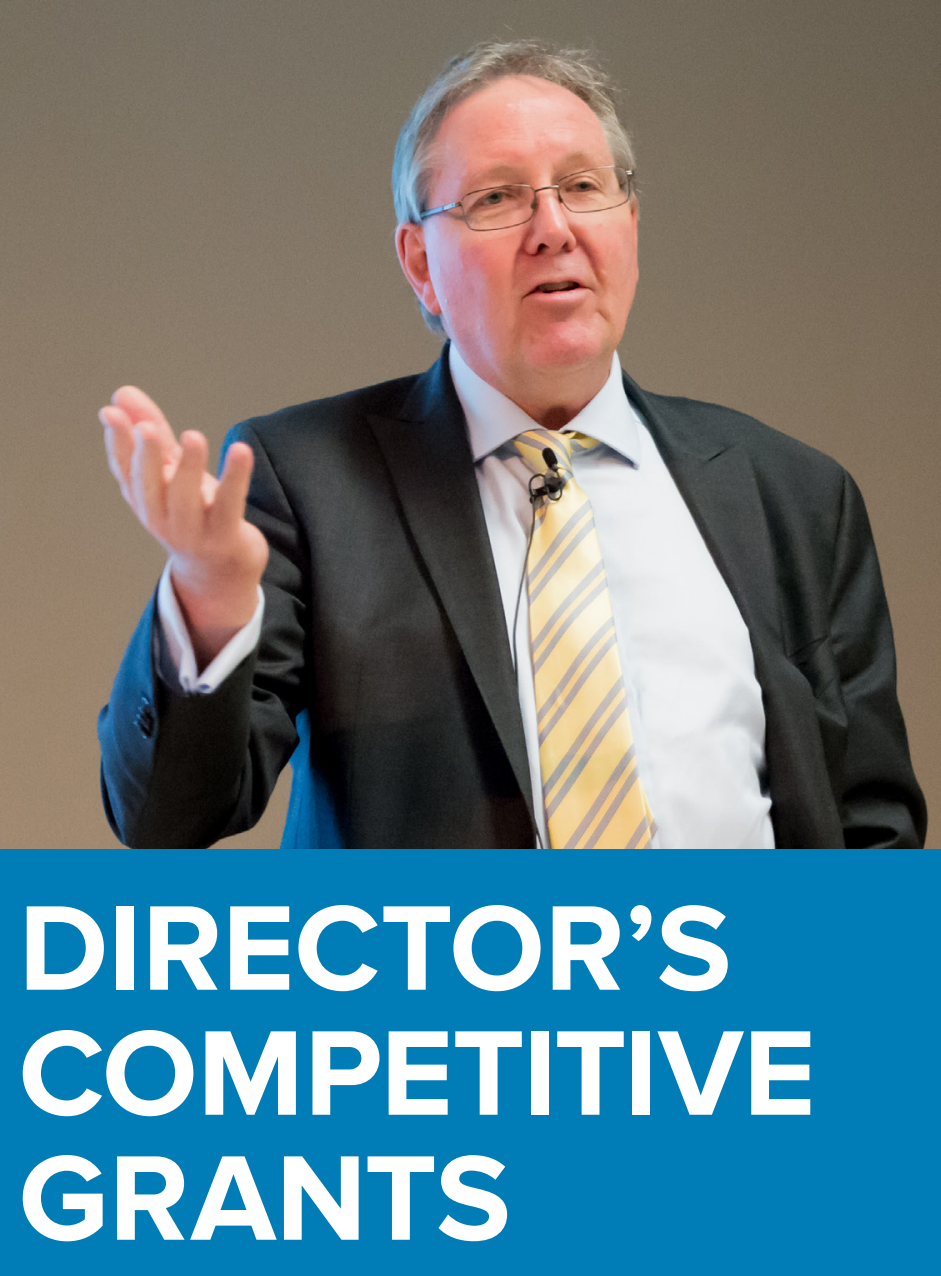




\section{Improving the Stability of Rubisco Activase, the Weak- Link in the Biological $\mathrm{CO}_{2}$ Fixation Machinery}

\section{3-013-R2}

\section{P. Raj Pokkuluri, Marianne Schiffer, and Rosemarie Wilton}

\section{PROJECT DESCRIPTION}

Plants convert atmospheric carbon dioxide $\left(\mathrm{CO}_{2}\right)$ into biomass with the help of sunlight in a process referred to as photosynthesis. However, plant photosynthesis faces reduced efficiency with the expected onset of global warming. One of the main determinants of the reduced efficiency is the temperature sensitivity of a protein called Rubisco activase. Rubisco activase is a helper protein that maintains the enzyme Rubisco in an active state. Rubisco is the key enzyme in the carbon fixation cycle. Rubisco is a heat-stable protein, but many plant Rubisco activases lose their abilities to function at even modestly higher temperatures $\left(\geq 5-10^{\circ} \mathrm{C}\right)$ than their optimums. We stabilized the Rubisco activase by using rational protein engineering principles. We predict that the incorporation of stabilized variants of Rubisco activase in plants (teaming with researchers at the University of Illinois) will lead to increased photosynthetic efficiency and greater yields of biomass.

\section{MISSION RELEVANCE}

Our research aims to find solutions to two major problems: excess atmospheric $\mathrm{CO}_{2}$ and risks to crop security. Engineering plants to better assimilate atmospheric $\mathrm{CO}_{2}$ and to increase the crop security with higher photosynthetic yields can mitigate both problems in one step. The utilization of excess $\mathrm{CO}_{2}$ by plants is relevant to DOE's environmental quality mission, and obtaining higher plant biomass yields is relevant to DOE's energy security mission.

\section{RESULTS AND ACCOMPLISHMENTS}

Cloning and Expression of Rubisco activase. Previously, we cloned, expressed, and purified full-length short and long forms of Rubisco activase from soybeans and Arabidopsis thaliana. In addition, we prepared the $\mathrm{AAA}^{+}$ domains of each activase and focused our stabilization strategy on the $\mathrm{AAA}^{+}$domain from the soybean short form. From a panel of 48 single-point mutations made, 16 were found to be stabilizing with their $T_{m}$ value (temperature midpoint of folded-unfolded transition) being at least $1-5^{\circ} \mathrm{C}$ higher than that of the native $A A A^{+}$domain.
The top variants were tested for their functions using an adenosine triphosphate (ATP) hydrolysis activity assay.

In FY 2015, our research included the following:

Combining stabilizing mutations in $\mathrm{AAA}^{+}$domain. The top single stability mutations found for the soybean short $\mathrm{AAA}^{+}$domain were combined to produce proteins carrying multiple mutations. Five variants carrying multiple mutations were successfully cloned and expressed, and the thermal stabilities of the purified proteins were measured. All the variants carrying combinations of single site mutations had higher stability (i.e., their $\mathrm{T}_{\mathrm{m}}$ values were higher) than the native ones, as well as those of the corresponding single mutants.

ATP (adenosine triphosphate) hydrolysis activity assay. One of the single stability mutants used in producing combination mutants had reduced the ATP hydrolysis activity compared to the native $\mathrm{AAA}^{+}$domain. But most of the combination mutants produced included this mutant. Therefore, four of the five combination mutants-although they had higher stabilities-were less active than the native $\mathrm{AAA}^{+}$domain. We therefore determined not to subject this mutation with reduced activity to further study.

Stabilizing full length soybean short form. The top three of the most stabilizing single-site mutations that were found to be active by the ATP hydrolysis activity assay were cloned into the full length soybean Rubisco activase short form. The single mutants were also combined into one protein to produce a "super-stable" Rubisco activase. The single mutants and the triple mutant protein were expressed and purified by nickel-2+ $\left(\mathrm{Ni}^{2+}\right)$ affinity chromatography followed by size exclusion chromatography. Each of the single mutations increased the $T_{m}$ value of the native protein (in the range of $4-8^{\circ} \mathrm{C}$ ), and the combined triple mutant had a $T_{m}$ value $14^{\circ} \mathrm{C}$ higher than the native protein. The ATP hydrolysis activity assay of all the single mutants and the triple mutant showed that the function of the proteins is not affected by these stabilizing mutations.

Crystallization trials. The purified native and single-site variants of all $\mathrm{AAA}^{+}$domains and the full-length soybean short form, including the native and the three single site variants (as well as the combined "super-stable" variant), were used to survey for crystallization conditions using commercial reagent screens. With one exception that is mentioned below, the crystallization trials did not yield any positive results. The recalcitrant nature of Rubisco activase toward crystallization is expected because it selfassociates to form higher-order complexes in solution. 
Small crystals for one of the $\mathrm{AAA}^{+}$domain single site variants were observed, which were tested for diffraction at the 19BM beamline at the Advanced Photon Source. However, diffraction of $x$-rays from these samples was either too weak to observe or nonexistent.

Rubisco activase from Miscanthus giganteus, a bioenergy crop. The genomic data for the Rubisco activases from Miscanthus giganteus were obtained from our collaborators at the University of Illinois. There are two forms of Rubisco activase, a long and short form, encoded by different genes similar to soybeans. Synthetic DNA was purchased for both long and short forms and was cloned into expression vectors as was done previously for the activases from soybean and Arabidopsis thaliana. The $A A A^{+}$domains of each activase were also cloned. All clones were confirmed by DNA sequencing. These constructs will be useful for further studies.

\section{Spin-Based Thermal Power Generation}

\section{3-016-R2}

\section{Axel Hoffmann}

\section{PROJECT DESCRIPTION}

Thermoelectric devices rely on the Seebeck effect to generate electric voltages from temperature $(T)$ gradients. This effect has been employed not only in temperature sensors but also for power generation, especially as energy scavengers that convert waste heat into useful electric power. To make thermoelectric generators efficient, it is desirable to decrease thermal conductivity while concurrently improving electrical conductivity. In conducting materials, the ratio of thermal conductivity $\kappa$ to electric conductivity due to electron transport $\sigma$ is universally given by the Wiedemann Franz law, $\kappa / \sigma=L T$, where the Lorenz number $\mathrm{L}$ is a constant of about $2 \times 10^{-8}$ watt-ohm/kelvin ${ }^{2}\left(\mathrm{~W} \Omega \mathrm{K}^{-2}\right)$. For this reason, despite decades of research, the efficiencies of thermoelectric power generation at room temperature are still rather low, and the dimensionless figure of merit, $\mathrm{ZT}=(\sigma / \mathrm{\kappa}) \mathrm{S}^{2} \mathrm{~T}$, has increased by a factor of only 3 to 4 (with $S$ being the Seebeck coefficient). $Z$ is the figure of merit describing the efficiency of the power conversion. Larger numbers are better. By multiplying $Z$ by $T$, it becomes dimensionless, which is a convenient way to compare the thermoelectric performances of a variety of materials.

A possible solution to this dilemma is spin-dependent charge and thermal transport. Spin-based effects allow charge conductivity to be decoupled from thermal conductivity; that is, a heat gradient over a ferromagnetic insulator can generate a spin current that is transferred into a metallic conductor, where the spin current is converted into a charge current. Recently, it was shown that heat currents result in concomitant spin currents in both ferromagnetic metals and ferromagnetic insulators. This is now known as the spin Seebeck effect. It is also possible to concurrently convert spin currents into charge currents through the so-called spin Hall effect. It has also been demonstrated that spin currents from ferromagnetic insulators can generate spin currents in adjacent metals through spin pumping. This approach allows thermal conductivity to be reduced and electrical conductivity to be increased, independently from each other.

The goal of this project is to investigate the conversion of heat current to charge current in various combinations of materials and geometries. Besides providing a better fundamental understanding of the underlying physics of spin-caloric effects, this work will allow for the development of thermoelectric devices that are superior to those that currently exist.

\section{MISSION RELEVANCE}

This project is relevant to DOE's energy and science missions, and it will have both fundamental and applied impacts. In terms of basic science, this work will lead to an improved understanding of the coupling between heat, spin, and charge currents. With respect to applications, it will explore the feasibility of the spin-mediated conversion of heat current to charge current for efficient thermoelectric power generation. The project will directly address, at a fundamental level, energy conversion-an issue that directly relates to today's grand challenge of achieving environmentally sustainable energy.

\section{RESULTS AND ACCOMPLISHMENTS}

In the first two years of the project, we established the growth of high-quality extended yttrium iron garnet films. Furthermore, we established that magnetically ordered systems, either due to proximity effects or intrinsic exchange coupling, can have spin Hall effects that are dependent on the magnetic order. In the case of metallic antiferromagnets, the spin-to-charge current conversion can be comparable in magnitude to some of the best non-ordered spin Hall effect materials. Furthermore, we developed a macroscopic experimental setup for characterizing spin Seebeck effects.

During FY 2015, we further optimized the fabrication of high-quality yttrium iron garnet thin films and developed a process that enables the lithographic patterning of sub-micron structures. The patterning was achieved via 
a lift-off process for films grown at room temperature. Subsequently, the patterned yttrium iron garnet films were annealed in oxygen gas to recover the right crystallinity and oxygen stoichiometry. This preparation resulted in structures with sub-micrometer dimensions and magnetic damping comparable to extended films. From the perspective of spin Seebeck effects this outcome is of interest, since it enables the modification of the spin wave spectrum, and thus the magnetic heat current, through geometric confinement.

Building on our previous results, where we discovered large spin Hall effects in polycrystalline metallic antiferromagnets, we expanded this study by exploring epitaxial thin films. This work enabled us to quantify large anisotropies of the spin Hall conductivities, which could vary by a factor of two, depending on the crystalline orientation. Using antiferromagnets for the spin- to charge-current conversion is particularly attractive, since the coupling between antiferromagnets and ferromagnets, also known as exchange bias, enables in a practical application the stabilization of the magnetization in the ferromagnet. In this way, the spin Seebeck device becomes more robust against external magnetic field applications.

Finally, we developed a new spin Seebeck measurement approach by integrating a microstructured heater directly on top of the bilayers used for the spin Seebeck effect. This approach has the distinct advantage that it enables measurements with sufficient temperature gradients while using a significantly lower heater power. This, in turn, enables measurements at conditions (such as high magnetic fields and low temperatures) that were inaccessible with our previous setup. We investigated whether antiferromagnetic insulators coupled to large spin Hall effect materials can also give rise to spin Seebeck effects. Using manganese difluoride/platinum $\left(\mathrm{MnF}_{2} /\right.$ $\mathrm{Pt}$ ) bilayers, we indeed observed spin Hall voltages that could be clearly correlated to the magnetic properties of the antiferromagnet. Interestingly, the ratio of the observed spin Seebeck voltages to the applied heater power was about 25 times larger than that for the comparable bilayers of yttrium iron garnet/Pt. This may suggest that antiferromagnets actually may be better than ferromagnets for electric power generation via the spin Seebeck effect.

\section{Superlubricious Carbon Films Derived from Natural Gas for Home-Refueling Applications}

\author{
2013-022-R2 \\ Ali Erdemir, Osman Eryilmaz, and Giovanni Ramirez
}

\section{PROJECT DESCRIPTION}

The main objective of this project is to develop novel catalytically active nanocomposite coatings that can extract highly durable and lubricious carbon films directly from natural gas and deposit them on sealing and sliding surfaces that are typical of advanced compressor systems.

\section{MISSION RELEVANCE}

This project is relevant to DOE's missions on energy independence and protecting the environment. DOE has an interest in breakthrough technologies that will enable home-refueling of natural gas for vehicular use. Compressing natural gas from home outlets to as much as 5,000 pounds per square inch (psi) is a challenge, but it is essential for achieving a reasonable driving range out of a vehicle that is powered by natural gas. Sealing and sliding surfaces of compressors are always subject to high friction and wear losses. This project focuses on the design and development of catalytically active nanocomposite coatings that can extract superlubricious carbon films from natural gas and deposit them on sealing and sliding surfaces of compressor systems. If proven effective, these coatings can eliminate one of the major roadblocks in the development of next generation compressor systems in which natural gas can also act as a lubricating agent.

\section{RESULTS AND ACCOMPLISHMENTS}

During FY 2013, major effort was directed toward the synthesis of catalytically active nickel (Ni)-containing molybdenum nitride (MoN-Ni), copper (Cu)-containing vanadium nitride ( $\mathrm{VN}-\mathrm{Cu})$, niobium nitride ( $\mathrm{NbN}-\mathrm{Cu}$ ), and tantalum nitride ( $\mathrm{TaN}-\mathrm{Cu}$ ) coatings and testing them in methane gas. When uncoated $440 \mathrm{C}$ (a martensitic stainless steel) test pairs were tested in methane, the specific wear volumes were as much as $2 \times 10^{-11}$ cubic meters $\left(\mathrm{m}^{3}\right)$. When the same tests were repeated with a $\mathrm{VN}-\mathrm{Cu}$ coated $440 \mathrm{C}$, the wear volumes were reduced to $5 \times 10^{-15} \mathrm{~m}^{3}$. Hence, the catalytically active nanocomposite coating resulted in a more than three orders of magnitude reduction in wear. These findings were consistent with the original concept. 
During FY 2014, we completed the design, synthesis, and optimization studies for all of the nanocomposite coatings. Specifically, we further refined the structure and chemical composition of these coatings to provide a truly equiaxed and nanocrystalline morphology with catalytic metals dispersed evenly throughout the coating thickness. We found that the tribological performance of $\mathrm{Ni}$-containing VN was the best as it provided some of the lowest friction and wear coefficients. Ni-containing MoN coatings also worked well. Attempts were made to alloy these coatings with cobalt (Co) as well, but the resulting coatings were mechanically very soft and bonded poorly to the underlying steel substrates. Hence, they could not resist wear or last for very long before being removed from the rubbing surfaces.

In FY 2015, capitalizing on our past experiences, we focused our attention on combining $\mathrm{Cu}$ and $\mathrm{Ni}$ as alloying elements in the MoN and/or VN hard phases. We felt that this novel composition would provide much higher catalytic activity and hence, superior friction and wear performance in the end. These novel cupronickel alloyed VN coatings have indeed worked extremely well as they were able to reduce wear by 3 to 4 orders of magnitude and at the same time reduce friction by as much as $40 \%$, as shown in Figure 1. Compared to the earlier coatings, these cupronickel-alloyed VN coatings were much harder (i.e., microhardness of $\sim 22$ gigapascals [GPa]) and hence, their superior wear performance may have been due, in part, to the higher hardness. As for the lower friction, we determined that these coatings were able to produce a thicker and more protective blackish tribolayer on rubbing surfaces. As shown in Figure 2, Raman spectroscopy revealed the highly disordered graphitic nature of this layer. It was acting as a solid lubricant that lowered friction. The resulting high friction and high wear of the uncoated steel test samples were due to severe adhesive wear since there was no such tribofilm formation on their sliding surfaces.

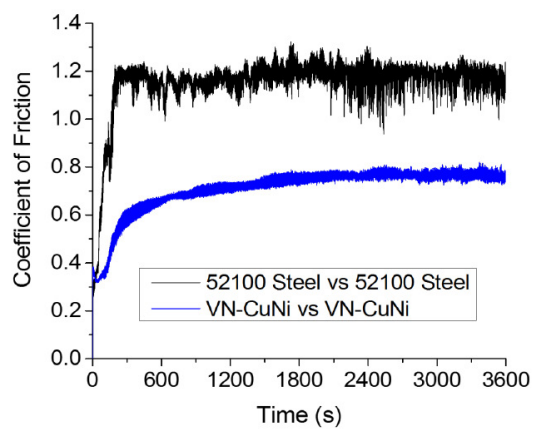

(a)
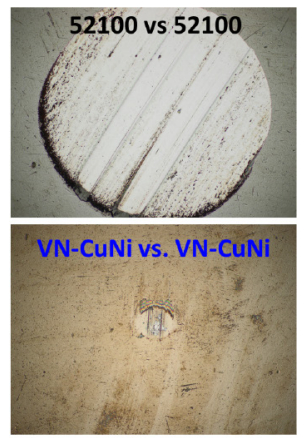

(b)
Figure 1. Friction (a) and wear (b) test results comparing the performance of uncoated steel against that of the $\mathrm{VN}$-CuNi-coated steel in methane.
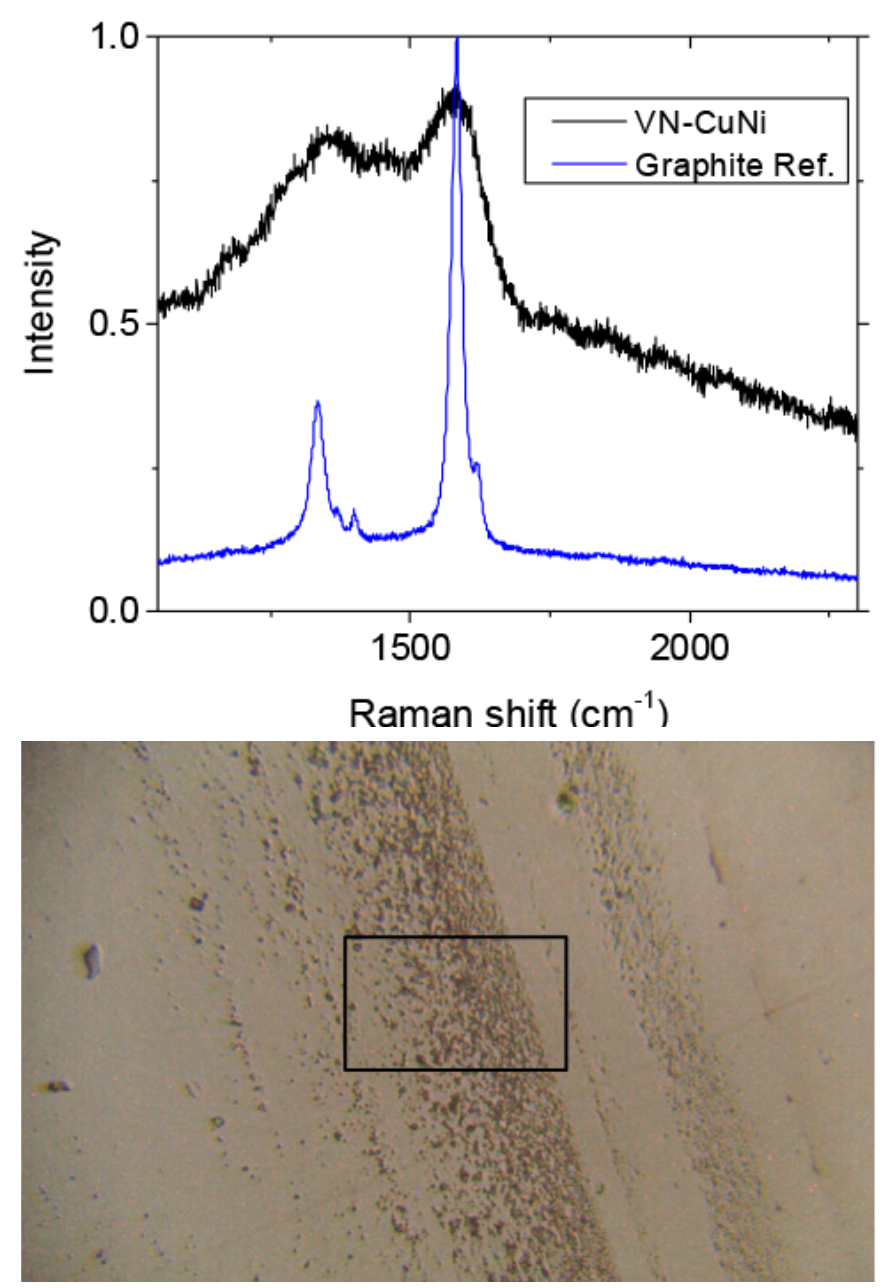

Figure 2. Raman spectrum of blackish tribofilm (top) in and around the rubbing surfaces of the VN-CuNi-coated test sample (bottom) after the tribological test.

Some of the optimized coatings were tested against sealing materials that are used in real compressors. The results were very positive as these coatings were able to afford very low wear to these sealing materials and provide friction coefficients of 0.05 to 0.2 . Furthermore, for actual sealing applications that may involve high temperatures (e.g., above $300^{\circ} \mathrm{C}$ ), we conducted a few additional tests with the highly optimized cupronickel-VN coatings. The friction and wear performance of these coatings was very impressive as they provided friction coefficients down to 0.1 at $400^{\circ} \mathrm{C}$ and the amount of wear was too small to quantify. Uncoated test pairs suffered severe wear damage and their friction fluctuated between 0.2 and 0.8 under the same test conditions. Overall, this project successfully met all of the original milestones by demonstrating very impressive friction and wear performance for catalytically active composite coatings in methane environments under a very wide range of test conditions that are very relevant to actual compressor applications. 


\section{Extreme Optomechanics}

\section{3-035-R2}

\section{Jeffrey R. Guest, Xiao-Min Lin, and Matthew Pelton}

\section{PROJECT DESCRIPTION}

This project involves the development of a new method for the real-time measurement of mechanical vibrations in nanometer-scale oscillators at frequencies above 10 gigahertz ( $\mathrm{GHz}$ ). The ability to precisely measure the frequency at which mechanical oscillators vibrate underlies a wide range of technologies. Present methods for rapidly and accurately measuring mechanical vibrations cover a vast range of length and frequency scales. On length scales from the macroscopic down to the microscopic, mechanical motion can be read out electrically, by directly connecting the oscillator to electrical leads, or by coupling it capacitively or magnetically to a readout circuit. On atomic- and molecular-length scales, optical spectroscopies, particularly Raman and Brillouin scattering, can be used to measure vibration frequencies. In between these two limits-for dimensions on the nanometer scale and frequencies in the 10 s of $\mathrm{GHz}$ range-none of these methods is applicable. The scientific opportunity addressed in this project is to fill this technological gap by developing a new method to measure vibrations in this mesoscopic regime.

Our new method will have important technological implications, particularly for mass sensing. Since the frequency of a mechanical oscillator is directly related to its mass, measuring this frequency can provide an accurate means of weighing material that is adsorbed onto the oscillator. The smaller the oscillator, the larger the fractional change in oscillation frequency a given mass will produce and, thus, the more sensitive the measurement. The demonstration of a method to monitor $\mathrm{GHz}$ vibration frequencies in nanostructures would then open up a new frontier in ultrasensitive mass measurement. We will be able to measure vibrations in nanoparticles that were synthesized using bottom-up chemical synthesis techniques, rather than by using the costly and complex top-down clean-room-based nanofabrication techniques that have been used so far. This, in turn, will facilitate the ability to functionalize the nanoparticles for binding to target molecules and will allow the sensors to operate in a liquid environment.

\section{MISSION RELEVANCE}

This project supports the DOE mission in use-inspired fundamental science. The investigations will yield new scientific insights into mechanical vibrations at the nanometer scale and will set the stage for future applications in ultrasensitive mass detection, enabling new nanoscale platforms for in-situ detection of targeted molecular species in fluids.

\section{RESULTS AND ACCOMPLISHMENTS}

The ultimate success of this project hinges on being able to develop the ability to monitor the vibrations of individual nanoparticles in real time and in solution at ultrahigh frequencies ( $20 \mathrm{GHz})$. In FY 2013, we designed the experimental setup, procured the necessary equipment, and began assembling it. In FY 2014, we optimized the experimental setup-including the laser, optics, and radio-frequency (RF) electronicsto achieve the ability to monitor the vibrations. The ultimate sensitivity of our approach will depend on how well we minimize noise in the detection of extremely small changes in transmission of light near the plasmon resonance at ultrahigh frequencies. We have successfully developed this monitoring capability and tested its efficacy, demonstrating a sensitivity to changes in transmission approaching $\Delta T / T \sim 4 \times 10^{-7}$. This allows us to detect changes in transmissions at ultrahigh frequencies to better than one part per million. In FY 2014, we also made progress in the synthesis and functionalization of tailored bipyramidal gold nanoparticles for these measurements, realizing different sizes of gold bipyramids with tunable plasmon wavelengths from the visible to the near-infrared, while improving their homogeneity. These different sizes of bipyramids will be ideal candidates for testing our optical setup, since different sizes of particles will have different mechanical frequency responses.

In FY 2015, we continued to improve the sensitivity of the experimental setup (achieving another factor of two improvement) and focused our attention on developing a deeper understanding of the plasmonic response to the vibrating nanoparticle with a larger goal of optimizing the nanoparticle design for these types of applications. Monitoring the minute vibrations in these nanoparticles remotely is possible because of the extreme sensitivity of the plasmonic optical response to the shape of the nanoparticle. This has been demonstrated experimentally many times, but to date, there has been no successful attempt to understand this connection theoretically. Performing both mechanical and optical modeling and linking these calculations, we were able to explain these experimental observations for the first time, achieving a deeper understanding of this nanoparticle probing technique that will lead to improved sensing capabilities. In addition, we have experimentally and numerically explored the responses of hybrid core-shell nanoparticles. These more complex nanostructures surprisingly reveal 
unexpected and multiple vibrational frequencies (see Figure 1) which may indicate a new regime in ultrahigh frequency nanomechanics.

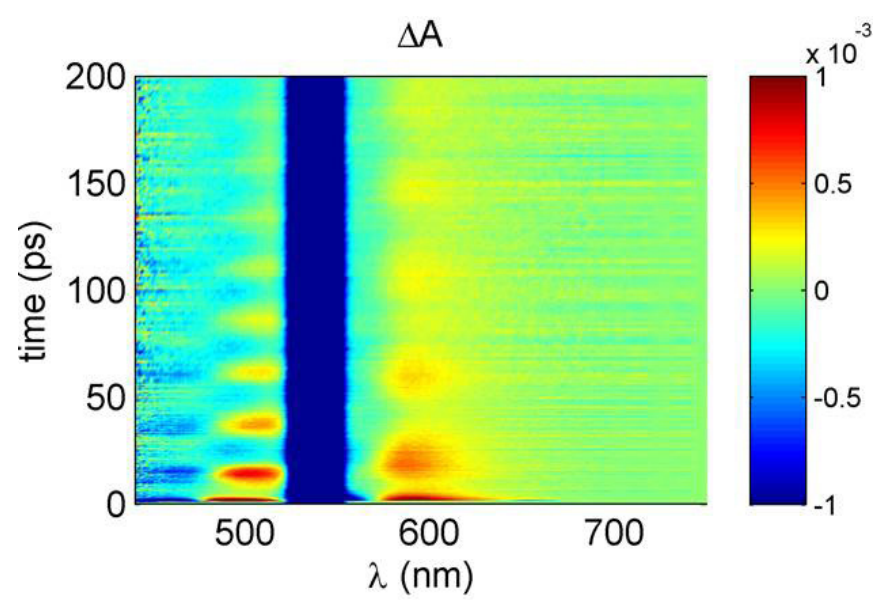

Figure 1. Transient absorption data showing the temporal dynamics of the Au-Ag core-shell nanoparticle plasmonic response. A pulse arriving at time zero rapidly heats the particle and initiates ultrafast vibrations, which we can monitor through changes in the plasmonic response. We show the fractional change $\Delta \mathbf{A}$ in transmission $T$ of a broadband probe pulse arriving at a later time $t$ as indicated by the color scale, which is a function of both the delay time $t$ and the wavelength $\lambda: \Delta \mathbf{A}(\lambda, t)=\mathbf{T}(\lambda, t) / \mathbf{T}_{0}(\lambda)$. The oscillations in time visible at $\lambda=500 \mathrm{~nm}$ have a period of about $25 \mathrm{ps}$, corresponding to a frequency of $40 \mathrm{GHz}$; similarly, at $\lambda=600 \mathrm{~nm}$, oscillations with a period of 42 ps (corresponding to $24 \mathrm{GHz}$ ) are observed.

\section{Development of Near-Field Enhanced Terahertz Pump X-ray Probe Techniques for Ultrafast Control of Strongly Correlated Materials}

\section{3-036-R2 \\ Haidan Wen, Zhonghou Cai, Matthew J. Highland, and II Woong Jung}

\section{PROJECT DESCRIPTION}

Strongly correlated materials exhibit complex coupling among multiple degrees of freedom, such as spin, charge, lattice, and orbital orders. The complexity not only offers rich physics, but also diverse functionalities. One may ask how researchers can characterize and control remarkable properties of matter that emerge from complex correlations far away from equilibrium? The recent development of intense terahertz $(\mathrm{THz})$ sources provides a new way of controlling mateirals without electronic excitation, enabling the investigation of a wider range of condensed matter phenomena. For example, the $\mathrm{THz}$ pulse can resonantly excite milli-electronvolt (meV) low energy modes in strongly correlated materials or provide ultrafast electric fields to manipulate ferroelectric polarization.
We developed terahertz-pump hard x-ray probe techniques for the control of strongly correlated materials in their electronic ground states. In particular, we designed and implemented a near-field approach based on nanoplasmonic enhancement to create a controllable 300 -femtosecond (fs) THz pulse with a peak field of 10 megavolts/centimeter $(\mathrm{MV} / \mathrm{cm})$ that is confined to a spatial region of several hundred nanometers $(\mathrm{nm})$. This will provide an unprecedented high-field condition that is extremely localized in the space and time domains for the control of the previously inaccessible phases of matter at ultrafast timescales.

\section{MISSION RELEVANCE}

This project is closely related to DOE's Science \& Innovation mission. The proposed project opens new scientific opportunities for in-situ studies of nonequilibrium dynamics of materials in real time under extreme field conditions. Without the complication of optical excitation, these studies allow novel selective ultrafast control of strongly correlated materials close to their electronic ground states. The time-resolved hard x-ray nanoprobe is a unique tool to explore the nonequilibrium states of matter using $\mathrm{THz}$ excitation, which is relevant to the development of next-generation energy materials, sensors, and devices.

\section{RESULTS AND ACCOMPLISHMENTS}

In FY 2013, we developed the laser-pump, hard x-ray nanodiffraction probe technique with 300-nm and 100-picosecond (ps) spatiotemporal resolution. This work set a firm foundation for the planned development.

In FY 2014, we designed and implemented the near-field THz pump, hard x-ray nanodiffraction probe capability on the 7-ID-C beamline at the Advanced Photon Source (APS). We studied the mesoscale structural phase transition in vanadium oxide $\left(\mathrm{VO}_{2}\right)$ upon optical excitation and investigated the transient grating induced structural changes in bismuth ferrite $\left(\mathrm{BiFeO}_{3}\right)$ and iron-rhodium (FeRh).

In FY 2015, we optimized the near-field enhanced $\mathrm{THz}$ pump x-ray nanodiffraction probe capability. Improved setup versatility and reproducibility shortened setup time from two days to one-half day. (The setup was ready to accept general users in January of 2016.) This capability enables a series of new experiments. In particular, we demonstrated a novel control of ferroelectric properties as discussed below.

The THz pump/hard x-ray microdiffraction probe setup was built at the 7-ID-C beamline of the APS (Figure 1a). 
An ultrafast Ti:Sapphire laser system provides 60-fs, 800-nm, 2-millijoule ( $\mathrm{mJ}$ ) laser pulses at a 1-kilohertz $(\mathrm{kHz})$ repetition rate and is synchronized with the storage ring of the APS. This laser system is used to pump a nonlinear conversion stage to generate broadband $\mathrm{THz}$ pulses with peak electric fields up to 200 kilovolts/centimeter $(\mathrm{kV} / \mathrm{cm})$ centered at $1 \mathrm{THz}$. The $\mathrm{THz}$ pulses are focused onto the sample surface by two parabolic mirrors. At the Center for Nanoscale Materials (CNM), gold split ring resonator (SRR) structures were fabricated on the sample surface by photolithography, to further enhance the $\mathrm{THz}$ fields. The square-shaped unit structure is $50 \times 50$ microns-square $\left(\mu \mathrm{m}^{2}\right)$, and the gap size is designed to be varied from 1 to $4 \mu \mathrm{m}$. For a $2.7-\mu \mathrm{m}$ gap as used in the experiments, a factor of 12 enhancement of the $\mathrm{THz}$ peak field is observed, corresponding to the enhanced $\mathrm{THz}$ peak field of $2 \mathrm{MV} / \mathrm{cm}$. The sample was mounted on a piezo stage for nano-positioning with SRR square unit cell edges aligned vertically. Monochromatic 11-keV x-ray pulses and 100-ps pulse duration were focused to $350 \mathrm{~nm}$ vertically by a Fresnel zone plate. The nanofocused $x$-ray beam is positioned in the horizontal gaps of the SRR structure to probe the structural dynamics upon $\mathrm{THz}$ excitation.

Using this new capability, we showed that an intense $\mathrm{THz}$ electric field, assisted by metamaterials, can directly couple to the ferroelectric polarization of a barium titanate $\left(\mathrm{BaTiO}_{3}\right)(\mathrm{BTO})$ film and yield large structural modifications. The 002 Bragg diffraction peak was measured by rocking scans before and after the THz excitation (Figure 1b). The shift of the Bragg peak is a result of a lattice expansion due to effective ionic excitation without excitation of electronic degrees of freedom. In this case, the $\mathrm{THz}$ field drives a titanium (Ti) atom in the unit cell of BTO, in resonance with the Slater phonon mode at $1 \mathrm{THz}$. [See T. Hoshina, et al., Jpn. J. Appl. Phys. 53, 09PD03 (2014) where this resonance was reported.] It represents a new approach to controlling material without activating the electronic degrees of freedom. To measure the timedependent response, the $\mathrm{x}$-ray incident angle was set at the half rising edge of the 002 Bragg peak, and the diffraction intensity was monitored as a function of time delay (Figure 1c). The increase of diffraction intensity is consistent with the Bragg peak shift to lower angles and recoveries in 10-ns timescales.

This spatiotemporally resolved instrumentation not only allows us to study time-dependent structural changes, but also local structural modification with a spatial resolution of $350 \mathrm{~nm}$. The spatially confined structural evolution, as a result of the near-field excitation, can be quantitatively measured. The differential signal before and after excitation was plotted at various delays (Figure 1d), showing the time- and spatial- dependent responses of the BTO film upon $\mathrm{THz}$ excitation. The double-peak structure is consistent with the nearfield distribution around the slit. At later delay times, the double-peak structure diminishes as the heat is transported transversely across the film. In addition, one can obtain a two-dimensional map of the $\mathrm{THz}$ induced strain in BTO film around the slit (Figure 1e). The doubleridge structures (red color) at the gold (Au) film edge is clearly visualized, representing the near-field imaging of the $\mathrm{THz}$ field distribution around a metamaterial with 350-nm spatial resolution. This demonstration opens new opportunities for nanoscale energy conversion and transport, which is important for both fundamental materials science and device applications.
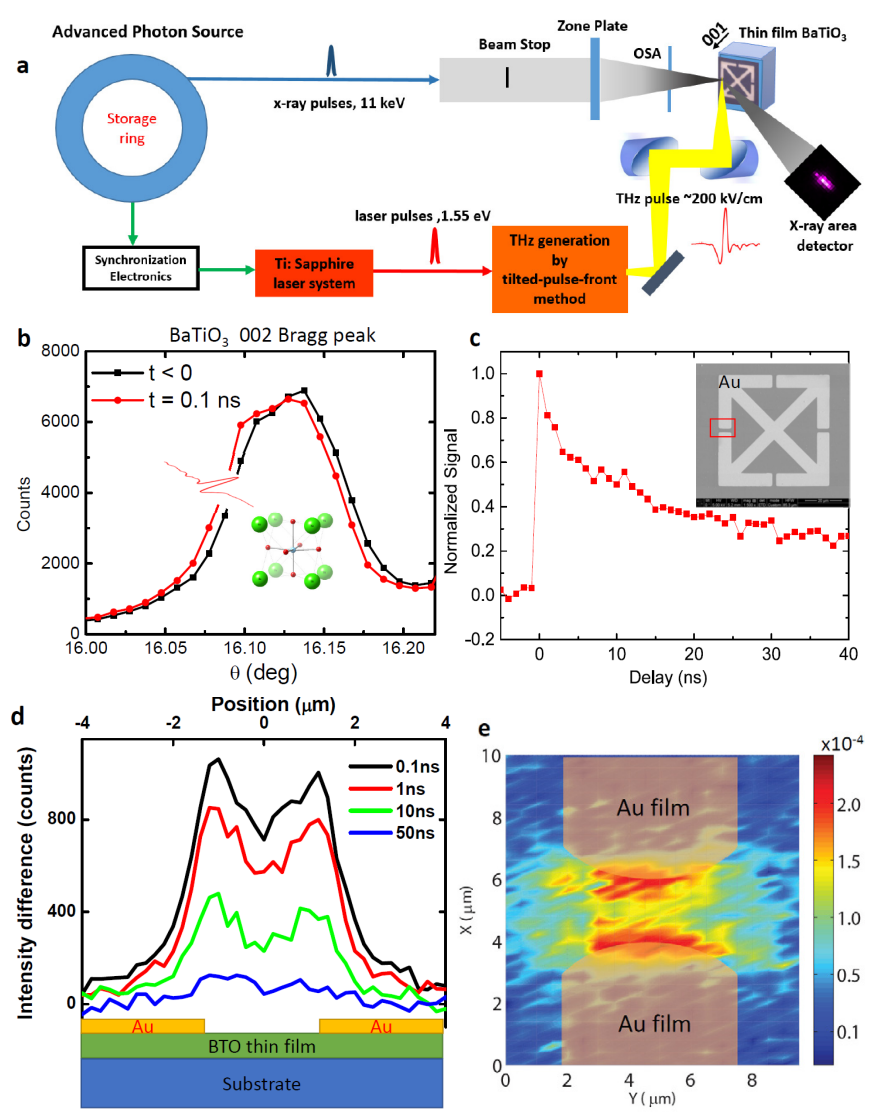

Figure 1. (a) THz pump/hard $x$-ray microdiffraction probe experimental setup at the Advanced Photon Source. (b) The 002 Bragg peak of the BTO film before and after the $\mathrm{THz}$ field excitation. (c) The diffraction intensity measured at 16.09 degrees as a function of time. The inset shows a unit structure of the split ring resonator. The red box indicates the area imaged in (e). (d) The structural response measured by the diffraction intensity difference at $\mathbf{1 6 . 0 9}$ degrees and at various delays as a function of probe position across the SRR gap. The bottom schematic plot shows the side view of the sample structure. (e) The map of THz induced strain around the gold gaps of the split ring resonator at 200 ps after the THz excitation.

The demonstrated new capability helped to attract DOE-Basic Energy Sciences (BES) support for studying complex oxides and two-dimensional transition metal dichalcogenides. 


\section{Visualization of Stress-Induced Polarization Switching in Electromechanically Coupled Ferroelectric Polymers}

\section{3-063-R2}

Seungbum Hong, YoonYoung Choi, and Charudatta Phatak

\section{PROJECT DESCRIPTION}

The goal of this project is to study the strain-induced phase transition and polarization switching in a polyvinylidene fluoride-triflouroethylene(P(VDF-TrFE)) polymer ferroelectric materials system by using piezoresponse force microscopy (PFM), in-situ transmission electron microscopy (TEM), and grazing incidence small-angle and wide-angle $\mathrm{x}$-ray scattering (GISAXS and GIWAXS) at the Advanced Photon Source (APS). The outcome of this research will lead to design guidelines toward the development of high-performance piezoelectric energy harvesting devices.

\section{MISSION RELEVANCE}

This project will help us to understand the science of new materials for clean and renewable energy harvesting and to achieve the technological goal of developing high-efficiency vibration energy conversion devices. It is therefore related to DOE's energy mission.

\section{RESULTS AND ACCOMPLISHMENTS}

In FY 2013 and FY 2014, we developed a nanoscale mechanical annealing process of polymer ferroelectric thin films, which can control nanoscale material properties and molecular orientation using intensive local stress. We directly applied mechanical stress, which can induce irreversible plastic deformation, to P(VDF-TrFE) thin film using a nanoscale tip at room temperature. Plastically deformed regions did not show any significant damage or delamination. Rather remarkably, the vertical and lateral piezoresponse amplitudes measured after the mechanical annealing were $28 \%$ and $102.4 \%$ higher than the pristine film in this study. In addition, randomly oriented lamellar crystals were well-aligned along the applied stress direction.

In FY 2015, x-ray scattering experiments were performed to determine the structure of pristine and mechanically annealed P(VDF-TrFE) thin films at the Advanced Photon Source (APS), as shown in Figures 1 and 2. We found a clear difference in the GISAXS data for the pristine versus the mechanically annealed region with a mechanical force of 1.0 micronewton $(\mu \mathrm{N})$, as shown in Figures 1a and $1 \mathrm{~b}$. While the GISAXS image for the pristine region is clear, the typical diffraction pattern from stripe domains is observed in the mechanically annealed region. We measured $d$-spacing of 79.7 nanometers $(\mathrm{nm})$ in the mechanically annealed region from the GISAXS image, which is in good agreement with the measured value of the line width of $78 \mathrm{~nm}$ from our previous results of the scanning electron microscopy (SEM) images. The diffraction pattern suggests that the stripe domains are aligned almost parallel to the $x$-ray beam in our setup for $\mathrm{x}$-ray analysis.
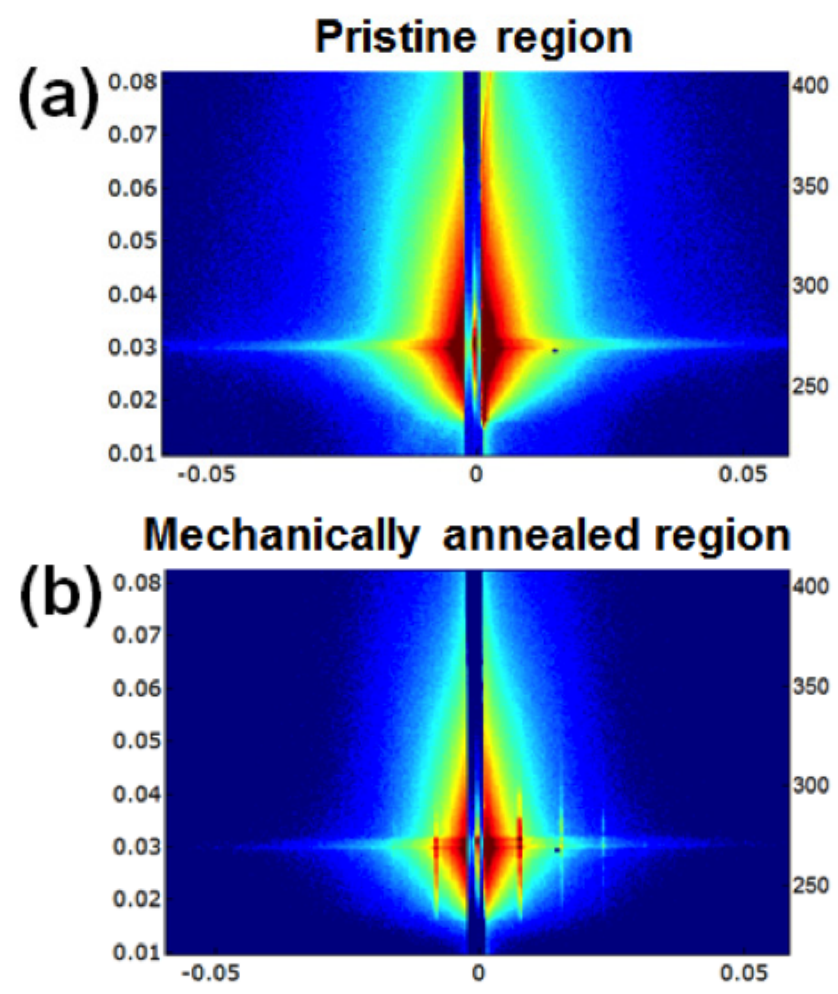

Figure 1. GISAXS data for the (a) pristine region and (b) mechanically annealed region.

In Figures $2 \mathrm{a}$ and $2 \mathrm{~b}$, grazing incidence wide-angle $\mathrm{x}$-ray scattering (GIWAXS) images are presented for the pristine and mechanically annealed regions. Both GIWAXS images show diffraction patterns possessing typical fiber symmetry, meaning that crystalline domains are randomly oriented in the plane, but aligned along the out-of-plane direction. The P(VDF-TrFE) unit cell is oriented such that (200) or (110) planes are oriented parallel to the substrate according to our GIWAXS intensity simulation shown in Figure 2c, which is the calculated diffraction pattern for (200) orientation. This indicates that P(VDF-TrFE) chains are aligned parallel to the substrate as shown in the top view of the unit cell in the inset of Figure $2 \mathrm{c}$. The unit cell used for GIWAXS simulation is shown in Figure $2 \mathrm{~d}$ for (110) orientation. Here, we confirmed that there was no a-phase 
(non-polar phase) before and after the mechanical annealing, which supports the enhancement of the piezoelectric properties of the mechanically annealed polymer ferroelectrics by molecular alignment. (a)

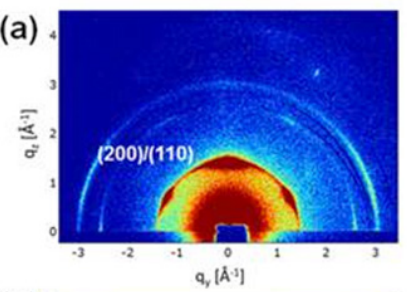

(c)

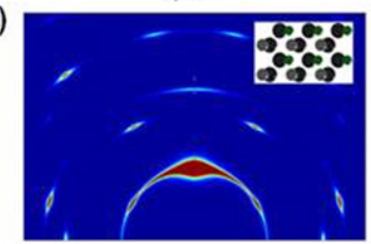

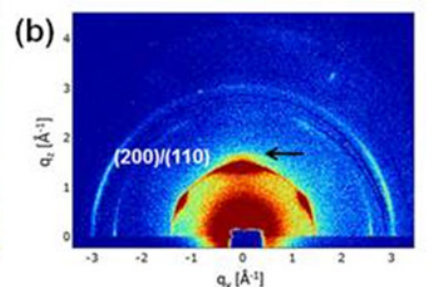

(d)

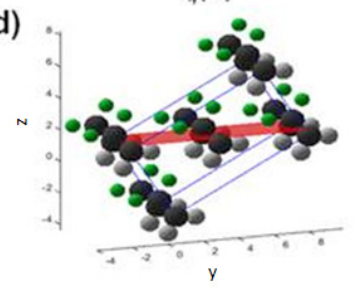

Figure 2. GIWAXS data of (a) pristine region, (b) mechanically annealed region with a mechanical force of $1.0 \mu \mathrm{N}$, (c) simulation of GIWAXS of (200) orientation of P(VDF-TrFE) film (inset: top view of the unit cell), and (d) side view along the $z$ direction for (110) orientation. Parameters of the orthorhombic lattice are $a=8.7, b=5.05$, and $c=2.55 \AA$. Weak peak (arrow in Figure $2 b$ ) was observed slightly above the $(200) /(110)$ peak, or higher $q_{z}$ position in the mechanically annealed region. The ferroelectric beta phase was observed in both the pristine and mechanically annealed regions and there was no alpha phase (non-polar phase) after mechanical annealing.

To continue this research, we have submitted an ARPA-E proposal entitled "Energy harvester based on electromechanical charge collecting." Future work will involve setting up a dip coating system to coat the polymer solution onto mechanical springs and cantilevers. The microscopic knowledge of the mechanical annealing effect obtained from the scanning probe (especially, charge gradient microscopy) and electron microscopy will be utilized to achieve an optimum thickness, direction of backbones, and straining direction in the polymer film.

\section{Imaging Ecological Engineers: A Novel Quantum Dots Approach to Map Microbes in Complex Soil Structures with X-rays}

\section{3-070-R2}

Sarah O'Brien, Dionysios A. Antonopoulos, Maxim Boyanov, Kenneth M. Kemner, and Edward J. O'Loughlin

\section{PROJECT DESCRIPTION}

This project aims to understand how soil microbes engineer their environments into dynamic three-dimensional complexes that promote microbial diversity, influence the terrestrial carbon cycle, support soil fertility, and impact the fate and transport of contaminants. The metabolic activities of soil microbes are the primary drivers of biogeochemical processes controlling the terrestrial carbon cycle, nutrient availability to plants, contaminant remediation, and other ecosystem services. However, we have a limited understanding of how microbes and microbial metabolism are distributed throughout the three-dimensional complex of the soil or how this phenomenon impacts emergent properties, such as microbial diversity or biogeochemical fluxes.

In this project, we are developing a novel application of quantum dots (QDs), which are engineered semiconductor nanoparticles that produce size-dependent fluorescence, that uses their relatively high electron density (rather than their optical fluorescence characteristics) to track the microbially facilitated incorporation of organic substrates into soil aggregates by using $x$-ray transmission and fluorescence microtomography. Combined with their distinctive ability to tag metabolically active molecules and thus gain entry into cells, the relatively high x-ray absorption of selenium (Se) in QDs will allow us to uniquely image the role of bacteria in building soil aggregates and pore spaces and provide new insight into the living conditions of microbial cells.

\section{MISSION RELEVANCE}

This project is relevant to DOE's environmental quality mission. The ability to track particular metabolic functions in a variable, opaque environment could be invaluable to any research program that investigates microbial processes in opaque materials-from soils to subsurface sediments, to arctic permafrost, to microbe-enabled industrial applications. It also meshes well with current DOE-supported work to characterize microbial metabolic functions by using metagenomic sequence data.

\section{RESULTS AND ACCOMPLISHMENTS}

Our initial experiments in FY 2013 determined that bacterial uptake of QDs is active and depends on the conjugated substrate and environmental conditions, which are prerequisites for our planned soil experiments. We grew two different non-pathogenic bacterial species commonly found in soil in one of four ecologically relevant experimental conditions: nitrogen $(\mathrm{N})$ limitation, phosphorus $(P)$ limitation, nitrogen and phosphorus limitation, or no-nutrient limitation. A slurry of conjugated QDs (representing each of five colors conjugated to five different organic substrates) was added to the cultures, and optical and confocal microscopies were used to evaluate bacterial preference for the various substrates under each of the four conditions. 
We found that growth conditions affected uptake, with significantly more uptake when either $\mathrm{N}$ (glycine, adenine, glucosamine) or both $\mathrm{N}$ and $\mathrm{P}$ (glycerol phosphate) were limited. In addition, phosphoserine appeared to be the preferred substrate, with greater uptake than any of the substrates that provided only $\mathrm{N}$ or only P. Uptake was similar for the two species, and we found no evidence for cytotoxicity issues at the QD concentrations we used. Cellular uptake of unconjugated QDs was negligible, indicating that bacteria actively consume the QD-labeled nutrient rather than passively entering the cells. These findings are the first direct empirical evidence showing bacterial substrate preference that varies in response to environmental conditions. X-ray fluorescence (XRF) imaging experiments performed in FY 2014 at the 2-ID-D beamline at the Advanced Photon Source (APS) suggest that the $x$-ray mapping approach can, indeed, detect bacterial uptake of cadmium selenide (CdSe)-core QDs.

In addition, we hypothesize that the combined confocal microscopy and XRF approach we developed is capable of detecting biofilms based on images we collected of bacterial cells growing on a solid surface, where XRF-based elemental maps for phosphorus, which is associated with organic substances, and Se, which is associated with QDs, were concentrated in similar patterns as in an optical image where cells were embedded in extracellular polysaccharides of a biofilm. Thus, we demonstrated that this XRF imaging approach enables the identification of cells (and cell products, such as extracellular polysaccharides).

In FY 2015, we gathered evidence that uptake of QDs by bacteria is indeed internal. Working with collaborators at the Environmental Molecular Sciences Laboratory at Pacific Northwest National Laboratory, we made aberration-corrected transmission electron microscopy (TEM) measurements of thin sections of $B$ subtilis and $P$. fluorescens that clearly demonstrate QDs in the cytoplasm of cells. Nanosized electron-dense objects containing crystal lattice planes consistent with CdSe lattice spacings were identified within cells. In addition, we imaged organisms in the three-dimensional, opaque medium of soil. XRF measurements were conducted at Sector 2-ID-E at the APS by mounting the sample on a stage, and then rotating it around an axis perpendicular to the $\mathrm{x}$-ray beam, with two-dimensional images collected at multiple angles. Computationally intensive tomographic reconstructions identified the distribution of elements in the aggregate, including the $\mathrm{Cd}$ and Se derived from QDs in labeled bacteria. Figure 1 shows a soil aggregate incubated for 120 hours with bacteria that had already taken up QDs. Green areas represent QD-derived Se, indicating the locations of the cells. Red areas represent Fe.

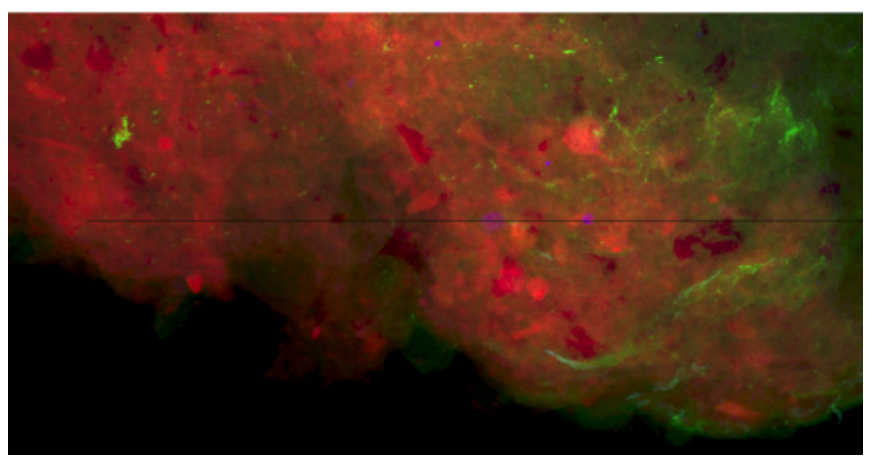

Figure 1. Two-dimensional elemental map of Se and Fe comprising a soil aggregate. QD-derived Se (green) identifies the location of $P$. protegens within the soil pore network. Red indicates iron.

Work on this project will continue as part of the DOE Biological and Environmental Research (BER) Biological Systems Science Division (BSSD) bioimaging project "Small Worlds", which aims to develop new imaging approaches for investigating plant-microbe interactions in soil. Now that we have demonstrated the utility of QD-labeling and/or XRF for identifying the location of bacteria in soil, we can use our new technique to study how bacteria respond to plants in the context of the soil matrix.

\section{Ultra-Low-Loss Superconducting Microstrip for Multi-Chroic Cosmic Microwave Background Detectors}

\section{3-080-R2}

\author{
Gensheng Wang, John Carlstrom, Clarence Chang, \\ Valentyn Novosad, Thomas Proslier, \\ and Volodymyr Yefremenko
}

\section{PROJECT DESCRIPTION}

This project aims to develop ultra-low-loss superconducting microstrip transmission lines, which are an essential technology for next-generation cosmic microwave background (CMB) experiments. A superconducting microstrip consists of a superconducting ground plane, a dielectric spacer, and a patterned superconducting transmission line. Loss in superconducting microstrips results from two known mechanisms. The first is two-level system (TLS) loss, which arises from the amorphous nature of the dielectric used in the microstrip. The second mechanism is quasiparticle loss associated with the breaking of Cooper pairs in superconductors with a gap that is comparable to the photon energies of interest. 
For this project, we will implement two complementary methods for studying and mitigating these losses. First, we will fabricate half-wavelength resonators for characterization at radio frequencies (RF) (1-10 GHz). Techniques for microstrip characterization at these frequencies are well established. The measurements will directly give the loss tangent of the superconducting microstrip and the transition temperature of the superconductor. Second, we will fabricate microstripcoupled transition edge sensor (TES) bolometers for measurements at millimeter wavelengths $(100-300 \mathrm{GHz})$. These measurements will help us understand and control loss at millimeter wavelengths in superconducting microstrip transmission lines.

\section{MISSION RELEVANCE}

This project is relevant to DOE's mission in basic science. Ultra-low-loss superconducting microstrip transmission lines are a critical technology for future measurements of the CMB. The CMB explores fundamental physics. Its polarization at angular degree scales is a unique probe of the energy scale of the cosmic inflation, expected to be near energies favored by Grand Unified Theories (GUTs). Its polarization at tens of arcminute angular scales is sensitive to the sum of the neutrino masses at a level relevant for resolving the neutrino mass hierarchy. CMB physics is an important dimension of DOE's High Energy Physics program and was recommended by the Particle Physics Project Prioritization Panel.

\section{RESULTS AND ACCOMPLISHMENTS}

In FY 2013, we successfully developed a low-loss dielectric recipe by sputtering silicon in oxygen plasma. We also developed techniques to control our fabrication at the nanoscale. These techniques eliminate nanometerthick etching residuals and nano-sized cracks, which cause microwave loss. In FY 2014, we studied $\mathrm{Nb}-\mathrm{SiO}_{2}-\mathrm{Nb}$ (niobium-silicon dioxide-niobium) microstrip resonators at radio frequencies and microstrip-coupled TES devices at millimeter wavelengths to understand the losses and material parameters of superconducting microstrip transmission lines. Our investigations were successful in that we were able to fully characterize our microstrip losses.

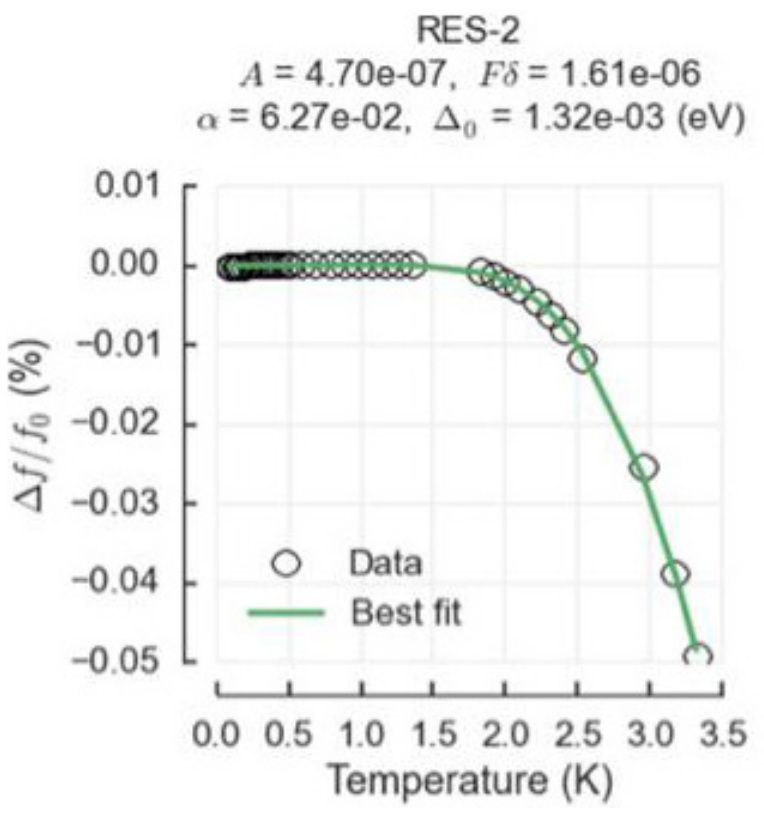

Figure 1. The fractional resonant frequency shift as a function of temperature of a quarter wavelength $\mathrm{Nb}$ CPW resonator. The model-dependent fit parameters are at the top of the figure. $A$ is an offset constant allowing for a non-zero cryostat base temperature. $F \delta$ is a geometry-dependent loss tangent that is small for this CPW resonator on an intrinsic silicon wafer. $\alpha$ is the fraction of kinetic inductance to the total inductance, including the magnetic inductance of the resonator. $\Delta_{0}$ is the superconducting energy gap parameter of the $\mathrm{Nb}$ film.

In FY 2015, we transitioned toward a focus on optimization and quality control of the superconducting microstrip fabrication process. We fabricated and tested halfwavelength microstrip resonators with a $\mathrm{SiO}_{2}$ dielectric made by plasma-enhanced chemical vapor deposition (PECVD), a process that is roughly five times faster than our traditional method of radio frequency (RF) sputtering silicon in an oxygen plasma. The measured loss tangent of the $\mathrm{SiO}_{2}$ made by PECVD is $\sim 0.0023$, which is larger than that of the $\mathrm{SiO}_{2}$ made by RF sputtering silicon in oxygen plasma, but still acceptable for multi-chroic $\mathrm{CMB}$ detectors. The PECVD process is also able to deposit oxide at a relatively low temperature of $110^{\circ} \mathrm{C}$ (contrast this with the $\sim 200^{\circ} \mathrm{C}$ required for RF-sputtered oxide). This lower temperature should have fewer negative impacts on the titanium/gold (Ti/Au) bilayer TESs, which constitute the active elements of the CMB detectors. We also studied the stability of the $\mathrm{SiO}_{2} / \mathrm{Nb}$ interface by depositing a thin layer of $\mathrm{Si}$ between the $\mathrm{SiO}_{2}$ dielectric and the $\mathrm{Nb}$ film in microstrip resonators. The intent was to determine whether free oxygen from the $\mathrm{SiO}_{2}$ was reacting with $\mathrm{Nb}$ and degrading it. However, the fresh silicon layer formed a SiNb compound that degraded the Nb transition temperature and resulted in additional Ohmic losses at the relevant microwave frequencies. The result of this study was that our $\mathrm{SiO}_{2} / \mathrm{Nb}$ interface is stable, at least compared to a $\mathrm{Si} / \mathrm{Nb}$ interface. Finally, we developed a fast test for verifying the quality of our $\mathrm{Nb}$ films. A quarter- 
wavelength coplanar waveguide (CPW) resonator, which is made from a single layer of $\mathrm{Nb}$ film, experiences a resonant frequency shift with temperature that is related to the superconducting energy gap of the $\mathrm{Nb}$ film. The CPW resonator can be assembled in less than a day, allowing us to verify the quality of our $\mathrm{Nb}$ film before investing roughly two weeks in the fabrication of a batch of multi-chroic CMB detectors. Figure 1 shows a fit to the measured fractional resonant frequency shift as a function of temperature of a 300-nm-thick Nb CPW resonator. The extracted value of the superconducting gap parameter $\left(\Delta_{0}\right)$, and therefore the transition temperature, are consistent with a high-quality $\mathrm{Nb}$ film.

The developed microstrip technologies in this project have been utilized in the fabrication of South Pole Telescope (SPT)-3G multi-chroic detectors. These detectors are expected to be deployed at the South Pole by the end of 2016 for observations of the CMB.

\section{3-D Compositional Control of Intermediate-Band Solar Cells}

\section{3-100-R2}

\section{Alex B.F. Martinson and Michael J. Pellin}

\section{PROJECT DESCRIPTION}

Intermediate-band (IB) solar cells are designed to retain the high-output voltages of large-bandgap semiconductors while harvesting significantly more of the solar spectrum, thereby exceeding the Shockley-Queisser (S-Q) limit of solar conversion efficiency. By using the IB as a stepping stone, electron-hole pairs may be generated from photons with insufficient energy to pump electrons directly from the valence band (VB) to the conduction band (CB). However, it appears likely that a new level of compositional control over these active layers will be required to tune the mid-gap states precisely while minimizing charge recombination. Advances in atomic layer deposition (ALD) and sulfide-based photovoltaics (PV) in our lab, combined with recently identified IB materials, reveal a new opportunity to make rapid advances in this emerging field.

In the course of this work, we have been (1) pursuing fabrication of thin films of the most promising bulk IB materials identified to date, leveraging the threedimensional (3-D) compositional precision of ALD; (2) probing the extent to which, as has been postulated, an indium-vanadium-sulfide $\left(V_{0.25} \operatorname{In}_{1.75} S_{3}\right)$ thin film satisfies the demands of an IB solar absorber in polycrystalline thin-film form; (3) investigating the complex photophysics expected from a multilevel system by using time-resolved optical spectroscopies; and finally, (4) fabricating and testing the first $V_{0.25} \ln _{1.75} S_{3}$ IB PV device stacks.

\section{MISSION RELEVANCE}

This project is relevant to DOE's missions in basic science and energy. Our basic science understanding of IB materials is incomplete, especially in the areas of thin-film synthesis and/or deposition. More generally, our control over the precise 3-D location of atoms in alloys and doped materials used in PV and other optoelectronic materials is lacking. If promising IB materials can be fabricated into efficient PV device stacks, there is an opportunity to exceed the theoretical limit of present PV technologies considerably. Higher PV efficiencies have a compounding economic effect due to reductions in the overall $\$ /$ watt of $P V$ that includes land use, hardware, installation, and maintenance.

\section{RESULTS AND ACCOMPLISHMENTS}

In FY 2013, we fabricated alloy indium-vanadium-sulfide thin films by using ALD. We developed and tested a novel In precursor and ALD process, which enables low-temperature growth without the risk of oxygen contamination. Through optical, electrical, and structural characterization, we explored the resulting material space and verified conditions under which a polycrystalline, lightly $n$-doped base is routinely produced.

In FY 2014, we investigated the optoelectronic properties and photophysical dynamics of semiconducting-base (indium [III] sulfide [ $\left.\ln _{2} \mathrm{~S}_{3}\right]$ ) and IB material (V: $\ln _{2} \mathrm{~S}_{3}$ [V-substituted $\left.\ln _{2} \mathrm{~S}_{3}\right]$ ) thin films. In addition, the thin-film synthesis was further refined to correlate the composition with distinct IB properties. An additional absorption feature is clearly seen upon $V$ substitution (Figure 1). Furthermore, the energy of this new transition (centered at $1.3 \mathrm{eV}$, see Figure 2) is in reasonable agreement with the one that was computationally predicted previously. Ultrafast transient absorption (TA) and photoluminescence (PL) experiments were performed on the $\ln _{2} S_{3}$. These spectra suggest that many photoexcited electrons have relaxed (radiatively and non-radiatively) back to the ground state within $\sim 25$ picoseconds (ps), but a measurable population resides for tens of nanoseconds in a density of states within the gap. These states are likely attributable to sulfur vacancies, which are expected in the $\beta-\ln _{2} S_{3}$ structure.

In FY 2015, the optoelectronic properties of $\mathrm{V}: \ln _{2} \mathrm{~S}_{3}$ thin films were investigated as a function of the substitutional $\checkmark$ doping levels, and the most promising compositions were incorporated into functional photovoltaics. An optical transition with onset approximately $0.8 \mathrm{eV}$ less 
than the lowest energy $\ln _{2} S_{3}$ band-edge transition $(2.1 \mathrm{eV})$, as deduced by reflectivity-corrected UV-Vis-NIR (ultraviolet-visible-near infrared) spectroscopy (Figure 1), is in good agreement with theory. However, the clear absence of a low-energy $(0.7 \mathrm{eV})$ transition, predicted to result from electronic excitation from VB to a partially filled IB and tentatively assigned in nanocrystalline powder experiments, suggests nearly complete IB density of state filling in these thin films (see Figure 2). Room temperature PL spectra also reveal a large NIR peak centered at $\sim 880 \mathrm{~nm}(1.4 \mathrm{eV})$ for vanadium-alloy that could be assigned to radiative recombination from the $C B$ to the IB; however, the possibility of enhancing defectmediated PL present in $\operatorname{In}_{2} S_{3}$ has not been excluded. The Fermi level of unintentially-doped $\ln _{2} S_{3}$ is often closer to the $\mathrm{CB}$ owing to intrinsic donors from sulfur vacancies and indium interstitials. Therefore, intrinsically $n$-type films based on $\ln _{2} \mathrm{~S}_{3}$ alloys seem unlikely to exhibit Fermi levels consistent with lightly $p$-doped films as required for the desired partially filled IB. In all cases, our Hall measurements reveal excess $n$-type carriers in $\ln _{2} S_{3}$ and $V_{x} \ln _{(2-x)} S_{3}$ thin films. Operational photoelectrochemical photovoltaics, also referred to as semiconductorsensitized solar cells, clearly confirm the presence of photocurrent originating from excitation across the primary bandgap but the absence of contribution from sub-gap absorption (Figure 2). In short, devices with the alloy absorber do not operate as IB photovoltaics.

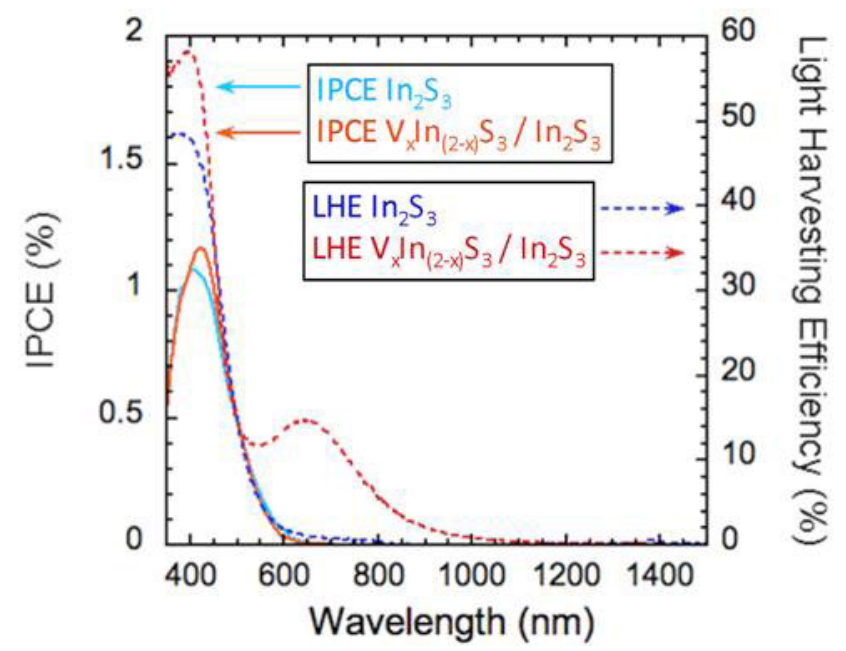

Figure 1. The incident photon to current efficiency (IPCE) (left axis) for $\operatorname{In}_{2} S_{3}$ $(60-n m)$ and $V_{x} I_{(2-x)} S_{3}(30-n m) / / n_{2} S_{3}(60-n m)$ films plotted next to their lightharvesting efficiency (right axis).

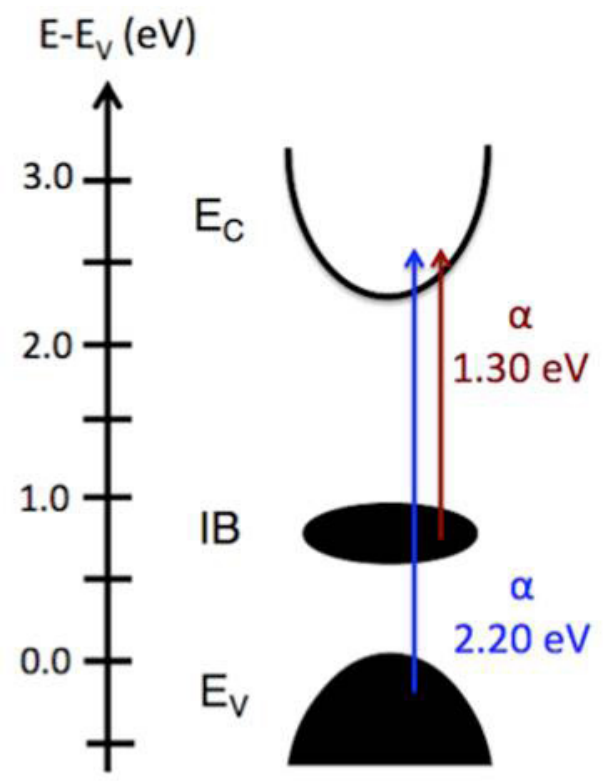

Figure 2. Idealized energy band diagram for $\mathbf{V}_{x} \mid n_{(2-x)} S_{3}$ films based on absorption data.

While sub-bandgap photoactivity has been previously inferred for $V: \ln _{2} S_{3}$ nanoparticle suspensions, we note several potential limitations to the use of these thin films in IB PV. First, we find no clear evidence for a partially filled IB given the absence of optical extinction at $0.8 \mathrm{eV}$. According to the principles of IB operation, this dramatically reduces the probability of refilling the IB and therefore, precludes the contribution of the smallest subgap absorption to steady-state photocurrent contribution. Second, that the IB is postulated (both theoretically and experimentally) to reside closer to the VB than to the $C B$ is in conflict with the need for a Fermi level within the IB when the parent material $-\ln _{2} S_{3}$-is naturally $n$-type. Finally, the relatively short excited-state lifetime of $\ln _{2} S_{3}$ thin films (that we deduced in FY 2014) will make the collection of photogenerated charge difficult.

Although a facile route to substitutional IB absorbers has been identified in ALD, we have conclusively demonstrated that several important shortcomings exist for this particular material system. As such, future work should pursue novel substitutional alloys with base absorbers that are clearly poised to avoid these pitfalls. 


\section{Nanolaminate Coatings for Improved Nuclear Fuel Cladding Performance}

\author{
2013-116-R2 \\ Michael J. Pellin, Michael Billone, and Abdellatif Yacout
}

\section{PROJECT DESCRIPTION}

As exemplified by the 2011 Fukishima reactor accident, nuclear materials-particularly in the claddings used to protect nuclear reactor fuel-are critical to a reactor's severe accident tolerance, ability to extend fuel burn-up, and cost of operation. Zircaloy, the material of choice for claddings in nearly all operating nuclear reactors, fails when driven beyond design limits under severe accident conditions such as a loss of coolant accident (LOCA) due to degradation of its passivating oxide layer. Zircaloy (in its many alloy forms) can burn either exothermically or produce explosive $\mathrm{H} 2$ gas mixtures when driven beyond normal operating temperature limits in a water environment. In addition, a fraction of the generated hydrogen embrittles the cladding alloy, ultimately leading to its mechanical failure.

This project has sought to enhance the passivation properties of the Zircaloy passivation layer by adding a nanolaminate $(\mathrm{NL})$ layer or layers, by atomic layer deposition (ALD) to the cladding. ALD is a unique synthesis method that enables the growth of pinhole-free films on large and convoluted substrates with nanometer precision. This approach of adding NLs to Zircaloy has the advantage of maintaining the use of traditional cladding materials instead of resorting to new cladding materials, such as silicon carbide ( $\mathrm{SiC}$ ), that might have the potential to reach improved-beyond-design-limits performance but at a much higher cost. It also has the flexibility to address, with the stepwise addition of different layers, the many material issues that limit the lifetime and operating conditions of current claddings, including pellet-cladding mechanical interactions (e.g., using nitride barrier layers), fretting corrosion (e.g., using super-hard nitride layer stacks) and potentially susceptibility to delayed hydride cracking.

\section{MISSION RELEVANCE}

Improved material performance is crucial if DOE'S Office of Nuclear Energy (NE) is to achieve its goals of accident tolerance, life extension, deeper fuel burnup, and improved cost. This is also true for the DOE Office of Science (SC), Basic Energy Sciences (BES), which has set forth the goal of understanding materials subjected to extreme environments and then using this knowledge to improve our nation's energy infrastructure.

\section{RESULTS AND ACCOMPLISHMENTS}

We made significant progress in demonstrating that nanolaminate materials represent a viable path toward improving the accident tolerance of nuclear cladding materials.

$\square$ High-quality, single-layer titanium nitride (TiN), zirconium dioxide $\left(\mathrm{ZrO}_{2}\right)$, silicon dioxide $\left(\mathrm{SiO}_{2}\right)$, zirconium nitride $(\mathrm{ZrN})$, and aluminum oxide $\left(\mathrm{Al}_{2} \mathrm{O}_{3}\right)$ films were applied to Zircaloy-2 samples (year 1). Also multi-layer stacks of $\mathrm{Al}_{2} \mathrm{O}_{3} / \mathrm{ZrO}_{2} / \mathrm{Zirc}-2, \mathrm{Al}_{2} \mathrm{O}_{3} / \mathrm{ZrO}_{2} / \mathrm{Al}_{2} \mathrm{O}_{3} / \mathrm{Zirc}-2$, ZrN/ $\mathrm{Al}_{2} \mathrm{O}_{3} / \mathrm{Zirc}-2$, and $\mathrm{ZrO}_{2} \mathrm{ZrN} / \mathrm{Al}_{2} \mathrm{O}_{3} /$ Zirc-2 were applied to Zircaloy-2 samples (year 2). This work includes significant optimization of sample conditions and precursor molecules. We found that the density of the applied film is a crucial factor in that film's ability to act as a barrier layer for oxidation (see some examples in Figure 1). The conformal ALD process allows coating the entire tube (inside and outside) in a single process step.

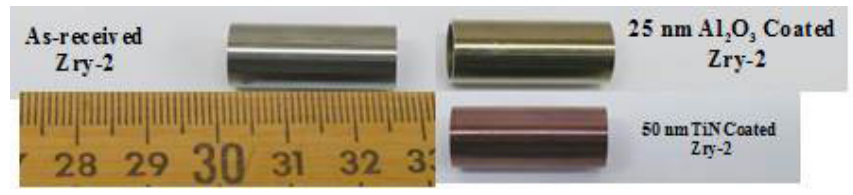

Figure 1. Examples of coated Zircaloy-2. We note that the coating also is applied to the inside of the tube as well as the outside by the ALD process.

$\square$ All samples were tested for weight gain as a function of temperature in an oxygen and steam atmosphere. We found synthesis conditions where each of the tested films prevented the onset of weight gain to $>550^{\circ} \mathrm{C}$ (untreated samples showed weight gain at $\mathrm{T}>300^{\circ} \mathrm{C}$ ). Layered stacks can achieve $>650^{\circ} \mathrm{C}$ without significant weight gain. Figure 2 shows the color change that accompanies weight gain in these samples (due to substoichiometric $\mathrm{ZrO}_{x}$ (years 1 and 2).

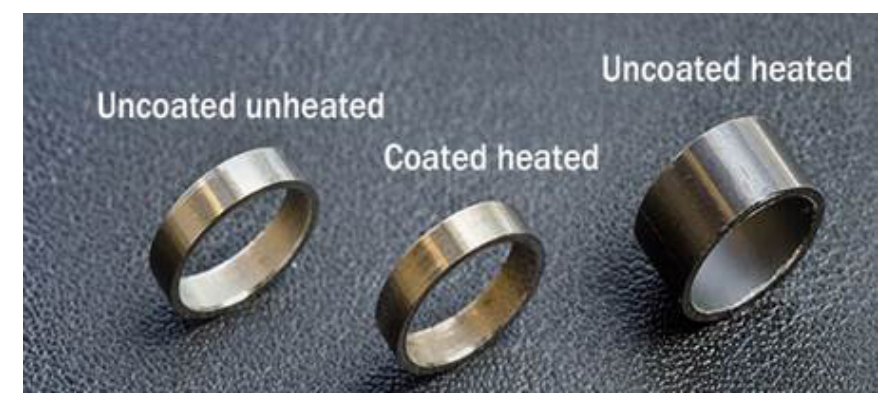

Figure 2. The oxidation behavior of Zircaloy-2 cladding tubes following 3-hour oxidation at $600^{\circ} \mathrm{C}$. The color change of the uncoated cladding is a result of an oxidative weight gain of $>3.5 \%$. The sample labeled "coated heated" $\left(\mathrm{Al}_{2} \mathrm{O}_{3}, 50 \mathrm{~nm}\right)$ shows no weight gain within experimental error $(0.07 \%)$.

$\square$ Extensive Advanced Photon Source (APS) and transmission electron microscopy (TEM) analysis of the heat-treated samples (also radiation-dosed at the Argonne Tandem Linac Accelerator System [ATLAS], see next item) demonstrated that the failure mechanism 
at $\sim 600^{\circ} \mathrm{C}$ for these samples comes from strain that appears as the underlying Zircaloy alloy undergoes a phase transition. The methods used include energy $x$-ray find structure (EXAFS), small angle $x$-ray scattering (SAXS) and wide angle $x$-ray scattering (WAXS).

$\square$ We demonstrated that high radiation doses were possible in just a few days of ATLAS beam time. The nanolaminate and nano films were not destroyed during these runs. We also discovered diffusion effects that allowed us to use diffusion to dramatically improve the performance of our coated materials.

$\square$ We were able to investigate the behavior of our coated samples at both APS Sector 1 and Sector 34 . An example of collected data can be found in Figure 3 where $x$-ray diffraction has been used. These results support our conclusion that diffusion can be used to reduce the ALD-applied layers and produce layered Zr MAX phase materials $(\mathrm{M}=$ an early transition metal, $\mathrm{A}=$ an $\mathrm{A}$-group element, and $\mathrm{X}=$ carbon and/ or nitrogen, in $M_{n+1} A X_{n}, n=1,2$, or 3 ) that can reduce oxidation up to $800^{\circ} \mathrm{C}$. Specifically, we used Sector 1's unique high-energy transmission beam ( $80 \mathrm{keV})$ to characterize in-situ oxidation kinetics at up to $800^{\circ} \mathrm{C}$. These studies included high-temperature steam and used line scans to obtain phase distributions across thicknesses of the Zircaloy tubes ex situ and in situ.

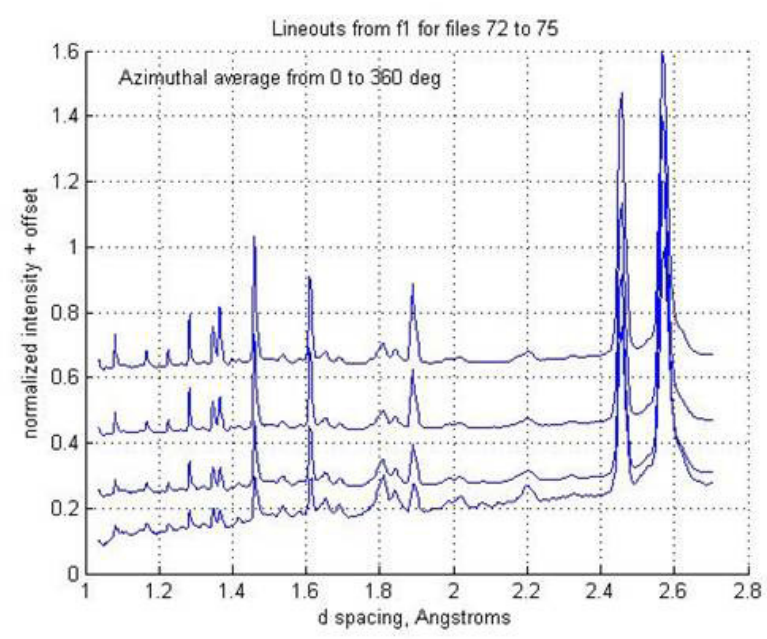

Figure 3. Ex-situ line scan result (integrated intensity versus d spacing, which gives volumetric phase information).These APS x-ray diffraction scans provide a clear indication of the phases formed upon heating the alloy. They also demonstrate that the phase is formed with an abrupt depth cutoff. The curves from bottom to top are a function of depth in 0.5 micron steps from the surface into the bulk. The diffraction lines at $\sim 2$ and $2.2 \AA$ (indicative of the new phase) show a rapid fall off with depth.
An example of what we found is demonstrated in Figure 4, which displays a TEM line scan of the relevant element concentrations of a cross-sectioned Zircaloy clad. This clad was coated with $50 \mathrm{~nm}$ of alumina and then heated to $500^{\circ} \mathrm{C}$ for 4 hours in an oxygen environment. The image is essentially unchanged from the original deposit. Above $550^{\circ} \mathrm{C}$, we observe slow diffusion changes to the films, as exemplified in Figure 5. In these films, we see the generation of several aluminum zirconium $\left(\mathrm{Al}_{x} \mathrm{Zr}_{\mathrm{y}}\right)$ phases. This results from aluminum diffusion into the Zircaloy. These phases do not have significant oxygen. Thus, diffusion is coupled with oxygen leaving as aluminum enters. The compositions match several MAX phase alloys. They have significantly higher thermodynamic stability than either $\mathrm{Zr}$ or Al and form in preference to the oxides. Their presence significantly strengthens the cladding. However, they also set the stage for the eventual film failure at temperatures below where one might otherwise expect alumina to remain resistant to oxygen transport. MAX phase stability drives the enthalpy of the process. Thus, for operating conditions below $550^{\circ} \mathrm{C}$, the alumina coatings provide significant failure resistance.
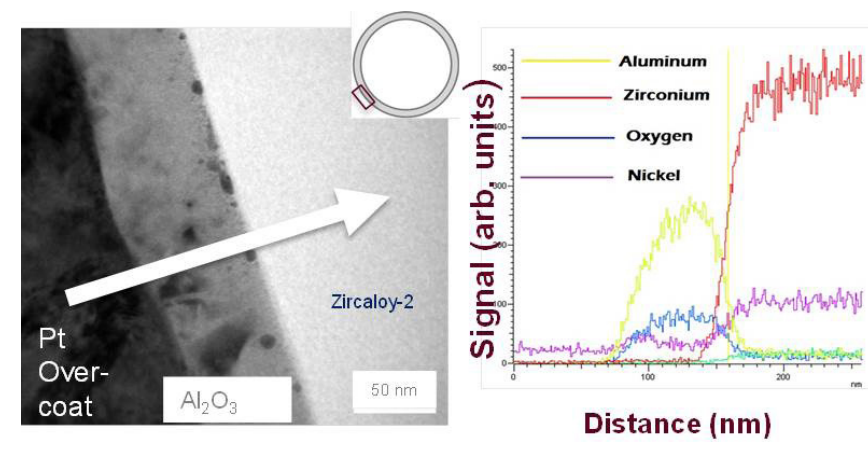

Figure 4. At left is a TEM picture of a 50-nm alumina-coated clad that has been annealed for 4 hours at $500^{\circ} \mathrm{C}$ in an oxygen atmosphere. At right is an energy-dispersive $\mathrm{x}$-ray traverse along the white arrow from the image at left. Note the smooth conformal coating and the sharp Zr/Al boundary limited by the traverse resolution. 


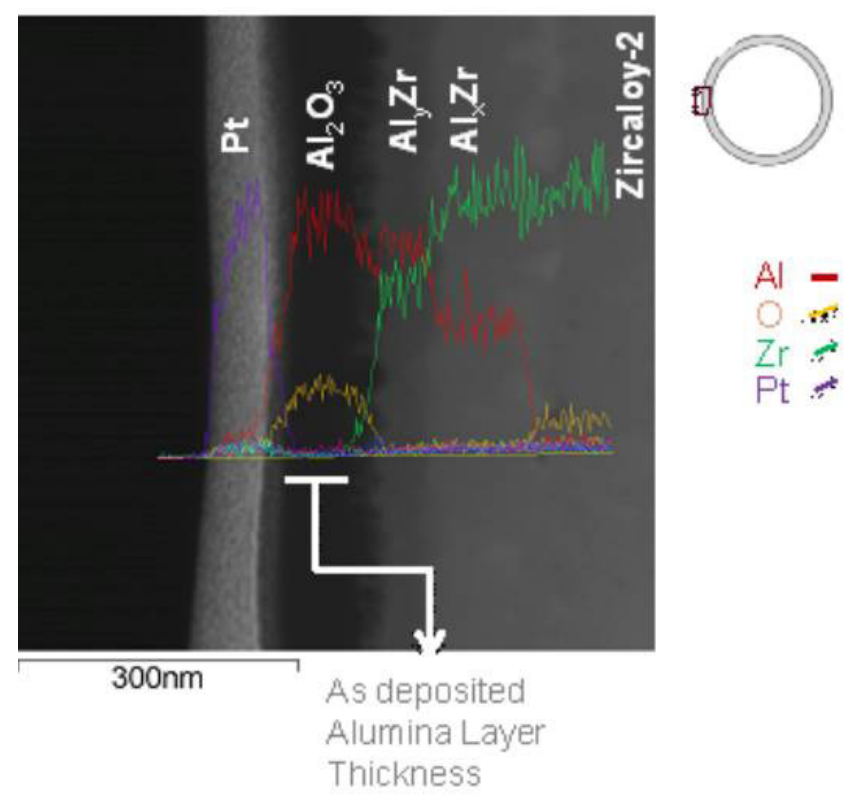

Figure 5. A TEM picture of a 50-nm alumina-coated clad that has been annealed for 4 hours at $550^{\circ} \mathrm{C}$ in an oxygen atmosphere. On top is an inregistry energy-dispersive $x$-ray traverse. At these temperatures, the stability of the two MAX $\mathrm{Al}_{\mathrm{x}} \mathrm{Zr}_{\mathrm{y}}$ phases promotes formation.

Our studies have resulted in our inclusion in a Westinghouse-led project sponsored by DOE/NE to enhance and test nuclear fuel accident tolerance.

\section{Magnetic Separation of Rare Earth Elements}

\section{4-004-R1}

Ross J. Ellis

\section{PROJECT DESCRIPTION}

Current techniques used to separate (refine) the lanthanides, also known as the rare earth elements, are inefficient and costly due to the very large volumes of liquid required in processing - a result of the lanthanides being chemically very similar, making them difficult to separate through chemical processes. Also, various expensive and hazardous chemicals are used in lanthanide refining to enhance their separation. The goal of this project was to look beyond chemical agents and instead explore the possibility of using magnetic fields to enhance the separation of lanthanides. The magnetic susceptibility of lanthanides changes a great deal across the lanthanide series, inviting new research into separating lanthanide ions based on large differences in their magnetic behavior (instead of small differences in chemical properties), or the use of magnetic fields to enhance existing separation technologies.
Recent publications point to a new class of organic fluids containing paramagnetic ions (especially lanthanides) that can be manipulated by an external magnetic field. These liquids are chemically similar to those often encountered in lanthanide-refining processes. The work performed under this project involved synthesizing new magnetically responsive lanthanide-containing organic liquids reminiscent of those also used in separation processes. These liquids consist of various lanthanide-binding organic ligands dissolved in alkane oils. The structures of the liquids were investigated to understand their relationships with magnetic properties.

\section{MISSION RELEVANCE}

The project is relevant to DOE's missions in energy and the environment. Rare earths are vital to a range of energy-related technologies, from wind turbines to nuclear power. Methods that effectively separate rare earth elements to yield purified materials are therefore crucial. Magnetic fields applied to responsive lanthanide-bearing liquids offer new opportunities to enhance lanthanide separations.

\section{RESULTS AND ACCOMPLISHMENTS}

Work in 2014 involved the synthesis of about 20 different kinds of organic liquid bearing concentrated lanthanide ions and testing their magnetic properties. These included ionic liquids, oils, and microemulsion phases, and their magnetic responses varied widely. Work in 2015 sought to understand the origins of these responses and to investigate whether different lanthanides could be separated within the same liquid phase by application of a field, or if magnetic fields could be used to manipulate phase properties to enhance existing approaches. Work focused on a category of lanthanide-containing liquids comprised of an organic oil (e.g., dodecane) bearing organic complexes of lanthanides (e.g., Ho(III), Gd(III), Eu(III)) bound to various ligands (organo-phosphates and organo-amides), with additions of nitric and hydrochloric acid and water. These were synthesized from lanthanide-chloride and lanthanide-nitrate salts and characterized using various spectroscopic methods, including extended $x$-ray absorption fine structure (EXAFS) spectroscopy. These confirmed that the lanthanides in the liquid were present as dissociated ions that had not formed precipitates or nanoparticles of some kind. The longer length-scale structure of these liquids varied a great deal, with lanthanide ions appearing to be associated with small water-segregated regions within the liquid. 
In FY 2015, small angle x-ray scattering (SAXS) showed that the bulk magnetic properties of liquid phases containing rare earth ions were correlated with fluid mesostructure. lons are solvated within aggregates of solvent molecules, and attractive interactions among the aggregates allow the ions to manifest a collective response. The bulk magnetic response derives from the long-range (aggregated) structure of the phase. The thermal motion of the ions, however, is substantially greater than the magnetic energy available from the magnetic field, making it impossible to separate individual ions using magnetic fields. However, enhancing the magnetic response of the liquid phase containing the dissolved lanthanide ions was found to be useful for controlling phase properties (e.g., magnetic pumping or phase separation). (See a schematic illustration of the collective behavior in Figure 1.)

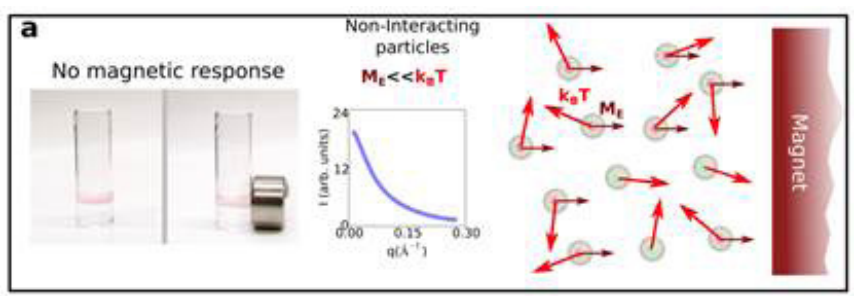

b
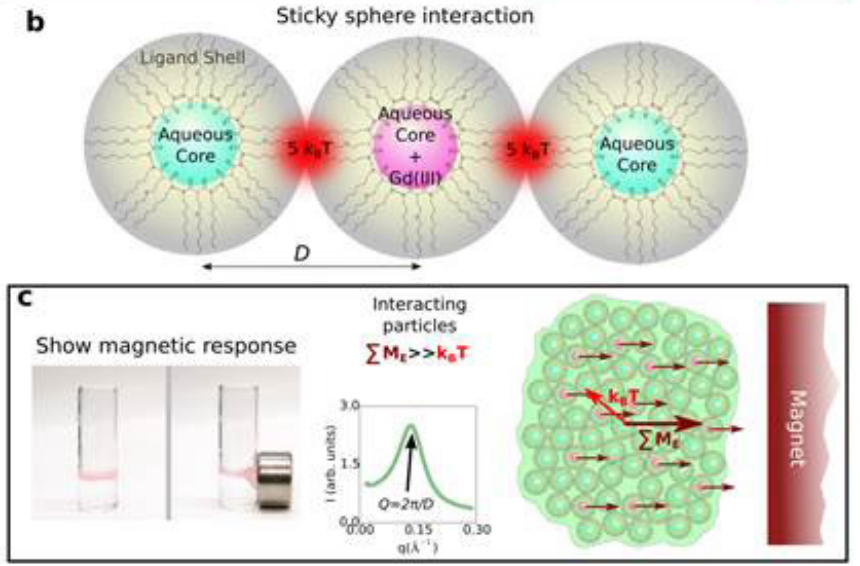

Figure 1. A summary of the correlation between solution mesostructure and bulk magnetic response. (a) In the uncorrelated 0.05 Molar Ho(III) reverse micellar solution: left figure shows no response to horizontally applied magnet; middle figure shows SAXS from this solution with typical profile of free particle scattering expected from a Brownian motion-dominated system; right figure shows the individual free reverse micelles where thermal energy $\left(k_{B} T\right)$ (red arrows) dominates the relatively weak magnetic force (brown arrows) exerted on the reverse micelles that contain Ho(III) (pink cores). (b) By increasing acidity, the reverse micelles interact and condense into a correlated phase stabilized by a cohesive interaction of $5 k_{B} T$. (c) In the correlated 0.05 Molar Ho(III) Winsor(III) phase: left figure shows a pronounced response to a horizontally applied magnet; middle figure shows SAXS collected from this solution with a typical profile for a correlated fluid evidenced by the correlation peak; right figure shows how the weak forces exerted by the magnet (small brown arrows) on the correlated reverse micelles manifest collectively in mesostructural domains (large brown arrow) overcoming $\mathrm{k}_{\mathrm{B}} \mathrm{T}$ (red arrow). SAXS data and photos of magnetic response were collected from: (a) 0.5 M DMDOHEMA in heptane solution containing 0.05 Molar Ho(III); (c) DMDOHEMA-heptane Winsor(III) microemulsion containing 0.05 Molar Ho(III). Pink Ho(III) phases are shown floating on water.
A patent has been filed based on this work, ANL-IN-15-014, describing how to enhance the magnetic properties of rare earth phases to facilitate phase separation (see Figure 2). A device has been conceptualized that could be used in industrial systems.

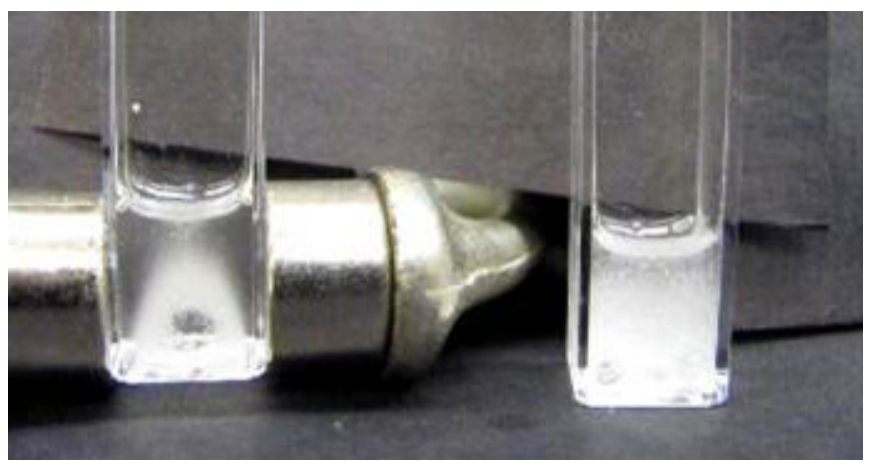

Figure 2. Magnetic fields enhance the separation of two phases used in the liquid-liquid extraction of Gd(III). This would enhance existing technology for lanthanide separations that relies on the mixing and de-mixing of phases.

\section{Dynamics of Spin Ice}

\section{4-018-R1}

\section{Axel Hoffmann and Olle Heinonen}

\section{PROJECT DESCRIPTION}

The dynamics of magnetic systems is fraught with complexities, making it an intriguing research topic from a fundamental point of view, but also attractive for applications, such as information processing. Toward this end, the nascent research field of magnonics aims at processing information with spin waves, the behavior of which can be controlled by the magnetic state of the underlying magnetic elements. In that respect, we have recently shown theoretically that so-called artificial spinice systems exhibit specific oscillations tied to peculiar magnetic defects.

Frustrated magnets, such as spin-ice systems, have been of scientific interest for a long time because of their highly degenerate ground states, which result in complex magnetic ordering and collective behavior. These frustrated magnets can be realized in crystalline materials, but analogous physics can also be observed in artificial spin ice, which consists of patterned nanoscale magnetic elements with dipolar interactions. Compared to crystalline systems, the artificial spin-ice systems have the advantage that the magnetization state of each individual "spin" (i.e., single-domain magnetic particle) is directly accessible through magnetic microscopy. 
Artificial spin ices exhibit large versions of elusive magnetic monopoles, which were originally predicted by Paul Dirac, and are connected by a chain of magnetic defects called a Dirac string. Up to now, experimental investigations of these systems have exclusively focused on the statistics of the defects and their quasi-static evolution after field and thermal cycling. But the new theoretical predictions open up new opportunities for characterizing topological defects in crystalline and artificially frustrated magnetic systems, as well as for using these defect strings and monopoles in information storage and computing devices based on magnetic oscillations.

The goals of our project are to experimentally investigate the localized dynamics due to topological defects in spinice systems and ultimately develop new approaches for their controlled manipulation. Toward this end, Brillouin light scattering (BLS) microscopy is an ideal tool, since it enables spatial imaging of the magnetization dynamics with high frequency and spatial resolution. Using this tool, we will test theoretical predictions at a microscopic level, which will be used to explore novel ways to control and manipulate the evolution of the topological defects.

\section{MISSION RELEVANCE}

This project is relevant to DOE's energy and science missions, and it will have both fundamental and applied impacts. The prospect that certain dynamic modes will only propagate along defect Dirac strings opens up exciting possibilities for guided energy and information flow in magnonic systems. This means that a controlled manipulation of the topological defects in spin-ice systems may enable fundamentally new ways of information processing with magnetization dynamics. The most direct impact of this work will be a better fundamental understanding of how topological defects impact the dynamic behavior of frustrated magnetic systems. Artificial spin-ice systems are ideal model systems for this, since they allow correlating statistical information from the whole ensemble, which is typically the only information available in crystalline frustrated systems, with a detailed microscopic arrangement of the individual spins comprising the frustrated system.

\section{RESULTS AND ACCOMPLISHMENTS}

In FY 2014, we developed the fabrication of artificial spin-ice systems integrated with co-planar waveguides, to enable their dynamic investigation, which at the same time have optical access for spatially resolved BLS microscopy. Furthermore, we refined the theoretical modeling of these systems.
In FY 2015, we obtained for the first time clear highfrequency magnetization dynamics spectra of artificial spin ices. As a function of magnetic field, we observed distinct changes in the absorption spectra, which can be directly correlated with different magnetization states. In fact, comparison to numerical simulations and a newly developed analytical model provide good agreement with the experimental observations. That differences in the magnetic field dependence of the dynamic spectra can be directly correlated with the presence or absence of specific topological defects was particularly exciting.

Early results with spatially resolved measurements using BLS spectroscopy reveal that indeed the magnetization dynamics of specific modes can be very inhomogeneous, which suggests that spatial distribution may directly reflect the locations of topological defects. However, we do not yet have a direct correlation between magnetization structure and local dynamic properties. In parallel with the optical investigations, we also developed an approach for the direct electrical detection of magnetization dynamics by using spin Hall effects. This provides an excellent signal-to-noise ratio, which suggests that electrical approaches may also be well suited for obtaining local information on magnetization dynamics. Furthermore, the preparation of such samples will provide a first step toward electric manipulation of the local magnetization structure and dynamics.

\section{PROPOSED FUTURE WORK}

$\square$ Adapting existing BLS microscope to imaging using the longitudinal magneto-optic Kerr Effect.

$\square$ Correlating local dynamics (measured with BLS microscopy) with actual magnetization structure.

$\square$ Extending investigations beyond square lattices to honeycomb and Kagome lattices.

$\square$ Fabricating samples with integrated microwave antennas for single-element excitation.

$\square$ Imaging the spatial distribution of defect dynamic modes upon local excitation.

$\square$ Exploring controlled manipulation of topological defects via microwave excitation. 


\section{Exploring the Universe with Full-Sky Simulations of the Cosmic Microwave Background}

\author{
2014-019-R1
}

Katrin Heitmann, Suman Bhattacharya, Hal Finkel,
and Samuel Flender

\section{PROJECT DESCRIPTION}

Our understanding of the cosmic microwave background $(\mathrm{CMB})$ is witnessing a paradigm shift through measurements of arc-minute scale anisotropies by a new generation of telescopes, such as the South Pole Telescope and the Planck Satellite. By combining these CMB observations with large-scale structural surveys, it is possible to address and connect some of the most fundamental questions in physics about the nature of dark energy, dark matter, the sum of the neutrino masses, and the number of relativistic species. To realize the potential of these measurements, next-generation studies in theory, modeling, and simulation are needed. Accurately capturing the physics of the real universe will be crucial for interpreting CMB experiments and answering questions pertaining to fundamental science.

This project is based on a set of state-of-the-art simulations of the CMB sky carried out on Mira, Argonne's petascale IBM Blue Gene/Q supercomputer. Extracting exciting science from the simulations is a major aim of this project. We are generating a set of CMB maps spanning different cosmological models and astrophysical effects.

\section{MISSION RELEVANCE}

The project addresses key areas in the DOE Office of High Energy Physics Cosmic Frontier focus area and touches upon related questions in the Intensity Frontier of particle astrophysics.

\section{RESULTS AND ACCOMPLISHMENTS}

In FY 2014, we built a first set of CMB maps including astrophysical effects and scaled up our analysis pipeline to enable efficient processing of our very large simulations. The first CMB map included foreground sources using a semi-analytic approach. Dark matter halos were identified and the Sunyaev-Zel'dovich (SZ) effect was added by using a semi-analytic model for gas physics. The $\mathrm{SZ}$ effect is a distortion of the CMB caused by the inverse Compton scattering of CMB photons from high-energy electrons in clusters. This scattering increases the average energy of those photons. Infrared sources were also added to the halos by using a semi-analytic approach calibrated to observations. The resulting synthetic maps in different wavebands were used to investigate the effects of different biases on the CMB lensing power spectrum. The lensing is due to the gravitational influence of intervening matter between the $\mathrm{CMB}$ and the observer.

In FY 2015, we finished work on the efficient construction of light-cone outputs, placing the particles and halos from the discrete snapshots in the simulation onto the surface that describes the temporal evolution of the universe. By placing an observer in the center of the simulation and building a light-cone, we basically mimic what an observer would see when looking out into the universe. First, we enhanced HACC (our Hardware/ Hybrid Accelerated Cosmology Code) to produce particle light-cones in situ. Second, a new, parallel stand-alone tool was written that can efficiently extract light-cones from output files. We integrated this light-cone capability with our SZ modeling pipeline, as optimized to work within the HACC framework. Figure 1 shows an example of an SZ map created from one of our light-cone halo catalogs. The pipeline has been applied to a large simulation and has been used to investigate possible systematic effects that could degrade the SZ signal.

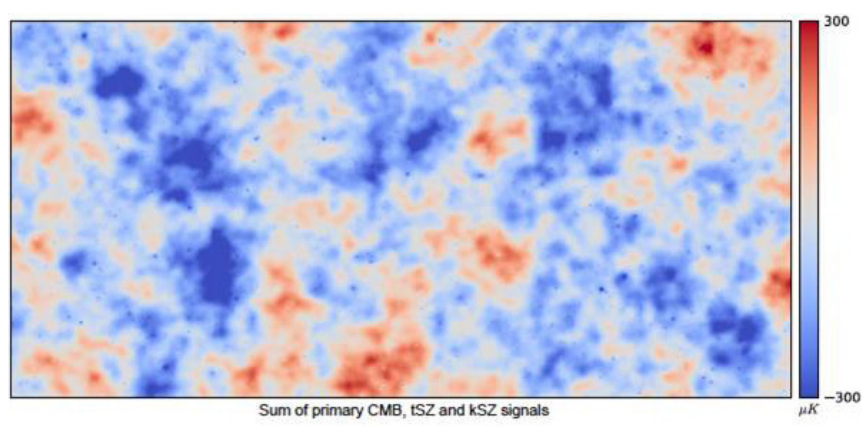

Figure 1. Simulated map of the primary cosmic microwave background and the summed thermal and kinetic Sunyaev-Zel'dovich signals (tSZ and kSZ, respectively) as observed by the South Pole Telescope. This synthetic map allows us to investigate the effect of systematic effects on the signal we can extract from the data. The colors show the temperature fluctuations with respect to the mean temperature. Clusters of galaxies can be identified by the SZ effect, which is seen as the very well defined temperature decrements (small blue dots).

Finally, the synthetic maps were used to aid the analysis of data taken from the South Pole Telescope (SPT) and the Dark Energy Survey (DES).

\section{PROPOSED FUTURE WORK}

During FY 2016, we will finalize the analysis of data from DES and SPT. We will apply our analysis pipeline to a set of different cosmologies and investigate the possibilities of extracting precision cosmological information from SZ measurements. We will also engage with upcoming surveys (we have begun work with SPHEREx and the Dark Energy Spectroscopic Instrument) to provide forecasts for their measurements. 


\section{Development of Large- Area Vacuum Ultraviolet Microchannel Plate Photodetectors for Use in Large Liquid Argon and Xenon Time Projection Chambers}

\author{
2014-023-R1 \\ Jonathan M. Paley

\section{PROJECT DESCRIPTION}

The goal of this project is to reconfigure and optimize the large-area photodetectors developed at Argonne for operation in a liquid argon time projection chamber (LArTPC). Noble liquid time projection chambers are used in many neutrino, dark matter, and neutrinoless double beta decay experiments. The vacuum ultraviolet scintillation light produced in these particle detectors is used for particle identification, calorimetry, and determination of the timing of charged particle interactions inside the liquid. With that information, it becomes possible to construct the complete threedimensional paths of such particles.

Traditional detector technologies, such as glass-based photomultipliers, silicon photomultipliers, and avalanche photodiodes, have served adequately in LArTPCs and liquid xenon time projection chambers, but they are very expensive per unit area. The core components of large-area photodetectors are a pair of microchannel plates (MCPs) that are coated with a resistive layer via atomic layer deposition (ALD) and a photocathode. The MCPs that we are developing will have similar or better performance and may cost significantly less per unit area. Since these components behave differently at cryogenic temperatures, significant effort was dedicated to optimizing device design for operation in a cryogenic environment.

\section{MISSION RELEVANCE}

The project is relevant to DOE's mission in science. LArTPC detector technology will be a central component of experimental neutrino physics over the next 1020 years and is a key driver of the DOE's Office of High Energy Physics investment in facilities and research. The main neutrino detector in the future Long Baseline Neutrino Facility, recently strongly endorsed by the Particle Physics Project Prioritization Panel (P5), will be a 10-40-kiloton LArTPC; many smaller neutrino experiments under development will also involve the use of this detector technology.

\section{RESULTS AND ACCOMPLISHMENTS}

During FY 2014, components that constitute the detector package and the MCPs themselves were tested in liquid nitrogen (LN2) to determine survivability and, in case of the MCPs, resistance change. The package consists of a borosilicate glass anode plate, sidewall, and top window. The anode plate is bonded to the sidewall with a glass frit (sealing glass). The top window is sealed to the sidewall using indium wire that is crushed between the surfaces and heated to promote a solid bond. Testing demonstrated that all components survive immersion in LN2, including a sealed detector under ultra-high vacuum, which continued to hold vacuum. As the MCPs were immersed in LN2, the resistance was observed to increase by approximately two orders of magnitude. As a result of this measurement, work was planned for FY 2015 to determine a new target resistance for MCP functionalization such that as the MCPs' resistance increases upon cooling, it comes into the resistance range required for usable operation, which is roughly 10-100 mega ohms (M $\Omega$ ).

The resistance of the tungsten-aluminum oxide ALD coating that is applied to the MCPs during functionalization, which forms the basis for voltage biasing the MCPs, may become prohibitively high at liquid argon temperature. The Argonne MCP detector group determined that the optimal resistance is in the range of 20-100 M $\Omega$. During FY 2015, the target resistance was successfully achieved by changing the ALD parameters, and two initial samples of the MCPs were produced. Testing the bare MCPs for gain during development necessitated that they be in a high-vacuum environment, which made it difficult to cool the MCPs to the temperature of liquid argon. So we fabricated an improved dewar that allows for much improved temperature control. We also verified that indium bonding, which is used for sealing room temperature photodetectors, is unaffected by the cryogenic environment.

FY 2015 was the final year of this project. Much additional development work will be required to realize the envisioned detection capability. We are actively seeking alternative sources of support. 


\section{New Paradigms for High $T_{\mathrm{c}}$ Superconductivity in Acene- Based Materials}

\author{
2014-025-R1 \\ Wai-Kwong Kwok and Scott M. Brombosz
}

\section{PROJECT DESCRIPTION}

The search for high critical temperature $\left(T_{\mathrm{c}}\right)$ superconductors (HTS) has recently been bolstered by the discovery of a new class of organic superconductors: alkali-doped acenes. Acenes (a class of polycyclic aromatic hydrocarbons) have been explored for application in thin-film transistors and organic photovoltaics for many years, leading to a wealth of knowledge about their synthesis and electronic properties. Doped acenes, however, are only just now coming under greater scrutiny because of their unique properties. A potential new application plus the extensive base knowledge leaves the field of superconducting doped acenes open for rapid exploration. Most efforts to produce alkali-doped acenes have relied on simple solidstate reactions and heating to high temperatures. We believe solution-based methods provide an opportunity to precisely control the doping levels, as well as to more accurately characterize these environmentally sensitive materials. In particular, the growth of single crystals of these materials will allow us to unambiguously assign not only the structure and doped state, but also the threedimensional arrangement of the constituents, upon which the development of superconducting phases depends.

\section{MISSION RELEVANCE}

The search for HTS has been highlighted by the DOE BES Advisory Committee as being critical for the energy security of the nation. A superconducting electrical grid would help reduce transport losses, reducing the need for localized power generation. Additionally, HTS would alleviate the need for cryogens, such as strategically important helium, to cool the high field magnets used in such instruments as magnetic resonance imaging scanners. Current early-stage research on alkali-doped acenes provides a basis for potential ground-breaking results. While the investment in a project at such an early stage incurs risk, the potential payoff of discovering new HTS would be substantial.

\section{RESULTS AND ACCOMPLISHMENTS}

Initial work in FY 2014 had focused on developing methods for removing significant impurities in commerically available acenes such as picene, coronene, and dibenzopentacene. For lower molecular weight acenes, vacuum sublimation provided high-quality crystals suitable for x-ray diffraction. However, the higher molecular weight acenes furnished pure but polycrystalline materials. Method development for solution-phase doping with alkali metals resulted in the characterization of potassium-doped coronene by x-ray diffraction.

In FY 2015, the solution-based method for producing alkali-doped acenes was optimized. This method was found to be useful for quickly producing doped alkali states under much milder conditions than the literature reports regarding solid-state reactions of acenes with alkali metals at high temperatures. Sonication allowed the reaction rate to increase substantially for less reactive acene-alkali metal combinations. From these solutions, doped acenes were isolated so they could be further processed to induce crystallization. It was found that adding a supporting ligand was important for the production of high-quality single crystals of the acenes.

An array of samples was produced by combining sodium, potassium, rubidium, or cesium with several acenes, including phenanthrene, pyrene, perylene, coronene, and dibenzopentacene. From the ligand-supported samples, crystal structures of many of the highly air-sensitive samples were obtained. As shown in Figure 1, the use of different alkali metals resulted in variations in the packing arrangement that became more dramatic as the atomic radius of the dopant increased.

To date, only samples with supporting ligands have provided high-quality single crystals. Samples produced by using non-coordinating solvents (such as toluene) have, however, produced doped acenes as amorphous solids. In literature reports of the high-temperature solid-state reactions, much attention has focused on the stoichiometry of the alkali metal dopant, as well as on the annealing conditions. Typically, an incorrect stoichiometry or insufficient annealing time has led to decreased superconducting transition temperatures or losses of superconductivity altogether. Based on Raman spectroscopic characterization (Figure 2), our method has provided phenanthrene reduced by potassium to the -3 oxidation state cleanly by precipitating it out of solution. Most notably, this -3 product formed even when only one equivalent of potassium was added, indicating that the $-1,-2$, and -3 states are in equilibrium and only the highly charged state is insoluble in the solvent system. This provides an excellent example of the advantages of solution-based production of alkali-doped acenes and highlights the need to produce these materials under a more homogenous, controlled environment. 


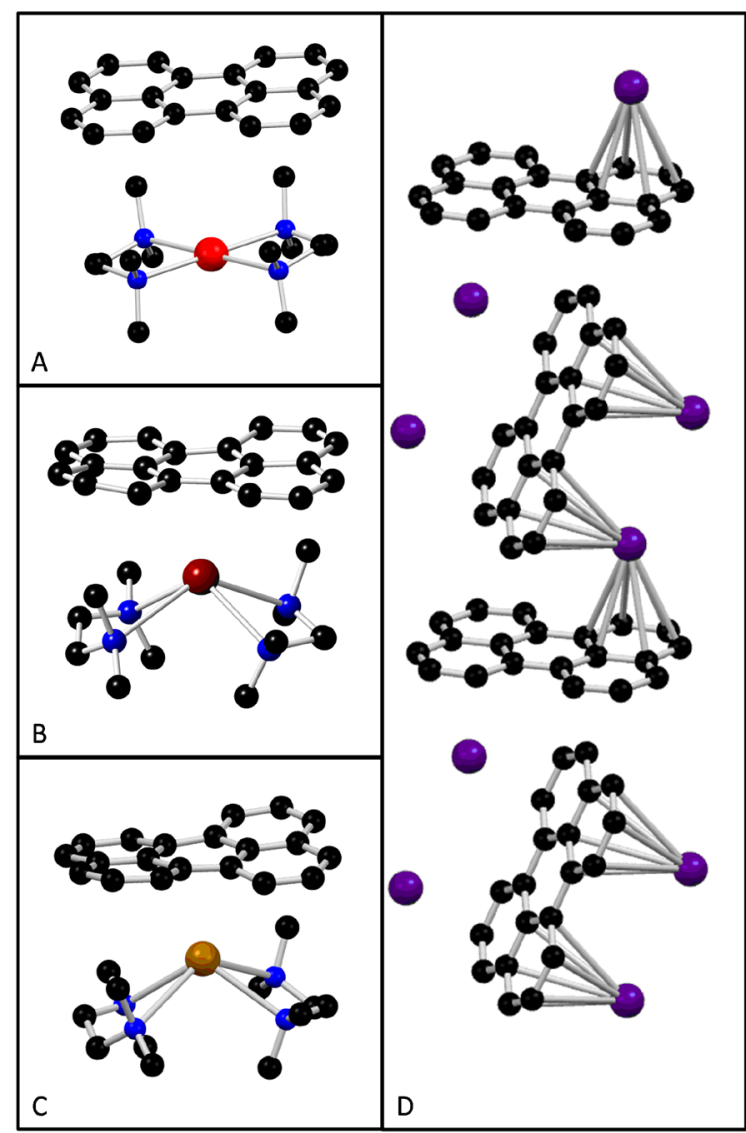

Figure 1. Crystal structures of perylene doped with (A) sodium (light red), (B) potassium (dark red), (C) rubidium (tan), and (D) cesium (purple). Solvent molecules have been removed from the structures in D for clarity. Sodium exhibits less direct interaction with the singly charged $\pi$-face of the acene than the potassium and rubidium analogues. While A-C show alignment of the alkali metal with the center face, $D$ demonstrates coordination of the cesium atoms to the outer rings due to the higher oxidation state (-1.5) of the acene, leading to a different packing arrangement, which indicates the oxidation state can strongly influence spatial arrangement and, potentially, bulk physical properties.

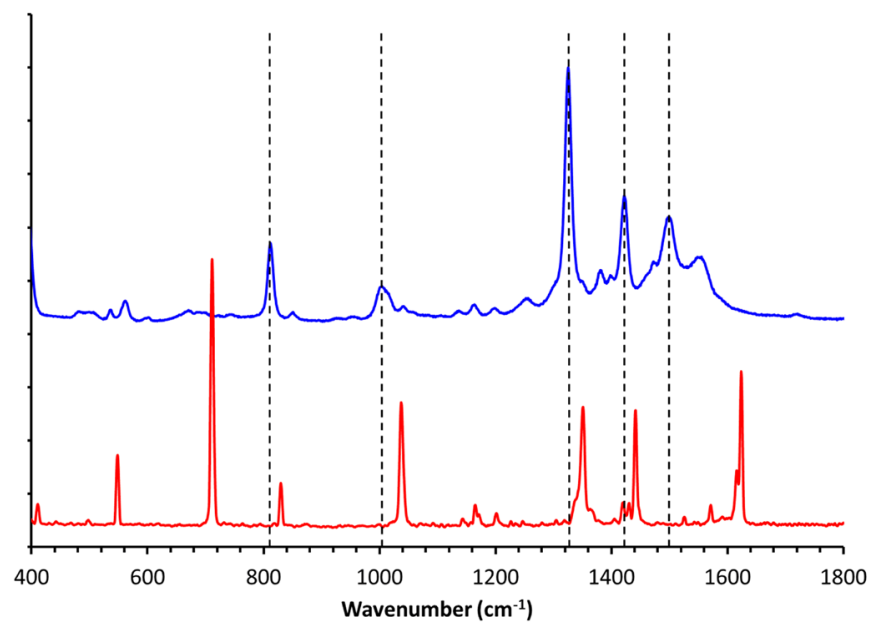

Figure 2. Raman spectrum of pristine phenanthrene (red) and potassiumdoped phenanthrene (blue). Peak positions of Raman modes identified in the literature as the -3 state are indicated by the vertical lines.

\section{PROPOSED FUTURE WORK}

Research in FY 2016 will focus on furthering the solution-based chemistry to achieve materials in the superconducting -3 state. Physical measurements, including magnetization, will be used to search for magnetic and superconducting states. We will explore sequential application of the precipitation and ligation methods as a means of first generating the desired state and then introducing supporting ligands to obtain single crystals of these highly charged species. We will continue to explore the electrochemical generation of acenes as a secondary pathway to the highly reduced states. Feedback from computational studies based on the elucidated crystal structures will further guide the discovery of new, promising alkali-acene pairs.

\section{Plastic Artificial Leaves for Water Splitting}

\section{4-046-R1}

Lin X. Chen

\section{PROJECT DESCRIPTION}

Best use can be made of the energy in sunlight by first storing it in the form of energy-dense chemical bonds (e.g., hydrogen $\left(\mathrm{H}_{2}\right)$ or methanol $\left(\mathrm{CH}_{3} \mathrm{OH}\right)$ ), which requires the development of highly robust and efficient photocatalysts. Additionally, photocatalysts that generate $\mathrm{H}_{2}$ can potentially be used as carbon dioxide- $\left(\mathrm{CO}_{2}-\right)$ reduction catalysts to generate $\mathrm{CH}_{3} \mathrm{OH}$, which would allow for the reduction of the greenhouse gas $\mathrm{CO}_{2}$ in the atmosphere. The main challenge in using sunlight as an energy source involves coupling single-photon absorption events with multiple electron/hole redox reactions for splitting water $\left(\mathrm{H}_{2} \mathrm{O}\right)$ into hydrogen and oxygen (i.e., $2 \mathrm{H}_{2} \mathrm{O} \rightarrow 2 \mathrm{H}_{2}+\mathrm{O}_{2}$ ).

In this project, we combine our knowledge of photoinduced electron transfer in artificial photosynthesis and the photophysics of conjugated polymers that have been used in organic photovoltaic devices in building a platform for photocatalytic water splitting, particularly to achieve hydrogen generation. We seek to integrate a single, organic photovoltaic $p-n$ junction and a water-splitting catalytic transition metal center into a bilayer organic "artificial leaf." An artificial leaf can be formed when the water oxidation and reduction catalytic reactions proceed simultaneously as the device absorbs sunlight. The scope of the project includes: (1) metallopolymer design and synthesis, (2) physical characterization of excited state properties and structures, and (3) testing of photoinduced hydrogen generation under various conditions using different materials and test devices. 


\section{MISSION RELEVANCE}

This project is highly relevant to the DOE missions in energy, the environment, and basic science. The project is especially relevant to the Solar Energy and Photochemistry and Catalysis programs in the Chemical, Biological, and Geological Science Division of DOE's Office of Basic Energy Sciences (BES) and may be also of interest to the Office of Energy Efficiency \& Renewable Energy (EERE) and the Air Force Office of Scientific Research with respect to the generation of fuel from renewable and clean energy sources.

\section{RESULTS AND ACCOMPLISHMENTS}

Polymer Synthesis, Characterization, and $\mathrm{H}_{2}$ Generation. Our previous work focused on designing, synthesizing, and characterizing novel photocatalytic architectures that can simultaneously combine the light-harvesting properties of conjugated polymers and transition metal catalysts. We initially focused on optimizing the hydrogen evolution half-reaction. The two synthesized polymer structures shown in Figure 1 (top) consist of an electrondonating benzodithiophene (BDT; PBB1) and electronaccepting perylene diimide (PDI; PPB1) copolymerized with a weakly electron-deficient bipyridine (bpy) block.

The ultraviolet-visible (UV-vis) absorption spectra of PBB1 and PPB1 (not shown) are direct spectroscopic probes of metal chelation. Indeed, upon Co(II) titration of divalent cobalt (Co(II)), the UV-vis features of PBB1 undergo a systematic red shift, and saturation is reached at a [cobalt chloride $\left.\left(\mathrm{CoCl}_{2}\right)\right] /[\mathrm{bpy}]$ ratio of 1.0/1.0. Thus, all available bpy sites bind Co(II). Theoretical calculations showed that these spectral changes are due to an energetic stabilization of the lowest unoccupied molecular orbital upon binding the cationic Co(II). Similar overall results were obtained for PPB1, but are reflected in higher energy absorption features. The polymer band gaps and energy level alignments, which drive photocatalysis for PBB1 and PPB1, indicate that both should be capable of $\mathrm{H}_{2}$ generation and that PPB1 should also carry out water oxidation. Indeed, we have shown in a recent breakthrough that both $\mathrm{Co}(\mathrm{II})$-chelated PBB1 and PPB1 catalyze the formation of $\mathrm{H}_{2}$ from $\mathrm{H}_{2} \mathrm{O}$ by using light and a sacrificial reductant (Figure 1, bottom). Thus, these metallopolymers represent novel constructs for photocatalysis, and, to the best of our knowledge, photo-catalytic $\mathrm{H}_{2}$ production from this type of artificial photosynthetic system has yet to be reported. Interestingly, $\mathrm{H}_{2}$ generation depends strongly on $\mathrm{Co}$ (II) catalyst loading [max 10 \% Co(II) for PPB1] (Figure 1, bottom), which proves the core idea of the project-that a conjugated polymer can successfully harvest photons and donate electrons to catalytic sites and carry out multi- electron/multi-proton reactions. Also, these results set the stage for detailed analyses of the reaction mechanism, including light harvesting, charge transport, and catalysis.
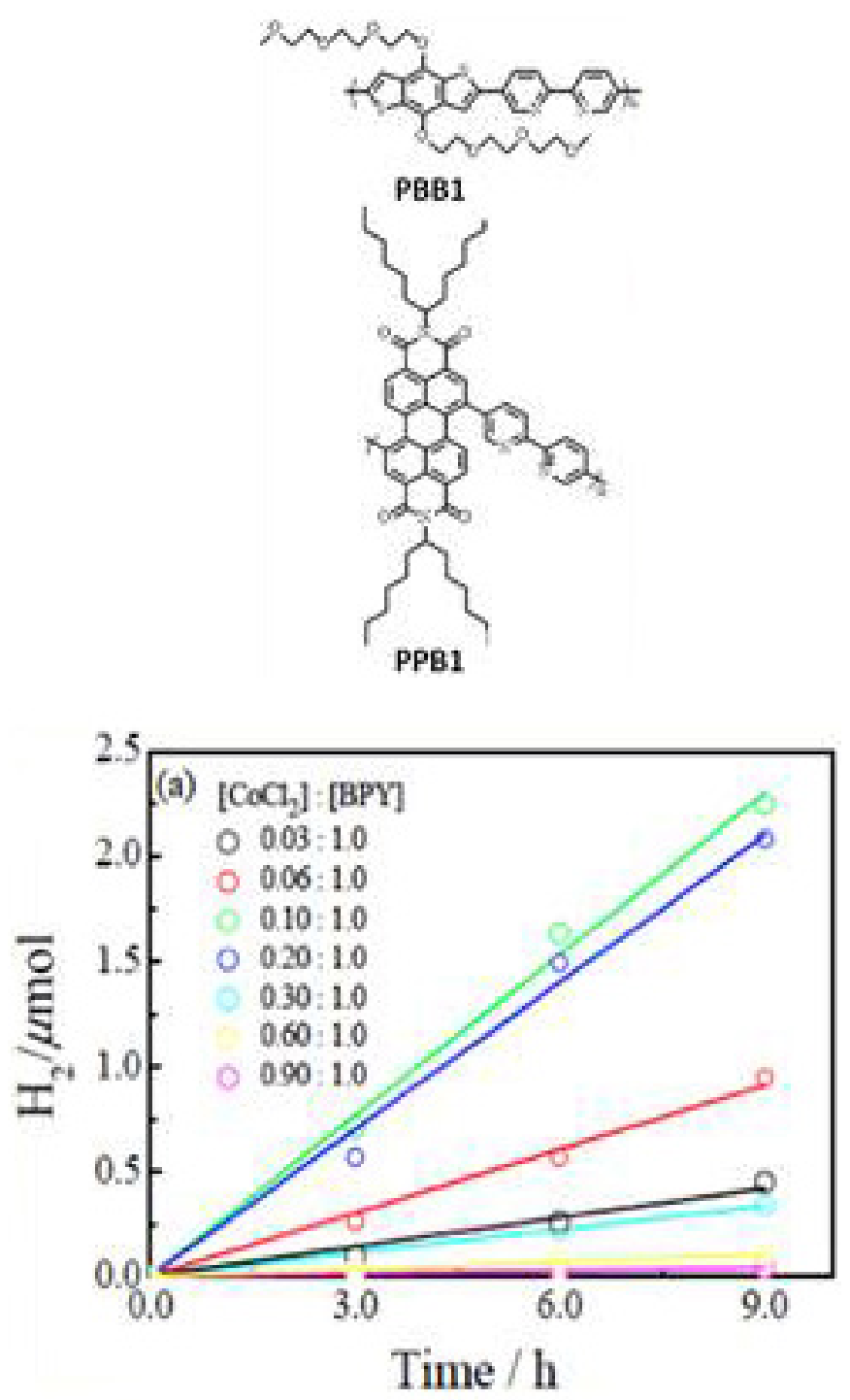

Figure 1. The PBB1 and PPB1 polymer structures investigated (top) and hydrogen generation vs. illumination times by light with different ratios of Co(II) and bpy (bottom), showing that the highest yields were achieved in the ratio range of $0.1-0.2$.

Polymer Excited State Properties and Charge Separation. The ability to carry out photocatalysis is governed by the ability of the conjugated polymer to harvest light and effect multiple electron/hole transfers to the transition metal catalyst. Both fluorescence and transient absorption spectroscopies indicate that electron/energy transfer is strongly favored in these metallopolymer systems. For instance, both free PBB1 and PPB2 are highly fluorescent in solution; however, upon $\mathrm{Co}(\mathrm{II})$ titration, the fluorescence is strongly quenched as a result of charge/energy transfer to $\mathrm{Co}(\mathrm{II})$. In transient absorption measurements carried out for PBB1 (Figure 2), excitation at a wavelength of 
491 nanometers $(\mathrm{nm})$ results in a strong ground state bleach at $\sim 477 \mathrm{~nm}$ and a broad excited state absorption at $\sim 600 \mathrm{~nm}$ (Figure 2A). Stimulated emission is observed at $\sim 550 \mathrm{~nm}$. Upon addition of Co(II), the stimulated emission is quenched (Figure 2B), which is consistent with the fluorescence quenching results described above and further confirms a charge/energy transfer process from the polymer backbone to the metal, which occurs upon Co(II) chelation. Similar results are obtained for PPB1 (not shown). These signals may be associated with a charge-separated intermediate and further indicate that time-resolved spectroscopic methods can be used to characterize the excited-state electron-transfer processes and how they are coupled to intermediate formation.
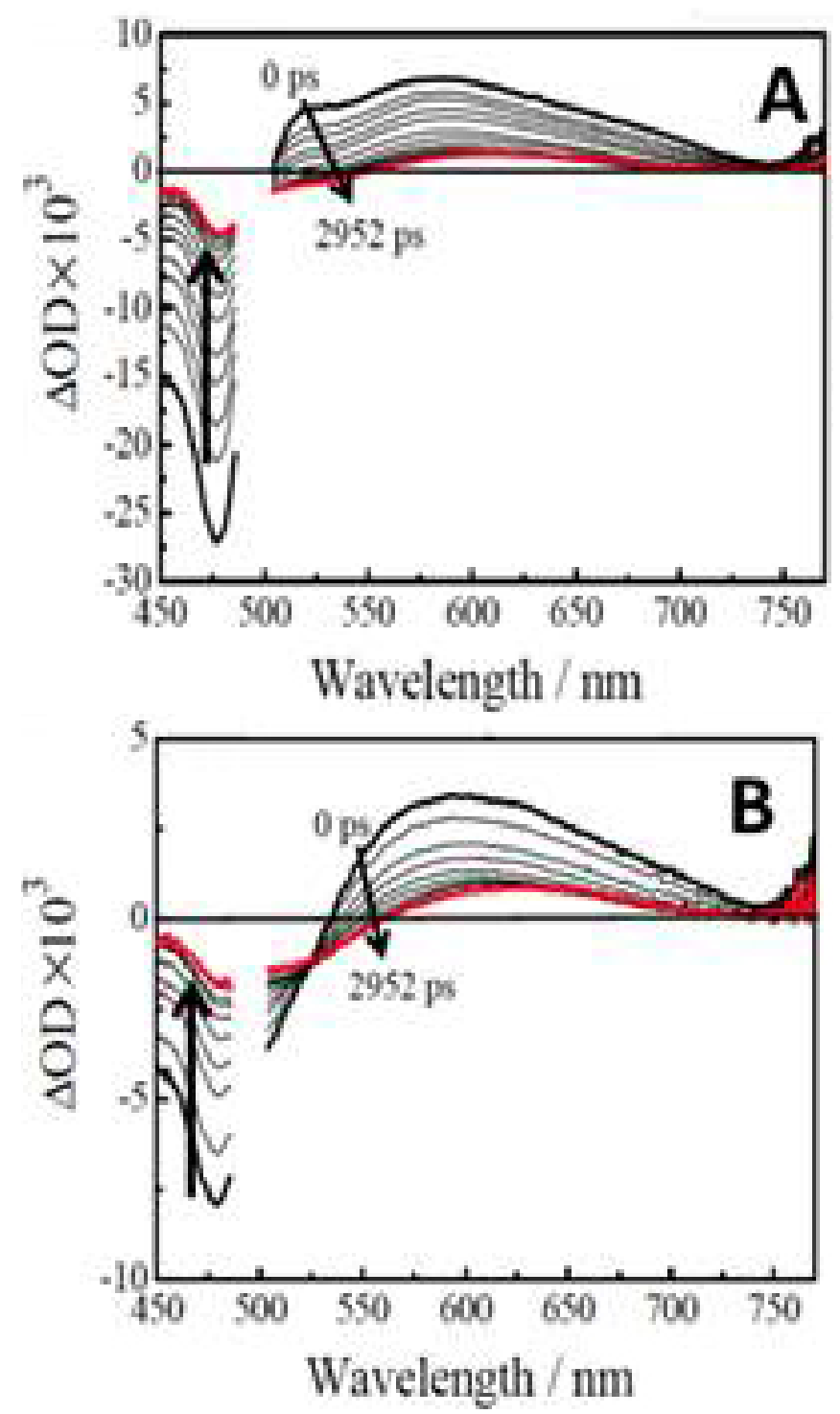

Figure 2. Transient optical absorption spectra for (A) PBB1 polymer only excited by 491-nm light and (B) PBB1 chelated with Co(II) excited by 491-nm light. The delay times are from 0 to 2,952 picoseconds (ps), and the changes in direction with the delay time are indicated by arrows.

\section{PROPOSED FUTURE WORK}

In FY 2016, our work will include efforts to:

$\square$ Change the relative ratio of conjugated polymer parts with metal chelating bpy sites in these metallopolymers to search for the optimal light harvesting/electron transfer and redox reaction efficiency.

$\square$ Investigate the synthesis of donor/acceptor polymers, as well as their excited-state properties with other thirdrow transition metals.

$\square$ Investigate the water oxidation half-reaction in the PPB1 system.

$\square$ Combine hydrogen and water oxidation half-reactions into a self-contained catalytic system.

$\square$ Carry out detailed multi-wavelength, time-resolved spectroscopic studies of metallopolymers under catalytic conditions from femtosecond (fs) to microsecond ( $\mu \mathrm{s})$ timescales.

$\square$ Receive beam time at Argonne's Advanced Photon Source and the Center for Nanoscale Materials to investigate the local geometric and electronic structures of the catalytic transition metal centers.

\section{Carbon Nano-Network as Next-Generation Support for Catalysis and Electrocatalysis}

\section{4-051-R1 \\ Di-Jia Liu and Jeff Miller}

\section{PROJECT DESCRIPTION}

This project focuses on developing a new type of catalytic substrate-a carbon nano-network-for catalytic and electrocatalytic applications. It contains macropores and micropores almost exclusively and therefore is fundamentally different from conventional carbon supports. It promises to overcome the shortcomings of traditional carbon supports by providing the following morphological advantages: (1) a highly microporedominant surface area and a pore volume crucial for enhancing the rate of heterogeneous and electrocatalytic reactions; (2) direct macropore-to-micropore connections that bypass the mesopores, thus lowering mass-transport resistances for reactants/products; and (3) catalytic active sites that are interconnected through a protective fibrous network and have better charge/thermal conductivities and chemical stability. The carbon nano-network substrate is being prepared through the electrospin method, which produces a finely dispersed metal-organicframework (MOF) encapsulated inside nanofibers. Upon 
thermolysis, a new structure forms that features activesite decorated catalytic carbon fibers connected by a network of "strings" and "knots" (Figure 1). The goal of the project is to design and fabricate several nano-network catalysts with different morphological properties and demonstrate their improved performance in fuel cells and in biofuel catalysis.

\section{MISSION RELEVANCE}

This project focuses on improving energy efficiency and renewable energy production. The project is therefore relevant to DOE's missions in energy supply and environmental quality. A pore-size-controlled carbon nano-network would not only have a strong impact on fundamental catalysis science but also have practical applications in next-generation catalysts and electrocatalysts. The new materials developed from this work could be applied directly to solve some of the most pressing problems in energy conversion and environmental protection.

\section{RESULTS AND ACCOMPLISHMENTS}

In FY 2014, we developed a "one-pot" synthesis method to prepare several MOF based electrocatalysts and successfully demonstrated their catalytic activities toward oxygen reduction in alkaline media. The objective of the project for FY 2015 was to complete developing the material chemistry for an MOF-based, nano-network nonprecious-metal electrocatalyst (NPMC) and demonstrate its application in a fuel cell. We have made the following major accomplishments:

Development of a Highly Porous Carbon Nano-network Catalyst. One key objective of this project is to develop a highly porous nanofibrous carbon network embedded with a high density of catalytically active sites. We achieved this objective by electrospinning a liquid mixture containing soluble transition metal organometallics, suspended MOF crystallite, and polymers to form an organic nanofibrous network precursor. The precursor was subsequently cured and activated at elevated temperature to be converted to an electrocatalyst with a carbon nano-network architecture (Figure 1). The new architecture provides facile mass/charge transfers to/from the microporous catalytic sites through a macroporous network and improves electrocatalyst durability by eliminating corrosion-prone inter-particle percolation. The electrocatalyst was fabricated into a membrane electrode assembly and was tested under fuel cell operating conditions. We found that, although the catalyst did not contain any precious metal, it produced a performance comparable to that of platinum-based material. In a single-cell test, the membrane electrode containing the nanofibrous non-precious metal catalyst delivered an extrapolated volumetric activity of 450 amperes/ centimeter ${ }^{3}\left(\mathrm{~A} \mathrm{~cm}^{-3}\right)$ at 0.8 volts $(\mathrm{V})$, representing the highest reported value in the literature.

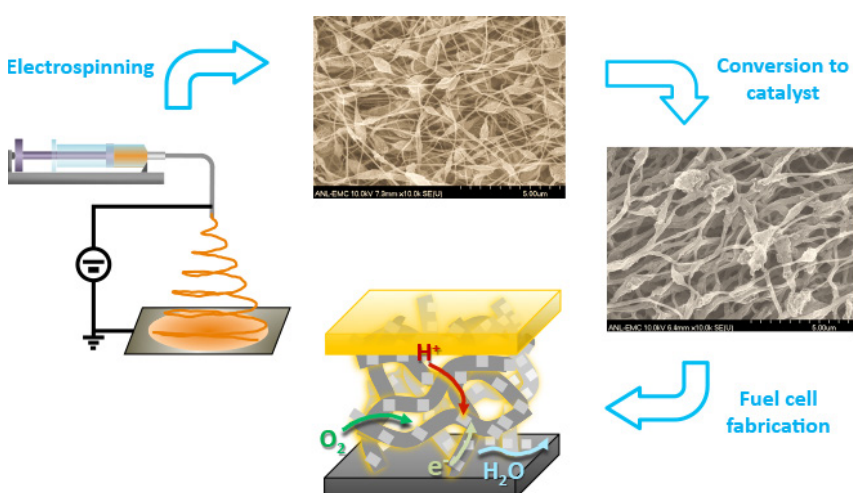

Figure 1. Diagram depicting the steps in preparing a non-precious metal nanofibrous network catalyst, including electrospinning, thermal activation, and activity testing in a fuel cell electrode.

Development of Novel Bimetallic Catalyst through Platinum Embedded MOF. The three-dimensional framework of an MOF is formed by repeating chelated transition metal-organic ligand entities. The voids between these entities can host secondary molecules, such as organometallics. Under proper activation conditions, highly dispersed, uniformly distributed bimetallic catalytic crystallites can be formed through the reaction between an MOF's metal and secondary organometallic components. We explored this approach by infiltrating an organometallic platinum (Pt) precursor into a cobalt (Co)-based MOF, followed by thermal activation and post treatment. We thus obtained a Pt-Co bimetallic catalyst having a very low Pt concentration. This catalyst was later incorporated into a fuel cell cathode and tested under both oxygen and air. A high oxygen reduction catalytic activity was observed over this catalyst, even though the Pt loading was substantially lower than those used in current commercial fuel cells. More importantly, excellent catalytic stability was observed under an accelerated stress test condition.

This work resulted in the following patent applications in FY 2015:

Liu, D.-J., S. Ma, G. Goenaga, D. Zhao, “Non-Platinum Group Metal Electrocatalysts Using Metal Organic Framework Materials and Method of Preparation." Patent Application No. 14/634,581 filed February 2015. [ANL-IN-14-079.] 


\section{PROPOSED FUTURE WORK}

We will continue to investigate and optimize the catalytic activity and durability of the bimetallic catalyst for the electrochemical application. We will also explore other bimetallic or trimetallic catalytic systems using MOFbased approaches. Additionally, we will investigate MOF-based and nanofibrous-based catalysts in the biofuel application. Various characterization tools will be applied to study the catalyst's physical and chemical properties during synthesis and under reaction conditions.

\section{Thin Film Skyrmion Spin Textures}

\section{4-054-R1}

Suzanne G.E. te Velthuis and Axel Hoffmann

\section{PROJECT DESCRIPTION}

A few years ago, it was discovered that certain magnetic materials lacking inversion symmetry could form complex magnetization patterns with magnetic spins, forming vortex-like structures. More surprisingly, this "skyrmion" structure of the magnetization could be manipulated with electric currents up to five orders of magnitude smaller than the currents required for current-driven magnetization dynamics in ordinary ferromagnetic systems. This feature makes magnetic skyrmions promising candidates as information carriers in low-power, ultra-dense memory and logic devices.

The goals of this project are to explore the possibility of creating and stabilizing room-temperature skyrmions in a variety of thin films and to then develop new approaches for skyrmion manipulation. We characterize the magnetic texture associated with skyrmions by means of optical imaging, neutron scattering, and electric transport measurements. New strategies for forming and manipulating individual skyrmions in patterned structures are being investigated, and the results may directly affect applications in information storage and computation.

\section{MISSION RELEVANCE}

This project is relevant to DOE's mission in science. The main impacts from this work will be on low-power information storage and logic device concepts. The focus on the underlying fundamental science is of interest to DOE's Office of Basic Energy Sciences. The demonstration of the controlled manipulation of skyrmions in magnetic thin films should generate opportunities for technology transfer.

\section{RESULTS AND ACCOMPLISHMENTS}

In FY 2014, we studied skyrmion bubbles in materials where long-range dipole interaction promoted stabilization of magnetic bubbles. Specifically, we illustrated how in films (6 micrometers thick) of the magnetic insulator yttrium iron garnet (Bi-doped $\mathrm{Y}_{3} \mathrm{Fe}_{5} \mathrm{O}_{12}$, YIG:Bi) covered by a thin layer of non-magnetic heavy metal platinum (Pt) (4 $\mathrm{nm}$ thick), skyrmion bubbles could be moved as well as made to shrink in size, by driving a current through the Pt. Measurements of the Hall resistivity also indicated the presence of an additional Hall resistivity superimposed on the ordinary Hall resistivity of Pt. In FY 2015, we determined that this additional Hall resistivity is due to the spin Hall effect and can be influenced by the magnetic texture in the adjacent $\mathrm{YIG:Bi}$, as well as by the strength of spin-orbit coupling and sign of the spin Hall angle of the heavy metal.

We studied a heterostructured system that consists of a thin layer of a ferromagnetic cobalt-iron-boron metal alloy, $\mathrm{CO}_{20} \mathrm{Fe}_{60} \mathrm{~B}_{20}$, with perpendicular magnetic anisotropy, sandwiched between nonmagnetic heavy metal tantalum (Ta) and insulating $\mathrm{TaO}_{\mathrm{x}}$ deposited on silicon/silicon oxide $\left(\mathrm{Si} / \mathrm{SiO}_{x}\right)$. The structural asymmetry in the present system is important with regard to stabilizing chiral domain walls due to Dzyaloshinskii-Moriya interaction (an anti-symmetric exchange interaction), which is necessary for stabilizing skyrmions and providing the efficient interfacial spin-orbit torques. The heterostructures were laterally patterned into a wire with a narrow constriction (or neck) in the middle. In FY 2014, we found that when a pulsed current was applied through the wire from left to right, stripe domains, initially present on both sides of the constriction, could be transformed into a dilute gas of skyrmions on the right-hand side. These micrometer-sized skyrmions were formed at room temperature, which is a prerequisite for potential device application.

In FY 2015, we extensively explored this exciting novel behavior as a function of current density, pulse width, magnetic field, and size and geometry of the constriction. We performed systematic studies of the evolution of the skyrmion formation as a function of the external magnetic field, applied perpendicular to the sample surface, and charge currents, which consequently established a phase diagram. The de-pinning and velocity of the skyrmions have been characterized at lower dc currents. We found that the skyrmions move at a relatively high speed after de-pinning. The direction of the motion of the skyrmions with current is consistent with them having Néel domain walls with a fixed chirality. Constrictions of different widths and shapes were tested, indicating the generality of the observed behavior and the concept that the formation of skyrmions after the constriction is linked to the divergent currents in that part of the wire. 
We made a first prototype skyrmion racetrack memory device (Figure 1), which is based on the constricted wire, but with an additional thin wire and current line attached orthogonally to the original. By sending pulsed currents though the constricted wire (marked in yellow), skyrmions are formed in the lower half of the wire. Subsequently, we found that a single skyrmion can be moved along the horizontal skyrmion bit line (marked in blue) by applying a current pulse along that direction (Figure 2). Systematic studies of the spin-orbit torque-induced skyrmion motion are under way, both as a function of the duration and amplitude of electrical pulses.

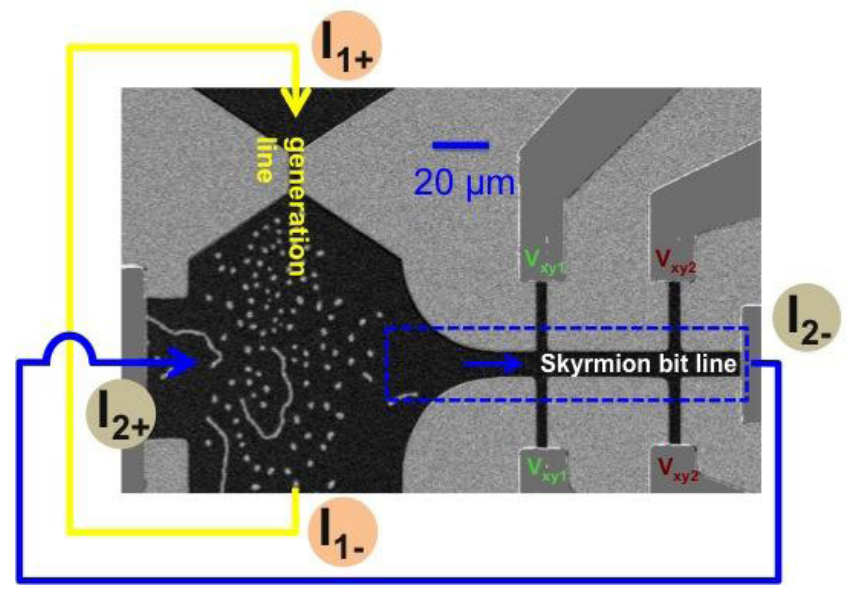

Figure 1. Conceptual skyrmion racetrack memory device consisting of a constricted wire in which skyrmions are created at the bottom by current pulses $I_{1}$ along the (yellow) generator line, with an additional wire attached (skyrmion bit line) along which skyrmions can be moved by applying current pulses $I_{2}$ along the blue circuit.

Because nanometer-sized skyrmions are desired for potential device applications, other heterostructures were explored as well. A strategy of interfacing thin ferromagnets between two different heavy metals, with stronger spin-orbit coupling than Ta, was employed. Additionally, by selecting two heavy metals with opposite signs of the spin Hall angle, complementary spin-orbit torques were created, which should stabilize smaller skyrmions and boost their motion. Following this strategy, a [Platinum(1.5 nm)/Cobalt(1 nm)/ Tungsten $(1 \mathrm{~nm})] \times 8$ multilayer was created for which we successfully observed Néel skyrmions with a diameter of about $100 \mathrm{~nm}$ at room temperature and in the absence of magnetic fields.

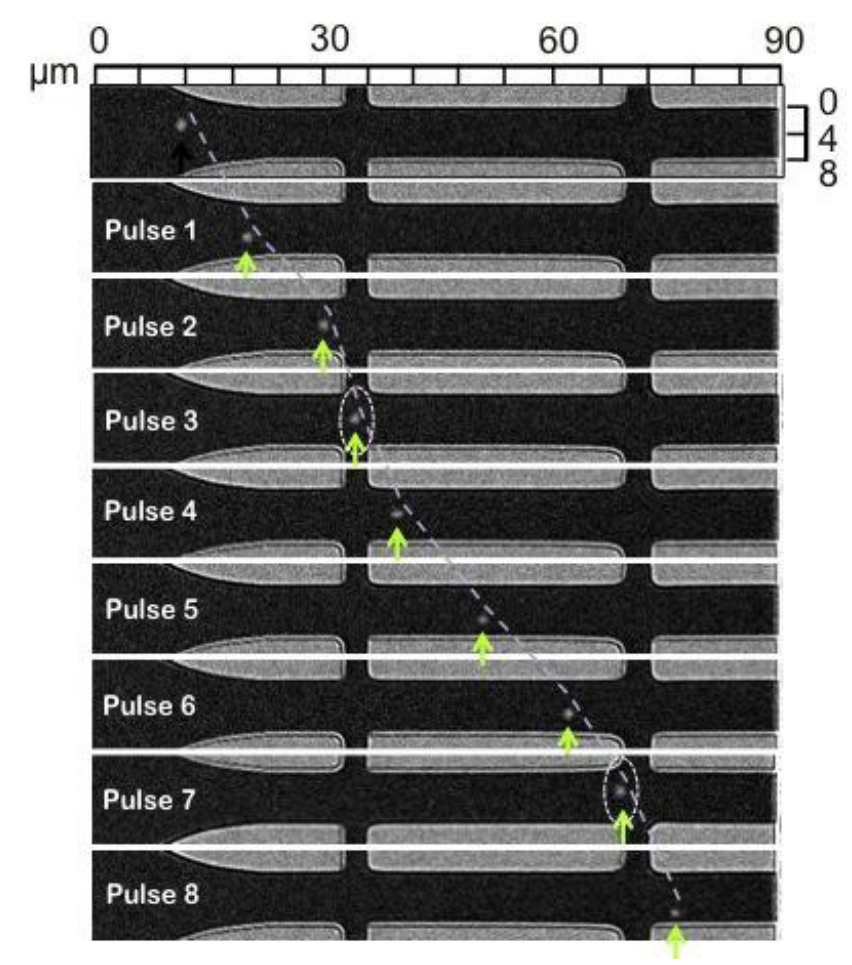

Figure 2. Series of polar magneto-optical Kerr effect images of the skyrmion bit line, taken after successive current pulses $I_{2}$. The green arrows point to the position of a single skyrmion in the bit line as it moved after each pulse.

\section{PROPOSED FUTURE WORK}

We will (1) investigate current-induced skyrmion dynamics, including the skyrmion Hall effect; (2) conduct a detailed characterization of the skyrmion spin texture with Lorentz transmission electron microscopy, spin-polarized low energy electron microscopy, and neutron scattering; and (3) explore heterostructures created with various combinations of heavy metals to optimize the stabilization of skyrmions with diameters below $100 \mathrm{~nm}$ at room temperature.

\section{Directly Probing Nanoscale Dynamics in Shear-Thickening Complex Fluids}

\section{4-077-R1}

Suresh Narayanan, Jonghun Lee, Xiao-Min Lin, and Alec Sandy

\section{PROJECT DESCRIPTION}

With increasing shear stress or shear rate, concentrated colloidal dispersions typically show shear thinning and shear thickening; such non-Newtonian behaviors arise from microstructural changes induced by shear. Shear thinning is believed to occur as particles rearrange 
themselves to reduce the resistance against flow. However, what causes shear thickening remains controversial. The proposed mechanisms include an order-disorder transition, hydrocluster formation by an increased lubrication force, and shear jamming caused by frustrated dilatancy in the constrained condition. Rheology has a limited capacity for understanding such non-Newtonian behaviors, as it measures macroscopic mechanical behavior but is not directly sensitive to the microstructural changes induced by shear.

In this project, we simultaneously measured rheological data while conducting small-angle x-ray scattering (SAXS) to understand the microscopic structure and dynamics of concentrated silica nanoparticle dispersions in different shear regimes. Specifically, we studied the dispersions under oscillatory shear that applies sinusoidal strain with certain strain amplitude and certain frequency. Oscillatory shear is a general method for understanding the elastic and viscous properties of fluids. Under oscillatory shear, the shear stress shows a mixture of an in-phase and out-of-phase response to applied strain. The storage modulus, or the degree of in-phase stress response, is a measure of the elastic or solid-like property, while loss modulus, or the degree of out-of-phase response, is a measure of the viscous or liquid-like property. Complex fluids under small strain show a linear stress response to the strain. However, if the strain is large enough to alter the microstructure, complex fluids enter a non-Newtonian regime, and the relationship between strain and stress is no longer linear. Recently, a theory of large amplitude oscillatory shear (LAOS) has been extensively studied to understand this nonlinear rheological behavior, but there remains a lack of experimental studies linking LAOS and the microstructures of concentrated colloidal dispersions. Because of its high spatial and temporal resolution, time-resolved in-situ coherent SAXS at beamline 8-ID-I of the Advanced Photon Source is ideally suited to linking LAOS and microstructure.

\section{MISSION RELEVANCE}

The scientific challenges being addressed by this project involve connecting macroscopic rheological properties to nanoscale motion in shear-thickening complex fluids under shear flow. At the nanoscale, non-equilibrium behavior can be observed directly, without averaging over many subsystems that are seen in macroscale measurements. The nature of such fluctuations is one metric for the existence of non-equilibrium in a system. Understanding how these fluctuations occur, measuring their evolution, and characterizing the approach to equilibrium was identified as one of five grand challenges by the DOE in 2007 (Directing Matter and Energy: Five Challenges for Science and the Imagination).

\section{RESULTS AND ACCOMPLISHMENTS}

Silica dispersions in polyethylene glycol (molecular weight $=200$ ) were prepared with varying particle sizes and volume fractions. This density-matching solvent prevents sedimentation of particles. As a representative example, Figure 1 shows the rheological behavior of 360-nanometer ( $\mathrm{nm}$ ) silica dispersions with a volume fraction of 52 percent. Under steady shear, with increasing shear stress, the viscosity $(\eta)$ of the silica dispersion initially decreases and then increases at the shear stress of 145 pascals $(\mathrm{Pa})$. Oscillatory shear was measured at a fixed frequency with increasing strain; in Figure 1, it is plotted as complex viscosity $\left(\eta^{*}\right)$ versus the maximum oscillatory shear stress. We observed unprecedented rheological behavior in that the oscillatory shear curves exhibited a two-step strain thinning and thickening with increasing shear stress. Interestingly, regardless of the oscillation frequency, the second local viscosity minima of these oscillatory curves coincided with the steady shear curve at the onset shear stress for shear thickening.

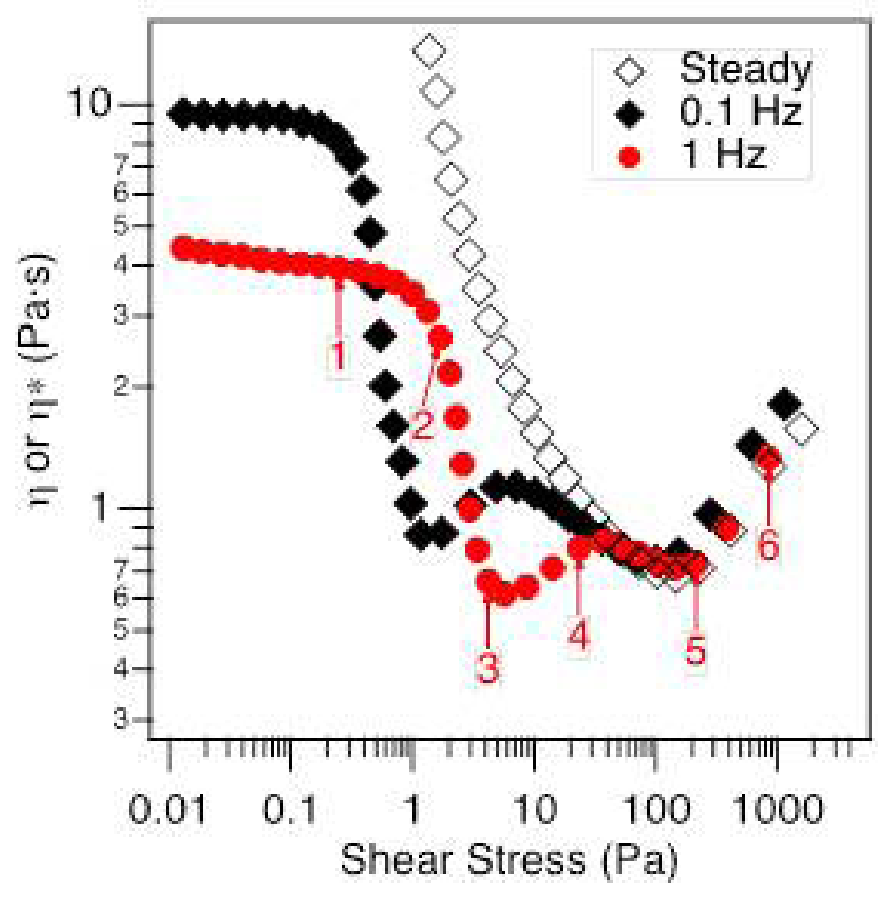

Figure 1. Rheological behavior of 360-nm silica dispersion with volume fraction of $52 \%$.

SAXS of the silica dispersions during shear were measured in the velocity-vorticity plane where velocity direction is parallel to shear direction and vorticity direction is normal to shear direction and to shear gradient direction. Figure 2 shows SAXS images at different shear stresses during oscillatory shear at a frequency of $1 \mathrm{~Hz}$. SAXS in the first strain-thinning regime shows that the silica particles are rearranged into an ordered structure (Figure 2: images 1, 2, and 3), and 
the ordered microstructure begins to disorder beyond this strain. According to the hydrocluster formation mechanism, shear thickening occurs at the same shear stress when the particle size is the same, which indicates that the second strain thickening may be attributed to shear thickening. It has long been debated whether an order-disorder transition is necessary for shear thickening. Monodisperse charge-stabilized dispersions often show an order-disorder transition coincident with shear thickening at the same shear stress. However, some colloidal dispersions are shear thickened without undergoing an order-disorder transition. Despite this counterexample, it was hard to exclude an order-disorder transition from the possible shear-thickening mechanisms for the monodisperse system. Our study directly proves that even for the systems that show the order-disorder transition, disordering can occur at a shear stress far different from the shear-thickening stress. This is the first direct observation that these two stress behaviors are unrelated to one other.
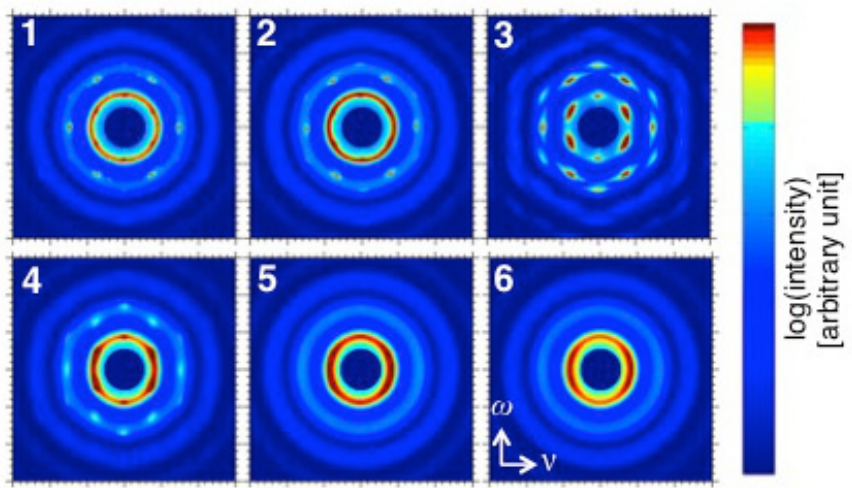

Figure 2. SAXS images of 360-nm silica dispersions under different oscillatory shear stress. Numbers correspond to the shear stress as indicated in Figure 1. The $v$ denotes flow direction, and $\omega$ denotes vorticity direction.

\section{PROPOSED FUTURE WORK}

We have gathered a significant amount of experimental data from the analysis of collective SAXS images to probe the dynamics of these dispersions by using $x$-ray photon correlation spectroscopy (XPCS). We have begun to work on quantifying the microstructure by using simulations based on structural models. Further, analysis of XPCS data is being carried out to extract nanoscale dynamical information, which will be correlated with the microstructure and rheological results. Further experiments are being planned to probe the effect of temperature on the above results.

\section{Pb-Assisted Corrosion/ Cracking Mechanisms at the Interface between $\mathrm{Pb}-$ Containing Solution and Nickel Oxide Surface}

\section{4-081-R1 \\ Seungbum Hong, Hawoong Hong, and Jongjin Kim}

\section{PROJECT DESCRIPTION}

The goal of this project is to study the fundamental mechanisms of lead $(\mathrm{Pb})$-assisted corrosion cracking by using both in-situ $x$-ray reflectivity and advanced in-situ/ex-situ atomic force microscopy (AFM) imaging techniques. This work will contribute to enhancing the reliability and safety of nuclear power plants. We performed in-situ $\mathrm{x}$-ray reflectivity imaging on liquid interfaces with a pre-oxidized 110 surface of nickel (Ni) and with molecular beam epitaxy (MBE)-grown nickel oxide ( $\mathrm{NiO}$ ) while the $\mathrm{Pb}$ concentration and the $\mathrm{pH}$ were varied. The interfaces were characterized by AFM in air and in $\mathrm{Pb}$-containing solutions. We will go on to identify an important mechanism of $\mathrm{Pb}$-assisted stress corrosion cracking in Alloy 690 (a high-chromium nickel alloy).

\section{MISSION RELEVANCE}

This project is relevant to DOE's energy security initiative. The primary benefits of the proposed work include identifying the fundamental mechanisms of $\mathrm{Pb}$-assisted stress corrosion cracking in the short term and developing mitigation methods for this problem in the longer term. The main beneficiaries/customers of the proposed work would be the nuclear power industry and DOE.

\section{RESULTS AND ACCOMPLISHMENTS}

We deposited an epitaxially grown $\mathrm{NiO}$ thin film on a magnesium oxide ( $\mathrm{MgO}$ ) substrate by using oxide MBE. The thin film of NiO was characterized by AFM as being a well-grown flat $\mathrm{NiO}$ surface with approximately 80-Ångstrom thickness and a root mean squared (RMS) roughness of 142 picometers (pm). According to this surface characterization, an epitaxially grown thin-film surface deposited by oxide MBE would be suitable for high-resolution $x$-ray reflectivity measurements with and without a $\mathrm{Pb}$ solution.

Using the MBE-grown NiO film, the high-resolution $x$-ray reflectivity was measured in helium gas, water, and a 10-millimolar (mM) Pb solution under different $\mathrm{pH}$ conditions. The experiment was conducted at the 
Advanced Photon Source's 33-BM-C beamline (in collaboration with Changyong Park of the Carnegie Institution of Washington). Figure 1(a) shows combined x-ray reflectivity results; we did not find any significant changes in the intensity of the oscillatory features as a function of the nature of the liquid or gas side of the interface. Figure 1(b) shows the combined $x$-ray reflectivity in the $\mathrm{Pb}$ solution at various $\mathrm{pH}$ levels $(\mathrm{pH} 3,7$, and 11). Although the $\mathrm{pH}$ values of the $\mathrm{Pb}$ solution were changed, there were no significant changes in either the intensity or the shapes of the curves. MBE-grown nickel oxide is therefore seen to be very inert both in pure water and in $\mathrm{Pb}$-containing solution at room temperature. We therefore designed experiments at higher temperature and pressure.
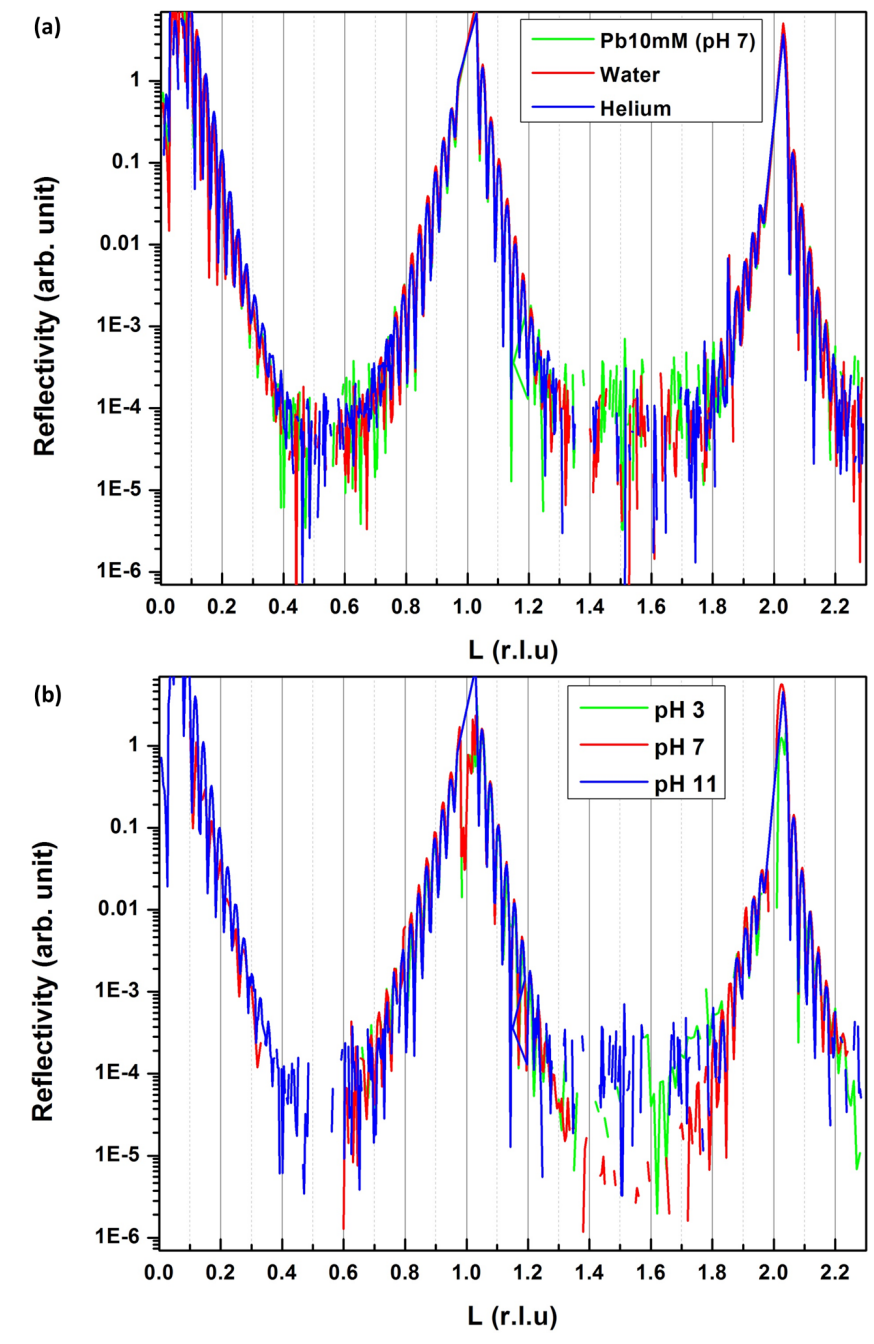

Figure 1. Measured $\mathrm{x}$-ray reflectivity of $\mathrm{NiO}$ on $\mathrm{MgO}$ substrate in (a) helium, water, and 10-mM Pb solution with $\mathrm{pH} 7$ and (b) in 10-mM Pb solution with $\mathrm{pH} 3,7$, and 11 (r. I. u stands for reciprocal lattice units).

We used AFM to measure the topographies of as-grown $\mathrm{NiO}$ on $\mathrm{Ni}(110)$ and $\mathrm{MBE}$-grown $\mathrm{NiO}$ on $\mathrm{MgO}$ samples both in air and after immersion in a 100-mM Pb solution for 15 hours. Figure 2 shows the results. For the case of the as-grown $\mathrm{NiO}$ on $\mathrm{Ni}(110)$, the RMS roughness dramatically increased from $601.06 \mathrm{pm}$ to 16.97 nanometers $(\mathrm{nm})$ with large changes in topography. Several crystalline compounds were formed on the surface. In case of the MBE-grown $\mathrm{NiO}$ on $\mathrm{MgO}$, the RMS roughness slightly increased from $718.19 \mathrm{pm}$ to $1.91 \mathrm{~nm}$, causing us to speculate that the surface was covered with $\mathrm{Ni}(\mathrm{II})$ hydroxide, which can form in the presence of water. The MBE-grown $\mathrm{NiO}$ on $\mathrm{MgO}$ and as-grown $\mathrm{NiO}$ on $\mathrm{Ni}(110)$ differed in the number of defects. The MBE-grown $\mathrm{NiO}$ is a model of defect-free $\mathrm{NiO}$ by the MBE method, while the as-grown $\mathrm{NiO}$ on $\mathrm{Ni}(110)$ has defects occurring naturally during the oxidation. We found by $\mathrm{x}$-ray reflectivity measurements that the as-grown $\mathrm{NiO}$ on $\mathrm{Ni}(110)$ reacts immediately when exposed to water. The $\mathrm{NiO}$ with defects was affected by the presence of $\mathrm{Pb}$, as evidenced by the morphology change of the $\mathrm{NiO}$ surface, which was probably due to the formation of a $\mathrm{Pb}$ compound on the surface, whereas the $\mathrm{NiO}$ without defects seemed to be very inert in the $\mathrm{Pb}$ solution.

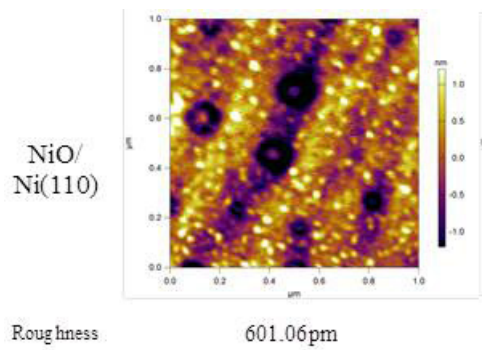

(a)

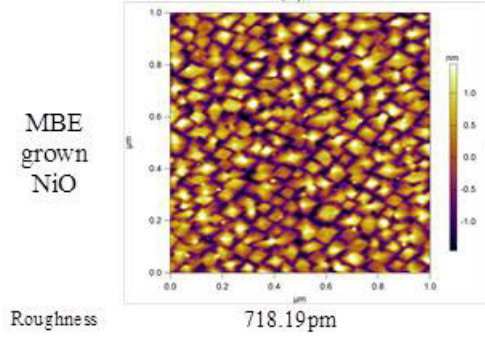

(c)

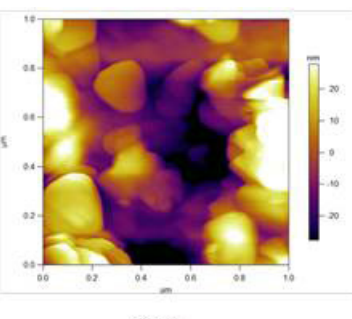

$16.97 \mathrm{~nm}$

(b)

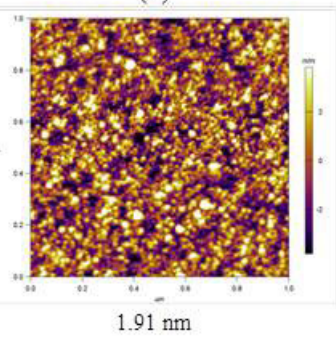

(d)
Figure 2. AFM topography images showing RMS roughness of one micrometer square areas for $\mathrm{NiO} / \mathrm{Ni}(110)$ in (a) air and (b) a 100-mM Pb solution for 15 hours, and for MBE-grown NiO on MgO in (c) air and (d) a $100-\mathrm{mM} \mathrm{Pb}$ solution for 15 hours.

\section{PROPOSED FUTURE WORK}

To understand $\mathrm{Pb}$-assisted stress corrosion cracking of a $\mathrm{Ni}$ alloy under real field conditions, we will measure and analyze the interface changes at high temperature $\left(\sim 300^{\circ} \mathrm{C}\right)$ and under high pressure ( $\left.15 \mathrm{MPa}\right)$ in a $\mathrm{Pb}$-containing solution by using a developed hydrothermal cell. Our results will identify the prevailing mechanism of $\mathrm{Pb}$-assisted stress corrosion cracking in Alloy 690. 
Probing the Chemistry of Atmospheric Dust Particles Using X-ray Spectromicroscopy: Implications for Climate Science

\section{4-084-R1}

Yan Feng, Rao Kotamarthi, Barry Lai, and Stefan Vogt

\section{PROJECT DESCRIPTION}

Iron (Fe) is a key micronutrient that is vital for all organisms. In many ocean regions, its availability controls primary productivity, affecting marine biogenic emissions and carbon dioxide fluxes between the ocean and the atmosphere. About $95 \%$ of the global Fe supply in the open ocean comes from atmospheric dust particles (aerosols), and most photosynthetic aquatic organisms can take up Fe only in its dissolved form. The water solubility and, in turn, the bioavailability of Fe in atmospheric dust aerosols must therefore be understood. Two key parameters determine Fe dissolution in dust: (1) the dust's Fe content and (2) the chemical mechanisms that produce Fe compounds dissolvable in water. Because of a lack of fundamental understanding about dust chemistry, it is conventionally assumed in current earth system models (ESMs) that the soluble Fe fraction of dust aerosols remains constant; soluble Fe fraction variability due to dust chemistry during transport is neglected, thus atmospheric influences on soluble Fe deposition from dust aerosols to the ocean are likewise ignored. This simplification has been subject to increasing scrutiny. For ESMs to be integrated as effective tools, they must address the uncertainties in modeled global soluble Fe inputs to ocean ecosystems by resolving the chemistry within the dust particle that transforms insoluble Fe forms into water-soluble (thus bioavailable) Fe. This is essential in order to understand the aerosol biogeochemical feedbacks in the changing climate.

This project strives to address the composition of $\mathrm{Fe}$ in dust and the lack of understanding about the chemical transformations involved in atmospheric transport, by using experimental studies to evaluate and improve the modeled representation of dust Fe chemistry. With modern microscopy and microanalytical techniques (such as X-ray fluorescence [XRF] microspectroscopy at Argonne's Advanced Photo Source [APS]), we can probe the chemistry of individual dust particles. Elemental compounds in mineral dust can be seen with hard x-ray microscopy at quantities down to the trace level. At beamlines with microspectroscopic instruments, we can determine the oxidization states of Fe(II) or Fe(III), which are linked to the solubility of Fe.

We are (a) mapping the chemical composition of dust particles collected from multiple locations (from the remote Southern Ocean to polluted urban regions and dust-source regions) with respect to mineralogy and Fe speciation and (b) deriving statistical relationships with other chemical components, depending on atmospheric conditions. We will use the derived datasets from different regions to evaluate and improve the existing process model for Fe dissolution and speciation. Then we will incorporate the process model into the Community Atmospheric Model (CAM), which is part of the ESM, and perform climate simulations of the bioavailability of Fe. Analyses will be performed at different temporal scales (seasonal, inter-annual, and decadal) to study variations in atmospheric Fe deposition as perturbed by changes in aerosol emissions projected for future climate scenarios.

\section{MISSION RELEVANCE}

The project is tied to DOE's environmental quality mission, particularly with respect to climate science. The success of this initiative will aid in developing DOE's nextgeneration ESM focused on the feedback between ocean biogeochemical cycles and atmospheric aerosols. This topic has been projected as a transformational research area of climate science in the next decade by DOE, the National Science Foundation, and the U.S. Department of Agriculture.

\section{RESULTS AND ACCOMPLISHMENTS}

In FY 2014, we primarily focused on developing a library of samples and data collection. Dust samples were gathered from six sites, representative for areas that are influenced by desert dust or anthropogenic sources, or both. The collected samples (ca. 40 micrometers square) were scanned by the $\mathrm{x}$-ray beam (energy $=7.2$ kiloelectronvolts (keV)) in the microscopic x-ray fluorescence mode. An energy scan of the Fe $x$-ray absorption near edge structure (XANES) region was also collected for ironcontaining spots identified from $x$-ray fluorescence maps as well as on large filter regions. In this way, both the filter area-averaged (bulk) and individual particle Fe oxidation states were characterized for the dust samples. Initial data analysis was performed on the Mediterranean dataset. Bulk Fe XANES showed that regardless of source region, namely Europe or North Africa, the composition of Fe in aerosols is uniform. Linear combination fitting further showed that some of the dust Fe particles consist of about $60 \%$ goethite (an Fe(III) oxyhydroxide) and $40 \%$ heterosite (an Fe(III) phosphate). The finding of heterosite indicates 
the acid processing of dust particles in the atmosphere because heterosite is not found in the original dust source.

Throughout FY 2015, we completed beamline tests on more than one hundred dust samples provided by collaborators from Rutgers University, Georgia Institute of Technology, Amity University (India), University of Hawaii, and University of Paris (France). These samples were collected from seven locations: the Southern Ocean, the Mediterranean, the Atlantic Ocean, Hawaii, Bermuda, Patagonia, and India. They represent a wide range of dust aerosol characteristics from different source regions and remote oceans.

Saharan dust analysis. With researchers from Georgia Institute of Technology, we conducted a comprehensive data analysis of the Saharan dust. This dataset includes samples collected at three sites located in the Mediterranean, the Atlantic Ocean, and Bermuda, as shown in Figure 1, which represent aerosol Fe at different stages of dust transport in the atmosphere with a wide range of observed iron solubilities (from $0.41 \%$ to $8.9 \%$ ). Fe solubility was also found to be higher in "aged" dust particles collected from the Atlantic Ocean and Bermuda, but with lower concentrations than the Mediterranean dust collected near the Sahara. Nearly half of the aerosol iron in the Bermuda samples was made of iron sulfates, which are a minor component in the original Sahara dust; the formation of these iron sulfates has been shown to significantly enrich iron solubility. In addition to indicators of acid processing on aerosol Fe solubility, the data also present strong evidence of a reductive Fe transformation (Fe(III) to $\mathrm{Fe}(\mathrm{II}))$ occurring as dust is transported. Because Fe reduction reactions occur in different environments from acid processing and Fe(III) is more soluble than Fe(II), our study highlights the importance of modeling both pathways in order to capture variations in iron dissolution.

CAM modeling. We also set up the latest CAM version 5 (CAM5) on the Argonne Leadership Computing Facility (ALCF) Blue Gene/Q test and development platform, Vesta. Eight different dust minerals (illite, kaolinite, montmorillonite, hematite, quartz, calcite, feldspar, and gypsum), each with a unique set of physical and chemical properties, have been added to the CAM5 model and will be compared to the chemical information derived from our observational data analysis and that found in the literature. We also upgraded the dust emissions in CAM5 with the new scheme, which has a substantial impact on the total Fe deposition flux to the ocean.

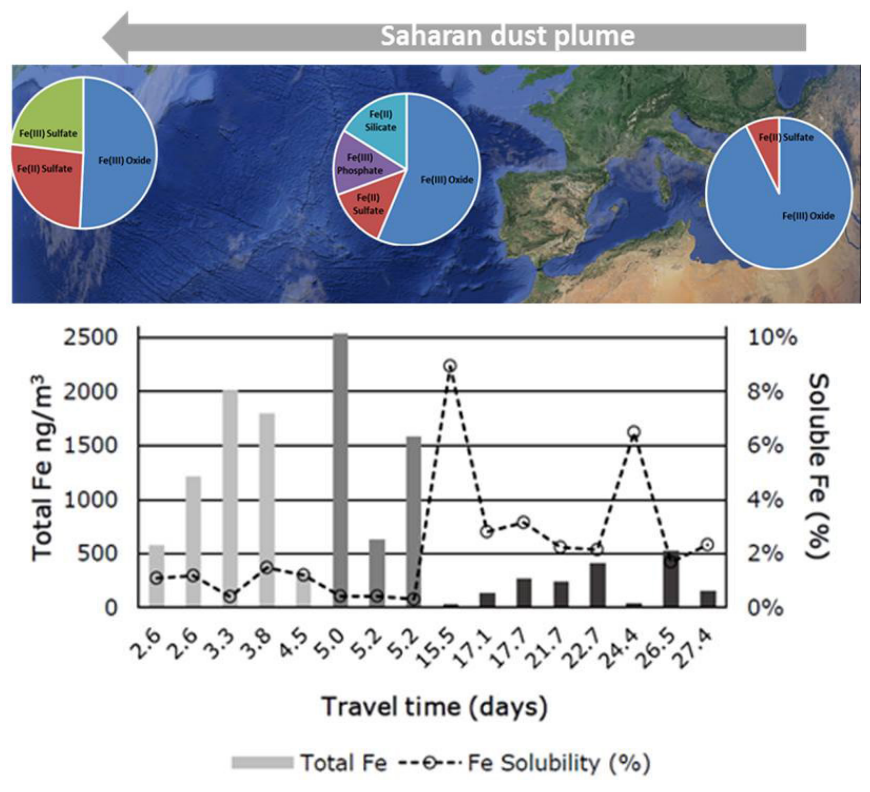

Figure 1. Variations of $\mathrm{Fe}$ (III) to $\mathrm{FE}$ (II) and formation of iron sulfates in atmospheric transport of dust (top) are consistent with the increase of solubility with longer travel times (bottom).

\section{PROPOSED FUTURE WORK}

In FY 2016, we will focus on bridging the data analysis to the modeling and impact studies. We will first implement an acid-based Fe dissolution scheme into CAM5. As suggested by our analysis of the Saharan dust data, there is also strong evidence of a ligand-promoted or photo-chemical reductive reaction in addition to the acid-induced Fe dissolution scheme. To date, only one model in the literature incorporates this pathway. We will establish this approach in CAM with observational constraints from our data analysis and those in the literature. Time-sliced sensitivity model experiments of global-scale soluble Fe deposition will be conducted with and without the inclusion of Fe dissolution chemistry in dust. We will also investigate the role of various iron dissolution schemes on soluble Fe deposition to the ocean by using model sensitivity studies. These simulations will demonstrate the enhanced capabilities of the DOE ESM, based on x-ray data analysis, in capturing the highly resolved temporal and spatial variabilities in the deposition of soluble (bioavailable) Fe to the ocean and its potential effects on ocean biogeochemistry and the climate and carbon cycles. 


\section{Tuning the Transport Properties of Coupled Majorana}

\author{
2014-095-R1
}

Konstantin Matveev, Lei Fang, and Wai-Kwong Kwok

\section{PROJECT DESCRIPTION}

Hailed as one of the top scientific discoveries of 2012 by Science magazine, the experiment that offered evidence of Majorana fermions in a superconducting wire by the Kouwenhoven group at Delft has set off a firestorm of interest in further exploring Majorana physics. This excitement is driven by the promise that these particles hold for applications to quantum computing. Like any computer, the operation of a quantum computer is based on the ability to store and manipulate information. In a quantum computer, the units of storage are microscopic degrees of freedom subject to the laws of quantum mechanics. Remarkably, quantum computers can perform operations "exponentially" faster than ordinary computers, and this feature has led to a great deal of research activity. One of the biggest challenges in quantum computing is minimizing the loss of information due to environmental noise. Majorana fermions are very promising because the information they store is expected to be immune to many of the forms of noise that plague other implementations. In this project, we are constructing a device that can probe thermal and electrical transport through a system of coupled Majoranas (Figure 1). The architecture consists of a topological insulator on a substrate that has a thin superconducting strip. The role of the topological insulator is to render the electronic degrees of freedom spinless-a crucial condition for isolating Majorana fermions. We are also constructing devices made from bismuth selenide (BiSe) in which we have detected signatures of superconducting order. These two ingredients-spin-polarized electrons and superconductivity-are sufficient to generate Majorana fermions in the cores of vortices.

\section{MISSION RELEVANCE}

This project supports DOE's science mission. Majorana fermions represent an exciting new aspect of superconducting order. The quantum information encoded by these exotic quasiparticles is expected to enjoy unprecedentedly long decoherence times. Applications to quantum computing arising from this project would strongly support the DOE mission of fostering leading-edge innovation in computer technology.

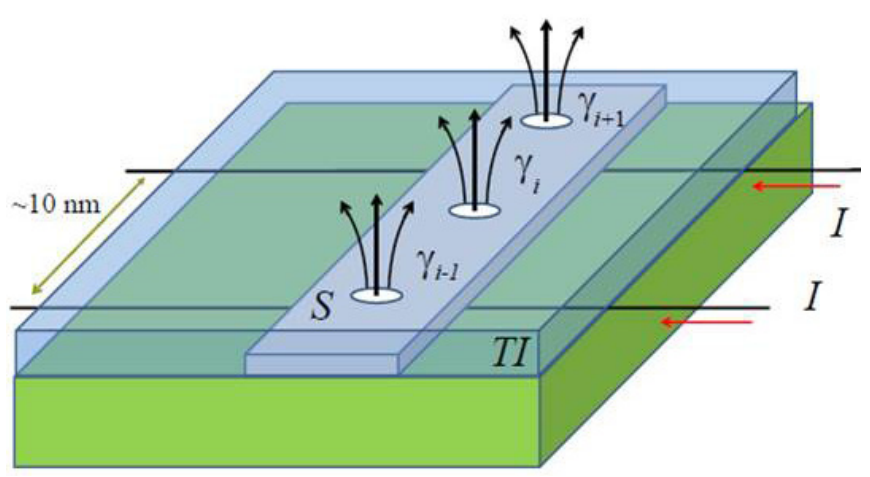

Figure 1. Diagram of proposed experimental setup. A topological insulator (TI) is deposited on a substrate with a thin strip of superconductor (S), which contains a line of vortices, forming a vortex lattice (vortices are shown as circles threaded by magnetic flux). Control currents (red arrows) would allow the tuning of the spacing between vortices.

\section{RESULTS AND ACCOMPLISHMENTS}

In FY 2014, we fabricated tellurium (Te)-doped $\mathrm{Bi}_{2} \mathrm{Se}_{3}$ as well as copper $\left(\mathrm{Cu}\right.$ )-doped $\mathrm{Bi}_{2} \mathrm{Se}_{3}$ crystals, nanoribbons, and nanowires. Resistance measurements confirmed that, through doping, we are able to control the effects of bulk transport. A theoretical study on systems of coupled Majorana fermions was published. This work forms the basis of the theoretical predictions laid out in this proposal.

In FY 2015, work focused on the synthesis and characterization of a number of BiSe-related compounds that show promise in hosting Majorana fermions. Specifically, our work on the novel two-dimensional (2D) conducting heterostructure $\left[\mathrm{Pb}_{2} \mathrm{BiS}_{3}\right]\left[\mathrm{AuTe}_{2}\right]$ (a naturally occurring mineral known as buckhornite, see Figure 2) revealed a number of interesting discoveries related to spin-orbit coupling. Spin-polarized electrons that arise from spin-orbit coupling are a crucial element of our proposal; thus, obtaining an estimate of the strength of the spin-orbit coupling in this material is an important milestone. In particular, through transport measurements, we found that scattering in the material is at odds with the Abrikosov-Gorkov model (Figure 3). Transport measurements revealed signatures of weak anti-localization, and these data were used to estimate the spin-orbit scattering time. These results are consistent with ab-initio calculations (performed by J. Im at the Korea Research Institute of Chemical Technology). 


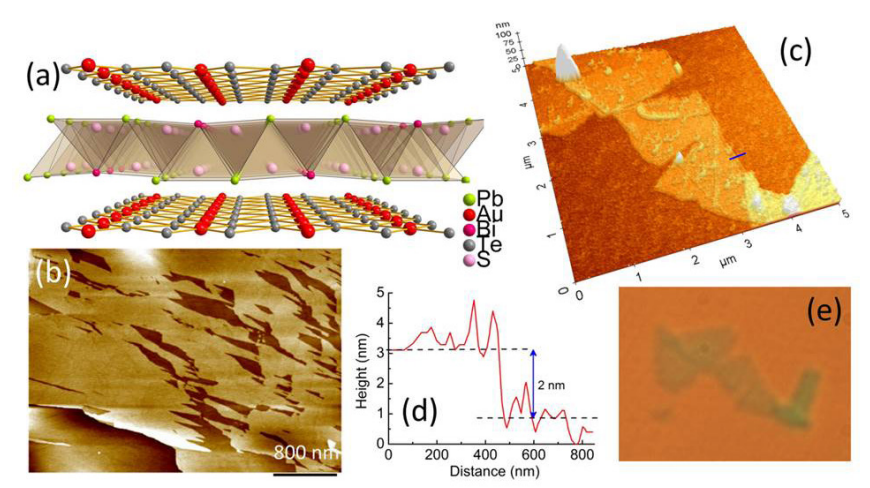

Figure 2. (a) Intergrowth structure of $\left[\mathrm{Pb}_{2} \mathrm{BiS}_{3}\right]\left[\mathrm{AuTe}_{2}\right]$ consists of two building blocks $\left[\mathrm{Pb}_{2} \mathrm{BiS}_{3}\right]$ and $\left[\mathrm{AuTe}_{2}\right]$, which stack alternately along the c-axis. (b) A high-resolution atomic force microscope (AFM) image of the cleaved surfaces of a single crystal of $\left[\mathrm{Pb}_{2} \mathrm{BiS}_{3}\right]\left[\mathrm{AuTe}_{2}\right]$. (c) An AFM image of ultrathin crystals on top of a silicon dioxide/silicon wafer. (d) Thickness of the crystal in Figure 2(c) (marked by the blue line). The 2-nm (determined by AFM) thickness corresponds to two unit cells of $\left[\mathrm{Pb}_{2} \mathrm{BiS}_{3}\right]\left[\mathrm{AuTe}_{2}\right]$. (e) An optical image of the same crystals shown in Figure 2(c). The ultrathin crystals are virtually transparent.

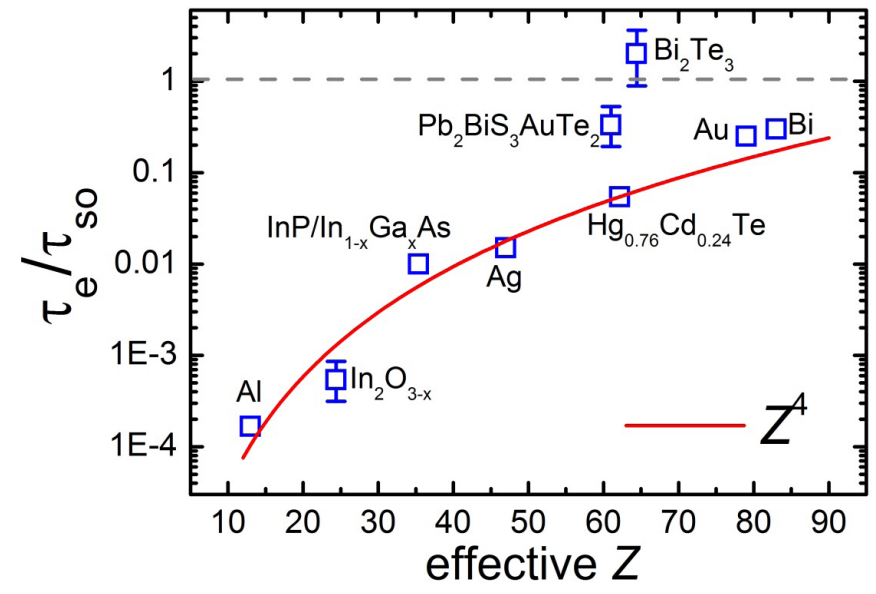

Figure 3. Graph of $\tau_{\mathrm{e}} / \tau_{\mathrm{so}}$, the ratio of elastic scattering time to the electron spin-orbit coupling (SOC) time, for various 2D materials (data for Al, InP/ In GaAs, $\mathrm{In}_{2} \mathrm{O}_{3}, \mathrm{Hg}_{0.76} \mathrm{Cd}_{0.24} \mathrm{Te}, \mathrm{Au}, \mathrm{Bi}, \mathrm{Bi}_{2} \mathrm{Te}_{3}$, and $\mathrm{Ag}$ are taken from the literature) and $\left[\mathrm{Pb}_{2} \mathrm{BiS}_{3}\right]\left[\mathrm{AuTe}_{2}\right]$ (our work) as a function of the effective atomic number $(Z)$. The red solid line is the predicted scaling according to the theory of Abrikosov and Gorkov. Like the topological insulator $\mathrm{Bi}_{2} \mathrm{~S}_{3},\left[\mathrm{~Pb}_{2} \mathrm{BiS}_{3}\right]$ $\left[\mathrm{AuTe}_{2}\right]$ exhibits anomalously large SOC. Due to the topology-related Berry's phase and the extremely large SOC, $\left[\mathrm{Pb}_{2} \mathrm{BiS}_{3}\right]\left[\mathrm{AuTe}_{2}\right]$ does not follow the $Z^{4}$ relation.

\section{PROPOSED FUTURE WORK}

Current theoretical work focuses on aspects of spin-orbit coupling in the material $\left[\mathrm{Pb}_{2} \mathrm{BiS}_{3}\right]\left[\mathrm{AuTe}_{2}\right]$. One aspect of particular note is that spin-orbit coupling gives rise to topologically protected edge states. We are investigating these states as well as non-topological states in this material. Such states have important implications for realizing Majorana fermions in this system.

\section{Single-Cell Structural Genomics of Uncultured Sediment Archaea: On the Trail for Novel Proteases}

\section{4-108-R1}

Karolina Michalska, Gyorgy Babnigg, Robert Jedrzejczak, Andrzej Joachimiak, and Joseph Mootz

\section{PROJECT DESCRIPTION}

Organic carbon is deposited ultimately as marine sediments, which comprise an ecosystem representing one of the richest microbial niches on Earth, which is predominantly inhabited by archaebacteria (archaea) and is estimated to constitute about one-tenth of the planet's total biomass. These microorganisms actively participate in sulfur, nitrogen, and carbon cycling. The latter process occurs predominantly through methanogenesis-a process converting simple organic molecules and carbon dioxide $\left(\mathrm{CO}_{2}\right)$ into methane $\left(\mathrm{CH}_{4}\right)$-and reverse methanogenesis-an anaerobic oxidation of methane. A recent study identifying novel archaea through single-cell genomic sequencing reports that these organisms encode putative extra- and intra-cellular protein-degrading enzymes (proteases) that utilize detrital proteins as basic nutrients. Thus, these newly discovered microorganisms may be key players in deep-sea biogeochemistry. However, their physiology and molecular adaptations to this unique environment are poorly understood. In particular, the predicted proteases have not been studied. The purpose of this project is to investigate the properties of these uncharacterized enzymes, which may reveal some unknown functional and/or structural features.

\section{MISSION RELEVANCE}

The project is relevant to the DOE's energy and environmental missions. DOE is committed to the characterization of ecosystems pertinent to carbon sequestration. Moreover, DOE funds several largescale projects for the characterization of highly specific classes of proteins. No such work is being done to study proteases, which are indispensable for the turnover of biomass-derived proteinaceous material. Characterization of archaeal enzymes will help researchers understand how these microorganisms contribute to global carbon cycling via protein remineralization in marine sediments.

\section{RESULTS AND ACCOMPLISHMENTS}

We previously analyzed genomes of four novel archaea derived from marine sediment. Fifty-five genes encoding putative proteolytic enzymes were cloned to facilitate recombinant expression in a laboratory strain of $E$. coli. 
To date, 16 proteins have been successfully purified at a large scale and three of these were crystallized to provide structural information. In addition, functional screening performed on six proteins resulted in the characterization of three representatives: a bathyaminopeptidase (BAP) and a TAE1 aminopeptidase (enzyme removing an $\mathrm{N}$-terminal residue from a longer peptide) from $T$. archaeon E09 and an M20 dipeptidase (enzyme cleaving dipeptides into two amino acids) from $T$. archaeon N05. Another characterized enzyme is a Xaa-His dipeptidase, which shows broad substrate specificity with respect to the Xaa position of the cleaved peptide. It belongs to the M20 family of proteases and requires either cobalt $\left(\mathrm{CO}^{2+}\right)$ or manganese $\left(\mathrm{Mn}^{2+}\right)$ ions for activity.

Recently, we made significant progress in characterizing the TAE1 enzyme. TAE1 is an aminopeptidase representing the M42 family of proteases. It displays activity toward aromatic and aliphatic amino acids, with the highest affinity toward tyrosine at the $\mathrm{N}$-terminus of the hydrolyzed peptide (Figure 1). Furthermore, this enzyme exhibits $\mathrm{N}$-terminal deblocking activity, as it is able to hydrolyze acylated $\mathrm{N}$-terminal residues. TAE1 requires the binding of divalent cations for catalysis and cation preference is $\mathrm{pH}$-dependent, with $\mathrm{Co}^{2+}$ being the most preferred ion at $\mathrm{pH} 6.5$ and zinc $\left(\mathrm{Zn}^{2+}\right)$ at 7.5. The optimum temperature for TAE1 activity is $\sim 45^{\circ} \mathrm{C}$. This finding is surprising, considering that the enzyme is produced by a psychrophile (an organism that grows at low temperature). However, it has a comparable activity profile with respect to temperature as previously characterized BAP. Future studies will examine whether the temperature profile is the same in the presence of other divalent cations and at other $\mathrm{pH}$ values.

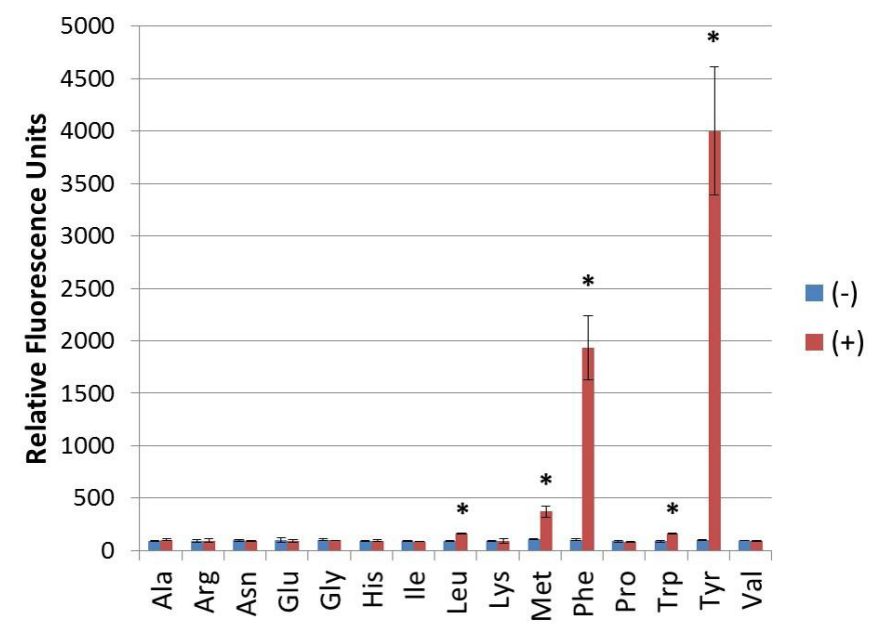

Figure 1. TAE1 substrate specificity measured with fluorescent dipeptide proxies with the $\mathrm{N}$-terminal residue of the Xaa-AMC substrate shown in the horizontal axis. The bars correspond to the sample with (+) or without (-) an enzyme. The asterisks are indicative of significance as measured with $95 \%$ confidence interval.
Similar again to BAP, TAE1 is a self-compartmentalizing enzyme, with 12 protein chains creating an oligomeric sphere with all 12 active sites located inside and several openings for substrates to diffuse in. The dimensions of the gates limit the size of the peptides that can access the active sites. The crystal structure was obtained by x-ray diffraction under conditions that displayed the highest enzyme activity-in the presence of cobalt ions and at a pH near 6.5. Therefore, our structure represents the active state of TAE1, with two cobalt ions participating in the catalytic reaction (Figure 2). To visualize binding of the substrate molecule in the active site, we designed an E214Q point mutation that abolishes enzyme activity. TAE1 E214Q was purified and successfully co-crystallized with a peptide substrate containing three amino acids (YLT). We are in the process of optimizing crystallization conditions in order to obtain high-resolution structural data. A manuscript describing TAE1 is in preparation.

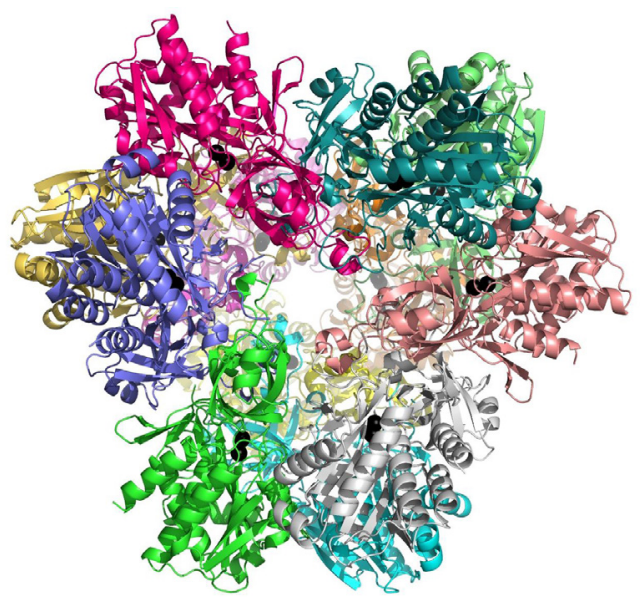

Figure 2. The crystal structure of TAE1. Cobalt ions are shown as black spheres. Each color represents a single protein chain. One of the gates leading to the active sites is located at the center of the assembly.

The results produced by this project were used in preparing a collaborative grant application submitted to the National Science Foundation together with Drs. Karen Lloyd and Andrew Steen of the University of Tennessee. The proposal has a broader scope than the current LDRD project and focuses on marine carbon cycle-related uncharacterized enzymes originating from organisms inhabiting specific niches. Besides proteases, other enzyme classes will be investigated, including cellulases, chitinases and carbonic anhydrases.

\section{PROPOSED FUTURE WORK}

In FY 2016, we will continue functional characterization of the remaining 13 proteins. In addition, we plan on optimizing crystallization conditions for the proteins we purified at a large scale. We are also working on 
optimization of expression constructs and crystallization of other targets. We will continue to investigate the structural aspects of M42 to achieve a better understanding of its mode of action.

\section{Detection of Dark Matter Directionality by Means of Columnar Recombination}

\author{
2015-015-NO \\ Richard Talaga, Karen Byrum, Marcel Demarteau, \\ Lisa Goodenough, and Stephen Magill
}

\section{PROJECT DESCRIPTION}

We seek to demonstrate that columnar recombination in a high-pressure gas can be used to detect the direction of ionized tracks as a replacement for direct imaging techniques. Use of this method could potentially detect the direction of dark matter particles. Their tracks would be just a few micrometers long, far shorter to detect than is possible by means of conventional direct imaging techniques. Dark matter is thought to be comprised of massive particles that are essentially at rest with respect to our galaxy. Their velocity relative to an observer on Earth can be attributed to our solar system's motion around the Milky Way, resulting in a velocity of about 220 kilometers/second toward the constellation Cygnus. The apparent kinetic energy of these particles is small, such that a collision with normal matter (such as the gas in a dark matter detector) produces recoiling atoms with very short recoil tracks, with directionality away from Cygnus.

Because the dark matter interactions are very weak, most detectors employ either solid or liquid detection media to increase the number of targets (atoms) in the detector volume. A high-pressure heavy gas composed mostly of xenon $(\mathrm{Xe})$ atoms has a mass that is reasonably close to a liquid or solid detector and also a critical advantage: the possibility of detecting track direction. Our ultimate goal is to detect such recoils, which produce a highly ionized but very short track. Although xenon is the primary gas target, a small percentage of an organic gas is necessary to enhance the likelihood of columnar recombination. The gas is TMA (trimethylamine). It enhances recombination by means of energy exchange with Xe, taking the energy of an excited (but un-ionized) xenon atom and ionizing the TMA. In addition, ionized Xe will charge-exchange with TMA. Otherwise, re-combined $\mathrm{Xe}$ atoms will emit vacuum ultraviolet (UV) photons (i.e., of wavelength shorter than about 200 nanometers [nm]). Therefore, all emitted light due to recombination will be around $320 \mathrm{~nm}$, in the near UV, and relatively easy to detect. We aim to show that using TMA will result in a much denser ionization column, replacing excited $\mathrm{Xe}$ atoms with ionized TMA molecules. This phenomenon is attributable to the Penning Effect, which we are trying to optimize by adjusting the small TMA admixture.

An electric field is used to determine the direction of the track in the following way. If the field is aligned with the track, columnar recombination is expected to be large and the resulting recombination will produce more photons from recombination. However, if the field is perpendicular to the track, the ions will quickly separate and recombination will be minimal, resulting in a lower yield of photons (see Figure 1). A fully functional dark matter detector utilizing this method would collect a maximum amount of light with its electric field pointed toward (or away) from Cygnus. The density/flux depends on the mass of dark matter particles. The mass of dark matter surrounding the galaxy is known, but the number of particles per unit volume depends on the mass of an individual dark matter particle. The largest detectors looking for WIMP (weakly interacting massive particle) dark matter are typically sensitive to a dark matter particle mass of around 10 to hundreds of giga-electron volts (GeV), whereas the mass of a proton is about $1 \mathrm{GeV}$. State-of-the-art detectors have targets in the range of 100 kilograms $(\mathrm{kg})$ of material, such as liquid xenon. The next generation of experiments will try to improve sensitivity by increasing the number of scattering targets by a factor of $\sim 10$, to $1,000 \mathrm{~kg}$ of liquid xenon. If a detector were built with high-pressure gaseous xenon (+TMA), the volume needed to contain $59 \mathrm{~kg}$ of this gas at 10 bar pressure is one cubic meter. A pressure vessel with dimensions on the order of $2.5 \mathrm{~m}$ would be needed to equal the target sensitivity of the next generation dark matter detectors, which cannot detect recoil tracks. Our goal is to show that a high-pressure, gas-filled detector is able to detect the direction of recoil tracks. The method of columnar recombination to determine recoil direction is localized to the track itself, and imaging the track by ionized electron collection or photographic means is not used. This approach allows us to scale up the volume to limits constrained only by pressure vessel strength.

\section{MISSION RELEVANCE}

The technique investigated in this project bears direct relevance to the DOE's Office of Science High Energy Physics missions, specifically, its particle physics science drivers of Energy, Intensity, and Cosmic Frontiers. Dark matter detection is a high priority in the Cosmic Frontier. 


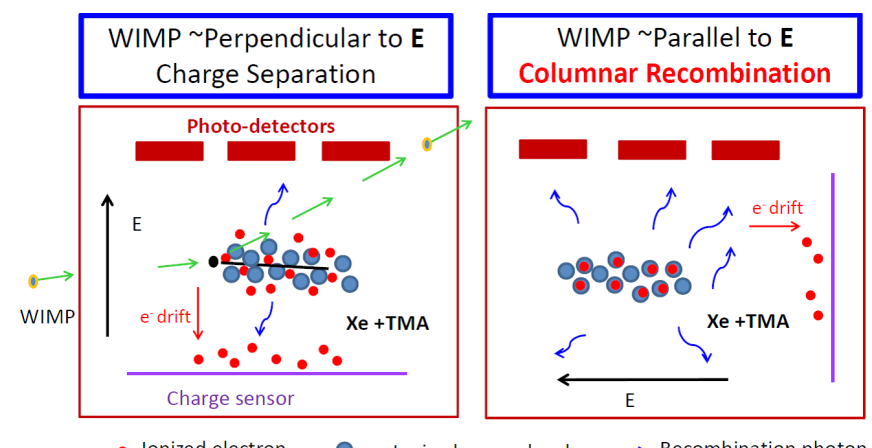

- Ionized electron $\bigcirc$ lonized gas molecule $\longrightarrow$ Recombination photon

Figure 1. Method of determining directionality using columnar recombination. As a WIMP enters the detection volume (left), it interacts with a Xe atom, producing a short, highly ionized track. With the electric field perpendicular to the track, charges are pulled apart (left). With the electric field along the track, more charges are recombined, resulting in fewer detected electrons and more emitted photons (right).

\section{RESULTS AND ACCOMPLISHMENTS}

We have obtained and assembled the components necessary as a first step to validating the columnar recombination method. Because the organic gas is hazardous, we are using a fume hood and a specially ordered gas closet for this purpose. The gas system, vacuum pump, demonstration detector, and gas analyzer are in place and functional. Other items have been fabricated or acquired and are now being installed, including photodetectors sensitive to UV light and a custom-made field cage to provide the electric field and charge collection electrodes. We anticipate collecting our first data by the spring of 2016. Most of our work in FY 2015 involved designing the apparatus, assembling the high-purity gas system, and obtaining the pressure vessel and gas analyzer, as well as determining the proper gas system to purchase for use with this hazardous gas mixture. We also investigated facilities where ion beams might be available to test the detector.

\section{PROPOSED FUTURE WORK}

The tasks for FY 2016 are to (1) demonstrate that this detector functions properly (holds voltage, holds pressure, has the proper gas purity); (2) demonstrate that recombination light and charge, when the detector is exposed to standard radioactive sources, are detected as expected and independent of the orientation of the electric field (because the ionization is not yet directional); and (3) optimize the admixture of TMA, which should result in a minimum of collected charge and a maximum of detected light. Our plans for FY 2017 are to expose the detector to an intense electron beam, a neutron source (several are available at Argonne), and a highly ionized, low-energy heavy ion beam, where the ionization density should be sufficient to demonstrate the hypothesis of columnar recombination as a method to determine directionality.

\section{Josephson Plasma Wave-Based Ultra-High-Frequency Electronics}

\section{5-078-NO}

\section{Alexei Koshelev and Vitalii Vlasko-Vlasov}

\section{PROJECT DESCRIPTION}

Operation at frequencies of 100 gigahertz $(\mathrm{GHz})$ and above is beyond the reach of conventional electronics. However, this frequency range is the next frontier for high-bandwidth communications and high-speed signal processing. Certain devices assembled from hightemperature superconductors promise to be naturally suited for operation at these frequencies. Extremely anisotropic high-temperature superconductors, such as $\mathrm{Bi}_{2} \mathrm{Sr}_{2} \mathrm{CaCu}_{2} \mathrm{O}_{8}(\mathrm{Bi}-2212)$ and $\mathrm{Tl}_{2} \mathrm{Ba}_{2} \mathrm{CuO}_{6}$, contain $\mathrm{CuO}_{2}$ (copper oxygen) planes alternating with insulating barrier layers. These naturally formed Josephson junctions can be used for direct-to-alternating current (DC-to-AC) conversion at terahertz $(\mathrm{THz})$ frequencies, enabling them to act as very compact sources and detectors of electromagnetic radiation in the "terahertz gap" range, spanning approximately 0.3 to $1.3 \mathrm{THz}$. These periodic structures are also predicted to act as nonlinear waveguides, allowing the propagation of so-called Josephson plasma waves (JPWs) with switchable transmission characteristics at frequencies of $100 \mathrm{GHz}$ and above. These various properties can thus be exploited for novel optoelectronic integrated circuits, incorporating such components as interferometers, ridge waveguides, and mixers for which the unique properties of layered superconductors can be fully exploited. Such systems would be of particular value for ultra-high-bandwidth telecommunications.

In the course of this project, we propose to demonstrate proof-of-concept for such devices by testing the mixing and controllability of JPWs in the Bi-2212 devices that we will fabricate. We also plan to test a new technique for growing high-quality thin films of Bi-2212, with a view to enabling wafer-scale fabrication of JPW devices.

\section{MISSION RELEVANCE}

This project is relevant to DOE's missions in national security, environmental quality, and science. The rapidly emerging field of $\mathrm{THz}$ science and technology holds immense promise for diverse areas, including high-speed electronics, high-bandwidth communications, environmental monitoring, security, manufacturing, and pharmaceuticals. Directly related to DOE's 
missions in basic science are the development of new materials platforms for high-speed electronics and the generation of electromagnetic nonlinearities in an energy-efficient way.

\section{RESULTS AND ACCOMPLISHMENTS}

The project requires sources for $\mathrm{THz}$ radiation. We have designed and fabricated a Bi-2212 mesa with dimensions of $60 \times 400 \times 0.64$ micrometers $^{3}\left(\mu \mathrm{m}^{3}\right)$. This mesa shows the strongest emission at a temperature of $\sim 65$ kelvin $(\mathrm{K})$ and voltage bias of 0.65 volt $(\mathrm{V})$, corresponding to an emission frequency of $f=0.75 \mathrm{THz}$ with a total emission power of $\sim 1.5$ microwatts $(\mu W)$. We used this radiation to investigate the excitation and propagation of JPWs in thin Bi-2212 crystals. Figure 1 shows the angle-dependent transmission curve through a 5 - $\mu$ m-thick Bi-2212 crystal at $0.75 \mathrm{THz}$ and $10 \mathrm{~K}$. The incident radiation is coupled through a $125-\mu \mathrm{m}$ gold ( $\mathrm{Au}$ ) grating into JPWs (see inset). The peaks at positive and negative angles are consistent with the anticipated excitation of JPWs traveling along the $\mathrm{CuO}_{2}$ planes. An estimate for the resonance angle, $\sin \left(\theta_{r}\right)=\left|n \sqrt{f^{2}-f_{p}^{2}}-c_{0} / a\right| / f \sim 15$ degrees, is in good agreement with the peak location evident in the experiment. Here, $n \sim 3.5$ is the far infrared (FIR) refractive index of $\mathrm{Bi}-2212, f_{\mathrm{p}}=0.13 \mathrm{THz}$ is the Josephson plasma frequency, and $a=125 \mu \mathrm{m}$ is the grating period. However, because the overall transmitted power is low, it is necessary to evaluate background signals and spurious scattering carefully.

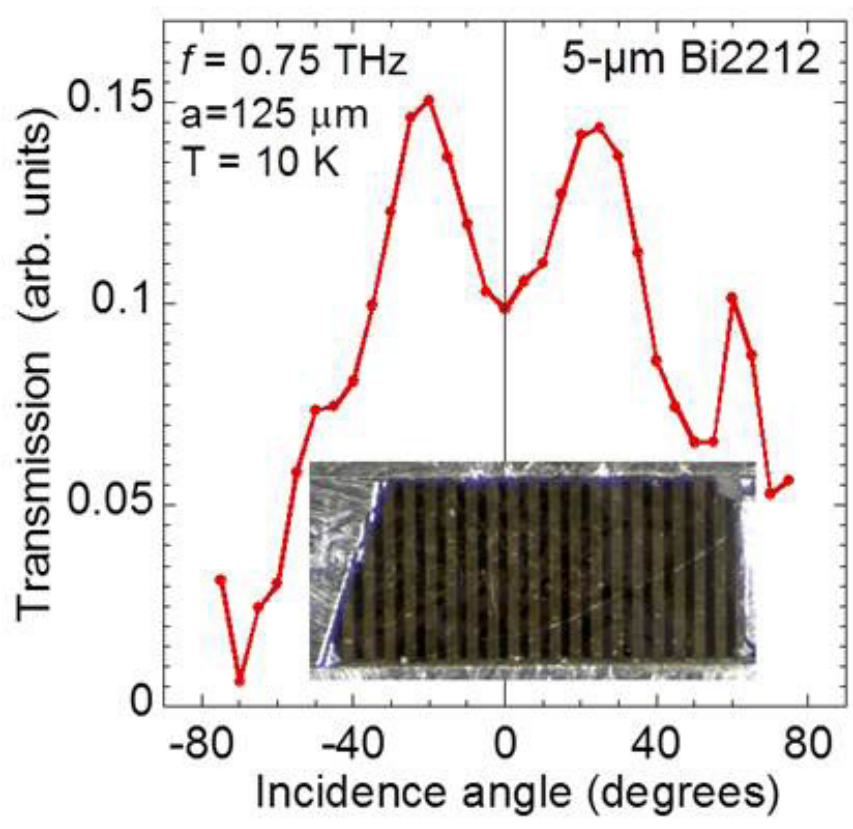

Figure 1. Experimental angular-dependent transmission through a 5- $\mu \mathrm{m}$-thick Bi-2212 crystal carrying a 125- $\mu \mathrm{m}$ Au grating as shown in the inset photo.
To develop a better understanding, we performed a theoretical calculation of the electromagnetic wave transmission through a $\mathrm{Bi}-2212$ crystal with the periodic metallic grating on the top and bottom surfaces. The calculation takes into account grating-induced conversion of the incident electromagnetic wave into the JPW inside the crystal and multiple reflections from the top and bottom surfaces. The transmission is controlled by two dissipation parameters, $v_{c}$ and $v_{a b}$, that are proportional to the $c$-axis and in-plane components of quasiparticle conductivity, respectively. The result was that for realistic parameters, the transmission is mostly sensitive to the in-plane dissipation parameter $v_{a b}$.

Figure 2 shows the computed angular dependences of the transmission coefficient for different values of $v_{a b}$. The transmission is characterized by a multiple-peak structure. The lowest-angle peak occurring at $\theta_{\mathrm{r}}=15$ degrees corresponds to excitation of the uniform c-direction mode. At low dissipation levels, $v_{a b}<0.1$. Fabry-Perot resonances at higher angles are also observed. The location of the first peak agrees with experiment; however, computation suggests sharper peaks.

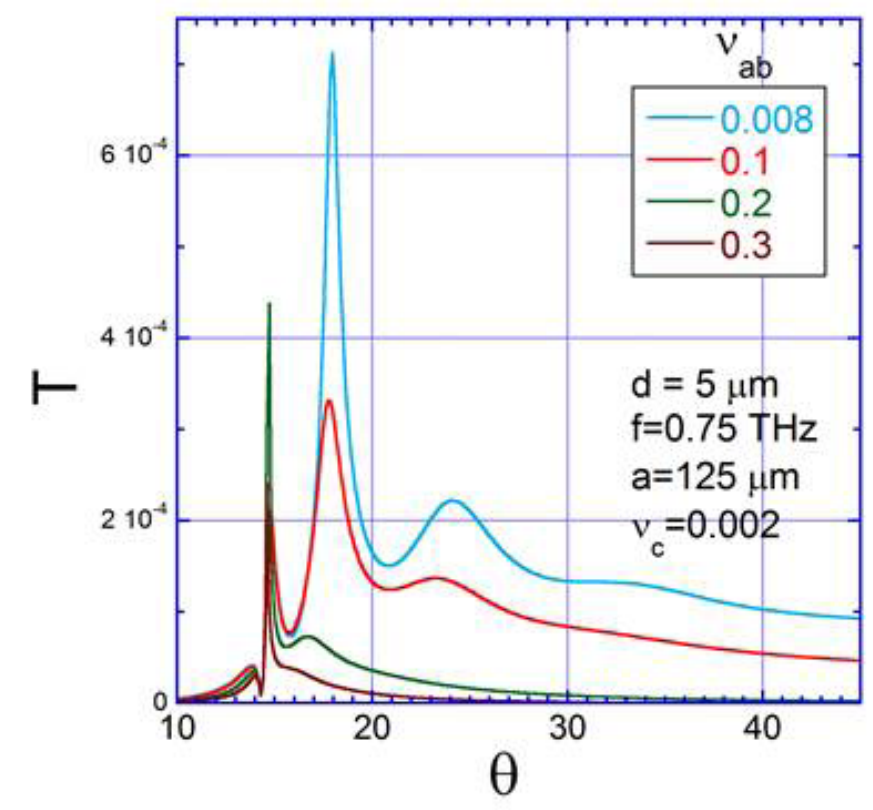

Figure 2. Computed angular dependence of transmission through a $\mathrm{Bi}-2212$ crystal with metal gratings for different in-plane dissipation parameters $v_{a b}$ and fixed $v_{c}=0.002$. 


\section{PROPOSED FUTURE WORK}

In FY 2016, we will grow epitaxial Bi-2212 films by using liquid-phase epitaxy and pattern gratings, such as those shown in Figure 1 to launch JPWs. We will fabricate ridge guides and interferometers for plasma waves on these films. We will investigate the nonlinear interaction of two beams and pursue the switching of one plasma wave train by another. The emission power of the emitter chip used for the experimental results shown here is comparatively low, at only $1.5 \mu \mathrm{W}$. We have therefore recently assembled $\mathrm{Bi}-2212$ mesa devices that have each been optimized to generate 5-10 $\mu \mathrm{W}$ with very high reliability, and we will employ synchronized arrays of these sources for future JPW experiments. Alternatively, we will pursue the use of backward wave oscillators as the source of incident radiation. Both of these approaches will result in a dramatic improvement in the achievable signal-to-noise ratio for experiments involving JPWs. We will also elaborate the theoretical description of these experiments.

\section{Next-Generation Natural Gas Adsorbent through Rational Design and Modeling}

\section{5-091-NO}

\section{Di-Jia Liu and Rajesh K. Ahluwalia}

\section{PROJECT DESCRIPTION}

This project focuses on developing a new synthesis strategy and fundamental understanding that could lead to the next-generation, three-dimensional porous polymeric network (PPN) materials for gas adsorption and separation applications. These could have such applications as storage of methane for a natural gas (NG) -fueled vehicle. A PPN is an all-organic framework, formed by the crosslinking of a molecular "strut" with a stereo-contorted molecular node. The scaffolding through covalent bonds between these building units creates unique properties. PPNs (1) have high surface areas and porosity when properly prepared; they could be significantly more porous with greater internal surface area than traditional zeolites; (2) are light weight because of their hydrocarbon-only frameworks; and (3) are extremely tolerant toward heat (up to $400^{\circ} \mathrm{C}$ ) and contaminants in natural gas due to their covalent bonds. In addition to physisorption, chemisorption based systems such as complex hydrides that can be integrated into PPNs or high surface area materials will also be explored for gas storage and separation.
We will prepare PPNs with high specific surface area and controlled micro-porosity. Molecular struts with different chelating functional groups will be introduced to prepare PPNs with desirable surface properties. Advanced characterization techniques, such as Brunauer-EmmettTeller (BET) analysis, high pressure Sievert isotherm, small and wide angle $x$-ray scattering methods, will be used to study gas adsorbance. We will determine how methane $\left(\mathrm{CH}_{4}\right)$ interacts with the PPN and the optimal structural/ physical properties for maximum usable storage capacity. The outcome of this project will be crucial in realizing the full potential of a PPN-based gas adsorbent. The new PPNs will also have broader potential applications in gas separation, catalysis, and other energy efficiency-related applications.

\section{MISSION RELEVANCE}

This project focuses on developing new natural gas storage technology for transportation applications. It aims at tapping into the vast NG reserve in the United States as a replacement for petroleum. The project is therefore relevant to DOE's missions in securing the national energy supply and reducing U.S. dependence on foreign oil. In addition to gas storage, the new PPNs from this project could also be applied to gas separation, catalysis, and other applications related to the energy conversion and environmental protection missions of the Department.

\section{RESULTS AND ACCOMPLISHMENTS}

Design and Synthesis of a PPN with Bi-dentate

Metallation Site. One key objective of this project is to develop a PPN containing a multi-dentate ligation site by incorporating functionalized molecular struts. Such multi-dentate sites could serve as host to chelates with transition metal ions during ion-exchange reactions. On the basis of theoretical insights, such exchange metal ions could modify the electronic property of the PPN surface to enhance the binding energy with a gas adsorbate such as methane. To explore this feasibility, we prepared a PPN containing bi-dentate ligation sites using bipyridine as the molecular strut. Figure 1a shows the synthesis scheme. The dibromo-bipyridine (1) through reaction step (i) and (ii), was converted to the diethynyl-bipyridine (3), which subsequently reacted with contorted molecular node tetraethynyl-spirobifluorene (4) to form PPN polybipyridine-spirobifluorene (5) through the trimerization reaction. Cobalt carbonyl was used as the transition metal dopant and was exchanged on the bi-dentate site of bipyridine. The PPN thus prepared has a relatively high specific surface area of 350 square-meters/ gram $\left(\mathrm{m}^{2} / \mathrm{g}\right)$ with narrow pore size of less than one nanometer $(\mathrm{nm})$. We are continuing to improve the surface 
properties through refinement of synthesis conditions and plan to measure methane uptake once the optimization is completed.

\section{Improving Onboard Hydrogen Storage through} Graphene-Encapsulated Hydride. Onboard storage of hydrogen represents another critical transportation technology for future zero-emission vehicles. Boron hydride has been regarded as a promising molecular hydrogen $\left(\mathrm{H}_{2}\right)$ storage medium because of its high theoretical capacity. Past studies found, however, that boron hydrides show poor regenerability and suffer by-product contamination during the hydrogen chargingdischarging cycle. With researchers from Shanghai Jiao Tong University, a novel approach for preparing stable and rechargeable boron hydride composite as a hydrogen storage material was developed. The new storage material was prepared by using a simple, robust wet chemistry method to encapsulate nano-dimensional boron hydride crystallites inside of lightweight, singlelayer graphene sheets (Figure 1b). Such a composite structure restricts the hydride phase segregation/ agglomeration during de/re-hydrogenation and prevents the escape of contaminates in hydrogen. A steady and highly reversible $\mathrm{H}_{2}$ storage capacity of $7.0 \mathrm{wt} \%$ was achieved. Furthermore, no by-product was detected in the so-produced hydrogen.

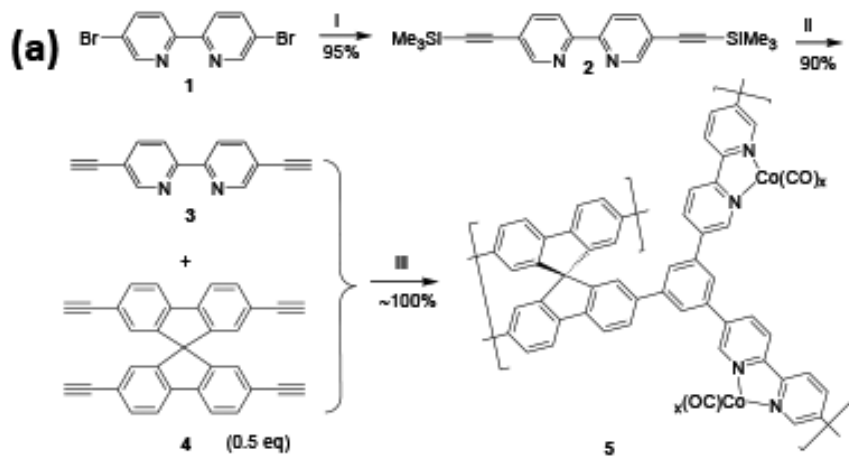

(b)

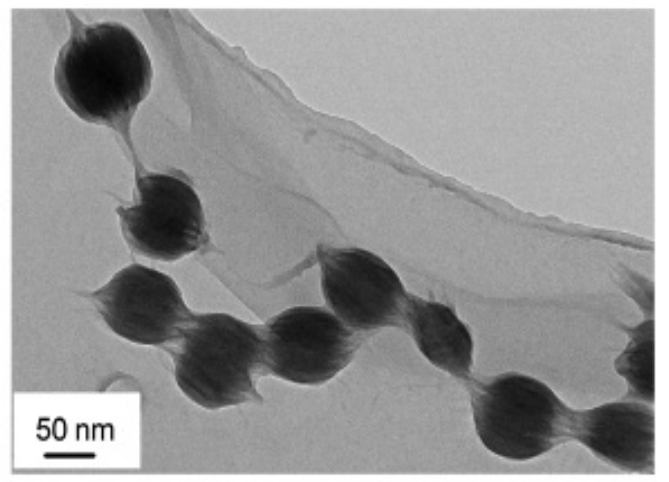

Figure 1. (a) The reaction scheme to prepare a PPN with a bi-dentate ligation site (polybipyridine-spirobifluorene) (details of the reaction sequence are described in the text) and (b) transmission microscopic images of graphene-wrapped boron hydride crystallites for hydrogen storage applications.

\section{PROPOSED FUTURE WORK}

We will continue to design, synthesize, and improve different PPN systems according to our original project plan. We will prepare, for example, PPNs with tetra-dentate ligation sites to further improve binding energy with transition metal and methane adsorption enthalpy. Other methods of enhancing surface properties and methane uptake capacity will be investigated. In addition to gas storage, we will apply surface functionalized PPNs to other clean energy-related applications. Advanced characterization tools, including BET analysis, Sievert isotherm, $x$-ray scattering methods will be employed to study the PPN framework structure and its interaction with gas adsorbates.

\section{Understanding Atomic-Scale Uranium Interactions under Severe Accident Conditions}

\section{5-096-NO}

\section{Chris Benmore and Mark Williamson}

\section{PROJECT DESCRIPTION}

A key safety concern in nuclear reactors is the melting and subsequent leakage of radioactive uranium dioxide $\left(\mathrm{UO}_{2}\right)$ as it reacts with its zirconium cladding. The atomic structure of the liquid state is the key to understanding the properties of molten nuclear fuel, yet until this work there were no published structural data on liquid $\mathrm{UO}_{2}$. Part of the reason for the lack of data is the very high melting temperature of $\mathrm{UO}_{2}, 3140$ kelvin $(\mathrm{K})$. The main objective of the proposed research is to develop a fundamental understanding of the physics of molten nuclear systems along with the high-temperature crystalline phases by using high-energy $\mathrm{x}$-rays and ultrahigh-temperature containerless melting techniques. To solve the problem of accessing samples at high temperatures, we used an aerodynamic levitator system with laser heating to attain temperatures above $3300 \mathrm{~K}$ in a radiologically controlled, hermetically sealed chamber, see Figure 1. The absence of a solid contact surface allows the chemical purity of the very hot sample to be maintained. The x-ray measurements were performed at the Advanced Photon Source (APS) and the results were interpreted using molecular dynamics simulations. 

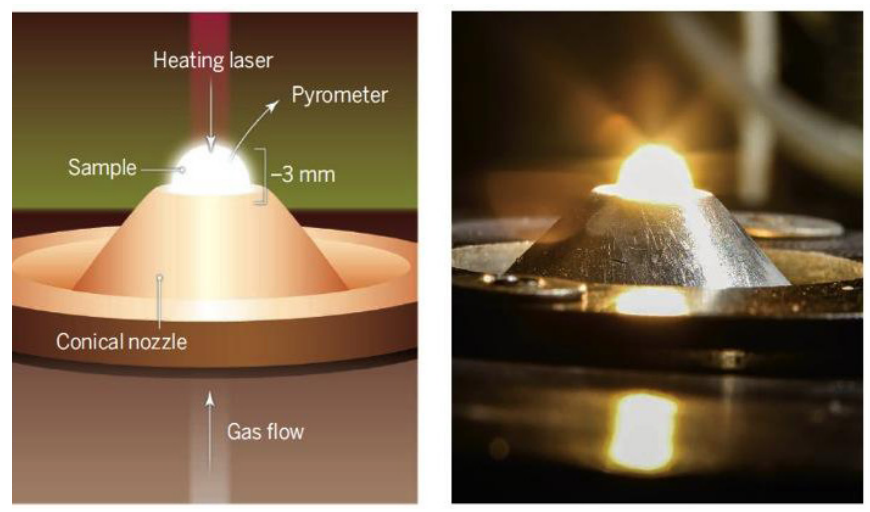

Figure 1. Laser heating of an aerodynamically levitated droplet.

\section{MISSION RELEVANCE}

Hazard and accident analysis of nuclear fuels is paramount to establishing nuclear safety requirements. The primary motivation for this study was the exclusive use of $\mathrm{UO}_{2}$ in nuclear power reactors in operation today, given that even mixed-oxide fuel is typically $90 \mathrm{wt} \% \mathrm{UO}_{2}$. The DOE Office of Nuclear Energy is actively pursuing the development of accident-tolerant fuels. Insight into the behavior of liquid $\mathrm{UO}_{2}$ will provide guidance in the selection of an optimum fuel composition, additives to stabilize the fuel, and cladding material. Since computer modeling of materials is an important component of the fuels development program, experimentally derived interatomic potentials are essential for model development. Similarly, reactor safety codes under development by the Nuclear Regulatory Commission would benefit from the experimental structural data from this research and development.

\section{RESULTS AND ACCOMPLISHMENTS}

The structures of high-temperature crystal and molten $\mathrm{UO}_{2}$ samples were probed by using high-energy $\mathrm{x}$-rays at the APS. The high atomic number $(Z)$ of $\mathrm{UO}_{2}$, and the 2- to 3-millimeter ( $\mathrm{mm}$ )-diameter droplet size of the molten sample, means that $x$-rays of greater than 100-kilo-electronvolt (keV) energy are required to minimize absorption and multiple scattering, which can distort the measured structure factor. A high flux of $x$-rays is also required to obtain sufficient statistical accuracy in short (a few seconds) measurement times. To avoid fluorescence from the uranium K-edge energy of $115.6 \mathrm{keV}$, a slightly lower energy of $114.7 \mathrm{keV}$ was used in the feasibility study to minimize multiple scattering. The scattered $\mathrm{x}$-ray data were analyzed and pair distribution functions extracted that characterize the local and longrange atomic structure of the material. The measurements of the hot solid show a substantial increase in oxygen disorder around the lambda transition at $2670 \mathrm{~K}$ (an anomalous behavior in the heat capacity), but negligible
$\mathrm{U}-\mathrm{O}$ coordination change. Upon melting, the average U-O coordination was found to decrease from 8 to $6.7 \pm 0.5$. The measured $\mathrm{U}-\mathrm{O}$ bond length was found to contract upon heating up to the melting point, despite normal volume expansion. These results indicate that a substantial change from the $\mathrm{UO}_{8}$ cubic polyhedra of the calcium fluoride $\left(\mathrm{CaF}_{2}\right)$-type crystal structure in the solid occurs in the melt. This change is associated with disordering of the local U-U arrangements, which describe the connectivity of $\mathrm{U}-\mathrm{O}$ polyhedra in the liquid. Theoretical interatomic potentials were tested and refined by using molecular dynamics simulations to reproduce the experimental results, see Figure 2.

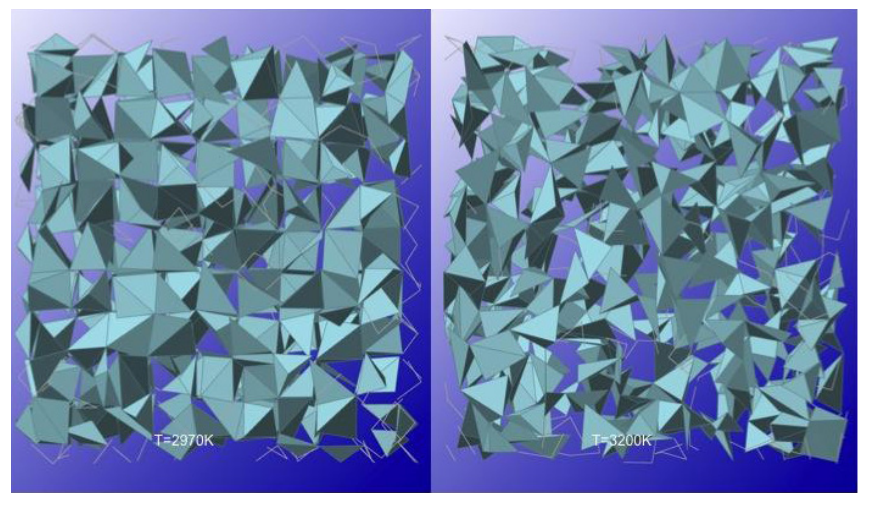

Figure 2. Snapshots of molecular dynamics simulations of the $\mathrm{UO}_{2}$ structure before and after melting.

\section{PROPOSED FUTURE WORK}

High-energy $\mathrm{x}$-ray measurements will be performed on the crystalline and liquid states of $\mathrm{UO}_{2}-\mathrm{ZrO}_{2}$ (zirconium dioxide) and $\mathrm{UO}_{2}-\mathrm{FeO}$ (iron oxide) systems to investigate the atomic-scale interactions of uranium at extreme temperatures. The proposed binary mixtures represent important components of model corium systems (e.g., the Chernobyl "lavas" that can form during core meltdown). As such, they are important benchmarks for modeling severe accident scenarios. To ensure that we obtain powdered averages from high-temperature solid $\mathrm{UO}_{2}-\mathrm{ZrO}_{2}$ as well as other systems, we plan to design and build a sample spinner system to be integrated into the system. This system will allow the solid sample to rotate freely without the instabilities normally associated with non-spherical materials in the levitator. The extracted $x$-ray pair distribution function data will enable local structural information to be obtained. Levitation will also be performed by using redox mixtures based on $\mathrm{CO} / \mathrm{CO}_{2}$ or $\mathrm{H}_{2} / \mathrm{Ar}$ gas mixtures. Various oxygen potentials will be used to stabilize $\mathrm{UO}_{2}$ and to adjust its stoichiometry toward and away from the mixed-valency triuranium octoxide $\left(\mathrm{U}_{3} \mathrm{O}_{8}\right)$. Molecular dynamics potentials will be refined against the experimental data to obtain the most realistic models. This approach will enable information on the topological and chemical ordering structure of the melt to be extracted. 


\section{Development of Advanced $\mathrm{VO}_{2}$ Nano-Composite Thermochromic Materials for High-Performance Smart Windows}

\section{5-121-NO}

Jie Li, Leah Guzowski, Ralph Muehleisen, and Yugang Sun

\section{PROJECT DESCRIPTION}

Buildings consume up to $40 \%$ of the world's primary energy. An emerging concept to reduce building energy consumption in hot weather is the so-called "smart window," which allows visible light to go through while selectively blocking infrared heat according to local temperature. The physics behind this temperaturedriven infrared-controlling behavior is the thermochromic (TC) mechanism: with an increase in temperature, the construction material experiences a (reversible) structural change that results in a corresponding electrical-property transition from a semiconductor to a metal. The metal-state material reflects the incoming long-wavelength light that passes through when the material is in its semiconductor state. Among various $\mathrm{TC}$ materials, $\mathrm{VO}_{2}$ (monoclinic $\mathrm{M}$-phase) represents the most promising material because of its large and sharp change in electric and optical properties around its transition. Currently, three key technological barriers prevent $\mathrm{VO}_{2}$ 's wide application for smart windows: (1) a high transition temperature of $68^{\circ} \mathrm{C}$, well exceeding the room temperature; (2) low luminous transmission (LT, less than 40\%); and (3) small solar transmission modulation (STM, typically less than 10\% between its high- and low-temperature operating modes). A recent theoretical calculation [J. Appl. Phys. 108 (2010) 06352] predicted that TC materials constructed from nanoparticles, rather than bulk film, can exhibit a remarkable increase in LT to as high as $80 \%$, and a doubling of STM to $21 \%$. These findings suggest the potential for a technological breakthrough using nanoparticle-based smart films.

The purpose of this project is to develop advanced $\mathrm{TC}$ materials and the related (continuous) processing technology based on nanocomposites of "core-shell" nanoparticles (rods) of silicon dioxide $\left(\mathrm{SiO}_{2}\right)$-coated $\mathrm{VO}_{2}$ that are uniformly dispersed in a visible-light-transparent host matrix. By synthesizing nanoparticles, engineering the surface, and dispersing them in a host dielectric material, we seek to develop new films that not only effectively enhance the heat reflection (metal state/high temperature) via a nano-confinement-enhanced surface plasmon resonance mechanism, but also selectively intensify light-material interactions only in the infrared region. The ultimate goal is to achieve the theoretically predicted performance levels. Our continuous process can be easily scaled up.

\section{MISSION RELEVANCE}

This project supports the DOE's missions of improving national energy security, supporting the U.S. economy, and protecting the environment. It aligns well with the Energy Secretary's emphasis on energy efficiency and with DOE's Office of Energy Efficiency and Renewable Energy (EERE) Building Technology Office's focus. The potential beneficiaries of this technology include building owners, window manufacturers, automobile makers, and sensor and detector producers, as well as the defense industry, where this $\mathrm{VO}_{2}$ material could be used to protect equipment from high-powered lasers.

\section{RESULTS AND ACCOMPLISHMENTS}

In FY 2015, $\mathrm{VO}_{2}$ nanorods with diameters of -100 nanometers ( $\mathrm{nm}$ ) and aspect ratios larger than 5 were initially synthesized through a microwave-assisted hydrothermal reaction by using an existing batch microwave reactor at Argonne's Center for Nanoscale Materials. The synthesis conditions were systematically examined to understand how to effectively control morphology and phase purity. The as-synthesized $\mathrm{VO}_{2}$ nanorods (in B-phase) were successfully coated with $\mathrm{SiO}_{2}$ shells via a Stöber sol-gel reaction to prevent agglomeration and sintering during post-treatment (thermal annealing). However, analysis shows that the final product is $\mathrm{V}_{6} \mathrm{O}_{13}$ rather than $\mathrm{VO}_{2}$ (M-phase); the cause has been identified as the low synthesis temperature, which reflects the limitations of the microwave reactor.

To address this issue and the issue of scale-up, we carried out systematic experiments by using a newly developed micro-reactor, which is capable of operating at much higher temperature and pressure and in a continuous mode. Because of the nature of continuous flow, the two steps of $\mathrm{VO}_{2}$ nanorod synthesis and $\mathrm{SiO}_{2}$ coating can potentially be integrated into a single system, significantly accelerating the process. The as-synthesized product from this micro-reactor is pure $\mathrm{VO}_{2}$ (A-phase) nanorods, as shown in Figure 1. The annealed product is proved to be $\mathrm{VO}_{2}$ (M-phase) (Figure 2). In particular, the synthesis time has been drastically reduced, from days to hours. This success enables a new direction for massively synthesizing high-quality $\mathrm{VO}_{2}$ nanorods, and this process can be used to synthesize other nanomaterials as well. An invention disclosure has been filed for this novel $\mathrm{VO}_{2}$ 
(M-phase) synthesis technology. Preliminary optical tests of the synthesized $\mathrm{VO}_{2}$ (M-phase) powders indicated that this material exhibits the TC effect (Figure 3). Further optimization is under way.

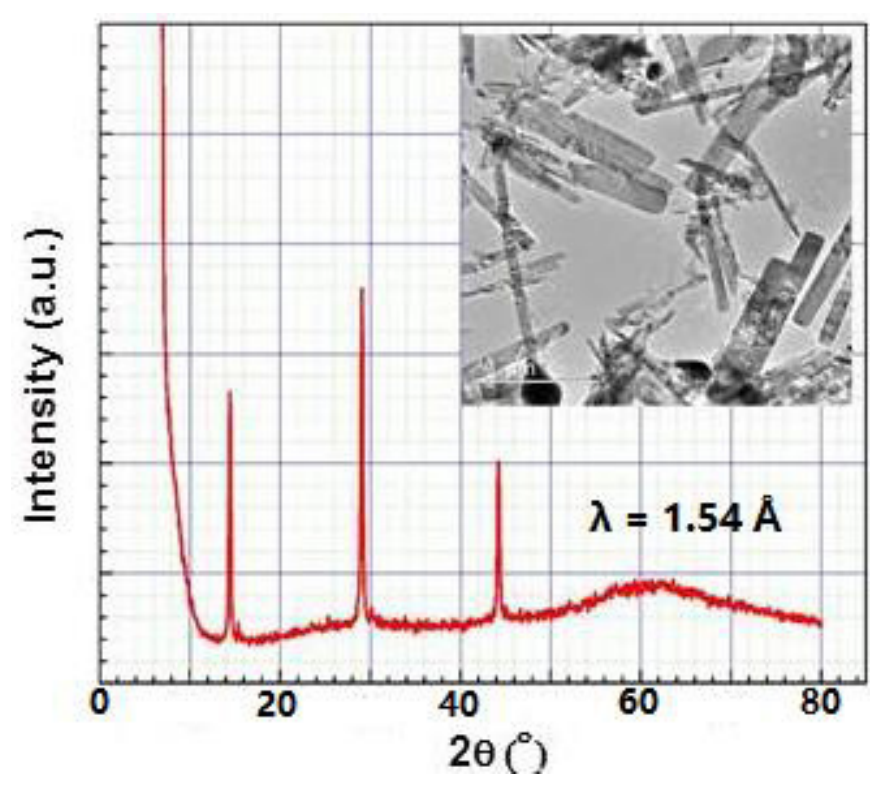

Figure 1. X-ray powder diffraction (XRD) data and transmission electron microscopy image showing $\mathrm{VO}_{2}$ (A-phase) nanorods as synthesized in a continuous-flow micro-reactor.

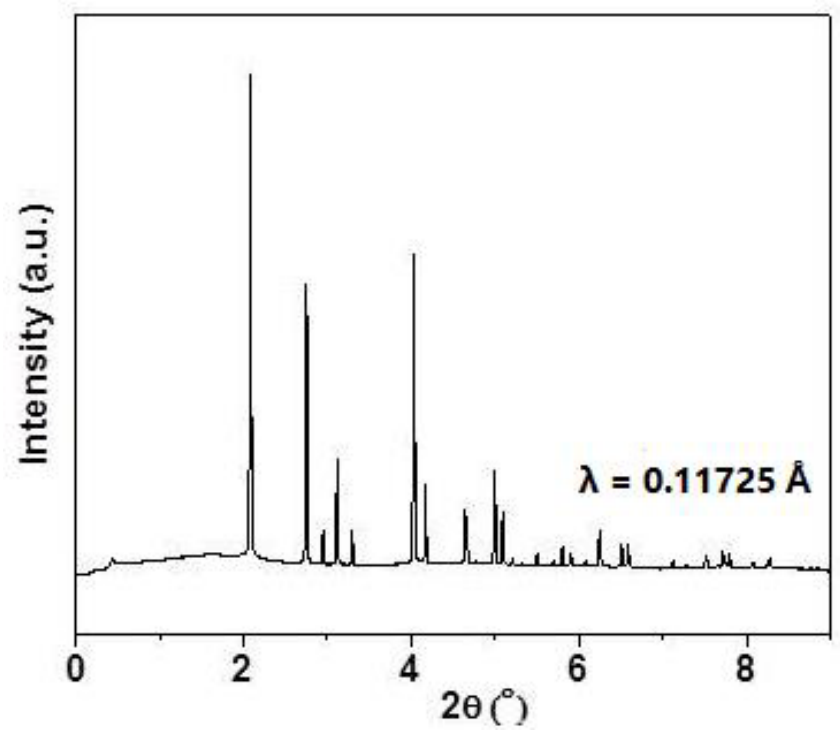

Figure 2. XRD shows the pure $\mathrm{VO}_{2}$ (M-phase) phase obtained via thermal annealing of the $\mathrm{VO}_{2}$ (A-phase) nanorods shown in Figure 1.

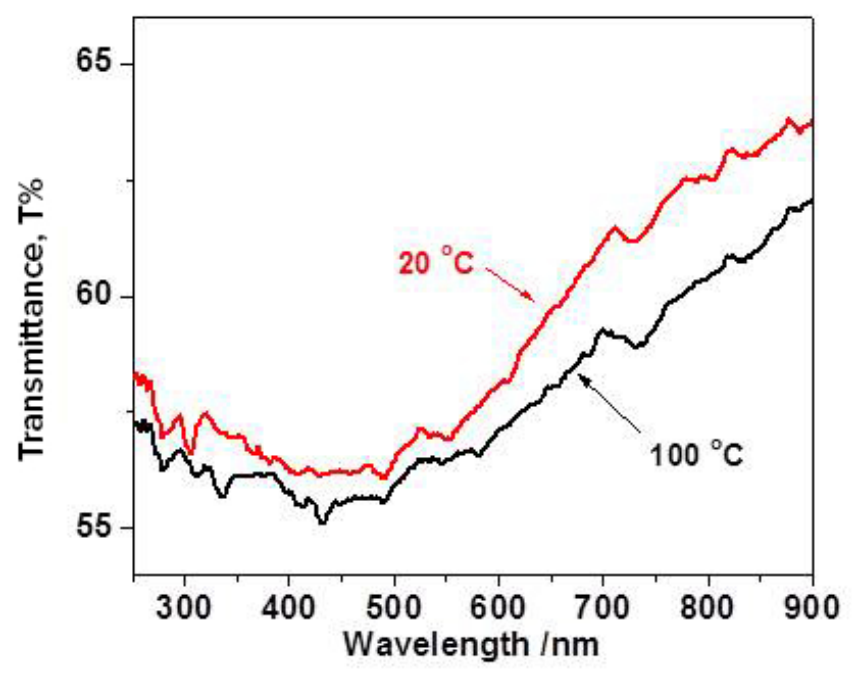

Figure 3. Ultraviolet/visible/near-infrared transmission spectra of the $\mathrm{VO}_{2}$ (M-phase) nanorods at low (red curve) and high (black curve) temperatures. The comparison shows that more near-infrared light is blocked at high temperature.

\section{PROPOSED FUTURE WORK}

In FY 2016, the major planned activities include the following:

$\square$ Improve the continuous-flow hydrothermal synthesis process for stable operation;

$\square$ Integrate nano-coating to make $\mathrm{VO}_{2}\left(\mathrm{~A}\right.$-phase) $/ \mathrm{SiO}_{2}$ core-shell nanorods;

$\square$ Prepare $\mathrm{SiO}_{2} @ \mathrm{VO}_{2}(\mathrm{M}$-phase) TC films by developing a self-assembly-based nano-coating technology to uniformly disperse the nanorods in a target transparent matrix in a fraction-, distance-, and orientation-controllable way; and

$\square$ Conduct performance tests with nanocomposite films prepared on the laboratory scale to identify the optimized conditions and achieve the targeted optical properties. 


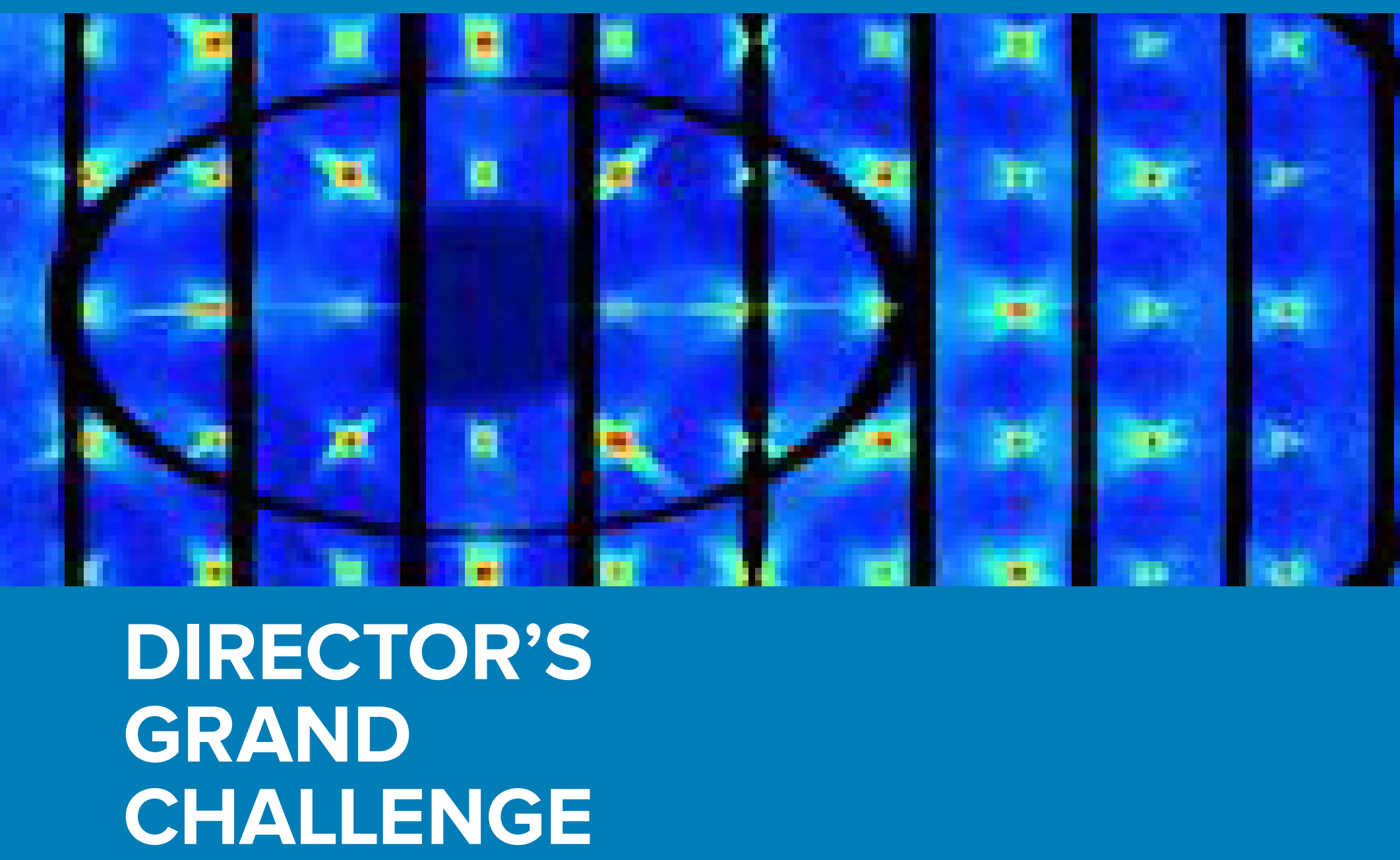




\section{Integrating Simulation and Observation: Discovery Engines for Big Data}

\section{3-165-R2}

Ian T. Foster, Jonathan D. Almer, Rachana Ananthakrishnan, Ben Blaiszik, Kyle Chard, Lin X. Chen, Peter Chupas, Emil M. Constantinescu, Hal Finkel, Nicholas Frontiere, Ben Gutierrez, Salman Habib, Katrin Heitmann, Guy Jennings, Raj Kettimuthu, Eve Kovacs, Ravi Madduri, Tanu Malik, Ray Osborn, Adrian Pope, Esteban Rangel, Stephan Rosenkranz, Hemant Sharma, David Tiede, Tom Uram, Peter van Gemmeren, Venkatram Vishwanath, Michael Wilde, Justin Wozniak, and Peter Zapol

\section{PROJECT DESCRIPTION}

This project was supported under the Argonne "Big Data" Strategic Initiative. In launching a big data initiative, we sought problems that were important to science, relevant to Argonne and DOE projects, and tractable given the state of the technology and our specific expertise. This perspective-plus feedback provided during the proposal development and review process-led us to adopt a dual focus for the project encompassing first, research problems in cosmology and materials science that involve the coupling of experiment and modeling and second, the acceleration of end-to-end workflows in our research.

In cosmology, we sought to build new methods capable of exploiting observational data from increasingly high precision digital sky surveys [e.g., the Sloan Digital Sky Survey (SDSS), Dark Energy Survey (DES), Dark Energy Spectroscopic Instrument (DESI), Large Synoptic Survey Telescope (LSST), and South Pole Telescope (SPT)] using high-resolution cosmological simulations run on supercomputers such as the Blue Gene/Q (Mira), to simultaneously determine the cosmological parameters and quantitatively evaluate the validity of the modeling process itself. In materials science, the goal was to develop the capability of managing big data on the Advanced Photon Source (APS) beamlines and to generate scientific models using computational simulation and other statistical techniques. A particular focus was the determination of complex defect structures derived from single crystal diffuse scattering experiments.

While cosmology and materials science differ widely in their scales and scientific foci, they share crosscutting concerns that make interactions between these two efforts fruitful. In particular, they share a need to deal with substantial analysis complexity (both must collect, organize, and manage data from many experiments, instruments, simulations, and analyses); analysis scale (both must perform complex analysis on large datasets, solve large statistical inverse problems, and manage big data); and deployment of new methods into the workflows of experimental and/or observational facilities. In both domains, the scientific progress can be accelerated via the creation of new discovery engines that can enable the rapid and collaborative acquisition, exploration, integration, and analysis of large quantities of observation and simulation data.

\section{MISSION RELEVANCE}

"Big data" problems are pervasive across DOE science. For example, extreme data volumes are encountered at DOE experimental facilities, such as light sources; in supercomputer simulations conducted at leadership computing facilities; in the digital sky surveys used in cosmology; and in high energy physics experiments. Furthermore, dramatically larger data volumes and velocities are expected from new light source detectors and exascale simulations. The need to integrate a challenging variety of data arises in biology, materials science, and sky surveys.

\section{RESULTS AND ACCOMPLISHMENTS}

In the first two years of the project, we developed new end-to-end high-performance APS data processing pipelines for two quite different experimental modalities (diffuse scattering and near- and far-field high-energy diffraction microscopy [HEDM]), engaged successfully with 10 APS sectors in various ways, and explored two new analysis methods that integrate theory with experiment. We also ran some of the world's largest cosmological simulations, producing a multi-petabyte simulation dataset that is bigger than any contemporary observational dataset in terms of raw size, and developed new density estimation, merger tree, and sub-halo tracking algorithms for the analysis of that data. We defined an overarching data services architecture, developed new services that enable tracking of complex end-to-end activities, led the definition of a Materials Data Facility, and informed the design of data publication and discovery services that are already being adopted by other disciplines.

We have continued to make good progress in FY 2015 in each of the project areas.

\section{DATA ACCESS, STORAGE, AND MANAGEMENT}

$\square$ We further refined a crosscutting data systems architecture for use in this project and others: see Figure 1. 


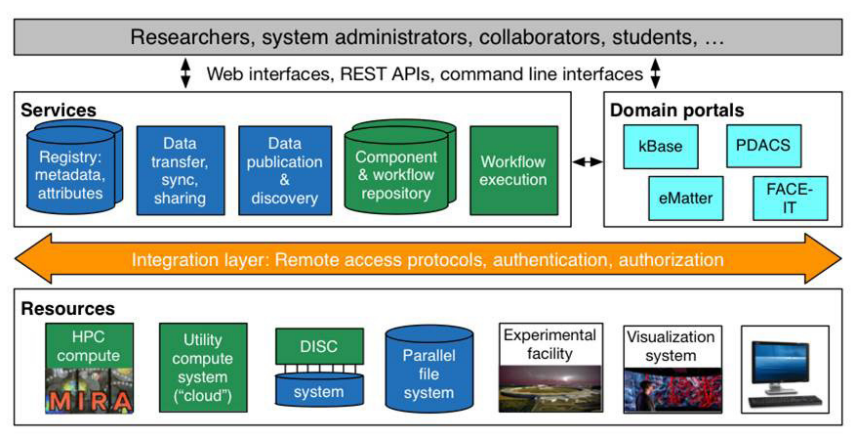

Figure 1. The integrating big data architecture that we have developed to support data-intensive science across computer systems, facilities, and disciplines. Important elements include an integrating layer permitting secure, high-speed access to remote data and services for tracking data products, transferring data, publishing data, and related purposes.

$\square$ We evaluated file system and management methods to support interactive parallel analysis, explored options including the Hadoop Distributed File System (HDFS) and direct use of Swift data management, and implemented Nexus-compatible remote data access operations. Nexus was developed as a common data format for neutron, x-ray, and muon science.

$\square$ We evaluated and optimized data transfer performance between various data systems at Argonne and beyond, including the Argonne Leadership Computing Facility (ALCF), the National Energy Research Scientific Computing Center (NERSC), and APS beamlines. The Globus data transfer service developed by Argonne and the University of Chicago was used for this work and was applied to link APS experimental runs with ALCF computing.

$\square$ We designed and tested an authenticated hypertext transfer protocol (HTTP) service for accessing remote data. The service leverages Globus authentication and authorization capabilities to manage access to data.

$\square$ Based on user feedback, we iteratively developed a new catalog user interface that makes it easier for users to interact with the catalog. This interface applies new web-development techniques to enable tabular and list views of results, expandable data set browsing, and a rich faceted search feature.

$\square$ We integrated the catalog with two suites of analysis tools: PyRO (Python remote objects) and NeXpy (which provides a high-level Python interface to Nexus data). We demonstrated that PyRO and NeXpy could discover datasets via integrated interfaces, remotely browse datasets, and launch analyses on cataloged datasets independent of location.

$\square$ Building upon our pluggable dataset ingestion and publication architecture, we developed metadata ingestors for the data types used at the APS Sector 1 beamlines. We used these ingestors for archived Sector 1 metadata. $\square$ We developed an automated metadata extractor for $\mathrm{x}$-ray photon correlation spectroscopy (XPCS) data with collaborators in Argonne's X-ray Science Division.

$\square$ We enhanced the Globus Catalog application programming interface (API) to associate provenance records with data stored in the catalog. These records describe the experimental configuration and computational steps taken to generate the data. In this way, we allow later users of the data to determine when and where it was produced.

Milestone: A catalog is used for substantial multi-experiment data collection by several groups. We cataloged datasets for HEDM, x-ray tomography, XPCS, and $x$-ray scattering. Our automated ingestion scripts have been used to ingest more than 300 datasets from a single beam-time run.

\section{DIFFUSE SCATTERING}

We demonstrated the scientific value and portability of our diffuse scattering data analysis pipeline by conducting three sets of experiments at two different synchrotron sources-APS and the Cornell High Energy Synchrotron Source (CHESS) -running our complete pipeline in both environments. Figure 2 shows some of the components and steps involved.

The diffuse scattering pipeline encompasses three steps, as follows. In the first step, raw images in a tagged image file (TIFF) or a crystallographic binary file (CBF) format are collected at the beamline, streamed to a remote server, and automatically stacked into large Nexus/HDF5 experiment data files, about 30 to $90 \mathrm{~GB}$ in size, along with relevant instrumental and sample metadata. See the top left of Figure 2 where TIFF files are collected from the detector (Pilatus 6M), stored, and processed with the NXSTACK program. These resulting data files are registered in the Globus Catalog, so that they can be easily accessed from the beamline or other locations using the PyRO server.

The second step, performed once per sample, involves the manual refinement of sample and instrumental parameters based on examination of the first dataset from the sample. This step can be performed by the scientist at the beamline using the PyRO links contained in the Catalog to access the data from the remote server.

The third step, performed automatically for each subsequent data set from the same sample, applies automatic peak searches, orientation adjustments, and transformations from instrumental coordinates to reciprocal space coordinates. See the top center of Figure 2 which shows the Crystal Coordinate Transformation Workflow (CCTW) program that we have developed for this purpose. 
After the automated reduction, we also demonstrated the ability to visualize and analyze the data on the beamline, using the extensible Python graphical user interface (GUI), NeXpy. This part of the workflow is shown on the right side of Figure 2. Thus, the scientist is able to review data during the experimental session, rather than (as is currently the practice) having to wait until after the experiment is completed.

The version of our diffuse scattering pipeline that we used for these experiments takes 20 minutes to perform data reduction for each $360^{\circ}$ sample rotation: a factor of three longer than the time required to collect the data. We have demonstrated, but not yet deployed, a parallelized Swift prototype of CCTW that produced a considerable speed-up.

In the two experiments at the APS, we collected 8.6 terabytes (TB) and 3.7 TB of data. In the experiment at CHESS, we collected 22.7 TB of data. We were able to process these large quantities of data with essentially the same network architecture shown in Figure 2.

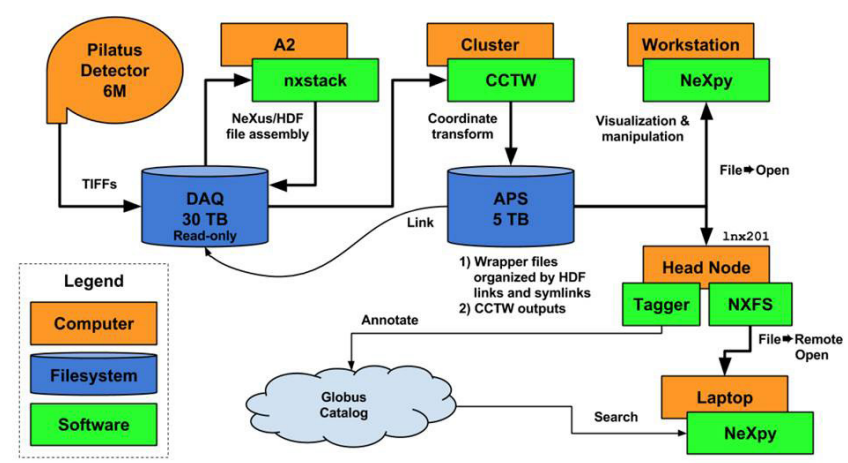

Figure 2. The network architecture developed to enable on-demand, automated analysis of diffuse scattering data at APS and Cornell. Raw data, collected at the beamline by using a Pilatus $6 \mathrm{M}$ detector (top left), is stored in a first repository (labeled DAQ). References to this raw data are gathered in a Nexus wrapper file, stored on a separate drive (labeled APS in the figure), so that both raw and computed data can be accessed from both the beamline and other locations. See the text for more details on the workflow.

\section{SCIENTIFIC HIGHLIGHTS:}

Sublattice Melting in a Battery Cathode. We performed measurements at temperatures of 100, 200, 300, 400, and 500 kelvin $(\mathrm{K})$ on sodium-intercalated vanadium oxide, $\mathrm{Na}_{\mathrm{x}} \mathrm{V}_{2} \mathrm{O}_{5}, \mathrm{x}=0.0,0.1,0.2,0.4$, which allowed us to observe, for the first time, a melting of the mobile cation's sublattice (i.e., the sublattice comprising the sodium intercalants, within an otherwise unperturbed parent lattice) $\mathrm{V}_{2} \mathrm{O}_{5}$ (see Figure 3). This is a demonstration of the value of performing high-throughput measurements of the structure factor, $S(Q)$, allowing multiple temperature measurements in a short time (i.e., five temperature readings in less than two hours). Complex impedance measurements are being conducted so that the growing crystallinity of the sodium sublattice can be directly correlated with the ionic mobility.
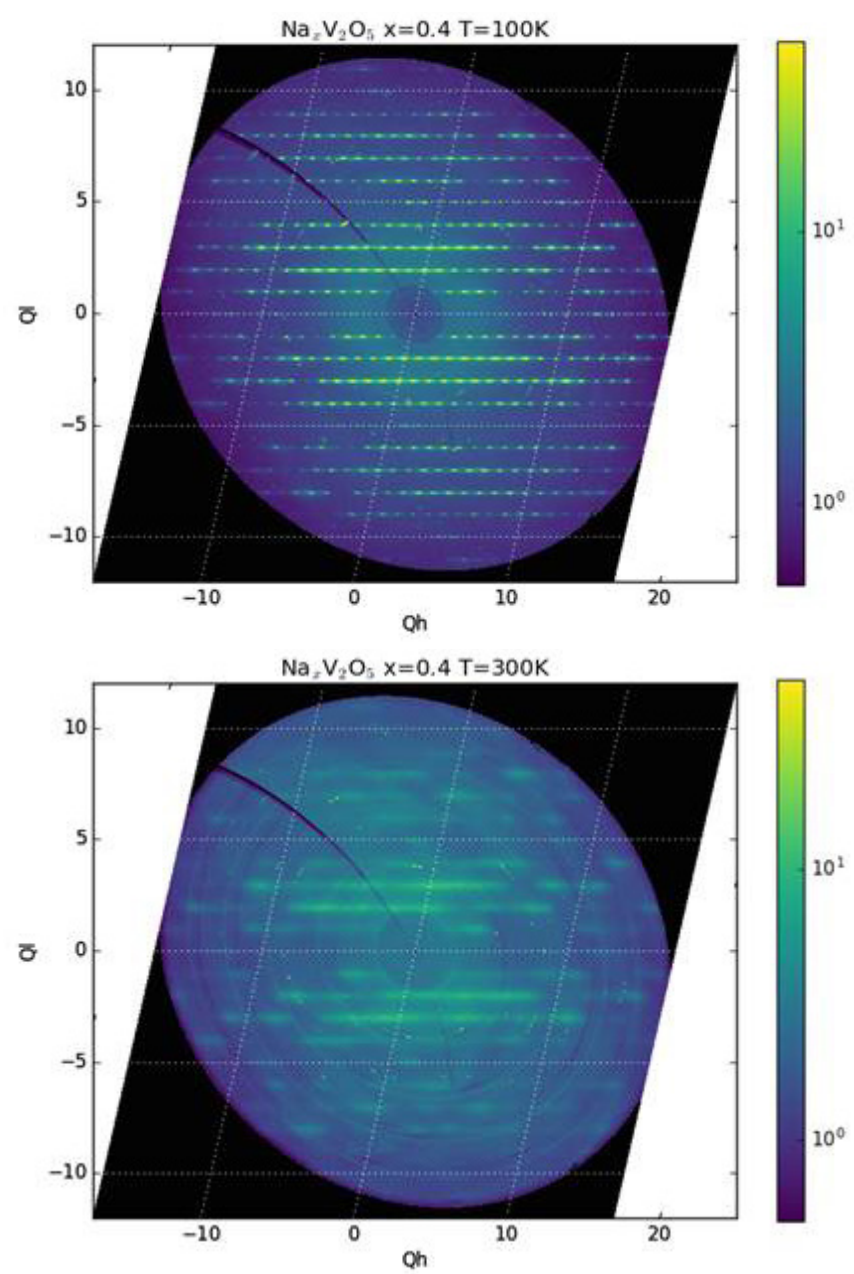

Figure 3. Diffuse scattering data taken at $100 \mathrm{~K}$ (top) and $300 \mathrm{~K}$ (bottom) in a $\mathrm{Na}_{x} \mathrm{~V}_{2} \mathrm{O}_{5}$ sample with $\mathrm{x}=0.4$. In both figures, there is evidence of diffuse rods of scattering along the $\mathrm{Qh}$ direction, representing strong two-dimensional disorder. However, at $100 \mathrm{~K}$, there are sharp Bragg peaks superimposed on these rods, indicating the crystallization of the sodium ions in three dimensions. This order-disorder transition will have a profound effect on the ionic mobility in this prototype battery cathode material.

An ab-initio cluster expansion of defect configurations in $\mathrm{Na}_{\mathrm{x}} \mathrm{V}_{2} \mathrm{O}_{5}$ has been completed as the preliminary step in modeling this sublattice melting. We have developed a novel Hybrid Reverse Monte Carlo (HRMC) method, to relax the atomic positions of defect structures generated by this cluster expansion. This has been successfully benchmarked against an aluminosilicate mullite, which has been extensively studied by diffuse scattering. The HRMC code has been parallelized to refine reciprocal space volumes in a few hours (see Figure 4). 


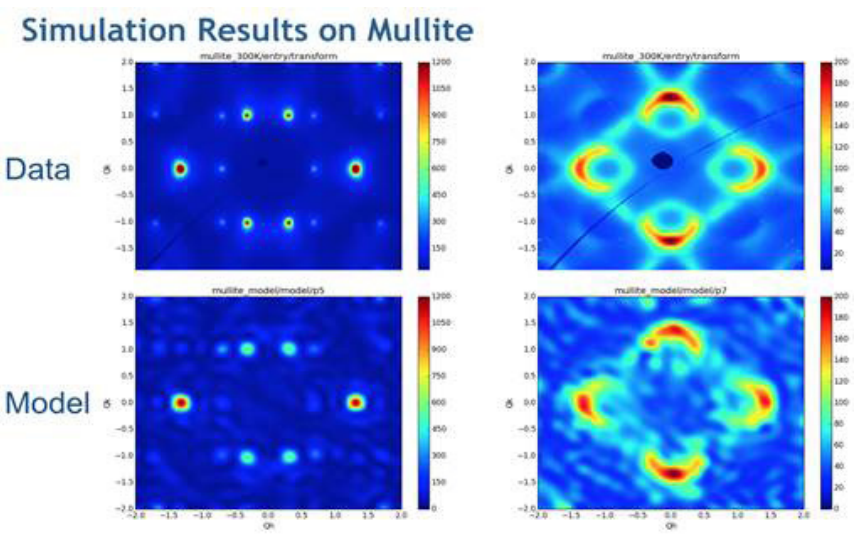

Figure 4. Results obtained with our ab-initio model of sublattice melting (bottom) match well with diffuse scattering data (top).

Charge Density Wave Order in $\mathrm{Sm}_{2} \mathrm{Ru}_{3} \mathrm{Ge}_{5}$. A previously unknown order was observed in an intermetallic compound of samarium (with ruthenium and germanium) being investigated as part of a project targeting charge density wave (CDW) materials as candidates for unconventional superconductivity. This observation resulted from measurements taken in $2 \mathrm{~K}$ temperature steps that were made possible through our support for high-throughput measurements. The CDW structure has now been solved and a publication is in preparation (see Figure 5).
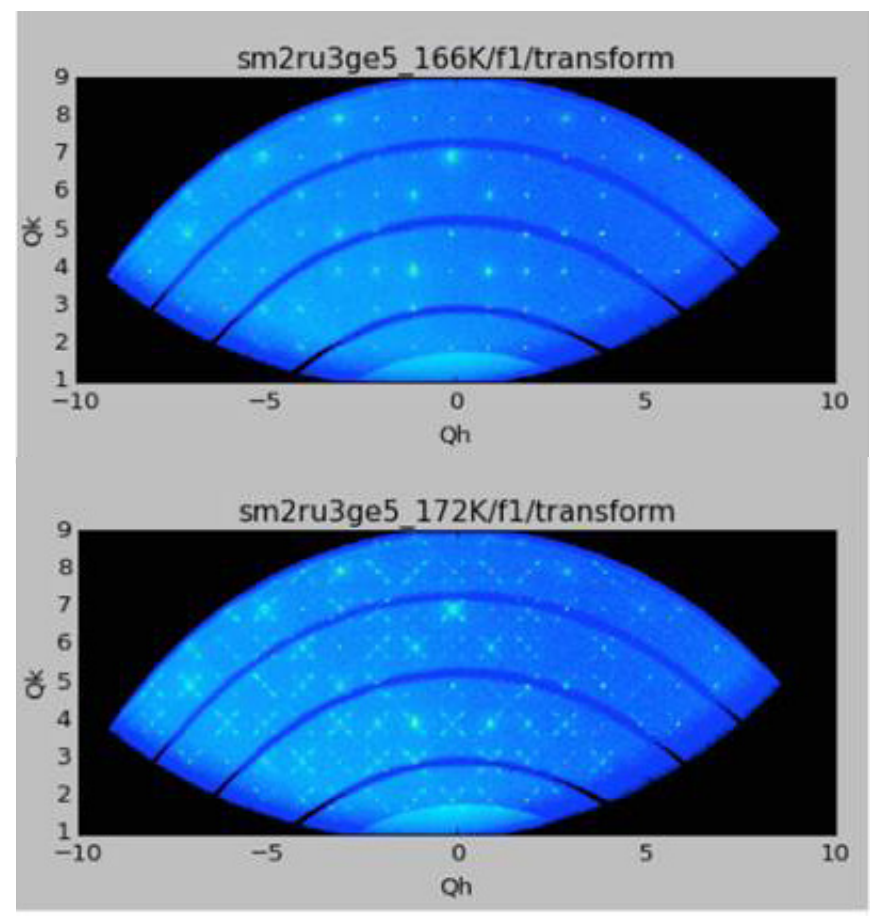

Figure 5. These data from an intermetallic compound of samarium (with ruthenium and germanium) at $166 \mathrm{~K}$ (top) and $172 \mathrm{~K}$ (bottom) illustrate the previously unknown transition.
High Energy Diffuse Scattering from a Relaxor

Ferroelectric. The generality and portability of our diffuse scattering pipeline has enabled new science. It is not possible to measure compounds with substantial quantities of heavy elements such as lead at the relatively low energies available on Sector 11-ID-D at the APS. However, at CHESS, we were able to use a 57 kiloelectron volt (keV) beam to measure diffuse scattering from a number of relaxor ferroelectrics, such as lead magnesium niobate, $\mathrm{PbMg}_{1 / 3} \mathrm{Nb}_{2 / 3} \mathrm{O}_{3}$ (see Figure 6 for a single slice of the CHESS data on this material). These measurements are complementary to neutron diffuse scattering data collected at the Spallation Neutron Source (at Oak Ridge National Laboratory), which are more sensitive to oxygen rather than lead displacements. We have identified some significant differences in the Q-dependence of x-ray and neutron diffuse scattering, which we are currently engaged in modeling.

Our demonstration of the ability to access the data at a granular level without transferring large data files from remote repositories is important in guiding the design of facility data servers in the future.

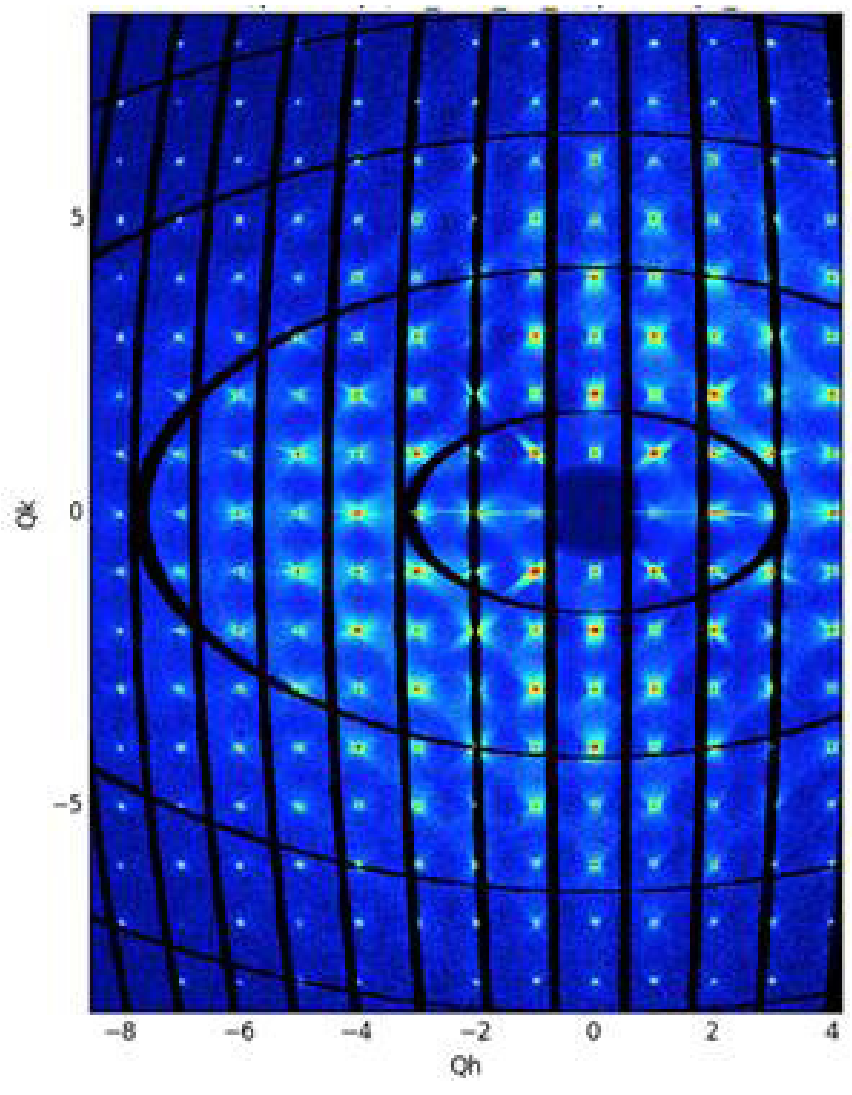

Figure 6. A single slice of the 3-D diffuse scattering dataset obtained at CHESS for lead magnesium niobate, $\mathrm{PbMg}_{1 / 3} \mathrm{Nb}_{2 / 3} \mathrm{O}_{3}$. 


\section{PARALLEL COMPUTATION}

We integrated Swift, Galaxy, MPI, and OpenMP for parallel computation, including resource provisioning, scheduling, and provenance. We have integrated Swift, MPI, and OpenMP in DIFFEV and used Swift pervasively in project workflows. We also developed additional pipelines and extended existing pipelines for materials applications. We developed a pipeline for batch correction that has received substantial use by over 30 beamline users at APS Sector 1-ID. We are developing a Swift workflow for high energy diffraction microscopy peak fitting and data reduction and a Swift pipeline for Coherent Diffraction Imaging of strain on the nanoscale at APS Sector 34-ID-C.

Milestone: End-to-end acquisition-to-analysis pipelines for APS data. We have completed end-to-end pipelines for diffuse scattering (see Figure 3 for examples of the results of such a pipeline, in which three different detector positions are merged and transformed to reciprocal space) and far-field HEDM.

\section{COSMOLOGY}

We established a dedicated, 50-node parallel computing system, called Jupiter, on which we use a new software packaging technology called Docker to run complex software pipelines. These pipelines include the South Pole Telescope (SPT) data pipeline and the Portal for Data Analysis Services for Cosmological Simulations (PDACS) analysis suite.

We implemented a new parallel merger tree code. In a cosmological large-scale structure simulation, matter clumps into localized halos; these halos in turn merge to form bigger halos and so on. The hierarchical formation of halos can be represented as a merger tree, where each tree node represents a halo merger. A parallel graph-theoretic algorithm is used to efficiently construct merger trees. We have implemented a new "reverse-time" algorithm (runs backward into the past) that is very efficient at recognizing unphysical anomalous events in merger-tree construction and eliminating them. This improvement solves a number of problems in running semi-analytic galaxy formation codes on merger trees.

The Delaunay Tesselation-based density estimator (a tool for reconstructing a volume-covering and continuous density field from a discrete point set) was enhanced with a stochastic sampling scheme and is the main driver behind two new results, a strong lensing pipeline for realistic image catalogs and a new pipeline for galaxy-galaxy lensing. The method was also used to investigate the efficacy of gravitational lensing to find large-scale structures in sky surveys ("superclusters").
PDACS has been ported to Edison at NERSC and is running on two parallel computer systems at Argonne, Magellan and Jupiter. There is interest at the National Center for Supercomputing Applications (NCSA) to run PDACS on its Blue Waters supercomputer.

A new parameter sampling scheme for building emulators was used to produce a convergent predictive method that has the property of improving interpolation accuracy smoothly as the sampling density increases. The success of the scheme was demonstrated on a new set of simulations run at ALCF and at the Oak Ridge Leadership Computing Facility (OLCF).

\section{PROPOSED FUTURE WORK}

Our high-level goals for the remaining three months of the project are to complete the functional initial data facilities and explore additional applications. In more detail, they are as follows:

\section{COSMOLOGY}

$\square$ Complete and/or optimize the suite of algorithms.

$\square$ Release the first production version of the software-as-a-service (SaaS) cosmology portal for analyzing simulation data.

$\square$ Complete database implementations.

$\square$ Carry out science analyses exercising the full system, including the observational data.

\section{MATERIALS}

$\square$ Evaluate research models, deployments, and applications developed during years one through three, collecting data for publication and promoting continued APS operation of the services that we have developed.

$\square$ Start developing scripts that handle other x-ray techniques within the APS and multiple techniques across different facilities.

\section{DATA AND WORKFLOW}

$\square$ Complete the work on user-defined analyses and the capture of derived data products. 


\section{ADVANCED COMPUTING}




\section{MADNESS for Materials}

\section{3-199-R2}

\section{Nichols A. Romero, Laura E. Ratcliff, and Álvaro A. Vázquez-Mayagoitia}

\section{PROJECT DESCRIPTION}

Although there are many feature-rich density functional theory (DFT) codes available, only a small subset of them are designed to run efficiently on petascale supercomputers. Even fewer of these codes are able to treat systems with greater than 10,000 electrons, because of the cubic computational complexity inherent in DFT (i.e., computational time increases with the cube of the number of atoms). The next generation of leadership-class computers will pose a number of new challenges to these codes in terms of weak and strong scaling, single-core performance, time to solution, and even precision. Further along the exascale roadmap, computational issues like managing ever-increasing amounts of node concurrency will require exploratory research.

We propose a massively parallel reduced-scaling DFT code for materials based on the well-established MADNESS (multiresolution adaptive numerical environment for scientific simulation) framework, which uses a multi-resolution, multi-wavelet basis in conjunction with a parallel task-based runtime. The goal of this project is to produce a reduced-scaling DFT code capable of running on up to millions of threads on Mira (Argonne's 10-petaflop Blue Gene/Q [BG/Q] computer) and permitting robust DFT calculations on materials containing in excess of 10,000 electrons.

\section{MISSION RELEVANCE}

Our work is relevant to DOE's missions in energy security and basic science. In particular, it is of interest to the ASCR (Office of Advanced Scientific Computing Research) and BES (Office of Basic Energy Sciences) programs in DOE's Office of Science. At present, no massively parallel reduced-scaling DFT code designed for the Blue Gene series architecture is available that meets the needs of the DOE materials research community. Such a code will be a strategic capability that will enhance our ability to deliver petascale science and beyond in key areas, such as energy storage and catalysis. In addition, we anticipate that industry partners who are performing exploratory materials research may benefit from this project.

\section{RESULTS AND ACCOMPLISHMENTS}

Work in prior years (FY 2013-FY 2014) was divided between investigating and improving performance issues on $B G / Q$ and adding to the existing scientific functionality of the molecular DFT code (called moIDFT). The thread scaling was improved by leveraging the BG/Q port of the Intel Thread Building Block (TBB) library in conjunction with a scalable memory allocator. The use of TBB, a task-based programming model, highlights moIDFT within the Office of Science's software portfolio. It is the only DFT code that scales to more than 32 threads "out of the box." Our investment in the TBB port of MADNESS will help to make moIDFT usable on the next generation of supercomputers. To address the scientific aspects, we worked with scientists at Stony Brook University to implement pseudopotentials (PSPs) in moIDFT, which allow the treatment of heavy atoms such as transition metals. A linear response code was implemented to calculate static and dynamic polarizabilities of small molecules. We also investigated and implemented a temporary solution to numerical precision problems that were encountered with DFT calculations using the generalized gradient approximation (GGA). Finally, we made progress in improving certain algorithmic kernels that exhibited quadratic or higher computational complexity, which is a step toward a linear-scaling DFT.

In FY 2015, we continued to improve the performance of moIDFT on BG/Q as part of our larger effort to enable high-impact materials science calculations on Mira and our next-generation supercomputers (Theta and Aurora). With the assistance of the Rice HPCToolkit developers, we are able to collect performance data on a MADNESS application. Unfortunately, this capability exposed a shortcoming in the design of TBB. We expect programmatic support to continue working with the developers of the Rice HPCToolkit and Intel to make progress on this issue. Recent modification to the wave function localization and load-balancing algorithms, performed in collaboration with the Stony Brook University scientists, resulted in scaling improvements to moIDFT.

Building on previous work on PSPs, moIDFT is now able to perform mixed all-electron (AE) and PSP calculations, wherein individual atoms can be specified as either $A E$ or PSP. The automatic refinement of the multi-resolution approach in MADNESS ensures that both full and pseudo-atoms are treated accurately and efficiently. This advance allows us to combine the benefits of PSPs (i.e., lower cost, treatment of heavy atoms) with those of $A E$ (i.e., direct access to core states) and is particularly useful for the simulation of electron energy loss spectra (EELS), where explicit core states are only needed for 
specific atoms of interest. We also implemented the calculation of EELS using the dipole approximation. An example is shown in Figure 1 for the cysteine molecule; note how the $A E$ and mixed $A E$ results are nearly indistinguishable from each other.

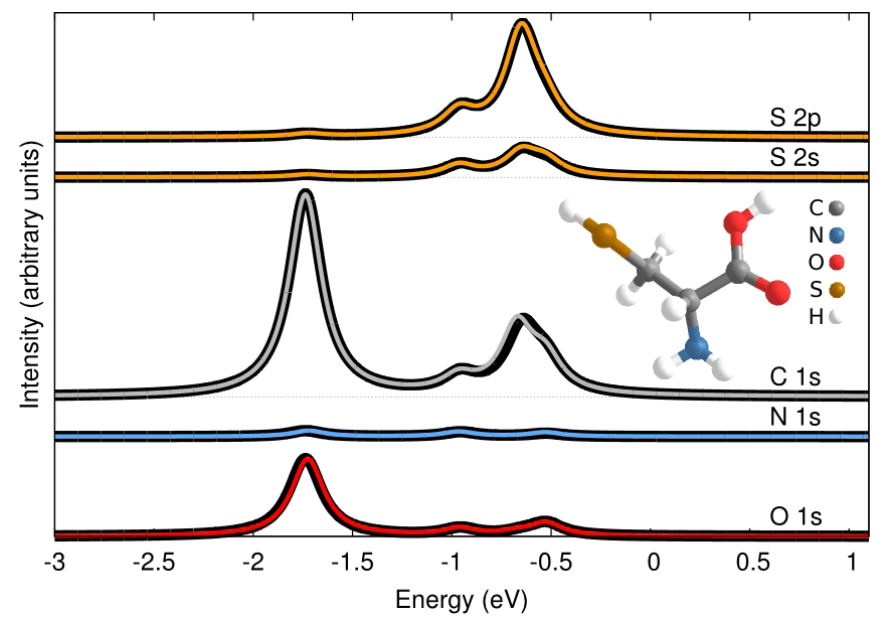

Figure 1. Electron energy loss spectra for the cysteine molecule, calculated using the dipole approximation, with both an all-electron (black lines) and a mixed all-electron/pseudopotential (colored lines) representation.

We developed a linear-response code in MADNESS, and it is now possible to calculate both static and dynamic polarizabilities, such as Raman spectra. The linear-response method implemented is the Adiabatic Local Density Approximation based on either an initial Hartree-Fock or DFT calculation. This code was benchmarked for organic molecules against Gaussian basis sets, which require large and diffuse functions to achieve the same accuracy as the multi-resolution approach in MADNESS.

We implemented a more robust truncation scheme for computing the exchange-correlation energy (and its derivatives) based on the ratio of the density to its gradient, rather than using a fixed-threshold truncation scheme. This scheme improved the numerical stability of GGA calculations, which had previously been problematic.

We helped prepare and support an INCITE project centered on MADNESS, led by Robert Harrison at Stony Brook University, which was awarded 15 million core hours in 2015 and was renewed for a further 20 million core hours in 2016.

\section{Mathematical Techniques to Model Urban Data}

\section{3-206-R2 \\ Charlie Catlett and Sven Leyffer}

\section{PROJECT DESCRIPTION}

Urbanization in developing economies such as China and India over the next three decades will drive the design and construction of about 60 billion square meters of floor space. Current practices and tools used for city designs and operations do not scale up to accommodate the magnitude and pace of such growth, portending megacities that, because of inefficiency, will have direct and indirect impacts on the natural environment, climate, and global energy supplies. Transparency and open data initiatives in many cities are exposing unprecedented detail about the urban environment and its systems, resulting in data from many independent sources and with diverse properties including resolution, content, and degrees of complexity. This project has focused on the significant volume of data for which two fundamental attributes-time and location-form the basis for common analysis. Increasingly, urban data are analyzed with machine learning techniques and, in some cases, via social network analysis, but these approaches are often limited in terms of scale. This project's goal was to develop and test strategies to represent urban social/ economic data so that a variety of mathematical methods and tools can be used for data analysis and visualization to discover, understand, and ultimately leverage the interactions and patterns among multiple independent urban processes and systems represented by these datasets. The results of this work have been captured in the architecture of an integrated data platform, which was subsequently implemented and evaluated as a data discovery resource used by the University of Chicago's Data Science for Social Good (a summer student fellowship program) and separately as a platform upon which the University of Chicago developed and tested a crime prediction system.

\section{MISSION RELEVANCE}

This project is relevant to DOE's missions in environment and energy, as well as emerging initiatives toward understanding metropolitan areas as complex multi-scale systems. The primary customer of the results from this work is the research community supported by the U.S. Government, particularly DOE, whose research portfolio is beginning to expand to investigate global urbanization and its impact on the planet's climate and energy supplies, as well as the practical urban design, 
planning, and operations challenges of U.S. cities. Similarly, city and state governments in the United States and governments abroad require tools and methodology to transform their growing, diverse data sources into understanding for both the operation of their cities and planning for growth and renovation.

\section{RESULTS AND ACCOMPLISHMENTS}

In FY 2013, we analyzed available urban data in the context of extensive discussions with sociologists, energy scientists, economists, and urban designers to select an initial set of data for the research. On the basis of this initial work, we elected to refine our strategy from "mashups of two-dimensional (2-D) surfaces" to time series vectors, which hold much greater analytic promise with respect to using machine learning and similar techniques. In FY 2014, we created a test system that allows a scientist to specify a set of data sources, a sampling period (e.g., daily, weekly, monthly, annually), a start and end date, and a location (e.g., address, latitude/ longitude, street segment, block, polygon). To evaluate this system, we leveraged a web-based portal at the University of Chicago called Plenario (http://plenar.io), a geospatial database with application programming interfaces, providing access to over 100 open datasets. Using this system, we were able to validate our designs with an initial 40 datasets in FY 2014, expanding to more than 120 in FY 2015. We further evaluated the general architecture in concert with the University of Chicago Harris School of Public Policy and through a collaboration with the University of Calabria (Italy) to incorporate machine learning and clustering algorithms to predict crime using historical crime data, associated data streams, and weather. This evaluation resulted in an implementation of a database architecture that supports integration of the database with computational workflows by using Argonne's Laboratory Computational Resource Center to train machine learning algorithms for the crime prediction system. Finally, our work exploring architectures of integrated data systems influenced the design of a City of Chicago open source data mapping system, called OpenGrid (http://opengrid.io), which was released in January 2016. OpenGrid uses the application programming interfaces developed by this project and implemented in Plenario.

We intend to continue to work with end-users to validate our architecture and techniques, beginning with the University of Chicago Harris School of Public Policy project on crime prediction, and expanding to include additional leading indicators. We will also work to understand how to best integrate real-time sensor data into the architecture. We have submitted proposals to the
National Science Foundation and the Arnold Foundation for potential follow-on funding.

\section{General-Purpose Technical Cloud Platforms}

\section{3-208-R2 \\ Daniel Murphy-Olson, Ryan Aydelott, Narayan Desai,
and Wolfgang Gerlach}

PROJECT DESCRIPTION

Our project has aimed to broaden the applicability of the Magellan system, an experimental cloud computing system, from its current bio-informatics domain to a larger set, including cosmology and materials. To do this, we set about improving the system in several ways. First, we needed to improve the overall system performance. Cloud systems have a thicker software stack compared with traditional high-performance computing (HPC) systems, which incurs some additional overhead. In this project, we are reducing the overheads to enable a wider range of applications to run effectively on this platform. Second, we are building a system where continuous availability is a key goal. This capability is desirable for supporting aggregated scientific services with distributed user communities. Finally, we have sought to develop methods that will effectively harness the extreme flexibility afforded by the cloud architecture to serve user communities ranging from computer scientists to domain scientists.

\section{MISSION RELEVANCE}

Our project is tied to DOE's mission in science. Cloud systems support a range of computing applications poorly served by traditional large-scale DOE computational platforms. These applications are primarily services where the scientific community at large can use a project's software platform to answer scientific questions. These projects have historically been implemented on small-scale computing infrastructure, limiting the scope and breadth of their activities. Cloud computing infrastructure has enabled large-scale comparative analysis and data integration activities that were difficult to accomplish on traditional platforms. The expansion of this approach will enable new computational approaches across a range of disciplines.

\section{RESULTS AND ACCOMPLISHMENTS}

In FY 2013, we performed the first production release of the Magellan system based on the Openstack Essex release. We developed a novel user coordination system called "poncho" that enables streamlined rolling 
maintenance and the development of high-availability user applications. This system provides an application programming interface for communication between users and the Magellan operations team that describes the impact of service operations and enables many operations to be conducted automatically, without manually coordinating with users. This non-invasive management technique has minimized the need for the preventative maintenance schedule that other large systems require.

We made excellent strides in improving the performance of virtual resources. We deployed experimental Ethernet emulation software that uses the system's quad data rate InfiniBand to provide high-performance networking to virtual machines (VMs). This software is alpha-quality and was released in a preliminary form. With this software, we experienced nearly 15 gigabits/second of network bandwidth and 25 microseconds of latency between nodes. While the latency was at the level we expected, the bandwidth was only half of what we expected to realize. We worked with the vendor to improve this performance to increase the level of other InfiniBand protocols (to nearly 30 gigabits/second), and we expected it to be improved substantially.

Similarly, we greatly improved the performance of persistent block storage ("volumes") in the system. This storage presents a block device directly to a VM. We built several high-density, high-performance storage targets that present aggregated virtual disks via the Internet Small Computer System Interface (iSCSI). We modified Openstack to make use of iSER, which is a set of remote direct memory access (RDMA) extensions to the iSCSI protocol. With iSER, RDMA is used for the data path, so little tuning is required. With this change, volume performance increased to 1,800 megabytes per second, with considerable latency improvements. We expected this single-node performance to be the best that we were likely to obtain from our current storage servers, although we expected to seek further improvements in aggregate throughput. We have not yet explored this issue with the current hardware; we expect more recent generations of hardware to provide higher aggregate throughput owing to improvements in InfiniBand and PCle bus speeds.

We also began to test model distributed, fault-tolerant storage. This new upgraded storage would enable the use of commodity components to build system-level aggregated storage resources.

We began working with new application groups at Argonne to test data-intensive model applications on Magellan. The first group we assisted was the Cosmology Group. This group wanted to perform high-speed, interactive analytics on its simulation time-step files, sizes of which at the time were about 40 terabytes each. Ultimately, members of the group exercised our test model system with a real application.

Finally, we developed a set of practices for building resilient applications on Magellan.

In FY 2014, we developed a new release of the Magellan system control plane based on Openstack Havana. This release included several improvements, most notably the migration of instances (virtual machines), improved network performance, and the ability for end users to download images from the system. This release was beta tested by several users.

We completed evaluations of Ceph, GlusterFS, and Sheepdog (distributed storage software) to improve storage performance and availability. We ultimately selected Sheepdog to host the ephemeral storage layer for the Openstack Havana deployment. This enhancement will improve availability by allowing us to migrate instances from hypervisors with hardware and performance issues by allowing ephemeral disk performance to scale beyond the bandwidth of a single disk.

One of the lessons learned from managing the KBase development environment on Magellan is that it was difficult for users and project administrators to determine what instances might be idle through the traditional Openstack user interfaces (here, "Openstack" is used generally-Havana is the version). We developed a periodic utilization report for projects using the system, which helps give users the visibility into the system that they need to use their allocation(s) optimally.

We developed a security group reporting utility that helps project owners view all of the network access control lists and how they map to nodes. This utility enables project owners to meet lab cybersecurity requirements, while still allowing a great deal of freedom for project owners to define network controls that meet the needs of their applications.

Finally, we developed a model building-block approach for scaling the system up and enabling buy-in. We selected several building blocks for high memory, compute, and storage. We procured several of the storage building blocks for the Cosmology Group, and began to test and tune them for throughput and manageability.

In FY 2015, we focused on streamlining operations, extending the capabilities of the existing system, and working with systems and software vendors to determine the best path forward for future systems. 
To streamline operations, we selected a commercially available Openstack distribution and deployed this software in a proof-of-concept environment. The Advanced Photon Source X-ray Photon Correlation Spectroscopy (XPCS) group, with whom we had previously been working on use of the existing Havana system, was the first group to use the new commercially available Openstack system. This system has been stable enough to support the XPCS group's production data processing. After the success we had with this approach, we developed a plan to redeploy the entire system using its underlying tool, "Fuel." To extend the capabilities of the current system, we replaced the InfiniBand interconnect adapter cards with newer Connect-X 3 cards with hardware pass-through capabilities. With these cards and some minor configuration changes to Openstack, we were able to obtain HPC-class message passing interface (MPI) performance from the system. The MPI performance in this configuration surpassed that of Connect- $X 2$ cards in the Argonne Leadership Computing Facility Tukey cluster using the Ohio State University MPI benchmark suite (http://mvapich.cse.ohio-state.edu/benchmarks). In addition to improving the performance, we added images to the system to support a wide range of operating systems, including Windows, FreeBSD, and OmniOS.

To prepare for future cloud systems, we researched several options from various hardware vendors.

\section{Multiscale Materials Modeling Using Accurate ab-initio Approaches $\left(M^{3} A^{3}\right)$}

\section{3-212-R2}

Álvaro A. Vázquez-Mayagoitia, Yuri Alexeev, and Anatole von Lilienfeld

\section{PROJECT DESCRIPTION}

In this project, we advanced the state-of-the-art of materials modeling by developing force fields (FFs) for complex materials using machine learning $(\mathrm{ML})$, which is a systematic method for transforming large datasets of accurate material properties-obtained from experiment or from quantum chemistry simulations-into accurate FFs for a broad class of systems.

FFs are molecular models that describe interatomic forces and properties within a functional form and a set of parameters. FFs are popular in modeling large systems, such as bulk materials and polymers. The quality of the FFs depends on how well their parameters are able to describe changes in molecular structures. The complex task of parameterizing FFs may be delegated to artificial intelligence models, such as ML models. The creation of $\mathrm{ML}$ models requires lots of training data. We generated high-quality training datasets employing petascale supercomputers, by running massively parallel quantum chemistry codes. We developed datasets of high-accuracy energies and properties (e.g., electrostatic moments, polarizabilities and hyperpolarizabilities, and torsional potentials) that covered a broad region of chemical space by using high-performance computing (HPC) resources and benchmark-quality methods, such as quantum Monte Carlo methods, many-body perturbation theory, coupled-cluster theory, and density-functional theory (DFT).

\section{MISSION RELEVANCE}

The project is relevant to DOE's mission in science. Atomic simulation of materials is a dominant use of HPC resources within DOE and around the world, with materials science and chemistry simulations using approximately one-third of the computer time of DOE Leadership Computing Facilities. Increasing the accuracy of atomistic simulation methods while decreasing their computational cost has an immediate practical payback. For example, our FFs and ML methods could compute in milliseconds the total internal energies of a molecule consisting of hundreds of atoms using only one processor.

\section{RESULTS AND ACCOMPLISHMENTS}

In FY 2013, we developed and tested the performance of our ML models by predicting several physical and chemical properties. We were able to predict DFT energies and polarizabilities within a dataset of 7,000 molecules. We also created multiple datasets of energies and forces to train our ML models by exploring the evolution of the structures of ions and small molecules in aqueous solutions.

In FY 2014, we calculated quantum chemistry data for the equilibrium geometries of more than 134,000 molecules

(Figure 1). We also devoted considerable effort to improving the predictive accuracy of the ML models. Toward this end, we applied and developed the " $\Delta \mathrm{ML}$-corrected" models to predict the deviations between molecular properties computed with highly accurate and baseline approximated quantum chemistry methods. The results suggest that we can easily attain chemical accuracy for thermochemical properties as well as electron correlations. 


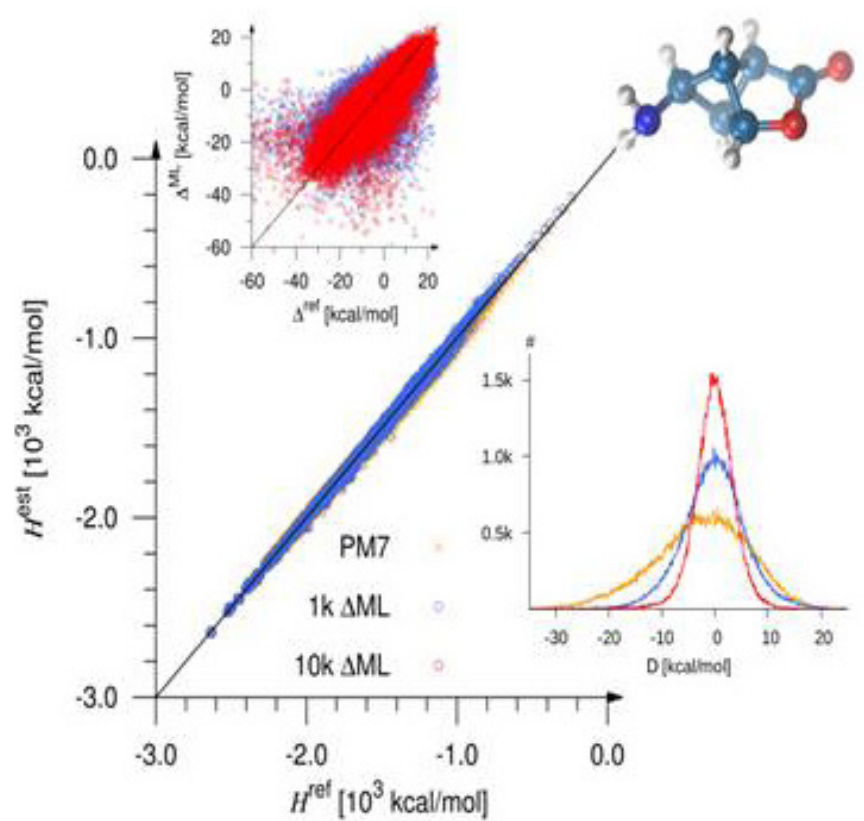

Figure 1. Scatter plot of enthalpies to separate all the atoms in a molecule (H) computed for 134,000 molecules; the $\Delta \mathrm{ML}$-model corrected and the semi empirical quantum chemistry method PM7 (vertical axis) are plotted versus the DFT reference values (horizontal axis). The upper inset shows the correlation between the $\Delta$ ML-models trained on $1 \mathrm{k}$ molecules (blue) and $10 \mathrm{k}$ molecules (red) and the actual correction DFT. The lower inset shows the change in error distribution $(D)$ for the baseline method, with $\Delta M L-m o d e l ~ 1 k$ (blue), $\triangle$ ML-model 10k (red), and PM7 (yellow). The most extreme outlier, the molecule 7-amino-3-oxatricycloheptan-4-one, is just an example of one of the 134,000 molecules studied.

Furthermore, we applied ML models for learning total energies and atomic forces. The results for forces on cis/ trans-butadiene derivatives are very promising. Figure 2 shows the correlation between ML-based and DFT-based predictions. We proposed a new model, using many-body perturbation theory and coupled-cluster theory methods, to correct the interatomic forces and binding energies of aqueous solutions of small molecules and ions computed with periodic DFT in the context of ab-initio molecular dynamics.

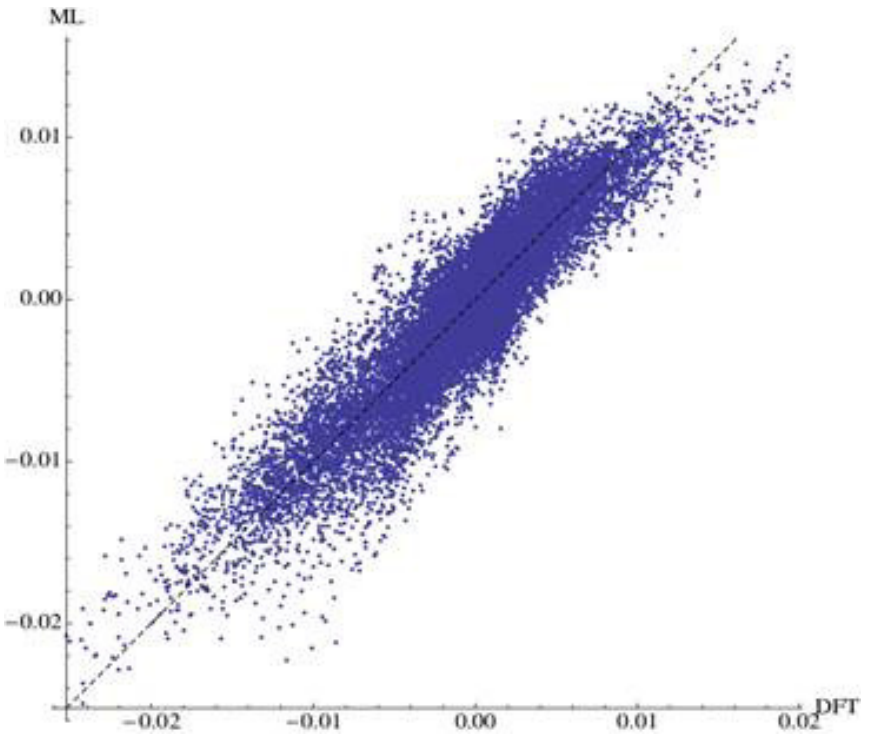

Figure 2. Scatter plot showing the correlation between the largest components of the atomic forces predicted with a ML method (vertical axis) and DFT (horizontal axis). The ML method was trained on 1,000 molecules drawn from over 2,000 cis/trans-butadiene derivatives. Forces shown are given in atomic units.

During FY 2015, we explored a wide variety of ML methods that were designed for different domains to see how they would work in the framework of atomistic simulation. We developed new approaches to investigating the performance of condensed-phase representations for $\mathrm{ML}$ models of the formation energies of solids. Also, we introduced a composite strategy that adds ML corrections to low computationally intensive quantum chemistry methods to achieve chemical accuracy of ground state properties. We validated our strategy, predicting the thermochemical properties of thousands of organic molecules. Furthermore, we applied our new composite models to predict vertical electronic excitation energies. Our findings showed promising results; eventually the strategies applied in this project could be used for the computational design of materials having new properties. 


\section{X-PECT: Performance Framework to Characterize and Transform Applications and Architectures at Extreme Scales}

\section{3-213-R2}

Kalyan Kumaran, Vitali Morozov, Venkatram Vishwanath, and Yao Zhang

\section{PROJECT DESCRIPTION}

In this work, we developed an integrated performance modeling and projection framework to study the performance of future scientific applications, highperformance computing (HPC) architectures, and the interactions between them. In particular, we contributed to the following technically challenging areas:

\section{$\square$ Abstracting the code structure of workloads to understand the code's potential behaviors; \\ $\square$ Modeling the performance of various hardware components, so that they can be combined to form complex systems; \\ $\square$ Developing mechanisms to explore workload transformations and hardware design options to better match workloads to machine characteristics; and \\ $\square$ Tuning and searching for optimized software-hardware configurations.}

The delivered framework projects the performance and power for future workloads on future systems. It will also guide application developers to tune their code and help hardware designers to evaluate the impact of hardware features on application performance at an early stage. The techniques developed herein have the potential to be incorporated into compilers and runtimes on production systems to optimize application performance.

Preeti Malakar (Leadership Computing Facility) and Prasanna Balaprakash (Mathematics and Computer Science) participated in the project, providing expertise in network/in-situ analysis and machine learning.

\section{MISSION RELEVANCE}

The framework is critical to the DOE's basic science mission. Leadership computing facilities, a strategic area for the DOE, accelerate scientific discoveries and technological innovations by enabling large-scale, computationally intensive projects that address "grand challenges" in science and engineering. A key challenge is to understand how to better design facilities to meet the needs of scientific applications, as well as how to effectively scale applications to fully exploit the features of future systems. The framework addresses these challenges by using performance models to provide insights for scalable science on leadership systems.

\section{ACCOMPLISHMENTS AND RESULTS}

In FY 2013, we did the following:

$\square$ We prototyped hardware performance models for Mira (IBM Blue Gene/Q) as well as Nvidia graphics processors. The models combine analytical modeling with empirical learning. The constructed model estimates the latency in computation, memory, and communication. We have validated our framework using a number of benchmarks extracted from production scientific applications.

$\square$ We produced a source-to-source translator that extracts the static code structure of a target application and automatically profiles statistics about the dynamic characteristics of the workload.

$\square$ We extended our framework to model complex loop structures in the workloads. The ability to model such behavior has enabled us to explore more code transformations.

$\square$ We developed a probabilistic model that captures the workloads' runtime behavior statistically. This workload model, together with the hardware model, allowed us to analyze the performance bottlenecks in several scientific applications.

In FY 2014, we did the following:

$\square$ We developed SKOPE, a framework for modeling and exploring workload behavior. SKOPE includes algorithm structure representations, a formalized code skeleton language, hardware performance models, and a mechanism to explore code transformations and architecture features.

$\square$ We used SKOPE for performance analysis, tuning, and projection for several use cases: (1) project the multinode scaling trends of scientific workloads; (2) explore code transformations including kernel fusion, loop partition, and cache optimization; and (3) detect hop spots of applications and use this information for hardware-software co-design.

$\square$ We developed Raexplore (Rapid Architecture Exploration), a software component of SKOPE for hardware modeling and exploration. It currently implements the Mira supercomputer and Intel Xeon Phi processors, and is easily extensible for other architectures. 
$\square$ We used Raexplore to analyze the performance of CORAL, which is developed according to DOE's mission needs. We explored the node-level design options for next-generation processors in terms of their major architecture features such as the number of cores, cache size, and the memory bandwidth. We also explored the optimal processor resource allocation between the number of cores and the last level cache size under the chip area constraint.

In FY 2015, we did the following:

$\square$ We developed network performance models to capture the impact of major interconnect components, including injection bandwidth, link speed, topology, and routing algorithms. These models were validated with communication-heavy CORAL scientific applications on the Argonne Leadership Computing Facility (ALCF) Mira BG/Q system.

$\square$ We designed an automatic dependency analysis heuristic and scheduling algorithm within SKOPE (SKeleton framewOrk for Performance Exploration). The developed algorithm is used for out-of-order scheduling instructions on the Hardware Accelerated Cosmology Code microkernel.

$\square$ We extended the SKOPE framework developed in the project for multi-site workflow scheduling. We used performance models within SKOPE to predict the execution time on resources and dynamic probes to identify the achievable network throughput between sites.

$\square$ We proposed a technique to model workloads with limited parallelism that can automatically explore and evaluate transformations involving cooperative graphics processing unit (GPU) threads. We implemented this framework within SKOPE to project the best achievable performance and the most promising transformations without implementing GPU code or using physical hardware.

$\square$ We developed dynamic analytical models to account for concurrency and external traffic in memory-to-memory data transfer over Ethernet media. We validated the model for transmission control protocols and used those models within Globus, a state-of-the-art transfer tool.

$\square$ We studied the impact of optimal multilevel checkpointing intervals on the trade-offs between run time and energy consumption. Our experimental study with Fault Tolerant Interface, a multilevel checkpointing library on Mira, shows that performance-energy tradeoffs are minimal, but may be significantly larger under certain future exascale HPC scenarios. $\square$ We developed an autotuning system for tensor contraction computations targeting GPUs. The system uses a tensor-specific mathematical representation as input and generates an autotuning search space that is customized to both the domain of tensors with small dimension sizes and GPU architectures. We explored the very large searches generated by these tools by using machine learning, resulting in search times that are practical.

$\square$ To tackle the data-centric requirements of exascale simulations, we designed models for in-situ analysis for large-scale science simulations on supercomputers. Next, we designed optimization strategies for optimal scheduling of in-situ workflows that fully exploited the characteristics of the simulation, the analysis workload, and the system characteristics. We demonstrated the efficacy of our approaches with scientific simulations on the ALCF Mira BG/Q supercomputer.

$\square$ We witnessed complex M-to-N data movement patterns in current applications such as multi-physics simulations, I/O, and in-situ workflows. We designed data movement algorithms to optimize the data movement while fully exploiting the underlying topology of the system interconnects together with the routing policies of the system.

DOE's Office of Advanced Scientific Computing Research is supporting continuation of aspects of this work.

\section{Developing an Integrated Sensor Network for Science}

\section{4-160-R1}

\section{Peter Beckman, Nicola Ferrier, Yuki Hamada, Katarzyna Keahey, and Rajesh Sankaran}

\section{PROJECT DESCRIPTION}

The availability of small, inexpensive, networked sensors is dramatically changing many data-driven science domains. At Argonne, modeling urban environments, terrestrial ecosystems, and soil biology are just a few research areas that are using sensors. However, several computer science challenges must be overcome: (1) the design of a secure and extensible sensor computing platform capable of in-situ data processing; (2) an architecture for data movement, caching, and verification from the sensor to the computing platform; and (3) a design that can support local autonomous actuation and control. 


\section{MISSION RELEVANCE}

Advanced sensors, computational science simulation and modeling, and climate science are all key parts of DOE's mission. Effectively leveraging these to provide near real-time data for predictive simulations will enable DOE to better understand energy usage, infrastructure threats, and our environment. Furthermore, DOE has started a "Smart City" initiative, and Argonne is well positioned to deploy our sensors as part of this initiative in urban environments.

\section{RESULTS AND ACCOMPLISHMENTS}

In FY 2014, we completed the first design of Waggle. The system comprises two components: the Waggle Fieldnode and Waggle Cloud-infrastructure. The Waggle Fieldnode affords a modular, scalable, fault-tolerant, secure, and extensible platform for hosting sensors and actuators in the field; supports in-situ computation; and works in concert with the Waggle Cloud-infrastructure. The Cloudinfrastructure is designed with the goal of scaling to several hundreds of thousands of Field-nodes, supporting real-time scientific simulation and analysis based on data sensed by the nodes, extending methods for inter-node communication and data exchange, and finally serving raw and processed data to end-users and applications running on other high-peformance computing resources. Prototype nodes were deployed indoors and tested outdoors for precision, accuracy, and robustness.

During FY 2015, we created and deployed a completely integrated Waggle sensor node (see Figure 1). The nodes were tested in real urban environments at the University of Chicago campus and are currently sending data (see www.wa8.gl). The team also assembled a desktop Waggle unit that can be used in the lab for testing and has deployed Waggle at the Indian Boundary Prairie near Chicago to study a natural ecosystem.

\section{PROPOSED FUTURE WORK}

We are working closely with the Array of Things project to deploy Waggle in Chicago. Our current node design is being updated slightly for deployment on Chicago street poles beginning in March 2016. We are also working with the Chicago Botanic Institute to deploy a Waggle node on the Chicago Botanic Garden's green roof.

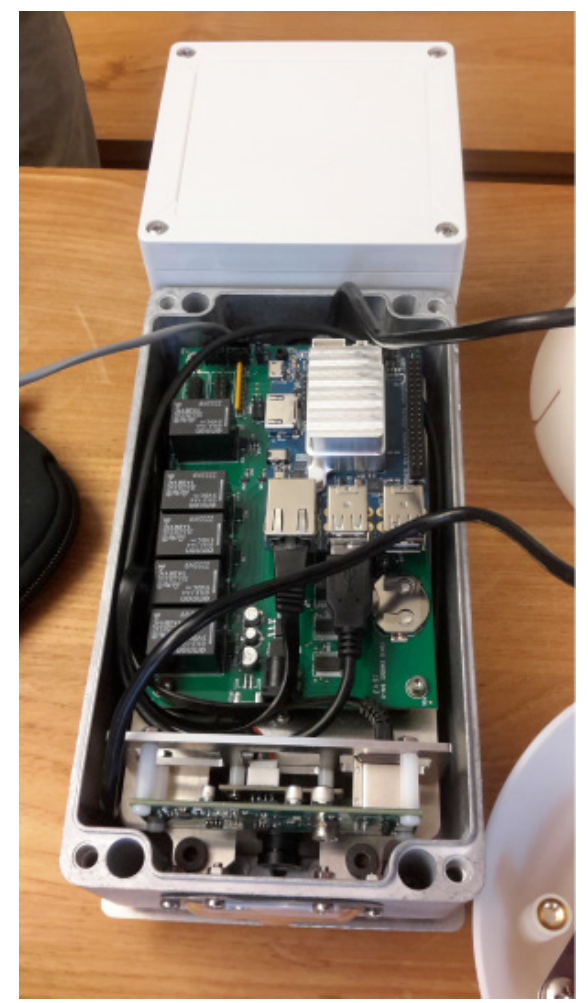

Figure 1. A Waggle computing container: The Waggle Field node assembled and ready for deployment. The green Waggle management (WagMan) circuit board ( 5.75 in. $\times 3.75$ in.) can be seen at the top portion of the metal enclosure. WagMan controls power and provides automated reboots in the event of failures. A custom-machined aluminum heatsink, in the top right corner, is spring-mounted to make contact with the metal lid of the enclosure, allowing the entire unit to be watertight and dissipate heat without the need for a fan.

\section{Emerging Compute and Data Infrastructure}

\author{
2014-163-R1 \\ Craig Stacey, William Allcock, and Linda Winkler
}

\section{PROJECT DESCRIPTION}

The relative maturity of computing has resulted in different markets driving the design of computational components. Mobile computing (advanced RISC [reduced instruction set computer] machines [ARMs]), the convergence of the gaming industry with supercomputing, and the rapid commoditization of storage and network spaces have all contributed components with vastly different performance, power footprint, and cost scaling. In this project, we evaluated how these components can provide capabilities for future high-performance computing (HPC) systems.

We procured hardware components that are likely to be useful in future systems and assessed them by using application kernels or embedding them into larger 
systems. We made use of existing test-bed infrastructure at Argonne as appropriate. We also collected proprietary vendor roadmap information to understand new hardware under development and engaged with vendors to drive product design toward our requirements.

\section{MISSION RELEVANCE}

This work addresses the DOE mission of innovation in extreme-scale computing for scientific workloads. This work benefits designers and users of future large-scale systems designed and built at Argonne and elsewhere. This work is of interest to DOE, the National Science Foundation, the National Institutes of Health, and the defense community.

\section{RESULTS AND ACCOMPLISHMENTS}

We evaluated the ARM Cortex-A15 processer-based architecture and defined a set of benchmarks for input/ output- (I/O-) and CPU-intensive applications. In addition, we examined the use of containers and virtualization with the kernel-based virtual machine software on the A15 system. We engaged HPC-oriented vendors of ARM systems to examine the requirements for test deployment.

In FY 2015, we investigated Cirrascale ARM-based platforms; however, their system specifications were not significantly different from the AppliedMicro X-Gene system in Argonne's Joint Laboratory for System Evaluation. In addition to ARM, OpenPOWER has emerged as a possible future architecture for HPC systems; however, we were unable to obtain an OpenPOWER Power 8 reference system.

We procured enterprise solid-state disks (SSDs) and benchmarked their use in two storage systems, ZFS and Ceph. With ZFS, we used the SSDs as a layer 2 adaptive replacement cache (L2ARC) with our existing virtual-block-device workload. The first configuration used SSDs as L2ARC cache, and the second used them as a ZFS intent log (ZIL). In both configurations, ZFS was not able to take advantage of SSD capacities beyond 20-30 GB, thereby negating the performance gains of larger SSDs.

As an L2ARC cache, we primarily saw I/O operations per second (IOPS) increase for short bursts of traffic; however, during simulations of random read traffic, the performance gain was negligible, and in some use cases, performance actually declined slightly. Configuring the SSDs as a ZIL, we again observed similar behavior such that, if the SSDs were servicing requests, the ZIL would provide us with a buffer of about 10-20 GB of high IOPS before falling back to the magnetic-media-only IOPS rate. Across both SSD configurations, we observed about a tenfold performance boost in both read and write IOPS.

We found possible value in configuring two storage systems provisioned with SSD only and hybrid/SSD configurations. Both endurance and capacity in modern SSDs are bringing them to a price/performance value that may favor deployment in smaller storage arrays, such as those used on Argonne's Magellan system.

We procured enough SSD to fully populate an IBM disk chassis and attempted to optimize the throughput through the Magellan Infiniband and Ethernet networks. We also targeted database and key-value store applications for this type of hardware deployment.

To evaluate software-defined networking, in late FY 2014 we procured a Big Switch Networks system and worked with the vendor to develop and extend the control software. If this system is shown to be viable, we will be able to increase bandwidth and throughput to the Advanced Photon Source, the High Energy Physics Division, and other high-output sources at a much lower cost.

In FY 2015, we completed deployment of the switch infrastructure. Obtaining adequate performance from the controller in a virtual machine was challenging, so the vendor provided us with purpose-built hardware with the controller software predeployed. These controllers performed adequately; however, their development delayed the start of experiments with OpenFlow capabilities. At the end of FY 2015, we were exploring ways of dynamically controlling the flows in this environment. In addition to the Big Switch infrastructure, we procured an Arista 7050 S switch and began integrating it with our open source OpenStack environment using its application programming interface.

We found the software from Big Switch to be difficult to configure, poorly documented, and not production ready for much of the testing period. However closer to the end of the project, we upgraded the controllers to their most recent version. Those were closer to being production quality. Based on the pace of improvement we observed, the product may be ready for use today, but was not determined to be the best option during the project. As well, we expect a Total Cost of Ownership evaluation on today's mainstream networking solutions versus non-branded or "white box" solutions such as Big Switch may demonstrate the branded solution to be the better option. This is likely due to competition from solutions such as that tested in our project. 
Scalable Stochastic Algorithms for Exascale Computational Mesoscience

\author{
2014-166-R1 \\ Dmitry Karpeyev, Juan J. de Pablo, and Olle Heinonen
}

\section{PROJECT DESCRIPTION}

This project aims to devise and implement scalable methods for computational materials mesoscience that take full advantage of modern computational hardware. We are focusing on scalable algorithms for simulating the dynamics of charged particles in electrolytes. Specifically, we want to devise fast computer codes that simulate translocation-slow, thermally driven transportof deoxyribonucleic acid (DNA) molecules (or similar polymers) through narrow channels. The molecules are modeled as a collection of point "beads" connected by springs and suspended in an electrolyte (a fluid with dissolved ions). Simulations of translocations are generally extremely time-consuming, in large part because the motion of polymers is dominated by thermal fluctuations, which "take one step back for each two steps forward." These simulations are also difficult to parallelize and are representative of current challenges in computational soft-matter science. We are pursuing this project in collaboration with the Argonne/University of Chicago Institute for Molecular Engineering.

\section{MISSION RELEVANCE}

This project intends to bring exascale-capable numerical algorithms to DOE's supporting sciences mission. This effort will help advance the energy sciences by enabling and expanding the use of leadership-class hardware in materials and chemistry. Research efforts that would benefit include the recently inaugurated Chicago Hierarchical Materials Design Center and the Argonne-led Joint Center for Energy Storage Research. In the long term, we expect broader benefits to accrue to DOE's energy sciences research, including modeling of nuclear fuels performance, global climate, and power grid optimization.

\section{RESULTS AND ACCOMPLISHMENTS}

In FY 2014, we developed parallel algorithms that identify and rapidly exchange particles that interact with each other but are located on different processors-the "halo" particles. The halo exchange contains most of the communication required by the calculation of the force on the particles and the fluid. We implemented a fully parallel algorithm that identifies and moves all of the interaction partners and has the complexity of the full-force calculation algorithms. A substantial achievement was that, irrespective of the size of the interaction halo, the time spent in the setup phase of the algorithm is essentially constant regardless of the number of processors used (Figure 1). This constancy implies that the algorithm is scalable.

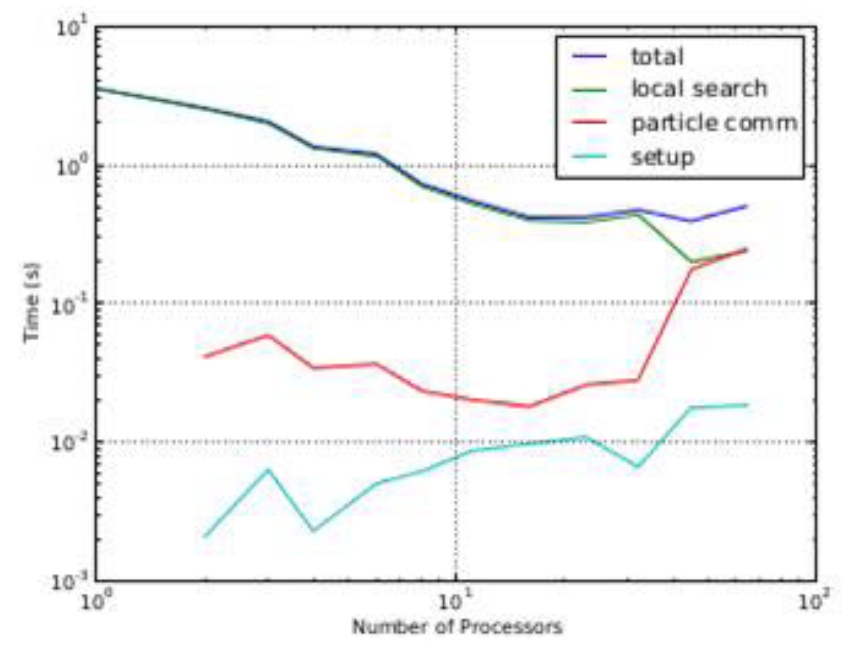

Figure 1. Particle computation runtime for different numbers of processors. The blue curve shows the total computational time, the green curve the time spent on locating particles within the halo of other particles, the red curve the time spent on communications between particles, and the light blue curve the time spent on initial overhead and set-up processes.

In FY 2015, we developed two complementary codes modeling the motion of charged DNA beads as they interact with (1) the fluid in which they are suspended and (2) an external electrostatic field. The fluid flow and electrostatic field are governed, respectively, by Stokes and Poisson's equations. Together, they dominate simulation computational time and account for the bulk of computer memory usage (on the order of 100 million to 10 billion bytes). Memory usage is driven by the need to resolve the so-called Debye length in the electrolyte (the size of the ion shells around the DNA beads) and the electrostatic field over very large surrounding material domains. We developed a fully parallel code that simultaneously solves the Stokes and Poisson's equations in the presence of point forces and charges. This code is fast and very accurate in the large domain around the particles and parallelizes very well.

To improve the accuracy of the flow and electrostatic field calculations near the particles, we developed a benchmark version of the General Geometry Ewald-like Method (GGEM), which essentially spends an optimal amount of time computing the particle-particle/ particle-fluid interactions. This benchmark version is a serial code that has been used extensively for bead suspensions in periodic and nonperiodic geometries. Our main near-term goal is to develop a parallel extension for GGEM suitable for massively parallel computers. 


\section{Improving and Validating Models of the Urban-Climate Connection with Dense Sensor Networks}

\author{
2014-167-R1
}

Robert Jacob, Charlie Catlett, Beth Drewniak, Rao Kotamarthi, and Rajesh Sankaran

\section{PROJECT DESCRIPTION}

Urban areas change their climates in these ways: by creating an urban heat island (UHI); altering the flow of air over, around, and through the city; and emitting pollutants that have strong negative local effects. To better understand how climate change will affect cities, it will be necessary to better understand and model the interactions of cities and their surrounding regional climate. Models of urban areas suffer from a common problem: lack of validation data from within the city. Most climatological and meteorological sensor networks are located outside of urban areas to better sample larger regions. They also have not taken advantage of new lowcost computing and sensor devices that the commercial cell phone industry has created. This project will design and deploy a dense sensor network that can measure urban climate in detail and provide validation data for simulations of urban weather and climate.

Our major task is to identify and develop a low-cost, low-power, and inexpensive sensor node for basic meteorological measurements. The node will also need software/hardware for managing power and communicating data. We will deploy about a dozen test nodes to test the hardware and software and assess data quality. We will also augment Argonne's existing weather station to provide measurements for comparison. If the initial deployment is successful, we will expand the network to cover more of Chicago. The data we collect will be combined and compared with data from citizen observer networks to make diurnal, monthly maps of the UHI. This data will be compared with UHI simulations.

\section{MISSION RELEVANCE}

This project is primarily relevant to DOE's science mission, as our sensor network will have the capability to dramatically improve our understanding of the urban boundary layer. This research is relevant to multiple programs in the Climate and Environmental Science Division of the Office of Biological and Environmental Research (BER). The BER Advisory Committee recently recommended that the first integrated field laboratory focus on an urban area. The project is also relevant to DOE's Office of Energy Efficiency and Renewable Energy, as cities are primary consumers of energy and data on their environment will be crucial to improved efficiency. The National Science Foundation and National Aeronautics and Space Administration also have interest in studying cities.

\section{RESULTS AND ACCOMPLISHMENTS}

In FY 2014, we developed a low-cost $(<\$ 300)$ weather sensor station with sufficient computing power for parsing and data manipulation. We worked with the "Integrated Sensor Network" LDRD project (2014-160-NO) to develop the data infrastructure. We integrated several similar sensors into the system in the first phase to evaluate them and understand their performance limitations. We designed a rooftop mountable platform and deployed two such "weather pots" at Argonne.

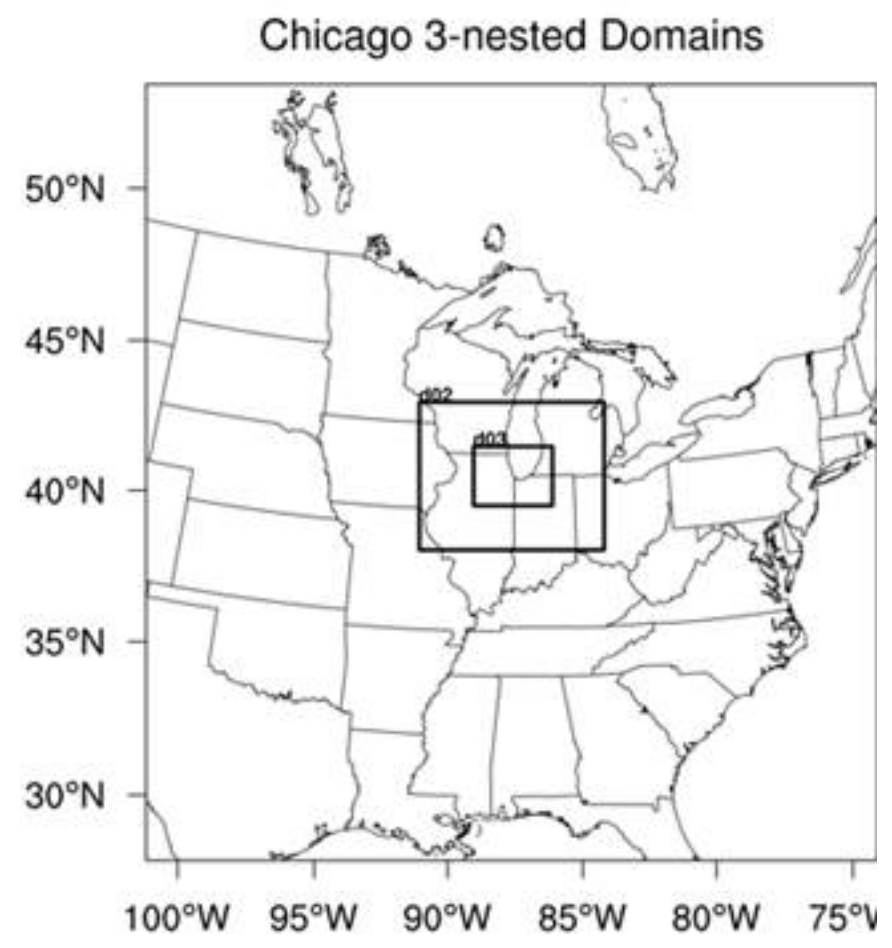

Figure 1. Three nested modeling domains for a Chicago configuration of the urban WRF system. The outer frame in the figure is itself nested in the parent domain which is not shown.

During late FY 2014 and early FY 2015, we began to explore how our network might improve the existing capabilities of Chicago's urban Weather Research and Forecast (WRF) system. We used WRF's nesting ability and built a fine-resolution (up to one kilometer) configuration over the Chicago area (Figure 1). We incorporated new land-cover data (from the National Urban Data and Access Portal Tool) and urban datasets 
(National Land Cover Database 2006) and tested various settings of urban canopy, lake scheme, vertical resolution, and other parameters (Figure 2). We also used WRF-Chem to examine the interaction between urban air quality and the climate.

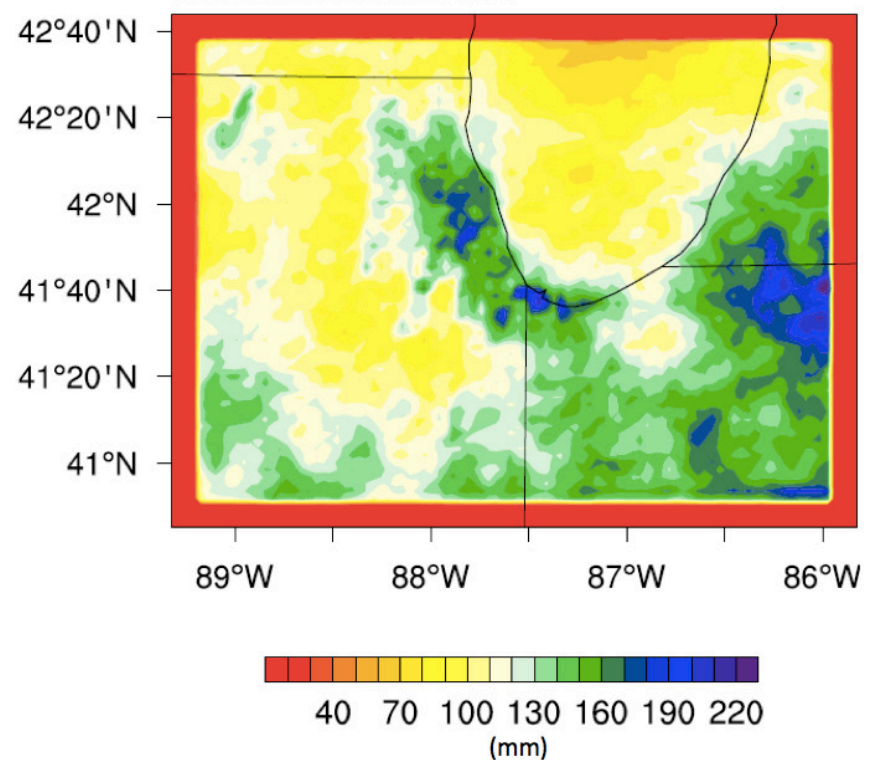

Figure 2. The effect of adding anthropogenic heating such as exhaust from air conditioners to total precipitation in the innermost domain of Figure 1 for a WRF simulation of July. Precipitation is greatly increased over the city.

In FY 2015, we also refined the design of our meteorological sensor board. Based on the summer, fall, and winter field tests of 2014 , we have readied our sensors for mass deployment. The testing included our first city rooftop location at DePaul University. We also evaluated chemical sealants for sensor boards and have greatly increased their durability. The new production boards will be commercially fabricated and sealed, therefore maintaining uniform production quality and sealant application. We have also eliminated sensors that were more prone to failure and designed the new boards with well-vetted sensors.

\section{PROPOSED FUTURE WORK}

In FY 2016, we will continue to expand our sensor network on rooftops in the Chicago area using the new versions of the sensor board and then analyze the resulting data.

\section{Advanced Pipeline for High- Throughput Digitization of Large-Scale Collections}

\section{4-174-R1}

Mark Hereld and Nicola Ferrier

\section{PROJECT DESCRIPTION}

A new merging of high-performance computing with realworld systems for high-throughput data collection and automated manufacturing requires technologies that are fast becoming usable. Integration of these technologies will allow us to apply them routinely to new kinds of science and engineering problems. In this project, we are designing and building components for scalable automated workflows in the context of a particularly challenging problem: digitization of the pinned insect collection of the Field Museum of Natural History in Chicago.

\section{MISSION RELEVANCE}

The beneficiaries of this work are many. Museums with complex object collections have already seen the need for automated digitization and data analysis. High-throughput biology, which falls into the DOE mission of "supporting sciences," also stands to gain from our efforts. Outside of the laboratory, field biology and other field sciences (including environmental research) would also benefit.

Many agencies have ongoing interest in development in this area, including DOE's current initiatives in automated manufacturing technologies and the National Science Foundation's wide-ranging interest in robotics. Within Argonne, increased competence in robotics and automation would benefit many research areas: sample handling (e.g., at the Advanced Photon Source); experiment automation in biology, chemistry, and physics; and machining and fabrication in the Laboratory's shops.

\section{RESULTS AND ACCOMPLISHMENTS}

Optical Digitizing Head. In FY 2014, we studied the Field Museum's insect collection to inform priorities, and we evaluated imaging modalities, cameras, and optical configurations. We determined that an array of light field cameras would capture sufficient three-dimensional (3D) data for a wide range of specimen orientation and occlusion configurations. (Light field cameras capture information about all the light in a scene, allowing adjustment of the focus and depth of field after the picture is taken.) We found that multiple views of the same text field can be automatically combined to remove occultation of the pin, compensate for variation in focus across the field, and provide a clean rectified image (Figure 1). 

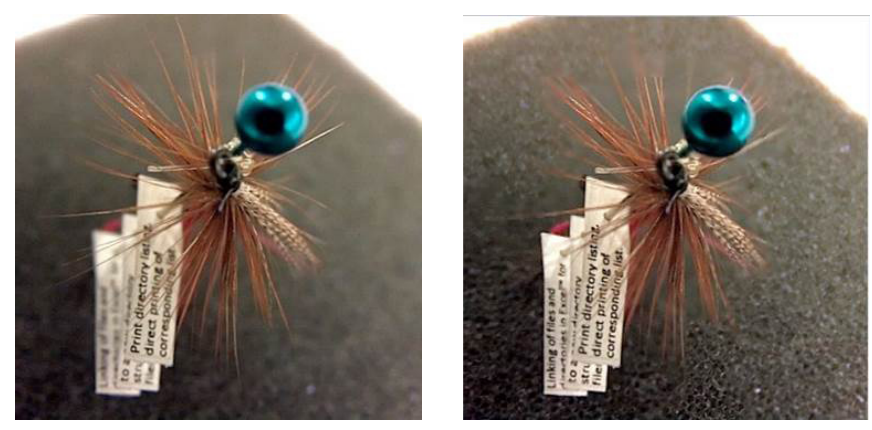

Figure 1. Infinite depth image (right) reconstructed from captured light field shows text on all labels in clear focus compared to typical image (left) with top label in focus.

In FY 2015, we tested a low-cost, commercial light field camera (Lytro) and found these advantages: focused image data from a large volume, ability to extract depth from a single snapshot, and low cost. A test multi-Lytro camera head is under development. We developed a software tool to explore camera placement and determine approximate volume of coverage.

Data Capture. In FY 2014, we explored database design, which includes some metadata and references to the primary image data. In FY 2015, computer-vision-based methods were developed to populate the database from each drawer.

Object Handling and Tracking. In FY 2014, we developed image analysis software to parse incoming drawers into identifiable boxes and bugs, which gives us a starting place for input to robotic handling, specimen tracking, and fault handling. The initial algorithms yielded about $90 \%$ accuracy, and further improvements are ongoing.

Image Analysis Software. In FY 2014, we evaluated optical character recognition software and developed algorithms to register and combine label data from multiple views (with some human assistance). We experimented with methods to automate extraction and alignment of label data within the images. This effort led to evaluation of methods to extract 3D information. We developed an approach using structured light to determine the 3D position of the planes for each label and used that 3D information to assist in image segmentation and label alignment.

In FY 2015, we developed software for various aspects of the imaging pipeline: calibration, label reconstruction, "unbundling" of the light field data, and 3D point cloud from light-field "stereo" cameras. We wrote software to handle the light field data, automatically build an "infinite depth" image, and store the image for further processing. We also developed software to obtain 3D data, using both the internal camera information and multiple light field cameras (for two cameras at this point) to build 3D point clouds (Figure 2).

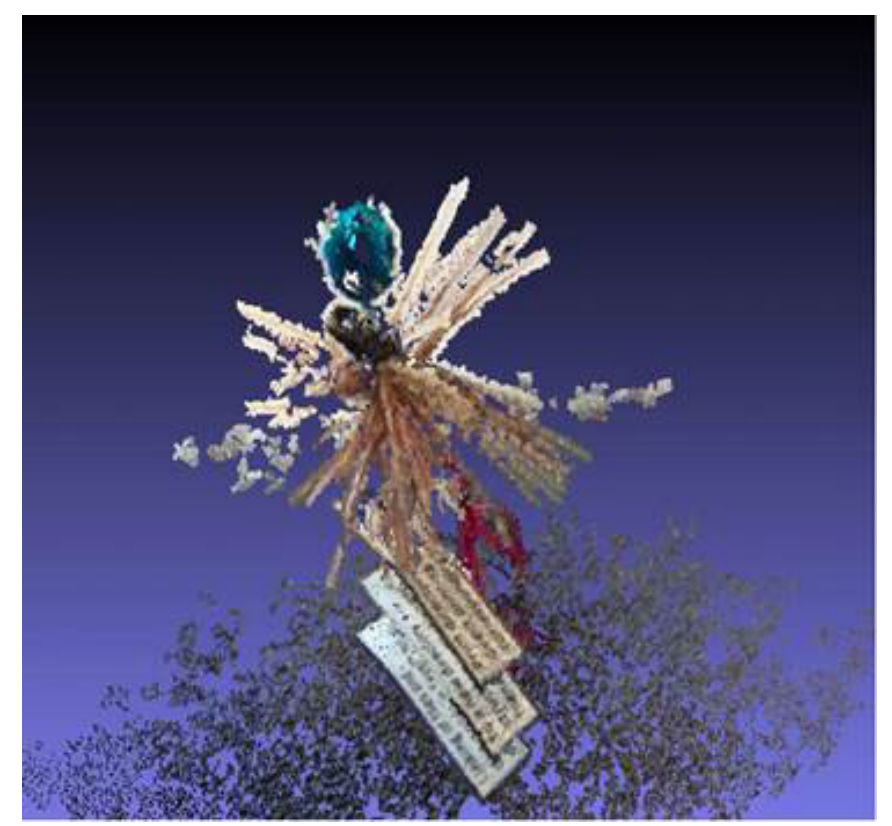

Figure 2. A 3D reconstruction using depth data recovered from the light field that allows 3D segmentation to separate each label from the field. Each pixel has been colored using captured data. Infinite or indeterminate depth is left transparent, allowing the artificial background gradient to show through.

\section{PROPOSED FUTURE WORK}

In FY 2016, we will pursue four sets of tasks:

$\square$ Optical Digitizing Head. We will complete fabrication of our 12-camera rapid capture head and a controlled lighting fixture that will surround the camera head and enable us to control the lighting.

$\square$ Data Capture. We will re-validate the statistical tools after automation tools have been completed. All of the tools are in place for a statistical analysis of the data once the database is sufficiently populated.

$\square$ Object Handling and Tracking. We will develop a mechanism to "feed" specimens to the camera and control the workflow.

$\square$ Image Analysis Software. We will continue to develop software tools for the Lytro cameras, including novel tricamera calibration and registration. Dense depth maps can be computed. We will complete our algorithms for $3 \mathrm{D}$ reconstruction to bring the overall effort together for a "best-in-class" imaging system. 
PARIS: Data Knowledge-Based Extreme-Scale Resilience

\author{
2014-181-R1 \\ Franck Cappello
}

\section{PROJECT DESCRIPTION}

With the increase in component number and the projected use of aggressive power-saving technologies, future extreme-scale computational systems will be less reliable than existing systems because of faults from internal or external causes. The high-performance computing community has recognized this "exascale resilience challenge" and is exploring solutions to ensure that numerical science applications provide correct results. The PARIS project explores the fundamental properties of numerical science applications to improve the resilience of extreme-scale executions and to provide efficient solutions to system failures and silent data corruptions (SDCs). Objects and phenomena studied using time-dependent numerical simulations and data analytics have important characteristics reflected in the data dynamics and data semantics that could be leveraged to discover new resilience techniques. Data dynamics refers to the steps of the data transformation applied during execution by the computational/analytical patterns of the application. Successive transformations of data could be represented by a trajectory that could be modeled, analyzed, and monitored. Data semantics considers the meaning of the data as well as correlations among data. Numerical science applications data are linked and should stay consistent during execution with the analyzed object or phenomena. We seek to leverage data dynamics and data semantics to devise new SDC-detection, data-compression, and self-healing techniques.

\section{MISSION RELEVANCE}

Extracting knowledge from large-scale numerical simulations and data analytics is key to the DOE mission in science. Silent data corruptions and execution failures are severe threats against producing timely and accurate DOE mission-critical results. The PARIS project provides lossless and lossy compressors to accelerate checkpoint/ restart and SDC detectors/correctors to reduce the risk of corrupted results. Application programmers are able to leverage PARIS mechanisms to reduce development effort for reducing checkpoint size and dealing with SDCs. This PARIS project provides data compressors and SDC detectors mechanisms for simulation codes involving cosmology (HACC), climate (ACME), nuclear reactors
(Nek5000), and shocks (FLASH). The results of the PARIS project are useful for other applications at Argonne, DOE, and other agencies.

\section{RESULTS AND ACCOMPLISHMENTS}

In FY 2014, we started the exploration of spatial and temporal properties of data sets generated by numerical simulations. We discovered that for many applications and data sets, the most important variables have relatively smooth trajectories in space and time. From this observation, we designed several SDC detectors leveraging the spatial smoothness or the temporal smoothness of the data set. These early detectors were instrumental to understanding the scientific methodology for SDC research and the different performance aspects of SDC detectors (overhead in memory occupation and computation time, false positive rate, false negative rate). We also noted that when the variable trajectories in time and space are not smooth enough, detection techniques closer to the numerical methods are needed. This first generation of SDC detectors set the basis for the design of the detectors developed in FY 2015.

In FY 2015, the project accomplished several of its important milestones: (1) We designed new lossy compression algorithms guaranteeing error bounds fixed by users. These algorithms are extremely efficient compared to other existing lossy compressors: they significantly outperform all other compressors in compression ratio and time to decompress. We tested these algorithms on 20 benchmarks from FLASH, NEK5000, and ACME and observed compression ratios of 3-5 for very hard-to-compress data sets and $\times 100$ for highly compressible data sets. (2) We implemented working versions of the compressors' software (named LZ and SZ: LZ for lossless and lossy compression and SZ for lossy compression with a different algorithm). The SZ software is tested at NCAR (the National Center for Atmospheric Research) on real CESM (Community Earth System Model) data sets. (3) We have started studying how restarting numerical simulations from lossy compressed checkpoints affect the end results of the simulations. We are investigating the diffusion cases using SZ for lossy compression. (4) We have designed and implemented two additional SDC detectors based on data analytics. These detectors use the spatial and temporal properties of the simulation data sets to perform detection. We have developed a machine learning (support vector machine)-based detector and a detector mixing prediction and partial replication. The computational and memory overheads are reasonable compared to SDC detection based on pure replication. 


\section{PROPOSED FUTURE WORK}

We will continue to develop new lossless and lossy compressor algorithms to further reduce the size of checkpoints. We will make public releases of the improved LZ and SZ compressors. Concerning SDC detection, SDC detectors have been developed in other contexts, with the results of a main numerical method compared with the results of the same numerical method on a coarser mesh or of a lower order. These detectors use different principles than the prediction-based detectors developed in this project. We will investigate how to combine these two types of detectors and will integrate the compressors and detectors in a popular multi-level checkpoint/ restart framework, such as Argonne/FTI (Fault Tolerance Interface) and Lawrence Livermore/SCR (Scalable Checkpoint Restart).

\section{Dynamic Data Mirroring for Data-Intensive Science}

\section{4-182-R1}

\section{Ian T. Foster and Steven Tuecke}

\section{PROJECT DESCRIPTION}

The project seeks to address challenges in managing and accessing large-scale scientific data on heterogeneous storage systems over the data lifecycle by providing a dynamic, high-performance data mirroring solution. Because there can be a diverse set of requirements for the type of storage needed for a given set of data, storage system characteristics such as performance, cost, and access capabilities determine suitable use. This approach to system design potentially results in generation of multiple copies of the data and different views into the same data. Managing such data on these systems such that it is readily available for processing or access is onerous for researchers. The proposed work will develop and evaluate options for dynamic data management and mirroring and will be driven by user policies as well as characteristics of the storage system.

\section{MISSION RELEVANCE}

This project is relevant to DOE's mission in science. These new methods will provide needed support for major data-intensive science projects of interest to DOE by contributing to an understanding of requirements and architectures for next-generation research campus storage solutions at institutions such as Argonne and other national laboratories. If this project is successful, DOE will gain significant knowledge about how to create a big data storage and analysis infrastructure that is able to meet the varying needs of scientists and their teams across a multi-program laboratory such as Argonne-a system that will provide those scientists with a competitive advantage. The existence of this solution will also enhance the value of other facilities, such as (at Argonne) the Advanced Photon Source (APS), the Argonne Leadership Computing Facility (ALCF), the Laboratory Computing Resource Center, and the Center for Nanoscale Materials.

\section{RESULTS AND ACCOMPLISHMENTS}

We have continued to build on the Petrel storage service, working with ALCF. The 1.7-petabyte storage system (Figure 1) was fabricated using repurposed disks and controllers from ALCF's Intrepid machine. In the previous year, we worked with ALCF to set up Petrel and establish a user-driven and project-based allocation mechanism. Researchers on several projects have been able to test use of this service and, in the last year, 230 terabytes of data have been moved into and out of Petrel. Others have used Petrel to back up and share data with external users, in some cases with restricted access.

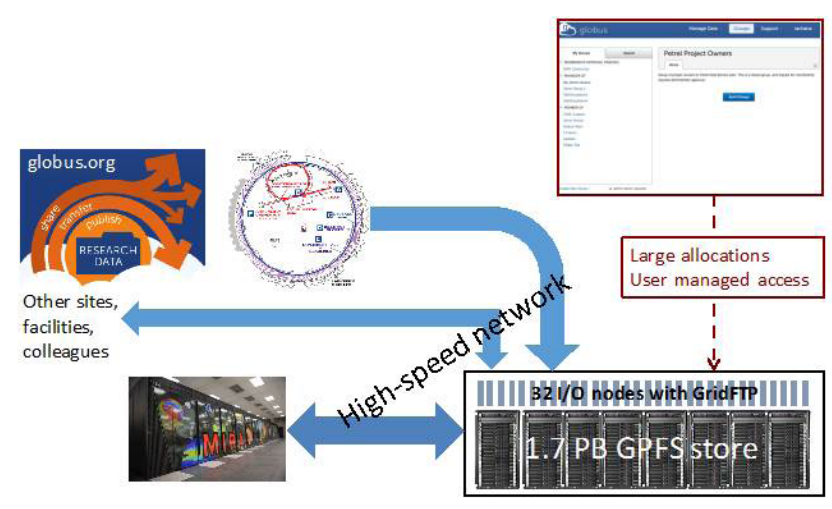

Figure 1. The Petrel data server developed as part of this project and its connection to other Argonne research infrastructure.

ALCF, APS, and Globus teams conducted extensive performance tuning to ensure that APS and Petrel transfers use the capacity effectively. On the basis of the success of using a data sharing infrastructure, select APS beamlines have also deployed Globus sharing on their acquisition infrastructure to move data directly from there to Petrel and to share it with their users. A command line tool to automate data movement was developed and made available, and APS scientists developed tools to apply APS identity and policies on shared data using Globus application program interfaces.

Petrel also proved to be an important resource for use by the "Discovery Engines for Big Data" (DEBD) LDRD project (2013-165-R2), which intends to accelerate the scientific process by connecting physical experiments 
to simulations. This project necessitates advanced data management, accommodating very large datasets that are rapidly produced by detectors or telescopes. Such data are transferred via Globus from or to other sites, such as APS beamlines and the ALCF, and are stored on Petrel for various future uses. Rapid data transfer and efficient data management are critical for joining experiment, analysis, and simulation. The large volume allows researchers to avoid working around data sizes; rather they make copious backups and can focus on aggressively manipulating and analyzing experimental, simulation, and derived data. In at least one significant case, raw experimental data from the APS was damaged during analysis and was successfully recovered from Petrel via a Globus transfer.

Under DEBD, APS scientists are able to use Petrel as a "hub" for transfers from the APS to the ALCF and group-specific analysis computers (e.g., NXRS of the Materials Science Division [MSD] or Orthros [APS]). Data on Petrel are cataloged using the Globus catalog for search and integration with application-specific tools (e.g., NeXPy). Petrel was also used as a data transfer hub by Argonne/MSD scientists using the Cornell CHESS synchrotron; data management techniques developed under DEBD were deployed at CHESS, while backups were moved to Petrel for safety and future use upon return to Argonne.

\section{PROPOSED FUTURE WORK}

Recently, new hardware was purchased to replace the existing Petrel stack. The new machines provide two 40-gigabyte connections, and initial studies show better performance with the new hardware. We will port data to the new machines and make that available for data sharing by the users.

In the final year of the project, we plan to explore use of Petrel as a storage hierarchy tier and automation of management of such a system. This functionality will allow researchers to set up tools to move subsets of their data automatically to Petrel, set up permissions for sharing, and synchronize between the systems based on their need. Enabling easy access to data on Petrel for computation is a key goal. We will explore options for rapidly and seamlessly staging data to and from compute nodes as part of the job directive and for managing the duration of storage on each tier. This work is scoped to integrate with the ACLF job scheduler system so that Petrel can be used to hold data prior to and after supercomputing runs.

We will also deploy alternate protocol access (HTTP/S) to allow direct access and reference to the files and anonymous access. This capability will facilitate integration that requires HTTP file access, such as inline viewers and browser downloads.

\section{Lab-Wide Research Analytics}

\author{
2015-135-NO \\ Ian T. Foster, Benjamin Blaiszik, Kyle Chard, Paul Domagala, \\ and Tijana Rajh
}

\section{PROJECT DESCRIPTION}

The goals of this project were to explore the feasibility and utility of an analytics platform for national laboratories that would (1) capture and map the institutional collaboration and domain expertise networks in conjunction with formal organizational structures within each laboratory; (2) analyze, visualize, and mine research output in conjunction with the organizational, collaboration, and expertise networks to derive deep institutional knowledge and identify unrealized internal and external opportunities; (3) facilitate end-user discovery of new research opportunities and points of potential collaboration; and (4) situate each laboratory's capabilities and contributions within the broader research landscape.

The initial phase of this research centered on building a graph for Argonne that combines researcher links, outputs, and competencies. This structure forms the foundation of a new and unique lab-wide ability to understand, visualize, and explore existing connections, competencies, and research products, as well as to find untapped opportunities for growth and new internal and external collaborations.

Building on network analysis techniques and naturallanguage processing, this project extended existing algorithms and software to analyze, investigate, and understand the Argonne network.

\section{MISSION RELEVANCE}

Recent high-profile DOE programs (Hubs, Energy Frontier Research Centers) have focused on accelerating breakthrough science by building wide collaborations between research institutions and researchers spanning academia, industry, and government. Our project provides a new capability to understand individual researcher competencies and complex research team compositions contextualized within the nationwide research organization. This capability simplifies and expedites matching researchers with collaborators nationwide, understanding the factors that lead to high-impact research teams, detecting projects that may complement 
each other across a variety of research agencies, and averting duplicative efforts.

\section{RESULTS AND ACCOMPLISHMENTS}

We synthesized data from a variety of sources, including Argonne's publication records, Argonne research staff data, and publication data indexed from Scopus (a citation database of peer-reviewed literature) to build a collaboration graph superimposed on the laboratory organizational structure. With this network, we completed social network analyses to identify and understand critical nodes and the relationship between these nodes in the collaboration structure and began to investigate key drivers of productive collaboration.

Research outputs (e.g., patents, journal papers) come in unstructured natural language. Extracting semantic understanding from these outputs is critical to understanding the research output of the Laboratory and tracking its dynamically evolving core competencies. As a first step, we built multiple author-topic models based on (1) Argonne's recent publications (2000-2015) and (2) a wider topic model, including recent publications (2000-2015) from Argonne, ORNL, and the University of Chicago. The Author-Topic (AT) model selected is based on Latent Dirichlet Allocation, ${ }^{+}$which, given a corpus of documents, yields representations of topics (as mixtures of words), documents (as mixtures of topics), and authors (as mixtures over topics).

The information obtained from the AT model allows for automated quantitative scoring of natural-language outputs into the model topics loosely corresponding to research topics. For ease of explanation, we show "energy storage" and "computing" (Figure 1) as examples from among the 50 topics in the model. Shown in Figure 1 are the top words in these two topics, along with the score associated with each word. To score a sample text, each word in the text (minus English stop words) is scored in each topic and sums are calculated. We assign scores to researchers (Figure 2) as an average of their scores across their publications. The resulting models are representative of Argonne and a subset of the DOE's wider research, with some initial reach into academic research. In the future, we hope to investigate application of this model toward understanding temporal changes, strategic directions, impact of funding (following vs. leading research direction), research outputs, and more.
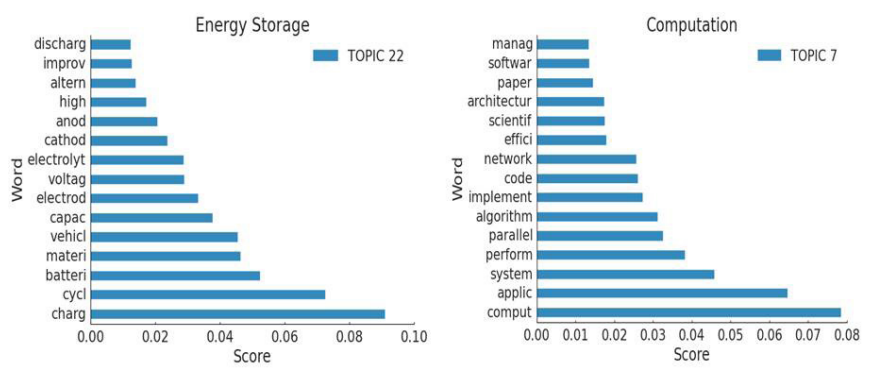

Figure 1. Examples of the top 15 words, with word stemming, associated with two of the $\mathbf{5 0}$ topics derived from the AT-model of Argonne's research since 2000 . While only the top 15 words are shown in these two examples, all topics have scores associated with every unique word in the corpus. Each topic from the model also has authors quantitatively associated with it (not shown).

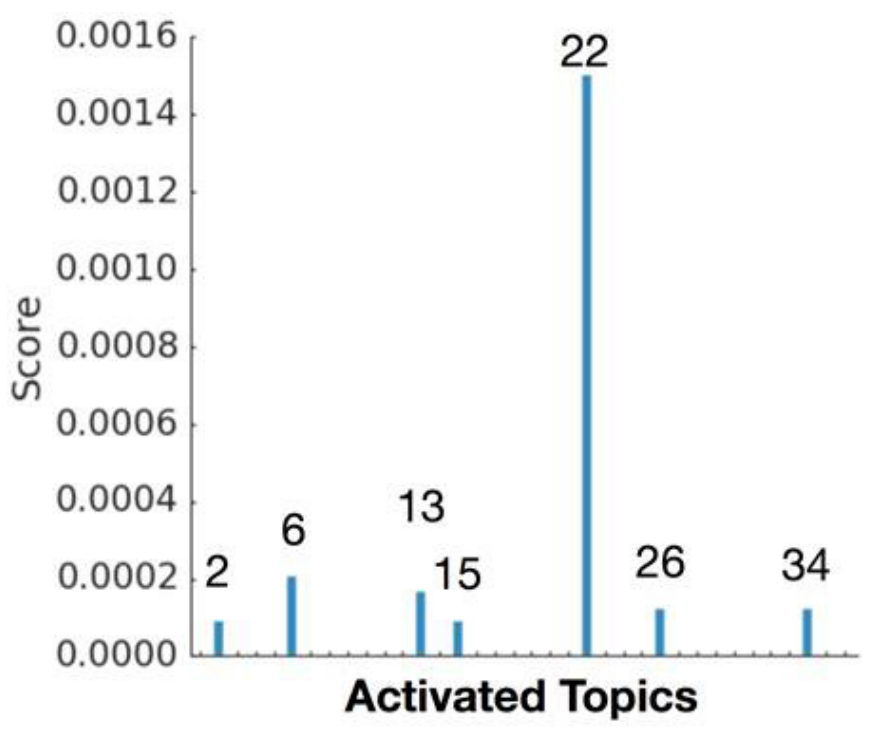

Figure 2. Each unique author is described as a weighted composite of his or her publications as scored into a topic model. In this example, the researcher is well-known in the field of energy storage, and shows a corresponding primary peak in the expected topic (topic 22-see Figure 1) and weaker peaks in topic 6 (loosely corresponding to experimental electrochemical testing) and topic 13 (loosely corresponding to nanoscience).

While the initial focus of the project was to develop the core of the analytics platform, we performed initial classification to predict observed collaborations for the year 2014 via an Extra Random Trees classification model trained on data from earlier years. Combining data from the network analysis with the topic model, our initial analyses showed that, as expected, past direct collaboration is the strongest predictor of future collaboration, followed closely by indirect collaboration (i.e., friend-of-a-friend collaboration), and that a researcher's topic similarity to a second researcher is a more important predictor of future collaboration than their organizational distance. Interestingly, sharing the same physical building was also a stronger predictor of future collaboration than organizational distance. These insights are representative of capabilities that the analytics platform may confer over time. 
In summary, we developed not only a network representation of research collaboration and researchers at Argonne built from heterogenous data sources, but also a means to cluster and classify natural-language research outputs by research domain into the same network for deeper analysis, and we have performed initial combined analyses of the natural-language research outputs and the collaboration network to understand key drivers of collaboration. These components form the initial core of an analytics platform to better understand the research output of Argonne, and eventually other research institutions, and they act as building blocks to support predictive analytics to investigate team formation, research directions, and collaborator matching.

Future work, contingent on acquiring additional support for this project, will include developing and deploying REST APIs (REpresentational State Transfer application programming interfaces) clients, and UIs (user interfaces) to allow researchers and a variety of stakeholders to interact with the data and analyses generated via the platform. It will also be critical to extend the completed social network analyses and models to dynamic networks and dynamic topic models. Further, it will be necessary to expand the scope of the analytics platform from Argonne to other institutions, individually and collectively, to increase the service utility.

${ }^{\dagger}$ Rosen-Zvi, Michal, Thomas Griffiths, Mark Steyvers, and Padhraic Smyth, "The author-topic model for authors and documents." In Proceedings of the 20th Conference on Uncertainty in Artificial Intelligence, pp. 487-494. AUAI Press, 2004.

\section{Implementing a New Extreme- Scale Parallel Programming Model with a Full Sample Application}

\section{5-139-NO}

Barry F. Smith and Junchao Zhang

\section{PROJECT DESCRIPTION}

The message passing interface (MPI) programming model is reaching the end of its viability for development of extreme-scale simulations and data analysis. The addition of threading or task-based parallelism to MPI has extended MPI's viability for a few years and increased the complexity of resulting codes, but it does not remove MPI's fundamental limitations. The time is ripe for developing a new simple, general-purpose model for extreme-scale parallelism that can replace the MPI + shared-X model. The push for ever-increasing levels of concurrency and the increasing complexity of simulation codes require a new parallel-programming model that both simplifies the development of extreme-scale codes and makes them more efficient and scalable on newly emerging architectures, as well as on graphics processing units (GPUs), many-core systems, and conventional computing systems.

For the past twenty years, careful attention to data locality has been crucial to obtaining high performance. The message-passing programming model succeeded in dominating the market because it provided a good approach for ensuring data locality. With exascale systems, data locality will remain crucial, but now resiliency, machine jitter issues, and more adaptive, heterogeneous simulations require a more flexible dynamic placement of data that simply is not achievable with the message-passing model. To expect the programmer to manage the extremely complicated data placement and movement needed in this environment is unrealistic. They can be handled only by having a runtime system that determines both the size and the placement of tasks and ensures that the data is where it needs to be when it is needed. But for a runtime to do this, it must be in control of the complex and generally unstructured data, and the user must be able to indicate to the runtime the relationships between the data items so that the runtime may manipulate, partition, and distribute them for efficient task performance. At the same time, one must keep in mind that HPC software is currently written in $\mathrm{C}, \mathrm{C}++$, and Fortran, with occasional high-level code written in Python and with computational kernels possibly written in CUDA and OpenMP. Expecting the entire HPC community to switch to a new (yet-to-be-developed) programming language for HPC is unrealistic and unnecessary. In fact, one of the most important reasons for the success of MPI as the HPC programming model was that it did not require any language changes.

The goal of this project is to develop and provide a sample implementation and sample application that utilize a new parallel programming model which overcomes many of the limitations of the current and previous parallel programming models, while preserving as much as possible the programming languages that application scientists are accustomed to. The model, called the Indexed Programming Model (IPM), is based on maintaining an abstract graph representation of the data dependencies for a set of computational kernels that implement the computations. A runtime system then uses the graph representation to partition the data across the machine and insure that all data dependencies are satisfied, and then launches the computational kernels. 


\section{MISSION RELEVANCE}

DOE has stated that exascale computing is crucial to its role in supporting science. The customers and beneficiaries of this project would include virtually everyone involved in HPC. Specifically, those developing scientific software libraries (e.g., those funded by DOE ASCR Applied Mathematics) will benefit in the short and long term by having a stable, portable development model for their libraries. Scientific simulation application developers (e.g., those funded by BES and BER) will similarly benefit. Hence, the scientists who utilize the simulation applications will benefit by having the applications available much sooner than otherwise possible. The exascale machine developers (e.g., IBM, Cray) will benefit almost immediately by having a specific common model for which to develop their hardware and software while still having a great deal of freedom in choosing what specific hardware (e.g., GPUs, many-core) to utilize to accomplish their goals. Those developing exascale operating system software (e.g., those in DOE ASCR Computer Science programs) will be able to focus their efforts on providing high-quality implementations of the model and infrastructure to support the model.

\section{RESULTS AND ACCOMPLISHMENTS}

We have made a great deal of progress on both the sample IPM runtime and sample application. The runtime now uses a very efficient, single-buffer, shared-memory communication protocol for use within shared memory nodes with MPI between nodes; this protocol provides a substantial (60\%) improvement in communication time compared to the full MPI four-buffer communication protocol. Yet the additional $\mathrm{C}$ code needed for this performance improvement amounts to less than 200 lines. In addition, we introduced a communication context that reduces the amount of indexing required for repeated communication with the same pattern. These contexts are automatically created and destroyed when the pattern changes, allowing higher performance without requiring additional application programming interfaces for the users. With these enhancements, we compared the time required for adaptive mesh refinement with over 100,000,000 elements on multiple shared memory nodes with that of the widely used libMesh package. The IPM sample application is orders of magnitude faster than the libMesh implementation for the same meshes. We have almost finished developing a finite element solver for time-dependent advection-diffusion equations in two dimensions that mimics an application provided with the libMesh/PETSc solver. Once that solver is complete, we can compare the performance of the entire solver, and we expect much higher performance with much less code using the IPM model.

\section{PROPOSED FUTURE WORK}

We will complete the application code that solves time-dependent advection-diffusion equations in two dimensions by using adaptive mesh refinement and compare its performance at scale with the libMesh/PETSc finite element solver, which uses the same discretization and solution techniques. This comparison will provide a good demonstration of the flexibility, performance, and simplicity of coding available with IPM. We will then prepare technical documentation, presentations, and publications explaining the design and sample implementations of IPM and demonstrating both its simplicity and its ability to obtain high performance. This will provide the HPC community an opportunity to evaluate IPM. Depending on the time constraints and interests of the community, we will also develop code for the application that utilizes GPUs for finite element computational kernels and/or kernels written in Fortran.

\section{Agent-Based Behavioral Modeling of Ebola Spread in Chicago and Other Large Urban Areas}

\section{5-169-NO}

Charles Macal, Matt Berry, Julie Carrera, Andrew Castiglioni, Nick Collier, Joe Insley, John T. Murphy, Jonathan Ozik, Emily Rosenblum, Vadim Sokolov, and Jessica Trail

\section{PROJECT DESCRIPTION}

It is generally recognized that the next outbreak of a pandemic-like disease is inevitable and that there are severe shortcomings in domestic preparedness. The goal of this project is to develop the Chicago Agent-based Model (ABM) to investigate implications for U.S. preparedness concerning pandemic infectious disease outbreaks. Initially motivated by the 2014 Ebola outbreak, the model is generally applicable to pandemic infectious diseases of importance to public health and national security.

The Chicago ABM includes synthetic populations of agents corresponding to everyone in Chicago and adjacent counties. Contact patterns among people are modeled, establishing possible disease transmission pathways, based on the flow of people moving from place to place in Chicago as they go through their daily routines, and people moving into and out of the region through various transportation modes-most notably by air travel through Chicago's major airports. The model explicitly incorporates the behaviors of the public, healthcare 
workers, and patients in response to an Ebola outbreak at a fine-grained level of detail. The inclusion of these behaviors will enable the generation of significantly more realistic contact networks than those generated by current models (which do not include these behaviors) that can be used to identify effective strategies for interventions to mitigate a pandemic.

\section{MISSION RELEVANCE}

In October 2014, the Secretary of Energy pledged his support to the President and to the Secretary of the Department of Health and Human Services (HHS) in responding to the Ebola outbreak in West Africa and its consequences, under the President's One Government Initiative. The Secretary asked the national laboratories to develop technical proposals based on each of their unique capabilities. Argonne proposed adapting an existing infectious disease model, the Chicago MRSA ABM, for Community Associated Methicillin-resistant Staphylococcus aureus (CA-MRSA), a virulent bacterial pathogen, to Ebola Virus Disease (EVD) and implementing the model on Argonne's high-performance computing (HPC) platform, Blue Gene/Q Mira. This model would be critical to identifying preparedness problems that would arise in the United States with an influx of suspected and actual EVD cases. The model uses the Chicago area as a test case for its application, since existing datasets for Chicago and existing disease transmission dynamic process models could be used as a foundation. The Argonne Ebola model, which began development in October 2014, was highlighted by DOE officials at a White House meeting on November 5, 2014, as an example of how DOE Labs are supporting DOE/HHS.

The Original DOE/HHS Ebola initiative has morphed into a long-term preparedness initiative focusing on epidemic and pandemic planning, investigation of the adequacy of community healthcare system response and resiliency, and other issues identified during the EVD crisis. Modeling the epidemic spread of a pandemic-like disease and gaining a science-based understanding of the strategies and resources required to mitigate such an event continue to be high national priorities. Ultimately, this research will help in understanding the state of pandemic preparedness in the United States.

\section{RESULTS AND ACCOMPLISHMENTS}

In FY 2015, we implemented Chicago MRSA ABM on Argonne's Blue Gene/Q Mira. Code optimization was done, and distributed computing algorithms were explored. This has so far resulted in a $50 \%$ reduction in clock time for a 10-year hourly simulation of $2.9 \mathrm{M}$ agents.
The memory footprint of the model was reduced from 22 GB to $4 \mathrm{~GB}$, allowing for efficient implementation on Mira. We scaled up the Chicago ABM from the City of Chicago (2.9 M agents) to Cook County (5.4 M agents). This entailed database expansion and restructuring. We surveyed all Ebola literature and identified relevant EVD disease states (e.g., susceptible, exposed, infected, recovered with immunity, and deceased) and transmission rate parameters. The ABM was recoded to allow the Ebola disease states and transmission rate parameters to be loaded into the model. We developed advanced visualization renderings (animations, etc.) of infectious disease spread and contact networks. See Figures 1 and 2 for model-generated visuals. We developed parallel workflows (using Swift/T) for ensemble simulations for modeling uncertainty propagation.

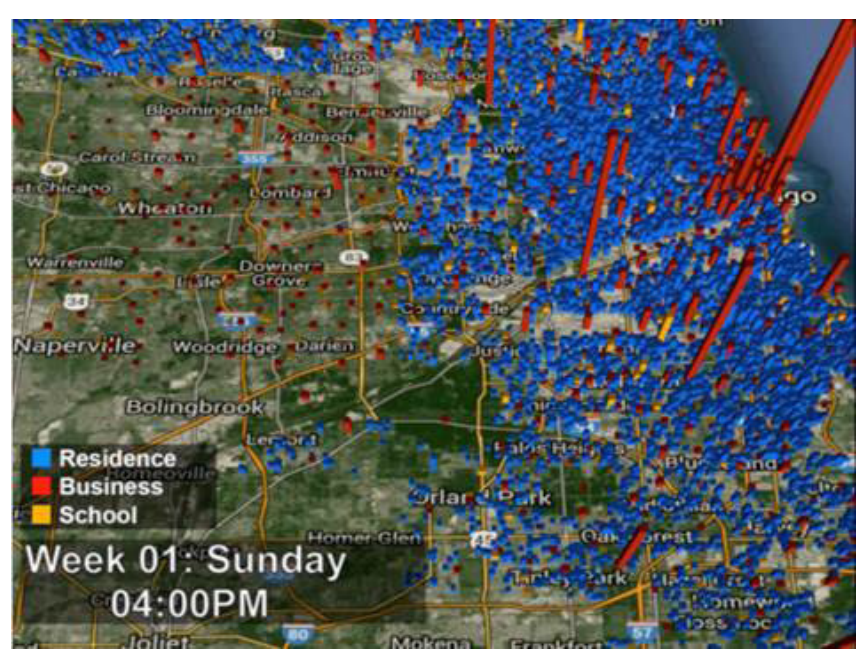

Figure 1. Hourly activity of people in Chicago as they go through their daily routines, moving from their homes to school, work, and other locations throughout the day. People located in the same places at the same time can be in social contact with each other, possibly leading to infectious disease transmission. Each person and each place in Chicago are included in the simulation. The height of the colored columns indicates the number of people at a location.

Finally, an invited presentation, “Designing Ebola Interventions for Large Urban Areas through Agent-Based Modeling and Network Analysis," was made on the project to an inter-agency Ebola working group led by the Biomedical Advanced Research and Development Authority (BARDA), within the Office of the Assistant Secretary for Preparedness and Response, HHS, April 1, 2015, by C.M. Macal. 


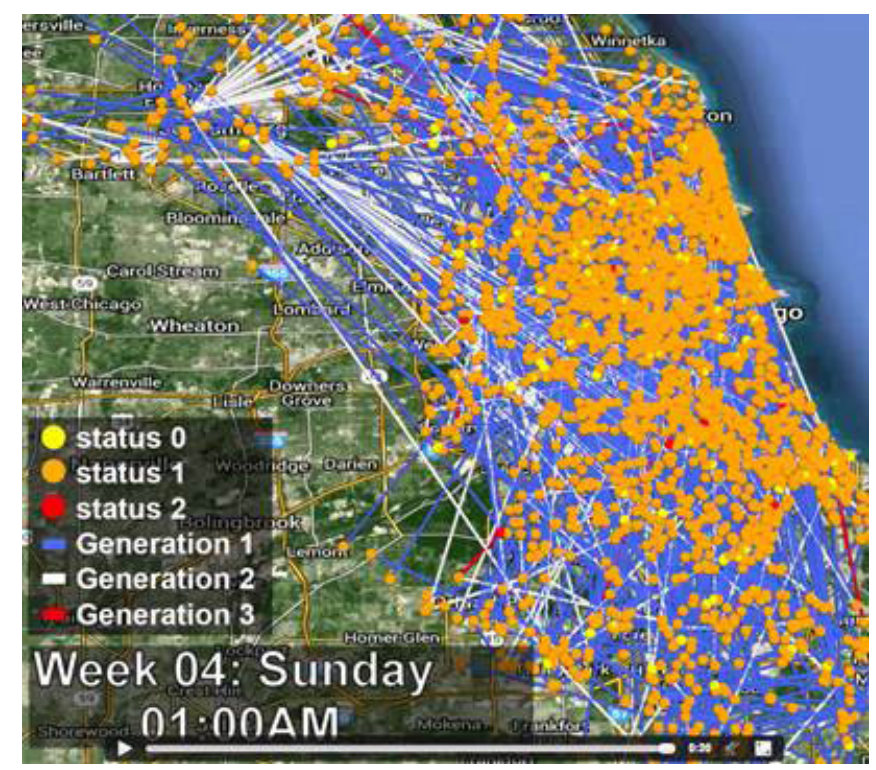

Figure 2. Disease transmission events as a result of personal contact as they occur in Chicago over a period of $\mathbf{2}$ weeks simulated by hour. Links show a contact tracing network as it develops in terms of who might have gotten a disease from whom. An agent's color indicates its disease state (yellow-not infected, orange-exposed, red-having an active infection).

\section{PROPOSED FUTURE WORK}

For FY 2016, we propose to generalize the Chicago Ebola ABM to a broad range of human-to-human contact diseases and urban areas. In addition to the existing large-scale ABM, we propose to develop a series of two other models of less complexity: a compartmental model (a differential equation analytical model that is standard in the field) and a simpler ABM with reduced structure in time and space that can be configured and run quickly. These models will be used to calibrate and inform searches in the parameter space for the full ABM. Preliminary work in FY 2015 validated the utility of work in this direction. In addition, we will advance multi-scale modeling to allow detailed process models (such as flows of patients and resources through hospitals) to be seamlessly integrated into area-wide population-based activity models.

Recently, the Centers for Disease Control and Prevention (CDC) announced that four Chicago hospitals (University of Chicago Medical Center, Northwestern Memorial Hospital, Rush University Medical Center, and Lurie Children's Hospital) have been designated as Ebola isolation facilities, able to receive Ebola patients and suspected cases. The inclusive scope and multi-resolution detail of the Chicago ABM will allow the model to incorporate explicit representations of patient and healthcare worker interactions (a primary source of secondary infections), hospital protocols, and procedures for using protective equipment. The model will also facilitate investigating required healthcare resources and waste requirements, general protocols for caring for patients, and coordination of hospitals across the region. In addition, the behavioral models will be included in population-based disease models. Behavior modeling consists of developing a framework for representing new agent behaviors. Agent behavior models consider agents' reactions to having the disease, hearing about the disease, reactions to proposed interventions, and agent-to-agent interactions. The Chicago Ebola ABM provides the capability to address numerous Ebola-related questions regarding public health and preparedness, as well as disease dynamics. We will also explore advanced technical methods for propagating uncertainty in large-scale simulation models through distributed ensemble simulation techniques using (Swift/T) workflows.

A key aspect of Argonne's contribution will be to generate large-scale scenarios that consider the full range of uncertainties in model parameters, agent behaviors, structural relationships, and assumptions. We will propagate the effects of these uncertainties into model forecasts through adaptive simulation techniques, by running ensembles of millions of stochastic simulation runs. We will continue to run the Chicago Ebola ABM on the Mira high-performance computer at Argonne to address the high simulation throughput requirements. Finally, we plan to advance technical methods for visualizing key information on infectious disease spread relevant to policy making. This consists of identifying the complete set of visualizations for conveying key information on infectious disease outbreaks to policymakers and implementing visualizations in the HPC environment.

\section{Determining Mechanical Properties of Material Systems Using Parameter-Free Metadynamics}

\section{5-172-NO \\ Carolyn Phillips and Juan J. de Pablo}

\section{PROJECT DESCRIPTION}

In this project, we are developing software and algorithms to perform a new free-energy calculation technique, a parameter-free metadynamics method. This method has the potential to calculate properties of material systems with higher accuracy and computational efficiency than 
prior computational methods. Using this method, we are calculating the values of mechanical properties that traditionally have been difficult to extract from either experiment or computation. We will extract values for these properties by implementing the parameter-free metadynamics method, in conjunction with a novel constrained molecular dynamics simulation test rig that is designed to isolate each mechanical property. Our first task is to calculate the elastic moduli of a liquid crystal and compare the result with the limited set of experimental values. Our second task is to calculate the shear coefficient of silicon using an atomistic model. If successful, this project will demonstrate how certain highly sought-after properties can be computed, create a general library to enable the rapid and accurate calculation of the mechanical properties for a diverse set of other material systems, and facilitate the use of large-scale computation in designing new materials. This project will also enable the use of a new, fast-converging metadynamics technique for performing other types of free-energy calculations in silico. This work is being performed with Jonathan Whitmer of the University of Notre Dame.

\section{MISSION RELEVANCE}

The methods to be developed in this project are designed to guarantee exhaustive sampling of phase space for free-energy calculations. These methods have applications in a wide range of research areas, ranging from mechanical calculations, phase transitions, and structural transitions, to calculating pathways in systems with complex free energy landscapes. They can also be applied to a wide range of materials, varying from metallic glasses to biomolecules. Computational rational design of these types of materials, as well as the design of new energy transfer and storage materials, is an important part of the mission of the DOE Basic Energy Sciences programs in materials and chemistry.

\section{RESULTS AND ACCOMPLISHMENTS}

In FY 2015, we selected and validated the coarsegrained atomistic model for the liquid crystal $5 \mathrm{CB}$ (4-Cyano-4'-pentylbiphenyl, $\left[\mathrm{C}_{18} \mathrm{H}_{19} \mathrm{~N}\right]$ ) in a chosen molecular dynamics simulation engine by demonstrating the correct temperature dependence of the order-disorder transition and density. We then created most of the software modules needed to generate a correctly constrained molecular dynamics test rig and tested each to verify that it functions as intended. In doing so, we identified limitations as to when certain constraints will work and developed a protocol for correctly preparing a test rig so that meaningful data are generated. We also performed initial simulation sweeps of a small system using a classic metadynamics technique parallelized at the node level via a highly parallelized molecular dynamics engine and at a multi-node level by using multiple walkers. Figure 1 shows the free-energy curve generated by these sweeps, confirming that a more aligned state (i.e., a nematic order parameter of 0.65) has the lowest energy at this temperature and is thus the preferred state of the system. For entropic reasons, perfect ordering (e.g., a nematic order parameter approaching 1.0) is not preferred. Inset images in Figure 1 show examples of the more and less aligned, or ordered, states for the liquid crystal.

\section{$2505 \mathrm{CB}$ at $\mathrm{T}=295 \mathrm{~K}$}

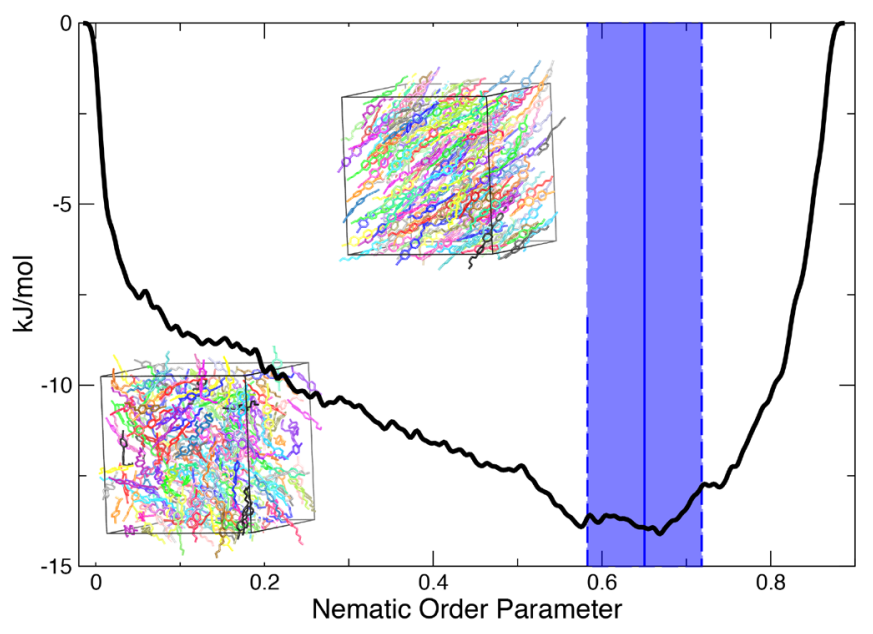

Figure 1. A converging free energy surface of the nematic order parameter for a small system of liquid crystals generated by well-tempered metadynamics.

\section{PROPOSED FUTURE WORK}

In the next stage of work in FY 2016, we will finalize the software necessary to generate the molecular dynamics test rig and use traditional metadynamics methods to extract mechanical properties. Then we will generate new software implementing the parameter-free metadynamics method in a reusable and distributable way. The computational performance of the new method will be compared with the traditional method. In FY 2017, we will select and validate an atomistic model for silicon in a molecular dynamics software package and develop a test rig for extracting mechanical properties from the system. In FY 2018, software modules will then be adapted so as to be incorporated into a generalized library of advanced statistical techniques. 


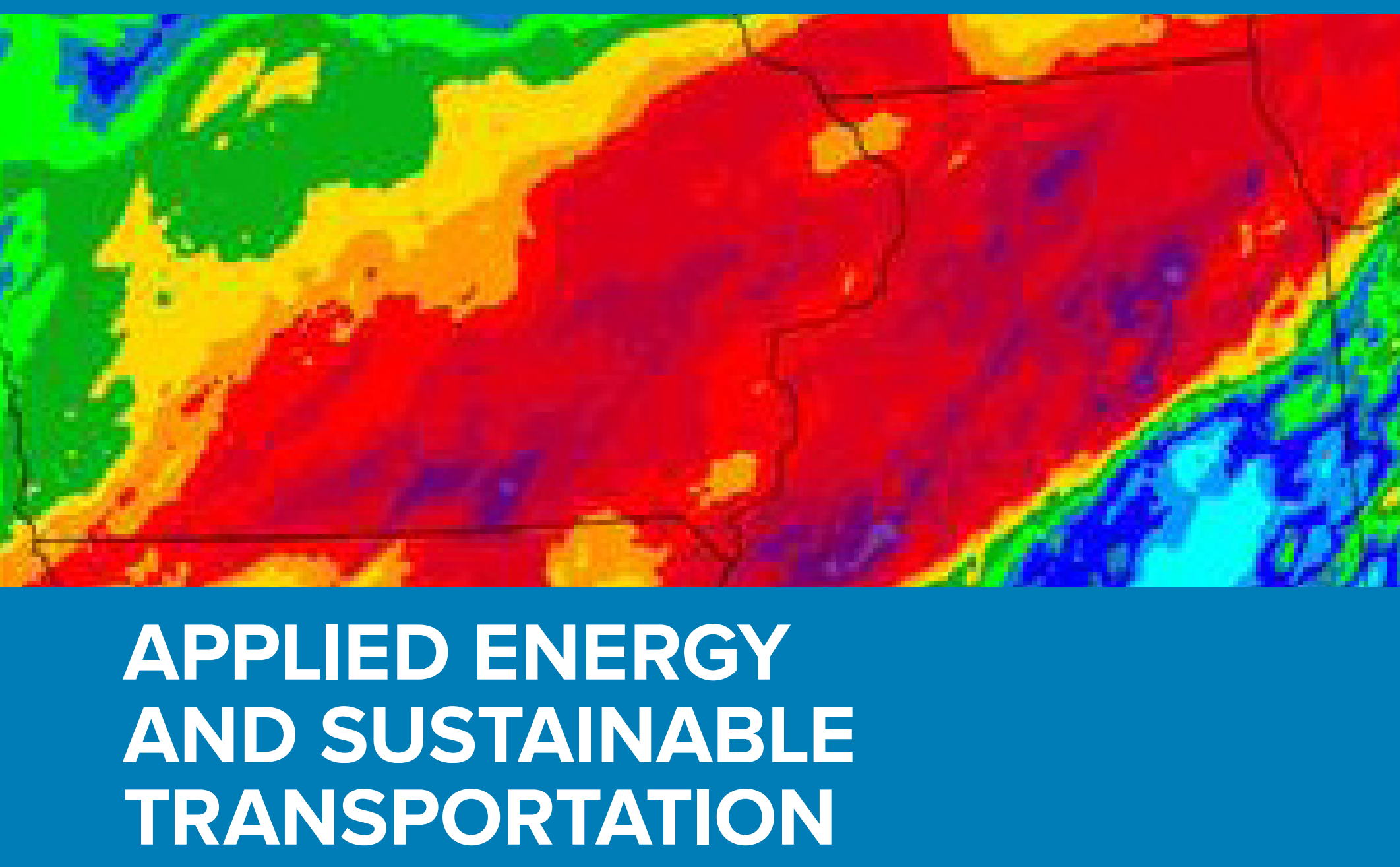




\section{Development of Predictive Multidimensional Combustion Modeling Capability with Detailed Chemistry}

\author{
2013-148-R2
}

Sibendu Som, Michael J. Davis, Marta Garcia Martinez, Douglas E. Longman, and Raghu Sivaramakrishnan

\section{PROJECT DESCRIPTION}

Computational modeling of internal combustion engines (ICEs) is an arduous task primarily because the length and time scales pose a variety of challenges. The predictive capability of modeling ICEs is hampered by the inability of the current spray and combustion models to capture the in-cylinder stochastics arising and evolving from the fuel spray injection and the interactions between fluid dynamics (turbulence) and the chemistry of fuel combustion. The main objective of our project has been to address this issue by developing a predictive multidimensional combustion modeling capability for compression ignition $(\mathrm{Cl})$ engine applications. This effort involved developing high-fidelity large eddy simulation (LES) turbulence and turbulence-chemistry interaction (TCl) models; developing a detailed chemistry of combustion for various $\mathrm{Cl}$ engine fuels and additives with different reactivities together with global sensitivity analysis (GSA); and improving the scalability of submodels and the CONVERGE ${ }^{\text {TM }}$ tool together with performance optimization on the IBM Blue Gene/P and $Q$ supercomputers. Experimental data for validation was obtained from engine and chemical kinetics experiments, for the different fuels and fuel additives. The predictive ability of these robustly validated models will enable combustion engineers and/or scientists to better predict the combustion and emission characteristics of fuels with different reactivities.

\section{MISSION RELEVANCE}

This project is directly aligned with the DOE Vehicle Technologies Office's (VTO) mission of developing high-efficiency, low-emission vehicles. This project supports this mission by developing predictive simulation capabilities for different processes occurring in an ICE. These simulation capabilities are then incorporated in software tools such as CONVERGE that are used extensively by original equipment manufacturers (OEMs). The use of the CONVERGE tool in the current project facilitates the transfer of our model enhancements and development approach to OEMs such as Cummins Inc., Caterpillar Inc., Chrysler Inc., the General Motors Research and Development Center, and Ford Motor Company, etc., as these OEMs use the CONVERGE tool.

\section{RESULTS AND ACCOMPLISHMENTS}

The results of our work over the past three fiscal years are presented in the following categories: (1) LES for turbulent combustion modeling, (2) the scaling of CONVERGE code on Mira and associated developments that made this feasible, and (3) further insights into chemical kinetics of fuels.

$\square$ LES for turbulent combustion modeling: An n-dodecane spray flame (Spray A from the Engine Combustion Network) was simulated using a detailed combustion model along with a dynamic structure LES model (developed for this project in FYs 2013 and 2014) to evaluate its performance at engine-relevant conditions and to understand the transient behavior of this turbulent flame. The liquid spray was treated with a traditional Lagrangian method, and the gas-phase reaction was modeled using a detailed combustion model. The LES was observed to have multiple ignition spots in the mixing layer simultaneously, while the main ignition initiates in a clearly asymmetric fashion. The temporal flame development also indicates that the flame stabilization mechanism is auto-ignition controlled and modulated by flame propagation. Multiple realizations for LES were performed in FY 2015 to understand the realization-to-realization variation and to establish the best practices for ensemble-averaging diesel spray flames. The relevance index analysis suggests that an average of 5 and 6 realizations can reach $99 \%$ of similarity to the target average of 16 realizations on the mixture fraction and temperature fields, respectively.

$\square$ Scaling of CONVERGE code on Mira: In FY 2014, we identified and addressed several computational bottlenecks associated with scaling up a high-fidelity engine computational fluid dynamics (CFD) simulation onto thousands of cores of a supercomputer (Mira in this case). These bottlenecks affect parallel scaling efficiency, and in extreme cases, significantly slow down critical portions of the simulation. The bottlenecks that were identified were (1) input/output (I/O),

(2) communication, (3) load balancing, and (4) memory. Of these, the first three were addressed, and, subject to obtaining follow-on support, we will be working on memory enhancements. On the I/O front, with the use of buffering and parallel file writes, the restarting time was brought down from more than two hours to under three minutes. The write operations were sped up by two orders of magnitude, rendering the overhead associated with the write operation insignificant. Communication was improved by reordering data structures and implementing collective communication, which brought down communication overhead during initialization from more than an hour to a couple of seconds, on 4,096 cores. A new stiffness-based, load- 
balancing scheme was developed and implemented for chemical kinetics calculations, which resulted in speeding up the calculations near ignition by more than three times.

$\square$ The improvements to code scalability and performance on Mira also led to the award of a 60-million core-hour Advanced Scientific Computing Research (ASCR) Leadership Computing Challenge (ALCC) award from DOE's ASCR office. We intend to use this allocation to perform global sensitivity analysis (GSA) on engine CFD simulations by performing thousands of medium-fidelity simulations (each on 64 cores) concurrently.

$\square$ Further insights into chemical kinetics of fuels: During FY 2015, a new representation of chemical kinetics was developed which we termed the "Sum Over Histories Representation.” In this approach, the chemical observables are represented in terms of molecular-level chemical pathways that emanate from the reagent species. A pathway is a sequence of species connected by elementary reactions where the product of one reaction becomes the reactant for the next. The reactions are taken as random events that obey a time-ordering. If the important chemical paths are enumerated and the associated probabilities are computed, the value of any kinetic observable can be determined. The key to the Sum Over Histories Representation was the development of an efficient scheme to compute pathway probabilities. We applied the Sum Over Histories Representation to the problem of $\mathrm{H}_{2}$ combustion. As a "proof of concept" demonstration, it was shown that the method could yield accurate concentration versus time profiles using 10 or fewer chemical pathways.

\section{Atomic Layer Deposition System for Continuous, High-Speed Thin-Film Processing}

\section{3-154-R2}

\section{Joseph Libera, Jeffrey W. Elam, Anil Mane, and Angel Yanguas-Gil}

\section{PROJECT DESCRIPTION}

Atomic layer deposition (ALD) is a thin-film coating technology that provides atomic-level control over thickness and composition and superb conformality over complex surfaces. Because of these attributes, ALD has been successfully integrated into the high-volume manufacturing of microelectronics. Furthermore, research at Argonne has demonstrated that ALD holds tremendous promise in applications that are far removed from microelectronics and could revolutionize nanomanufacturing in a broad range of energy technologies, including photovoltaics, solid-state lighting, lithium batteries, and catalyst manufacturing. However, a major barrier to the utilization of ALD technology in these fields is the need to speed up the throughput by factors of 10 to 100 and to move from batch processing to continuous processing.

To overcome these barriers, we have developed a concept that enables continuous, high-speed ALD, is compatible with roll-to-roll (R2R) manufacturing, and runs at speeds of up to 100 times that of the existing processes. In this project, we will design and build a bench-scale ALD tool to demonstrate this novel ALD technology. We will use computational fluid dynamics simulations to guide our design. We will base our design on modifications of our existing equipment to conserve laboratory resources and expedite development. We will validate our design by depositing high-performance moisture barrier coatings and transparent conducting (TC) coatings on continuous rolls. Our work will focus on establishing the necessary proof-of-concept to make feasible future private-sector and government investments that will eventually carry this technology through to a production scale. The situation is analogous to the birth of the microelectronics industry when the excellent semiconducting properties of silicon were understood, but the manufacturing (lithography, polishing, and deposition) had not yet been developed. Similarly, today there is a deficit of useful commercial tools and manufacturing processes for ALD films that are broadly accepted as having substantial technological promise.

\section{MISSION RELEVANCE}

Clean energy (i.e., carbon-free energy) industries are facing a common technological hurdle-the need for a continuous high-rate process to deposit precise, thin-film coatings-a process that can be integrated into their existing manufacturing practices. For instance, high-performance moisture permeation barriers and robust TC layers could radically transform the manufacturing of photovoltaics, solid-state lighting, and electrochromic windows. Potential industries that will benefit from our technology include technical glass, solar glass, photovoltaics, transparent conducting oxides for flat panel displays, solid-state lighting, smart windows, lithium battery manufacturing, and catalyst manufacturing. The successful completion of this project will facilitate the future realization in the manufacturing domain of existing Argonne intellectual property (IP) in ALD technology and will also permit other processes to be successfully scaled and implemented in industry. 
This project addresses the overarching DOE mission of advancing our nation's energy security. More specifically, the wide-scale deployment of clean, renewable energy will require breakthroughs in manufacturing technology so that devices such as solar cells can be produced with low costs and in high volumes. A common need that cuts across a wide range of clean energy technologies including solar cells, batteries, and lighting is the requirement for thin-film coatings. This project will develop a continuous, high-speed coating process that will serve as a platform technology that is capable of being adapted to address all of these thin-film requirements within the DOE mission.

\section{RESULTS AND ACCOMPLISHMENTS}

The major accomplishments achieved in FY 2013 were:

$\square$ Modifications to our research ALD reactor to enable its high-speed operation.

$\square$ Proof-of-concept demonstration of our high-speed technology.

$\square$ First trials of high-speed deposition.

$\square$ Modeling of our high-speed ALD using computational fluid dynamics.

The major accomplishments achieved in FY 2014 were:

$\square$ A high-speed virtual-valve concept was developed to overcome the limitation of reducing the purge times to very small values which was causing nozzle tip chemical vapor deposition (CVD). The high-speed virtual-valve concept was approximated using valves that were tested in this way.

$\square$ Reactant cross talk was eliminated by the high-speed virtual-valve arrangement. This was first tried with a single injector and then with a dual injector configuration. Successful depositions yielding an 8 in. wide deposition zone at $2 \mathrm{Hertz}(\mathrm{Hz})$ were demonstrated that could be applied to a R2R production machine.

$\square$ We discovered that local heating during static substrate tests results in temperature feedback to the ALD deposition rates. This results in nonuniformity when attempting to high cycle rates. Better performance is expected when a moving substrate is implemented so that the buildup of heat is avoided.

$\square$ The computational fluid dynamics (CFD) modelling was advanced to the point of creating a close prediction of ALD deposition patterns. The modelling takes into account all physical phenomena including mass transfer, reactive sticking coefficients, temporal-spatial tracking reactant concentrations, and product concentrations.
The major accomplishments achieved in FY 2015 were:

$\square$ The high-speed virtual-valve concept was tested to the limits of the technology assembled in this project for the task-namely, the maximum rate at which valves will produce a response by using the Fujikin valve driver. These tests indicate that ALD rates in the range of $20-50 \mathrm{~Hz}$ are achievable by pushing the current state-of-the-art production to the practical limits of readily available components. However, practical implementation as a production technique will require further development of a pulse-sequencing device that does not use conventional valve equipment, since the lifetimes of these components would only allow for a few days of use. At $20 \mathrm{~Hz}$, one million cycles would take just two working days of production operation to complete.

$\square$ The high-speed ALD equipment was tested on the trimethyl aluminum/ozone and diethyl zinc/water systems. In the trimethyl aluminum/ozone system, the rates were limited to several hertz due to the low concentration of ozone in the normal ozone production equipment. Thus, the minimum dose time required to deliver sufficient chemical to the growth zone far exceeds the high-speed valve switching rate. The high-speed ALD concept is therefore limited to systems whose precursors are available in the pure form, with a minimum vapor pressure of about 0.1 torr.

$\square$ Using the diethyl zinc/water system, an ALD deposition rate of $20 \mathrm{~Hz}$ was demonstrated. The test film of zinc oxide ( $\mathrm{ZnO})$ was $92 \mathrm{~nm}$ thick and was deposited in 25 seconds. The same operating parameters for the trimethyl aluminum/water system did not produce a good result due to the limited carrier gas velocity of our existing ALD deposition systems.

$\square$ In summary, regarding the high-speed R2R effort, we believe that a practical high-speed production unit with deposition rates of $20-50 \mathrm{~Hz}$ could be implemented with the following restrictions: (a) a larger pumping system, (b) new technology for long-term pulse creation (billions of cycles) using non-valve pulsing technology, and (c) ALD system precursors with relatively high vapor pressure.

$\square$ We also completed a high-volume ALD powder-coating reactor (PCR). This powder-coating reactor will facilitate a wide range of nanomanufacturing applications, including electrode materials for batteries in transportation, heterogeneous catalysts for the chemical industry, and "green" processing in microelectronics. With the PCR completed, we were able to establish industry-sponsored research contracts with a number of companies that require ALD coating of $\sim$ kilogram quantities of powders. 
This work resulted in the following inventions/patents:

Libera, J. and J. Elam. "Atomic Layer Deposition System for Continuous, High-Speed Thin-Film Processing." Invention report disclosed August 2012. [ANL-IN-12-078]

Yanguas-Gil, A., J. Libera, and J. Elam. "Method for Continuous ALD.” Patent Application No. 14/339,058 filed July 2014. [ANL-IN-13-042]

Libera, J., J. Elam, A. Mane, and A. Yanguas-Gil. "Chemical Vapor Processing for Additive Manufacturing." Invention report disclosed October 2014. [ANL-IN-14-074]

Libera, J. and J. Elam. "A Batch Reactor for Industrial Scale Atomic Layer Deposition onto High Surface Area Powder Substrates." Invention report disclosed January 2015. [ANL-IN-15-001]

Libera, J., J. Elam, A. Mane, and A. Yanguas-Gil. "Refractory Solar Selective Coatings." Invention report disclosed March 2015. [ANL-IN-15-016]

Elam, J., A. Mane, and T. Seidel. "Silicon Doping Source Films by ALD Deposition." Invention report disclosed August 2015. [ANL-IN-15-087]

\section{Fast High-Efficiency Process to Fabricate Aligned Nanotubes in Nano-Composite Membranes for High-Performance Filtration Applications}

\section{3-156-R2}

\section{Patricia Ignacio-de Leon, Xing Chen, and Meltem Urgun-Demirtas}

\section{PROJECT DESCRIPTION}

This project aims to develop and demonstrate a new and fast high-efficiency process for producing high-performance molecular transport layers on microporous supports to fabricate membrane nanofilters that will yield significant energy savings and have wide applicability to separation processes. Our goal is to eliminate the substantial performance gap between laboratory-scale and commercial membranes by developing processes that employ controllable force (e.g., magnetic fields) to manipulate responsive material phases or components to affect and/or direct nanostructure assembly. This technology would become the basis of a novel, high-throughput manufacturing process for producing nanostructured composite membranes useful for applications such as water purification and desalination, nanofiltration, gas separation, and combined catalytic transformation and separation of products from reactants.

In FY 2015, this project demonstrated (a) an optimized and reproducible method for preparing narrow-web filtration membranes that include a dynamic process to embed perpendicularly oriented nanotubes into the permselective layer via magnetic alignment and (b) successful filtration performance of our narrow-web membranes in terms of two-key membrane figures of merit, flux and percent rejection.

\section{MISSION RELEVANCE}

This project is related to two DOE mission areas: energy security and environmental quality. The benefits of the project include better membrane performance (greater permeation rate: equivalent or better rejection performance toward salts, pathogens, and pharmaceuticals); lower operating pressures; and reduced energy consumption, operation cost, and waste. Current reverse osmosis (RO) technology provides potable water by desalinating three main sources of input water: brackish inland waters, municipal wastewater, and seawater. Combined worldwide desalination provides 13 billion gallons of potable water daily, yet meets less than $1 \%$ of the world's demand for water. The global market for RO membrane elements is forecast at $\$ 1.4$ billion by 2015 . However, given the improved economics offered by the proposed nanofiltration technology (30-50\% less energy use, up to 10 times higher flow, 90\% less membrane area needed, one-third cost reduction), the water purification market is expected to expand beyond projections.

Commercial membrane filtration original equipment manufacturers (OEMs) that license the technology would realize near-term benefits. In the medium term, engineering, procurement, and construction companies and engineering consulting service providers that deploy this technology in their systems would benefit from this technology advance. In the long term, two-thirds of the world's population would have greater access to water for drinking, industry, and agriculture. Mitigating the scarcity of safe, potable water by wastewater treatment via an energy- and cost-efficient manufacturing system allows both an energy savings and a decreased impact on the environment. 


\section{RESULTS AND ACCOMPLISHMENTS}

In FY 2013, a scalable, robust process to produce aligned $\mathrm{Fe}_{2} \mathrm{Co}$ nanofibers was identified by performing a series of two-level Plackett-Burman design experiments to evaluate the significant nanotube processing parameters. The process employs vertically-configured electrospinning electrodes under field-mediated deposition conditions. The fibers were then thermally treated to initiate nitrate salt oxidation and remove the polymer component in the as-spun fibers (about 20-nanometers [nm] in diameter). A second thermal treatment under reducing conditions converts the metal oxide (hydrophilic) to an $\mathrm{Fe}_{2} \mathrm{Co}$ (hydrophobic) magnetic nanotube. $\mathrm{Fe}_{2} \mathrm{Co}$ has a high saturation magnetization and is relatively inert to oxidation and was explored throughout FY 2013. Magnetic alignment of $\mathrm{Fe}_{2} \mathrm{Co}$ nanorods has also been demonstrated.

In FY 2014, the process described above to fabricate nanotubes of controllable diameter and surface chemistry was demonstrated. The electrospinning system was scaled up, increasing the production rate from $0.01 \mathrm{mg} /$ day to $25 \mathrm{mg} /$ day (a 2,500-fold increase). This new system is an automated, continuous process that integrates nanofiber production and the first thermal treatment. Samples from the new configuration evidently formed nanotubes after heat treatment. Nanotubes of different diameters were also produced by changing the variables associated with the electrospinning process, such as the composition of the precursor solution containing the iron ( $\mathrm{Fe}$ ) and cobalt (Co) salts. We can create nanotubes of various lengths (less than 1 to about 7 microns $[\mu \mathrm{m}]$ ) via sonication in a quick-drying solvent (such as ethanol) and by adjusting the power level (20 to 100 watts [W] at 30 kilohertz [kHz]) and duration (10 seconds to 30 minutes) of sonication.

Also in FY 2014, we began to develop a dynamic process to magnetically align nanotubes perpendicular to the permselective layer of a narrow-web nanofilter. Nanotubes were incorporated via encapsulation in a thin polymer onto a microporous support such as polyethersulfone (PES) or regenerated cellulose. The permselective layer consists of nanotubes that function as pores and/or nanochannels, embedded in a nonporous polyamide resulting from interfacial polymerization between a nonpolar phase (trimesoyl chloride dissolved in hexane) and a polar aqueous phase containing m-phenyldiamine. Magnetic nanotube (MNT) orientation occurs during the interfacial polymerization upon application of an external magnetic field with lines perpendicular to the support. By optimizing the monomer concentrations, we achieved continuous, uniformly thin coverage of the polymer over the entire support (Figure 1).
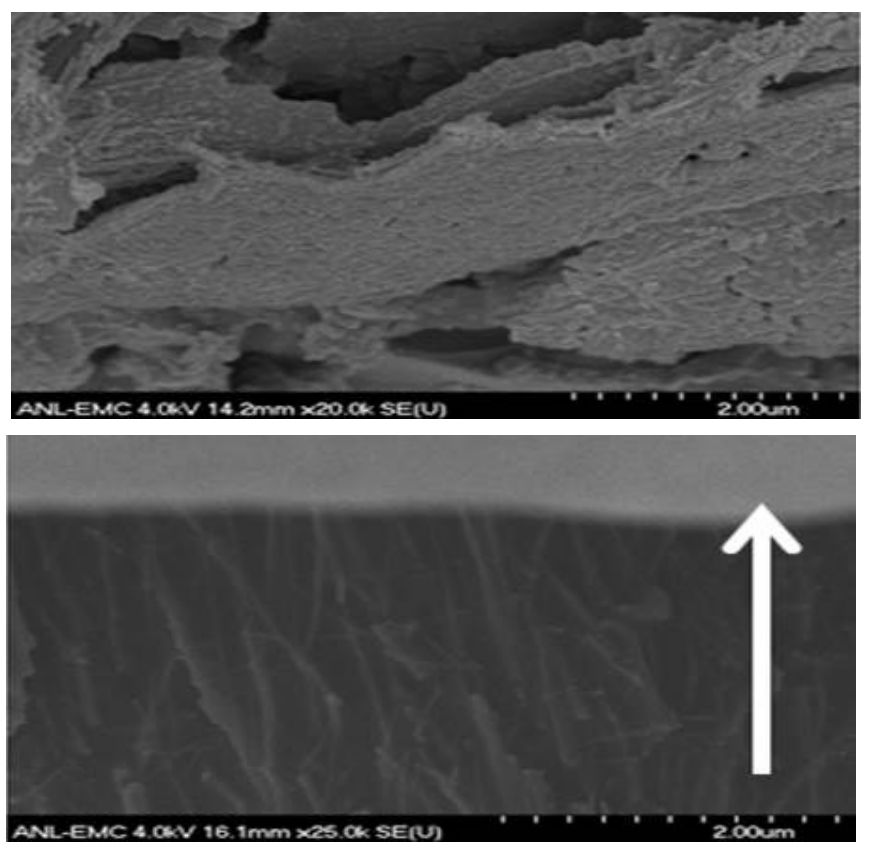

Figure 1. Scanning electron microscope (SEM) images of cross sections of nanocomposites show (top) plates of randomly oriented polyamide-encapsulated MNTs in the absence of an external magnetic field; (bottom) vertically-aligned MNTs with long axes parallel to applied magnetic field lines (white) (scale bars $2 \mu \mathrm{m}$ ).

Optimized and Reproducible Method for Preparation of Narrow-Web Magnetic Nanotube Membrane Composites. In FY 2015, we further optimized the method that we began to develop the previous year for nanotube incorporation into the membranes via interfacial polymerization. To ensure that the nanotube ends remain unblocked to enable the flow of the feed solution through the nanotubes, nanocomposite membranes were treated post-synthesis with a dilute solution of Protease $\mathrm{E}$, an enzyme that specifically cleaves amide bonds via catalyzed hydrolysis. To optimize conditions for etching and better control of the polyamide thickness, the polyamide and/or support composites were subjected to varying exposure times and concentrations of the enzyme (Figure 2). 

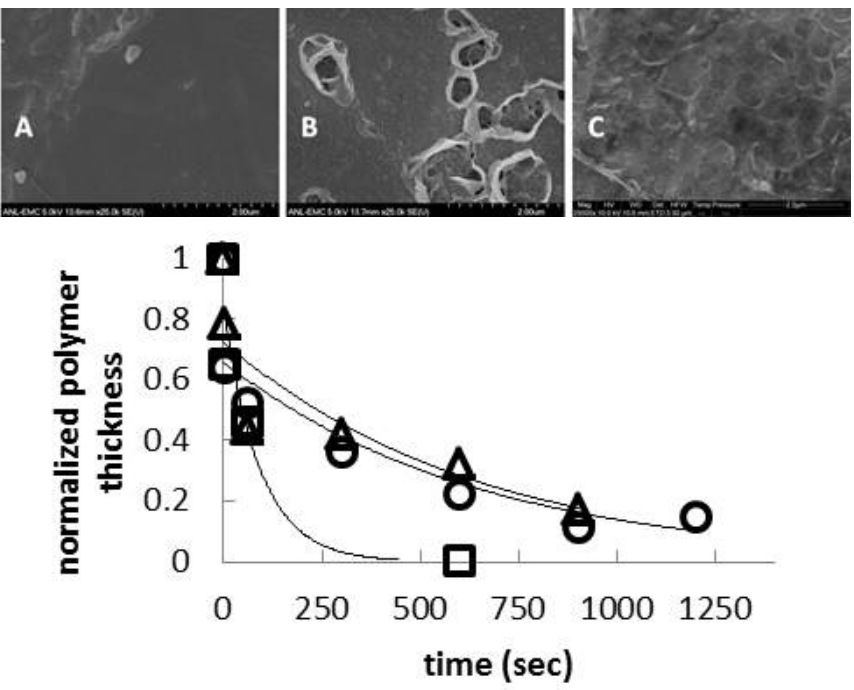

Figure 2. (Top) Top view SEM images for polyamide/support composites (A) before enzyme treatment and after immersion for 1 min in 500 micromolar (uM) Protease E, (B) without shaking, and (C) with use of shaker plate (scale bar 2 um). (Bottom) Polyamide etching activity of Protease $E$ as a function of concentration and reaction time $(\square 500 \mu \mathrm{M}, \circ 25 \mu \mathrm{M}, \Delta 1 \mu \mathrm{M})$.

Successful Demonstration of Filtration Performance of Magnetic Nanotube Composite Membranes. To determine the efficacy of the addition of MNT and its orientation on membrane performance, pure water flux was measured through various membrane samples containing MNTs prepared in the presence and absence of an external magnetic field, as well as membranes without MNTs (bare support and with the polyamide alone). Figure 3 shows a trend in pure water flux according to expectations that an extra layer will act as an additional barrier to mass transfer. Similar patterns in flux were observed for feed solutions containing 1000 and 10,000 parts per million (ppm) lignin. To measure the extent to which our membrane samples can retain contaminants in feed solutions, we used the prepared lignin solutions. Lignin with an average molecular weight of 10 kilodaltons $(\mathrm{kDa})$ was chosen as a model compound suitable for our demonstration. Lignin is a polyphenol and represents a main class of structural materials found in plants and some algae; it is therefore a typical contaminant in process water produced from lignocellulosic biofuel production processes and wastewaters produced from paper and mill industries. The lignin percentage rejection from a $10,000 \mathrm{ppm}$ feed at 40 pounds/square-inch (psi) pressure through a PES support increases from $7.3 \%$ to $39.1 \%$ (5.3 times higher) and 62.8\% (8.6 times higher) through membranes prepared with and without a magnetic field, respectively.

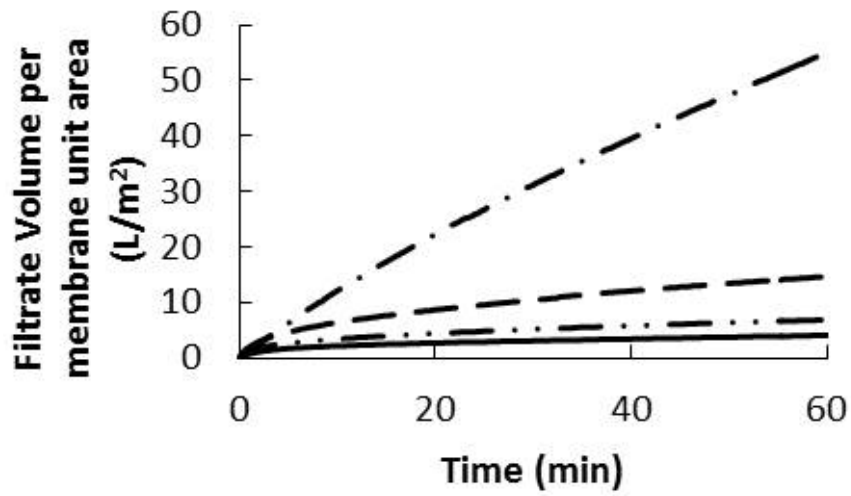

Figure 3. Pure water flux through various membranes (- - PES support, - - MNTs in magnetic field, - - - MNTs without magnetic field, polyamide only).

\section{Grid-Level Energy Storage for Integration of Renewable Energy}

\section{4-120-R1}

\section{Audun Botterud, Julie Bessac, Emil M. Constantinescu, Fernando de Sisternes, Kevin Gallagher, Prakash Thimmapuram, and Canan Uckun}

\section{PROJECT DESCRIPTION}

Energy storage is widely considered as a key solution for the large-scale expansion of renewable energy. However, most energy storage technologies are expensive and do not scale up to the magnitudes required for grid-level storage. To better understand the role of energy storage in the power grid, we are conducting a detailed analysis of the possible applications of such technologies in power systems dominated by variable renewable resources. Toward this end, we are developing advanced methods for storage and grid planning and operations considering the uncertainty and variability of wind and solar power. This comprehensive modeling framework enables us to analyze the type of energy storage, the amount, and where it should be placed in the grid to enable high levels of renewable resources. We are also investigating the economics of several storage technologies and the extent to which investor incentives are aligned with the benefits provided to the power system.

\section{MISSION RELEVANCE}

The comprehensive analytical framework and advanced methods developed in this project directly support DOE's Grid Energy Storage Plan, as well as its mission to ensure America's security and prosperity by addressing its energy, environmental, and nuclear challenges through 
transformative science and technology solutions. DOE's Offices of Electricity Delivery and Energy Reliability (OE) and Energy Efficiency and Renewable Energy (EERE) are both very interested in energy storage for the power grid and have relevant research programs. Moreover, energy storage is receiving increasing levels of attention from the electric power industry; our work is also of interest to developers of batteries and other energy storage technologies.

\section{RESULTS AND ACCOMPLISHMENTS}

Building on and extending the analytical framework established in the first year of the project, we made advances along several research paths.

We developed a new strategy for generating realistic wind speed scenarios that are used in the characterization of wind speed resources. This was accomplished through a statistical space-time model for predicting atmospheric wind speed based on deterministic numerical weather predictions and historical measurements. We considered a Gaussian multivariate space-time framework that combines multiple sources of past physical model outputs and measurements along with model predictions in order to produce a probabilistic wind speed forecast within the prediction window. Test results for a region near the Great Lakes (Figure 1) show that the prediction is improved in the mean-square sense relative to the numerical forecasts as well as in probabilistic scores. Moreover, the samples are shown to produce realistic wind scenarios based on the sample spectrum.

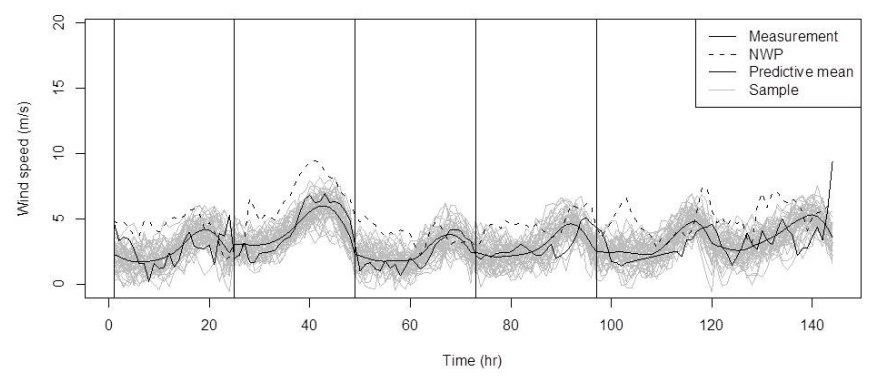

Figure 1. Time series of forecasted and measured wind speed at one weather station in Illinois for six days during August 2012.

We also investigated the value of storage as a system asset providing energy and operating reserves in a power system with renewable energy. We developed a novel stochastic programming approach for day-ahead scheduling along with a new operational strategy for batteries in real-time dispatch. In a case study of a smallscale power system with high wind penetration levels, we found that the proposed operational strategy provides better results than multiple benchmarks, and we also found significant benefits for battery storage.
For long-term planning of electric power systems with stringent carbon emissions limits, we analyzed the value of energy storage considering detailed operational constraints. We found that energy storage can substantially reduce generation costs by increasing utilization of generation assets and enabling greater penetration of the lowest-cost carbon-free resources. Moreover, the value of longer-duration energy storage, such as pumped storage hydro, appears sufficient to justify deployment at current costs; however, opportunities are geographically limited. Conversely, the value of shorter-duration storage technologies, such as Li-ion batteries, is only justified under the most stringent carbon emissions limits (Figure 2). Hence, continued innovation and cost reductions for battery technologies will be necessary for the economic justification of largescale deployment.

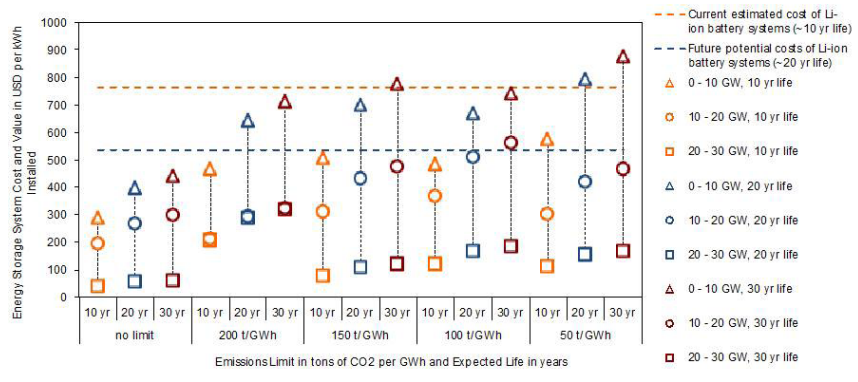

Figure 2. Estimated grid benefit of 2-hour energy storage for various $\mathrm{CO}_{2}$ emissions limits compared to current and future battery costs (Li-ion), based on projected wind, solar, and load for Texas in 2035.

Considering the storage owner's perspective, we studied the energy arbitrage maximization problem considering uncertainty in market prices and developed a novel approach for bidding into the day-ahead and real-time electricity markets using stochastic programming. The proposed model helps storage owners in market bidding and operational decisions for batteries. We validated the stochastic bidding model in several case studies. According to the results, the proposed bidding approach performs significantly better on average than deterministic benchmarks.

Grid optimization models like the ones discussed above have an unmet need for a simple self-consistent and physics-based battery representation that can be applied to any chemistry. To respond to this need, we are developing a generic framework to capture the physics that controls electrochemical energy storage with straightforward equations that may be rendered piecewise linear for implementation in grid models. This approach enables the use of a single model to represent the primary behavior of many different battery chemistries by the specification of only a minimum number of technology-specific parameters (i.e., less than 10). 
The storage representation developed in this task will improve power grid models in which the current battery representation is taken to the Oth order, risking unphysical performance behavior.

\section{PROPOSED FUTURE WORK}

We will complete the comprehensive analytical framework, focusing on improved representation of battery electrochemistry and degradation effects in grid optimization models and how energy storage can benefit from improved spatiotemporal forecasts of renewables.

\section{Magneto-Dielectric Composite Substrates Comprised of High-Aspect- Ratio Magnetic Nanofibers for Smart Antennas Operating at Microwave Frequency}

\section{4-169-R1 \\ Xing Chen and Ziyao Zhou}

\section{PROJECT DESCRIPTION}

We hypothesize that electrical permittivity $(\varepsilon)$ and magnetic permeability $(\mu)$ may be tailored in an advanced composite material structure. To implement magnetodielectric materials with desired $\mu$ and $\varepsilon$ values, highperformance magnets with high $\mu$ and low loss at gigahertz frequencies are needed. High-aspect-ratio nanostructured magnetic materials present a significant opportunity to improve the magnetic properties, particularly at ferromagnetic resonance (FMR) frequencies in the gigahertz range (i.e., microwave frequencies). Very small magnetic particles coated with an insulator have been demonstrated to control eddy current loss. The demagnetizing effect of a finite-sized magnetic particle also affects its apparent permeability. Highaspect-ratio shapes (long rods and thin films) can achieve full permeability in their magnetic easy-axis direction. Compared with other systems, nanofibers have fundamental advantages, such as higher shape anisotropy, lower eddy current loss, and lower stress/ defects in magnetic crystals.

The objective of this project is to demonstrate high and tunable $\varepsilon$ and $\mu$ in magneto-dielectric composites at microwave frequencies and demonstrate the fabrication and scalability of the materials in a microwave patch antenna. We are fabricating magneto-dielectric nanocomposites on flexible substrates, comprised of ferromagnetic or ferrimagnetic nanofibers with diameters between 20 and 30 nanometers ( $\mathrm{nm}$ ) embedded in dielectric polymer matrices. Nanoparticles of high $\varepsilon$ materials are dispersed in the matrix to tailor the system permittivity using known polymer processing methods. Electrospinning and heat-treatment are used to produce the metallic and ferrite nanofibers.

\section{MISSION RELEVANCE}

This project is relevant to key DOE missions in national security and energy. The technology being developed in this program has the potential to create much more energy-efficient and secure antenna technology for wireless communication, as well as wireless power transmission. It is also relevant to the U.S. Department of Defense's mission in microwave communications, as well as industries in the same technological area.

\section{RESULTS AND ACCOMPLISHMENTS}

In FY 2014, we fabricated magnetic nanofibers with diameters less than $30 \mathrm{~nm}$ and quantified the magnetic properties as a function of fiber diameter. Electrospinning produced metal-salt-containing polymer fibers, which were heat-treated to produce metal oxide fibers and then reduced to metal nanofibers. x-ray diffraction, electron microscopy, and direct current (DC) magnetic measurement were used to characterize their morphology and magnetic properties.

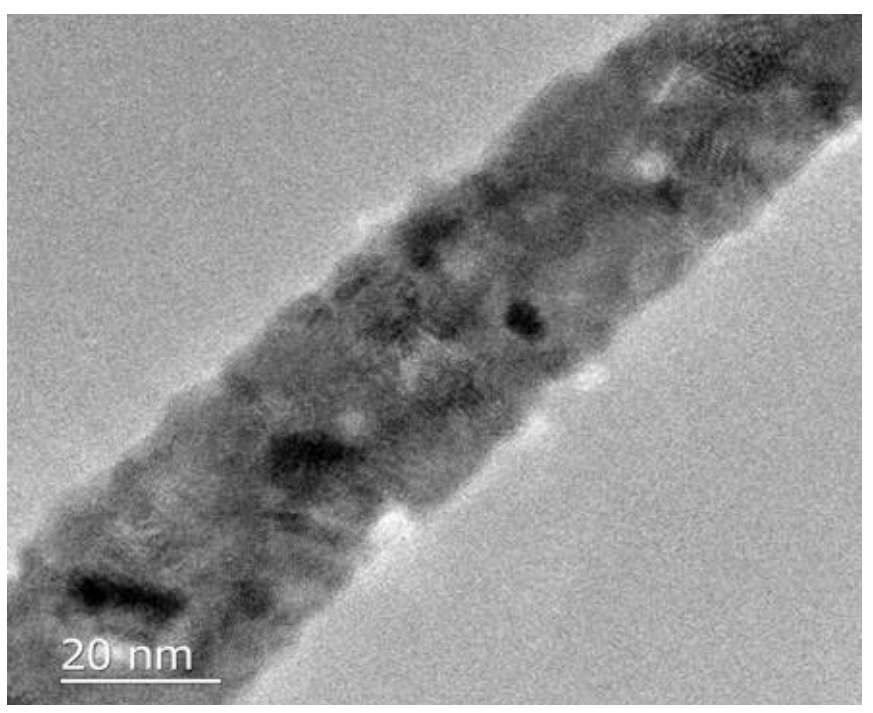

Figure 1. Nickel-ferrite $\left(\mathrm{NiFe}_{2} \mathrm{O}_{4}\right)$ nanofibers prepared by the new integrated electrospinning system. The different contrast spots within the fiber shows the polycrystalline nature of the nanofiber.

In FY 2015, a scalable and robust process of fabricating magnetic nanowires was established. Figure 1 shows a transmission electron microscopy image of a nickelferrite oxide nanofiber prepared in our new integrated system. The fibers were consolidated to test the magnetic 
properties at DC and microwave frequencies. The microwave property results guided our enhancement of the fiber morphology. Initial trials were also made to embed magnetic nanofibers and dielectric nanoparticles in polymer matrices.

So far, this work has generated five inventions, with three patent applications filed and two authorized for filing. The project has established a significant and unique capability at Argonne in magnetic materials for microwave applications and has attracted significant interest from industry and government agencies.

\section{PROPOSED FUTURE WORK}

In FY 2016, we will test magnetic permeability and dielectric permittivity in the microwave regime using similar techniques as in FY 2015. Nanofiber production will be scaled up, and an antenna will be fabricated to demonstrate using a DC magnetic field to bias the antenna substrate for active beam steering. We plan to further improve material properties of our samples. New printing technology will be explored for better performance and integration with semiconductor manufacturing. Tunability through magnetic-electric coupling will also be explored.

\section{A Novel Reactor for the Continuous Manufacturing of Metal Oxide Particles}

\section{5-132-NO \\ Gregory Krumdick, Ozgenur Kahvecioglu Feridun, and Youngho Shin}

\section{PROJECT DESCRIPTION}

This project aims to develop and implement an advanced mixing reactor for the continuous manufacturing of highly crystalline metal oxide particles. A novel reactor using periodic Taylor Vortex Flow motion, which enables an intense micro-mixing zone, was established to overcome the difficulties (flow pattern due to design, insufficient mixing, long reaction time, broad particle size distribution, complex morphology of the particles) associated with conventional tank reactors used by industry. Because of the simple and flawless design of the Taylor Vortex Reactor (TVR), the elimination of dead zones contributed to the improvement of purity, morphology, and degree of crystallinity of the particles. The particles manufactured in the TVR were compared with the conventionally made particles produced by a continuous stirred tank reactor (CSTR) and a batch tank reactor.

\section{MISSION RELEVANCE}

The project relates to DOE's energy security mission by enabling the United States to produce next-generation battery materials, rather than relying on manufacturers in Asia to provide the materials. Beneficiaries of materials synthesized by this process include a wide range of industries, such as manufacturers of catalysts, battery materials, metal compounds (e.g., oxides, carbonates, hydroxides), polymers, pigments, electronics, coatings, and filtration and biological systems. In addition, several DOE programs, including the Office of Energy Efficiency \& Renewable Energy (EERE) Vehicle Technologies and Advanced Manufacturing program, would benefit from the success of this project. Establishment of this capability would enable advances in materials synthesis and production in a number of strategic areas, including energy storage, transportation, and transformational manufacturing. Successful production of concentration gradient (core-shell) materials would immediately benefit a wide number of industries looking for higher-capacity, faster-charging batteries for use in, for example, electric vehicles, consumer electronics or grid applications.

\section{RESULTS AND ACCOMPLISHMENTS}

The TVR was established at Argonne's Materials Engineering Research Facility on March 25th, 2015. We used three co-precipitation reactors-batch reactor, CSTR, and TVR-under the same experimental conditions to prepare the quaternary intermetallic oxide $\mathrm{Li}_{1.067} \mathrm{Ni}_{0.61} \mathrm{Mn}_{0.33} \mathrm{CO}_{0.06} \mathrm{O}_{\mathrm{y}}$ cathode materials for lithium ion batteries. In the batch reactor, the particles' tap density was increased from $1.25 \mathrm{~g} / \mathrm{cc}$ to $1.73 \mathrm{~g} / \mathrm{cc}$ after two months of initial optimization studies. The CSTR, after 6 months of optimization, gave a tap density of $2.06 \mathrm{~g} / \mathrm{cc}$. The TVR, on the other hand, was not optimized but gave a value of $2.04 \mathrm{~g} / \mathrm{cc}$ at its preliminary run. These results can be elucidated by examining the bulk and cross-sectional morphology, as shown in Figure 1. TVR-made particles, with less internal porosity and a well-defined spherical morphology, show homogeneous grain size distribution internally and on the surface as well, while CSTR-made particles have heterogeneous grain sizes decreasing from the center of the particle to its surface, creating internal porosity. This characteristic is more obvious in batch-made particles, where a higher degree of porosity with a distorted sphericity could be easily seen. 


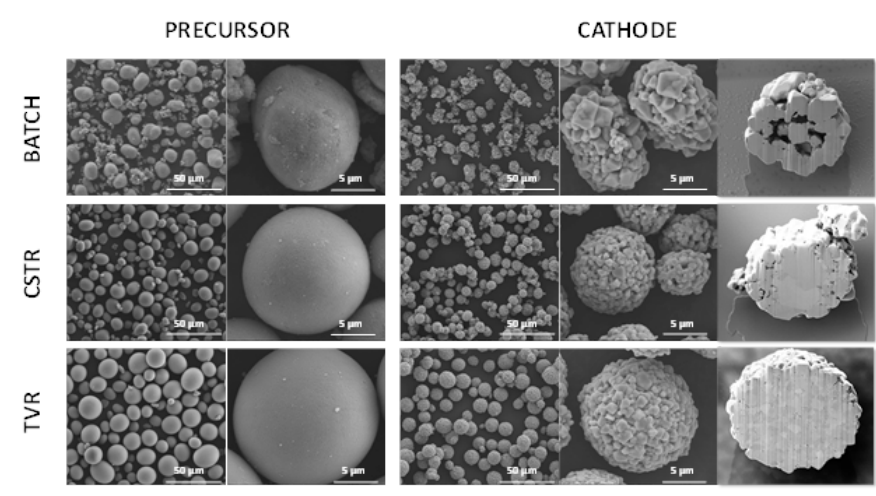

Figure 1. The emerging Taylor Vortex Reactor (TVR) technology has been shown to improve the sphericity of the precursors, reduce the porosity of the cathode metal oxide particles, and result in cost and time savings.

There is interest from several companies as well as DOE's Office of Energy Efficiency \& Renewable Energy in supporting future development work using this reactor.

\section{Chemical Vapor Processing for Additive Manufacturing}

\author{
2015-151-NO \\ Jeffrey W. Elam, Seth B. Darling, and Randall Winans
}

\section{PROJECT DESCRIPTION}

We are in the midst of what is arguably the most significant transformation in manufacturing since the industrial revolution: the advent and proliferation of additive manufacturing. Additive manufacturing, also called three-dimensional (3D) printing, creates solid 3D objects from digital models by depositing a series of thin, patterned layers. Since the 1980s, 3D printing has been used primarily for rapid prototyping, but recent advances in printer size, speed, and printable materials are pushing this technology toward manufacturing in a range of industries, including defense, aerospace, automotive, construction, and medicine. A variety of 3D printing technologies have emerged, but all have limitations. For instance, the most common 3D printing medium is organic polymers, which are cheap, light, and versatile. But for applications such as engine parts, electronic components, or medical implants, polymers typically lack the necessary robustness, electrical properties, and chemical compatibility.

To address these problems, we propose to develop chemical vapor processing for additive manufacturing (CVPAM, Figure 1). In this technique, the desired part is first 3D-printed and then post-processed by treating with chemically reactive vapors to (1) deposit a coating, (2) infuse the bulk of the part to enhance the material properties, or (3) densify the part. This project will develop CVPAM to the point of attracting industrial interest. The first task will be to perform a targeted survey of 3D printing media and chemical infiltration methods. This survey will allow us to evaluate the suitability of CVPAM for surface coating, infiltration, and densification of 3D-printed parts. Next, we will down-select the most promising processes for development and refinement and evaluate coating equipment, chemistry, and methodology. Finally, we will pursue applications for CVPAM technology. We will work with industrial partners to identify opportunities where CVPAM will provide the greatest benefit and value.

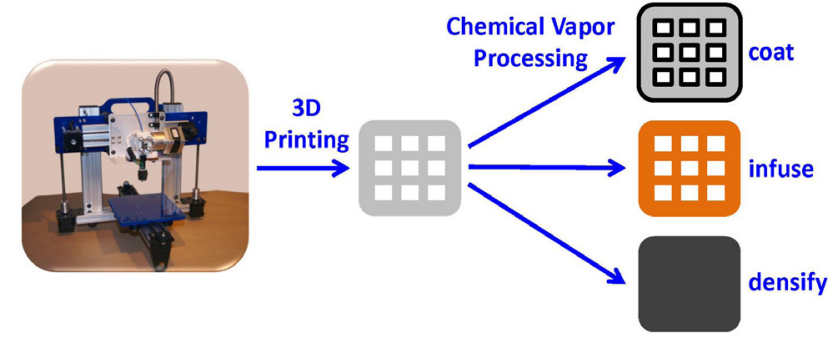

Figure 1. Chemical Vapor Processing for Additive Manufacturing (CVPAM): After 3D printing, a part is subjected to chemically reactive vapors to (1) coat all exposed surfaces (e.g., for biocompatibility), (2) infuse the part with inorganic material (e.g., for added stiffness or conductivity), or (3) densify the part (e.g., to fill voids in a sintered metal).

\section{MISSION RELEVANCE}

This project supports the DOE mission of energy security. Additive manufacturing has great potential to reduce energy intensity by eliminating production steps, enabling re-use of byproducts, and producing lighter products. In addition, remanufacturing of parts through additive manufacturing and surface treatment can reduce energy consumption by up to $25 \%$ compared to manufacturing new parts. Industrial beneficiaries will include 3D printer suppliers that can incorporate the CVPAM technology into their products, industrial coating equipment manufacturers, and chemical suppliers. Industries such as aerospace, automotive, and medicine will also benefit through the numerous advantages of additive manufacturing, including shorter lead times, easy customization, and reduced materials usage.

\section{RESULTS AND ACCOMPLISHMENTS}

In this project, we have engaged local suppliers of 3D printers for production to discuss their needs and the relevance of our technology to their industry. We acquired and installed a development-scale 3D printer capable of manufacturing parts in a wide range of polymers and with a build volume of $11 \times 8 \times 12$ inches. We printed a variety of test structures in acrylonitrile butadiene styrene 
plastic; coated them with thin films of zinc oxide, aluminum oxide, and tungsten via atomic layer deposition (ALD); and subsequently examined the parts using scanning electron microscopy and four-point-probe conductivity measurements. To probe the infusion of polymers by ALD precursors, we performed in-situ grazing-incidence small-angle $x$-ray scattering measurements of trimethyl aluminum and water reacting with polystyrene-block-polymethyl methacrylate (PS-b-PMMA) block copolymer lamellae. These measurements revealed, for the first time, structural changes during the densification of the PMMA portion, and "breathing" of the structure upon precursor infiltration and desorption.

This work resulted in the following inventions:

Elam, J., A. Mane, A. Yanguas-Gil, and J. Libera. "Chemical Vapor Processing for Additive Manufacturing." Invention report disclosed October 2014. [ANL-IN-14-074]

Elam, J., A. Mane, J. Libera, and A. Yanguas-Gil. "Refractory Solar Selective Coatings." Invention report disclosed March 2015. [ANL-IN-15-016]

Elam, J., and A. Mane. "Atomic Layer Writer (ATW)." Invention report disclosed April 2015. [ANL-IN-15-031]

\section{PROPOSED FUTURE WORK}

Our expected milestones for the coming years are as follows:

$\square$ Year 2: Down-select the most promising processes for development and refinement. Evaluate coating equipment, chemistry, and methodology.

$\square$ Year 3: Pursue applications for CVPAM technology. Work with industrial partners to identify opportunities where CVPAM will provide the greatest benefit and value.

This project will no longer pursue work that will continue under follow-on funding provided by the DOE EERE SunShot Program for the "Refractory Solar Selective Coatings" activity, which will explore the suitability of 3D printed parts as templates for selective solar-absorbing coatings in concentrated solar power applications.

\section{Sustainable Transportation: Novel Bio-Derived Fuel Additives for Improved Vehicle Efficiency}

\section{5-157-NO}

\section{S. Scott Goldsborough, Xing Chen, Stephen Ciatti, Phillip Laible, Raghu Sivaramakrishnan, and Robert Tranter}

\section{PROJECT DESCRIPTION}

The objective of this project is to discover/develop novel, bio-derived fuel additives that can be used at small concentrations (e.g., $\sim 1 \%$ by volume [v/v]) to tailor the chemical kinetic pathways controlling autoignition of petroleum-based fuels. Such additives could be employed in internal combustion (IC) engines to help meet evolving performance targets and legislated mandates, significantly increasing overall vehicle efficiencies, while at the same time augmenting the use of biomass feedstocks for, and reducing lifecycle greenhouse gas emissions of the transportation sector. Though the replacement of IC engine-based powertrains is envisioned in the long term, this work will facilitate near-term gains.

Advanced IC engine designs are expected to employ a range of robust combustion systems, including downsized, boosted spark-ignition (SI) approaches, as well as low-temperature combustion (LTC) schemes. However, fuel-engine interactions can considerably constrain potential advancements. SI configurations are knock limited, while LTC schemes, which modulate the extent of mixing and fuel reactivity in order to mitigate pollutant formation, are limited by the difficulty in controlling ignition timing and rates of heat release over the entire engine operating map.

Fuel additives such as tetraethyl lead and ethylhexyl nitrate have been used historically to control fuel reactivity, as either octane boosters or cetane improvers. However, toxicity, air/water pollution issues, component incompatibilities, and the inability to properly tailor combustion in novel engine concepts prevent the use of such chemicals. Bio-derived fuel additives, if appropriately designed, could be effective alternatives. The required production scale for such high-value bio-products is more relevant to projected biofuel capacities, while there is greater flexibility in fuel "purity" since the additives will not be required to be blended at large quantities (e.g., $10-30 \%$ v/v).

This project integrates fundamental and basic research as well as applied efforts. Emphasis is placed on additive production scenarios where negative-value feedstocks 
(e.g., cellulosic and biosolids waste streams) can be converted into chemical precursors which can be separated and upconverted into fuel additives. Production approaches are targeted whereby the additives can be synthesized renewably and economically at scale.

Major tasks include (1) utilizing a heuristics-based approach to identify and screen molecules/classes of molecules that can boost octane or improve cetane, and evaluating the autoignition behavior of various additive/fuel blends via rapid compression machine (RCM) tests; (2) characterizing the decomposition kinetics of the targeted molecules using shock-tube experiments, ab-initio theory, and modeling, and investigating additive-fuel interactions using chemical kinetic modeling; (3) biosynthesizing and separating bench-scale levels of select targets; and (4) scaling up sustainable, carbon-neutral biosynthesis efforts and chemical upgrading processes to produce sufficient volumes. Full-scale engine demonstration will depend on the project time frame and budget.

\section{MISSION RELEVANCE}

This project directly addresses urgent national needs to improve the sustainability of the U.S. transportation system via improved IC engine/vehicle efficiency and the utilization of sustainably produced bio-derived components. Significant fuel economy gains are projected using bio-derived fuel additives compatible with combustion technologies that are expected to dominate in the near and medium terms. This work is related to the DOE energy and environmental mission areas of a number of DOE Offices, including Energy Efficiency and Renewable Energy (EERE), Basic Energy Sciences (BES), and Biological and Environmental Research (BER).

\section{RESULTS AND ACCOMPLISHMENTS}

Six classes of fuel molecule were targeted during FY 2015 screening activities: alcohols, esters, ethers, ketones, furans, and aromatics. Comparisons were also made to ethanol. The intent was to quantify and understand the influence of various molecular functional groups on autoignition when blended into conventional fuels. The focus is to discover/design chemicals that can drastically modulate the chemical kinetic pathways responsible for autoignition and thereby improve engine performance.

Measurements were conducted in Argonne's twin-piston RCM, where pure, single-component additives were blended into a full-boiling-range gasoline at $10 \% \mathrm{v} / \mathrm{v}$. Wide ranges of temperature and pressure were covered to characterize perturbation effects at conditions relevant to conventional SI combustion, as well as LTC schemes.
Seventeen different compounds were tested, and the results were correlated to identify structural features that provide resistance to autoignition, or conversely, promote it.

High-level quantum chemical calculations were undertaken targeting three additive candidates. Calculations were performed to determine bond strengths and potential decomposition pathways, and thus the tendency to resist or enhance autoignition. The rotational-vibrational properties of the reactants and products were determined for the bond dissociations. Energies required for ring openings at either carbon-carbon (C-C) or carbon-oxygen (C-O) bonds were obtained. The analyses provided key insights into the trends observed in the RCM datasets.

Strains of purple, non-sulfur bacteria were isolated that can grow on renewable waste streams and accumulate chemical precursors at up to $70 \%$ of their total cell mass. The bacteria were found to grow at rates comparable to those on purified carbohydrates. Interactions were initiated with six local companies that can provide waste streams convertible by this bacterial platform, and we are partnering with Archer-Daniels-Midland Company to source glycerol as one material, since this is a major by-product of biodiesel and ethanol production. High-throughput fluorescent assays were also established in order to conduct non-invasive quantitation of the chemical precursor in live cell cultures, which is not trivial in pigmented photosynthetic bacteria. The precursor levels determined by these fluorescent assays were confirmed by gas chromatography-mass spectrometry.

Currently, the biopolymer from the cells is harvested via a two-step, one-pot chemical route that involves heat-assisted cell lysis to release the polymer, which is then broken down into monomers using an acid-catalyzed methyl esterification. The methyl ester monomers are then separated from cell debris via solvent extraction. Guided by initial RCM results and theoretical calculations, we identified at least a dozen molecules that can be produced via monomer valorization. We also identified two to three candidates and structurally relevant analogues on the basis of optimal upgrading conditions such as consideration of ease of synthesis (fewer steps, higher yield) and little to no need for external additional carbon source (minimizing carbon footprint).

\section{PROPOSED FUTURE WORK}

Additional molecular structures have been selected for testing in FY 2016. The search will be narrowed to one to two compounds that will be studied in detail. Some new candidates will be synthesized specifically for this project, 
since they are not available commercially. In addition to the ignition-perturbing characteristics of potential candidates, properties important for deployment such as corrosivity, toxicity, and material incompatibility will be examined.

With success in production of chemical precursors using glycerol waste streams, experimental plans for FY 2016 include exploring production potential from two additional negative-value waste streams (from brewery and dairy industries). In addition, to expand the repertoire of molecules available for upgrading, we will explore conditions that lead to the biosynthesis of larger carbon chains or reactive substituents. Using in vivo quantitation methodologies that were developed in FY 2015, we will determine culture conditions that allow: (1) growth of bacteria at $75 \%$ of native rates using the new reclaimable carbon sources and (2) polyhydroxyalkanoates (PHA) production at $>40 \%$ of cellular mass.

Alternative methods for biopolymer and monomer extraction from cells have been identified and will be developed in FY 2016. Specifically, a nanoadsorbent-based biopolymer/monomer separation from cell debris is considered more economically attractive compared to solvent extraction methods. Synthetic routes and upscaling optimization for monomer valorization for additional fuel additive candidates will also be identified.

\section{Large-Scale Modeling and Simulation for an Adaptive and Resilient Power Grid}

\author{
2015-159-NO \\ Jianhui Wang, Edwin Campos Ortega, Chen Chen, \\ James Kavicky, Sven Leyffer, and Feng Qiu
}

\section{PROJECT DESCRIPTION}

The purpose of this project is to implement state-of-the-art algorithms combining weather forecasting, power system modeling and simulation, stochastic optimization, and high-performance computing to enhance the U.S. power grid's capability to integrate clean and distributed energy, eliminate large-scale and long-term blackouts, and recover and survive after major weather events.

\section{MISSION RELEVANCE}

This project contributes to DOE's energy security mission to ensure a resilient, reliable, and flexible electricity system. DOE recognizes the need for electric grid modernization and for resiliency in the energy infrastructure, and this project provides a complete suite of solutions for enhancing power grid resilience.

\section{RESULTS AND ACCOMPLISHMENTS}

In FY 2015, we made significant progress on this project. Following are the tasks performed in FY 2015.

Extreme Weather Event Characterization and Advanced Nowcasting Techniques. In this task, the team reviewed specialized datasets from state-of-the-art short-term weather forecasting techniques. The objective was to look for applications relevant to power grid resilience. In particular, we investigated the applicability of weather data coming from both research and operational forecasting technologies. On the research side, we experimented with tools for analyzing the weather event associated with the record Chicago floods in April 2013, which were the worst floods experienced by residents of the Chicago area in the last three decades. We analyzed various customized simulations from the Weather Research and Forecasting (WRF) model at high temporal and spatial resolutions (10 minutes and 3 kilometers, respectively) with nested domains. To evaluate the model performance, we employed observations from various meteorological instruments at Argonne and multi-sensor (weather radar and rain gauge) precipitation estimates from the National Oceanic and Atmospheric Administration's National Weather Service.

Figure 1 compares the multi-sensor estimates with our WRF model simulations. The study was published in the Journal of Hydrology. On the operational side, we identified public (internet) data repositories with operational nowcasting products on wind speeds, wind gusts, precipitation intensity, tornado occurrence probabilities, and hurricane paths and winds. 

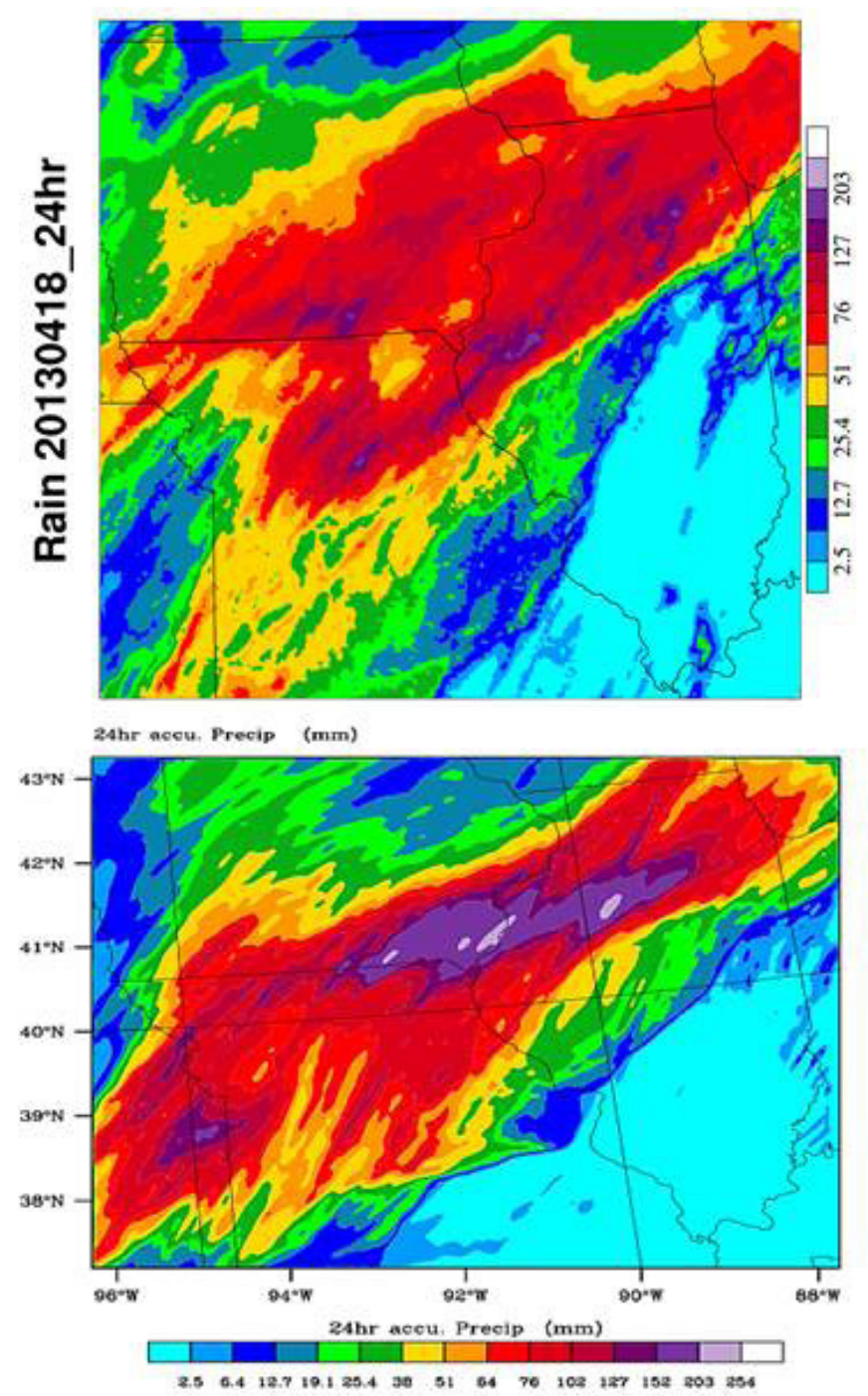

Figure 1. Observed (top) and simulated (bottom) 24-hour accumulated precipitation (millimeters) for April 18, 2013, during the Chicago 2013 spring floods.

Development of Advanced Power System Simulation Modules. The structures and operations of modern power systems exhibit highly nonlinear properties. Many operation models used in the power industry are simplified to be able to obtain satisfactory solutions in a reasonable amount of time. Though efficient, they cannot capture many critical features of power systems when it comes to system security and stability. Take the generator rotor angle stability as an example: it is governed by the dynamics of nonlinear electrical-mechanical equations. Incorporating these nonlinear equations into the operational models creates immense computational complexity and undermines the efficiency of the solution process, which results in problems that are impractical to solve. To avoid this difficulty, simulations are used to perform certain analytical tasks that involve significant nonlinearity, instead of adding the system nonlinearity into operational models. In this task, we created a state-of-the-art power system simulator that can simulate the entire electrical/mechanical process of power system restoration with unprecedented credibility and fidelity.

\section{PROPOSED FUTURE WORK}

In FY 2016, we will add automatic adjustment of restoration plans to satisfy stability and security requirements in our model, and expand our model's functionalities in bulk power-system restoration and distribution-level restoration.

\section{Connected and Automated Vehicles}

\author{
2015-176-NO \\ Aymeric Rousseau, Joshua Auld, Dominik Karbowski, \\ Eric Rask, and Vadim Sokolov
}

\section{PROJECT DESCRIPTION}

Connected and Automated Vehicles (CAVs) are bound to transform on-highway transportation in the near future.

Vehicles will be able to communicate with each other and with the infrastructure as some or all of the tasks incumbent upon drivers will gradually shift to automated controllers. The R\&D in the underlying technology is many years in the making, but the impact CAVs may have on energy use has been largely overlooked, whether it is positive through more efficient driving and reduced congestion or negative through increased travel demand. Advanced high-fidelity simulation tools are needed not only to evaluate impacts before actual implementation, but also to identify system-level control strategies that will improve mobility and reduce energy use. The objective of this project is to develop an integrated model combining both traffic flow models and advanced vehicle models.

\section{MISSION RELEVANCE}

Most of the research in the area of intelligent transportation systems is targeted on mobility and safety. Similarly, vehicle systems researchers focus on improving energy efficiency on standardized cycles. However, it has not been possible so far to assess global energy performance in a real-world transportation network scenario. The main benefit of the integrated model we propose would be to quantify the energy impact of advanced vehicle technologies, one of the main focuses of DOE. 


\section{RESULTS AND ACCOMPLISHMENTS}

Traffic Flow Model. As shown in Figure 1, a new traffic flow model was developed to represent detailed vehicle movements on arterial roads. Such a model is capable of generating microscopic vehicle speed profiles necessary to estimate the energy profiles of each vehicle trip while including an explicit spatial representation of vehicles within a road segment. This enables us to implement new traffic flow control strategies that rely on connectivity and automation.

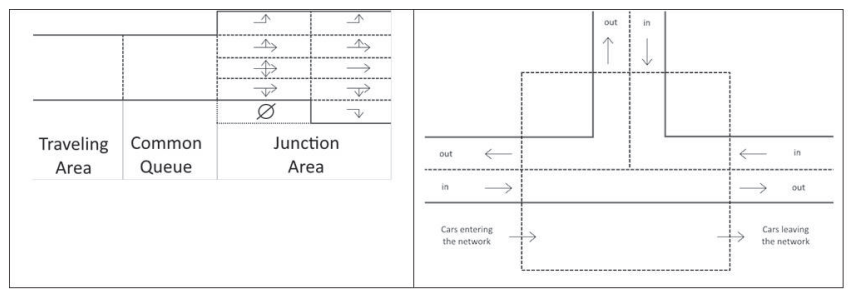

Figure 1. New road intersection model supporting turn lanes and queue spill-over.

Development of Ann Arbor Model for Connected Vehicle Simulations. A Polaris model of the Ann Arbor, Michigan, area was developed for regional travel simulations in connection with the connected vehicle safety pilot. The work had three primary motivations: (1) demonstrate the development of a Polaris model for an urban area by outside researchers (in this case, a student hired from the University of Michigan); (2) develop processes for integrated data collected from connected vehicles into the Polaris environment for model calibration, validation, and future simulation of connected vehicles; and (3) demonstrate the viability of the CAV simulation in Polaris (http://www.anl.gov/energy-systems/project/ tool-transportation-system-simulation-polaris). An example of the output from this work is shown in Figure 2.

Vehicle Control through Man-In-the-Middle (MiTM) Control of Adaptive Cruise Control (ACC) Systems. When gauging the impacts of CAV technologies, laboratory testing is an important component of an overall assessment plan. A wider range of experimental plans can be executed within the laboratory, some of which would never be able to be executed on-road.

We decided to focus on enabling and emulating the behavior of a vehicle's ACC system as a first step toward using a dynamometer to evaluate specific CAV functionality. We first identified the components related to ACC system sensing, control, communication, and vehicle actuation. An entry point from which to broadcast an emulated signal was then chosen to operate the vehicle using an emulated lead vehicle. Looking at the structure of the communications shown in Figure 3, it was decided to provide the emulated signal between the vehicle's millimeter wave radar (MMWR) and the actual ACC system controller.

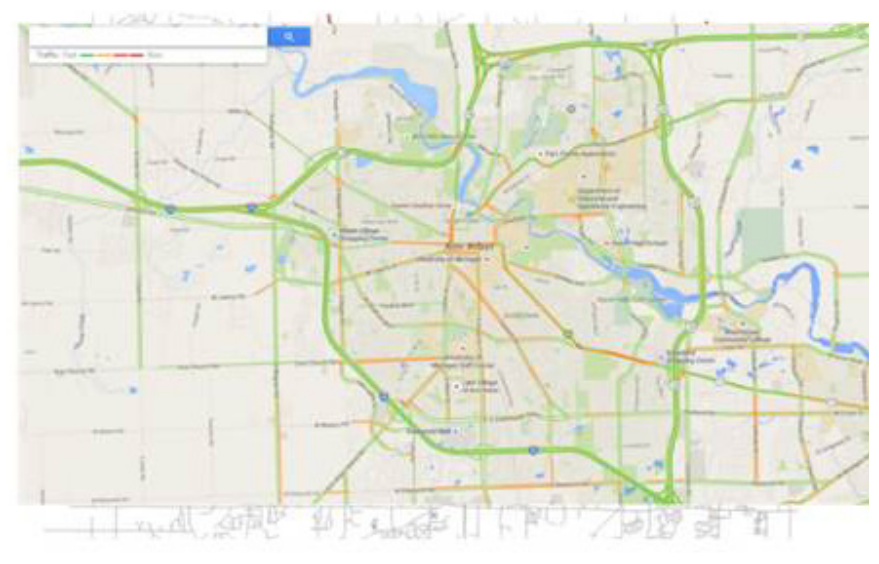

traffic map, 9 am -10 am, Sunday, 6/2/13

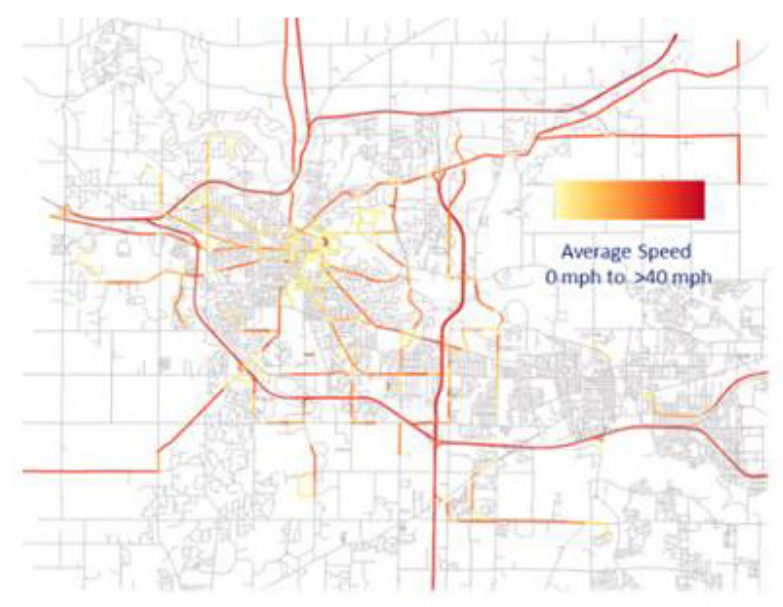

traffic map, 9 am - 10 am, Monday, 6/3/13

Figure 2. Comparison of modeled traffic flow with measured vehicle speed profiles.

The communication bus was tapped and an emulation box was used to provide the emulated signals as well as any required calculations needed to facilitate the relative speed of the lead and following (test object) vehicles. 


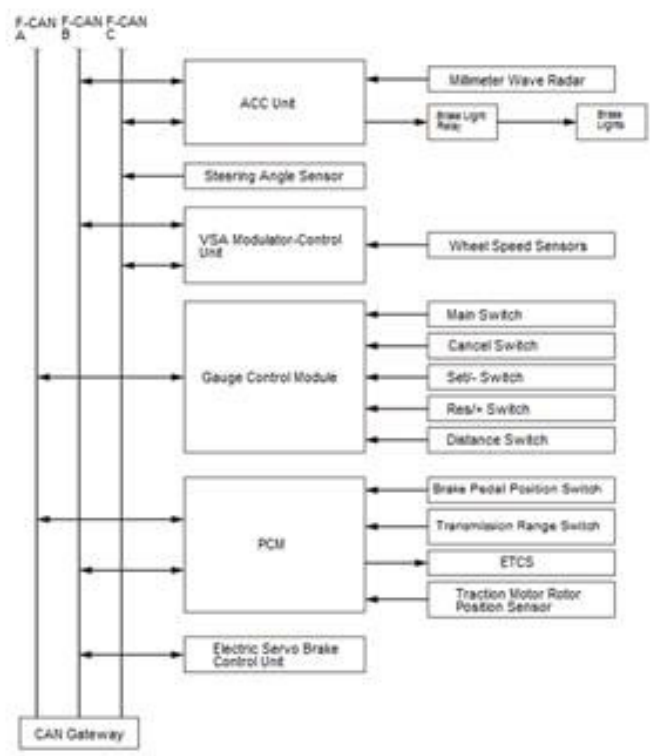

Figure 3. Honda Accord ACC system and related components communications diagram.

\section{PROPOSED FUTURE WORK}

In FY 2016, one main focus will be to continue the development of Polaris models, including:

$\square$ Traffic flow simulation. (1) Improve algorithms for arterial roads, (2) support managed lanes and vehicle classes, and (3) produce microscopic speed trajectories.

$\square$ Activity modeling update for dynamic activity generation process, sensitive to network level-of-service (LOS). Improve the activity generation components to incorporate trip chaining and intra-personal interactions. Ensure that the various activity choice dimensions are sensitive to CAVs LOS changes, including start time, destination, etc.

$\square$ Vehicle choice model. Introduce discrete vehicle types with different performance and capabilities and distribute at the household level.

The tools processes will also be enhanced to accelerate the simulation time by further leveraging Argonne high-performance computing capabilities as well as the analysis through advanced post-processing/visualization. 


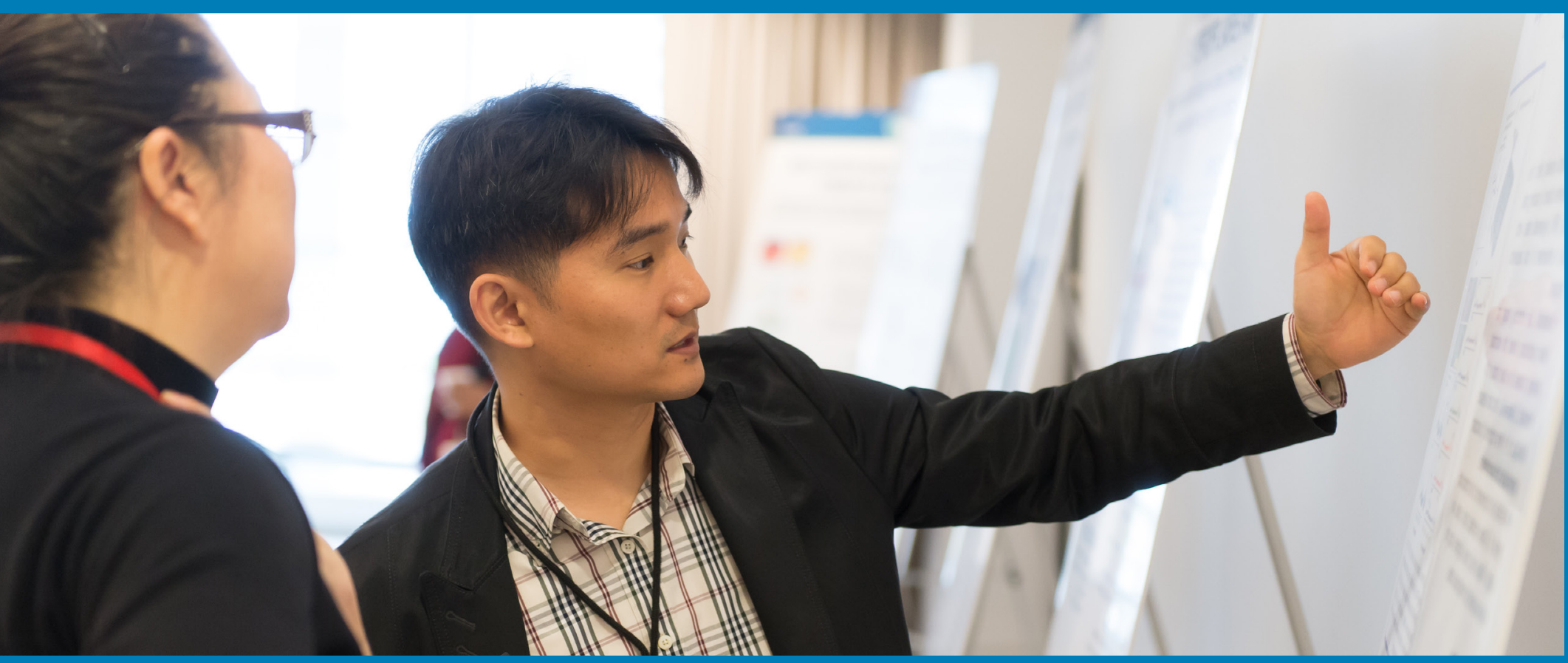

\section{BIOLOGY AND ENVIRONMENT}




\section{Modeling the Interactions of Biophysical, Biogeochemical, and Microbial Dynamics in Permafrost-Affected Soils: From Pore Scale to Regional Scale}

\author{
2012-205-R3 \\ Zhaosheng Fan
}

\section{PROJECT DESCRIPTION}

High-latitude regions contain approximately $30-60 \%$ of the world's terrestrial soil organic carbon. Over the past four decades, the mean annual air temperature in high-latitude permafrost regions has increased by $0.2^{\circ} \mathrm{C}$ or $0.3^{\circ} \mathrm{C}$ per decade, and it is projected to increase further from $4^{\circ} \mathrm{C}$ to $7^{\circ} \mathrm{C}$ per decade by the end of this century. As a result of the huge carbon stocks and great warming potential, high-latitude ecosystems have the great potential to drive important interactions and feedbacks with the Earth's climate.

The objective of this work is to improve our quantitative and predictive understanding of high-latitude soil carbon cycling and its potential role in altering greenhouse gas concentrations in the atmosphere and thus, in the global climate. To achieve this goal, mechanism-based models will be developed to represent and simulate the fundamental and important interactions of the soil's physical, hydrological, geochemical, biogeochemical, and microbial community dynamics. The developed models will then be used to investigate the mechanisms controlling high-latitude soil carbon dynamics and to better evaluate the feedbacks between high-latitude regions and the global carbon cycle.

\section{MISSION RELEVANCE}

This project is relevant to DOE's mission in Basic Energy Sciences and the environment. One of DOE's primary missions is to provide quantitative and predictive understanding of the terrestrial ecosystems that can affect atmospheric greenhouse gas concentration changes due to the large carbon stocks in these ecosystems. There is a need to understand the impacts of and feedbacks from a changing climate on the terrestrial ecosystems. The outcome of this work will be helpful in improving projections of the roles of high-latitude ecosystems in the context of climate change.

\section{RESULTS AND ACCOMPLISHMENTS}

In Fiscal Years 2012, 2013, and 2014, we developed (1) a mechanistic model that can simulate simultaneously occurring physical, chemical, biological, and hydrological processes in the soil and their interactions, (2) a porescale oxygen transport and consumption model to investigate factors affecting the distribution of anaerobic microsites within the unsaturated soil zone, and (3) a coupled soil water-carbon-microbial model to simulate the production and release of carbon dioxide from the soil and the corresponding soil carbon dynamics in an African savanna ecosystem.

Modeling soil carbon-microbe interactions. Scientists disagree on how soil organic carbon dynamics will respond to global warming. This disagreement is in part because research on litter decay and the formation and/ or stabilization of soil organic carbon is often uncoupled. To address this issue, we developed a novel biologically realistic model based on established ecological theories and known microbial processes. In the model shown in Figure 1, microbes function as valves controlling carbon sequestrated from plant litters versus carbon released into the atmosphere. This model is believed to be the first to simultaneously investigate the (1) origins of soil organic carbon (plant-derived versus microbial-derived), (2) responses of soil and microbial biomass carbon pools to litter inputs, and (3) feedbacks between soil organic carbon and the microbial community (fungal versus bacterial functional groups).

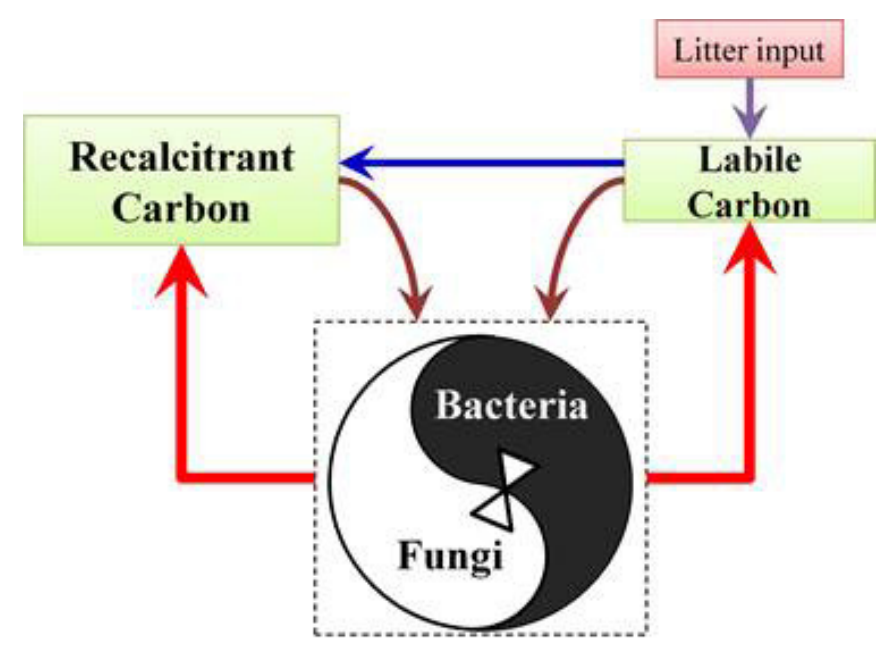

Figure 1. Model schematic used to simulate the dynamic interactions among litter inputs, microbial biomass, and soil carbon pools.

A series of model simulations revealed that plant litter quality (i.e., how easily plant litter is decomposed by microbes)-not quantity-regulated long-term soil carbon dynamics. The impacts on soil organic carbon stocks were eventually controlled by asynchronous responses 
in the acceleration of biosynthesis rates by fungi versus bacteria. These responses were driven by litter quality and were independent of the amount of microbial biomass and the quantity of litter inputs. The simulations also demonstrated the significant contribution of microbialderived carbon to total soil organic carbon (from $47 \%$ to $80 \%$ at the steady state). The critical importance of litter quality on the acceleration of microbial biosynthesis rates-identified for the first time by this study-offers new insights into fundamental soil processes and suggests new research directions for understanding controls on soil carbon dynamics.

Ecosystem biogeochemistry model. The environmental controls over gross primary production can be roughly separated into physical (temperature and moisture) and nutrient (nitrogen and phosphorus) controls. It remains very difficult to examine the relative importance of environmental physical controls versus nutrient controls over the ecosystem's primary production. This challenge arises from both the inherent limitations of field studies and the complicated temporal interactions between physical and biogeochemical control of productivity under field conditions. Such limitations may be overcome with quantitative models that can improve the understanding of the ecosystem functions. In FY 2015, we also developed a mechanistic ecosystem biogeochemistry model (shown in Figure 2) that simulates the coupled cycling of carbon, nitrogen, and phosphorus and their interactions with gross primary production. The model was then used to investigate several key limiting factors that control alpine tundra productivity. This simulation provides testable predictions of the shifting nature of physical and nutrient limitations on plant growth in the context of climate change.

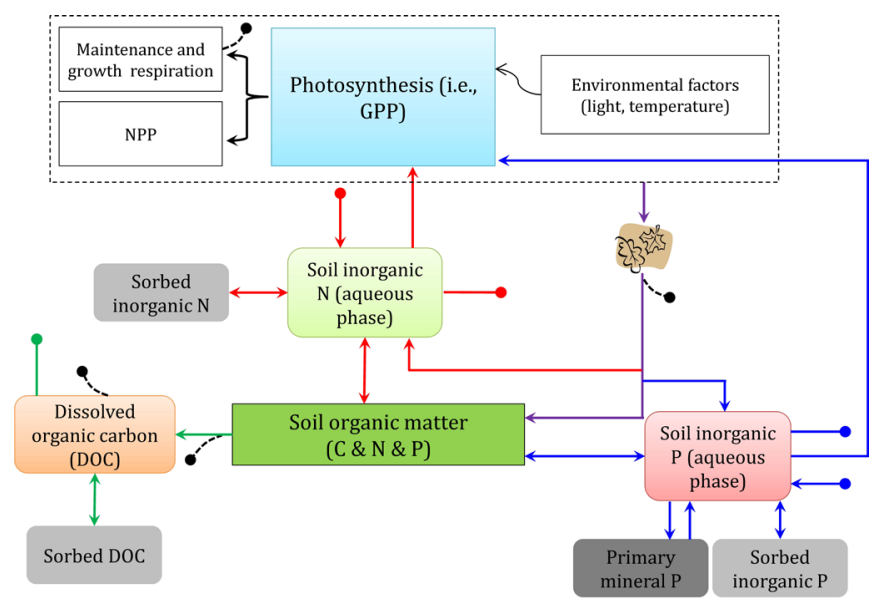

Figure 2. Model schematic that outlines the coupled cycling of carbon, nitrogen, and phosphorus, and their interactions with ecosystem primary production.

\section{Developing, Improving, and Testing Methods for Predicting Spatial and Vertical Distributions of Soil Organic Carbon at Regional Scales}

\author{
2012-206-R3 \\ Umakant Mishra and John Krummel
}

\section{PROJECT DESCRIPTION}

Perennially frozen soils -19 million $\mathrm{km}^{2}$ or $16 \%$ of the global soil area-of the northern circumpolar region (land area above $60^{\circ}$ north latitude) store the largest quantity of soil organic carbon $(\mathrm{SOC})$ in the terrestrial biosphere. Soils of this area are expected to experience much greater increases in their temperatures over the next century $\left(3.2-3.5^{\circ} \mathrm{C}\right.$ by 2100$)$ than soils in temperate or tropical regions. Therefore, high-latitude SOC stocks-which are preserved, in large part, because of low temperaturesare a potentially vulnerable component of the global carbon cycle.

Current global estimates of SOC stocks substantially underestimate permafrost-affected SOC, mainly because of the paucity of observations at high latitudes. Moreover, current estimates of SOC stocks in permafrost-affected soils have been made by stratifying the study area, averaging point observations of SOC stocks within each stratum, and multiplying by the areal extent of that stratum. In addition, most observations rarely extend below the upper meter of the soil profile, even though high-latitude soils often contain considerably deeper SOC because of cryoturbation (i.e., frost churning-the mixing of materials from the various soil horizons (distinct layers) down to the bedrock due to freezing and thawing). As a result, estimates of total SOC stocks in high-latitude regions remain highly uncertain, with current assessments suggesting that the total amount of frozen carbon in permafrost soils could be equal to or even double the amount of carbon presently in the atmosphere. Recent studies have also recognized the need for more accurate assessments of the spatial heterogeneity of SOC stocks in permafrost-affected soils.

The objectives of this project are to (1) estimate spatially resolved SOC stocks in bedrock, distinguishing active-layer and permafrost-layer stocks on the basis of geospatial analysis of soil profile observations and spatially referenced environmental variables for permafrost affected soils; (2) quantify the prediction errors and uncertainties associated with the estimates; and (3) investigate the environmental controls that determine regional-scale SOC stocks. 


\section{MISSION RELEVANCE}

The objectives of this study are relevant to DOE's missions in science and the environment. DOE, as well as other agencies, are now demanding tighter coupling and iteration between empirical studies and model development, in which the uncertainties in model predictions will help in identifying priorities for new observations and experiments. The outcome will be used to improve and test new models. The results of this study will produce spatially distributed estimates of permafrost SOC stocks, which are important for developing and testing earth system models (ESMs).

In addition to DOE's Office of Biological and Environmental Research, agencies such as the National Science Foundation, National Aeronautics and Space Administration, and U.S. Department of Agriculture, as well as private foundations, are giving increasing priority to research related to climate change, coupled natural and human systems, ecosystem services, and land-use/landcover change impacts on SOC stocks.

\section{RESULTS AND ACCOMPLISHMENTS}

In FY 2013, we completed a study entitled "Empirical estimates to reduce modeling uncertainties of soil organic carbon in permafrost regions: a review of recent progress and remaining challenges" that was published in the Environmental Research Letters journal. This study discussed the state of current knowledge about the characterization of soil carbon in permafrost-affected soils and its potential vulnerability to changing climatic conditions. We determined existing differences between empirical and ESM estimates of the size and distribution of permafrost-region SOC stocks, and research efforts that will reduce this discrepancy were identified. Five research challenges for improving empirical assessments of the distribution and potential mineralization of SOC stocks in the northern permafrost region were highlighted. These challenges include (1) improving the number of and robustness of observations, (2) predicting the lateral and vertical distributions of SOC stocks, (3) characterizing existing carbon forms to better predict their fates, (4) using improved observation-based SOC estimates to inform model development, and (5) quantifying uncertainties in observations and predictions. These challenges are interlinked and suggest opportunities to organize, prioritize, and coordinate future permafrost soil carbon research efforts.

In FY 2014, we completed a study where we used geospatially referenced soil-profile description data coupled with environmental data-such as topography, climate, and land-cover types-to predict the variability of active-layer thickness at fine-scale resolution across Alaska. The predicted active-layer thickness ranged from 0.14 to 0.93 meters $(\mathrm{m})$, with a spatial average of $0.46 \mathrm{~m}$ and a $30 \%$ coefficient of variation (Figure 1). Surface air temperature, land cover type, and slope angle were the primary controllers of the spatial variability. While comparing our results with projections of active-layer thickness produced by the Coupled Model Intercomparison Project Phase 5 (CMIP5) ESMs for Alaska, we found CMIP5 projections had lower coefficients of variation but substantially larger prediction errors than the observation-based estimates. In addition, CMIP5 projections showed large inter-quartile ranges in predicted active-layer thickness (0.35-4.4 m), with some models substantially overestimating the predictions derived from geospatial estimates. These comparisons call attention to the need for better process and spatial heterogeneity representations in ESMs to enable more realistic estimates of regional-scale active-layer thickness and improved predictions of permafrost carbon feedback to climate under future warming scenarios.

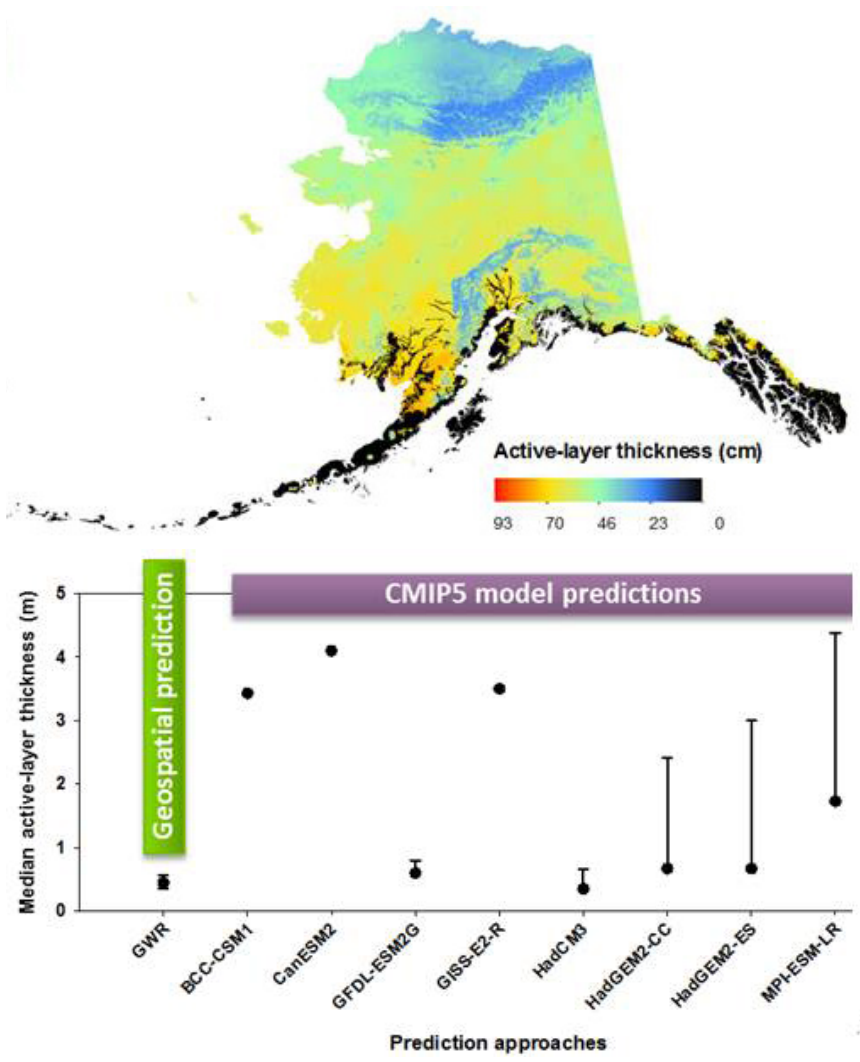

Figure 1. Predicted active-layer thickness (on top) and comparison of observation-based predictions with earth system model predictions (on left).

In FY 2015, we completed a study where we used soil profile observations, environmental factors (such as topography, climate, land cover types, surficial geology) and geospatial modeling to study the impact of spatial 
scaling on environmental controls, spatial structure, and statistical properties of SOC stocks in Alaska. We found that different environmental factors were significant predictors of SOC stocks at different spatial scales. Of the nineteen environmental factors evaluated, only fourelevation, temperature, potential evapotranspiration, and scrub land cover types-were significant predictors at all of the investigated scales. The strengths by which these four environmental variables controlled SOC stocks decreased with increasing scale. The spatial structure of SOC stocks also changed with scale. The spatial heterogeneity of predicted SOC stocks decreased with spatial scale over the range of $50 \mathrm{~m}$ to $\sim 500 \mathrm{~m}$ (Figure 2) and remained constant beyond this scale. Moderately accurate linear relationships were found between the mean and other statistical properties of predicted SOC stocks. Current ESMs operate at coarse spatial scales (50-100 km) and are therefore unable to represent environmental controllers and spatial heterogeneity of high-latitude SOC stocks in a manner that is consistent with observations. Improved knowledge of the scaling behavior of environmental controls and statistical properties of SOC stocks is critical for ESM land model benchmarking and ultimately might enable the spatial heterogeneity of soil biogeochemistry to be represented at scales finer than those currently resolved by ESMs.

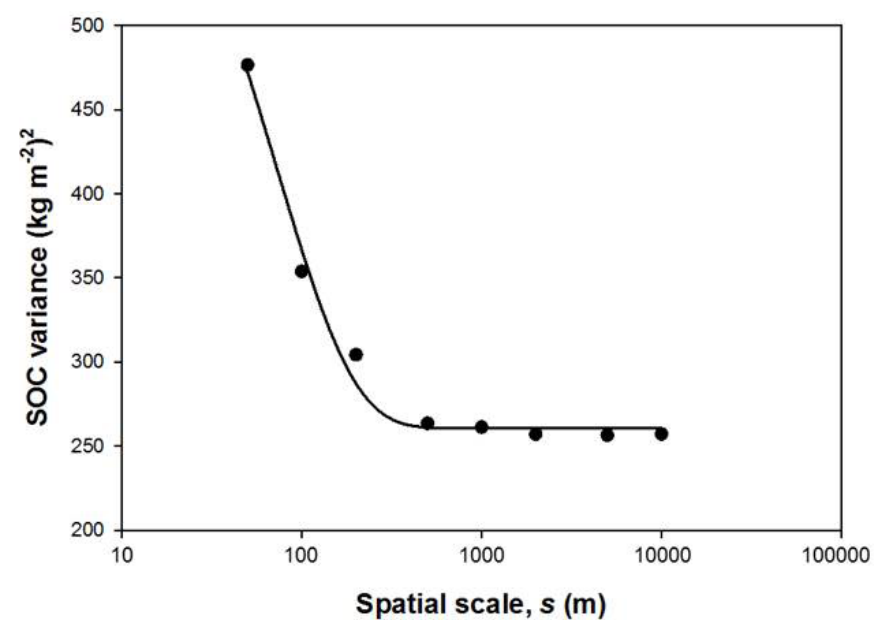

Figure 2. Variance of soil organic carbon stocks as a function of spatial scale. Each dot is the predicted variance of SOC stock at each spatial scale across Alaska.

\section{Identifying Novel Pathways for Anaerobic Microbial Oxidation of Methane}

\author{
2013-177-R2 \\ Edward J. O'Loughlin, Dionysios A. Antonopoulos, \\ Theodore M. Flynn, and Kenneth M. Kemner
}

\section{PROJECT DESCRIPTION}

Although not as prevalent in the atmosphere as carbon dioxide $\left(\mathrm{CO}_{2}\right)$, methane $\left(\mathrm{CH}_{4}\right)$ is an important greenhouse gas (GHG) that accounts for approximately $20 \%$ of human-induced radiative forcing. Atmospheric methane concentrations have increased by nearly $160 \%$ since 1850 , largely because of human activities relating to large-scale land management and agricultural practices (e.g., wetland rice production, the raising of ruminant livestock, and mining operations). Moreover, increased methane emissions due to the warming of Arctic permafrost have been identified as a potentially significant factor resulting from (and contributing to) global climate change.

Extensive biogeochemical research has been conducted that focuses on the causes and effects of variation in global fluxes of methane throughout the Earth's history. Although it is known that methane formation (methanogenesis) and consumption (methane oxidation) in soils are the result of highly specialized microorganisms, the underlying microbial processes and key microbial players are poorly understood. Methane formed during methanogenesis can be oxidized to carbon dioxide via aerobic methane oxidation by methanotrophic bacteriaobligate aerobes that use methane as a sole carbon and energy source. While the aerobic oxidation of methane by microorganisms has been known since the early 1900s, anaerobic microbial methane oxidation has only recently been identified. In environments devoid of sufficient oxygen to support aerobic methane oxidation (suboxic to anoxic conditions), other oxidants commonly found in aquatic and terrestrial systems (e.g., sulfate, nitrate) are thermodynamically capable of serving as terminal electron acceptors for anaerobic methane oxidation.

This project focuses on the identification of anaerobic methane-oxidizing microbes from a diverse range of aquatic and terrestrial environments, characterization of the relevant metabolic pathways of anaerobic methane oxidation in these organisms, and assessment of the effects of key environmental parameters on the contribution of anaerobic methane oxidation to overall methane and carbon dioxide flux from surface and near-subsurface environments. 


\section{MISSION RELEVANCE}

This project is relevant to DOE's missions in science and the environment. An improved understanding of the global terrestrial carbon cycle has become a policy imperative, both domestically and internationally. It is crucial in efforts to model, predict, and potentially mitigate the effects of increasing concentrations of GHGs, such as carbon dioxide and methane, on global climate. Identification of the microorganisms and the relevant metabolic pathways controlling anaerobic methane oxidation will provide fundamental insight into a key component of net methane flux to the atmosphere and will refine our understanding of the linkages between the biogeochemical cycling of carbon and key major/minor elements.

\section{RESULTS AND ACCOMPLISHMENTS}

In FY 2013, we collected soils/sediments from 25 separate locations in 12 different states from habitats representing a diverse range of terrestrial and aquatic (freshwater, estuarine, and marine) environments throughout the continental Unites States and one site in Costa Rica. We used six of these samples to create microcosms designed to promote the growth of microorganisms that can use nitrate/nitrite, sulfate, sulfur, iron(III) oxide, manganese(IV) oxide, or humic substances as terminal electron acceptors for anaerobic methane oxidation. In the nitrate-amended incubations, we observed depletion of methane and increased carbon dioxide, coupled with the reduction of nitrate to nitrogen gas, which is consistent with nitrate-dependent anaerobic methane oxidation.

Building on FY 2013 sampling efforts, in FY 2014 we collected additional soils/sediments, expanding our sample library to include soils/sediments from 36 separate locations in 13 different states and Costa Rica. We used 11 of these samples to create additional enrichment microcosms. In several of the longest-running incubations, we observed oxidation of methane to carbon dioxide, coupled with the reduction of nitrate to nitrogen gas. We generated inventories of the microbes in these enrichments by targeting the 16S rRNA gene, a broadly occurring gene found in all microorganisms.
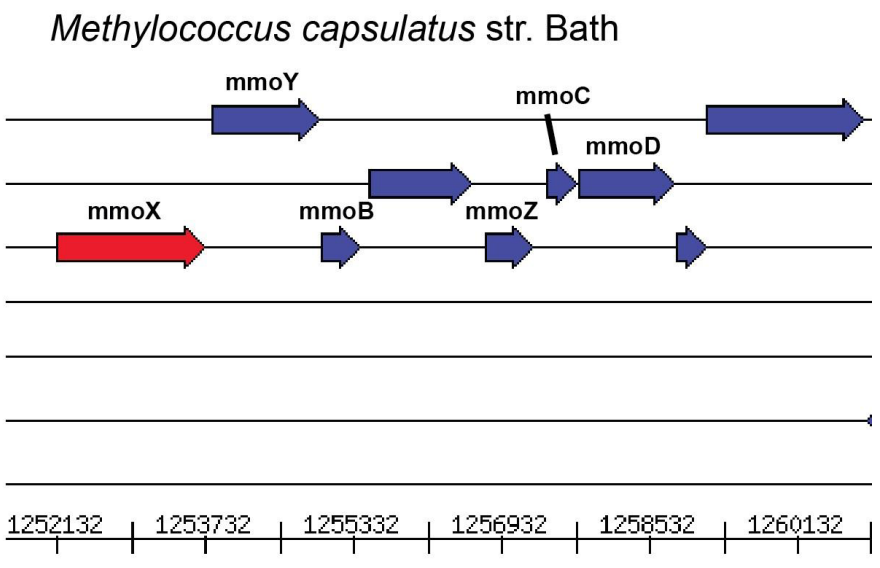

Bradyrhizobium japonicum USDA 110
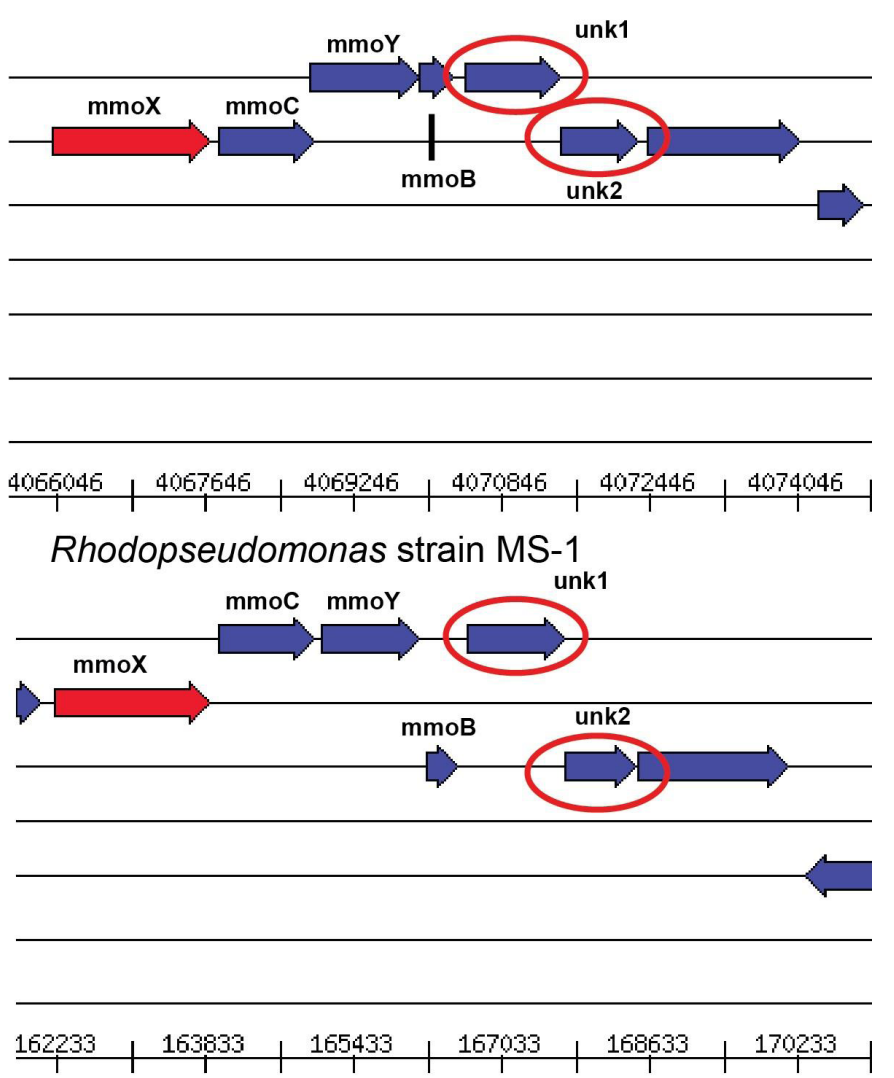

Figure 1. Comparison of the SEED subsystem for soluble methane monooxygenase (sMMO) among a known methane oxidizer (Methylococcus capsulatis), our phototrophic isolate (Rhodopseudomonas strain MS-1), and Bradyrhizobium japonicum, which is not known to oxidize methane but shares the same number and order sMMO genes with strain MS-1. Circled genes have no known function but may be related to the putative sMMO pathway in strain MS-1, B. japonicum, and others.

In FY 2015, we used whole-genome shotgun sequencing of the metagenome of one of the microorganisms from these enrichments (designated MS-1) to reconstruct its 6.1-megabase genome. Comparison of the full-length $16 \mathrm{~S}$ rRNA gene from the reconstructed genome to a reference database of $16 \mathrm{~S}$ rRNA genes of known isolates showed that this organism was most closely related to the 
genus Rhodopseudomonas. By comparing the annotated genome of MS-1 to existing Rhodopseudomonas genomes, we found that in addition to phototrophy genes found in all known strains of Rhodopseudomonas, MS-1's genome contains metabolic genes for the oxidation of methane that are absent from other known members of this genus. Figure 1 shows that the arrangement of the genes that encode this enzyme (soluble methane monooxygenase, or sMMO) also differs from previously characterized methane oxidizers, such as Methylococcus capsulitis. The previously characterized pathway for sMMO in M. capsulatis and other methanotrophs has six genes: $\mathrm{mmoX}, \mathrm{mmoY}, \mathrm{mmoZ}, \mathrm{mmoB}, \mathrm{mmoC}$, and $\mathrm{mmoD}$, whereas strain MS-1 has only four of these six genes (mmoX, mmoY, mmoB, and $\mathrm{mmoC}$ ). There are two genes that encode for proteins of unknown function adjacent to these genes on the MS-1 genome, however, suggesting that MS-1 and other organisms (15 identified in the SEED database) that also share the same four of six genes in the sMMO pathway (e.g., Bradyrhizobium japonicum and Rhodobacter sphaeroides) may have a previously unrecognized ability to metabolize methane.

\section{Annotating, Modeling, and Exploring Enzyme Promiscuity}

\section{3-194-R2}

Christopher Henry, Andrzej Joachimiak, Ross Overbeek,
Samuel Seaver, and Fangfang Xia

\section{PROJECT DESCRIPTION}

Recent structural and mechanistic studies have demonstrated that enzymes often mediate many side reactions. Documented side reactions can get sidelined, however, because enzymes are seen as highly specific agents. Despite increasing evidence for the catalysis of side reactions by metabolic enzymes, the side reactions have failed to appear in genome annotations or metabolic models, where they might be considered by researchers to correct misannotations, design metabolic engineering strategies, identify new pathways to engineer, and select enzymes to form components of a new pathway.

Enzyme promiscuity is one reason why it is so challenging to eliminate undesirable by-products in industrial strains: even when the primary genes associated with undesirable activities are identified and removed, enzymes with secondary activities associated with the undesirable pathway remain. However, the "promiscuous" activities of enzymes also hold great potential, because they can represent new pathways that make the production of known products more efficient or lead to novel products.
Obtaining better knowledge of the secondary functions of enzymes will unveil the potential these enzymes hold if properly engineered.

There has not yet been any systematic study of alternative possible functions for enzymes from central metabolism. "Phylogenetic gene trees" assembled from well-annotated reference genomes provide a mechanism to study all of the possible functions that may be catalyzed by enzymes with similar amino acid sequences. Cheminformatic techniques make it possible to identify all reactions that involve a similar class of biochemical transformation and to generate novel reactions automatically. Structural proteomics makes it possible to explore how protein structure governs enzyme specificity. In experiments, high-throughput pipelines for protein expression, purification, and functional assay enable the rapid validation of proposed secondary functions for enzymes, and metabolomics provides a means for observing the substrates and products of proposed secondary functions.

The objective of this project is to identify and predict alternative functions of promiscuous enzymes by:

$\square$ Fabricating phylogenetic trees of enzyme function, in which the distances between "leaves" indicate the probability that an enzyme whose primary function lies at one leaf will perform the functions found at the other leaves;

$\square$ Proposing novel enzymatic reactions based on patterns of enzyme activity, rules of biochemistry, and substrate similarity and adding these reactions to the phylogenetic trees of enzyme functions;

$\square$ Characterizing the conformational diversity of selected enzyme functions through molecular dynamics simulations and using this biophysical feature for annotating enzyme promiscuity;

$\square$ Applying promiscuous trees of enzyme functions to propose new annotations for poorly annotated gene clusters found in genomes and metagenomes;

$\square$ Obtaining and analyzing protein structures for a variety of enzymes contained within a few protein families; and

$\square$ Designing and synthesizing one or two ancestral enzymes and testing for increased promiscuity, then validating developed models of enzyme promiscuity/ sequence relationships by designing, synthesizing, and characterizing one to five enzymes with increased/ decreased promiscuity. 


\section{MISSION RELEVANCE}

The project is relevant to DOE's mission in science. Improving our understanding of enzyme promiscuity will have an impact on many objectives of the DOE Office of Biological and Environmental Research. The database of promiscuous functions and improved annotations is relevant to the DOE-funded KBase project. The improved capacity for supporting metabolic engineering and studying the link between promiscuity and protein structure also directly applies to an area of growing interest within DOE: synthetic biology.

\section{RESULTS AND ACCOMPLISHMENTS}

In this project, we are taking a combined bioinformatic, cheminformatic, molecular dynamics, and molecular biology approach to understanding enzyme promiscuity. We are combining many orthogonal data sources to attack a single problem and obtain a more complete picture of enzyme promiscuity.

Bioinformatic exploration of enzyme promiscuity. We assembled phylogenetic gene trees for more than 100,000 enzyme families. We applied these trees to curate annotations and improve the consistency of our functional annotations. These trees are then instrumental in predicting promiscuity. We examined mutations that correlate to the loss and gain of genes in various closely related organisms. This approach enables us to identify the specific residues that control the substrate specificity of enzymes. This ability is highlighted in the specific case of the PriA gene family (Figure 1).

Cheminformatic exploration of enzyme promiscuity. We applied the BNICE (Biochemical Network Integrated Computational Explorer) algorithm to explore potential novel reactions for the TrypC enzymes, predicting nine potential novel reactions for TrypC that involve known biochemical substrates. Three of these reactions were subsequently validated experimentally (including the promiscuous activities of TrypC in the histidine and tryptophan pathways described elsewhere in this report). To further expand on this work, we applied BNICE to explore potential novel reactions starting from every native metabolite in the known biochemistry databases. From this effort, we built and populated a large database (>500,000 compounds) of all potential novel biochemistries that could arise given our enzyme reaction rules and the known substrates (http://minedatabase. mcs.anl.gov). This Metabolic In Silico Network Expansion (MINE) database serves as a source of cheminformatic predictions for a wide variety of applications, such as interpreting metabolomics data, annotating novel genes, and predicting novel pathways for new organic product biosynthesis. Although other large compendiums of biochemical compounds exist (e.g., PubChem), the MINE database is notable for having compounds that more closely resemble natural products (Figure 2a). We subsequently searched the MINE database for four compounds using MS-MS spectra, obtaining four strong candidates for previously unidentifiable compounds, two of which were subsequently validated (Figure $2 b$ ).

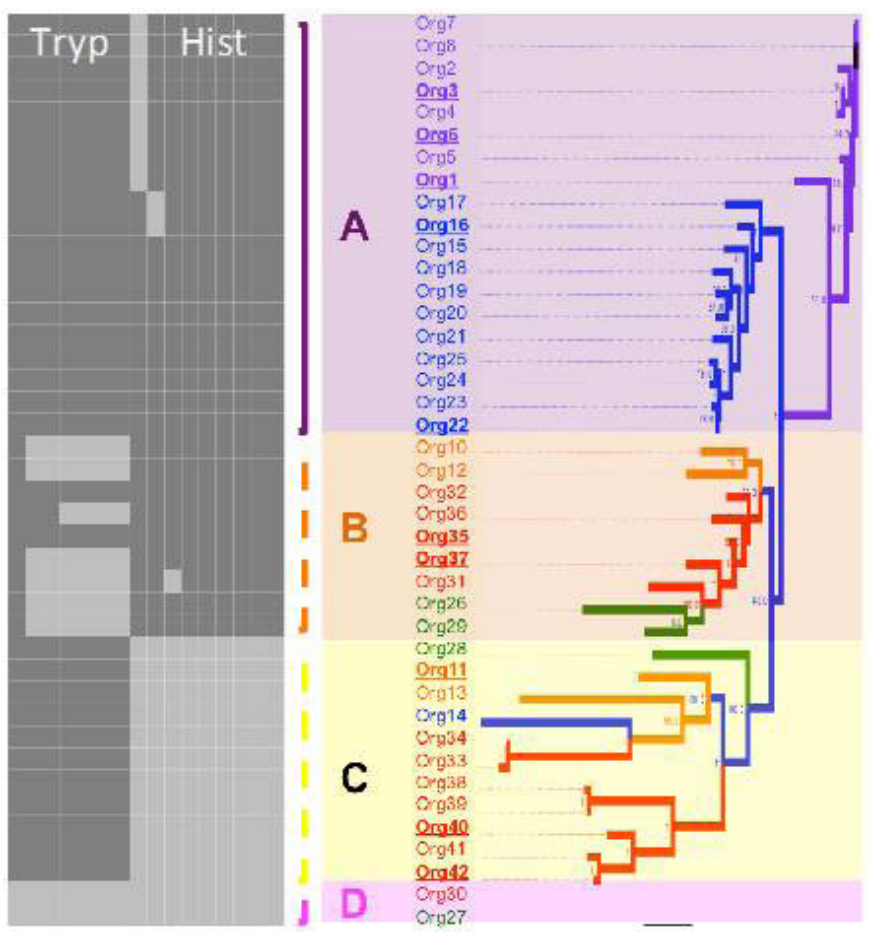

Figure 1. Coevolution of PriA and amino acid pathways. In examining the coevolution of the PriA gene with the tryptophan and histidine biosynthesis pathways, we find that (A) a promiscuous PriA occurs in species with both pathways but no histidine gene; (B) a PriA gene that is specific for histidine exists in species with no tryptophan pathway; (C) a PriA gene that is specific for tryptophan exists in species with no histidine pathway; and (D) no PriA gene occurs at all in a species with neither the histidine nor tryptophan pathway.

Molecular biology exploration of enzyme promiscuity. With researchers at the National Laboratory of Genomics for Biodiversity (Guanajuato, México), we identified 48 distinct TrypC enzymes from various organisms with widely varying levels of promiscuity. Sequences and data were sent to the Advanced Protein Characterization Facility laboratory, where genes were cloned, synthesized, purified, and crystallized. This effort produced a library of purified proteins of varying promiscuity, which were tested for promiscuous functions proposed by our bioinformatic and cheminformatic approaches. Three predicted functions involving natural metabolites were ultimately experimentally confirmed. The protein purification and crystallization process also produced 
three-dimensional structures to feed into our molecular dynamics analysis. We subsequently conducted a molecular dynamics-based substrate-docking and binding affinity analysis of four structures obtained for variants of the TrypC enzyme (Figure 3). Specifically, we used results from our bioinformatics analysis to identify the specific variants of the TrypC enzyme that display all the forms of TrypC promiscuity: (1) promiscuous activity in tryptophan and histidine biosynthesis; (2) specific activity in the histidine pathway only; or (3) specific activity in the tryptophan pathway only. As expected, we found that the critical residues identified as key controllers of enzyme specificity all fell within the binding pocket, and changes in the residues at these amino acid locations resulted in changes in hydrogen-bonding affinities that disrupted or enhanced enzyme activity with the histidine and tryptophan substrates.

\section{A \\ B}
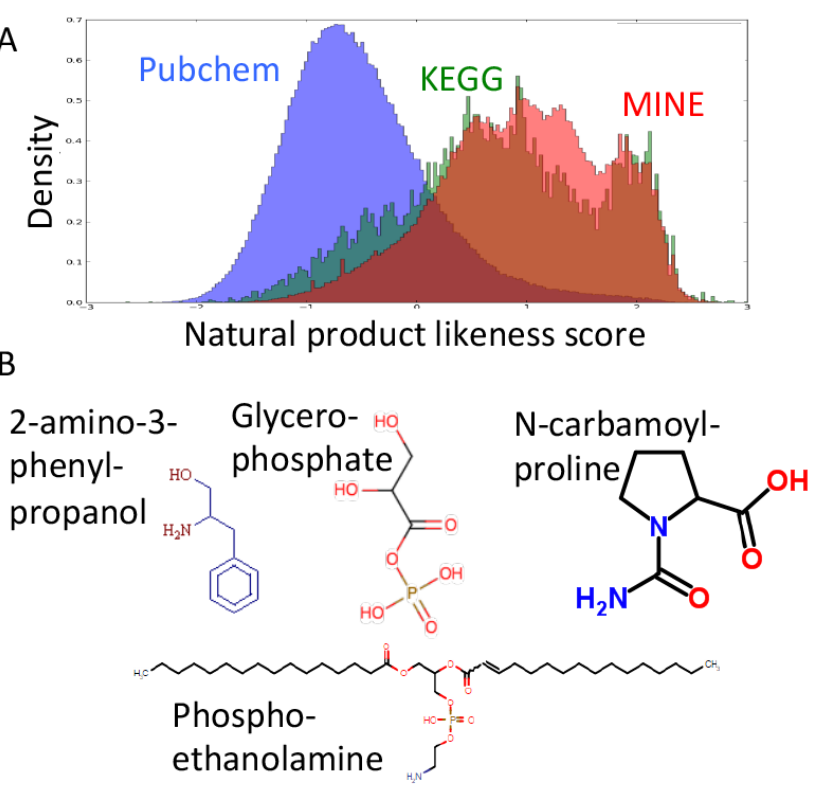

Figure 2. Cheminformatic prediction of enzyme promiscuity. (A) We applied our cheminformatics tools to predict potential novel reactions and metabolites by expanding on the KEGG database of known biochemistry. The resulting database (red distribution) compared very favorably to KEGG (green distribution) in Natural Product Likeness, in contrast to existing chemistry compendiums like PubChem (blue distribution). (B) The four compounds depicted were predicted to occur in $E$. coli by our cheminformatics tools. Then these compounds were subsequently identified in MS-MS spectra from samples of $E$. coli biomass.

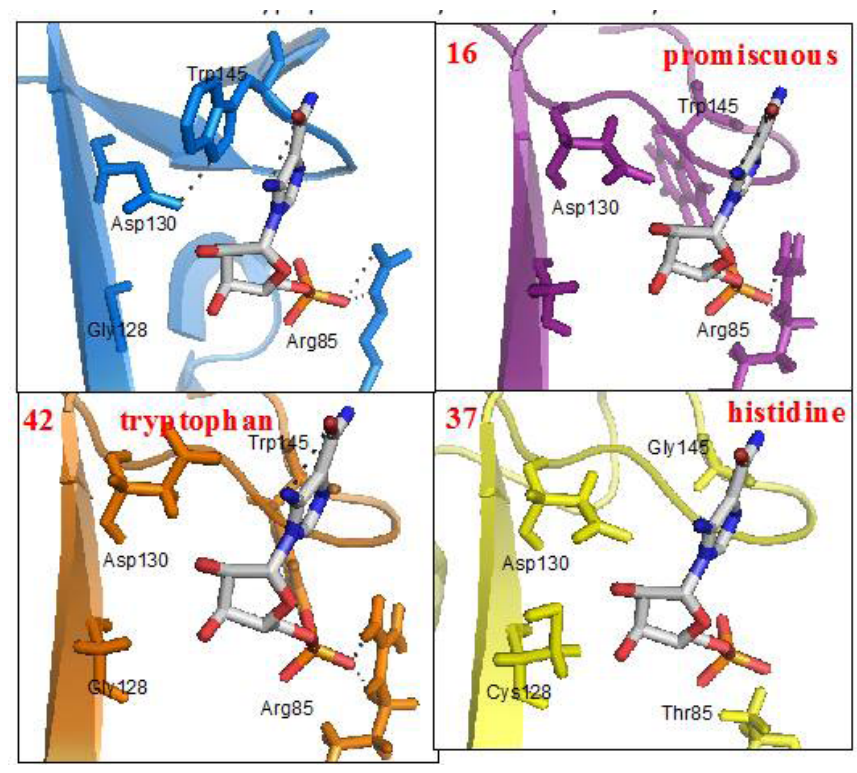

Figure 3. Molecular dynamics-based analysis of various structural variants of the TrypC enzyme binding with the substrate of the tryptophan biosynthesis pathway.

This work demonstrates how the integration of bioinformatics, cheminformatics, and structural proteomics enables the prediction of promiscuity, the prediction of key residues in protein sequences that control promiscuity, and the prediction of how changes in specific amino acids at key locations in protein structures result in changes to promiscuity. With an extended application of these principles to many more protein families, we will gain the ability to predict, control, and design new promiscuous activities for countless enzymes, opening up vast new applications for biochemistry, metabolic engineering, and synthetic biology. 


\section{Identifying Patterns and Associations among Hyperspectral Data and Meteorological and Biological Measurements for Investigating Near-Surface Atmosphere-Biosphere Interactions}

\author{
2014-132-R1
}

\author{
Yuki Hamada, David Cook, Nicola Ferrier, \\ and Roser Matamala
}

\section{PROJECT DESCRIPTION}

Atmosphere, plants, and soils control terrestrial carbon and water cycles. Researchers need to gain a better understanding of ecosystem dynamics at the biosphereatmosphere interface to enable more accurate climate forecasting. The goal of this project is to explore analytics for investigating temporal patterns and associations between optical measurements and meteorological and biological measurements by using high-temporalfrequency, hyperspectral reflectance measurements of the land surface. These associations will aid in understanding the indicative power of hyperspectral data in carbon and water exchange between the near-surface atmosphere and biosphere and facilitate parameterization of land components that will help improve climate modeling. The objectives are to (1) construct an environmentally resistant optical tower system to support autonomous data collection and streaming of land surface properties; (2) collect high-temporal-frequency, hyperspectral reflectance measurements of land surfaces; (3) determine associations and temporal patterns between hyperspectral measurements and meteorological/ biological measurements; and (4) determine the effectiveness and limitations of hyperspectral measurements for indicating ecosystem functions and processes, such as carbon and water fluxes.

\section{MISSION RELEVANCE}

This project is relevant to DOE's initiative in biology and the environment because it addresses the resolution of current roadblocks in collecting and analyzing hightemporal-frequency hyperspectral data for developing ecosystem measurements for improving current climate models. Outcomes of this project will benefit DOE Biological and Environmental Research (BER) Terrestrial Ecosystem Science programs by contributing new technologies to the AmeriFlux network. It will also benefit the DOE-BER (1) Systems Biology KnowledgeBase by integrating hyperspectral data into cloud computing to study plant/ecosystem activities and (2) Atmospheric Radiation Measurements (ARM) program by providing insight into the integration of land surface measurements into current atmospheric measurement capability.

\section{RESULTS AND ACCOMPLISHMENTS}

In FY 2014, we developed the robustly equipped towerbased optical sensor (EcoSpec) system and Linux-based software to support autonomous data collection and streaming in a synchronized manner. We performed rigorous testing of the system in indoor and outdoor environments. We completed all development and testing in order to deploy the system to a data collection site in FY 2015.

In FY 2015, we deployed the EcoSpec system to the Fermilab Agricultural AmeriFlux Site. We collected spectral reflectance measurements and other optical measurements approximately every minute from dawn to dusk from June 24 to October 8, 2015, and transmitted the data to an Argonne server daily. The 2015 data collection produced approximately $300 \mathrm{~GB}$ in raw data on ecosystem properties, functions, and processes across biological and temporal scales.

Seasonal analysis of the mid-day average of the 2015 data showed that the red-edge normalized vegetation index (RENDVI) calculated from the reflectance measurements consistently corresponded to gross ecosystem productivity (GEP) throughout the growing season (Figure 1), and that these optical and biological variables are strongly correlated $\left(R^{2}=0.854\right)$. RENDVI was also strongly correlated with ecosystem respiration $\left(R^{2}=0.830\right)$. The photochemical reflectance index was strongly correlated with $\mathrm{CO}_{2}$ flux $\left(\mathrm{R}^{2}=0.833\right)$. Three-dimensional visualization of diurnal spectral reflectance showed characteristic patterns, such as large spikes created by noise early and late in the day due to low solar angles and wavy features in the afternoon that likely correspond to sky conditions (Figure 2). 


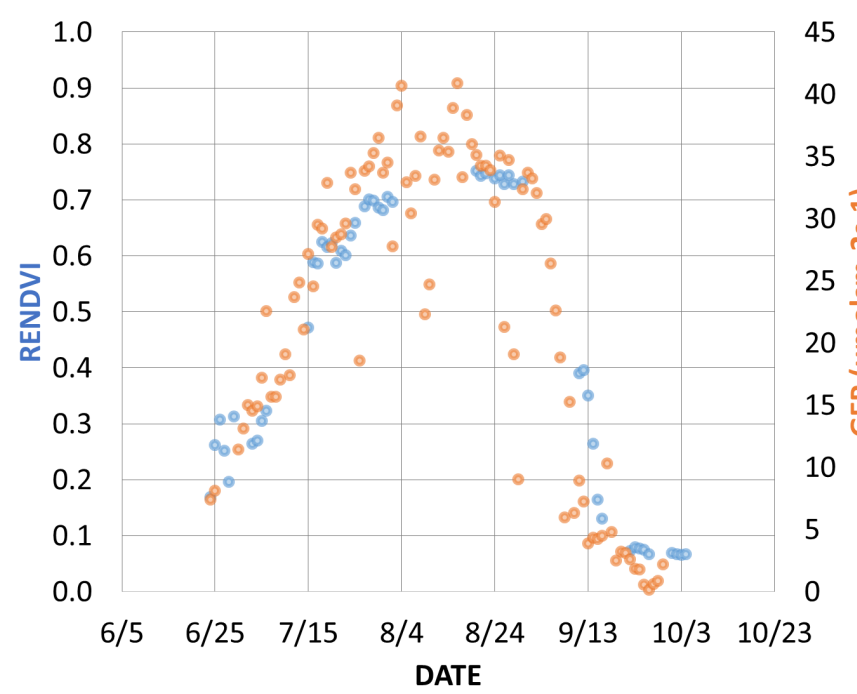

Figure 1. Mid-day average of RENDVI and GEP values at the Fermilab soybean field during the $\mathbf{2 0 1 5}$ growing season (June 24-October 8).

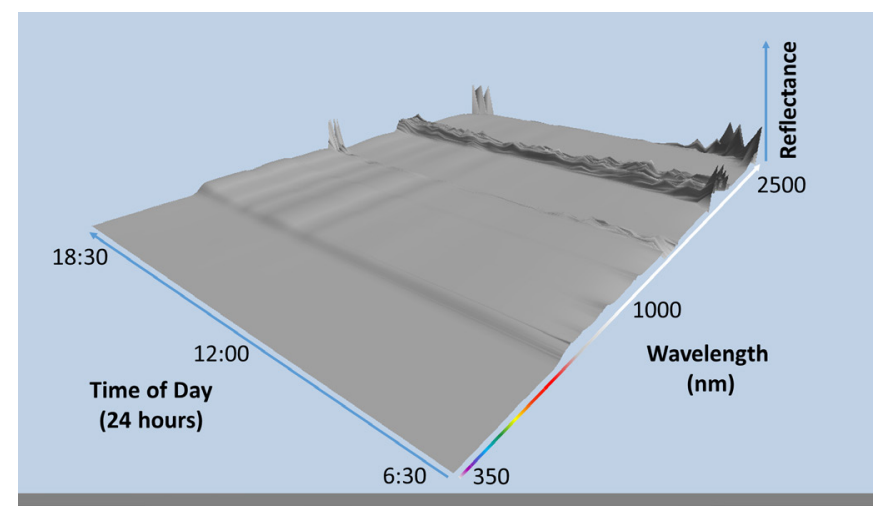

Figure 2. Diurnal spectral reflectance values at the Fermilab soybean field on August 22, 2015 (6:21 am to 6:58 pm).

\section{PROPOSED FUTURE WORK}

In FY 2016, we will continue to model effects of factors influencing hyperspectral reflectance measurements, including sun angle, cloud cover, atmospheric conditions, and incoming radiation characteristics. Specific tasks include (1) improving database management,

(2) collecting and analyzing 2016 growing season data,

(3) developing protocols for data quality assurance and control, (4) developing hyperspectral data analytics, and (5) determining the indicative power of hyperspectral data for ecosystem functions across scales. In fall and winter, we will calibrate, refine, and maintain the optical tower system to improve robustness and reliability.

\section{Minimizing Environmental Microbial Community Complexity at the Bench: Isolating and Characterizing Minimal Stable Communities (MSCs) Over Time}

\author{
2014-141-R1
}

Dionysios A. Antonopoulos, Theodore M. Flynn, Kenneth M. Kemner, Folker Meyer, and Edward J. O'Loughlin

\section{PROJECT DESCRIPTION}

Many natural environments (e.g., soil) exhibit ultra-high levels of microbial community diversity, obfuscating the interactions between organisms and how these interactions contribute to a community's stability. This project is focused on uncovering components of microbial community stability by isolating and studying what we term "minimal stable communities" (MSCs) via a high-throughput screening, cultivation, and monitoring platform.

\section{MISSION RELEVANCE}

This project has direct relevance to development of the DOE Systems Biology KnowledgeBase platform, as well as other components within the DOE Genomic Science Program. Notably, the research described here would contribute to the development of enabling technologies to achieve a dynamic, systems-level understanding of organism and community functions.

\section{RESULTS AND ACCOMPLISHMENTS}

We utilized parallel bioreactors to examine the effect of nutrient amendment on microbial communities in soil taken from five disparate environments (temperate prairie and forest, tropical forest, subalpine forest, and palustrine emergent wetland) by enriching for MSCs in each bioreactor and then coupling it with massively parallelized DNA sequencing to compare the profiles of the enrichments en masse. In FY 2014, we honed our approach for uncovering hypothetical MSCs from environmental materials by testing "washings" and dilutions of material in order to separate microorganisms from the environmental particulate matrix. As a result, we were able to determine the optimal incubation time required for respiration and/or growth in subsequent experiments. 
Presently, dilute subsamples of material from each environment were used to inoculate 96-well microtiter plates amended with one of 31 carbon sources from 6 different classes of organic compound in triplicate. After incubation, we measured microbial activity and performed microbial community analysis. DNA sequencing libraries were generated to inventory the organisms present in each well by targeting ribosomal RNA, which is critical to the synthesis of proteins.

Non-metric multi-dimensional scaling (NMDS) was used to display the differences between the communities from each enrichment well (Figure 1). The most divergent communities were those started with tropical and subalpine soil and wetland water. Based on the analysis of similarity (ANOSIM), the tropical soil enrichments differed the most from the others. The subalpine forest enrichments produced a gradient distinct from that of the tropical and temperate enrichments, while the temperate enrichments showed significant overlap with one another.

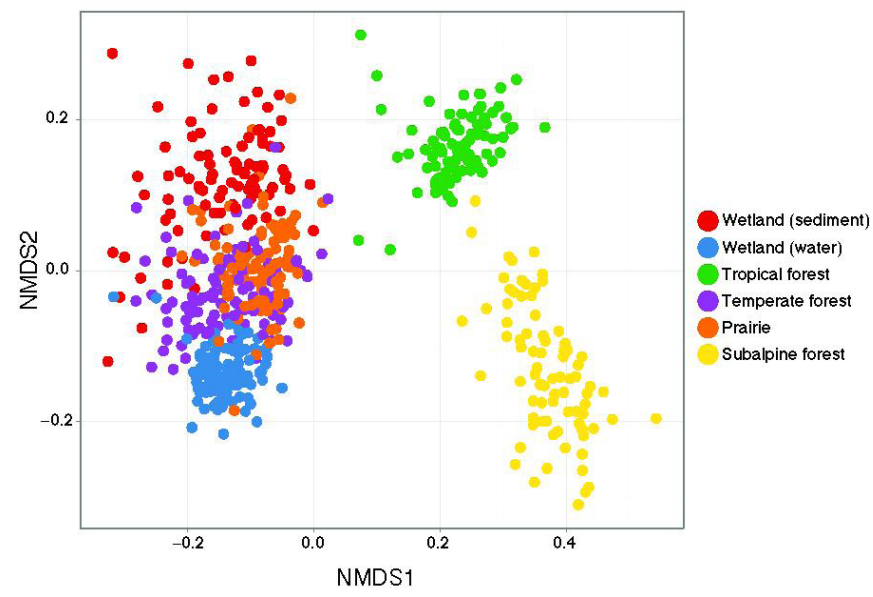

Figure 1. NMDS analysis based on the weighted UniFrac distance metric showing how microbial community structure varies in different environments.

The most abundant organisms in the enrichments were not the most abundant organisms in the environments. Sequences from Pseudomonas accounted for 36\% of all sequences in the enrichments but had an average environmental abundance of only $0.26 \pm 0.17 \%$. In five of the six experiments, Pseudomonas was enriched 61- to 300-fold relative to the inoculum (Figure 2). Others, like Ralstonia, were considerably enriched in some environments (tropical and subalpine forest) but absent or depleted in others. Overall, the operational taxonomic units (OTUs) most abundant in the enrichments were Proteobacteria and Bacteroidetes, while OTUs from Actinobacteria, Verrucomicrobia, and Acidobacteria were considerably less abundant. However, each individual well comprised multiple organisms, validating this approach for enriching MSCs.
Pseudomonas

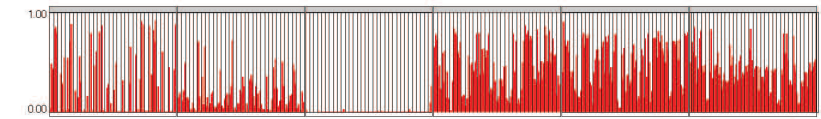

Ralstonia

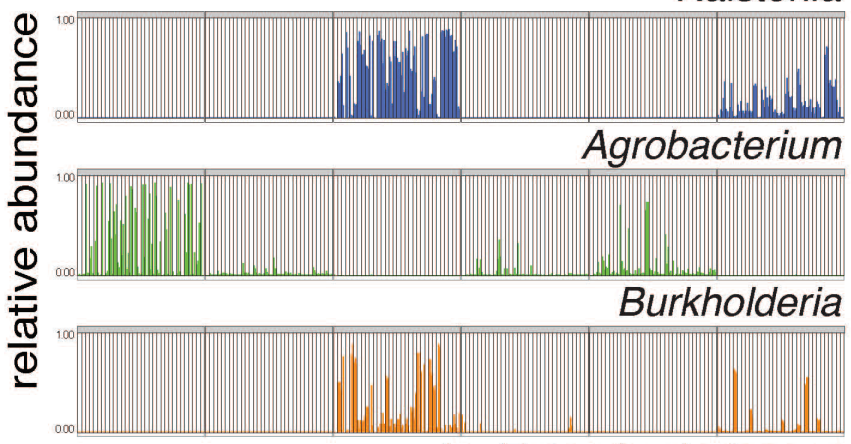

fa. Enterobacteriaceae

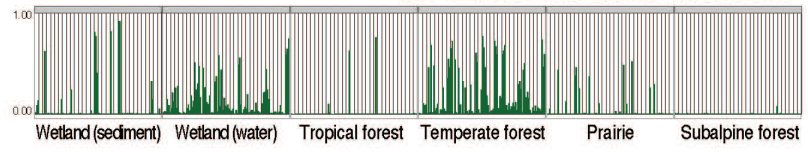

Figure 2. Relative abundance of the top five taxa in the enrichments, with triplicate samples grouped by carbon amendment.

Global ANOSIM values were calculated to compare microbial communities within each substrate category and individual substrate. Most significantly, comparison of carbohydrate and phenolic enrichments showed statistically significant ANOSIM values in three enrichments: wetland water, temperate forest, and subalpine forest. None of the other within-environment comparisons yielded significant results for more than one of the six environmental sources.

That the initial abundance of an organism does not determine its abundance after enrichment provides evidence for niche adaptation by organisms better adapted to nutrient-rich conditions. Yet we found that the environmental source used to inoculate the well, not the carbon source it was amended with, was the strongest factor influencing the overall community structure. This suggests that neutral, stochastic factors (the original community composition) also shape how the community composition responds to environmental changes.

\section{PROPOSED FUTURE WORK}

We now have a novel dissection approach for studying natural complex communities by uncovering natural subcommunities (candidate MSCs) based on substrate utilization. In FY 2016, we will monitor their stability and expression dynamics over time in response to abiotic/ biotic perturbations using continuous cultivation systems. These systems, unlike static (batch) systems, provide a constant influx of influx and efflux of biomass in such a way that the flow rate of the system is commensurate 
with the growth rate of its organisms. In this context, the response of the MSCs to an abiotic/biotic perturbation will be monitored over time via the efflux biomass as it is collected.

\section{Developing Remote Automated Sensors to Direct Sampling of Aerobic-Anaerobic Switching in Floodplain Ecosystems to Characterize the Response of Microbial Carbon Metabolism at High Temporal Resolution}

\author{
2014-145-R1
}

\section{Jack A. Gilbert, Yuki Hamada, Christopher Henry, Kenneth M. Kemner, Christopher L. Marshall, Sarah O’Brien, and Edward J. O'Loughlin}

\section{PROJECT DESCRIPTION}

Variation in the localized concentration and availability of terminal electron acceptors, such as iron(III) compounds and nitrates, are likely to influence the abundance of anaerobic respiratory microorganisms that utilize the reduction of these compounds for energy. Thus, changes in the concentration and availability of terminal electron acceptors will alter the onset of methanogenesis and flux of methane because of the competition between methanogenic archaea and anaerobic respiratory bacteria for the same electron donors (e.g., hydrogen and acetate). Additionally, terminal electron acceptors are required for anaerobic methanotrophy that will also modulate methane flux to the atmosphere. However, the genomic and regulatory response to different initial concentrations of electron acceptors is poorly characterized,

especially regarding their influence on methanogenesis time-to-onset and duration and the resultant methane flux. The genotypes and functional markers for these processes are likewise poorly characterized. Thus, this project will capture the genomes of the key organisms involved in methanogenesis and determine how they will respond to changing concentrations of terminal electron acceptors.

\section{MISSION RELEVANCE}

The DOE's Biological and Environmental Research Program is developing new initiatives aimed at advancing understanding of Earth's integrated biogeochemical processes to identify key factors controlling the storage; release; or transport of carbon, nutrients, or contaminants in the environment. The Genomic Science Program specifically has highlighted the value of genome sequencing of microbes and complex environmental assemblages of organisms. Microbial communities are essential components of all ecosystems but particularly at the interface between terrestrial and aquatic ecosystems, such as floodplains. Elucidating the metabolic capabilities and interactions that enable bacterial, archaeal, and viral assemblages to adapt to the dynamic change in redox potential in these environments is essential to our understanding of ecosystems.

\section{RESULTS AND ACCOMPLISHMENTS}

Following our project planning discussions in early FY 2014, we began the development of the microcosm system that was used in the experiments that are currently ongoing. We also collected samples from a series of floodplains in Holland, MI, representing a gradient since restoration that enables us to test our hypotheses about the influence of the microbial biogeographic distribution on methane turnover and flux in these environments. We collected samples from four floodplain sites that were restored $2 \mathrm{yr}, 6 \mathrm{yr}, 20 \mathrm{yr}$, and $>20$ yr ago; we collected more than 60 soil samples from three depths at each location. In FY 2015 we extracted DNA and created $16 \mathrm{~S}$ ribosomal RNA libraries from these studies. In addition, soil physicochemistry was characterized to understand how soil properties influence specific microbes. Some of the key findings from this field campaign were that soil carbon:nitrogen ratio and $\mathrm{pH}$ were the main drivers of soil microbial community structure and that soil depth strongly influences microbial diversity (Figures 1 and 2). Interestingly, a poorly described methanogenic family, Methanomassilicoccaceae, was significantly upregulated at deeper soil depths and was the predominant methanogenic family at three of the four sites. We are currently identifying a series of these samples for shotgun metagenomics and genome assembly to explore strain-level variation in methanogens and methanotrophs through this system. 

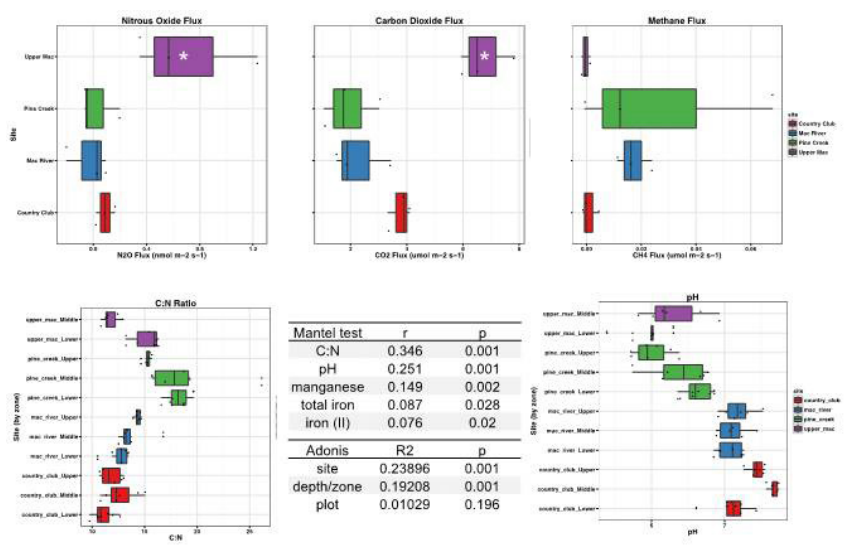

Figure 1. Gas flux and soil properties for each of the Holland field sites.

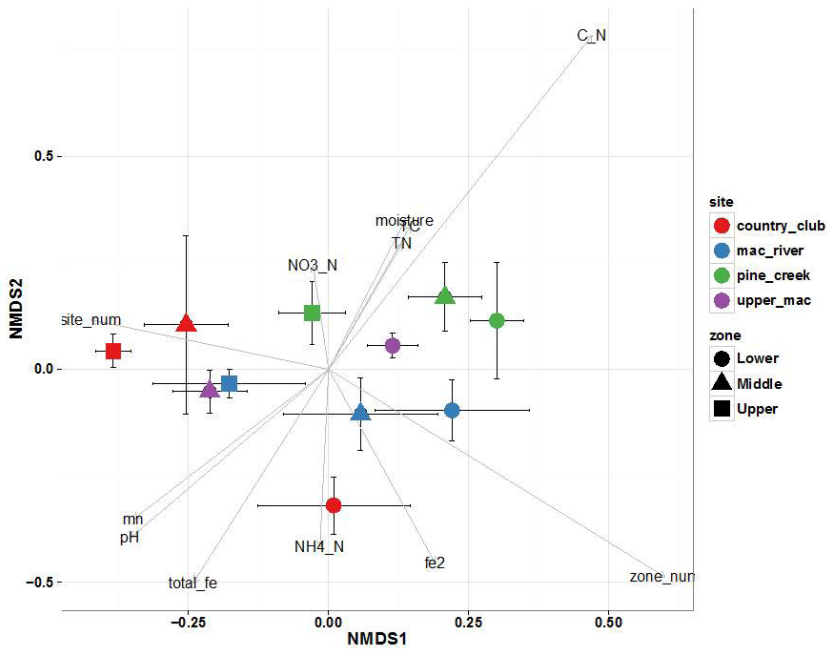

Figure 2. Non-metric multidimensional scaling (NMDS) of the Bray-Curtis diversity index. Vectors are the soil properties influencing the microbial communities.

In addition to the field campaign, a laboratory experiment is under way to investigate cycling redox conditions on methanogenic activity. Soil samples from the top and bottom layer at each site were used as inocula. In most cases, the microcosms generated methane in the initial anoxic phase of the experiment and were then subjected to an oxygen surplus followed by a return to an anoxic state (Figure 3). This anoxic-oxic transition simulates the redox cycling of flooding and will shed light on the methane-generating capacity of these sediments following these transitions.

\section{PROPOSED FUTURE WORK}

Samples from both the field and lab experiments will be selected for deep metagenomic sequencing to understand the role of the Methanomassilicoccaceae and other novel methanogenic clades in methane flux. This is an underexplored methanogenic family with a potential for widespread implications on methane production in response to flooding. We plan on assembling draft genomes of these taxa to characterize their competitive advantage over other methanogens in these systems. The laboratory experiments will continue with high-frequency sampling to monitor fine-scale changes in the microbial community in response to nutrient limitations and environmental insults.

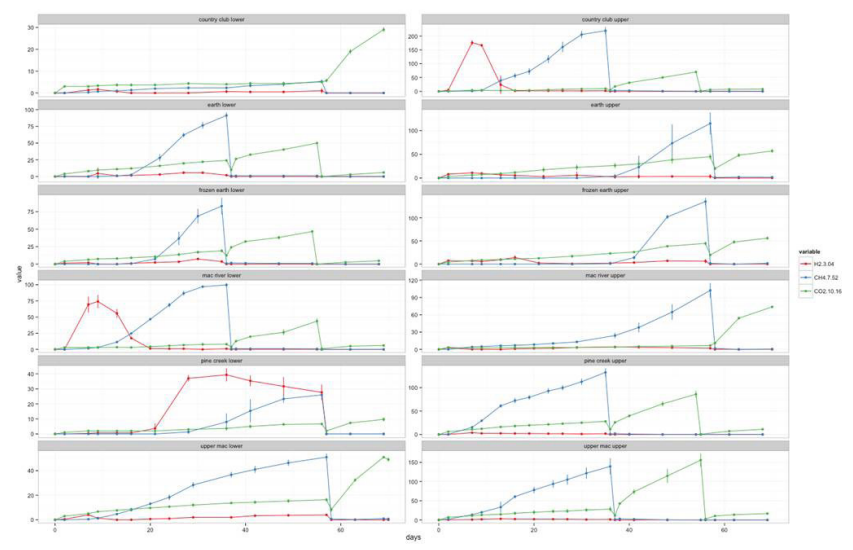

Figure 3. Hydrogen, carbon dioxide, and methane production in each of the enrichments. The points where production steeply drops are when oxygen was either added or removed from the system.

\section{Biology@Speed: D-Factory, A Novel Experimental Framework}

\section{4-157-R1}

\section{Gyorgy Babnigg, Andrew Binkowski, Robert Jedrzejczak, Andrzej Joachimiak, Karolina Michalska, and Boguslaw Nocek}

\section{PROJECT DESCRIPTION}

Future gene sequencing and bioinformatics studies will require more automation, significantly higher throughput, and direct translation of in-silico ideas to executable workflows on a highly modular platform. The aim of this project is to develop the technology to enable high-throughput (HTP) "biology at speed." This platform will consist of a hardware layer, including modular microfluidics core engines coupled with detectors, and an abstraction layer describing workflows coupled with an application programming interface. The platform will use off-the-shelf products, incorporate in-house technologies designed specifically for a given task, and involve the Center for Nanoscale Materials (CNM) and outside vendors. The microfluidics-based HTP assay platform will be benchmarked against traditional assay setups. 


\section{MISSION RELEVANCE}

The project is relevant to DOE's mission in science. Biology@speed will reduce current bottlenecks in experimental biology. The modular and reconfigurable design makes it compatible with current and future science needs. The project is, therefore, relevant to the DOE Systems Biology Knowledgebase (KBase) and other biology projects funded by DOE and the National Institutes of Health (NIH).

\section{RESULTS AND ACCOMPLISHMENTS}

In FY 2014, we explored several avenues to establish the microfluidics infrastructure: (1) the use of the CNM, (2) the use of fabrication resources offered by universities and industry, and (3) the testing of off-the-shelf microfluidics products. We set up dedicated laboratory space for the microfluidics work at Argonne's Advanced Protein Characterization Facility. We also established techniques for the cultivation of anaerobe microbes, whole-genome amplification, and isolation of messenger ribonucleic acid (RNA). We constructed several expression vectors and miniaturized and optimized assays in the microfluidics setting for glycoside hydrolases, which are important in DOE bioenergy missions, and beta-lactamases, which are relevant to $\mathrm{NIH}$ biomedical initiatives.

In FY 2015, we designed new microfluidic HTP devices, namely, a new droplet generator and sorting chips. These were fabricated commercially. We also started to fabricate a microchemostat (Figure 1). Additional microfluidic devices-droplet mergers and double-emulsion generators-were acquired from vendors. We finished installing the dedicated confocal microscope for detecting fluorescent signals in microfluidic chips and developed electronic control mechanisms for droplet sorting.

We further optimized the cultivation of anaerobe microbes. Genomic deoxyribonucleic acid (DNA) and messenger RNA were isolated from three actively growing anaerobes rich in carbohydrate-active enzymes (CAZymes): Bacteroides intestinalis, Bacteroides cellulosilyticus, and Bacteroides salanitronis. The samples were used for regular and complementary DNA library generation by a commercial service. Unfortunately, the yield of library DNA was poor, and the fragments were too short to contain full-length CAZyme genes when tested via targeted polymerase chain reaction (PCR). We are currently optimizing an alternative method for expression library production.

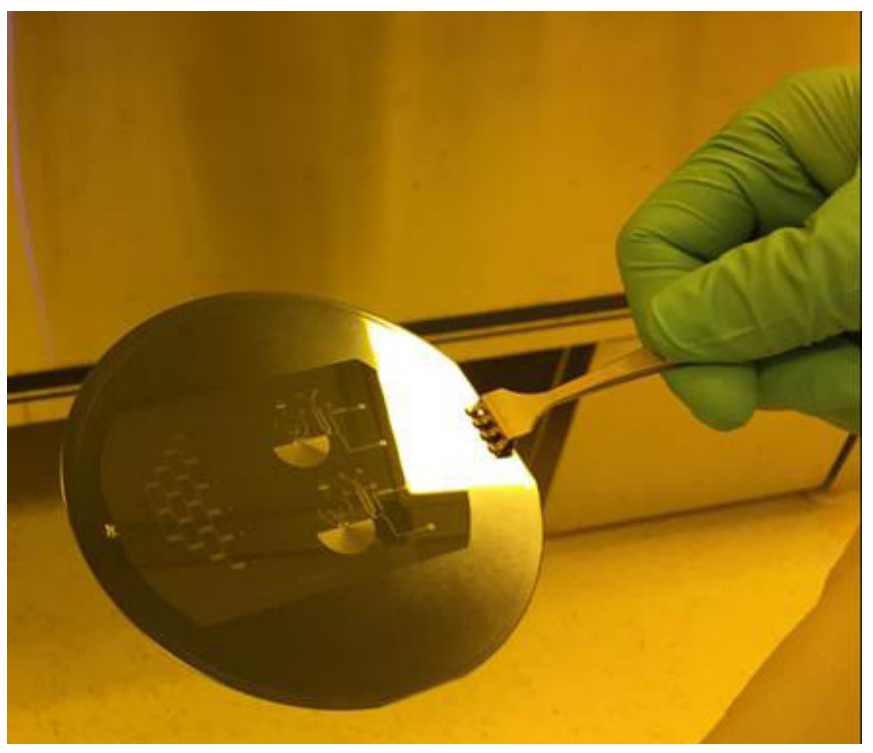

Figure 1. Microchemostat fabrication at the CNM.

We characterized several enzymes in 96-well plate assays. These included assays for beta-lactamases and glycoside hydrolases. We showed that fluorocillin, a fluorescent beta-lactamase substrate, can be used in both cell-free and cell-based microfluidic assays (Figure 2). The microfluidic assay requires less than 1 millionth of the volume of reagents used in traditional 96-well assays. We also tested the feasibility of using fluorocillin to indirectly measure enzyme activities against mixed-in beta-lactam antibiotics. We characterized several glycoside hydrolases via direct assays using fluorescent substrates or via coupled assays. We established a protocol to assay the activity of the heterologously expressed protein from a single cell encapsulated in a droplet. Several combinations of fluorinated oils, surfactants, and detergents were identified and tailored for a given single-cell biological assay. We also optimized conditions to culture single cells in microfluidic droplets and developed ways to monitor cellular activities and fitness (Figure 3).
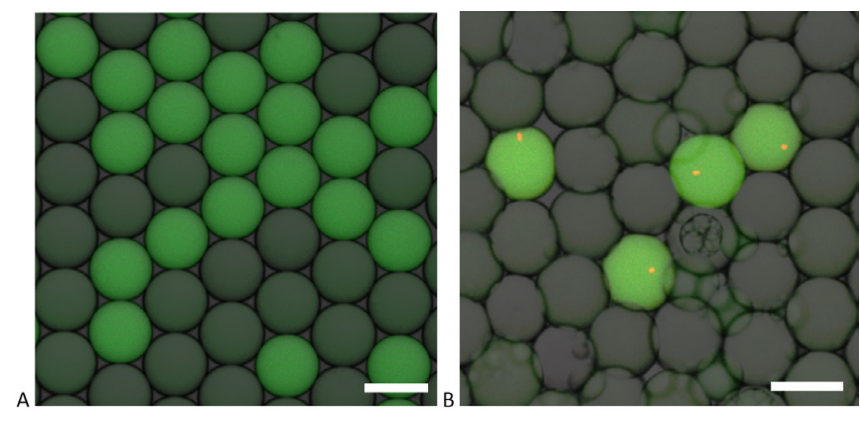

Figure 2. Cell-free (A) and cell-based (B) enzyme assays optimized for beta-lactamases and glucoside hydrolases. Bar length is $35 \mu \mathrm{m}$. 

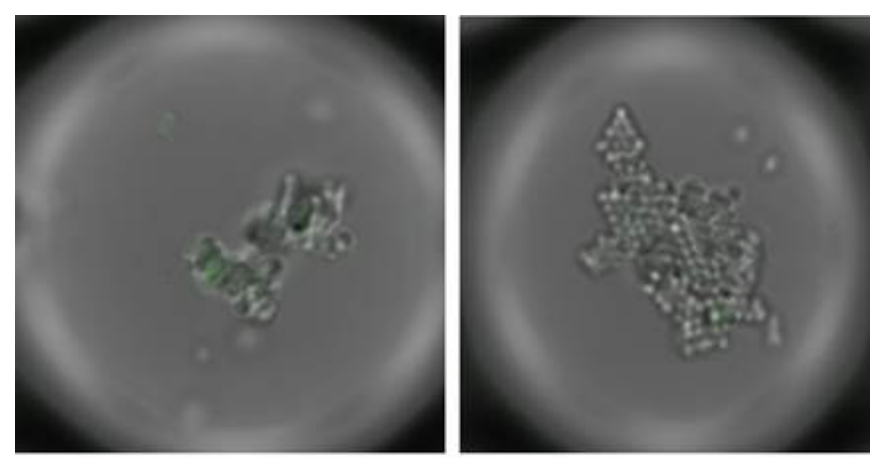

\title{
Impact of Radiation and Surface Turbulent Fluxes on the Transition from Stratocumulus to Cumulus Cloud Regime
}

\author{
2014-183-R1
}

Virendra P. Ghate, Rao Kotamarthi, and John Krummel
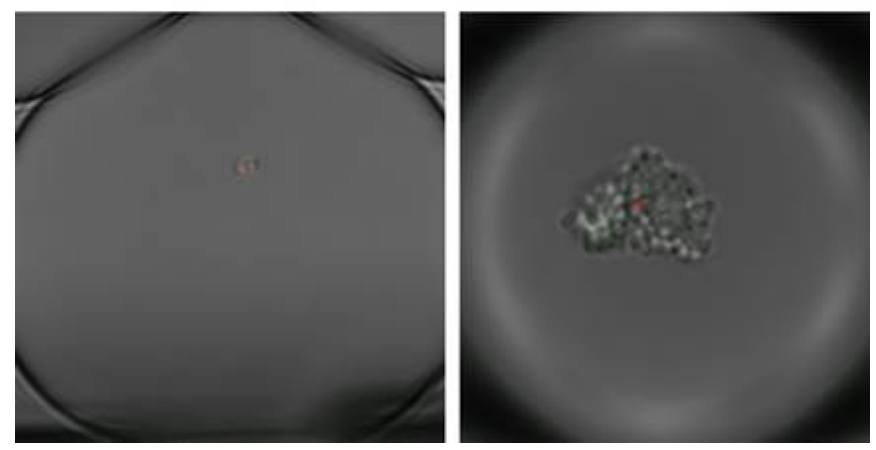

Figure 3. Droplet-based single-cell cultivation.

In order to multiplex assays, barcoding of the droplets is necessary so the original condition can be recalled during downstream processing. After exploring various droplet barcoding techniques, we obtained optimal barcoding with a mix of fluorescent beads and dyes.

Current quantitative droplet-PCR techniques are designed to amplify shorter DNA fragments (less than 500 nucleotides). We explored combinations of various microfluidic devices, fluorinated oils, surfactants, and PCR reaction mixtures to successfully optimize the amplification of a 3-kilobase test DNA fragment. This method will allow us to generate long DNA templates used in droplet-based, cell-free protein expression-coupled enzymatic assays.

\section{PROPOSED FUTURE WORK}

The microfluidics sorting framework will be finalized and tested with the beta-lactamase and glucosidase assays using expression libraries generated from several anaerobes. The droplet PCR method will be applied to generate bead-based expression libraries, which will be used for the cell-free protein expression-based assays. A demonstration automation hardware and software layer will be developed.

\section{PROJECT DESCRIPTION}

Marine stratocumulus clouds cover vast areas of Eastern subtropical oceans. These clouds reflect greater amounts of solar (shortwave) radiation back to space compared to the amounts reflected by the ocean surface, while emitting similar amounts of terrestrial (longwave) radiation as the surface because of the lower heights of their cloud tops. Hence, they are a significant component of the Earth's radiation budget and need to be represented accurately in the global climate models (GCMs) used to predict future climate conditions and energy needs. The overall goal of this project is to improve the representation of marine stratocumulus clouds in GCMs and specifically to understand the transition of stratocumulus clouds to the cumulus cloud field in the context of changes in boundary layer radiation and surface energy fluxes.

\section{MISSION RELEVANCE}

This project is relevant to the DOE's energy, environment, and basic science missions. The research uses data collected at the Atmospheric Radiation Measurement (ARM) climate research facility to quantify the interactions among aerosols, clouds, precipitation, radiation, and atmospheric dynamics and thermodynamics to improve our fundamental, process-level understanding, with the ultimate goal of reducing uncertainty in global and regional climate simulations and projections.

\section{RESULTS AND ACCOMPLISHMENTS}

The main factors responsible for affecting the lifetime of marine stratocumulus clouds and eventually causing their transition to cumulus clouds are (1) a reduction in boundary layer radiative cooling, (2) an increase in surface buoyancy, (3) a decrease in lower tropospheric stability, and (4) an increase in precipitation-induced evaporative cooling. In FY 2014, we used the data collected by multiple instruments in the Southeast Pacific, Eastern North Atlantic, and the U.S. Southern Great Plains during stratocumulus cloud conditions to characterize the 
boundary layer radiative and thermodynamic structure during well-mixed and decoupled conditions. Our results suggested the stratocumulus-topped boundary layers differ significantly among the three regions, with multiple processes being responsible for causing boundary layer decoupling, which is the first step toward the cloud transition. To gain further insights into the processes responsible for producing decoupling and the relative impact of the above factors on the transition of a stratocumulus to a cumulus cloud field, in FY 2015, we used data collected during one trip from Los Angeles to Hawaii during the Marine ARM GCSS Pacific Crosssection Intercomparison (GPCI) Investigation of Clouds (MAGIC) field campaign (Figure 1). (GCSS stands for the GEWEX Cloud Systems Study, and GEWEX stands for the Global Energy and Water Cycle Experiment, which is a core project of the World Climate Research Programme.) Data from the vertically pointing cloud radars operating at two different wavelengths were combined to retrieve the cloud and drizzle liquid water contents. The observations of surface-sensible heat flux and latent heat flux were used to characterize the buoyancy production at the surface, while the observations made by balloonborne radiosondes were used to quantify the lower tropospheric stability. Our preliminary results suggest the boundary layer (1) gradually deepens westward with a corresponding increase in the height of the lifting condensation level (LCL), (2) increases in the surface buoyancy, (3) decreases in large-scale subsidence, and (4) weakens the boundary layer inversion.

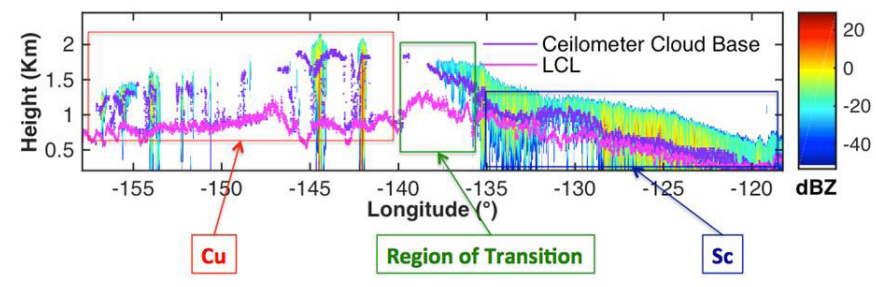

Figure 1. Longitude vs. height mapping of the radar reflectivity as reported by the vertically pointing Ka-band cloud radar onboard a ship during the MAGIC field campaign. The ceilometer recorded cloud base height, and the lifting condensation level (LCL) is also shown. The stratocumulus (Sc), Cumulus $\mathrm{(Cu}$ ), and the transition region are also marked. The unit $\mathrm{dBz}$ (decibels of reflectivity) is a meteorological measure of the strength of radar return from, for example, precipitation.

\section{PROPOSED FUTURE WORK}

During FY 2016, we will use the developed estimates of liquid water content to characterize the cooling induced by drizzle evaporation. These estimates will also serve as an input to a one-dimensional radiative transfer model, the output of which will be used to characterize boundary layer radiative cooling. Finally, we will compare the evolution of the four different factors during the transect to assess their relative impact on the transition from stratocumulus to cumulus clouds.

\section{Self-Assembled Functional Membranes for Filtration and Photocatalytic Water Treatment}

\author{
2014-187-R1
}

\section{Seth B. Darling and Jeffrey W. Elam}

\section{PROJECT DESCRIPTION}

We are applying a novel approach for the synthesis of photocatalytic low-fouling membranes, wherein the top layer is a nanoporous titania $\left(\mathrm{TiO}_{2}\right)$ membrane. These photocatalytic membranes with controllable nanopores (size and shape) have the potential to overcome the limitations of current filtration systems. We prepare nanoporous titania membranes using cylinder-forming block copolymer thin films on a solid substrate as templates. The poly(styrene-block-methyl methacrylate) (PS-b-PMMA) system is the primary material of our focus. Polystyrene (PS) cylindrical domain size and spacing can be varied from $\sim 15$ to 50 nanometers ( $\mathrm{nm}$ ), depending on the molecular weight of the starting polymer material. Orientation of the self-assembled microdomains is manipulated using surface chemistry, such as through the application of brush layers of random copolymers to obtain a neutral interfacial interaction and therefore vertical orientation of the cylindrical domains. These oriented block copolymer films serve as a template for the growth of nanoporous photocatalytic material. Titanium dioxide (i.e., titania, $\mathrm{TiO}_{2}$ ) is selectively and controllably grown within the polymethyl methacrylate (PMMA) domains using a self-limited, gas-phase technique developed at Argonne called sequential infiltration synthesis (SIS). SIS entails alternating exposure of a polymeric film to gas-phase precursors using an atomic layer deposition (ALD) reactor operating in a semistatic mode; the precursors penetrate into the film and chemically react with moieties on the polymer chains. In the case of titanium dioxide, typical precursors are titanium tetrachloride $\left(\mathrm{TiCl}_{4}\right)$ and water. Under these conditions with a PS-b-PMMA film as the substrate, $\mathrm{TiO}_{2}$ grows selectively in the PMMA domains because of chemical interactions between the $\mathrm{TiCl}_{4}$ molecules and the carbonyl moieties along the PMMA chains. Subsequent reaction with water completes one SIS cycle and leaves hydroxyl groups available, which in turn seed the $\mathrm{TiO}_{2}$ growth in subsequent SIS cycles. An important advantage of SIS is that the reaction is self limited, meaning that a well-defined amount of material will be grown with each cycle; thus, by selecting the number of cycles, the amount of material grown-and hence the nanostructural dimensions-can be precisely tuned. The 
residual polymer is then removed by thermal treatment, which also serves to crystallize the titania into the anatase phase, thereby rendering it photocatalytically active.

\section{MISSION RELEVANCE}

Our society is entering a period of water crisis, as highlighted in the DOE Water-Energy Nexus Report in 2014. As energy and water are intimately intertwined, this project helps advance the energy security of the United States through delivery of scientific discoveries. New filtration technologies will be a central piece of the solution to this grand challenge. Here we are using a low-cost combination of block copolymer self-assembly and a novel technique called sequential infiltration synthesis to prepare nanoporous membranes with unique and tunable mechanical, filtration, and catalytic properties.

\section{RESULTS AND ACCOMPLISHMENTS}

We developed two methods to fabricate a multi-functional $\mathrm{TiO}_{2}$ membrane: in method I, $\mathrm{TiO}_{2}$ membrane formation occurs via SIS using PS-b-PMMA as a template, and in method II, nitrogen (N)-doped and un-doped $\mathrm{TiO}_{2}$ depositions via atomic layer deposition (ALD) occurs using various ceramic filters as templates. In the case of method I, which was the focus in FY 2014, we established the optimal experimental parameters to form vertically oriented PS cylinders in a PMMA matrix, which serves as a template for selective $\mathrm{TiO}_{2}$ deposition. We then successfully established SIS conditions for $\mathrm{TiO}_{2}$ deposition. Membranes were fabricated following method I exhibiting a pore diameter of $41.9 \pm 6.3 \mathrm{~nm}$. In the case of method II, which was the focus in FY 2015, we successfully developed a fabrication method for $\mathrm{N}$-doped and undoped $\mathrm{TiO}_{2}$ membranes via ALD using ceramic alumina and zirconia/titania filters as templates. Figure 1 shows ultraviolet (UV)-visible optical absorption spectra of doped and undoped $\mathrm{TiO}_{2}$ membranes. A clear absorption edge spanning toward visible transitions was observed for the novel $\mathrm{N}$-doped $\mathrm{TiO}_{2}$ membrane, and this observation is in good agreement with the calculated imaginary part of the permittivity of $\mathrm{N}$-doped $\mathrm{TiO}_{2}$. In order to test the visible-light activity of the doped $\mathrm{TiO}_{2}$ coating, photocatalytic degradation experiments were performed. Aqueous solutions of a model organic dye were prepared and placed with the coated membranes in a stirred cell under exposure to simulated sunlight from a xenon lamp with an infrared filter. Figure 2 shows the results of this experiment in which uncoated membranes were unable to break down any of the organic dye molecules while the membranes coated with doped titania successfully degraded the dye. The number of cycles as shown in the legend pertains to the number of ALD layers, with thicker coatings exhibiting reduced dye degradation as a result of reduced fluid flow in the more restricted nanopores.

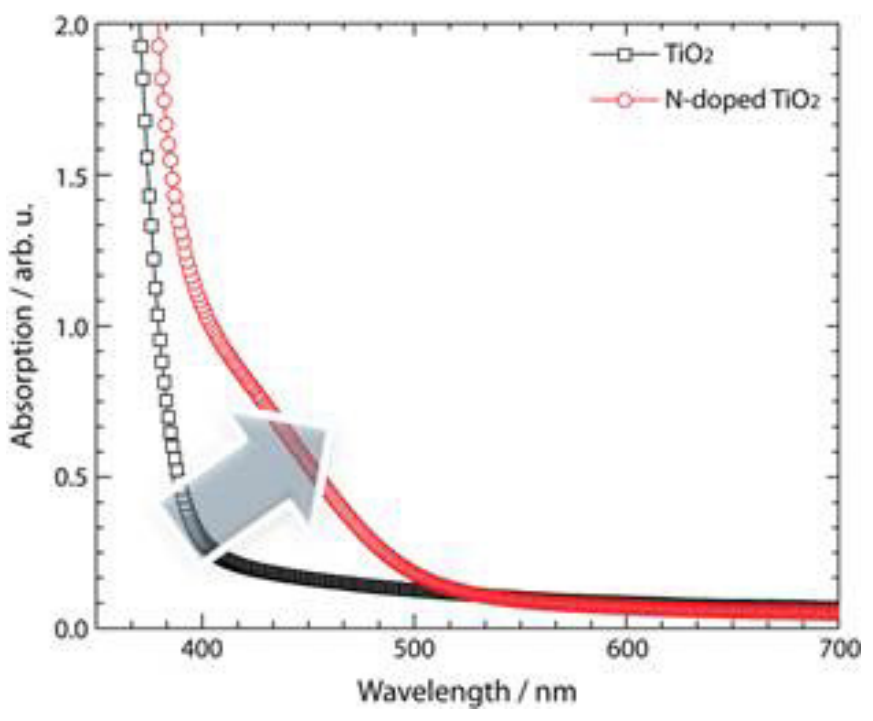

Figure 1. UV-visible optical absorption spectra of a traditional titanium dioxide coating on a membrane compared with a doped titanium dioxide coating, showing improved harvesting of light in the visible region of the spectrum by the $\mathrm{N}$-doped coating.

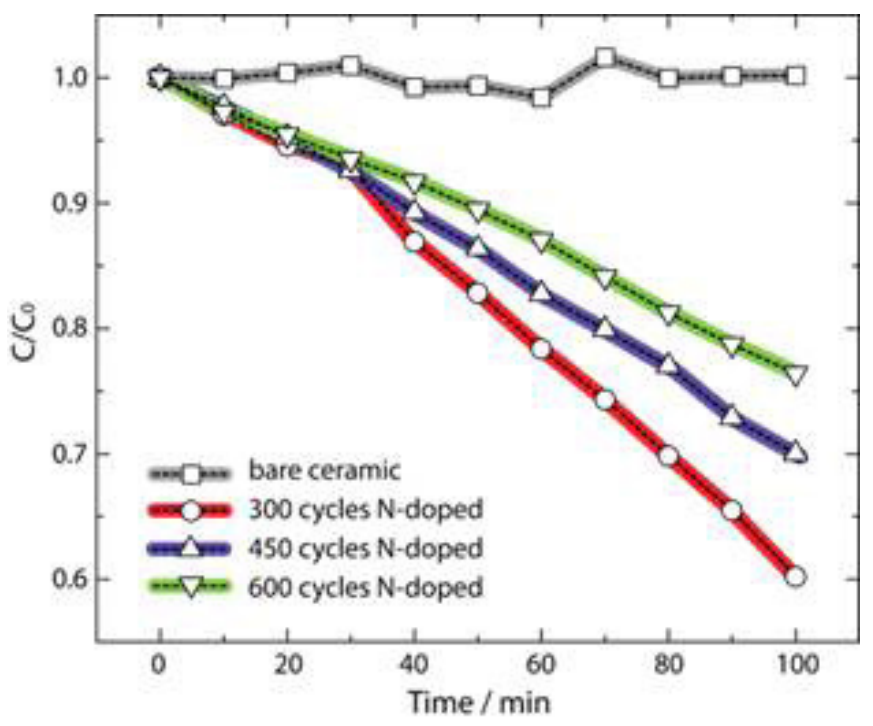

Figure 2. Degradation of a model organic "pollutant" (plotted as a normalized concentration where $C$ is the measured concentration and $C_{0}$ is the original concentration) under simulated solar illumination showing strong photocatalytic performance from the membrane coating.

This project concluded in FY 2015. The Institute for Molecular Engineering will support follow-on studies to quantitatively characterize the filtration capabilities of these membranes. 


\section{Timescale of Groundwater Transport: A Prerequisite for Developing and Maintaining Groundwater Reservoirs}

\author{
2014-188-R1
}

Zheng-Tian Lu, Wei Jiang, and Peter Mueller

\section{PROJECT DESCRIPTION}

Assessing groundwater behavior in aquifer systems is an indispensable part of developing and maintaining groundwater reservoirs. With the recent development of the Atom Trap Trace Analysis (ATTA) method at Argonne National Laboratory, measurements have been enabled of the rare noble gas isotopes krypton-81 $\left({ }^{81} \mathrm{Kr}\right.$ half-life $=230,000$ years) and ${ }^{85} \mathrm{Kr}$ (10.8 years), which possess nearly ideal chemical and physical properties for determining the timescale of groundwater transport up to one million years. Our project applies these tracers to investigate a number of aquifer systems that are important sources of groundwater exploitation in Israel and that range in age from approximately 10 to 500 thousand years. This project is being conducted with researchers from the University of Chicago and Ben Gurion University. Our research involves pursuing technical developments to enhance sample throughput capacity and promote accurate data interpretation. Once successfully implemented in Israel, this new methodology can also be used to characterize groundwater reservoirs from many hydrogeologically complex basins around the world.

\section{MISSION RELEVANCE}

This project supports missions in DOE's Office of Nuclear Physics, which has long fostered the development of the novel isotope trace analysis method as an application within the field. By using laser spectroscopy and the atom-trapping tools of nuclear physics, we have developed a sensitive instrument with a wide range of applications in the earth and environmental sciences.

\section{RESULTS AND ACCOMPLISHMENTS}

For the first time, the ages of samples of very old groundwater in Israel have been revealed and studied in detail. During the two years of this project, we completed sampling and analysis of 32 water wells across Israel (see Figure 1 for resulting krypton-81 ages at 20 sampling locations in southern Israel), of which many were found to be older than 30,000 years. The large measured age differences reflect slow underground flow and mixing patterns and can be used to obtain total capacity and recharge rates of the respective aquifers. Analysis of this data and comparison to numerical aquifer models is ongoing. Our results are expected to lead to significant reevaluations of groundwater flow patterns and available water resources in Israel. They have also guided the planning for a second major sampling campaign. This time, a total of 40 well locations were targeted, and the field work was completed as of November 2015. Support for sample preparation and analysis in the follow-up years was obtained through the United States-Israel Binational Science Foundation.

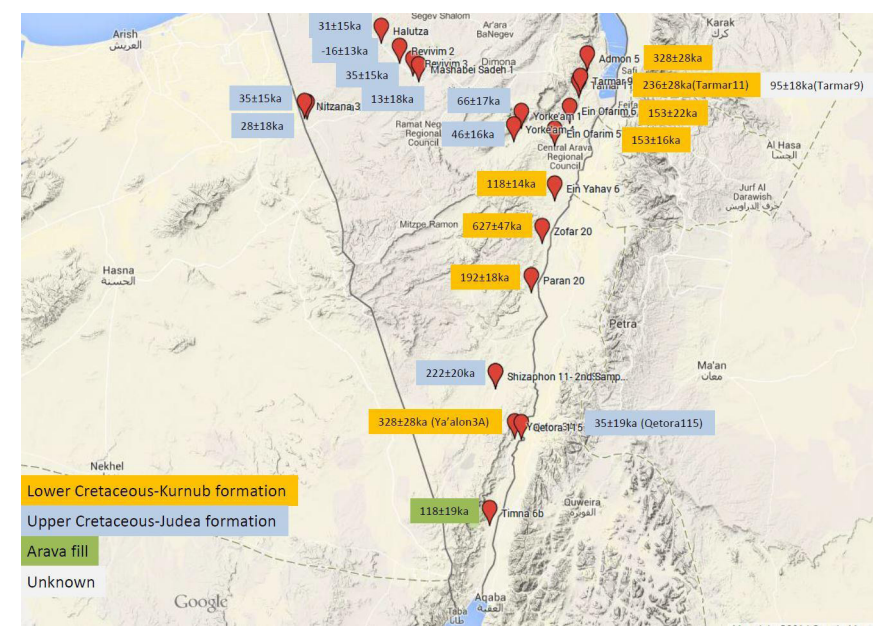

Figure 1. ${ }^{81} \mathrm{Kr}$ ages of groundwater in Negev Desert region of Israel; "ka" denotes "one thousand years."

Regarding development of instrumentation for ${ }^{81} \mathrm{Kr}$ dating, we implemented several upgrades to our apparatus to improve the reproducibility of its isotope ratio measurements and its sample throughput. Key changes concerned stabilization of laser beam intensities and alignments, as well as more frequent reference sample measurements. We also developed a new scheme to reduce sample cross-contamination. Previously, a 36-hour "wash cycle" was required between consecutive measurements to minimize instrumental memory effects and obtain reliable results. The new approach has reduced this time to 12 hours, effectively doubling our sample throughput to one sample per day. This new measurement protocol is now in routine use and has resulted in a significant improvement in ATTA as an analytical tool. 
Membrane-Biofilm Nexus: Advanced Membrane Autopsy as a Tool for Revealing Membrane Biofouling and Development of New Membrane Materials and Structures

\author{
2014-189-R1
}

Meltem Urgun-Demirtas, Jack A. Gilbert, and Seth W. Snyder

\section{PROJECT DESCRIPTION}

The interdependency of water and energy requires development of cost-effective water treatment technologies. Technological advances in membrane separations have been extensively used in water and wastewater treatment applications; however, membrane biofouling is a major obstacle in the application of membrane filtration processes in water purification and reclamation. Obtaining a fundamental understanding of the nature of biofouling is crucial to operating membranes sustainably to meet desired water quality and performance criteria. To this end, research partners Ben Gurion University (BGU) (PI: C.J. Arnusch), Argonne National Laboratory, and the University of Chicago are seeking to develop a tool to diagnose, predict, and provide guidance for the prevention and control of membrane biofouling. Three tasks were included: (1) determining the metagenomics of fouled membranes, (2) modeling biofouling mechanisms, and (3) developing new anti-fouling membrane surfaces.

\section{MISSION RELEVANCE}

The project is tied to DOE's mission in energy security and environmental quality. This project offers energy savings in water treatment, which is a critical focus of the energy-water nexus. This project's most critical strength is its alignment with DOE's energy-water mission. The Water-Energy Tech Team has determined that development of cutting-edge water treatment technologies is one of the most important areas. The core understanding of biofouling will draw significant attention from water/wastewater utilities and the membrane industry. This project seeks to provide essential industrial knowledge for the development of more robust "smart" membranes and more efficient water/wastewater treatment processes.

\section{RESULTS AND ACCOMPLISHMENTS}

An extensive literature search and a review of utilization of membrane types and materials at wastewater treatment plants were conducted in FY 2014. A "toolbox" of methods for the surface modification of membranes and identification of microbial community structure was developed. A series of polysulfone membranes was modified using ultraviolet (UV)-initiated graft polymerization via a traditional approach (UVA Cube) and a specially developed approach (three-dimensional [3D] printed membranes). The performance of the modified membranes was tested using secondary effluent water samples from a wastewater treatment plant.

In FY 2015, we expanded the application of the $3 \mathrm{D}$ printing technique and technology protocol to different membrane technologies. BGU developed new fabrication and modification techniques using two-dimensional inkjet printing, as well as 3D printing technology. Printing-assisted fabrication/modification (PAF/PAM) of ultrafiltration (UF) and thin-film composite membranes was demonstrated at the lab scale. PAF included the hybridization between inkjet printing technology and the interfacial polymerization process for the production of novel thin-film composite membranes. Furthermore, both PAF and PAM incorporated either inkjet printing or 3D printing for graft polymerization from nanofiltration/ reverse osmosis (NF/RO) and UF membrane surfaces. The patterned membrane surfaces using newly developed 3D printing techniques were tested with different water samples. The use of modern printing techniques for the fabrication and modification of polymer membranes has improved membrane surface properties and performance. Performance testing showed that membrane fouling depends on pattern type (Figure 1). Hydrophilic stripes parallel to water flow yielded the least amount of membrane biofouling compared with perpendicular stripes or control membranes. Experimental results also showed that membrane biofouling depended more on pattern type than coating composition.

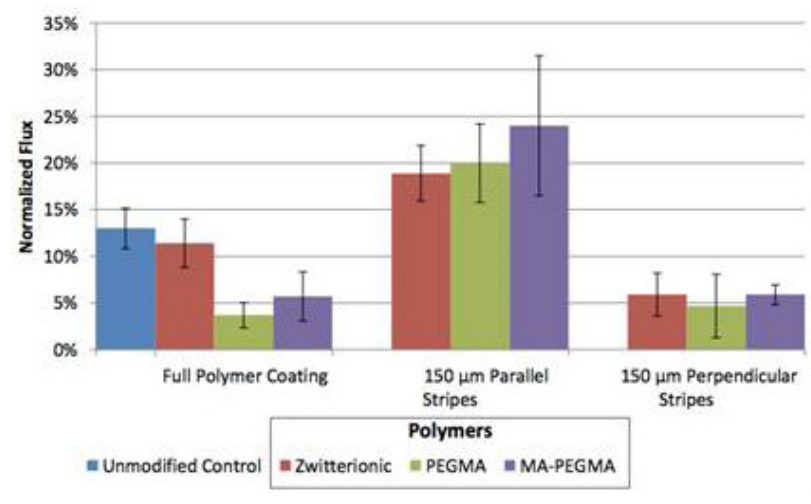

Figure 1. Performance of tested membranes. Error bars denote standard deviations for 4 replicate membranes tested with different wastewater samples. 
Metagenomic testing of fouled new membranes shows lower alpha diversity (species diversity) compared to their respective controls (Figure 2a). Alpha-diversity measurements in terms of Shannon indices clearly illustrates that unmodified membranes (in the control group) have higher microbial diversity (higher Sharon indices) compared to membranes with modified filters. The newly patterned membrane surfaces, hydrophilic parallel stripes, are less prone to membrane fouling (Figure 2b) since principal coordinate analysis (PCOA) plots of beta-diversity represented less diversity based on the taxonomic composition.
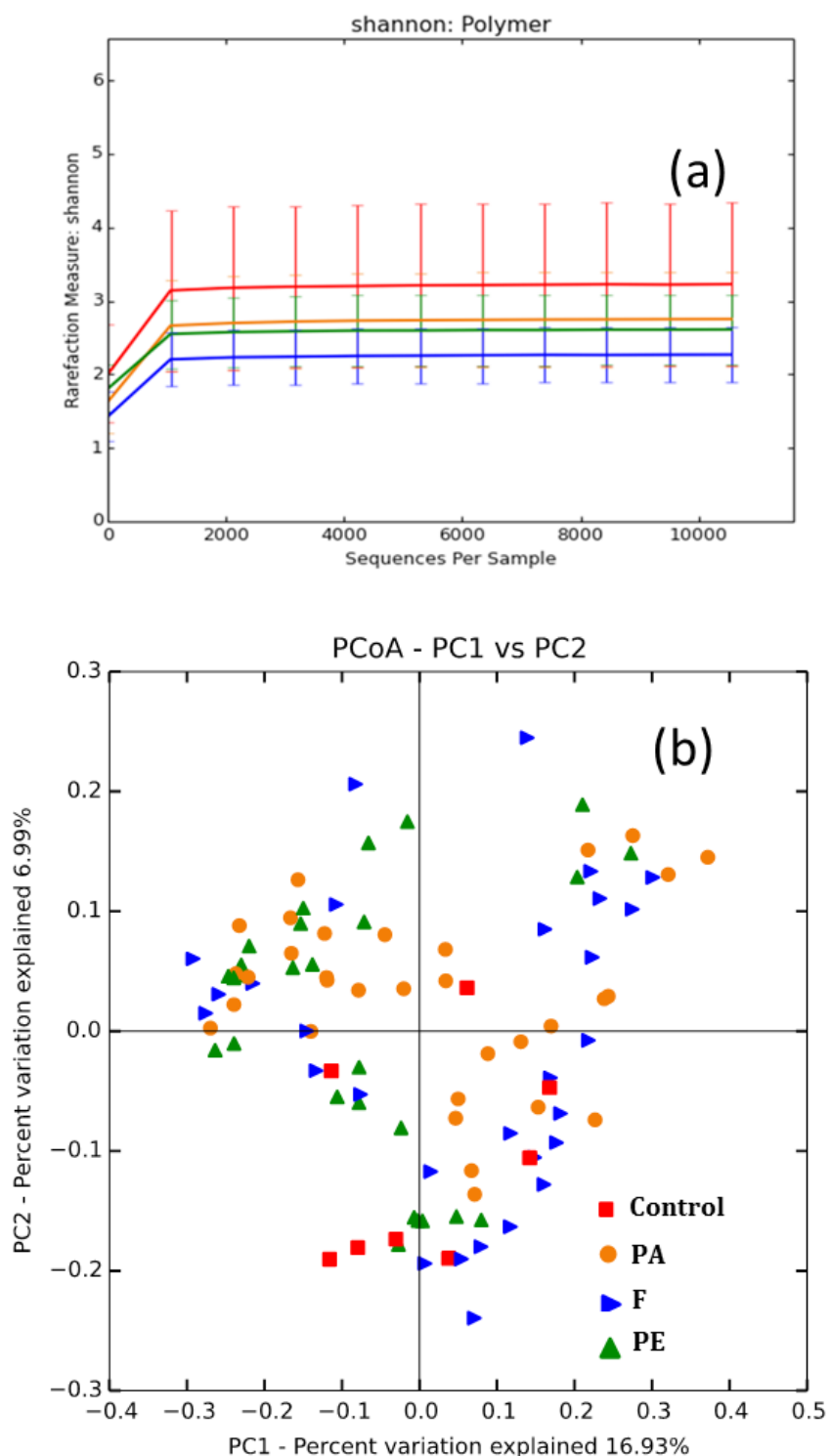

Figure 2. (a) Alpha rarefaction plot demonstrating bacterial community diversity grouped by polymer type (Key: red - control, orange - PEG-MA, green - zwitterionic, blue - PEG). (b) PCoA of beta-diversity representing a comparison of filter sample microbial communities based on their taxonomic composition. Samples labeled by filter pattern (Key: red - control, orange parallel, green - perpendicular, blue - fully modified).
In order to better understand membrane fouling behavior, eight different membrane fouling models that are typically used to describe membrane fouling were used to analyze the experimental data sets. Overall, of the eight fouling models, the "cake complete" model fits best to the experimental data obtained from the operation of UF membranes.

Combining printing technology with these membrane fabrication and modification techniques facilitated the fabrication of unique selective layers and coatings and proved to be a potentially useful new tool for laboratory-scale membrane fabrication. Further development may accelerate the discovery of more efficient and robust membranes and promote the exploration of new material combinations, the potential of patterned compositions, and polymer morphologies that are not possible with conventional methods.

\section{Turbulent Transports in Cumulus-Topped Boundary Layers}

\section{4-193-R1}

\section{Virendra P. Ghate, Rao Kotamarthi, and John Krummel}

\section{PROJECT DESCRIPTION}

Boundary-layer cumulus clouds are intimately tied to the turbulence in the boundary layer and form when enthalpy, moisture, and momentum are transported upward from the Earth's surface. These clouds are a crucial component of the Earth's radiation budget and need to be represented accurately in global climate models (GCMs) used to predict the future climate, extreme weather events, and the demand for energy. As these clouds occur at spatial and temporal scales that are much smaller than can be modeled in the GCM grid box and temporal resolution, their effects and the associated processes need to be parameterized in the GCM.

The overall goal of this project is to improve the representation of cumulus clouds in GCMs. The specific objectives of the project are to (1) characterize the vertical velocity structure of the entire cumulus-topped boundary layer, (2) explore the similarities and differences between subtropical and tropical shallow cumuli, and (3) characterize the turbulent transport of water vapor within the cumulus-topped boundary layer. 


\section{MISSION RELEVANCE}

This project addresses DOE's energy, environment, and basic science missions. The research uses data collected at two of DOE's Atmospheric Radiation Measurement (ARM) climate research sites to quantify the interactions among aerosols, clouds, precipitation, radiation, and atmospheric dynamics, and thermodynamics and thus improve our fundamental, process-level understanding, with the ultimate goal of reducing uncertainty in global and regional climate simulations and projections.

\section{RESULTS AND ACCOMPLISHMENTS}

To achieve our objectives, we used data collected at the ARM observing site previously located on the island of Manus in Papua New Guinea in the tropical western Pacific, as well as data collected during deployment of the ARM mobile location on the island of Graciosa in the Azores in the North Atlantic. In FY 2014, from the data collected at the two locations, we identified cases of cumulus clouds purely of marine origin that were devoid of any heavy precipitation. Fourteen cases lasting for 200 hours, and 19 cases lasting for 306 hours were identified at Azores and Manus, respectively. In FY 2015, we used the data to contrast the boundary layer thermodynamic, radiative, and vertical-velocity structure of cumulus-topped marine boundary layers observed at the two locations, along with the clouds' macro-physical and microphysical properties. Our analysis shows the cumuli over Manus to be deeper, to have higher surface fluxes, and to have higher boundary-layer radiative cooling compared to the cumuli over the Azores. At both locations, about $70 \%$ of cloudy profiles were updrafts with the number of updrafts stronger than one meter per second $(\mathrm{m} / \mathrm{s})$ being greater in the cumuli over Manus than over the Azores. The mean radar reflectivity within updrafts and the magnitude of updrafts was higher in the cumuli over Manus than in the cumuli over the Azores (Figure 1). Our results broadly suggest that the cumuli at the two locations differ significantly in their macrophysical and microphysical structures.
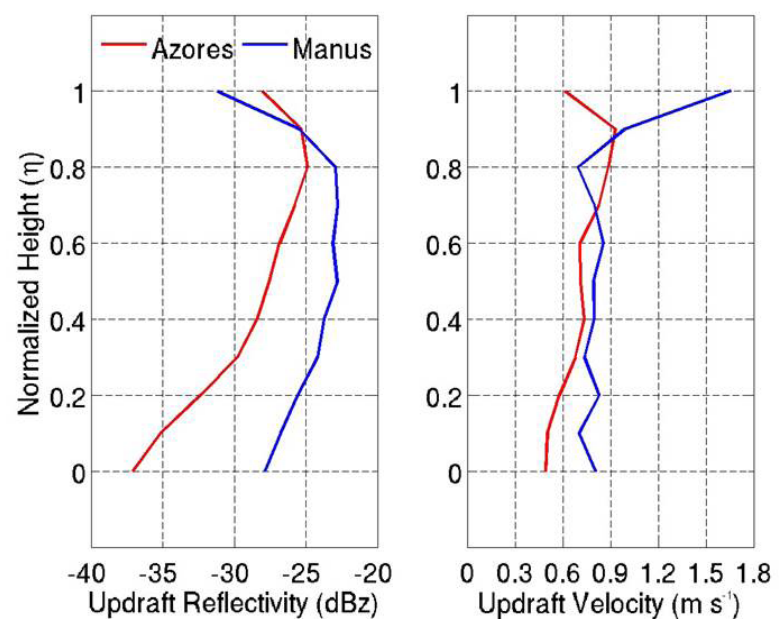

Figure 1. Cloud depth-normalized profiles of average radar reflectivity within updrafts (left) and updraft velocity (right) for clouds over Manus (blue) and the Azores (red). The cumuli over Manus had higher radar reflectivity and updraft velocity at the cloud base than those over the Azores. The unit dBz (decibels of reflectivity) is one meteorological measure of the strength of radar return, for example, from precipitation.

\section{Incorporating Fungal and Bacterial Community Metabolisms to Improve Carbon Cycle Predictions of Earth System Models}

2015-124-NO

Zhaosheng Fan, Dionysios A. Antonopoulos, Beth Drewniak, Jack A. Gilbert, and Peter Larsen

\section{PROJECT DESCRIPTION}

Average global surface temperatures increased by approximately $0.85^{\circ} \mathrm{C}$ between 1880 and 2012 . In addition, the simulation results of various climate models suggest that average global surface temperature will increase by another $\sim 1$ to $\sim 6^{\circ} \mathrm{C}$ by 2100 . Terrestrial ecosystems have played an important role in the climate system since the last glacial maximum by storing a substantial amount of carbon (C) in soil and plant biomass. Environmental drivers have the potential to alter the amount and stability of $C$ stored in soils, and substantial releases of soil $C$ to the atmosphere in response to climate change have implications for atmospheric concentrations of carbon dioxide $\left(\mathrm{CO}_{2}\right)$ and methane $\left(\mathrm{CH}_{4}\right)$, both important greenhouse gases. Therefore, terrestrial ecosystems are a key component of global $\mathrm{C}$ cycling, and their response to climate change may generate large positive or negative feedbacks affecting global climate. 
Soil $\mathrm{C}$ balance is mainly controlled by $\mathrm{C}$ inputs through plant photosynthesis and subsequent production of dead plant residues (litter) and by $\mathrm{C}$ losses through plant respiration and microbial decomposition of soil organic C (SOC). Therefore, microbes are a key agent of SOC cycling. However, the roles of microbes in the cycling of SOC are still poorly represented in Earth System Models (ESMs), athough the dynamics of SOC are ultimately driven by microbial activities. Therefore, the goal of this project is to account for microbial dynamics in ecosystem models and ESMs in order to more reliably predict SOC dynamics and thus future global climate.

\section{MISSION RELEVANCE}

In the short term, this effort would directly benefit DOE's Biological and Environmental Science Capability Development strategic initiative by investigating the integrated responses of biogeochemical systems to environmental forcing factors. This effort will also address DOE's mission area of basic science in relation to climate change research by contributing to the development of predictive microbial-ecosystem models that represent ecological processes and mechanisms and improve the performance of regional-scale and Earth-system models, including the new DOE climate modeling initiative, Accelerated Climate Model for Energy.

\section{RESULTS AND ACCOMPLISHMENTS}

We collected and compiled the necessary experimental data that will be used for the development, parameterization, testing, and analysis of a C-microbial model that simulates the interactions between soil microbes and carbon cycling. These experimental data are from 300 soil incubation experiments (four study sites $\times$ three soil layers $\times$ five replicates $\times$ five temperature conditions) conducted by members of Argonne's Environmental Science Division. To date, we have compiled and completed preliminary analysis of sequencing data for 16S rRNA marker genes (bacteria and archaea). In addition, we have compiled other data to characterize the soils' physical and chemical properties.

We developed a rough strategy for coupling our developed mechanistic microbial model with the statistical modeling framework developed by colleagues in Argonne's Biosciences Division. This strategy comprises three steps. The first step is to transform the taxonomic composition (marker gene) obtained from the high-throughput sequencing data (i.e., the 16S rRNA gene) to predict the metagenome functional composition using PICRUSt, a bioinformatics software package, along with a reference genome database. After the functional composition of the microbial community is predicted, the second step is to generate an interaction network of the soil microbial community using Banjo, a software package for Bayesian network analysis. The last step is to integrate the generated functional interaction network with our developed mechanistic C-microbial model.

To date, we have completed the step-one compilation of the high-throughput $16 \mathrm{~S}$ rRNA sequencing data. We learned how to use the Banjo software by generating a genetic interaction network based on the compiled microbial-community information for the 240 completed sequenced samples. These Banjo exercises with the genetic interaction network have allowed us to become familiar with the software package and, importantly, will provide useful information on the microbial diversity of the different soils and how this diversity is impacted by warming.

\section{Biomimetic Approaches for Water-Smart Landscapes}

\section{5-170-NO}

\section{Cristina Negri, Liliana Fernandez-Gonzalez, Herbert Ssegane, Olivia Tse, and Colleen Zumpf}

\section{PROJECT DESCRIPTION}

This project, performed with researchers at Israel's Ben Gurion University (BGU), aims at understanding how vegetation self-arranges in arid environments and then mimicking this strategy to develop vegetative systems that survive drought. We develop experimental data to study the spatial self-organization patterns of vegetation in green infrastructure (GI). The experimental data we are generating help validate the models developed at BGU and provide guidance on how to develop more efficient GI systems for U.S. cities. GI is vulnerable to drought, even in temperate regions, as its shallow soil limits water storage and exposes vegetation to potentially long dry spells. It is important to develop efficient $\mathrm{Gl}$ as it is an energy-efficient, low-cost, resilient approach to managing stormwater and reducing ambient temperature in cities. Insight from this work will also be used to improve agroforestry systems in arid regions and xeriscapes in arid cities, such as those in the western United States.

This project has four tasks: (1) defining model case scenarios (BGU and Argonne), (2) conducting experimental studies at GI sites (Argonne), (3) developing a mathematical model (BGU), and (4) testing and validating the model (BGU and Argonne). 


\section{MISSION RELEVANCE}

Adaptation to climate change is critical to the mission of DOE and other federal and local government organizations. The development of energy- and water-efficient GI is critical to ensuring that cities will be (1) adaptable to the changed storm patterns forced by climate change, and (2) energy efficient in cooling indoor and outdoor spaces. Results from this work will be of interest to a number agencies and have already enabled participation in a large City of Chicago proposal to the U.S. Department of Housing and Urban Development (HUD) to develop resiliency to flooding. Similar expertise is of interest to the City of Detroit, with which we have had exploratory discussions. Relevant DOE mission matches are found in DOE - Energy Efficiency and Renewable Energy (EERE) programs in bioenergy, and the Energy Water Nexus. This work is also relevant to the National Science Foundation (NSF) (landscape adaptation to climate change and biogeochemical cycles), U.S. Department of Agriculture (USDA) (crop water efficiency and drought tolerance), and U.S. Environmental Protection Agency (EPA) (stormwater management). Internationally, this work has potential to help support efficient water management technology in developing countries, which is important for global security.

\section{RESULTS AND ACCOMPLISHMENTS}

We coordinated with BGU to identify parameters of interest of the model to be empirically measured in the field. We are studying the Chicago Botanical Garden's 16,000-square-foot $\left(\mathrm{ft}^{2}\right)$ research-grade green roof system in Glencoe, Illinois, USA. We collected extensive experimental data over the summer until the first hard frost in November 2015. Soil moisture sensing provided us with a spatial understanding of moisture dynamics after rainfall events (Figure 1). Results show that the depth of the soil medium in a green roof influences the water storage capacity and ultimately plant survival. We developed a methodology to relate water content to vegetation growth, survival rates, and diversity. We gathered recordings of temperature, relative humidity, radiant energy, precipitation, nitrogen deposition, and so forth, so that moisture balances can be calculated. After a detailed vegetation survey on the green roof, we isolated areas for focused monitoring, representing specific planting patterns, the presence of a single versus multiple (herbaceous and woody) plant species, and vegetation densities. We also selected a few sample plants for sacrificial, end-of-season analysis of aboveground and belowground biomass. We characterized the soil structure in terms of particle size distribution and textural class to model water movement and gathered detailed information on root length density in a spatially defined volume of soil (Figure 1). These results will be converted to a uniform digital format over the winter to develop a detailed model of root development as a function of moisture in the green roof and will be provided to BGU together with all metadata collected.

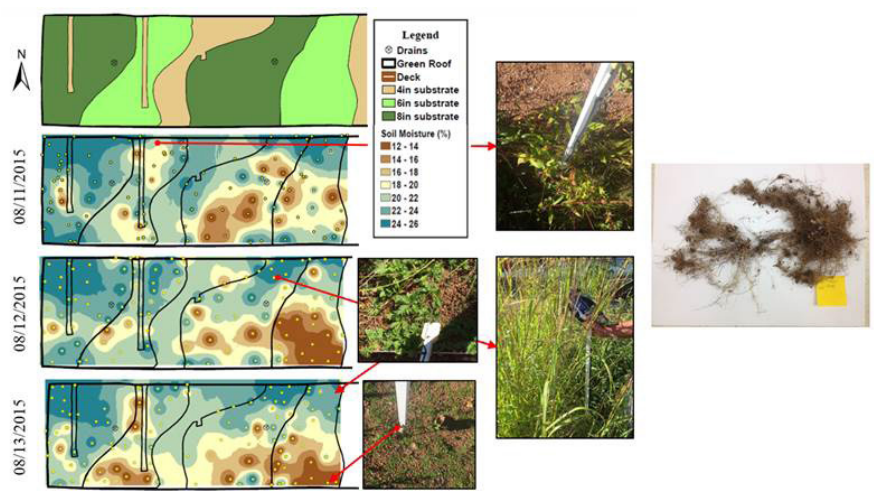

Figure 1. Soil moisture dynamics in green roof portions with different soil depths, and Big Bluestem root system retrieved from the green roof. Spatial root distribution is being digitized and related to ground cover and moisture gradients.

\section{PROPOSED FUTURE WORK}

In FY 2016, we will continue with the development of field data at the Botanical Garden site, refine our methodologies, and produce robust data spanning a two-growing-seasons period. To this end, we are liaising with other researchers at Argonne who will connect part of our sensing apparatus with their Array of Things, a cloud-based network of nodes, so that we can improve our monitoring efficiency and also use our field station as a test facility to develop next-generation monitoring networks. We will evaluate the model developed by BGU and propose general guidelines for exporting the knowledge generated to relevant landscape scenarios identified at project onset. We will pursue scaling up the improved designs in real-life translational demonstrations.

\section{Genome Engineering of Environmental $P$. fluorescens to Investigate Bacterial Interactions with Plants and Other Microbes}

\author{
2015-171-NO \\ Philippe Noirot, Gyorgy Babnigg, Frank Collart,
and Christopher Henry
}

\section{PROJECT DESCRIPTION}

This project aims to develop genome engineering approaches for environmental Pseudomonas fluorescens strains, which are bacteria that associate with fungi and 
roots and provide benefits to plants. Bacteria from the environment are generally very poorly tractable; thus studies of the mechanisms by which bacteria provide benefits to plants have low throughput and are labor intensive. Availability of efficient genome engineering tools in $P$. fluorescens will help bolster our understanding of the molecular mechanisms underpinning plant growth promotion (PGP). Such tools are not currently available. The major tasks pursued are (1) genome engineering in $P$. fluorescens strains, (2) mapping of transcriptional responses of $P$. fluorescens to plant and/or fungus, (3) functional validation of PGP and development of specific reporters for cellular processes of microbe-plant interactions (MPIs), and (4) development of a P. fluorescens platform for MPI studies.

\section{MISSION RELEVANCE}

The project has direct relevance to components of the DOE Genomic Science program, specifically by addressing the first objective, "Determine the molecular mechanisms, regulatory elements, and integrated networks needed to understand genome-scale functional properties of biological systems." In addition, it directly addresses two "Decadal Thrusts" from the Office of Biological and Environmental Research's (BER's) Molecular Science Challenges (April 2015): "Elucidate mechanisms by which plants benefit from microbiomes" and "Characterize signaling or communication pathways between microbiomes and plants." BER has sponsored research in systems biology that includes MPIs. Thus, this project, which focuses on understanding the benefits plants receive from microbiomes, is relevant to the missions of BER.

\section{RESULTS AND ACCOMPLISHMENTS}

Genome Engineering in P. fluorescens strains. We selected four fully sequenced PGP strains that phylogenetically cover the diversity in the $P$. fluorescens group. From published plasmids, selection markers, phage-encoded recombinases, and from genes endogenous to our strains, we constructed a suite of vectors expressing various recombinases as well as integration cassettes composed of a resistance gene (tetracycline or kanamycin) and a gene encoding a green or red fluorescent protein. As illustrated in Figure 1, chromosome loci were selected as integration sites, and appropriate deoxyribonucleic acid (DNA) fragments were amplified by polymerase chain reaction (PCR) from genomic DNA. The expression of some of our recombinase constructs promotes integration of a selectable cassette at the targeted chromosomal locus, establishing genome engineering in $P$. fluorescens. The efficiency of chromosome integration varies considerably between strains. However, the limiting factor appears to be DNA uptake rather than recombination efficiency. Several strains were fluorescently labeled to be used as reporters of bacteria-plant root interactions.

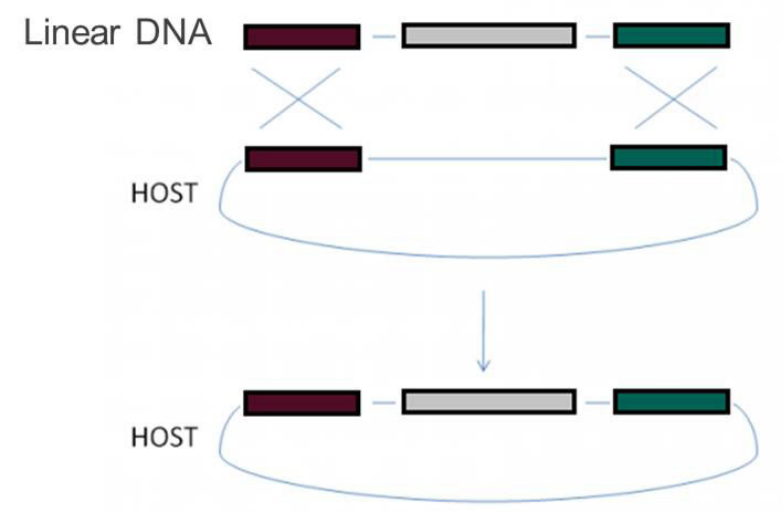

Figure 1. Target chromosome loci (brown and green boxes) are PCR amplified and joined with a cassette comprising a selectable marker and a gene encoding a fluorescent protein (grey box). The resulting linear DNA is introduced in the $P$. fluorescens host cell where it is directed to its target locus by the phage-encoded recombinase, leading to a replacement of the intervening chromosome region (thin blue line between brown and green boxes) by the cassette (grey box). This reaction is entirely dependent on the activity of the phage-encoded recombinase in the cell.

Mapping of Transcriptional Responses of P. fluorescens to Plant and/or Fungus. We encountered a methodological problem. Specifically, the bacterial ribonucleic acid (RNA)-sequence reads from plant/fungus/bacteria communities are not abundant enough to obtain reliable gene expression data. However, we could obtain expression profiles from a simpler plant/bacteria community. Methods for RNA extraction and ribosomal RNA depletion in samples are currently being refined.

Functional Validation of PGP and Development of Specific Reporters for MPI Cellular Processes. We purchased a fluorescence microscope for which we defined the technical specifications for observation of interactions between our genetically engineered bacteria with plant roots. We determined that we need nondestructive simultaneous acquisition of two or three channels of fluorescence, often in combination with confocality, at high magnification, high speed, and on a platform offering stability as well as the ability to step through the sample by automated means. In addition, we need the capability to expand our system as the project progresses and the needs of our work evolve. The specified Nikon microscope was delivered in September and assembled late November 2015. 


\section{PROPOSED FUTURE WORK}

We will optimize transformation procedures to overcome limitations in DNA uptake. We will map the ability of our genome engineering method for creating various types of mutations (e.g., large deletion, single base change, and insertion) and adapt the procedures to microtiter plate format for higher throughput. An RNA-sequence experiment involving bacteria growing on plant roots under various conditions has been designed and initiated. This experiment will take place over eight weeks and will enable us to identify root-responsive bacterial promoters and genes. These root-responsive promoters will be fused to gfp (green fluorescent protein) gene variants and will be integrated into the $P$. fluorescens chromosome, enabling microscopic observation of how expression of a particular gene/pathway is modulated in the bacterial population within the rhizosphere. Personnel were trained in the operation of the Nikon microscope which enabled full operation from mid-December 2015.

\section{Illuminating Linkages between Microbial Diversity and Biogeochemical Cycling in a Redox Dynamic Environment}

\section{5-179-NO \\ Theodore M. Flynn, Jack A. Gilbert, Kenneth M. Kemner, and Edward J. O'Loughlin}

\section{PROJECT DESCRIPTION}

Periodically flooded environments, such as floodplains and wetlands, can be both major sources and sinks of greenhouse gases. To a large extent, the flux of methane from these environments is controlled by microbial metabolism. Both methane-producing and methane-consuming organisms inhabit the water and sediments there, although the extent to which the activity of each is controlled by environmental variables (e.g., pH, temperature) is poorly understood. In particular, the dynamic redox conditions of these environments are thought to cause large shifts in both the rates of greenhouse gas emissions and, concomitantly, the structure of microbial communities. As a result, the flux of methane from periodically flooded environments is a significant source of uncertainty in global climate models (see Kirschke et al., 2013, Nature Geosci. 6:813-823).

Our project seeks to understand the microbial processes that regulate greenhouse gas emissions in a periodically flooded environment at spatial and temporal scales relevant to earth system modelers. We are leading a rigorous field sampling effort to measure relevant biogeochemical parameters (e.g., concentrations of dissolved gases in water and sediment), as well as the composition of the microbial community by using targeted gene sequencing and metagenomics. This will not only improve our understanding of the feedback between microbial metabolism and greenhouse gas emissions, but also provide us with a more quantitative understanding of microbial ecology by monitoring changes in native microbial communities over space and time.

\section{MISSION RELEVANCE}

DOE's Genomic Science Program has 5 key objectives, three of which are addressed by this project.

$\square$ Aim 1: "Determine the molecular mechanisms, regulatory elements, and integrated networks needed to understand genome-scale functional properties of biological systems."

$\square$ Aim 3: "Flexibly scale understanding of biological processes from defined subsystems to individual organisms, consortial assemblies of multiple organisms, or complex communities operating at ecosystem scales."

$\square$ Aim 4: "Understand the foundational rules and 'design principles' governing living systems and develop tools for more sophisticated biosystems design, enabling the targeted modification of functional properties at the genome scale."

In this study, we aim to use omics-enabled techniques to uncover basic organizing principles of biological systems in terrestrial environments. Combining this knowledge with detailed geochemical measurements will provide key variables to inform improved models of the critical biogeochemical processes that control the storage, release, and transport of carbon, nutrients, and contaminants in the critical zone, which are all critical mission challenges recently identified by DOE's Biological and Environmental Research program (BER). As an example, this project is directly applicable to a recent funding opportunity announcement (FOA) from BER's Genomic Science Program, "Systems Biology Enabled Research on the Roles of Microbial Communities in Carbon Cycle Processes," which specifically solicits applications for the development and application of omics approaches to investigate microbial community functional processes involved in carbon cycling in terrestrial ecosystems. 


\section{RESULTS AND ACCOMPLISHMENTS}

This project began late in FY 2015. The primary results and accomplishments involved planning to identify the locations and frequency of the field-sampling program. A local wetland site was identified as a prime location for high-frequency temporal sampling of water and sediment to monitor changes in biogeochemical parameters and microbial community composition over time. Potential collaborations with researchers at Argonne (Atmospheric Research Measurement group) and outside experts (microbiologists at The Ohio State University) were evaluated. These collaborations would enable us to expand the scope of our biogeochemical measurements as well as make available additional environmental material (e.g., sediment and water) to better understand the variability within similar ecosystems in different geographical locations.

Field equipment was ordered and a test trip to the wetland site was taken in order to obtain test measurements and initial samples of the microbial community to better determine the scope and extent of sampling that will be required. 16S rRNA amplicons were sequenced from this initial dataset, showing both the complexity of the microbial community in this system as well as establishing the reproducibility of our sampling methodology (Figure 1). Determining a consistent sampling volume is essential for the type of longitudinal study we plan to make.

\section{PROPOSED FUTURE WORK}

We are now in the process of conducting high-frequency sampling trips in order to monitor changes to the microbiology and geochemistry of the test wetland environment across short (diurnal), moderate (weekly), and long (seasonal) time scales. These efforts will be combined with laboratory incubations of wetland sediment and water to test specific hypotheses related to the effect of redox cycling and electron shuttles on greenhouse gas emissions and microbial community composition. Analysis of both laboratory microcosms and the microbial communities from the field will be conducted first by using $16 \mathrm{~S}$ rRNA gene amplicons, then by using whole-genome metagenomics, whereby portions of all microbial genes present in a sample are sequenced. The former will provide us with a "fingerprint" of the overall microbial community, while the latter will enable us to characterize the specific metabolic capabilities of these microbes through gene annotation and genome re-assembly.

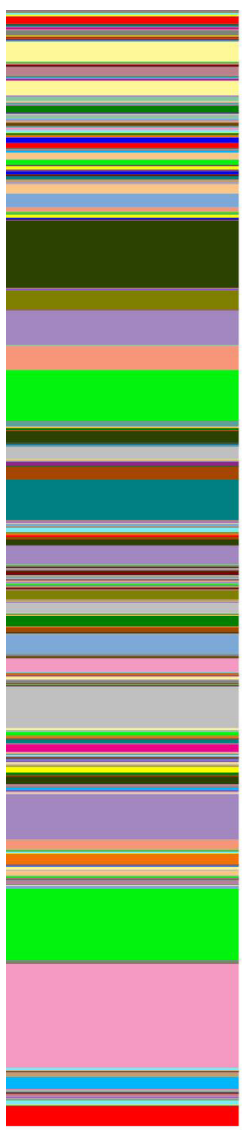

$60 \mathrm{~mL}$

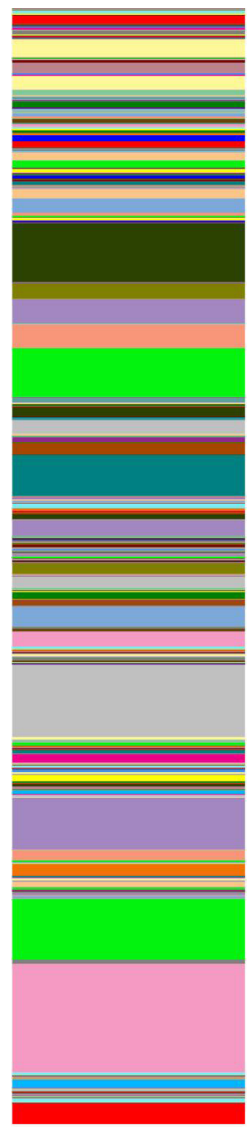

$120 \mathrm{~mL}$

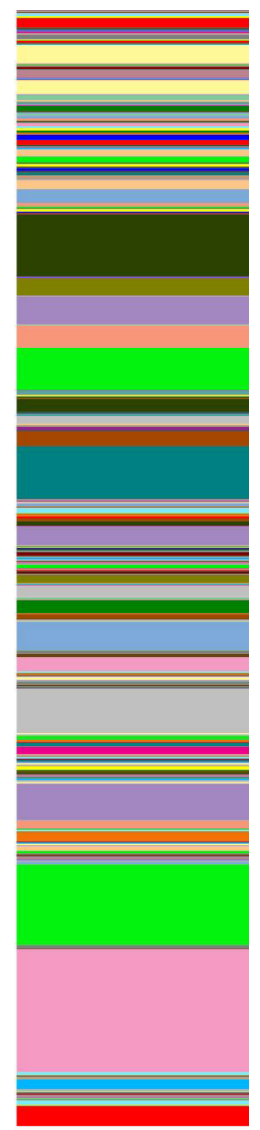

$180 \mathrm{~mL}$
Figure 1. Diversity of 16S rRNA gene amplicons (classified at the genus level) of microorganisms filtered from wetland water in test samples, highlighting the reproducibility of this approach across different sample volumes. Over 700 unique genera of microorganisms were detected in wetland surface water. The height of each color band represents the relative abundance of a particular genus.

\section{Functional Analysis of Proteins from a Key Signaling Network Involved in Plant Growth-Promoting Bacteria}

\section{5-180-NO \\ Marie-Francoise Gros}

\section{PROJECT DESCRIPTION}

Plant growth-promoting (PGP) rhizobacteria exert their beneficial effects through direct and indirect interactions with plant roots, including mobilizing soil nutrients, producing antibiotics, and eliciting plant defense mechanisms. However, while PGP activities have been extensively characterized at a physiological level, the underlying molecular mechanisms remain poorly characterized. This project addresses this knowledge 
gap by identifying protein complexes that decipher environmental stimuli and initiate signal transduction pathways in rhizobacteria to trigger PGP effects. In many bacterial species, the second messenger cyclic diguanylate (c-di-GMP) has emerged as a ubiquitous regulator of bacterial physiology, such as biofilm formation, developmental transition, production of exopolysaccharides, and control of virulence. The object of this project is to characterize the protein-protein network underpinning bacterial response to c-di-GMP in a PGP bacterial strain of Pseudomonas fluorescens SBW25.

In bacterial cells, intracellular levels of c-di-GMP result from the balance between synthesis by diguanylate cyclases (DGCS) and degradation by phosphodiesterases (PDEs). Upon binding c-di-GMP, these proteins can exert a regulatory action on gene expression at transcriptional, post-transcriptional or post-translational levels, either directly or through interaction with other proteins (Figure 1). A comprehensive inventory of all the players (DGCs, PDEs, and c-di-GMP-binding regulators), their regulatory interplay, and targeted processes is thus necessary to fully understand the molecular logic underlying c-di-GMP circuitry in PGP bacteria. This study combines interactomic approaches to identify protein complexes involved in c-di-GMP regulatory pathways with the analysis of their cellular function to provide mechanistic insight into the basic principles of plant growth promotion mediated by rhizobacteria.

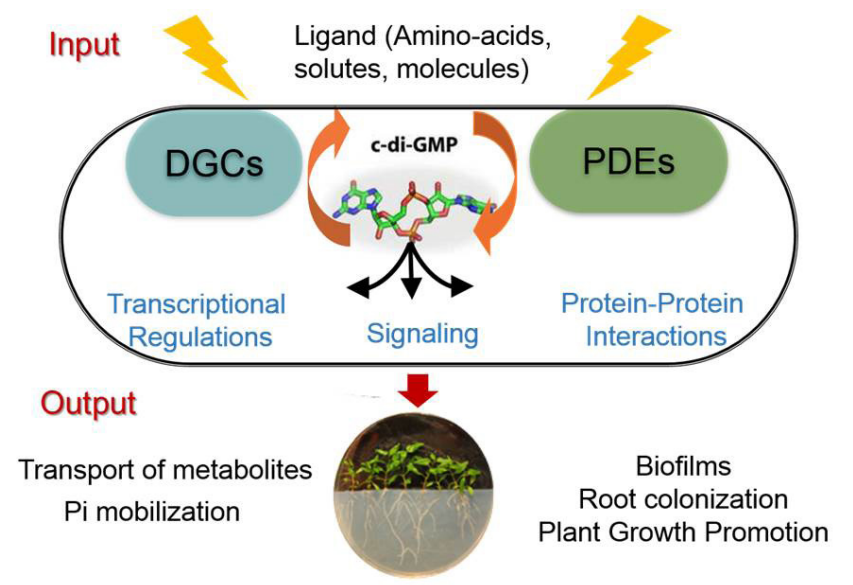

Figure 1. C-di-GMP signaling in PGP bacteria. Regulation of c-di-GMP homeostasis performed by diguanylate cyclases (DGC) and phosphodiesterases (PDE) upon receiving environmental signals.

Biologically validated interactions will also be used to benchmark the development of a microfluidic-based approach to identify protein complexes at high throughput. By leveraging recent developments in microfluidic capability, establishing a proof-of-concept, in collaboration with Gyorgy Babnigg of Argonne's
Biosciences Division, will be pursued for a high-throughput microfluidic methodology based on fluorescence detection of transient protein-protein interactions (PPIs) involved in signaling. Such an approach is expected to provide a leading-edge methodology to map PPIs on a genomic scale.

\section{MISSION RELEVANCE}

This project is relevant to the DOE's mission in science and the environment. The determination of the molecular mechanisms, regulatory elements, and integrated networks needed to understand the functional properties of microbes and their interaction with plants is one important aspect of the research for sustainable bioenergy promoted by the DOE Office of Biological and Environmental Research. Within this framework, the understanding the c-di-GMP signaling cascade of $P$. fluorescens will prove invaluable in learning how to design plant-microbe communities with more efficient PGP properties. Our exploration of the development of microfluidic-based interactomics is also fully consistent with the objectives of the genomic science program to develop omics capabilities to achieve system-level understanding of living organisms.

\section{RESULTS AND ACCOMPLISHMENTS}

The identification of the PPI network centered on c-di-GMP signaling in P. fluorescens was subcontracted to the company Hybrigenics Services, which possesses a unique capability and a strong expertise with yeast two-hybrid (y2HB) technology. A large-scale purification of $P$. fluorescens genomic DNA was done and provided to the subcontractor for the construction of a y2HB genomic library (delivered in December 2015). The genome of $P$. fluorescens SWB25 encodes for 48 predicted c-di-GMP-associated signaling proteins (Protein Supercluster SC_37130).

The first cornerstone of the project is to identify the main players of the c-di-GMP signaling pathway involved in PGP and related phenotypes, such as phosphate solubilization, biofilm formation, and swarming properties. These candidates will be used as "baits" for the identification of protein partners through y $2 \mathrm{HB}$ genomic screens and will be functionally characterized. To identify these proteins, two strategies were adopted. The first one was to leverage the available transcriptomic data on plant root colonizing versus non-colonizing bacteria in the Aspen/P. fluorescens model system in the frame of the Environmental Sensing and Response-SFA (ESR-SFA) project in collaboration with colleagues in Argonne's Biosciences Division. Analysis of the variation of gene 
expressions revealed six candidates among the genes encoding proteins of the SC_37130 supercluster. These genes will be targeted for future functional analysis combining interactomic, genetic, and subcellular localization approaches.

The second strategy was to identify, among the SC_37130 supercluster set, the proteins with DGC or PDE activities. Recently, strains of the Gram positive bacteria Bacillus subtilis were engineered as c-di-GMP responsive biosensors to assess the effect of heterologous proteins on c-di-GMP intracellular levels (X. Gao, et al., "Engineering of Bacillus subtilis Strains to Allow Rapid Characterization of Heterologous Diguanylate Cyclases and Phosphodiesterases" Appl. Environ. Microbiol. 2014; 80:19 6167-6174). These strains were obtained from the laboratory of Prof. Daniel Kearns (Indiana University) and genetic constructions were done to allow the conditional expression of the $48 \mathrm{c}$-di-GMP-associated genes of P. fluorescens.

\section{PROPOSED FUTURE WORK}

Work in FY 2016 will be focused on exploring the role of c-di-GMP in plant growth promotion in P. fluorescens. We will follow the multipronged strategy to (1) Identify the PPI network centered on c-di-GMP signaling in $P$. fluorescens, and (2) use this PPI network to drive functional studies of selected protein candidates, aiming at validating the role of c-di-GMP signaling in PGP and colonization of roots. Knockout (KO) mutants of individual genes encoding c-di-GMP-binding proteins will be generated using chromosome engineering methods developed here. A particular focus will also be on the development, in $P$. fluorescens, of a CRISPR (Clustered regularly-interspaced short palindromic repeats) -based interference genetic tool for the targeted silencing of gene expression. The fitness of the KO (or silenced) mutant stains will be assessed by evaluating their ability to promote growth of aspen seedlings in a vertical plate assay and to solubilize phosphate. Cell mobility and chemotaxis toward roots will also be tested by spinning disk confocal microscopy. The c-di-GMP gene sensors exhibiting the strongest PGP phenotypes will be selected for further functional studies.

During FY 2016, we will also start leveraging functionally validated PPIs to establish proof-of-concept for a high-throughput microfluidic methodology based on fluorescence detection of transient PPIs involved in signaling. 


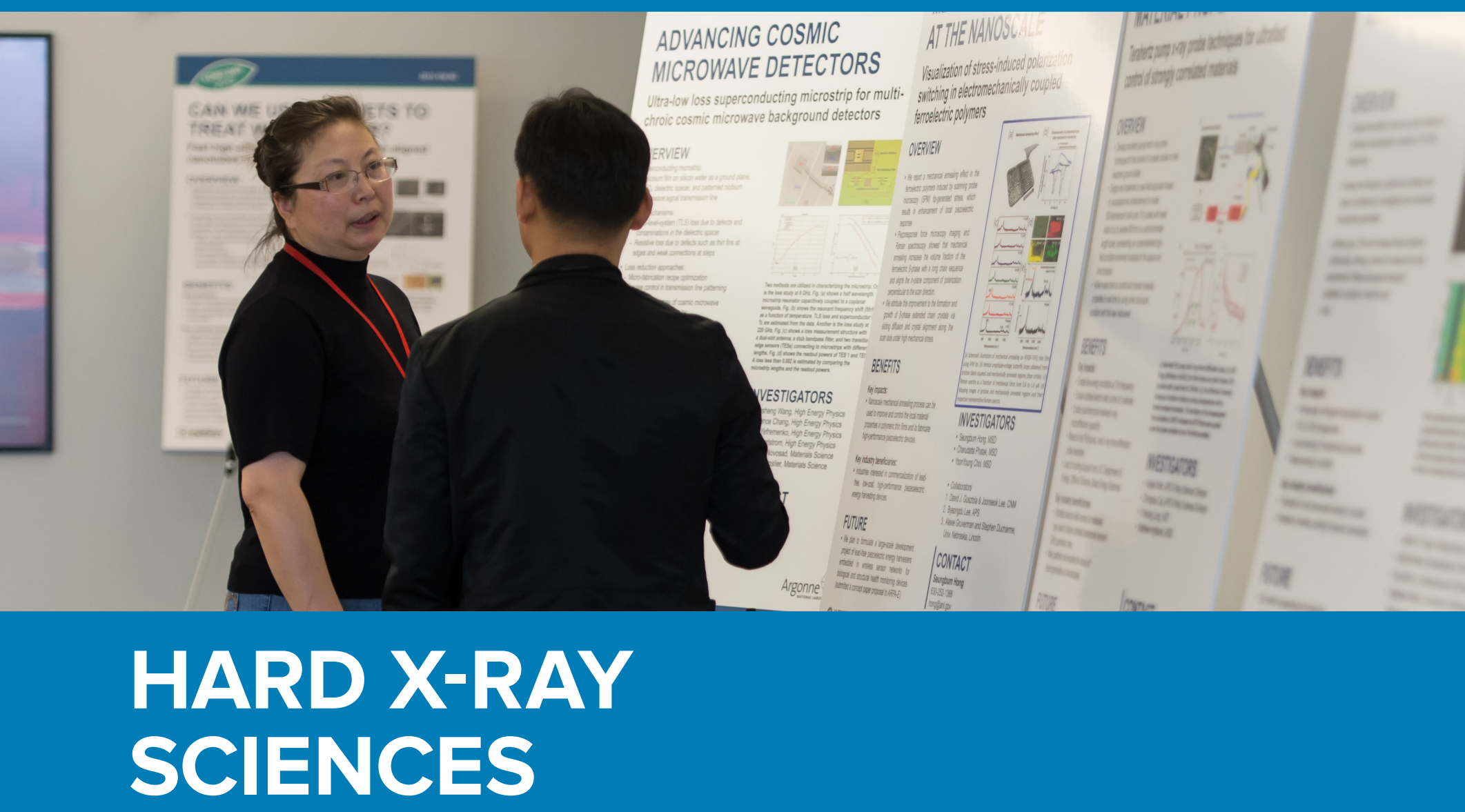




\section{Feasibility Study of Applying Thin-Film High-Temperature Superconducting Films on Copper or Niobium-Sputtered- on-Copper for the Purpose of Achieving Helium-Free Operation with Cryocoolers}

\section{3-111-R2 \\ Alireza Nassiri and Robert Kustom}

\section{PROJECT DESCRIPTION}

This project focused on feasibility studies of the application of magnesium diboride $\left(\mathrm{MgB}_{2}\right)$ thin films with high superconducting critical temperature $\left(T_{c}\right)$ to radio frequency (RF) resonators (cavities). Studies were directed toward applying the films to niobium and copper cavities with the goal of increasing the accelerating gradients to greater than $50 \mathrm{MV} / \mathrm{m}$ (megavolts/meter). RF cavities are elements of a particle accelerator that provide electromagnetic fields that add kinetic energy to the beam of charged particles as it passes through the accelerator.

Researchers Xiaoxing Xi, Matthaeus Wolak, and Wenura Withanage of Temple University and Grigory Eremeev, Fay Hannon, and Robert Rimmer of Thomas Jefferson National Accelerator Facility have also contributed their expertise to this project.

\section{MISSION RELEVANCE}

This project supports DOE's mission in science. By achieving the successful thin-film coating of $\mathrm{MgB}_{2}$ or other possible high- $\mathrm{T}_{\mathrm{c}}$ material films, it would be possible to design and build helium-free superconducting RF (SRF) systems for synchrotron light source storage ring cavities, future $x$-ray free electron lasers (XFELs), energy recovery linear accelerators (linacs) (ERLs), and the next linear collider (NLC). These future projects would benefit from lower design, equipment, and fabrication costs that easily could constitute $10-20 \%$ of the current production costs for a liquid helium system.

\section{RESULTS AND ACCOMPLISHMENTS}

In FY 2013, we developed a design concept for the integrated cavity (resonator), cryomodule and cryocoolers. We explored using the atomic layer deposition (ALD) technique to grow $\mathrm{MgB}_{2}$ on sapphire and metallic small samples. We established a collaboration with Temple University on $\mathrm{MgB}_{2}$ coating using a high pressure chemical vapor deposition technique developed by Prof. Xi and his team at Temple University. Several two-inch diameter sapphire samples coated with $\mathrm{MgB}_{2}$ were produced and measured.

In FY 2014, we succeeded in depositing $\mathrm{MgB}_{2}$ films on commercial thin copper foils and on two-inch copper (Cu) disks. A dielectric resonator working at $18 \mathrm{GHz}$ was fabricated and tested for the purpose of testing small $\mathrm{MgB}_{2}$ films to provide feedbacks for materials optimization.

In FY 2015, we made significant progress with very encouraging results pursuing hybrid-physical chemical vapor deposition (HPCVD) techniques to coat a $\mathrm{MgB}_{2}$ thin film onto $\mathrm{Cu}$ coupons. In the initial stage, $\mathrm{MgB}_{2}$ was grown on small $\left(5 \times 5\right.$ millimeter-square $\left.\left[\mathrm{mm}^{2}\right]\right) \mathrm{Cu}$ foil substrates, which were protected by buffer layers. The buffer layers that were tested in the deposition series included sputter-deposited niobium (Nb) and magnesium oxide $(\mathrm{MgO})$, as well as boron (B) deposited by CVD. The quality (i.e., surface smoothness and uniformity) of the $\mathrm{MgB}_{2}$ films grown on these buffer layers was characterized using scanning electron microscopy (SEM). Out of the three buffer layers we employed, $\mathrm{Nb}$ appeared to be the most promising. While $\mathrm{MgB}_{2}$ films on $\mathrm{Nb}$-buffered $\mathrm{Cu}$ samples showed uniform coverage (as shown in Figure 1), which was further improved by lowering the deposition temperature, depositions on $\mathrm{MgO}$-buffered samples showed an increased number of cracks and pinholes in the $\mathrm{MgB}_{2}$ film, which were attributed to the much larger difference in the thermal expansion coefficients between film and substrate.

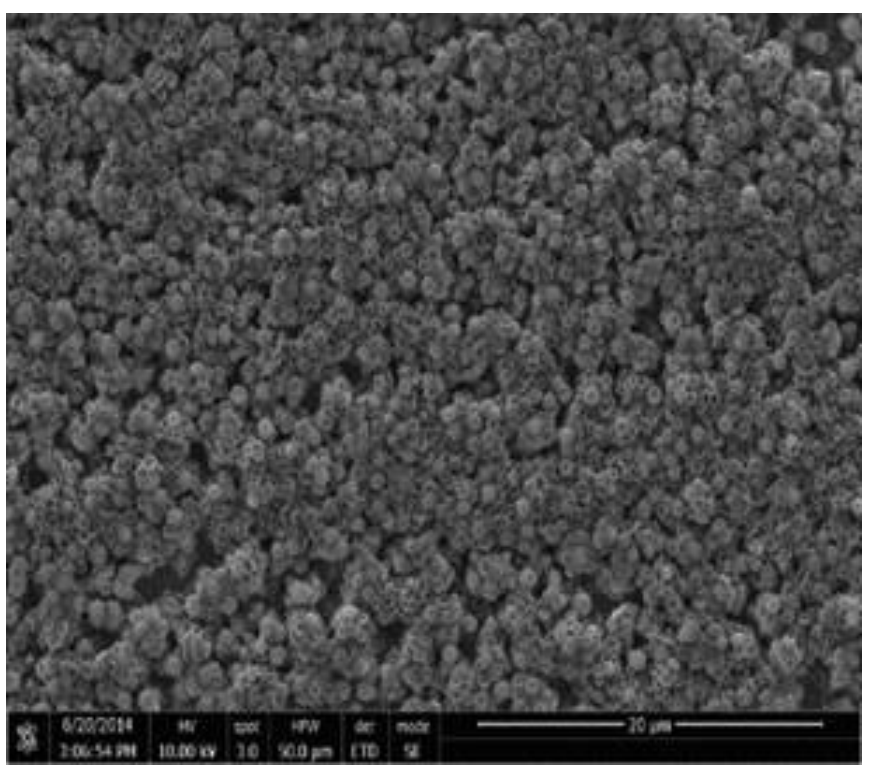

Figure 1. SEM image of $\mathrm{MgB}_{2}$ film deposited on Nb-buffered $\mathrm{Cu}$. Substrate was deposited at $630^{\circ} \mathrm{C}$ shows a uniform film coverage with small-sized grains. 
The transition to lower temperatures not only led to the growth of high-quality $\mathrm{MgB}_{2}$ films on $\mathrm{Nb}$-buffered $\mathrm{Cu}$ disks, but eventually it allowed for the direct growth of $\mathrm{MgB}_{2}$ on bare $\mathrm{Cu}$ (see Figure 2), once the deposition temperature was low enough to limit residual $\mathrm{Mg}$-Cu alloy formation on the surface of the $\mathrm{MgB}_{2}$ film which increases the total surface resistivity.

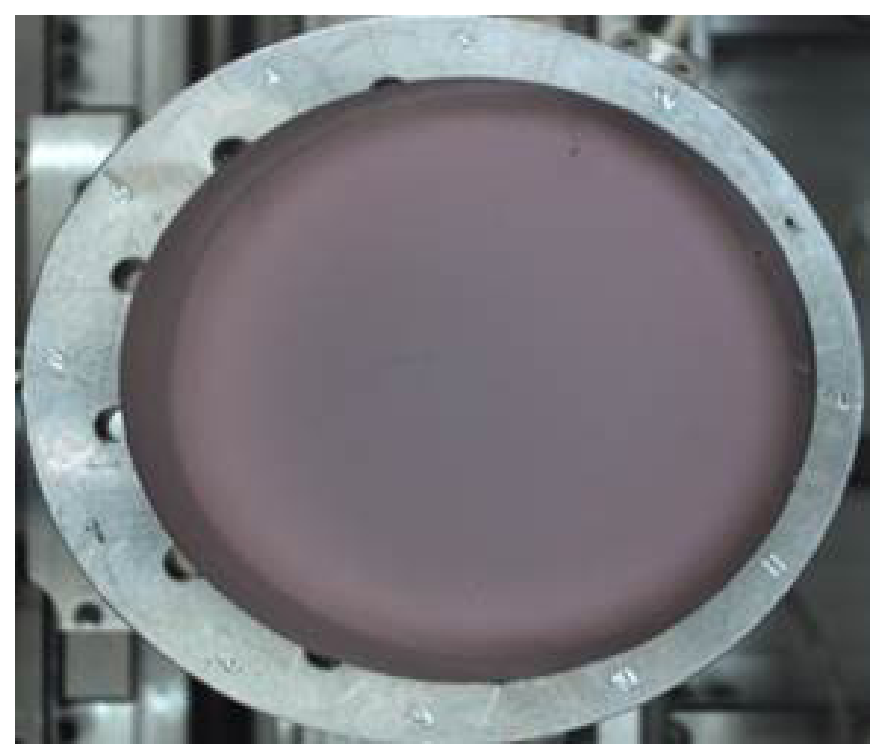

Figure 2. $\mathrm{MgB}_{2}$ film grown on a bare copper disk.

$\mathrm{MgB}_{2}$ depositions on both polished and unpolished $\mathrm{Cu}$ led to dense coverages, where the unpolished substrates facilitate a much smaller grain growth. However, the small grain growth is not expected to be detrimental to the electrical quality of the film as $\mathrm{MgB}_{2}$ does not exhibit intergranular weak-link behavior, thus grain boundaries have no significant impact on the film's electrical characteristics.

All of the deposited films were tested for their RF performance in a dielectric resonator. In comparison to the various reference samples $\left(\mathrm{Cu}, \mathrm{Nb}\right.$, and $\mathrm{MgB}_{2}$ on aluminum oxide $\left[\mathrm{Al}_{2} \mathrm{O}_{3}\right]$ ), it is clearly evident that the $\mathrm{MgB}_{2}$ film deposited on a bare $\mathrm{Cu}$ disk displays an enhanced quality factor ( $Q$ factor)-a metric for power efficiencywhile exhibiting a high $\mathrm{T}_{\mathrm{c}}$ of 39 kelvins $(\mathrm{K})$ as well.

\section{The Tao of Fusion: Pathways for Big-Data Analysis of Energy Materials at Work}

\author{
2013-168-R2
}

Chris Jacobsen, Francesco De Carlo, lan T. Foster, Sven Leyffer, Todd Munson, Suresh Narayanan, Tom Peterka, Nicholas Schwarz, Stefan Vogt, and Stefan Wild

\section{PROJECT DESCRIPTION}

The Advanced Photon Source (APS) at Argonne is the largest single scientific user facility in the United States. It generates 100-300 terabytes (TB) of data per month among 60 different experimental end-stations serving about 5,000 researchers per year. We are developing data workflow management tools to allow for automated transfer of data from the computer, tied in with an x-ray detector to a central storage system with cataloged metadata and experiment-based access control. In addition, we are developing optimization methods for combining images taken at different length scales and from different instruments. These activities are aimed at the imaging of energy materials via tomography and fluorescence microprobe analysis and work toward real-time analysis of the per-pixel time correlations at the heart of x-ray photon correlation spectroscopy. Our goal is to pioneer capabilities that might then serve as a model for facility-wide implementation and for follow-on computational research.

\section{MISSION RELEVANCE}

This project is relevant to DOE's mission in Basic Energy Sciences (BES) and in Advanced Scientific Computing Research (ASCR). It aims to test a possible approach for facility data management and access, where beamline data are automatically transferred to a central storage system (and, upon confirmation, deleted from the beamline computer to free up its storage space). Access permission is to be automatically configured based on users on the Experiment Safety Approval Form (ESAF) and provided through APS User Accounts, as well as Globus Online. This will allow for far more efficient management of data, freeing up BES facility staff to concentrate on user support and analysis, and better utilization of the data through easy dissemination. Multiscale and multimode $\mathrm{x}$-ray imaging leverages expertise from ASCR-supported research at Argonne and serves as an example of the "big data" analysis capabilities needed for more effective utilization of the data taken at BES light source facilities. 
RESULTS AND ACCOMPLISHMENTS

In FY 2013, we achieved the following:

$\square$ Acquired a 210-TB data storage system with about one-tenth the cost and rack space per terabyte as previous systems used at the APS.

$\square$ Began testing the Spade (nest.Ibl.gov/projects/spade/ html/) data transfer system from Lawrence Berkeley National Laboratory as a possible code base that we may want to contribute to in the future. This was tested as an approach for automatic data transfer from a beamline data acquisition computer to a central data store. We also identified a method for organizing data access, as described previously, and initiated the software development effort required to test it and put it into production. We worked with the Globus Catalog team to provide specifications first, and early testing later, of the capabilities it can provide for metadata catalogs to enable searches through datasets.

In FY 2014, we achieved the following:

$\square$ Developed and published a software package TomoPy that provides a streamlined workflow for tomographic data reconstruction and analysis. This program is proving popular and is being adopted by Lawrence Berkeley and Brookhaven National Laboratories.

$\square$ Developed a supercomputer implementation of TomoPy, with a factor of 700 speedup on reconstructions.

In FY 2015, we achieved the following:

$\square$ Demonstrated an approach for automatic data transfer from a beamline data acquisition computer to a central data store.

- This included completing a daily database update to create user accounts on our storage system from the APS User database. (As a result, our storage server now has 16,000 user accounts.) We also established a daily database update from the APS ESAF database, which is separate from the user database. With these two connections, we can have Unix accounts for each user and Unix groups for the data from each experiment. Access to data will be initially limited to those users who are listed on an ESAF form. A web interface will then be provided to let the experiment's Principal Investigator, as well as the responsible APS beamline scientist, add or remove users to/from the Unix group and to add or revoke data access privileges.

- Developed and tested a system for automated data transfer from APS beamlines to the APS local data store, and from there to Argonne's "Petrel" data storage facility (http://petrel.alcf.anl.gov/\#about) via Globus data transfer tools developed by Argonne and the University of Chicago (http://www.globus.org).

$\square$ Carried out further enhancements to the software package TomoPy.

$\square$ Explored a supercomputer implementation of TomoPy, with a factor of 700 speedup on reconstructions.

$\square$ Enhanced a flexible HDF5 (hierarchical data format) file schema for the storage of multimodal imaging data.

$\square$ Developed a model-based reconstruction scheme for x-ray fluorescence tomography that is also able to incorporate other data modalities, such as differential phase contrast imaging. This work is continuing with support on NIH project R01 GM104530.

$\square$ Delivered (via a contract to 2ndlook Consulting, Hong Kong) an open source software package MANTiS, written in Python/Qt, which provides a story-board-guided approach to spectromicroscopy analysis. This package has since been enhanced by the addition of non-negative matrix analysis capabilities.

\section{Development of a Novel Analyzer System for Resonant Inelastic X-ray Scattering with Better Than 10-meV Resolution}

\section{4-127-R1 \\ Jung Ho Kim, Thomas Gog, and Xianrong Huang}

\section{PROJECT DESCRIPTION}

State-of-the-art resonant inelastic x-ray scattering (RIXS) will require development of the next-generation analyzer system, which will overcome intrinsic energy resolution limits of conventional spherical analyzers. This project is focused on developing an analyzer system having better than 10 milli-electron volt (meV) energy resolution-more than three times better than the current best. That will enable studies of a class of emerging materials that have remained inaccessible due either to (a) inherently small energy scales, unresolvable by current RIXS analyzers, or (b) the lack of large enough single crystalline samples as are required by inelastic neutron scattering. The new analyzer exploits multiple $x$-ray bounces from crystals rather than the single bounce of spherical analyzers. The incident photons are highly monochromatized by a four-bounce silicon (Si) crystal monochromator. A two-dimensional multilayer Montel mirror is placed between the sample and the proposed analyzer to collect 
scattered $\mathrm{x}$-rays with a sufficiently large solid angle of 10 milliradian (mrad) by 10 mrad. The first crystal collimates the scattered beam emerging from the sample, while subsequent crystals disperse the beam, selecting a specific energy and reflecting it into the detector.

\section{MISSION RELEVANCE}

The significant improvement of the energy resolution of RIXS proposed in this project has broad scientific implications for materials science, and is well aligned with one of the Grand Challenges highlighted by DOE's Office of Basic Energy Sciences and its division of Materials Sciences and Engineering. Over the last few decades, a revolution has occurred with the emergence of novel, complex materials displaying a variety of electronic phenomena that require fundamental scientific study and may be immediately useful for device development. The RIXS technique is uniquely suited for studying the electron dynamics of these materials; however, improvements in the energy resolution of RIXS are needed to discern the subtle new electronic features.

\section{RESULTS AND ACCOMPLISHMENTS}

All essential optics and Si crystals were designed and manufactured in FY 2014. The Montel mirror for use at $11.215 \mathrm{keV}$ was manufactured by Incoatec Company and delivered in FY 2014. All three Si crystals of the new analyzer system were designed and manufactured by the Optics Group of Argonne National Laboratory's $X$-ray Science Division. The 7-meV monochromator was designed and implemented, and its working principles and performance were thoroughly characterized and tested.

In FY 2015, the angular acceptance and collimation of a laterally graded parabolic collimation Montel mirror were successfully characterized at beamline 27-ID of the Advanced Photon Source. Through comparisons with $x$-ray tracing simulations, the measured $x$-ray divergence could be well understood, and it was found that the Montel mirror achieves the required quality of $x$-ray collimation for use in an angular dispersion multi-crystal RIXS analyzer system; see Figure 1(a).

A multi-crystal RIXS analyzer system was then tested using a direct, highly monochromatized incident beam generated by two symmetric $\mathrm{Si}(844)$ monolithic channel-cut crystals. Three different combinations of flat crystals were studied to find the optimum combination in terms of good energy resolution and reliable/easy operation. The first combination consisted of a sequence of three asymmetric crystals-Si(111), Si(844), and $\mathrm{Si}(440)$-in the collimating-collimating-dispersing mode.
The energy resolution was found to be about $15 \mathrm{meV}$. In the second combination, the Si(440) crystal was omitted, while the remaining $\mathrm{Si}(111)$ and $\mathrm{Si}(844)$ crystals were arranged in the collimating-dispersing mode. The associated energy resolution was found to be about $13 \mathrm{meV}$. In the third combination, the asymmetric Si(844) crystal was replaced with a symmetric quartz(309) crystal. It was found that the third combination gave the best energy resolution of $11 \mathrm{meV}$ with high efficiency; see Figure 1(b).

\section{(a)}

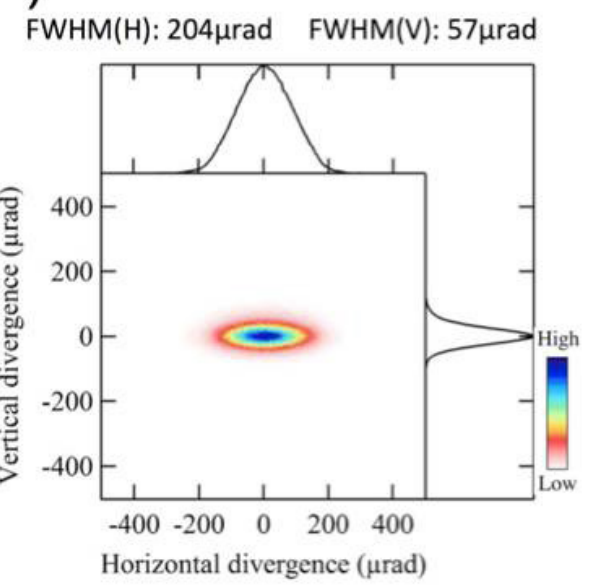

(b)

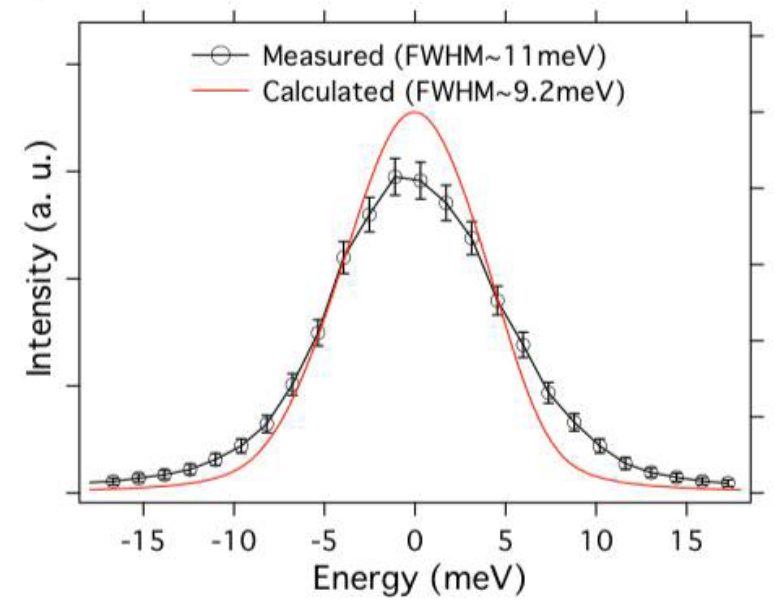

Figure 1. (a) The x-ray divergence of the actual source sizes at beamline 27-ID; $x$-ray divergence on the scattering plane (vertical) of the multi-crystal analyzer is better than the required $100 \mu \mathrm{rad}$. (b) The measured energy scan from the combination of asymmetric $\mathrm{Si}(440)$ and symmetric quartz(309) crystals in comparison with calculations using dynamical diffraction theory.

\section{PROPOSED FUTURE WORK}

In FY 2016, the multi-crystal RIXS analyzer system consisting of asymmetric Si(111) and symmetric quartz(309) crystals will be tested in an actual scattering setup, 
consisting of a two-symmetric-Si(844) channel-cut crystal monochromator, a Kirkpatrick-Baez focusing mirror system (FWHM=10 microns [ $\mu \mathrm{m}]$ and $40 \mu \mathrm{m}$ for the vertical and horizontal directions, respectively), and the x-ray collimation optics (Montel mirror). The whole analyzer system will be enclosed in a helium-filled housing to reduce x-ray attenuation and air-scattering background. Actual measurements will be carried out on some strontium-iridium and sodium/lithium-iridium oxides: square-lattice $\mathrm{Sr}_{2} \mathrm{IrO}_{4}$ and honeycomb-lattice $(\mathrm{Na}, \mathrm{Li})_{2} \mathrm{IrO} \mathrm{O}_{3}$. In the case of $\mathrm{Sr}_{2} \mathrm{IrO}_{4}$, magnon gaps will be measured that contain information about anisotropic magnetic interactions but cannot be measured by the current 25-meV energy resolution. $\mathrm{Na}_{2} \mid \mathrm{IO}_{3}$ and $\mathrm{Li}_{2} \mid \mathrm{IO}_{3}$ show exotic ground states and novel electronic phenomena such as quantum spin liquids. Preliminary measurements showed that low-energy magnetic excitations of those iridium compounds are suitable for the 10-meV analyzer system because their energy scales fall in the 10-meV range.

\section{Three-Dimensional Coherent Diffraction Imaging Using Polychromatic Hard X-rays}

\section{4-134-R1}

Stephan O. Hruszkewycz, Paul H. Fuoss, Ross J. Harder, Matthew J. Highland, Wenjun Liu, and Jörg Maser

\section{PROJECT DESCRIPTION}

In this project, we are developing a three-dimensional coherent $x$-ray diffraction imaging (3D-CDI) technique that uses the energy spectrum and integrated flux delivered by an Advanced Photon Source undulator to rapidly measure volumetric coherent diffraction and provide about a 100-fold reduction in data acquisition time. The central concept of the technique is to scatter a range of $x$-ray energies from a sample and use a series of very thin analyzer crystals to extract simultaneous slices of coherent scattering. This imaging technique has implications beyond the coherent diffraction imaging and materials communities, including incoherent ultrafast timeresolved measurements at dedicated timing instruments and certain ultrafast pump-probe experiments, especially "one-shot" pump-probe studies.

\section{MISSION RELEVANCE}

This project is relevant to DOE's mission in science and innovation. Polychromatic coherent diffraction methods will provide a fundamentally new capacity for the study of novel nanoscale materials and will address outstanding challenges in the field of 3D-CDI, making advances toward in-situ, real-time materials studies. Polychromatic 3D-CDI will be useful at DOE light sources that emphasize both coherent imaging and time-resolved physics, including the new National Synchrotron Light Source and potential diffraction-limited synchrotron facilities.

\section{RESULTS AND ACCOMPLISHMENTS}

In FY 2014, we designed and commissioned an initial mock-up of the instrument to develop the basic principles of the pink beam analyzer system. We demonstrated that a single-crystal silicon (Si) membrane can be used as an analyzer and performed preliminary tests on x-ray scattering from a ferroelectric thin film.

In FY 2015, we built and commissioned a second dispersive pink beam analyzer system in the Sector 34-ID-E endstation, improving significantly on the original mock-up. The underlying design of the new system is shown in Figure 1. A critical design element for this instrument was the compact five-analyzer Laue system that offsets the angle of each Si membrane by one degree.
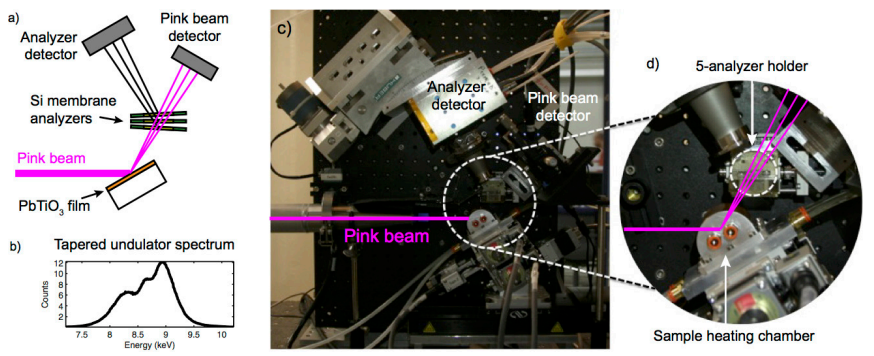

Figure 1. (a) Schematic of analyzers arranged in Laue diffraction geometry. (b) Typical tapered undulator spectrum. (c) Completed diffractometer system. (d) Close-up of the sample chamber and five-analyzer holder.

This diffractometer system successfully demonstrated the multi-analyzer approach by resolving the twin domain peaks of a ferroelectric lead titanate $\left(\mathrm{PbTiO}_{3}\right)$ thin film grown coherently on a dysprosium scandate $\left(\mathrm{DyScO}_{3}\right)$ single-crystal substrate. A 2D reciprocal space map of this system is shown in Figure 2a. We illuminated this film with an unfocused "pink" beam containing photon energies from 8 to $9.5 \mathrm{keV}$ from a tapered undulator. By using our five-analyzer system, five 2D cuts of the $3 D$ reciprocal space map were recorded simultaneously with a single detector without engaging any motors (Figure 2b). Using a heating stage we developed for this system, we monitored the diffraction from the $\mathrm{PbTiO}_{3}$ film as it underwent a phase transition at about $800 \mathrm{~K}$, demonstrating the capability of this approach for in-situ measurements. 


\section{a) Mono beam reciprocal space map}

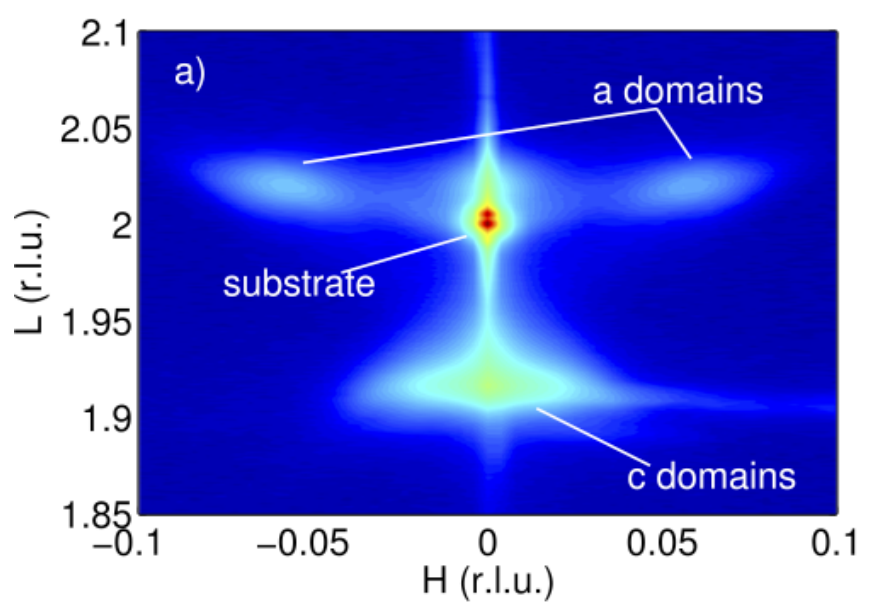

b) Single exposure of 5-analyzer system

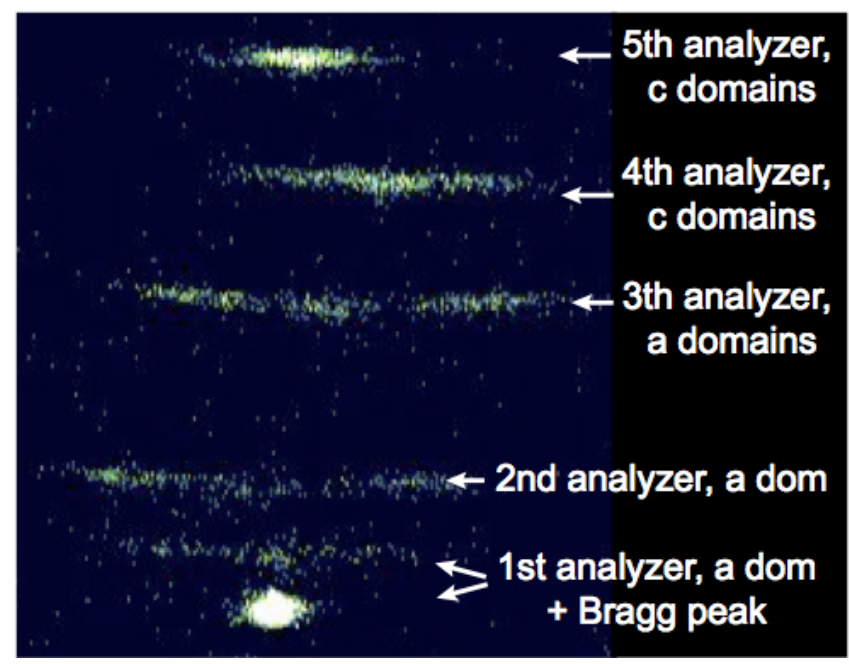

Figure 2. (a) 2D reciprocal space map of a $\mathrm{PbTiO}_{3}$ film grown on $\mathrm{DyScO}_{3}$, measured with a traditional monochromatic beam at room temperature. (r.l.u. = reciprocal lattice unit) (b) Elements of the 3D reciprocal space map corresponding to the tilted ferroelectric domain structure resolved with a single 5-s pink beam exposure.

Another major FY 2015 accomplishment was developing a theoretical framework for reconstructing a 3D image from a Bragg coherent diffraction data set obtained by scanning energy (rather than scanning angle, as is typically done). This was an important first step toward enabling 3D coherent Bragg diffraction imaging with polychromatic $\mathrm{x}$-rays.

\section{PROPOSED FUTURE WORK}

In FY 2016, our aim is to demonstrate single-exposure CDI reconstructions of single nanocrystals. In the experimental effort, we will develop precision sample manipulation in the test-bed system and use it to illuminate a single gold crystal with a focused pink beam. This capability will enable single-particle polychromatic 3D-CDI imaging.
Furthermore, incorporating precision sample control into our detector system also opens the door to 3D Bragg ptychography of continuous nanostructured thin films. We will also enhance the analyzer system by increasing the number of Si membranes from 5 to 10 .

The major computational challenge will be to develop a reconstruction algorithm that relaxes the current requirements of reciprocal space sampling. The limited number of energy-angle slices that can be measured in a single beam exposure with our system is insufficient for current reconstruction algorithms. We will build upon preliminary success in sparse-sampling reconstruction for Bragg ptychography and extend these ideas to the multi-analyzer experiments.

\section{$\mathrm{YBa}_{2} \mathrm{Cu}_{3} \mathrm{O}_{7}$ High-Temperature Superconducting Prototype Undulator}

\author{
2014-137-R1 \\ Ulrich Welp and Yury Ivanyushenkov
}

\section{PROJECT DESCRIPTION}

The goal of this project is to develop a short superconducting undulator by using magnet coils having conductors coated with yttrium barium copper oxide $\left(\mathrm{YBa}_{2} \mathrm{Cu}_{3} \mathrm{O}_{7}[\mathrm{YBCO}]\right)$. Undulators are at the heart of $\mathrm{X}$-ray synchrotron storage rings, as well as free-electron laser-based light sources. Advances in brilliance and tunability in next-generation light sources are, therefore, intimately tied to progress in undulator technology. Current superconducting undulators are fabricated with niobium-titanium (NbTi) superconducting wires, a mature technology. However, NbTi has nearly reached the limits of its performance. Tremendous progress has been made in high-temperature superconductors (HTSs), in particular YBCO-coated conductors. The YBCO tapes currently under development meet or exceed the performance of $\mathrm{NbTi}$ under operating conditions typical for undulators, which would enable a higher on-axis field and, therefore, a higher undulator strength. Furthermore, $\mathrm{NbTi}$ undulators operate at liquid helium temperature, which requires complicated and expensive cryogenic installations. In contrast, HTS undulators could meet the performance of NbTi while running at a higher temperature, about 10 kelvin $(\mathrm{K})$, thereby enabling simpler and cheaper cooling systems. 


\section{MISSION RELEVANCE}

This project addresses novel insertion devices within advanced light sources and is related to DOE's mission in science and innovation. In this mission, user facilities such as the Advanced Photon Source play a central role, since they provide new knowledge about structure and function of new materials for innovative devices. Our project will establish the technology for a new generation of HTS undulators with enhanced $\mathrm{x}$-ray brilliance and tunabilty.

\section{RESULTS AND ACCOMPLISHMENTS}

In FY 2014, we developed the layout for the undulator coil that uses a unique $U$-slit conductor geometry. In this geometry, the header section of the U-slit conductor establishes a superconducting joint between neighboring winding stacks and, at the same time, reverses the current flow direction. The functioning of undulators is based on alternating current flow directions in successive winding stacks. Due to the superconducting joint dissipation, demands on the cooling system are greatly reduced. We also established a winding assembly for the undulator coils and developed the hardware and protocol for the reliable fabrication of solder joints between winding stacks.

In FY 2015, we successfully implemented the fabrication and winding of U-slit YBCO-coated conductors (CCs). The U-slit wires were fabricated by SuperPower, Inc., by slicing a commercial $7.5 \%$ Zr-doped 12 -mm wide CC. A new magnetic core was designed and fabricated, and a test winding comprising four winding stacks with 55 layers each was produced. At $4.2 \mathrm{~K}$, the windings reached a critical current of $I_{c}=545$ amperes (A), corresponding to an critical current density of $J_{e}=1,360$ amps per squaremillimeter $\left(\mathrm{A} / \mathrm{mm}^{2}\right)$. This is the highest value achieved yet for this type of magnetic structure. Magnetic field profile scans showed a peak field of 0.75 tesla $(T)$ for a complete undulator, thus matching the performance of $\mathrm{NbTi}$ devices. These results showed that an undulator magnetic structure using YBCO HTS CCs would be compatible with the existing cryostats and typical cooling power requirements.

However, the sliced edges of the CC in long wire lengths were found to be prone to delamination. We therefore devised an alternative wiring scheme that allows for the continuous winding of standard 4-mm CCs without the need for resistive joints and wire slitting (see invention disclosure ANL-IN-15-054). By incorporating turnaround pieces into the structure of the magnetic core, the winding sense of neighboring stacks was reversed, yielding a "figure 8" wire pattern (Figure 1). A second test device containing seven winding stacks with 30 layers each was wound using 4-mm CCs. This coil reached a critical current of $I_{c}=660 \mathrm{~A}$ at $4.2 \mathrm{~K}$, corresponding to $J_{\mathrm{e}}=1,646$ amps per square-millimeter $\left(\mathrm{A} / \mathrm{mm}^{2}\right)$. Figure 2 shows the current-voltage curve for the second coil. Magnetic field scans indicated an on-axis field of 0.8 T, surpassing the performance of the initial test device, as well as $\mathrm{NbTi}$ devices. We are building a final test device by using this architecture and have already designed and manufactured a new magnetic core optimized for this layout.

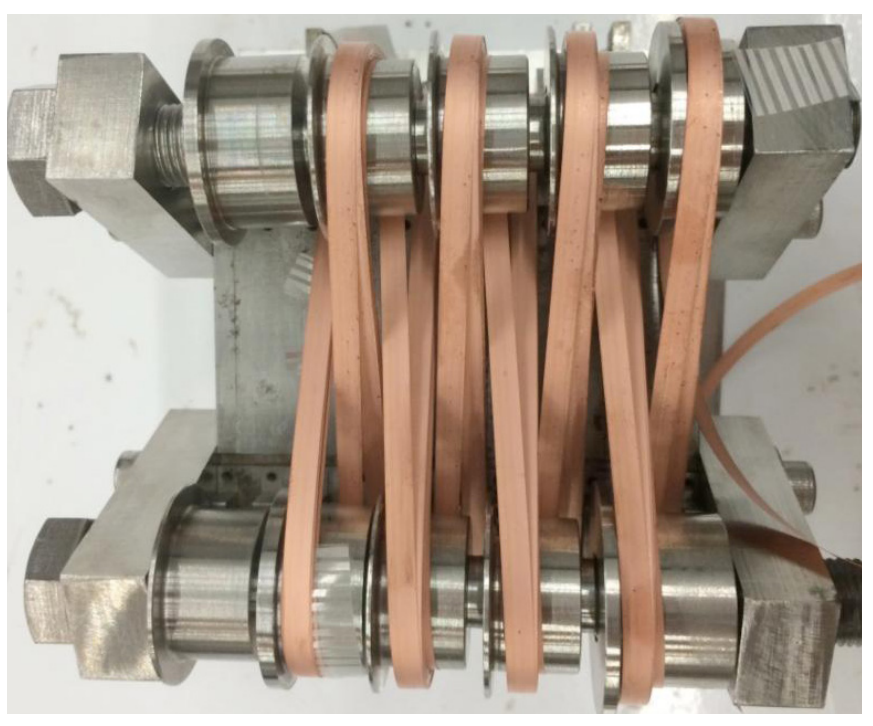

Figure 1. Top view of second undulator test device containing seven winding stacks with 30 layers each. The brown ribbon is the 4-mm CC. The winding sense in neighboring stacks is reversed with the help of turn-around pieces seen along the top and bottom edges of the picture. The beam pipe in the final undulator will run horizontally underneath the middle line of the winding.

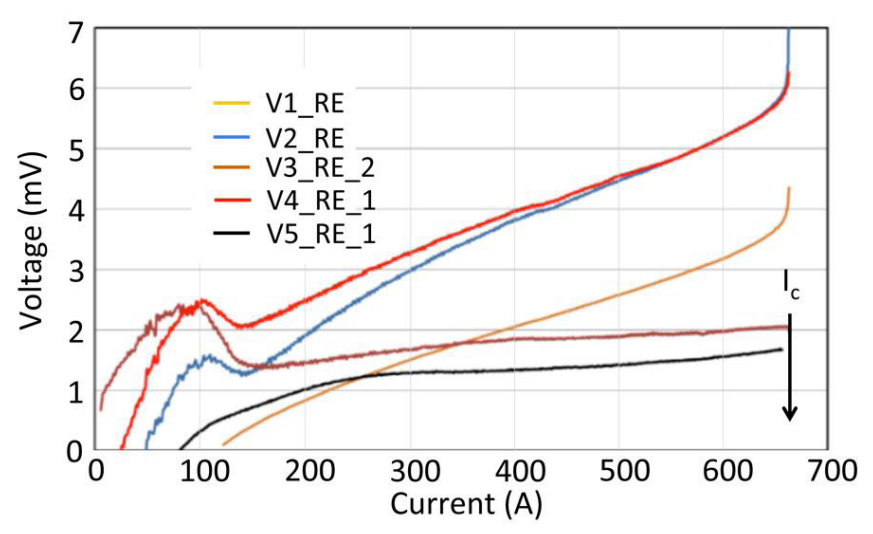

Figure 2. Current-voltage curves at $4.2 \mathrm{~K}$ measured across various taps of the second test device. The arrow marks the critical current $\left(I_{c}\right)$ of $650 \mathrm{~A}$, the highest current achieved in HTS undulators. The voltage signals at low current are due to the magnetization of the core and resistance in the cryostat current leads.

\section{PROPOSED FUTURE WORK}

In FY 2016, we will develop procedures for wet-winding (applying epoxy while winding layer on top of layer) of undulator magnets. The final undulator will operate 
in a vacuum, and cooling is accomplished via thermal conduction. This is facilitated by securing all the windings to the core with epoxy using a diamond/aluminafilled formulation. We will acquire high-performance 15\% Zr-doped CC, perform initial tests of this wire's field and temperature dependence of $J_{e}$, and wind the final undulator using the "figure 8" configuration. We will evaluate the performance of the undulator windings via tests at $77 \mathrm{~K}$ and $4.2 \mathrm{~K}$. We will begin the design of a complete planar and helical HTS undulator.

\section{Automation of In Situ Crystallization Plate Screening and Data Collection at Room Temperature}

\author{
2014-175-R1 \\ Craig M. Ogata and David Kissick
}

\section{PROJECT DESCRIPTION}

Conventional macromolecular crystallographic procedures consist of freezing a protein crystal to liquid nitrogen temperatures, mounting the frozen samples, and collecting data from a few small crystals or many positions on a large crystal. The freezing reduces radiation damage and facilitates shipping to synchrotron facilities for remote-control operations. However, macromolecular samples have grown in complexity, and single proteins have been replaced by multi-component molecular machines that are difficult to assemble and crystallize. Another important class of molecules, membrane proteins, requires crystallization mixtures that mimic their lipidic environment in order to preserve their conformation, increasing the probability for crystallization. The capability to screen through small crystals by characterizing their $x$-ray diffraction in their original crystallization conditions, prior to mechanical manipulation and the introduction of chemical cryosolvents, will provide important early leads in the crystallization process. This project is focused on providing automated diffraction screening capabilities for samples in a variety of in-situ crystallization formats. The second goal is to collect room-temperature data for in-situ samples. The recent development of room-temperature serial femtosecond crystallographic (SFX) approaches at the Linac Coherent Light Source, a free electron laser (FEL) source at the Stanford Linear Accelerator Center, has reignited interest by expanding the feasibility for room-temperature data collection. We are adapting these techniques for use at synchrotron sources, specifically the Advanced Photon Source.

\section{MISSION RELEVANCE}

This project is relevant to DOE's mission in science. It serves as an early investigation into possible new experimental techniques that will be applicable to experiments at future synchrotron sources. Commercial detector technology has also progressed. New pixel array detectors have no electronic noise, smaller pixels, and higher frame rates. New sample delivery systems combined with data collection and reduction procedures that have been pioneered at FEL sources are being exported to synchrotron sources. These room-temperature techniques employ the single diffraction image per crystal, "diffract before destroy" philosophy. The main drawbacks for this technique are the amount of sample needed to collect a data set and access to a FEL that operates at hard $\mathrm{x}$-ray (>5-kiloelectron volt $[\mathrm{keV}])$ energies. This project explores methods that take advantage of the properties of synchrotron sources while incorporating the quickly developing technologies of the FEL. Specifically, it will provide data collection from limited amounts of precious material, early diffraction characterization for improving crystal quality, and easy access to these capabilities.

\section{RESULTS AND ACCOMPLISHMENTS}

The work in FY 2014 served as a building block for the developments of FY 2015. Many of the earlier experiments concentrated on the use of established automated data collection tools following a 2-dimensional grid or along an explicitly defined 3-dimensional vector. These were applied to crystals in a variety of fixed-target sample holders. The conventional approaches are well suited to large relatively radiation-insensitive samples but insufficient for small or large radiation-sensitive samples. One of the experiments initiated in FY 2014 has continued to completion in FY 2015. In this collaborative experiment, a prototypical sample-holding chip was used to deliver hundreds of 10- to 15-micrometer $(\mu \mathrm{m})$ roomtemperature crystals to the $\mathrm{x}$-ray beam (Figure 1). The data collection mimicked the FEL data collection style-a single still diffraction image per crystal. A complete data set for lysozyme was collected from 324 crystals to 1.55-Ångstrom (Å) resolution, and a molecular replacement hen egg white lysozyme structure was solved and refined. This experiment demonstrated the feasibility and sample efficiency-hundreds of images versus a hundred thousand images-of incorporating the fixed-target serial crystallographic approach at synchrotron sources. Attempts to collect high-resolution data from high-symmetry virus crystal samples grown in commercial crystal-cards using the microcapillary protein crystallization system are ongoing. 


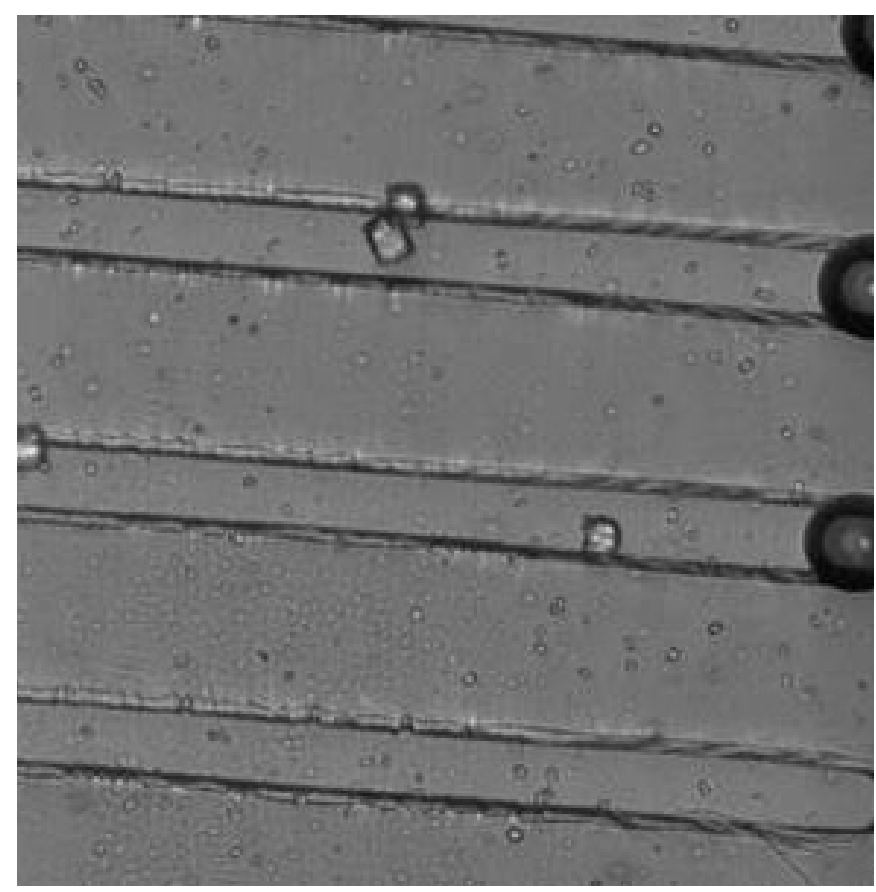

Figure 1. Lysozyme crystals in prototypical sample chip.

A second experiment characterized the $\mathrm{x}$-ray dose response of room-temperature crystals. These experiments exploited the high frame rates of a 100-hertz $(\mathrm{Hz})$ Pilatus3 6M detector (Figure 2), as well as a smaller, $800-\mathrm{Hz}$ Eiger detector to explore decay rates of roomtemperature protein crystal samples. These data analysis tools will be used to explore metrics for optimizing data collection strategies.

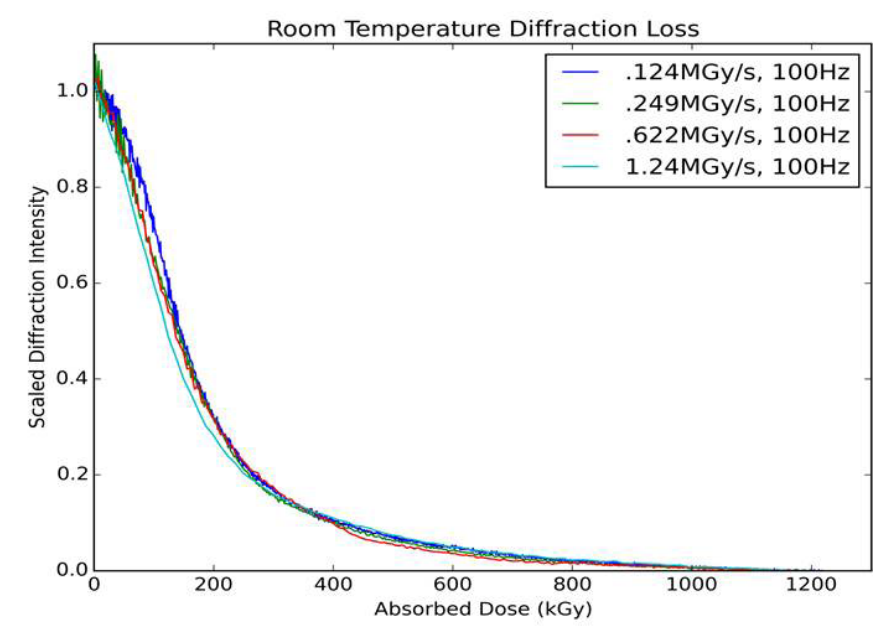

Figure 2. Diffraction kinetic studies at $100 \mathrm{~Hz}$ as a function of absorbed dose (kilo-grays) and incident dose rate (mega-grays/second).

In regard to automation, the FY 2014 designs of plate-holding assemblies for larger crystallization formats that can be quickly installed onto the experimental set-up have been manufactured, and equipment modifications to accommodate the larger position translation range are complete. Another aspect of the automation is the identification of crystals prior to mounting. Offline ultraviolet images have been used to locate the crystals in their mounts, and the locations have been transformed onto the data collection coordinate system. This feature will further increase the efficiency of data collection.

\section{PROPOSED FUTURE WORK}

Experiments on room-temperature sample delivery systems will continue with a focus on optimizing serial crystallographic approaches at synchrotron sources. Previous experiments emulating FEL approaches will be refined to reduce sample material and improve data quality. Studies on microfluidic sample holders will be expanded to include larger crystallization plates. Automated data collection techniques incorporating offline sample identification and radiation damage analysis are planned.

\section{Long SCU Magnets}

\section{4-184-R1}

Yury Ivanyushenkov, Charles Doose, Joel Fuerst,
Efim Gluskin, Quentin Hasse, Matthew Kasa,
Yuko Shiroyanagi, and Emil Trakhtenberg

\section{PROJECT DESCRIPTION}

The first superconducting undulator (SCU), the "SCUO" prototype, has been in operation in Sector 6 of the Advanced Photon Source (APS) storage ring since January 2013. It has been experimentally confirmed that this device, with a magnetic length of only 0.33 meters $(\mathrm{m})$, generates a higher photon flux at photon energies above 80 kiloelectron volts (keV) than a conventional 2.4-m-long undulator-proving that superconducting undulators, even short ones, outperform conventional undulators at the high-energy $x$-ray range. Calculations predict that SCUs with magnetic length above $1 \mathrm{~m}$ will outperform conventional undulators over the entire x-ray spectrum. Therefore, it is important first to confirm that prediction and then to extend the length of SCU magnets even farther. The goal of this project is to design and fabricate a 1.1-m-long SCU magnet that could be used in the future as a building block for longer magnetic assemblies. The magnetic performance of the magnet will be measured in a horizontal cryostat (some parts of which already exist), and the cryostat will be completed. An existing horizontal magnetic measurement system will be used for the magnetic measurements. The experimental data will be used for simulation of a long two-magnet structure with a phase shifter. We will then propose pursuing the design of a long SCU magnet with 1.1-m-long sections. 


\section{MISSION RELEVANCE}

This project supports development of brighter synchrotron x-ray radiation sources-an important part of DOE's mission in Basic Science. APS users will benefit from much higher photon fluxes as compared to the fluxes available from the existing insertion devices. Developed designs will establish a strong and reliable foundation for future SCUs planned for the APS Upgrade.

\section{RESULTS AND ACCOMPLISHMENTS}

The first year of the project was devoted to fabricating and testing the superconducting undulator magnet cores, as well as fabricating the horizontal cryostat.

In FY 2015, the fabrication of the 2-m-long horizontal cryostat was completed. The previously fabricated magnet was assembled into the cryostat, and the complete undulator was subjected to stand-alone testing. The cryogenic part of the system performed reliably with the observed heat leaks not exceeding their design values. The magnetic measurements with the horizontal measurement system confirmed the early results-the measured root-mean-square phase error was 5.3 degrees at a maximum design current of 450 amperes. The design specification for magnetic field quality was met. Following successful completion of a stand-alone cold test, the superconducting undulator (known as "SCU1") was installed into Sector 1 of the APS storage ring during the April-May shutdown of 2015. After a short commissioning period, it became a user device and has since been in full-time operation.

Another part of the work was magnetic simulation of a long two-magnet structure. Magnetic models of a general planar SCU, as well as of a 3-pole electromagnetic phase shifter, were created using the magnetostatic simulation package RADIA. The calculated independent field profiles were then used to create an overall magnetic field of a system consisting of two SCU magnets with the phase shifter located between them. The synchrotron radiation from such a system was calculated using the computer program UR developed at the APS for calculation of radiation from undulators. Zero-emittance computed spectra of the angular flux density was used to determine both (1) the initial phasing distance between the undulators and (2) the required magnetic field strength (and current) of the phase shifter to set the radiation from the two undulators in phase. It is computed that when the radiation from the two SCUs is in phase, the maximum reduction in the photon flux density is about $3 \%$ compared to a single-segment undulator of twice the length, which is consistent with radiation coming from fewer full-strength magnetic poles. When the radiation is 180 degrees out of phase, complete destructive interference is seen. Without the phase shifter turned on, the radiation goes out of phase causing significant reduction in the flux density (Figure 1). Thus, a framework of computer codes and models was created and tested with success. Overall, as a result of this two-year project, a 1.1-m-long, superconducting magnet was fabricated, tested successfully, and used for completion of superconducting undulator SCU1. The magnetic simulation of a long, two-magnet system was also performed.

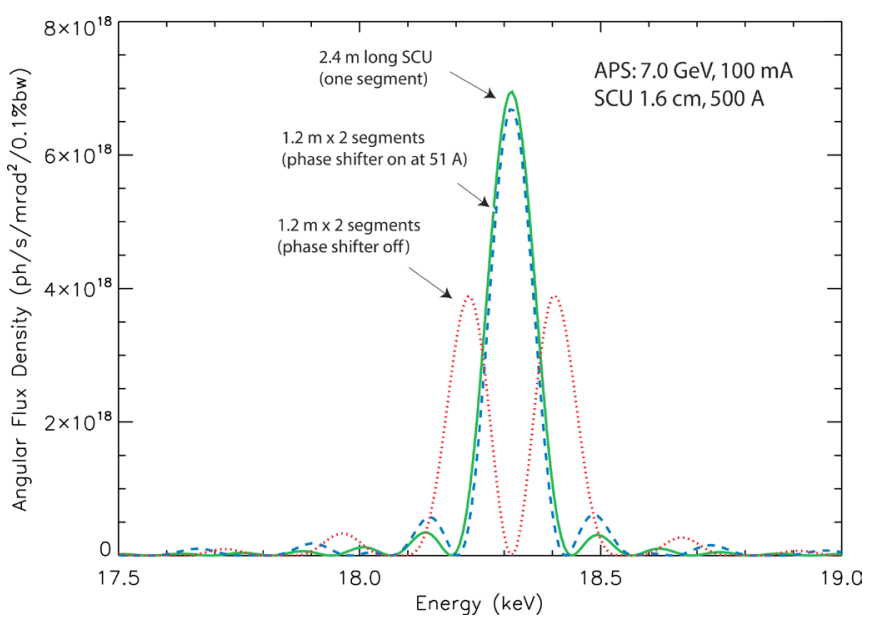

Figure 1. Calculated photon radiation spectra from two-segment superconducting undulator with (dashed line) and without (dotted line) the phase shifter. The single segment case (solid line) is shown for comparison.

\section{Using Hard X-rays to Accelerate the Synthesis of Materials}

\section{5-141-NO}

Peter Chupas, Karena Chapman, Gregory Halder, Guy Jennings, Charles Kurtz, Kenneth Poeppelmeier, and Xianbo Shi

\section{PROJECT DESCRIPTION}

Our ability to deliver a battery with 5 times higher energy density, a catalyst with 5 times longer lifespan, or a material that is $30 \%$ more efficient at converting sunlight to electricity is predicated on our ability to both discover and synthesize the next generation of functional materials. Computational approaches, such as the Materials Genome and Inverse Design initiatives, have been successful in identifying an expanded library of potential materials with predicted stability and attractive functionality; however, few of these materials have been physically realized beyond the confines of a computer. The critical unresolved bottleneck in materials discovery is the synthesis and physical realization of 
these new predicted materials. Our goal is to eliminate this bottleneck by developing hard-x-ray-based in-situ tools that allow synthetic reactions to be studied as they occur and to identify new materials as they appear. This is an advance over the trial and error approach used in laboratory synthesis where reaction pathways are only studied "post-mortem" (i.e, after some predetermined time). By identifying new materials in-situ during synthesis, this eliminates the possibility that metastable phases are not observed. Thus, in-situ $x$-ray methods will accelerate the synthesis of new materials by a factor of 1000 .

\section{MISSION RELEVANCE}

Materials discovery and synthesis science-critical to developing new energy technologies-is a traditional area of strength at Argonne and a high priority within DOE, motivated by the mission to promote energy security. This project addresses this area and provides a strategic path to accelerated discovery of new materials by changing the process we use to search for new materials. This project complements existing programs at Argonne (e.g., flux crystal growth and emergent materials in the Materials Science Division, battery electrode development in the Chemical Sciences and Engineering Division/Joint Center for Energy Science Research, and new ceramics for nuclear applications in the Nuclear Engineering Division) and will have significant impact on the broader materials community (both industrial and academic) by providing an accessible platform for accelerated materials discovery at the Advanced Photon Source (APS). This community includes companies and universities interested in a broad set of materials from catalysts to batteries and ceramics, among others.

\section{RESULTS AND ACCOMPLISHMENTS}

Several R\&D activities have been ongoing in parallel over the last year with the expectation that they will coalesce in the final year; these comprise both $\mathrm{x}$-ray optics development and high-temperature sample environment development. The developments in x-ray optics are needed to provide a high flux of high energy $x$-rays (which are able to penetrate reaction cells) from a bending magnet beamline (which are more readily accessible than insertion device beamlines for longer duration synthetic studies). The multi sample environment being developed will allow the phase distribution in multiple reaction compositions to be monitored in parallel using high energy $x$-ray powder diffraction. Together these developments will allow the temperature and compositional space for a synthetic target to be simultaneously monitored, thereby accelerating the preparation of new materials (compared to conventional new materials synthesis efforts where the product from a single reaction temperature and composition would be documented with diffraction after the reaction is complete.

A critical aspect of the R\&D was the development of monochromator optics, optimized for the energy range from 30 to 55 kiloelectron-volts (keV), that maintain the current flux and energy resolution required for quantitative powder diffraction on an APS bending-magnet beamline. Simulations and optimization of various configurations were undertaken and a model version was fabricated and tested at the optics test beamline at the APS. A monochromator with a vertically focusing bent Bragg and horizontally focusing bent Laue configuration was fabricated and demonstrated to provide the necessary focusing and flux while eliminating the need for additional optical components (i.e., mirrors).

The second activity was the development of experimental equipment (i.e., sample-environments) that allows materials reactions to be probed by hard x-rays during the synthetic process. Environments were designed to accept the standard reaction vessels and volumes used in lab-based synthesis (e.g., sealed quartz/tantalum tubes) so that insights will be directly transferrable to subsequent lab efforts. A compact furnace was designed around sample vessels with suitable apertures for the incident and scattered $\mathrm{x}$-rays. The furnace can accommodate multiple reaction tubes to allow more efficient, parallel reaction studies. Test measurements demonstrated data acquisition time scales of a few seconds, allowing multiple samples to be studied in parallel and at fine reaction intervals (e.g., 10 data points for each of 10 reactions for a 10 -min synthesis). Initial designs have allowed temperature control up to $900^{\circ} \mathrm{C}$ and provide access to a broad range of materials synthesis conditions.

\section{PROPOSED FUTURE WORK}

Final designs and assembly of the optics and the furnace will improve stability for both optics and temperature control. These developments will be applied to proof-of-principle studies focusing on a series of complex transparent conducting oxides based on the $\mathrm{Ga}$ (gallium)/In (indium)/Sn (tin)/O (oxygen) (GITO) system.

Transparent conducting oxides are materials that find use in devices such as solar cells, displays, and opto-electrical interfaces. The first experiments will evaluate what substituents assist in optimizing the synthesis of GITO. The conductivity of any new phases will be assessed. Subsequent tests will be expanded to substituted GITO systems, where distortions from the cubic lattice due to lattice distortions, vacancies or atomic displacements are 
of particular interest. Potential structural transitions driven by temperature would be valuable in understanding how to manipulate materials composition to achieve a target structure. In-situ studies are critical as they allow rapid identification of desired products and metastable phases that may be missed in otherwise standard synthetic approaches.

\section{Development of a Compact 352-MHz/150-kW CW Solid State RF Power Amplifier System for Accelerators}

\author{
2015-147-NO

\begin{abstract}
Alireza Nassiri, David Bromberek, Aditya Goel, Douglas Horan, and Geoff Waldschmidt
\end{abstract}

\section{PROJECT DESCRIPTION}

This project is focusing on developing trial designs for high-power radio frequency (RF) amplifiers operated at 352 megahertz (MHz), employing highly efficient, solid-state power amplifier technology, that can be used at DOE accelerator facilities.

\section{MISSION RELEVANCE}

Following Executive Order 13514, the DOE has committed to meeting sustainability goals by, among other things, improving energy efficiency at DOE laboratories. Among the systems with high energy consumption at DOE laboratories are the RF sources that power accelerators. Significant energy savings can be realized if high-efficiency alternatives are installed as existing RF sources reach end-of-life or as new facilities are brought on-line. This project aims to address these issues.

\section{RESULTS AND ACCOMPLISHMENTS}

In FY 2015, significant progress was made on both thermal and electrical design of a 352-MHz/2-kilowatt (kW) continuous-wave amplifier module utilizing a single LDMOS (laterally diffused metal oxide semiconductor) transistor device that will be utilized in a compact 12-kW amplifier system. Initial design and analysis of a 6-way $\mathrm{RF}$ power combiner to be used in the $12-\mathrm{kW}$ system was started.

A carrier/cold plate assembly to be used as the cooling system for the first 2-kW amplifier was designed and fabricated to provide sufficient cooling of the transistor; cooling is a critical element of the design, as it has a direct effect on transistor lifetime. A thermal simulation model using finite element analysis was developed using the LDMOS transistor geometry and material thermal properties of the device. Direct-current power dissipation tests were performed using the transistor, carrier, and cold plate. The thermal model developed was within $\sim 2$ degrees Celsius of actual test data. Other novel cooling schemes were explored, including a nano-bonding technique as an alternative to soldering for the fabrication of solid-state power amplifiers.

The 352-MHz/2-kW amplifier design was based on the manufacturer-supplied transistor model. Simulations were performed to obtain first-pass impedance values for optimal performance, efficiency, and spectral purity. On the basis of these simulation results, the input and output matching networks were designed and simulated to verify satisfactory performance. This verification led to fabrication and testing of a 2-kW model amplifier (see Figure 1).

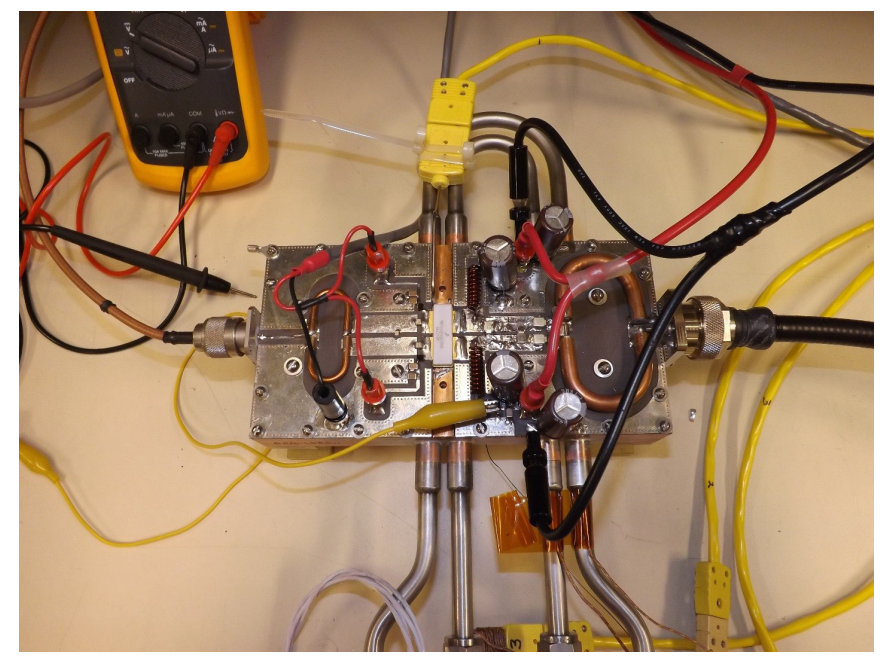

Figure 1. Prototype 2-kW amplifier mounted on test bench for power testing.

To produce multi-kilowatts of RF power with solid-state amplifiers, we began the design of a resonance cavity RF power combiner. Preliminary design was focused on a 6-way combiner (combining six 2-kW amplifiers) using inductive coupling to produce $12 \mathrm{~kW}$ of output power. A preliminary design of the cavity combiner with a waveguide transition output is shown in Figure 2a; a tulipshaped matching element was used to achieve low return loss and minimum reflection (see Figure $2 \mathrm{~b}$ ). 

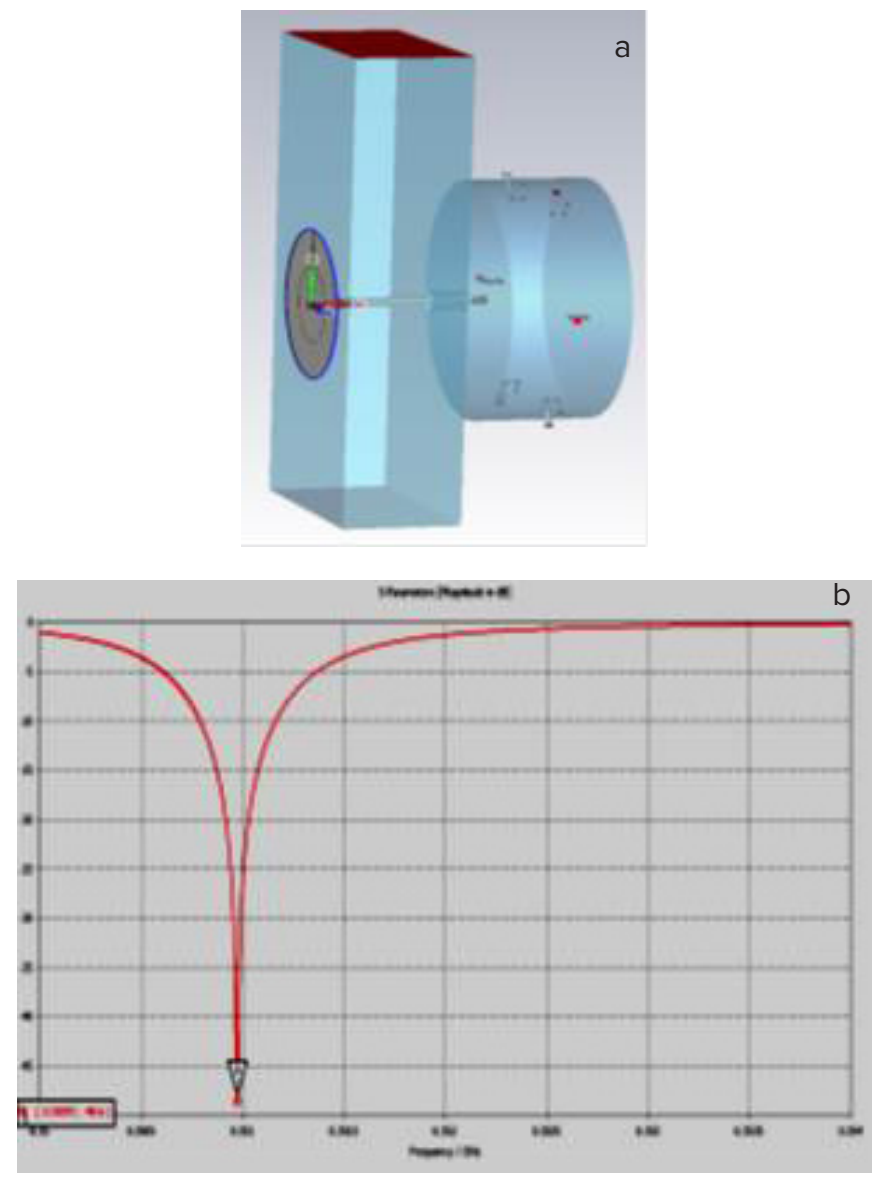

Figure 2. Full simulation model of cavity-to-waveguide output: (a) geometry and (b) return loss from waveguide to amplifier inputs.

\section{PROPOSED FUTURE WORK}

In FY 2016, we began the design and fabrication of a suitable 6-way rf cavity (resonator) combiner that will combine the outputs of six 2-kW rf amplifiers to produce $12 \mathrm{~kW}$ of combined output power. This unit will be fully power tested in FY 2016. We will also start the initial design of a 150-kW unit.

In FY 2017, we plan to complete the design of a 352-MHz/150-kW rf power combiner system and be ready to start fabrication, assembly, and evaluation of this unit if the project receives the requested funds.

\section{Unraveling Mesoscale Spatial-Temporal Correlations in Materials Using Coherent X-ray Probes}

\author{
2015-150-NO
}

Alec Sandy, Robert Bradford, Anthony DiChiara, Eric Dufresne, Paul H. Fuoss, Zhang Jiang, Alan Kastengren, Meimei Li, Xiao-Min Lin, Timothy Madden, Antonino Miceli, Suresh Narayanan, Paul F. Nealey, Nicholas Schwarz, Joseph Strzalka, Donald Walko, Jin Wang, John Weizeorick, Haidan Wen, and Hoydoo You

\section{PROJECT DESCRIPTION}

Our project is focused on (1) advancing x-ray photon correlation spectroscopy (XPCS) to very fast time scales, and (2) creating and deploying the infrastructure necessary to develop coherent surface imaging (CSI) beyond its current demonstration state. The overarching goal is to develop coherent $x$-ray scattering capabilitieswell aligned with the proposed Advanced Photon Source (APS) upgrade - that will enhance understanding of structure and dynamics at the mesoscale.

\section{MISSION RELEVANCE}

Our project is a first step in creating programs that will lead to full utilization of the increased coherent flux from the APS multi-bend achromat upgrade. It will advance coherence-based techniques for surfaces and for measuring fast energy-dissipative dynamics. Our project supports the large investment that DOE-BES has begun making to upgrade the APS into a 4th-generation light source. In addition, our project greatly enhances basic science capabilities that DOE-BES already supports through programmatic funding at national laboratories and universities.

\section{RESULTS AND ACCOMPLISHMENTS}

We performed fast time-resolved coherent x-ray scattering measurements (via x-ray photon correlation spectroscopy [XPCS]) with a new design of a photon-counting detector called VIPIC. Using this detector, we achieved multi-speckle XPCS measurements to a time delay of 10 microseconds ( $\mu s)$, which is approximately 5-times faster than had been achieved previously. Figure 1 shows correlation decays as a function of temperature for 70-nanometer ( $\mathrm{nm}$ )-diameter latex particles in glycerol, measured using the VIPIC detector. As the sample temperature is increased, the viscosity of the solvent changes rapidly, yielding increasingly faster correlation decays. The measurements were performed 
using an unfocused, narrow-bandpass coherent x-ray beam. Unfortunately, the VIPIC is no longer functioning, so to further advance fast XPCS, we worked with our colleagues to modify the firmware of a novel Voxtel detector to perform dual gate measurements with delay times that can approach the bunch length of the APS (100 picoseconds). Our demonstration of the overall utility of this approach succeeded; however, access to short delay times was limited by other shortcomings in this particular detector. On the basis of our efforts using both of these detectors, we worked with a research group from the Akademia Górniczo Hutnicza (AGH) University of Science and Technology in Krakow, Poland, to apply a powerful new dual-gate detector to measurements of the dynamics associated with the gelation of "hairy" nanoparticles in a solvent where the nanoparticle interactions depend sensitively on temperature. At higher temperatures, where the interparticle interactions are very weak, we were able to observe simple fluid-like dynamics at fast time scales. At lower temperatures, we observed arrested dynamics corresponding to a gelled state. At intermediate temperatures, we observed novel correlation decays that have previously been attributed to unusual glassy-liquid states. Detailed analysis and understanding of this data are ongoing.

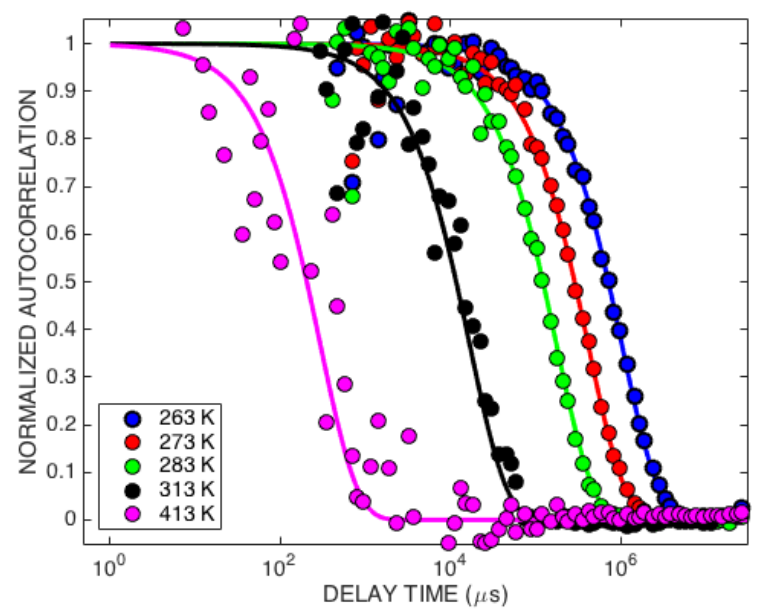

Figure 1. Correlation functions measured for 70-nm-diameter latex particles in glycerol as a function of temperature. The wavevector transfer was $0.024 \mathrm{~nm}^{-1}$.

We advanced CSI by developing a method for providing fine, in-plane resolution for exact near-surface reconstructions in all azimuthal directions. First, we performed simulations that showed that azimuthal rotations $(\phi)$ about the surface normal of only several degrees-so-called angular ptychography-provide dramatic gains in resolution. The inset in Figure 2 summarizes the results of this study, namely, the finding that a rotation about the surface normal of $10^{\circ}$ increases the in-plane resolution by 20 times. Experimentally, to accomplish this rotation with the necessary reproducibility, we determined that a new type of flexure-based rotation stage was required. With APS Senior Engineer Deming Shu, we designed such an assembly and have procured and received all necessary parts (assembly and installation will be performed in FY 2016). Figure 2 shows a model of this positioning assembly.

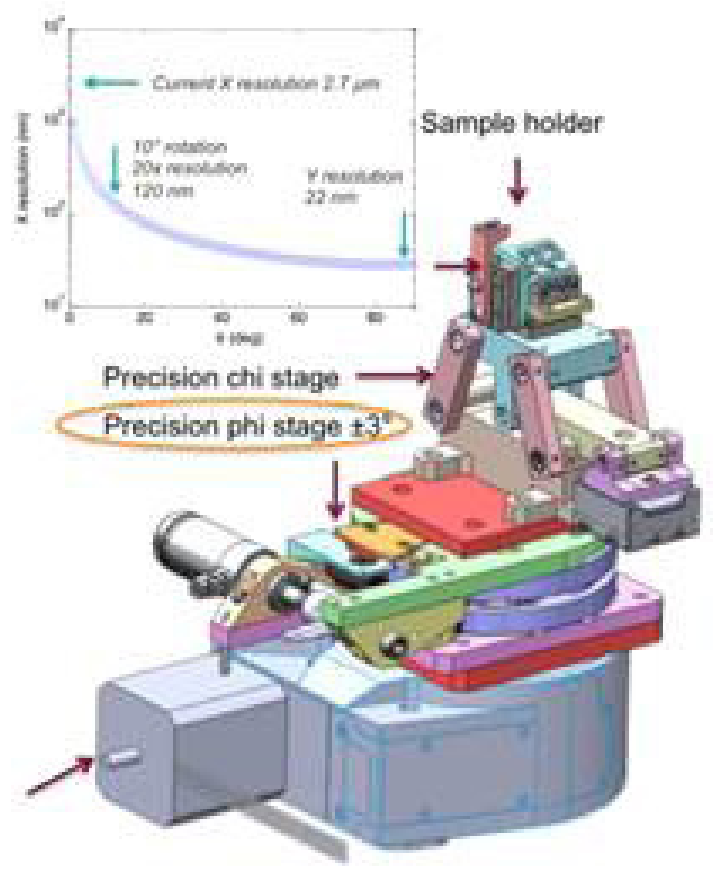

Figure 2. A model of the apparatus designed and procured for providing precision rotation about the surface normal to facilitate fine CSI resolution in all directions in the surface plane. The inset shows the calculated resolution as a function of rotation angle about the surface normal.

\section{PROPOSED FUTURE WORK}

In FY 2016, we will perform coherent surface imaging measurements that can be inverted to demonstrate angular and lateral ptychography in the CSI geometry. We will accomplish this by reconfiguring our experiment station to enable high resolution via the assembly and installation of recently designed and procured hardware that allows both longer sample-to-detector distances and fine-resolution angular positioning. We will continue our efforts to acquire XPCS data at increasingly shorter delay times. This effort will require modeling and understanding detector response at delay times approaching the APS storage ring bunch spacing and the detector pulse-shaping time. We also will perform XPCS measurements with much higher flux (enabling access to shorter delay times) by incorporating focusing and broader-bandpass $\mathrm{x}$-ray beams. 


\section{The VelociProbe: Ultra-High-Resolution Ptychographic Hard X-ray Nanoprobe}

\author{
2015-153-NO \\ Shane Sullivan, Chris Jacobsen, Barry Lai, Jörg Maser, Curt \\ Preissner, Chris Roehrig, and Stefan Vogt
}

\section{PROJECT DESCRIPTION}

The goal of the VelociProbe project is to design, build, and demonstrate a new type of x-ray microscope that can achieve sub-10-nanometer $(\mathrm{nm})$ spatial resolution using ptychography and scan one-square-micron areas in under 10 seconds so as to take advantage of the Advanced Photon Source (APS) Upgrade. Currently, no instruments exist that can do this. Our novel approach employs new hardware designs, new positioner control designs, and new data acquisition (DAQ) strategies. The hardware design maximizes dynamic stability to enable high-speed scanning. The controller design will push the limits of scanning bandwidth and enhanced disturbance rejection, also taking advantage of the high-frequency dynamics to employ scanning strategies other than the simple raster scan. The new DAQ strategies may relax the constraints on the precision of the hardware positioning while still allowing for the highest resolution.

The challenge of high resolution and fast scanning will be met in two fundamental ways: (1) development of a nanopositioning system with high dynamics and low drift, and (2) control and metrology systems that offer scanning and disturbance rejection heretofore unavailable in the $\mathrm{x}$-ray microscope world. Our nanopositioning design incorporates a commercially available low-mass, high-dynamics scanner integrated into a novel and ultra-stable coarse-positioning system. This hardware provides the necessary long travel ranges to accommodate real samples and variable-energy operation while remaining highly stable during scanning. Our advanced control design is the product of joint efforts with Professor Vasu Salapaka of the University of Illinois at Urbana-Champaign. It will take advantage of bandwidth-enhancing and disturbance-rejection schemes employed by scanning probe instruments (e.g., atomic force microscopes).

\section{MISSION RELEVANCE}

The VelociProbe (an ultra-high-resolution ptychographic hard x-ray nanoprobe) is being designed to enable science that has never been performed before at
DOE-SC's Multi-Bend Achromat (MBA) lattice upgrade of the APS. This upgrade represents a major change for nanofocusing experiments. The increased brightness of the APS MBA lattice translates directly to coherent flux. With improved nanofocusing optics, increases in flux density approaching tens of thousands are expected. These dramatic changes will enable scientific studies that are not possible today using methods such as ptychography to study structure and x-ray fluorescence to study elemental composition in three dimensions (3D) at $20 \mathrm{~nm}$ and better resolution. The increased flux density both invites and necessitates high-speed microscopy; if the sample is not scanned rapidly enough, radiation damage will occur. Likewise, if the instrument is not stable enough to use the new optics, no resolution advantage will be evident. Our new instrument overcomes those barriers and will support a variety of DOE science mission-related R\&D at the APS.

\section{RESULTS AND ACCOMPLISHMENTS}

The VelociProbe project was planned to take place over three years. The first year has included an extensive design phase, moving into a final design. The second year is focused on assembly and integration of the hardware, development of the control system, and extensive off-line testing. The third year includes at least two periods of beam-time use at the APS.

The major accomplishments in the first year of the project were the design and procurement of the ultra-stable coarse-positioning system shown in Figure 1. This system will be fully assembled in the first quarter of CY 2016. An Argonne invention report (ANL-IN-15-072) was filed for the coarse-positioning-stage system.

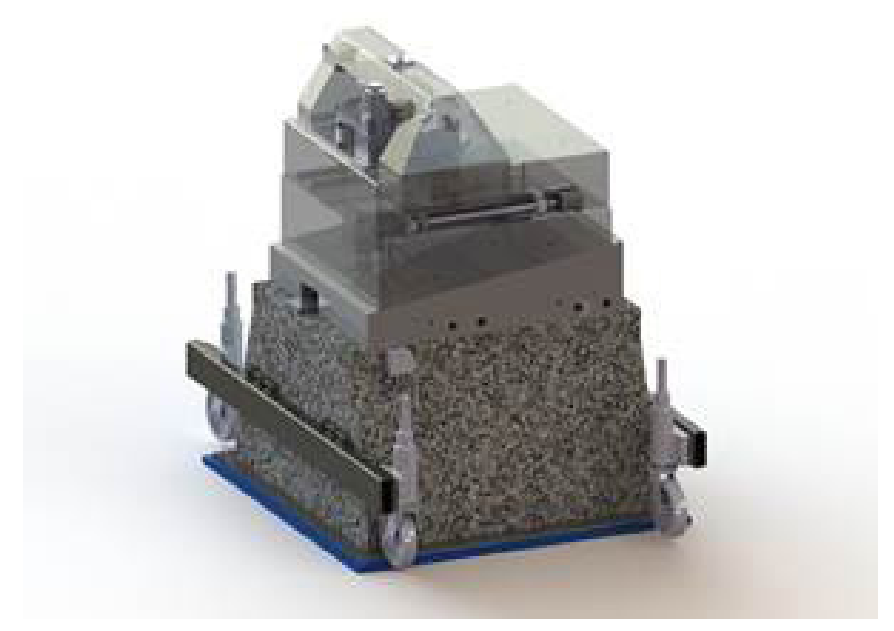

Figure 1. Rendering of the VelociProbe design, with a footprint of $\sim 1 \times 1 \mathrm{~m}^{2}$. The granite coarse-positioning stage is shown as being partially translucent to reveal the details of the motion and guiding mechanisms. The fast-scanning stage is located on the gantry along with the interferometers for sample and optics position sensing. 
In addition to the coarse-motion platform, the fast-scanning stage and one of the two three-axis interferometer systems have been acquired. The electronics to develop both our baseline controller and the advanced controls have been acquired, including those for one of the two three-axis interferometer systems (with 0.1-nm position accuracy). Testing of these components is ongoing both to verify that they meet specifications and we are developing the initial control setup.

\section{PROPOSED FUTURE WORK}

Assembly of the instrument will be completed once all components have arrived. Development of a basic control system and testing will be performed off-line. Lab testing of a conventional parameter ID (PID) scanning control system will be performed off-line using a laser optical system to simulate a coherent $\mathrm{x}$-ray source. That will allow us to fine-tune the instrument design as needed, as well as collect data for ptychography reconstruction algorithms and test on-line (real-time) reconstruction techniques. The advanced control system will be developed in parallel with instrumentation refinement. The timeline for construction, off-line testing, and control system development is set to allow for first APS beamline use (x-ray imaging) at the beginning of the Fall 2016 APS run.

\section{Ion Beam Figuring with In-situ Metrology: Diffraction Limited X-ray Optics and Dynamic Aperture for Three-Dimensional Control of Thin-Film Deposition and Ion-Beam Erosion}

\section{5-161-NO}

\begin{abstract}
Raymond Conley, Lahsen Assoufid, Jason Carter, Mark Erdmann, Kurt Goetze, Tim Mooney, Jun Qian, and Bing Shi
\end{abstract}

\section{PROJECT DESCRIPTION}

This project comprises two design efforts: an ion-beam figuring (IBF) with an in-situ metrology component and a dynamic aperture (DA) component. Both efforts leverage the Advanced Photon Source's (APS's) new Modular Deposition System (MDS), which is described below.

The APS is constructing a unique $x$-ray optics processing system that is designed to produce advanced multilayer-based $x$-ray optical elements. The machine is built around a precision in-vacuum brushless direct current (DC) servo drive that provides 1-nanometer (nm) position resolution over a travel length greater than 4 meters (m). By integrating this machine with an in-situ Fizeau interferometer and ion-milling equipment, the APS may begin in-house fabrication of mirrors capable of taking full advantage of the improved coherence $x$-ray beams from the multi-bend achromat (MBA) lattice. This MDS will be the world's first thin-film deposition system capable of in-situ metrology and ion milling for 1.5-m long mirrors. The combination of ion milling, deposition, and in-situ metrology will allow rapid turnaround for mirrors with the figure errors appropriate for the MBA lattice. At present, such optics must be fabricated by cycling between two separate instruments. In addition to inducing inherent mirror repositioning and registration errors, there is a 2-month turnaround time for a profile-coat-plus-metrology cycle, a period that can be substantially reduced with this new system. In 2014, the X-ray Science Division (XSD) alone owned 55 long beamline mirrors, with a median age of 10 years, none of which meet the specifications required for the MBA. Since existing efforts utilize separate ex-situ metrology stations, there is no capability for iterative figure correction by either material removal or material addition when using reactive materials or metals. By incorporating an in-situ metrology station, this machine will be uniquely positioned to handle both standard optical materials, such as silicon or glass, as well as materials that oxidize or form nitrides.

The second goal of this project is to develop dynamically actuated baffle arrays, comprising multiple, identical actuated baffle modules, for real-time control of thin-film deposition and/or ion-beam erosion in three dimensions. We wish to develop a modular actuated baffle mechanism that can be easily adapted to a variety of similarly sized planar, rectangular magnetron cathodes, and rectangular ion sources, regardless of their specific dimensions.

\section{MISSION RELEVANCE}

A successful project (both the DA and IBF components) would benefit the majority of the beamlines at the APS, both existing and planned. This effort would also benefit the majority of users who utilize reflective $x$-ray optics, especially those requiring diffraction-limited focusing or minimal coherence distortion. Through higher performance optics and the ability to produce new optical geometries through the use of the DA, all DOE mission priorities in fundamental science will be impacted directly; that is, the discovery of new materials, predictions of new physical processes, and the advancement of major scientific user facilities. 


\section{RESULTS AND ACCOMPLISHMENTS}

During FY 2015, several research and development (R\&D) topics and instrumentation design goals were accomplished. By using the existing APS 6-inch Fizeau interferometer, single-frame interferogram measurements of a conventional transmission flat to surface under test (SUT) setup were obtained. Following this measurement, an ultra-high vacuum (UHV) window was then inserted into the optical path before the transmission flat for a second measurement. Both measurements were structurally similar. Carrying on with these tests, quantitative data taken with an actual mirror under vacuum and a static transmission flat indicated a below 2-nm root-mean-square (RMS) difference. While this value is sufficiently small for a wide range of optics, this difference could possibly be attributed to shifts by the transmission flat relative to the SUT and therefore eliminated. This served to demonstrate a reliable method for measuring a flat mirror which is within a vacuum system, while the interferometer is isolated from the UHV environment-a FY 2015 goal of this project. These results guided the development of a UHV gimbal that provides tip, tilt, and full rotation of the transmission flat under vacuum. The gimbal shown in Figure 1 is based around rotational flexure joints with the tip and tilt axes controlled by picomotors. The transmission flat is suspended vertically by a steel band in order to eliminate single force points on the optic which will produce figure distortions. Stepper motors lift the transmission flat off the band and provide rotation. Mechanical design, fabrication, parts procurement, and assembly were completed in FY 2015. Secondly, a software foundation was developed that allows for convolution and trajectory solution of an arbitrary ion flux profile with a measured mirror surface. While this has been done by groups worldwide over the last few decades, an in-house capability must be implemented for final deployment in the MDS.

The first phases of the DA component for this project are primarily instrumentation design and development. The first version DA was based around a miniature recirculating linear guide and brushless DC motor coupled to a lead screw. Two different motors were tested-a directly coupled motor, as well as a motor with an integrated gear reduction assembly for higher torque. These motors are shown on the left side of Figure 2. The version shown includes five finger elements; however, only one was driven at a time. As can be seen in the figure, the mechanical stiffness comes from the linear rail mounted to the base of the unit. Flaws found in the first design (including unsatisfactory travel rate, poor reliability, and lack of mechanical stiffness) led to the development of a second version which is currently undergoing testing in a UHV sputtering environment.

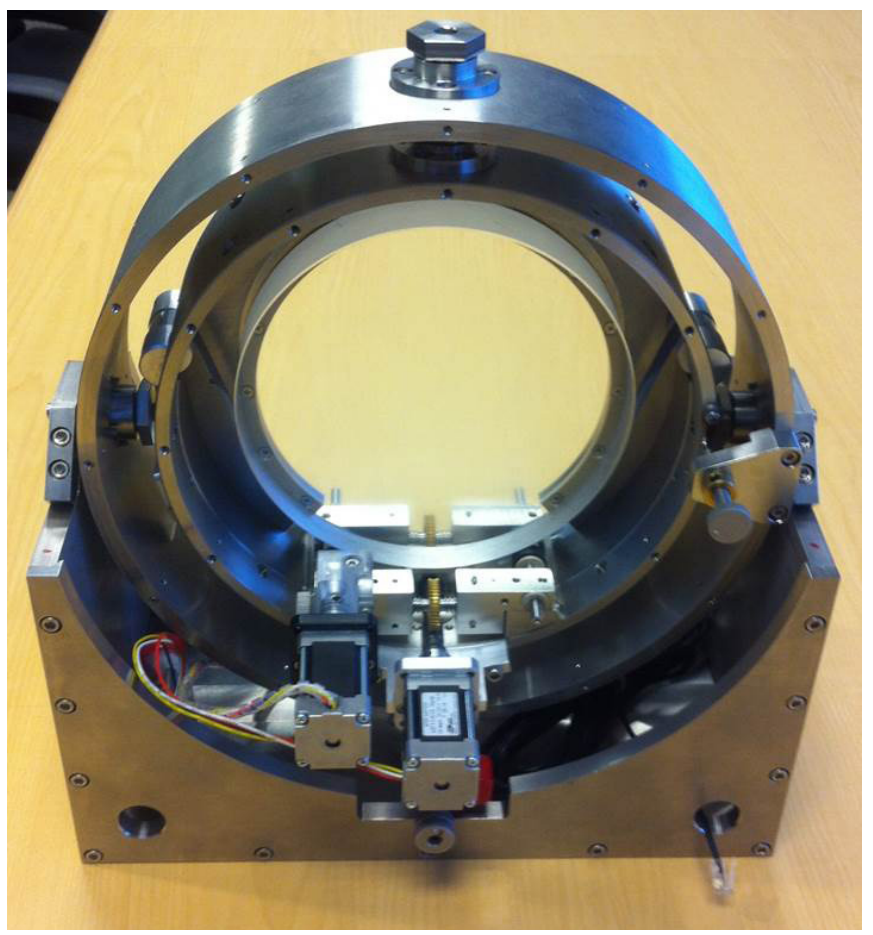

Figure 1. Photograph of the assembled gimbal awaiting testing. Piezo-based picomotors (not shown) actuate the tip and tilt axes, the central stepper motor at the base of the unit elevates the transmission flat off the steel support band, and the stepper to the left provides rotation.

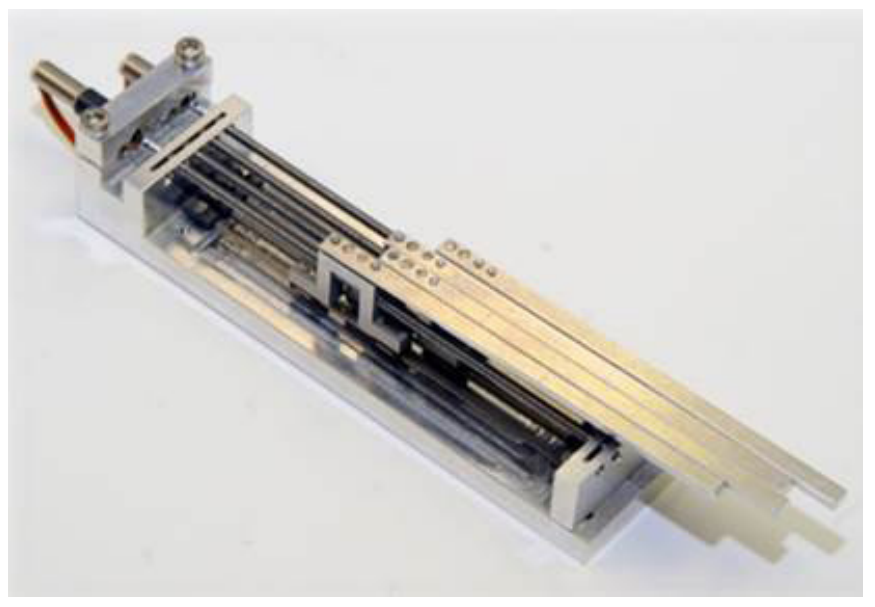

Figure 2. Dynamic aperture-assembled prototype version 1.0. Five finger elements were fabricated, although only two are driven by motors at the left of the unit during testing. The larger motor incorporates an integrated planetary gear reduction assembly for increased torque.

Two "Record of Intention" forms have been submitted for Intellectual Property protection. One has been filed as a provisional patent.

\section{PROPOSED FUTURE WORK}

In FY 2016, we plan to investigate the effects of utilizing a transmission flat as a vacuum to atmosphere pressure interface for interferometry. This includes quantifying the relative inclusion of true surface height errors as well as 
raymapping errors. It is possible that atmospheric effects such as barometric pressure fluctuations will introduce error. Testing during FY 2016 will aim to identify and quantify these. The DA component will focus on two questions-how reliable the DA is during use in an actual sputtering environment, and how accurately our modeling reflects the use of a dynamically moving mask in front of the flux. We will also procure ion mills and continue development of hardware integration and automation.

\section{Next-Generation Mössbauer Spectroscopy}

\section{5-164-NO}

Thomas S. Toellner, Ahmet Alatas, E. Ercan Alp, Michael Hu, and Jiyong Zhao

\section{PROJECT DESCRIPTION}

Using synchrotron radiation as a radiation source to perform Mössbauer Spectroscopy (MS) offers many advantages for materials research. The inherent small beam size and excellent collimation, which do not exist for traditional MS, allow samples from a wide range of scientific disciplines to be probed on a microscopic scale. Currently, MS is performed at beamline 3-ID of the Advanced Photon Source (APS); however, the data collection rates are limited. This project aims to improve the data collection rates significantly, as well as to expand this measurement technique to include simultaneous x-ray diffraction.

The project involves building a novel Mössbauer spectrometer at beamline 3-ID at the APS that will operate during the "hybrid-fill mode" by combining micro-focusing with high-speed shutters that will significantly improve data collection rates and allow simultaneous $\mathrm{x}$-ray diffraction to be collected on samples containing Mössbauer isotopes. During the hybrid-fill mode at the APS, a pulse of synchrotron radiation excites the nuclear level in a sample once every 3.7 micro-seconds, leading to a $270-\mathrm{kHz}$ repetition rate. This rate is "low" enough to allow periodic mechanical blocking of the beam with a high-speed shutter placed between a sample and the detector. This offers a means of protecting the detection system from being overwhelmed by the synchrotron radiation pulses, while then opening fast enough to allow a time spectrum of the decay of the nuclear excitation to be detected in a coherent scattering channel (e.g., forward scattering). Furthermore, because this mitigates any detector "overload" problems, the sample can be excited with very broadband synchrotron radiation containing high flux, thus allowing simultaneous $x$-ray diffraction data to be collected with an image detector placed after the sample but before the high-speed shutter. This will allow structural studies to be directly combined in situ with measurements of hyperfine interactions.

In addition to this spectrometer, the project involves building a hyper-monochromatic source (nano-electron volt bandwidth) of 14.4-keV synchrotron radiation by using $x$-ray polarization filters and a single crystal containing Fe-57 that will allow MS to be performed in a manner that is more familiar to spectroscopists by collecting energy spectra, as opposed to time spectra, which is the norm when using synchrotron radiation as the source. Our approach to producing a hyper-monochromatic gamma-ray beam is accomplished in three basic steps. First, synchrotron radiation is prepared in a pure linear polarization state with the use of a filter made from crystalline silicon. Second, the beam then scatters coherently (e.g., into the forward direction) from a paramagnetic single crystal containing Fe-57 (e.g., potassium ferricyanide) that has a small externally applied magnetic field. This induces polarization switching for the nuclear resonant radiation, but not for the non-resonant radiation. Third, the forward scattered radiation from the Fe-57-containing crystal is directed through a second linear polarization filter that is oriented orthogonally with respect to the first filter. The orthogonal filters prohibit the transmission of non-resonant radiation (if made well enough) and transmit only resonant radiation. The resulting hyper-monochromatic gamma-ray beam may be scanned by Doppler shifting the resonant crystal. This approach avoids many of the problems other approaches present. For example, this method produces a narrow band of radiation with a centroid and spectral shape that is insensitive to small angular and temperature changes of the resonant crystal. Also, this way of performing MS at a synchrotron does not rely upon time discrimination as is usual, and so does not depend on the time structure of the pulses, or "fill mode," of the synchrotron.

\section{MISSION RELEVANCE}

This project is carried out in support of a DOE synchrotron user facility by realizing a novel approach that provides improved measurement capability with higher data collection rates. This project is also relevant for the proposed multi-bend achromat (MBA) upgrade of the APS, which involves reducing the emittance of the source and offers a number of benefits for x-ray measurements, including the potential for a significantly higher spectral intensity. The proposed MBA upgrade entails a time structure for the delivery of $\mathrm{x}$-ray pulses that leaves less time between pulses than what is currently provided at the APS. This is detrimental to some x-ray measurements 
that rely on a longer time between $x$-ray pulses. Exploring the viability of high-speed shutters to perform x-ray pulse selection and isolation offers a potential means of recovering the favorability of a longer time between pulses for some measurements, while maintaining the benefits of a lower emittance source.

\section{RESULTS AND ACCOMPLISHMENTS}

All FY 2015 milestones stated in the original proposal have been met:

$\square$ Designed and procured components for both vertical and horizontal focusing,

$\square$ Procured platform for sample environment and high-speed shutters,

$\square$ Identified and procured Fe-57-enriched candidate materials,

$\square$ Designed linear x-ray polarization filters for $14.4-\mathrm{keV}$ synchrotron radiation, and

$\square$ Specified design parameters for beryllium refractive lenses. These are to collimate the synchrotron radiation to allow the polarization filters to perform more efficiently.

In addition, we assembled and installed a complete vertical focusing assembly with motion control in the C station of beamline 3-ID of the APS. Naturally abundant ( $2 \% \mathrm{Fe}-57$ ) single crystals of potassium ferricyanide were grown in the lab. We demonstrated the viability of our new approach to producing a hyper-monochromatic $\left(\Delta E / E=7 \times 10^{-13}\right)$ source of gamma rays using those crystals to measure a Mössbauer absorption spectrum of alpha-Fe (see Figure 1).

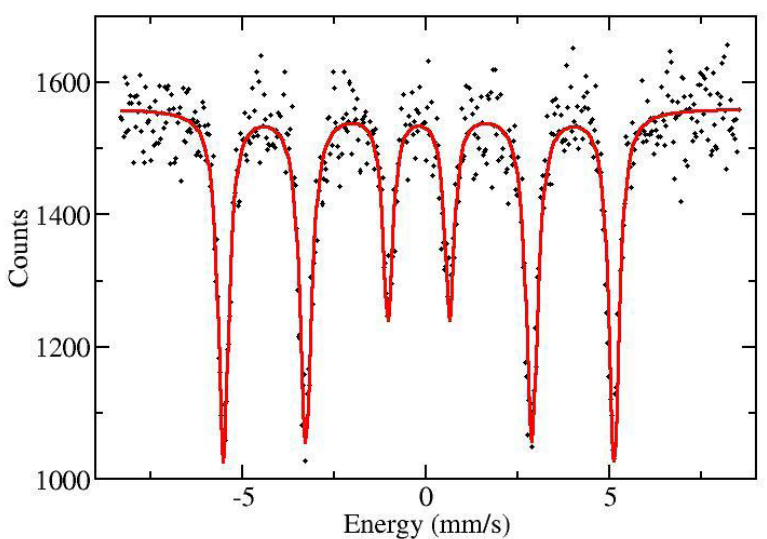

Figure 1. A Mössbauer spectrum of alpha-Fe measured with synchrotron radiation using linear $x$-ray polarization filters and a single crystal of potassium ferricyanide to generate the hyper-monochromatic $\left(\Delta E / E=7 \times 10^{-13}\right)$ source. Data were taken at beamline 3-ID of the APS.

\section{PROPOSED FUTURE WORK}

A reduced scope of this project for FY 2016 will include only those aspects that directly impact the implementation of high-speed shutters for MS. The development of the hyper-monochromatic source of gamma rays for energy-domain MS will not be pursued further within this project.

Milestones for FY 2016:

$\square$ Procurement of necessary components to upgrade the double-crystal monochromator at beamline 3-ID,

$\square$ Installation and commissioning of both vertical and horizontal focusing systems, and

$\square$ Installation and commissioning of a platform for sample environment and high-speed shutter system.

\section{Isotope Geochemistry via Sn Isotope Fractionation Using Inelastic X-ray Scattering of Synchrotron Radiation}

\section{5-173-NO}

\section{E. Ercan Alp and Michael Hu}

\section{PROJECT DESCRIPTION}

Siderophile (iron-loving) elements provide clues to the conditions under which terrestrial planets differentiated into metallic cores and silicate mantles. The concentrations of such elements (e.g., nickel [Ni], cobalt [Co], tungsten [W], and tin [Sn]) in the Earth's mantle were set by metal-silicate equilibrium in an early magma ocean. The thermodynamic conditions that obtained prior to this differentiation (pressure, temperature, and $\mathrm{fO}_{2}$ [fugacity or effective partial pressure of oxygen]), however, are a matter of intense debate.

Sn is generally classified as a chalcophilic (sulfur-loving) element; however, classification as siderophilic or chalcophilic is a matter of degree and depends on the nature of the host matrix. For example, there is a large variation in the thermochemical behavior of $\mathrm{Ni}-\mathrm{Sn}$ and $\mathrm{Ni}$-iron(Fe) alloys. Very large changes occur in the trace element activity coefficient of $\mathrm{Sn}$ in Fe-Ni alloys as a function of composition versus pure Fe. Furthermore, the behavior of two stable oxidation states of $\mathrm{Sn}$ as $\mathrm{Sn}^{+2}$ and $\mathrm{Sn}^{+4}$ creates a further complication in interpreting oxygen fugacity data (see C.J. Capobianco et al., 1999, Geochemica et Cosmochemica Acta, 63:2667). The thermodynamic conditions that were obtained prior to this differentiation (pressure, temperature, and $\mathrm{fO}_{2}$ ), however, 
are a matter of intense debate. For example, it is reported that $\mathrm{Ni}$-Fe meteorites contain up to 100 parts per million (ppm) Sn, while Fe meteorites contain 0.2 to 7 ppm Sn. The variation in the $S n$ content in rocks and meteorites indicates the siderophilic nature of Sn (see G. Wenkui, 1988, "The Problem of Tin Metallogeny" in Geology of Tin Deposits in Asia and the Pacific, C.S. Hutchinson (editor), Springer-Verlag, pp. 50-58).

One new approach to addressing this question is based on the isotopic composition of siderophile elements (mainly silicon [Si] and Fe). A key limitation of this approach is the knowledge of equilibrium fractionation factors between coexisting phases (metals, silicates, and sulfides) used to interpret data on natural samples. These properties are difficult to determine experimentally.

As explained above, our plan is to measure $\mathrm{Sn}$ and $\mathrm{Fe}$ phonon density of states (pDOS) in several Fe-Ni-Sn compounds under pressure to determine force constant and isotope fractionation. Different isotopes of the same element segregate or fractionate just as if they were different chemical elements, due to mass differences.

\section{MISSION RELEVANCE}

This project is relevant to DOE's mission in advancing the basic science of minerals, which are natural resources. The more we learn about the nature of phase separation during the formation of Earth, the easier it is to develop best geological practices for mineral search and exploitation.

\section{RESULTS AND ACCOMPLISHMENTS}

We synthesized three compounds of Fe-Ni-Sn, isotopically enriched with ${ }^{119} \mathrm{Sn}$ and ${ }^{57} \mathrm{Fe}-\mathrm{Fe}_{0.93} \mathrm{Ni}_{0.04} \mathrm{Sn}_{0.03}$, $\mathrm{Fe}_{0.96} \mathrm{Ni}_{0.03} \mathrm{Sn}_{0.01}$, and $\mathrm{Fe}_{0.97} \mathrm{Sn}_{0.03}$. We measured the pDOS and determined the force constants of these three compounds. Figure 1 shows the results of these measurements. The data evaluation with comparative methods using PHOENIX and SciPhon programs is under way. We are now in a position to determine temperature-dependent isotope fractionation for $\mathrm{Sn}$ in these compounds.

We developed a synchrotron-based method to circumvent difficulties related to the determination of isotope fractionation. It is quite valuable to develop a new proxy for isotope geochemistry studies that will indicate redox conditions at the time of formation. About a decade ago, it was realized that one can measure the force constant that binds atoms together via an inelastic $x$-ray scattering method. We had earlier successfully exploited nuclear resonant inelastic $x$-ray scattering to study iron isotope fractionation in silicate melts relevant to rock formation. From the phonon excitation spectrum, the force constant and pDOS can be calculated. These quantities can then be used to derive thermoelastic properties such as specific heat, vibrational entropy, sound velocity, and isotope fractionation factors.

The next geologically relevant and experimentally practical isotopic system to develop and exploit is Sn. Only the lack of fractionation factors limits its use in planetology. Here, we exploit a new capability at Sector 30-ID-B of the Advanced Photon Source (APS) in terms of Sn-specific spectroscopy for isotope geochemistry work. We will extract the required fractionation factors to make $\mathrm{Sn}$ isotopes a new probe of the early evolution of the Moon, Earth, and Mars. The behavior of Sn oxides in glassy and crystalline silicates, iron-based alloys, and sulfides of geochemical interest has yet to be studied.

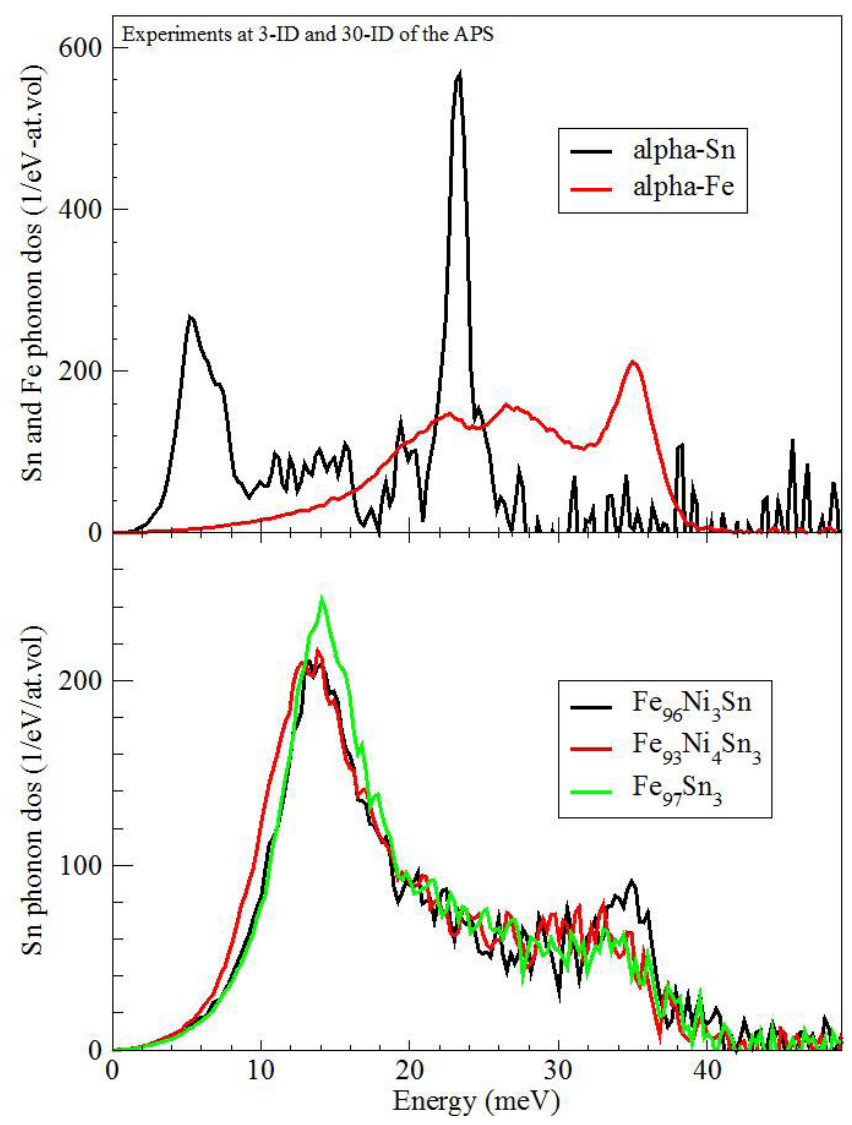

Figure 1. Top panel: Phonon density of states of alpha-Sn and alpha-Fe. Bottom panel: the Sn partial phonon density of states of FeSn and FeNiSn alloys of different compositions. The high-quality data obtained from these measurements will enable the extraction of isotope fractionation, which will help us develop ${ }^{119} \mathrm{Sn}$ as a new proxy for studying the partial pressure of oxygen (fugacity) as the Earth was forming. 


\section{PROPOSED FUTURE WORK}

It is clear that we can measure Sn pDOS even if $\mathrm{Sn}$ is a tiny fraction of the total alloy, as low as one atomic percent. It is also clear that Sn does not precipitate out by itself and remains in the solid as part of the body-centered cubic (bcc)-iron lattice, as evidenced by significant vibrational modes at 36 milli-electron volts (meV), the cut-off frequency of iron metal (Figure 1). We will continue evaluating the data to extract the force constant and temperature dependence of Sn isotope fractionation.

\section{Developing New Schemes for Nuclear Resonant Scattering Measurements at an Upgraded APS}

\section{5-182-NO}

\section{Bogdan Leu}

\section{PROJECT DESCRIPTION}

Nuclear resonance scattering (NRS) is a relatively new, isotope-sensitive, synchrotron-based technique that provides information about the local atomic environment, vibrational dynamics, and electronic and magnetic states. While ${ }^{57} \mathrm{Fe}$ is the isotope most studied with NRS, due mostly to the importance of iron in biology and geophysics, the implementation of capabilities for other isotopes, such as ${ }^{119} \mathrm{Sn}$-which plays important roles including thermoelectric materials, nanomaterials, and geophysical applications-will substantially broaden the applicability and usefulness of the NRS method. The aim of this project is to develop an Sn-based NRS capability at beamline 30-ID of the Advanced Photon Source (APS), home of the high-energy resolution inelastic x-ray scattering (IXS) technique, by taking full advantage of the technical and scientific synergies between NRS and IXS. The APS is perfectly suited for timing experiments such as NRS due to its characteristic electron bunch filling. The working energy for Sn-based NRS (23.88 kiloelectron volts (keV)) is generally outside the optimum performance of most undulators. Beamline 30-ID, however, has a new undulator that provides peak flux in the first harmonics at this particular energy. Also, detectors and associated electronics with nanosecond time resolution are available at the beamline.

Soon after the discovery of the Mössbauer effect (ME) in 1958 (a nucleus bound in a solid has a large probability of no recoil when absorbing or emitting a photon that will therefore have the precise energy of the nuclear excited state involved), theorists predicted the existence of energy shifts on the order of tens of milli-electronvolts (meV) due to lattice vibrations (phonons) in the system. However, these shifts were beyond what conventional ME experiments could detect. Third-generation synchrotron sources circumvented the technical limitations and were able to measure phonon densities of states and to expand Mössbauer spectroscopy into the time domain. Sub-meV resolution monochromators have been developed for energies near particular nuclear resonances. Due to the sub-nanosecond duration of synchrotron pulses, fast detectors, and time discrimination techniques, a nuclear resonance signal can be observed.

Synchrotron Mössbauer spectroscopy (SMS) has several distinct advantages over conventional ME experiments: (1) it can excite isotopes for which there are no good radioactive parent nuclei; (2) the signal-to-noise ratio is on the order of $10^{6}$ times better, so monolayers, dilute samples, etc., can be measured; (3) small samples under extreme conditions can be studied (high pressure, thin layers, buried layer, interfaces) with a synchrotron beam whose brightness is $10^{7}$ times more intense; and (4) the time domain measurements make extremely small nuclear state energy splitting observable.

An isotope's suitability for NRS is dictated by the energy and lifetime of its resonances, which restrict the number of isotopes that can be used in nuclear resonance experiments. Sn is a particularly good subject for NRS studies due to its presence in a variety of materials of practical importance, such as tin oxides; $\mathrm{Sn} / \mathrm{Si}$ multilayers (for understanding Schottky barriers in metal/semiconductor systems); tin clathrates and SnSe (as materials with improved thermoelectric properties); stanene and stanane (large-gap quantum spin Hall insulators); tin halides (as subjects for understanding the pressure-induced amorphization and recrystallization mechanism); superconductors (e.g., tin nanowires, to probe the role of phonon confinement in the enhancement of superconducting properties); Sn-bearing minerals, such as granitic and basaltic glasses (to probe the isotope fractionation, which would allow for the use of tin in planetology); and tin halide perovskites as photovoltaic materials. Such a long, albeit incomplete, list explains our interest in Sn-based NRS.

\section{MISSION RELEVANCE}

The project supports DOE's science mission by enabling new areas of fundamental research on the atomic and electronic structures of materials relevant to a host of energy and environmental systems. 


\section{RESULTS AND ACCOMPLISHMENTS}

We investigated two promising thermoelectric materials. Also, preliminary results on a new tetra-nuclear antitumor drug entity, Sn(IV)-oxo-\{di-o-vanillin\}dimethyl dichloride, have been successful. The main rationale for developing this program at beamline 30-ID is the six-bounce, cryogenically cooled monochromator. It yields a flux of $4 \times 10^{9}$ photons/second at the sample with an energy resolution of $1 \mathrm{meV}$. The high flux not only shortens the duration of experiments, but also makes certain experiments possible that could not be performed in the past. Experiments on $\mathrm{Snl}_{4}$ at high pressures demonstrated that. The high flux becomes even more important for materials that are not enriched with the NRS-suitable ${ }^{119} \mathrm{Sn}$ isotope (natural abundance of about $9 \%$ ).

We recently attempted to measure $\mathrm{Ba}_{8} \mathrm{Zn}_{\mathrm{x}} \mathrm{Ge}_{46-\mathrm{x}-\mathrm{y}} \mathrm{Sn}_{\mathrm{y}}$-with $\mathrm{x}=7.66, \mathrm{y}=1.79$-a material with improved thermoelectric properties and very little tin content. Not unexpectedly, we could not detect any NRS signal. However, if the same sample were ${ }^{119} \mathrm{Sn}$-enriched, effective (i.e., NRS-useful) Sn content would be sufficient. Once a sample becomes available, tin will act as an impurity at germanium lattice sites and Sn-based NRS will be sensitive to the Ge dynamics, otherwise technically impossible at this time.

\section{PROPOSED FUTURE WORK}

The remainder of this project will be dedicated to adapting low-temperature and high-temperature capabilities to NRS needs and to investigating some of the tin-containing materials mentioned above (several General User Proposals have been submitted on tin halides and tin-containing minerals, among others).

\section{Implementing New Microscopy Capabilities at the APS}

\section{5-183-NO}

\section{Lydia Finney}

\section{PROJECT DESCRIPTION}

The overall goal of this project is to develop capabilities for multi-scale and multi-modal imaging across different beamlines and instruments. In this project, we implement and enhance three new microscopy techniques at the Advanced Photon Source (APS) in preparation for the APS upgrade. Firstly, a commercial transmission x-ray microscope (TXM) recently relocated from National Synchrotron Light Source (NSLS) will be commissioned on beamline 8-BM and will be made available to general users. This system provides full-field imaging capabilities with 30-nanometer $(\mathrm{nm})$ resolution in either the 2D or 3D mode. This system can be used for in-operando imaging of the microstructure of materials such as battery electrodes, solid oxide fuel cells, and microelectronics, to name a few. Secondly, an x-ray scanning tunneling microscope (XS-STM) system jointly developed by the APS and Argonne's Center for Nanoscale Materials will also be permanently installed and commissioned in an experimental station. This combination will provide truly transformational capabilities for understanding surfaces and/or nanostructured systems. Lastly, a system for wide-field fluorescence analysis with moderate spatial resolution ( 10 microns $(\mu \mathrm{m})$ ) will be developed. This system will enable rapid fluorescence measurements for statistical analysis studies of a large number of samples or sample screening prior to higher spatial resolution measurements on an optimized insertion device beamline.

\section{MISSION RELEVANCE}

The mission of the DOE is to address America's energy, environmental, and nuclear security challenges through transformative science and technology solutions. As part of this mission, the Office of Basic Energy Sciences supports world-class, open-access, and complementary scientific user facilities such as intense $\mathrm{x}$-ray sources, neutron scattering facilities, electron beam characterization centers, and research centers for nanoscale science. The instruments developed under this proposal will benefit the entire U.S. scientific user community that uses $x$-ray spectro-microscopy for its research. This community is extremely large and varied, since spectro-microscopy can be used to addresses a wide range of problems such as cellular function, medical drug delivery, energy conversion in catalysts, carbon sequestration, complex materials manufacturing, microelectronics failure, and understanding cultural heritage items. Enhancement of the capabilities of the APS will benefit DOE by enabling science to meet its mission and to keep the APS at the forefront of the world's most advanced x-ray user facilities, as well as to replace capabilities lost as a result of the closure of the NSLS at Brookhaven National Laboratory.

\section{RESULTS AND ACCOMPLISHMENTS}

This project was started in late 2015. We initiated the installation of the TXM at beamline 8-BM and began preparations for the instrument to accept users. This instrument will provide additional full-field imaging capacity in the United States, thereby accommodating displaced NSLS users, until the corresponding instrumentation becomes available at NSLS II. At the close of the fiscal year, it was anticipated that the TXM would be accepting users upon beamtime availability in October. 
A double multi-layer monochromator (DMM) was also installed and will be ready for the next cycle of beamtime. While use of this monochromator leads to a slight loss in spatial resolution of the instrument due to chromatic aberrations, it can create 100 times more incident flux on the sample, and this allows one to study dynamic processes out of reach today. Because of the aberrations introduced by the DMM and the greater divergence of the beam at beamline 8-BM relative to the beams where these instruments were piloted, further development work will be required for all three instruments-the wide-field fluorescence instrument, the TXM, and the XS-STM-to make them operational at 8-BM. Some examples may include the development of additional components as part of the system such as slits, filters, and collimators.

\section{PROPOSED FUTURE WORK}

In ongoing work, a trace-element large field-of-view fluorescence microscope for high-throughput sample screening or for examining samples not suitable for current insertion device beamlines at the APS will be developed. This will enable rapid fluorescence measurements for statistical analysis studies of a large number of samples or sample screening prior to higher spatial resolution measurements on an optimized insertion device beamline. Also, an XS-STM will be permanently installed and commissioned on an APS beamline. This instrument will provide unique capabilities for atomic-scale imaging with chemical, charge, and magnetic specificity. This combination will provide truly transformational capabilities for understanding surfaces and/or nanostructured systems.

\section{Development of Novel X-ray Tools for Understanding Extreme-Pressure Magnetism and Electronic Ordering at Fourth-Generation Synchrotron Storage Rings}

\section{5-184-NO}

Yejun Feng

\section{PROJECT DESCRIPTION}

We seek to develop $\mathrm{x}$-ray magnetic diffraction at high pressure. In contrast to normal diffraction which studies the charge order, magnetic diffraction directly probes magnetic order. Combined magnetic and charge order resulting from the electronic structure of the material is referred to here as electronic ordering. Typically such types of order are very weak in amplitude and thus very difficult to observe with $\mathrm{x}$-ray diffraction techniques, not to mention when a sample is held under pressure in a device that severely limits the available sample volume. There are non-resonant and resonant types of magnetic diffraction. Non-resonant $x$-ray magnetic diffraction under pressure was developed in the past decade at the Advanced Photon Source (APS). However, resonant $x$-ray magnetic diffraction has not been developed. Not only does the sample environment need to be drastically different in design, but the general diffraction geometry is also unsettled, mainly due to the demand of varying the $x$-ray energy for different elements in various samples. The project is pursued in two stages. In the first stage, we will perform diffraction in the transmission geometry. In the second stage, we will try the reflection geometry. Both involve different high-pressure setups. This capability of performing resonant magnetic diffraction under pressure is not available anywhere in the world, and we plan to build it at the APS Sector 4-ID-D beamline, and possibly also at Sector 6-ID-B, if the $x$-ray focusing capability is improved.

\section{MISSION RELEVANCE}

This project is relevant to DOE's mission in basic science. The project attempts to develop a fundamental technique that would benefit a significant amount of research on magnetism-especially the study of magnetism emerging at zero temperature and the competition between magnetism and other types of collective electron phenomena such as superconductivity.

\section{RESULTS AND ACCOMPLISHMENTS}

The project was active for the final two months of FY 2015. Most effort was spent on planning, including identifying the sources of samples, choosing the APS beamline, deciding on the first scientific tasks to be pursued, acquiring high-pressure cells, designing diffraction setups, and interacting with collaborators.

\section{PROPOSED FUTURE WORK}

A few resonant magnetic diffraction experiments under pressure are planned for the coming year. We mainly focus on osmium- or iridium-based compounds, such as $\mathrm{Na}_{2}\left|\mathrm{rO}_{3}, \mathrm{Li}_{2}\right| \mathrm{rO}_{3}$, and $\mathrm{Cd}_{2} \mathrm{Os}_{2} \mathrm{O}_{7}$, where the spin-orbital coupling physics is of high interest. They also offer a resonant edge that is high enough to alleviate the difficulty of $x$-ray experiments. These projects are driven by new science based on mutual interest in collaboration with external groups, such as one already identified at Caltech. These experiments will naturally resolve equipment issues as they arise. 


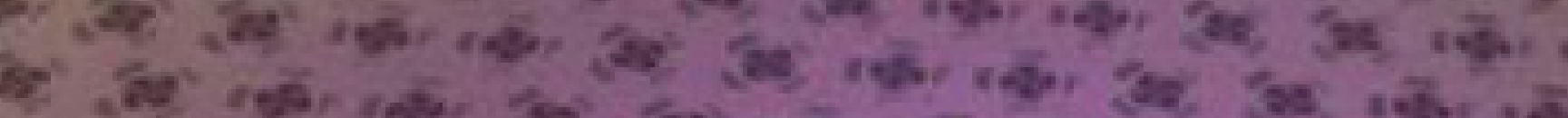

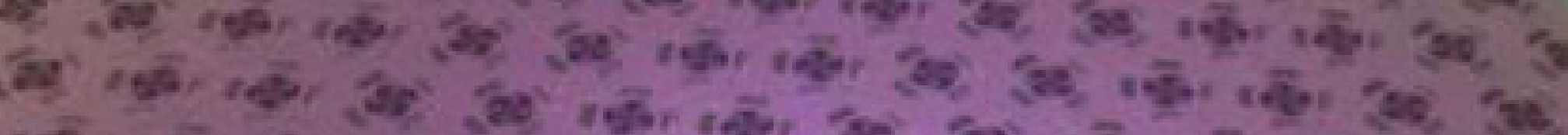

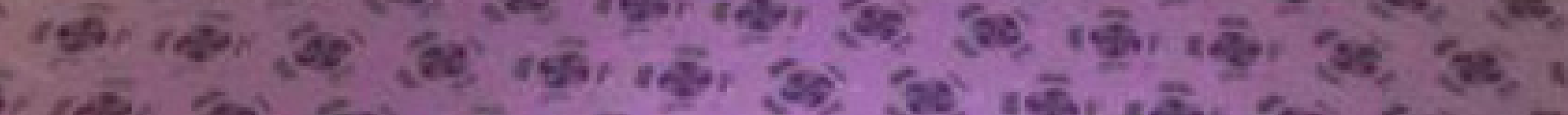

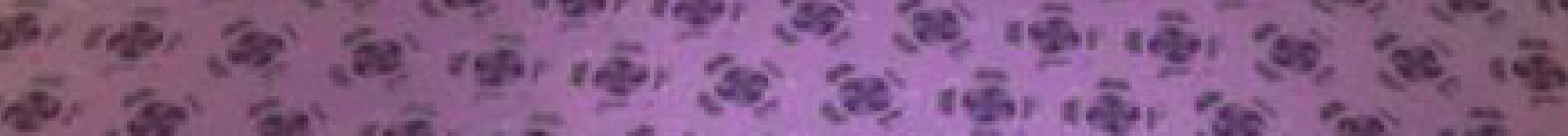

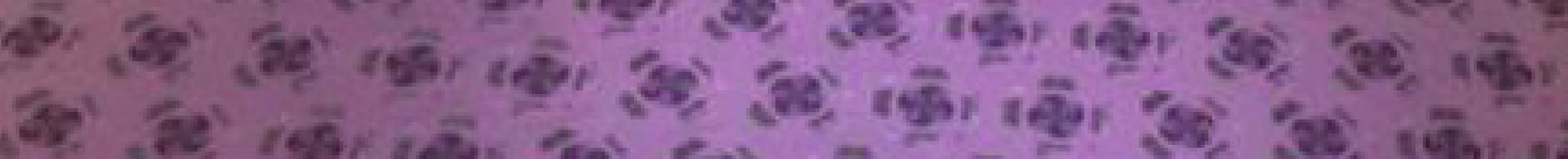
(3) के

MATERIALS FOR ENERGY 


\section{Atomistically Informed Mesoscale Modeling for Advanced Electrical Energy Storage Systems}

\author{
2012-209-R3
}

Larry A. Curtiss, Mihai Anitescu, Olle Heinonen, Dmitry Karpeyev, Marius Stan, Michael Welland, and Dieter Wolf

\section{PROJECT DESCRIPTION}

The most critical processes that limit the reversibility of the charging-discharging cycle and, hence, the performance and lifetime of advanced electrical energy storage (EES) devices are microstructurally controlled; that is, by the electrochemical and kinetic processes that give rise to dynamically evolving microstructures within the various components of the batteries. One of the major challenges is understanding and, thus, predicting these highly coupled processes across all the relevant length scales and timescales involved. An example is the case of a lithium (Li) -air battery where the distinct length scale and timescale regimes and the underlying processes play key roles. At the center is a microstructure-level ("mesoscale") battery model that incorporates the lowerscale electronic and atomic mechanisms associated with the electrochemically induced interface motion and defect and diffusion processes during nanocrystalline lithium peroxide $\left(\mathrm{Li}_{2} \mathrm{O}_{2}\right)$ growth or dissolution into a unified mathematical framework. This mesoscale model, in turn, provides the link to the system ("continuum" or "engineering") level, enabling a predictive understanding of battery performance based on the underlying defect and interfacial processes and mechanisms. At present, such a mesoscale model does not exist, although the development of mesoscale approaches capable of bringing a more fundamental materials physics-based and materials chemistry-based understanding to the continuum level could revolutionize the engineering design of the distinct components of advanced EES devices.

The goal of this project is to develop such a mesoscale modeling capability and a code suitable for leadershipclass computational platforms that capture the most important microstructural processes taking place during electrochemical crystal growth and dissolution during discharging and charging at the cathode of a Li-air battery. As input, such a mesoscale model requires lower-scale information on the fundamental reaction, diffusion, and interfacial processes that control these microstructural processes. In this work, insights from lower-level electronic structure calculations on the underlying mechanisms are being used to develop a state-of-theart mesoscale modeling approach based on phase-field theory that will incorporate the applied-math algorithms and computer-science tools necessary for leadershipclass computational platforms. The lithium-oxygen system serves as a test bed for systematically building an atomistically informed mesoscale battery-materials modeling project at Argonne. The approach and the code to be developed as part of the project will be applicable to similar advanced EES systems involving the making and breaking of chemical bonds at the electrode-electrolyte interface.

\section{MISSION RELEVANCE}

This theoretical and computational project addresses the key challenges associated with bridging the mesoscale with lower-length-scale approaches in hierarchically structured systems that are relevant to the DOE mission in energy. The insights gained from the simulations enable the interpretation and understanding of the microstructure level needed to develop advanced battery systems that are a key part DOE's goal for energy independence. The scale-bridging methodologies developed in this project will be applicable not only to Li-air but also to Li-ion batteries and electrolysis, catalysis, and fuel-cell materials that are also part of the DOE mission to develop renewable and alternative energy sources.

\section{RESULTS AND ACCOMPLISHMENTS}

Various different mechanisms have been proposed for the growth of the discharge product in $\mathrm{Li}-\mathrm{O}_{2}$ batteries. Some researchers have postulated a surface-growth mechanism with the basic reaction step involving two-electron oxygen reduction and the addition of two Li cations on the cathode surface. Others have postulated that after oxygen reduction on the surface, subsequent reactions occur in the solution phase involving $\mathrm{Li}_{2} \mathrm{O}_{2}$ or $\mathrm{LiO}_{2}$ followed by nucleation and growth of one of these species on the cathode surface. In some cases, the $\mathrm{Li}_{2} \mathrm{O}_{2}$ formation mechanism is postulated to form by the disproportionation of lithium superoxide $\left(\mathrm{LiO}_{2}\right)$ in solution, which can account for the lithium peroxide nucleation and growth from solution. A through-solution mechanism has also been proposed involving heterogeneous nucleation from a supersaturated solution of $\mathrm{LiO}_{2}$. The factors that control the morphology and/or microstructure of the discharge products are difficult to determine experimentally.

In this project, we developed a phase-field code for the model case of electrochemical crystal growth at the carbon electrode in the Li-air system. Phase-field models 
have been used extensively before in solidification studies. However, their application to chemical-reaction driven systems is still largely unexplored because crystallization from the melt involves near-equilibrium states (i.e., driving energies of the order of $k_{B} T$ ). By contrast, in electrochemical crystal growth the system is driven far from equilibrium by an applied voltage-induced dissolution and/or precipitation reaction, with a large energy, resulting in a highly non-linear behavior. Because there are various different reaction mechanisms that may occur in a $\mathrm{Li}-\mathrm{O}_{2}$ battery depending on cell configuration (e.g., electrocatalyst, electrolyte, carbon substrate), we chose one specific system to investigate that has been well studied experimentally. This system is based on activated carbon as a cathode material and tetraglyme (TEGDME) as an electrolyte. Systematic experimental studies have revealed that the disproportionation of $\mathrm{LiO}_{2}$ occurs after its deposition as a bulk material on the surface so that the discharge product is a mixture of $\mathrm{LiO}_{2}$ and $\mathrm{Li}_{2} \mathrm{O}_{2}$.

The mesoscale model developed in this project considers $\mathrm{LiO}_{2}$ formation through reaction of $\mathrm{O}_{2}{ }^{-}$and $\mathrm{Li}^{+}$in the electrolyte, which deposits on the cathode surface when the $\mathrm{LiO}_{2}$ concentration reaches the supersaturation limit in the electrolyte. Our model incorporates a rateequation approach for capturing the production of $\mathrm{LiO}_{2}$ in the electrolyte by a diffusion-reaction process and a phase-field model that describes the formation of a solid microstructure of $\mathrm{LiO}_{2}$ through deposition. Together, the two approaches capture the diffusion-controlled evolution of the system in two distinct length-scale regimes: whereas reaction-diffusion occurs on the scale of millimeters in the electrolyte between the cathode and anode, microstructure formation and evolution occur on the scale of hundreds of nanometers on the cathode surface. The two regimes share a common boundary: the cathode surface in the electrolyte and the top of the microstructure regime. At this boundary, the two regimes are coupled strongly by the concentration of $\mathrm{LiO}_{2}$. A three-stage scheme for particle nucleation, growth, and coarsening is developed, based on a simplified nucleation model.

The mesoscale model requires as input key material properties, which were either extracted from experiments or determined by electronic structure calculations. Few material properties for this system are experimentally available because $\mathrm{LiO}_{2}$ is thermodynamically unstable to disproportionation. Therefore, most of the input properties come from density functional theory calculations, such as $\mathrm{LiO}_{2}$ solubility and elastic properties of the particles. These calculations also provided insight into significant atomic-level mechanisms. Moreover, by using our model to fit some experimental data, we extracted previously unknown material properties, such as the attachment kinetic parameter, the surface scaling parameter, and the bulk $\mathrm{LiO}_{2}$ solubility.

The following are the major conclusions for nucleation and growth of $\mathrm{LiO}_{2}$ in a $\mathrm{Li}-\mathrm{O}_{2}$ battery from this work:

$\square$ The model predicts that coarsening, in which large particles increase in size and small ones decrease in size and disappear, has a substantial effect on the size distribution of the $\mathrm{LiO}_{2}$ particles during the discharge process.

$\square$ The size evolution during discharge is the result of the interplay between this coarsening process and particle growth from the discharge process. This growth through continued deposition of $\mathrm{LiO}_{2}$ from the discharge process has the effect of causing large particles to grow faster and delaying the dissolution of small particles from the coarsening process described in the first conclusion.

$\square$ The predicted size evolution is consistent with experimental results for a previously reported cathode material based on activated carbon during discharge and when it is at rest.

$\square$ The model, even without consideration of more complex microstructural features including $\mathrm{LiO}_{2}$ disproportionation, can capture the evolving size and number of $\mathrm{LiO}_{2}$ particles, but not their shape (e.g., toroid formation).

The mesoscale model developed here should have applications in studying other related discharge processes such as $\mathrm{Li}_{2} \mathrm{O}_{2}$ deposition in $\mathrm{Li}^{-} \mathrm{O}_{2}$ batteries and nucleation and growth in lithium-sulfur (Li-S) batteries.

\section{Hierarchical Modeling of Self Assembly in Nanostructured Soft Materials at Equilibrium and Far from Equilibrium}

\section{3-184-R2}

Juan J. de Pablo

\section{PROJECT DESCRIPTION}

This project seeks to develop multiscale simulation algorithms that will enable design of self-assembled functional materials and will facilitate development of processes to prepare such materials. The particular focus of the project is on soft matter, including polymers, colloids, and liquid crystals. 


\section{MISSION RELEVANCE}

This project addresses DOE missions in fundamental materials science, and has important potential applications in energy research, including development of energy storage systems, light harvesting systems, and separations media for a wide array of applications, ranging from water purification to gas concentration. Beyond the mission of DOE, the research is of interest to industry (semiconductor, battery, polymer), and to other federal agencies including the Department of Defense, the National Institutes of Health, and the National Science Foundation.

\section{RESULTS AND ACCOMPLISHMENTS}

In past years, we developed a multiscale formalism for simulation of nanostructured polymeric materials. That formalism, the so-called "Theoretically Informed Coarse-Grained (TICG)" simulation approach, relies on Hamiltonians for materials of interest that have traditionally been solved by computationally complex self-consistent field theoretic approaches. In TICG, one relies on stochastic simulations of particle-based representations of the systems of interest to arrive at more realistic, and more efficient, representations of materials. Since their inception, TICG methods have gained considerable attention and have been adopted by dozens of research groups around the world, both from academia and industry. In FY 2015, we finalized the originally proposed work on theory and simulation of macromolecular self-assembly, where we made progress on multiple materials platforms amenable to directed assembly, including proteins, deoxyribonucleic acid (DNA)-based complexes, liquid crystals, and block copolymers. In each, we developed models and methods to probe emergent assembly processes and materials characteristics.

In the area of protein assembly, we focused on two aspects of the modeling of unstructured polypeptides. Such molecules are of scientific interest for applications and they are also of medical importance. In FY 2015, we focused on islet amyloid polypeptide (amylin), a molecule that is implicated in the onset of type 2 diabetes. We also completed a study of dimerization of amylin and another study of misfolding of amylin fragments and mutants in the vicinity of bilayer membranes.

We made significant advances in the development of coarse-grained models of DNA. We completed a study of systematic coarse-graining of explicit ions in the vicinity of DNA, and a study of the mechanical properties of DNA-based nanomaterials. We also completed a study of nucleosome unfolding, which forms the basis for subsequent studies of chromatin structure and properties.
For the particular case of liquid-crystalline-based materials, we demonstrated that nanoparticles can be controllably assembled at the interface of liquid crystal droplets. We also demonstrated that the morphology of liquid crystals can be manipulated by confinement in droplets, thereby enabling applications in biosensing.

In the area of directed assembly of block polymers, we published a comprehensive study of the pathways for defect annihilation in directed block-copolymer assembly. A significant accomplishment of this work was to demonstrate conclusively that the defects that are encountered in directed block-copolymer assembly by chemoepitaxy represent metastable states, as opposed to equilibrium configurations. By relying on string-method simulations, we were able to explain that such metastable states are kept in place by large free energy barriers that, at low temperatures, are difficult to overcome. We identified the minimum free energy pathways (MFEPs) that molecules must follow in order to climb such barriers, and we proposed processing strategies designed to traverse such pathways. Our predictions were confirmed by experimental characterization of thin self-assembled films using three-dimensional (3D) transmission electron microscope (TEM) tomography.

Figure 1 shows a representative result from our simulations, where the free energy along the reaction pathway is plotted in units of Boltzmann's constant times temperature $\left(k_{B} T\right)$. As can be seen in the figure, the free energy of the defective state is higher than that of the defect-free state by several hundred $\mathrm{k}_{\mathrm{B}} \mathrm{T}$. One can also see that multiple barriers must be crossed in order for the system to eliminate its defects. The first and most important of these barriers corresponds to the formation of a "bridge," consisting of just a handful of molecules connecting different polymeric domains. Once a sufficiently large bridge is formed (i.e., the transition state is achieved), the process of annealing can continue in a "downhill" manner until all defects disappear. Figure 2 shows simulated images and experimental TEM images of a defect at various positions along a film. In those images, one can appreciate that the agreement between predicted morphology and experimentally observed states is quantitative. 

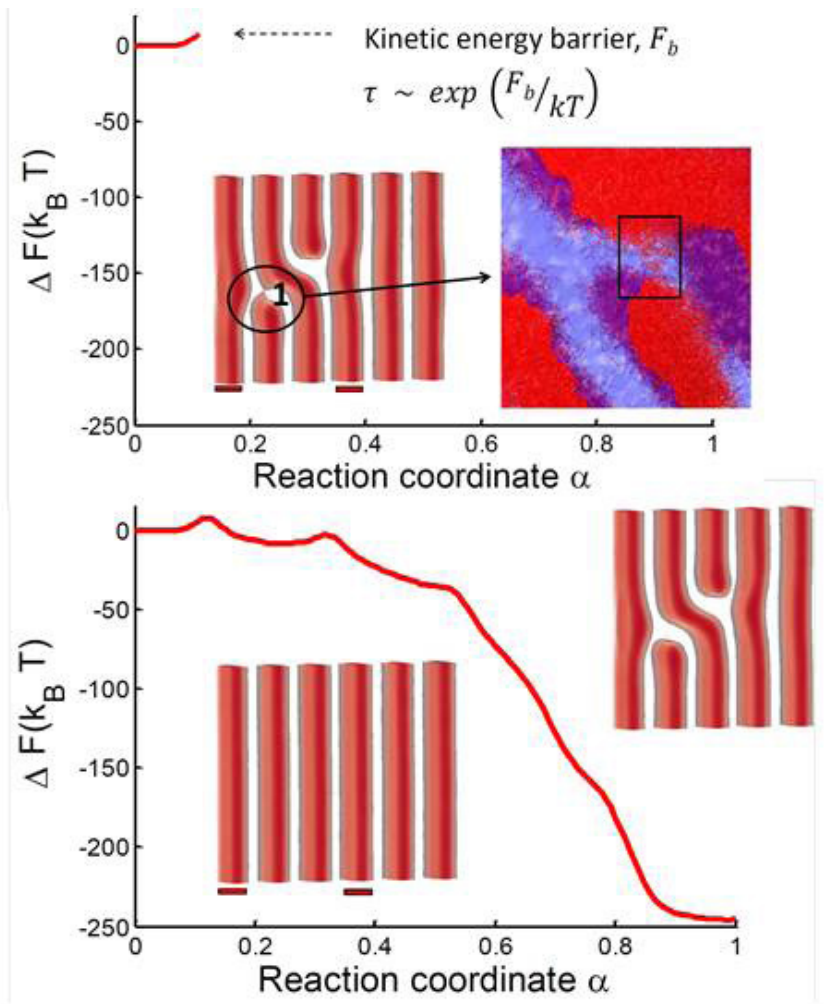

Figure 1. Free energy for defect annihilation in directed assembly. The red and white domains represent two blocks of a diblock copolymer. The inset in the top panel shows the transition state, with the formation of a molecular bridge. The insets in the bottom panel show the defective and defect-free states, along with the entire free energy profile.

\section{Experimental Tomography Data}

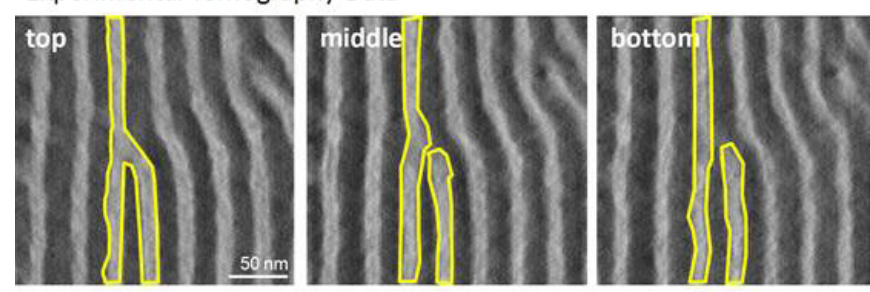

Meta-stable states from MFEP

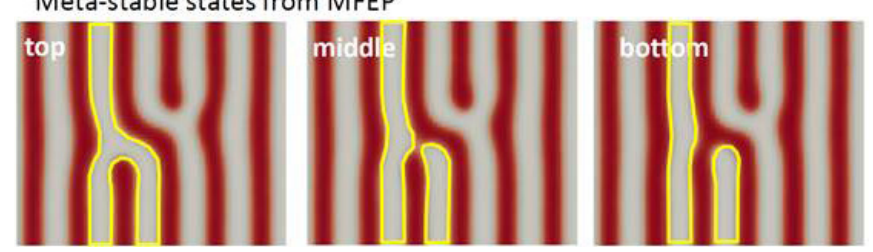

Figure 2. Experimental 3D TEM images and predicted configurations of a defect in directed self-assembly of Poly(styrene)-Poly(methyl methacrylate) (PS-PMMA). The panels correspond to the top, middle, and bottom (near substrate) of thin films. The yellow line is used to highlight the defective lamellae.

\section{Computational Molecular Science}

\author{
2013-202-R2 \\ Gregory A. Voth and Christopher J. Knight
}

\section{PROJECT DESCRIPTION}

An understanding of chemical, biological, and material systems containing multiple species, phases, and interfaces and undergoing chemical reactions requires accurate knowledge of interaction potentials. Additionally, the sizes of the systems studied must be large enough to properly account for both the local neighborhood and more distant environmental effects on chemical dynamics. The accurate simulation of phenomena across several length and time scales requires both models and software that are uniquely designed for the task. The main objectives of this project are to develop methodologies that can accurately and efficiently parameterize molecular simulation models, compute reactive simulations, and enhance configurational sampling. In this work, condensed-phase electronic structure calculations generate reference data (e.g., energies and forces) on representative systems. Using genetic optimization algorithms, reactive molecular dynamics models are parameterized to reproduce results of reference calculations at significantly reduced computational cost. The developed algorithms were applied to better understand the structure of electrolyte/electrode interfaces and proton transport mechanisms in enzymes.

\section{MISSION RELEVANCE}

The development of simulation tools useful at both the smallest molecular scales and the largest mesoscopic scales supports DOE missions in energy security and science. Understanding charge transport mechanisms in inhomogeneous systems (e.g., fuel cell membranes) is critical to optimizing performance of electrochemical conversion and storage devices, which supports the DOE mission of energy security. Facilitated by the tools developed in this project, obtaining detailed atomic-scale information on material properties, such as the modulation of charge transport dynamics near complex interfaces, will enable a greater understanding of critical molecular mechanisms, such as proton transport and degradation reactions at electrode interfaces. 


\section{RESULTS AND ACCOMPLISHMENTS}

Achievements in FY 2013 and 2014 included the parameterization of an electrolyte/electrode model and design of new parallelization strategies for reactive simulations and cellular-scale, coarse-grained simulations. The parallel performance of reactive simulations with the RAPTOR code was further improved with improved multi-threaded performance and optimizations to a multiple-copy parallelization framework for reactive simulations of concentrated systems. Functionality was also added to RAPTOR to model polarization of electrodes by reactive ions and the application of a constant bias voltage across simulation cells. The computational efficiency afforded by this effort enabled calculation of the exact Hellmann-Feynman forces due to long-range electrostatic interactions with a modest computational overhead compared to a previously adopted approximation. These improvements led to the granting of a 2014-2015 DOE ALCC (ASCR [Advanced Scientific Computing Research] Leadership Computing Challenge) award of computing time at the Argonne Leadership Computing Facility (ALCF). Also completed in FY 2014 was a study on the proton transport mechanisms of [FeFe]-hydrogenase, which belongs to a class of enzymes that reversibly catalyze the reduction of protons and oxidation of molecular hydrogen. In this study, water pathways and corresponding free energy surfaces for proton transfer between different sites were examined for three important oxidation states to identify conditions resulting in increased enzymatic activity.

In FY 2015, work continued on improving the computational performance of RAPTOR. Compared to FY 2014, there was a 45\% improvement in its performance on Mira at the ALCF, based on optimizations to the multi-threaded kernels and calculation of long-range electrostatic interactions. Additional effort was focused on developing efficient, on-the-fly load-balancing strategies for simulations with many reactive species to reduce the amount of time that compute nodes were idle in large-scale calculations. The optimization efforts in RAPTOR led to receiving an award of computing time at ALCF in 2016 through the DOE Innovate and Novel Computational Impact on Theory and Experiment (INCITE) program. The workflow and functionality of the FitEVB code were extended to support new types of interaction potentials and functional forms supporting those recently implemented in RAPTOR. FitEVB uses genetic algorithms to optimize model parameters for simulations and has been efficiently parallelized. Support for multi-threaded parallelism was implemented with parallel efficiencies of $96.8 \%$ and $73.4 \%$, respectively, on 8,192 and 16,384 Blue Gene/Q (BG/Q) cores on Mira for a benchmark of 1,024 configurations (Figure 1). These improvements to
FitEVB were used to start development of reactive models for proton transport in membrane proteins and near polymer/catalyst interfaces. Working with experimental colleagues, we also used quantum chemistry calculations to investigate the binding properties of lithium sulfides to several inorganic surfaces used in lithium-sulfur batteries, with examples shown in Figure 2.

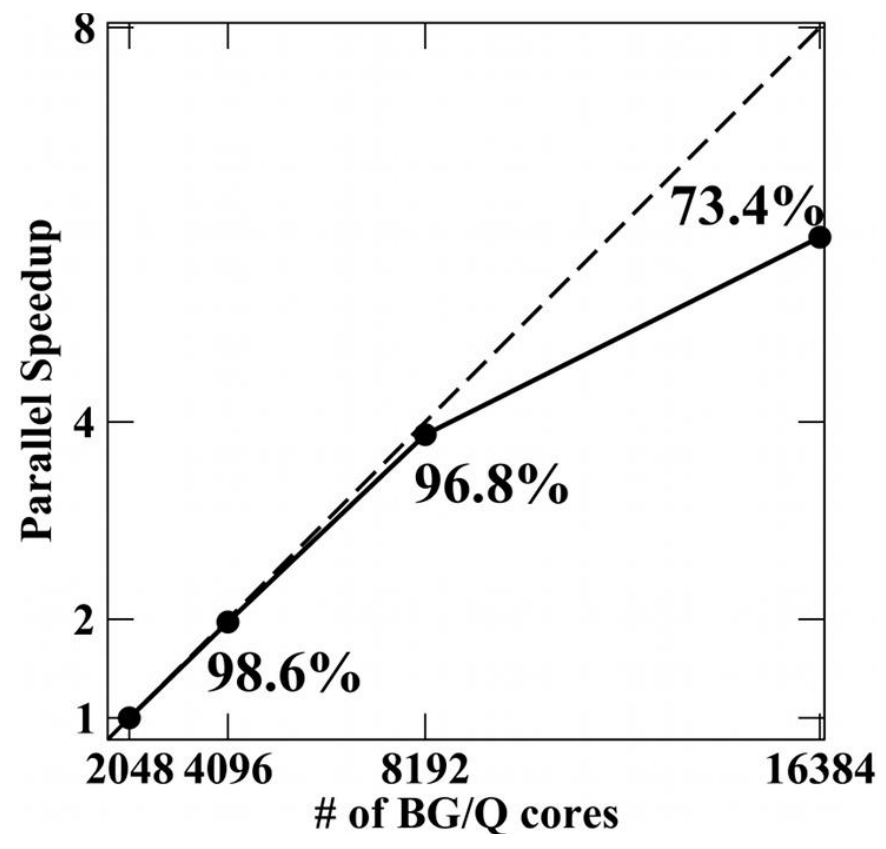

Figure 1. Parallel speedup of the FitEVB code on Mira using 1,024 configurations.

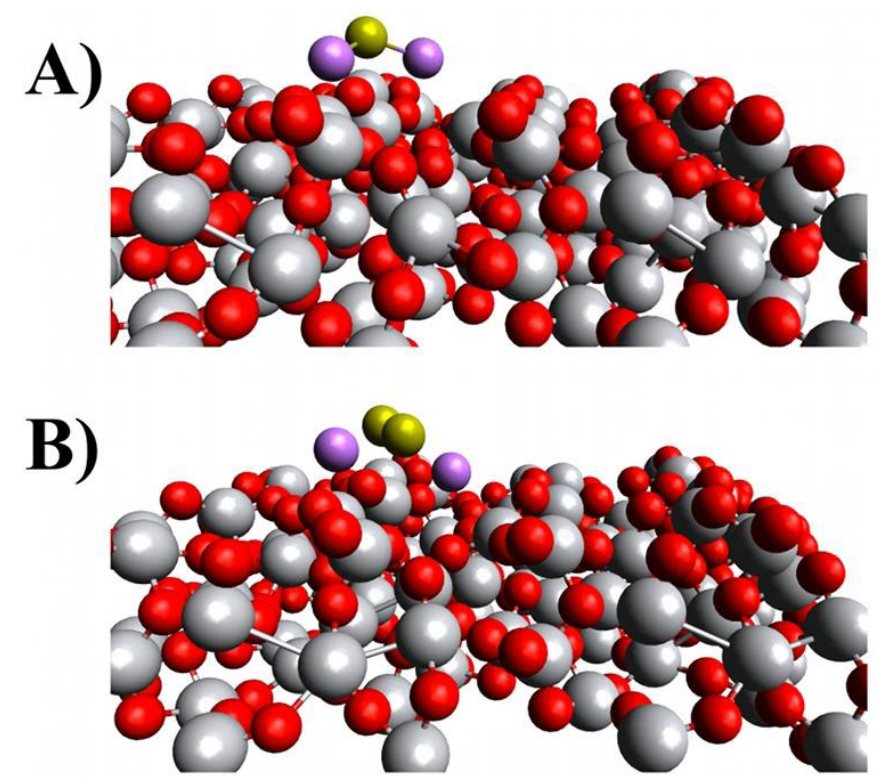

Figure 2. Representative configurations for (A) lithium sulfide $\left(\mathrm{Li}_{2} \mathrm{~S}\right)$ and (B) $\mathrm{Li}_{2} \mathrm{~S}_{2}$ polysulfide on a titanium oxide $\left(\mathrm{Ti}_{4} \mathrm{O}_{7}\right)$ surface showing preferential binding of sulfur to titanium sites in both cases. Oxygen (red), titanium (gray), lithium (magenta), and sulfur (gold) atoms are rendered as spheres. 


\section{Directed Self-Assembly and Three-Dimensional Characterization of Block Copolymers in Semi-Thick Films}

\author{
2013-216-R2
}

Paul F. Nealey

\section{PROJECT DESCRIPTION}

The central aim of this work is the advancement of tools capable of characterizing structures formed during directed copolymer assembly in semi-thick films. Intrinsically coupled with the goal of the project is the development of a fundamental understanding of the thermodynamic and kinetic factors controlling the assembly process. The project will form the basis for design rules for the creation of equilibrium and non-equilibrium nanostructured materials with precisely controlled architectures at the scale of 10 nanometers $(\mathrm{nm})$ in three dimensions. Characterization of the three-dimensional (3D) structures of the films and how they evolve will require advancing the capabilities of small-angle $x$-ray scattering (SAXS), grazing-incidence small-angle $x$-ray scattering (GISAXS), and resonant soft $x$-ray scattering (RSoXS), as well as additional complementary techniques, such as scanning transmission electron microscopy (STEM) tomography. A unique aspect of the research relates to the direct comparison of dynamic experimental scattering data to simulated scattering profiles that are generated from dynamic structures that are predicted by molecular simulations.

\section{MISSION RELEVANCE}

The project is relevant to DOE's missions in science and energy. Beyond contributing new knowledge to the scientific community, this study of directed copolymer assembly will find a wide variety of potential practical applications relevant to DOE's missions. We also anticipate that the project will be the impetus for the development of a suite of state-of-the-art 3D nanoscale characterization tools for soft materials at Argonne. The block copolymer systems we are developing, with their well-defined architectures, are ideal for the development of such tools. Thus, the materials and tool development included in this project are broadly applicable in science-based research on structure-property relationships in nanoscale material architectures for energy transfer, chemical sensing, and information technology.

\section{RESULTS AND ACCOMPLISHMENTS}

In FY 2013-2014, progress was made toward designing new components (sample chambers) for the instruments at beamline 8-ID-E of the Advanced Photon Source, as well as the development of software to analyze the 3D structures of soft materials as probed by $x$-ray scattering. The capability of $x$-rays to probe sample characteristics with the desired resolution throughout the polymer films was also established with GISAXS experiments.

Within FY 2015, we further developed our tools to characterize morphology in semi-thick films, employing designed samples prepared at Argonne as well as samples provided by our industrial partner, IMEC. Specifically, we designed polymer grating samples with programmed line edge roughness to showcase our newly developed methodology that is capable of providing quantitative measures of structures and fluctuations in polymer films. Figure 1 presents a direct comparison between scattering patterns measured at beamline 8-ID-E and calculated intensities by using 3D models, as shown in the lower panel of the figure. A complete characterization of such samples with atomic force microscopy, scanning electron microscopy, and GISAXS was performed during FY 2015 to critically ascertain the accuracy of each method.
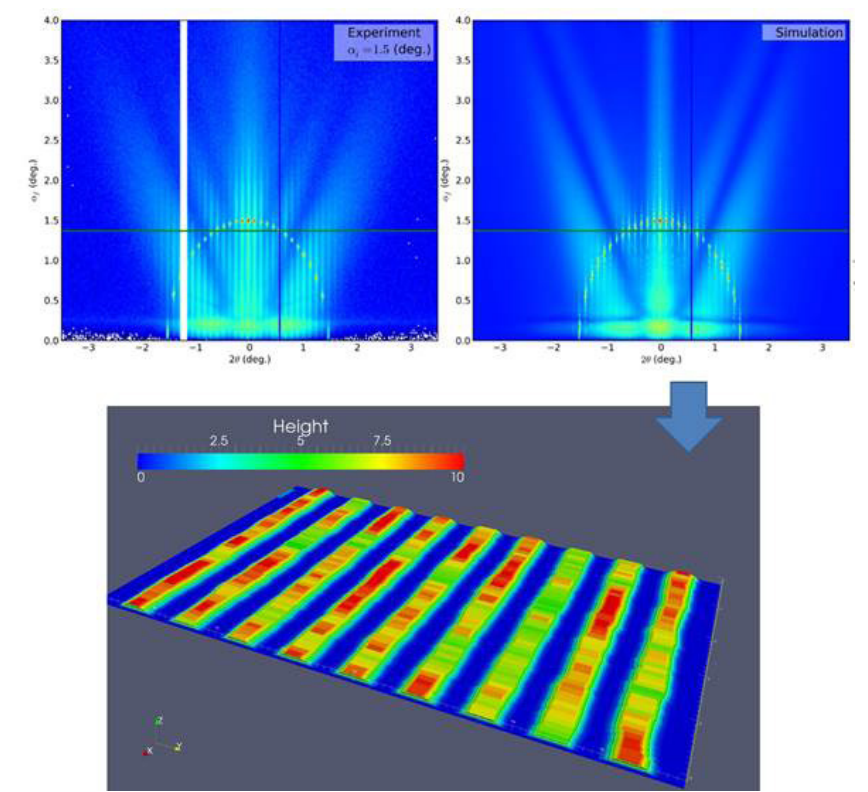

Figure 1. Analysis of recorded GISAXS data from polymer grating samples (top, left) using calculations of scattering from 3D models extending over tens of micrometers. The top, right panel presents the calculated scattering intensity (with high-intensity grating truncation rods along the arc of the Ewald sphere), while the bottom figure is a representation of a small area (approximately $\mathbf{5 0 0} \times \mathbf{5 0 0}$ nanometers square; height is also in nanometers) of the 3D model. Detailed information on the characteristics and fidelity of these structures is critical, as these patterns direct the assembly of diblock copolymer films. 
A second major accomplishment was the ability to characterize structural features and defects throughout directed self-assembled copolymer films. We further developed an ability to probe the morphology in real space by using STEM tomography. Figure 2 presents sample data on triblock copolymer films employing $4 \times$ density multiplication. The real-space information provided critical details for the local arrangement of the polymer molecules and the sensitivity of the process to the geometry of the underlying guiding pattern. Using such methods, we were able to examine the assembly on top and between the guides. We found that under specific conditions, roughness is more pronounced toward the lower part of the film, in proximity to the chemical pattern.

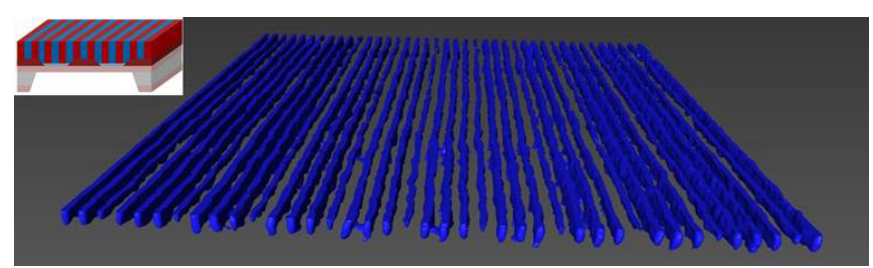

Figure 2. STEM tomography of a triblock copolymer film assembled with $4 \times$ density multiplication. The method provides detailed data on the 3D morphology of these structures and the dependence on such process parameters as the geometry of the underlying guiding pattern. The inset provides a sketch of the ideal structure (with a spacing of 28 nanometers) assembled on top of a guiding chemical pattern.

The last milestone of our investigation was to couple detailed molecular simulations to experimental investigations. The aim of this effort was to extract direct quantitative information on the thermodynamic and kinetic factors that drive the formation of the morphologies probed with any of the previously mentioned techniques. We advanced toward this aim by deploying large-scale calculations with established molecular modeling methods such as the theoretically informed coarse-grained simulations developed by our collaborators (J.J. de Pablo group at Argonne/University of Chicago). The outcome of the simulations provided structure as a function of the chemical design of the pattern and the copolymers employed. The results demonstrated that an accurate description of the interactions with the pattern is necessary and sufficient to provide morphologies whose predicted scattering is in agreement both with RSoXS and GISAXS experiments performed during FY 2015 at beamline 8-ID-E. In addition, simulations enabled us to directly answer questions regarding the effect of polymer chemistry on the structure and investigate the origin of defects revealed by microscopy and scatterings. The results of this approach have a direct impact on both our fundamental understanding and our ability to direct and control the structure in block copolymer films.

\section{PROPOSED FUTURE WORK}

For the final year of the project, we will focus on morphologies present in block-copolymer films with the next generation of materials employed in the semiconductor industry, and we will optimize the coupling between large-scale computations and scattering experiments. The development of high- $\chi$ block copolymers (where $\chi$ is the Flory-Huggins interaction parameter) drives current efforts to pattern features with sizes below 10 nanometers. However, the fidelity of the assembled structures depends on avoiding kinetically arrested states. The combination of simulations, scattering and tomography will allow us to advance metrology and characterize the critical molecular factors, such as the chemistry of the underlying pattern, in achieving optimal assembly for these materials.

\section{Transition Edge Sensors for Fundamental Physics}

\author{
2013-219-R2
Clarence Chang, Valentyn Novosad, Gensheng Wang, and Volodymyr Yefremenko

\section{PROJECT DESCRIPTION}

This project aims to develop large arrays of superconducting transition edge sensors (TESs) for applications in fundamental physics. The TES is an ultra-sensitive thermal detector that exploits the sharp superconducting-to-normal transition of a superconducting film. The fabrication of large TES detector arrays requires engineering of material transport properties, tuning of superconducting properties, and nano-machining of superconducting circuitry and mechanical and thermal structures. This project involves studies of precision fabrication techniques and novel production processes, all with the goal of mass-producing large arrays of TES detectors. Developing this capability will immediately open new follow-on opportunities in both fundamental and applied science. For example, our detector development program will enable the fabrication of a 16,000-element TES detector array for the South Pole Telescope. Such an array will not only revolutionize our understanding of the cosmic microwave background (CMB), but will also position us to address the technical challenges confronting future experiments requiring large arrays of TES detectors. For example, large TES arrays are relevant for $\mathrm{x}$-ray spectroscopy, gamma ray spectroscopy, and the detection of long-wavelength photons and heavy particles. 


\section{MISSION RELEVANCE}

This project is directly relevant to DOE's basic science mission. Specifically, it develops new technology required for building large TES detector arrays, including the largest CMB focal plane in the world. This detector array will push the boundaries of CMB science, which explores the physics of our universe, the nature of cosmic inflation, and the neutrino sector. CMB measurement is a cornerstone of modern cosmology and a critical element of high-energy physics in the United States. Developing the capability to mass-produce large TES detector arrays will not only sustain leadership in CMB science, but will also lay the foundation for exploring new opportunities across a broad spectrum of applications. Potential future applications range from particle physics (e.g., bolometer arrays for CMB studies and low-threshold detectors for dark matter searches) to national security (e.g., nuclear nonproliferation applications using high-resolution calorimeters for measuring the isotopic composition of plutonium samples).

\section{RESULTS AND ACCOMPLISHMENTS}

This project started at the end of FY 2013, and the first year was devoted to specifying and procuring new capital equipment required for TES detector array development. We acquired five new tools, including four microfabrication tools (stepper, inductively coupled plasma [ICP] etcher, and two AJA International Inc. sputtering systems) and one adiabatic demagnetization refrigerator for detector testing. The tools were received, installed, and commissioned at the end of FY 2014.

In FY 2015, we used these new tools to migrate our detector processing from fabricating individual detectors on 100-mm wafers to fabricating full detector arrays on 150-mm wafers (see Figure 1). An individual array consists of 271 dual-polarization multichroic pixels arranged in a hexagonal close-packed configuration and includes the full wiring layout for reading out the complete array (see Figure 2). Each pixel has six TES detectors coupled via superconducting microstrip circuitry to a broadband polarization-sensitive antenna. Our array fabrication involves a total of 16 processes, including reactive and magnetron sputtering, reactive ion etching, stepper and contact lithography, ICP etching, and chemical etching. Our smallest feature is 1 micrometer wide, and all of the processing layers are aligned to less than $200 \mathrm{~nm}$. We process five 150-mm wafers in parallel with a single wafer having 1,600 detectors, a detector number comparable to the entire focal plane of currently fielded CMB instruments. Cryogenic testing of our initial detector arrays shows reasonable performance with optical bandpasses within $15 \%$ of our desired values and detector saturation powers within $25 \%$ of target. Some improvements are needed to achieve the high sensitivity required for precision measurements of the $\mathrm{CMB}$, and improving the array performance of our design is the goal of our upcoming work.

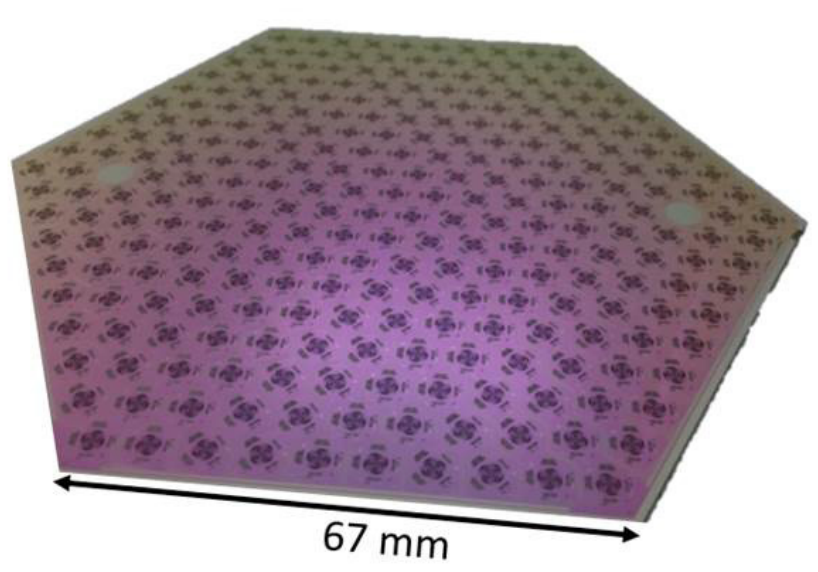

Figure 1. Image of a hexagonal array of mm-wave TES detectors. Each array consists of 271 pixels and is fabricated from a single 150-mm wafer in 3-4 weeks.

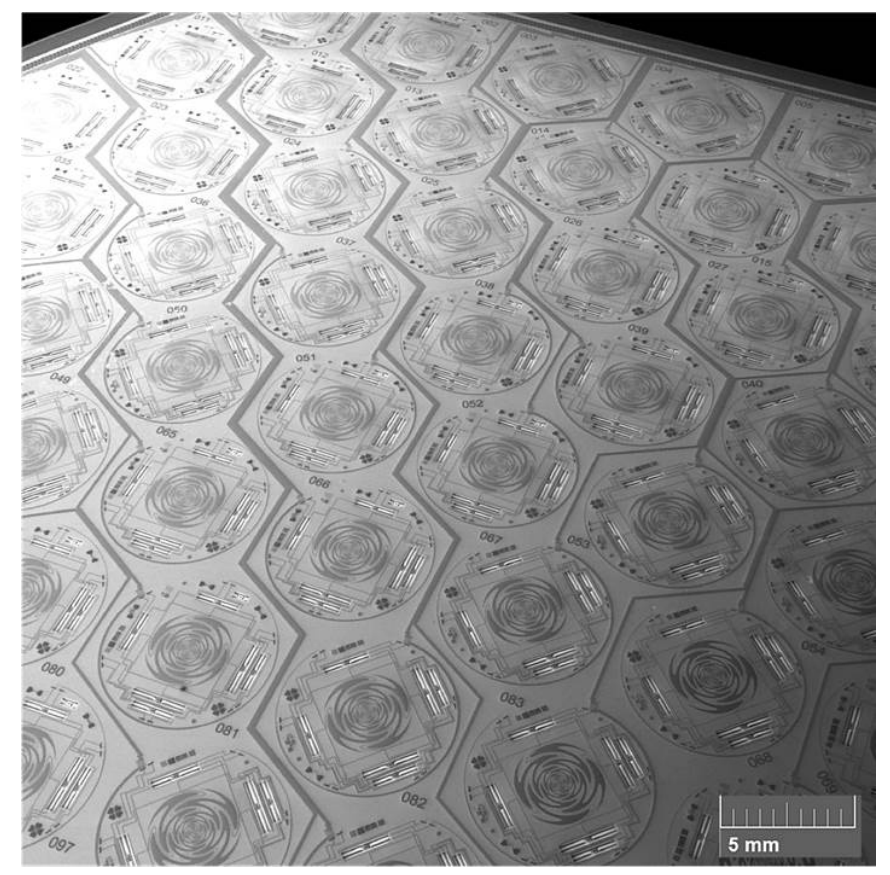

Figure 2. Scanning electron micrograph showing some of the dualpolarization multichroic pixels from a TES detector array. Each pixel has a broadband sinuous antenna, microstrip transmission line with in-line bandpass filters, and six TES bolometers.

\section{PROPOSED FUTURE WORK}

In the upcoming year, we plan to continue our iterative testing and fabrication strategy to realize detector arrays with optimal performance. We will also explore process improvements, with the goal of reducing array fabrication 
time. Lastly, we will pursue new applications for TES detector arrays. One specific opportunity we will target involves developing TES detectors for use in experiments searching for neutrinoless double beta decay.

\section{Integration of Scalable Microwave Reactor with High- Energy X-ray Beamline for High-Throughput Screening of Energetic Nanomaterial Synthesis}

\author{
2014-121-R1 \\ Yugang Sun, Jonathan D. Almer, Zonghai Chen, \\ Joseph Gregar, John Okasinski, and Yang Ren
}

\section{PROJECT DESCRIPTION}

This project focuses on integrating a specialized in-situ microwave reactor and high-energy x-rays from Argonne's Advanced Photon Source (APS) in order to directly probe the complex chemical and physical processes involved in synthesizing colloidal nanoparticles that have energy-related applications. The ultimate goal is to provide a generic platform for studying the reaction kinetics, intermediate species, structural transitions, and defect evolution of nanoparticles in real time and under reaction conditions. Using this platform, we will be able to create predictive and rational designs for functional nanoparticles with controlled architectures that range from the atomic and molecular scales to the bulk scale.

Microwave-assisted synthesis has the potential for scalable synthesis because it combines fast heating and uniform temperature distribution in reaction solutions. These preferable characteristics motivated us to systematically screen the microwave reaction conditions required for the synthesis of functional colloidal nanoparticles by using in-situ high-energy x-ray techniques. These are crucial for large-volume reaction systems because of the strong penetration of high-energy $x$-rays in solutions. The rapid reaction kinetics in large, statistically relevant sample volumes can be captured by seamlessly integrating (1) microwave nanoparticle synthesis; (2) high-energy, small- and wide-angle x-ray scattering (SAXS/WAXS) at APS beamlines; and (3) glassware fabrication; the results can then be applied to energy storage and catalysis applications. Specifically, integrating an in-situ microwave reactor on a high-energy $x$-ray beamline will establish the capability to carry out high-throughput screening of the synthesis of functional nanomaterials at a large scale, and this capability will likely have a significant impact on our understanding and optimization of functional nanomaterials for energy applications.

\section{MISSION RELEVANCE}

This research is pursuing a key component needed for discovering and designing a wide variety of energyrelevant materials. As such, it aligns well with the mission of one of the three core research areas-materials discovery, design, and synthesis-in the Materials Sciences and Engineering Division of DOE's Office of Basic Energy Sciences, tying in closely with research in synthesis and processing science. Because of the importance of synthesized nanomaterials for energy applications, this project is also relevant to programs in DOE's Office of Energy Efficiency and Renewable Energy and Energy Frontier Research Centers, which focus on energy research using, for example, high-quality nanoparticles for catalysis, energy conversion, and energy storage. Success in developing such a key instrument will also benefit the Materials Genome Initiative.

\section{RESULTS AND ACCOMPLISHMENTS}

In FY 2015, we continued to work on the development of the in-situ microwave reactor with the addition of an enclosure and an automated sample handling system. The controllability and efficiency of the updated in-situ microwave reactor was systematically evaluated for real-time probing of the synthesis of colloidal nanoparticles. Studied with the updated in-situ microwave reactor were the synthesis of colloidal plasmonic silver nanoparticles that are useful for nanophotonics, nanostructured 2D molybdenum disulfide $\left(\mathrm{MoS}_{2}\right)$ with edge-terminated and interlayer-expanded features that are promising as non-noble-metal electrocatalysts for the hydrogen evolution reaction. The major achievements are summarized as follows.

$\square$ Further development of the in-situ microwave reactor with an automated sample handling system. The in-situ microwave reactor was updated with an enclosure to improve its mobility, safety, and compatibility with other useful features, such as an automated sample handling system that is very useful when a large number of reactions have to be studied under different reaction conditions. The updated in-situ microwave reactor was evaluated at APS beamlines (Figure 1) with the collection of time-resolved WAXS and SAXS data. 


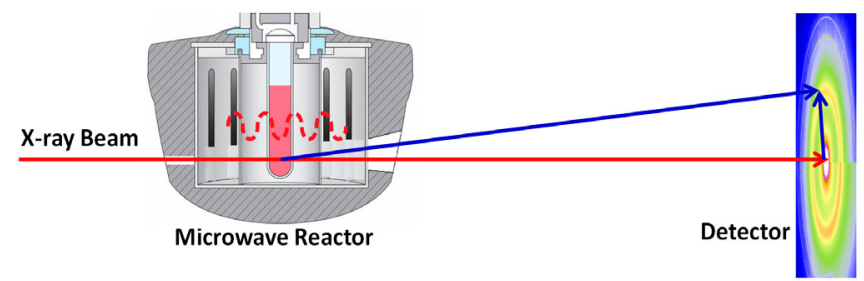

Figure 1. Schematic illustration of the in-situ microwave reactor integrated at high-energy synchrotron $x$-ray beamline.

$\square$ Real-time probing of the nucleation/growth kinetics of microwave synthesis of silver nanoparticles. The fast reaction kinetics during the microwave synthesis of colloidal silver nanoparticles were quantitatively studied for the first time using the in-situ microwave reactor. WAXS patterns, corresponding to signals away from the $\mathrm{x}$-ray beam center, provided information on crystalline phase, crystalline domain size, and the amount of crystalline nanoparticles, whereas the SAXS patterns, corresponding to the signals close to the beam center, were sensitive to the size, size distribution, and morphology of the nanoparticles. Figure 2 presents the time-resolved WAXS (equivalent to x-ray powder diffraction) patterns recorded from a microwave reduction of silver nitrate $\left(\mathrm{AgNO}_{3}\right)$ in ethylene glycol containing polyvinylpyrrolidone (PVP) at $140^{\circ} \mathrm{C}$. Comprehensive data analysis (see Figure 3 ) reveals two different types of reaction kinetics corresponding to the nucleation and growth of the Ag nanoparticles. The formation of seeds (nucleation) follows typical first-order reaction kinetics with an activation energy of $20.34 \mathrm{kilojoules} / \mathrm{mole}(\mathrm{kJ} / \mathrm{mol})$, while the growth of seeds follows typical self-catalytic reaction kinetics. Varying the synthesis conditions indicates that the microwave colloidal chemistry is independent of surfactant concentration. These discoveries reveal that the microwave synthesis of Ag nanoparticles proceeds with reaction kinetics significantly different from the synthesis present in conventional oil bath heating. The in-situ $\mathrm{x}$-ray diffraction technique reported in this work promises to enable further understanding of crystalline nanomaterials formed through microwave synthesis.
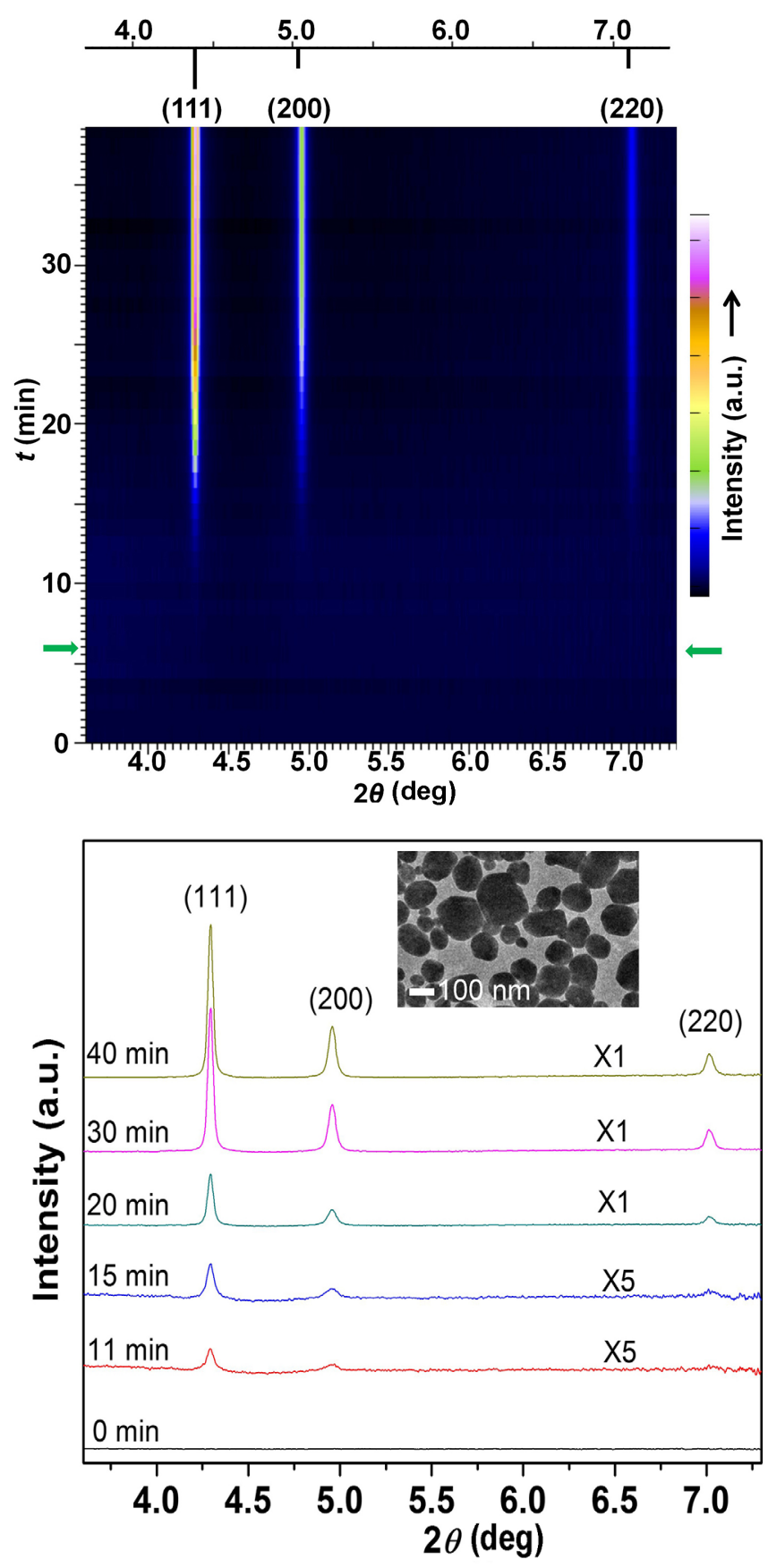

Figure 2. Time-resolved WAXS patterns recorded from a microwave reduction of $\mathrm{AgNO}_{3}$ in ethylene glycol at $140^{\circ} \mathrm{C}$ (x-ray wavelength, $\lambda=0.1771 \AA$ A). (top) Contour plot of the WAXS patterns recorded in the course of the reaction. The standard diffraction pattern of crystalline Ag (ICDD PDF 04-001-3180) is highlighted with sticks for reference. (bottom) Typical WAXS patterns at a number of key reaction times. The inset presents a typical transmission electron micrograph of the synthesized Ag nanoparticles. 

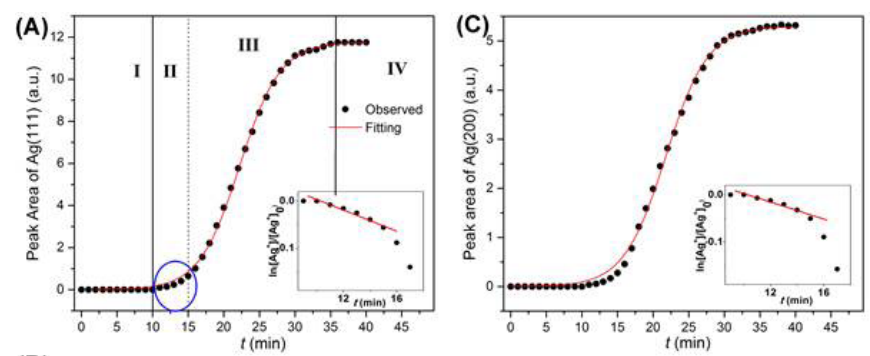

\title{
Length-Scale Bridging Computational Scheme for Structure and Transport
}

\author{
2014-128-R1 \\ Olle Heinonen, Dmitry Karpeyev, Nichols A. Romero, \\ and Peter Zapol
}
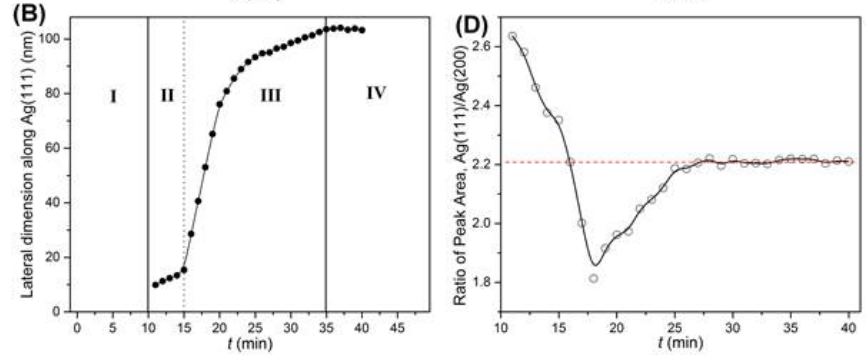

Figure 3. Detailed analysis of the in-situ WAXS data shown in Figure 2. (A) Dependence of peak area of the $\mathrm{Ag}(111)$ on reaction time. The insert shows $\ln \left(\left[\mathrm{Ag}^{+}\right] /\left[\mathrm{Ag}^{+}\right]_{0}\right)$ (the concentrations are determined by the timedependent $\mathrm{Ag}(111)$ peak area) as a function of reaction time at an early reaction stage. $(B)$ Dependence of crystalline lateral dimension along the $\mathrm{Ag}<111>$ direction. (C) Dependence of peak area of the $\mathrm{Ag}(200)$ on reaction time. The inset highlights $\ln \left(\left[\mathrm{Ag}^{+}\right] /\left[\mathrm{Ag}^{+}\right]_{0}\right.$ ) (the concentrations are determined from the time-dependent $\mathrm{Ag}(200)$ peak area) as a function of reaction time at an early reaction stage. (D) The peak area ratio of $\mathrm{Ag}(111) / \mathrm{Ag}(200)$ as a function of reaction time, indicating anisotropic growth along different crystalline directions at different reaction times. The dotted lines in (A and B) highlight the distinguished reaction stages (I, II, III, and IV) corresponding to nucleation and growth of Ag nanoparticles.

\section{$\square$ Real-time probing of the synthesis of nanostructured} $\mathrm{MoS}_{2}$. Layered $\mathrm{MoS}_{2}$ has demonstrated great promise as a low-cost alternative to platinum-based catalysts for electrochemical hydrogen gas $\left(\mathrm{H}_{2}\right)$ production from water. Research effort on this material has focused mainly on synthesizing highly nanostructured $\mathrm{MoS}_{2}$ that allows the exposure of a large fraction of active edge sites. We developed a promising microwave-assisted strategy for the synthesis of narrow $\mathrm{MoS}_{2}$ nanosheets with edge-terminated structure and a significantly expanded interlayer spacing, which exhibit striking kinetic metrics with onset potential of -103 millivolts $(\mathrm{mV})$ versus standard hydrogen electrode (SHE), Tafel slope of $49 \mathrm{mV} /$ decade, and exchange current density of $9.62 \times 10^{-3}$ milliamperes/square-centimeter $\left(\mathrm{mA} \mathrm{cm} \mathrm{cm}^{-2}\right)$; performance is among the best of currently developed molybdenum disulfide catalysts. Besides the benefits from the edge-terminated structure, the expanded interlayer distance with modified electronic structure is also responsible for the observed catalytic improvement, which suggests a potential way to design newly advanced $\mathrm{MoS}_{2}$ catalysts through modulating the interlayer distance.

\section{PROJECT DESCRIPTION}

This project addresses the many-fold computational challenges encountered in systems in which a local domain has to be treated quantum mechanically but is interacting with and influenced by larger regions that cannot be treated quantum mechanically because of extremely high computational cost. Examples are metal/insulating-oxide/metal (MIM) heterostructures, where electrostatics and strain from the larger structure significantly influence the atomic structure and charge transport. Such structures are ubiquitous in resistive random access memories, battery electrodes, and catalysis systems. This project will develop a scalable computational framework that concurrently combines and merges first-principles quantum mechanical calculations for structure and transport with molecular dynamics and mesoscale calculations for quenched disorder at interfaces, as well as elastic strain and electrostatic fields on large irregular domains. The research will focus on MIM systems in which the insulating oxide is a transition metal oxide.

Aspects of the work is conducted in collaboration with Ivan Rungger at the U.K. National Physical Laboratory, Michael Wilde and Ketan Maheshwari of Argonne's Mathematics and Computer Science Division (MCS), Subramanian Sankarayanayaran and Badri Narayanan of Argonne's Nanoscience and Technology Division (NST), and Yoshihiro Asai and Hisao Nakamura at the Japan National Institute for Advanced Industrial Science and Technology.

\section{MISSION RELEVANCE}

The project contributes to the DOE energy mission that supports research into areas relevant to energy efficiency, including use-inspired R\&D on new materials for information storage and energy efficiency. Linking the micro-, meso-, and macro-scales in theory and computation is also relevant to DOE's mission in fundamental supporting science. 


\section{RESULTS AND ACCOMPLISHMENTS}

In FY 2014, we focused on adapting density functional theory (DFT)-based methods to titanium oxides. We demonstrated, using self-interaction corrections within DFT, that the Magnéli-phase titanium oside $\left(\mathrm{Ti}_{4} \mathrm{O}_{7}\right)$ has four different low-energy states at low temperatures; we also found a new antiferromagnetic state as the ground state. We also worked with our collaborators to construct a massively parallel workflow linking molecular dynamics and DFT modeling.

In FY 2015, we focused on heterostructures that comprised a hafnium oxide $\left(\mathrm{HfO}_{2}\right)$ layer sandwiched between titanium nitride ( $\mathrm{TiN}$ ) electrodes and a thin layer of tantalum (Ta) between one TiN electrode and the oxide (Figure 1). This structure is being investigated in academic and industrial labs because if its apparent superior performance in devices. We used a version of the electronic transport code Smeagol, in which electronic correlations beyond the standard local spin density approximation are included to account for the appreciable electron interactions in $\mathrm{HfO}_{2}$. By modeling large heterostructures that consist of over 500 atoms, our calculations showed that, in contrast to the TiN electrode, the Ta metal layer readily incorporates oxygen atoms removed from the $\mathrm{HfO}_{2}$ through oxide formation, which then dramatically enhances the conductance of the heterostructure.

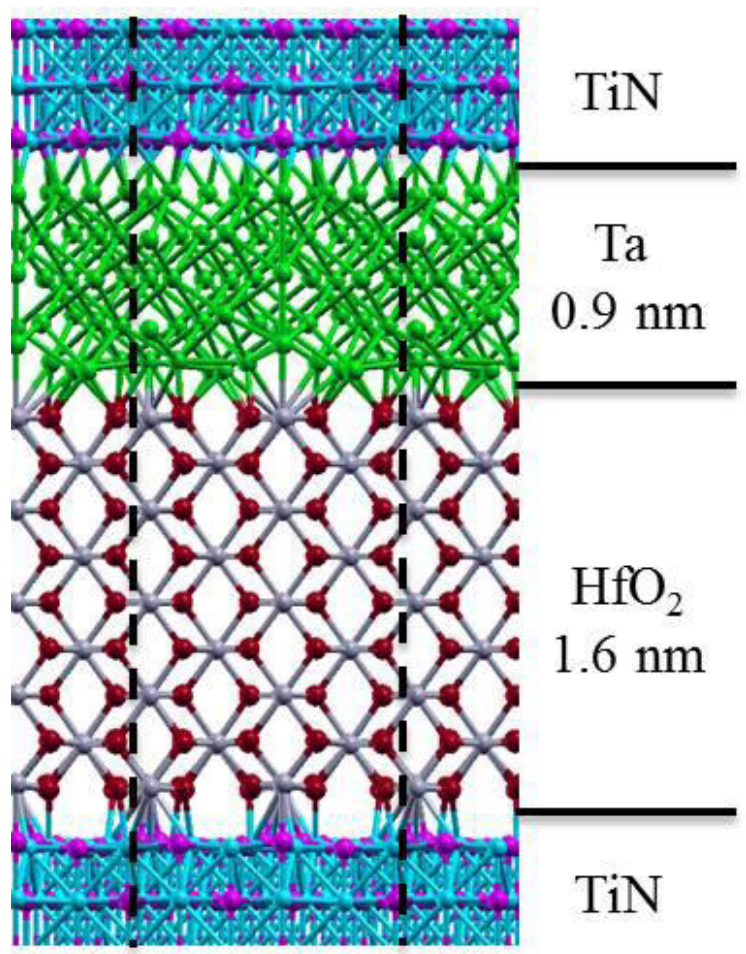

Figure 1. Model of $\mathrm{HfO}_{2}$ heterostructure. $\mathrm{Ti}$ is depicted in blue, $\mathrm{N}$ in purple, $\mathrm{Ta}$ in green, $\mathrm{Hf}$ in grey, and $\mathrm{O}$ in red.
An issue with the quantum mechanical simulations is the assumption that the interfaces are nearly disorder-free, while experimental systems typically have considerable disorder at the interfaces. To be able to model many different configurations of disorder, we devised a workflow that starts with molecular dynamics simulations of the heterostructure after quenching, which traps some disorder at interfaces. The disordered configurations serve as input to a massively parallel quantum mechanical calculation of the electronic structure and the low voltage bias transport. Finally, to evaluate transport properties of mesoscale systems with heterostructures, we developed a scalable parallellized code based on a boundary matrix method to calculate the electrostatic potentials and fields. For additional speed, we combined this method with a kernel-independent fast multipole method (FMM) that uses low-rank approximations to accelerate dense matrixvector multiplications.

We have tested the accuracy, efficiency, and scalability of our code in two cases. In the first case, we calculated the electrostatic potentials and fields in polarized regions (spheres or cuboids) in a vacuum for ferroelectric/ ferromagnetic simulations. The second case was a polarization problem (Figure 2) that originates in a dielectric contrast between regions with different dielectric constants. To solve this problem, we combined a Krylov-subspace iterative solver (GMRES) and the FMM to enable extremely efficient calculation of the electrostatic fields due to polarization and the resulting forces on polarizable objects.

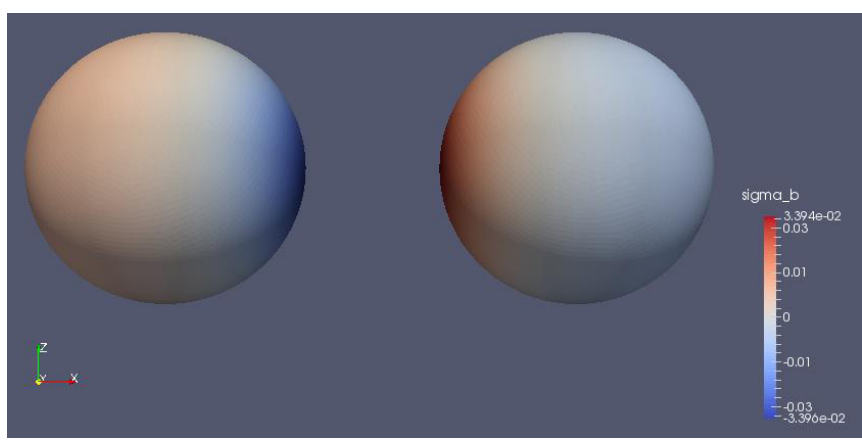

Figure 2. Magnitude of the bound surface charge density (sigma_b, in $\mathrm{C} / \mathrm{m}^{2}$ ) on two $1-\mu \mathrm{m}$ spheres in a medium with a different dielectric constant. The bound surface charge density originates in the discontinuity of electrostatic fields across the interface between the spheres and the medium. The centerto-center distance between the spheres is $3 \mu \mathrm{m}$, and the relative dielectric permittivities of the sphere and matrix are 5 and 1, respectively. The free negative and positive charges on left and right spheres are $-1 \mathrm{C}$ and $+1 \mathrm{C}$, respectively, distributed uniformly throughout the spheres.

\section{PROPOSED FUTURE WORK}

For FY 2016, we will work with our collaborators at MCS and NST to perform a large number of simulations of $\mathrm{HfO}_{2}$-based heterostructures with quenched interfacial 
disorder to gain a quantitative understanding of its effect on conductance. We will also perform so-called nudged elastic band calculations of the energy barriers for oxygen diffusion from the $\mathrm{HfO}_{2}$ to the Ta or Ta-TiN interface. Finally, we will combine mesoscale calculations of the electrostatic potentials with quantum mechanical calculations to model the impact of inhomogeneous electric fields from the metal electrodes.

\section{The Design and Synthesis of Novel Oxides: Coupling Materials Informatics with a Next-Generation Deposition System Employing In-situ $X$-ray Scattering and Photoemission Spectroscopy}

\section{4-129-R1}

Dillon D. Fong, Richard Rosenberg, and Jonathan Z. Tischler

\section{PROJECT DESCRIPTION}

The primary goal of this project is to upgrade an existing diffractometer-mounted chamber and build the world's first in-situ pulsed laser deposition (PLD) system with both surface $x$-ray diffraction (SXRD) and hard x-ray photoemission spectroscopy (HAXPES) capabilities at the Advanced Photon Source. This new experimental capability is being combined with computational materials design (in collaboration with J.M. Rondinelli, Northwestern University [NU]) focused on the development of novel $\mathrm{A}_{2} \mathrm{~B}_{2} \mathrm{O}_{5}$ materials (so-called " 225 " compounds) displaying metal-insulator transition (MIT) behavior. This work is also performed in collaboration with $\mathrm{G}$. Reyes and C.M. Rouleau of Oak Ridge National Laboratory.

\section{MISSION RELEVANCE}

A central goal of DOE-Basic Energy Sciences is to accelerate the design and discovery of materials able to address the nation's energy and environmental challenges. The key strategy is to close the feedback loop between experimental synthesis and predictive materials theories. In this project, we address this challenge by focusing on complex oxide materials, an area with outstanding potential for meeting many of our technological needs.

\section{RESULTS AND ACCOMPLISHMENTS}

During the first year of this project (FY 2014), we completed the redesign of the in-situ PLD system at Sector 33 of the Advanced Photon Source (APS) and received the mechanical components of the hemispherical electron analyzer (Scienta EW4000). The electron analyzer geometry permits easy control over the attenuation length by permitting variable $x$-ray incidence angles and energies, allowing variable depth profiles for HAXPES, protecting the HAXPES lens system from the deposition flux, and avoiding interference with the current scattering geometry. Before the availability of the in-situ PLD-HAXPES system, we performed real-time growth studies of $\mathrm{A}_{2} \mathrm{~B}_{2} \mathrm{O}_{5}$ materials, in this case $\mathrm{Sr}_{2} \mathrm{CO}_{2} \mathrm{O}_{5}$, with in-situ PLD-SXRD. We found that the successful growth of this system is extremely sensitive to the strain state, temperature, and oxygen partial pressure.

In FY 2015, we received the electrical components of the electron analyzer and worked with R. Engel-Herbert (Penn State) on upgrading the deposition facility into a hybrid PLD system (PLD + metal-organic chemical vapor deposition). This significantly expanded our synthesis capabilities by enabling the growth of layered oxides (e.g., Ruddlesden-Popper compounds) and the growth of 225 oxides and superlattices with controlled doping of the $B$-site; neither material would be possible without the hybrid capability. Furthermore, with J.M. Rondinelli (NU), we studied the general properties of 225 superlattices.

An interesting handedness is associated with tetrahedral chain alignment in these structures, as shown in Figure 1 (top). When grown as a superlattice, the cation ordering combined with the tetrahedral chain alignment can lift inversion symmetry, leading to an intrinsic polarization with a magnitude (in microcoulombs/sq. centimeter) ranging from $<1 \mu \mathrm{C} / \mathrm{cm}^{2}$ to $6 \mu \mathrm{C} / \mathrm{cm}^{2}$. The energy of the different chain-ordered $\mathrm{Sr}_{2} \mathrm{Fe}_{2} \mathrm{O}_{5}$ structures as a function of epitaxial strain is shown in Figure 1 (bottom, top). Here, the parallel vacancy ordering (filled symbols) is stabilized under tensile strain, while perpendicular (open symbols) is stabilized under compressive strain. This change in stabilization occurs at the point where either the parallel or perpendicular phase maximizes the average intralayer tetrahedral chain separation ( $R$, middle panel) and minimizes the octahedral distortion effect $(\Delta$, bottom panel). 

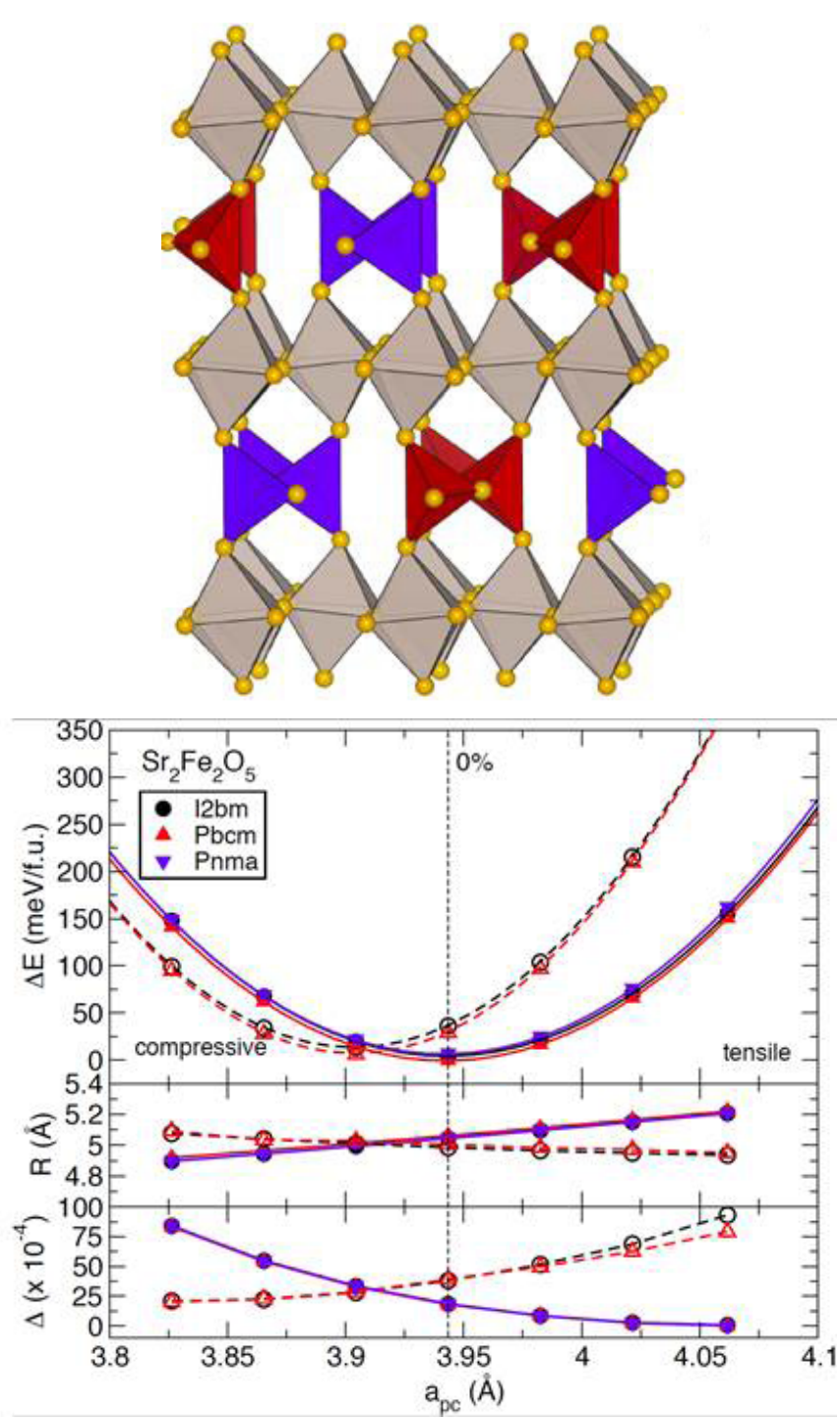

Figure 1. Top: Brownmillerite structure showing alternation of left- and right-handed chains within each layer $(\mathrm{Pbcm})$. [from J. Young and J.M. Rondinelli, Phys. Rev. B 92, 174111 (2015)]. Bottom: Energy, average intralayer tetrahedral chain separation, and octahedral distortion (top, middle, and bottom, respectively) of the different chain-ordered $\mathrm{Sr}_{2} \mathrm{Fe}_{2} \mathrm{O}_{5}$ structures as a function of epitaxial strain. Filled symbols denote parallel vacancy ordering, and open symbols denote perpendicular ordering. $\mathrm{Pbcm}$, Pnma, and $12 \mathrm{bm}$ refer to the crystallographic space group symmetry of the different phases.

\section{PROPOSED FUTURE WORK}

In FY 2016, we will install a recently purchased ultraviolet (UV) source (Specs model UVS300) for low-energy, high-resolution angle-resolved photoemission spectroscopy (ARPES) after each layer of growth; concurrently, we will modify the PLD chamber to permit both hard $x$-ray ARPES and UV ARPES. While the Scienta EW4000 has been specially designed to permit hard x-ray photoemission studies (up to $12,000 \mathrm{eV}$ ), its focusing optics and $60^{\circ}$ angular detection range work over an energy range from $10 \mathrm{eV}$ to $12,000 \mathrm{eV}$, with an angular resolution of $0.2^{\circ}$. By integrating both low- and high-energy capabilities within the deposition chamber, we have a singular opportunity to study and distinguish the electronic structure at the surface from that in the "bulk" of the film. With J.M. Rondinelli, we will continue to study the properties of brownmillerite materials, focusing on the construction of a digital materials library for $A_{2} B_{2} O_{5}$ structures, including elemental descriptors, stability parameters, and materials properties.

Looking ahead toward support for continuing this work, we will respond to calls from DOE in Energy Frontier Research Centers; from ARPA-E, which will solicit proposals in new solid ion conductors; and from the Defense Advanced Research Projects Agency (DARPA), which has stated aims of developing next-generation electronic devices.

\section{In-situ X-ray Characterization of Doped Materials Synthesized by Atomic Layer Deposition for Energy Applications}

\author{
2014-133-R1
}

Thomas Proslier, Jeffrey W. Elam, Steve Heald, Adam Hock, Jeffrey Klug, Michael J. Pellin, Christian Schlepuetz, Matt Weimer, and Angel Yanguas-Gil

\section{PROJECT DESCRIPTION}

In this project, we have developed a unique instrument for performing in-situ studies of dopant concentration, local chemical environment, and structure (including shortand long-range order) during atomic layer deposition (ALD) growth by using synchrotron x-ray measurements. This project was leveraged by powerful, complementary in-situ characterization tools developed at Argonne, such as Fourier transform infrared spectroscopy, quadrupole mass spectrometry, and quartz crystal microbalance techniques to study, in real time, ALD growth and doping mechanisms.

\section{MISSION RELEVANCE}

This project provided powerful research tools that can be applied to a large palette of materials and energy-related research areas. A non-exhaustive list of such areas encompasses: thermoelectrics, solar cells, lasers, catalysis, and batteries which are at the heart of DOE clean energy strategic research priorities. More specifically, the development of portable ALD systems suitable for in-situ synchrotron measurements did 
benefit Argonne's Materials and Molecular Design and Discovery program and provide a powerful and unique tool to the materials research community. Moreover, improvement of our 3-D synthetic dopant control and a detailed understanding of the application range of these techniques have provided the program with an important demonstration of Argonne's ability to play a central role in synthetics development.

\section{RESULTS AND ACCOMPLISHMENTS}

During the two years of this project, we:

$\square$ Built two portable ALD systems and four growth chambers for performing in-situ studies at the Advanced Photon Source (APS):

- Chemistry (bond length, oxidation, coordination): Extended x-ray absorption fine structure (EXAFS) (APS Sector 9-BM) and grazing-incidence small-angle x-ray scattering (GISAXS) (Sector 20-ID).

- Structure (density, roughness, crystalline structure, island formation): $x$-ray powder diffraction (XRD) (Sector 33-BM, 33-ID) and GISAXS (Sector 12-ID).

$\square$ Successfully tested a variety of ALD precursors and materials with different growth/operating temperatures from $50^{\circ} \mathrm{C}$ to $450^{\circ} \mathrm{C}$ : manganese oxide (MnO), zinc oxide $(\mathrm{ZnO})$, magnesium oxide $(\mathrm{MgO})$, erbium oxide $\left(\mathrm{Er}_{2} \mathrm{O}_{3}\right)$, yttrium oxide $\left(\mathrm{Y}_{2} \mathrm{O}_{3}\right)$, aluminum oxide $\left(\mathrm{Al}_{2} \mathrm{O}_{3}\right)$, titanium nitride ( $\mathrm{TiN}$ ), and molybdenum nitride (MoN).

$\square$ Achieved the following:

o Studied dopant/host systems: $\mathrm{MnO}: \mathrm{ZnO}, \mathrm{Er}_{2} \mathrm{O}_{3}: \mathrm{MgO}$.

- Measured for the first time half ALD cycles in situ on flat samples (as opposed to nano-powders).

- Produced nucleation/island formation of ALD ZnO and $\mathrm{Al}_{2} \mathrm{O}_{3}$ on graphene.

- Developed infiltration processes of ALD ZnO and $\mathrm{Al}_{2} \mathrm{O}_{3}$ in block copolymers.

- Produced epitaxial growth of $\mathrm{ZnO}, \mathrm{MgO}$, and $\mathrm{TiN}$ on sapphire.

\section{Fast Electronic Structure Methods for Rapid Reaction Screening for Inorganic Materials Synthesis and Particle Formation}

\author{
2014-139-R1
}

\section{Albert Wagner, David Dixon, Murat Keçeli, Álvaro A. Vázquez-Mayagoitia, and Peter Zapol}

\section{PROJECT DESCRIPTION}

If simulations are to assist experimental programs for the synthesis of new materials and chemicals, new electronic structure codes must be capable of studying systems with very large numbers of atoms. We are developing highly parallelized codes for simulations involving up to tens of thousands of atoms. One code involves semi-empirical molecular orbital (SEMO) theory, and the other involves tight binding based on density functional theory (TB-DFT). Two computational needs must be addressed:

$\square$ An efficient method to calculate the energy of molecular systems by the massive parallelization of available eigensolver methods.

$\square$ An improved training set for optimizing the "empirical" SEMO/TB-DFT parameters by supplementing scattering measurements of molecular energies with systematic and accurate $a b$-initio electronic structure calculations on small molecular systems.

\section{MISSION RELEVANCE}

The project is relevant to DOE's missions in energy and science. Reliable computational methods that predict reaction mechanisms are needed for condensed-media synthesis planning and the management of particle growth in a broad variety of environments. The making and breaking of chemical bonds means that electronic structure must be explicitly included in the simulation. At present, SEMO/TB-DFT electronic structure methods are the fastest available. Success in developing efficient semi-empirical code will enable basic science in areas relevant to DOE's missions, such as combustion (soot formation), heterogeneous catalysis, nuclear chemistry (aggregation phenomena), materials phase changes, and multilayered materials.

\section{RESULTS AND ACCOMPLISHMENTS}

Parallelized Eigensolver. In FY 2014, we developed a new, highly parallelized eigensolver called shift-and-invert parallel spectral transformations (SIPs). This method takes full advantage of the sparse TB-DFT or SEMO Hamiltonian 
matrix, the eigenvalues of which we seek. "Sparse" here means that most of the matrix elements are zero, a feature prominent in semi-empirical methods. In FY 2015, we further developed this eigensolver and submitted this new method for publication, along with favorable comparisons to conventional parallelized eigensolve methods for dense matrices and two recent methods suitable to sparse matrices. (The recent methods were pole expansion and selected inversion [PEXSI] and multiple shift-and-invert Lanczos [MSIL], an inefficient, less stable SIPs-like method.) These comparisons indicate that SIPs is a highly competitive method for sparse eigensolvers.

Our eigensolver must be imbedded in a highly parallelized method for constructing the Hamiltonian matrix. Figure 1 shows the speedup of the method we have developed and implemented for matrix assembly with respect to number of processors. The speedup deviates from the ideal curve (100\% efficiency) only when the number of matrix rows is comparable to the number of processors. With this level of parallelization, the computational cost of the Hamiltonian construction is less than $5 \%$ of the overall cost.

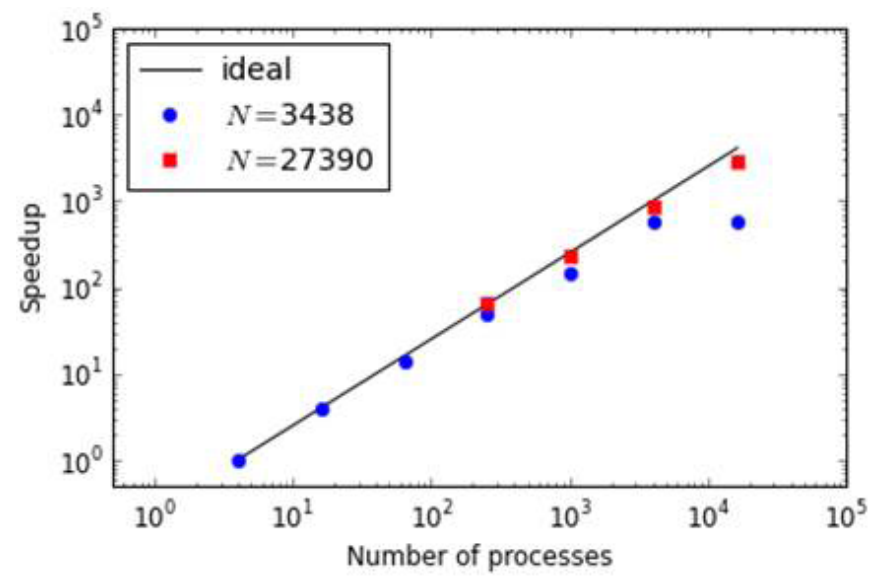

Figure 1. Speedup versus number of processors for two different matrix sizes. Speedup is $t_{o} / t_{p}$, where $t_{o}$ is computational time with 4 or 64 processors $\left(N=3438\right.$ or 27390 , respectively) and $t_{p}$ is the computational time with p processors.

Training set improvements. We used the FellerPeterson-Dixon composite approach based on ab-initio coupled cluster CCSD(T) electronic structure calculations extrapolated to the complete basis set limit to predict the heats of formation of transition metal oxide clusters. The resulting systematic set of structures and energies can be used to improve the training set for transition metal SEMO parameters (Table 1). Our calculations have led to the development of a more rapidly convergent extrapolation sequence based on DFT orbitals or Brueckner orbitals, produced unexpected equilibrium structures, and provided the training set information necessary to improve the reliability of SEMO applications to transition metal problems.

Table 1. Transition metal SEMO parameters for which calculations are complete.

\begin{tabular}{|c|l|}
\hline \multicolumn{1}{c|}{$\begin{array}{c}\text { Transition } \\
\text { Metal }\end{array}$} & \multicolumn{1}{|c|}{ Species } \\
\hline $\mathrm{M}^{\prime}=\mathrm{V}, \mathrm{Nb}, \mathrm{Ta}$ & $\begin{array}{l}\mathrm{M}^{\prime} \mathrm{O}_{3} \mathrm{H}, \mathrm{M}_{2}^{\prime} \mathrm{O}_{5} \mathrm{M}_{2}^{\prime} \mathrm{O}_{4}(\mathrm{OH})_{2}, \mathrm{M}_{2}{ }_{2} \mathrm{O}_{3}(\mathrm{OH})_{4,} \\
\mathrm{M}_{3} \mathrm{O}_{8}{ }^{-1}, \mathrm{M}_{3}^{\prime} \mathrm{O}_{7}(\mathrm{OH})\end{array}$ \\
\hline $\mathrm{M}=\mathrm{Mn}, \mathrm{Tc}, \mathrm{Re}$ & $\begin{array}{l}\mathrm{MO}_{4}{ }^{-1}, \mathrm{MO}_{3}, \mathrm{MO}_{4} \mathrm{H}, \mathrm{M}_{2} \mathrm{O}_{7}, \mathrm{M}_{3} \mathrm{O}_{11} \mathrm{H}, \\
\mathrm{M}_{3} \mathrm{O}_{11}{ }^{-1}\end{array}$ \\
\hline
\end{tabular}

\section{PROPOSED FUTURE WORK}

The following tasks are planned for FY 2016:

$\square$ Completion of first-generation effective parallel code for both SEMO and TB-DFT. This will include a massively parallelized Hamiltonian builder that hands off the Hamiltonian to the parallel eigensolver and a self-consistent iteration process that takes advantage of knowledge developed in previous iterations to improve the load balance of the eigensolver in the current iteration. For SEMO, the code will feature a "menu" of Hamiltonian types, each with a customized set of optimized semi-empirical parameters.

$\square$ Formulation and solution of two illustrative science problems that requires our software. The first is the growth of transition metal oxide (TMO) nanoparticles from TMO clusters. There are structural changes that occur in this growth process and the goal would be to identify at what size particle those structural changes occur. The second problem is the evolution of changes in force field descriptions in polymers as the number of repeating units in the polymer grow. The force field becomes simpler with polymer growth and the goal would be to determine how rapidly that occurs.

\section{Developing Predictive Models of Wide Bandgap Semiconductor Synthesis and Processing}

\section{4-151-R1}

Paul H. Fuoss, Jeffrey A. Eastman, Jeffrey W. Elam, Ross J. Harder, Matthew J. Highland, Martin V. Holt, Stephan O. Hruszkewycz, Angel Yanguas-Gil, and Peter Zapol

\section{PROJECT DESCRIPTION}

This project seeks to understand nanoscale physical and chemical processes during the growth of wide-bandgap 
(WBG) semiconductors, such as gallium nitride (GaN) and aluminum nitride (AIN), and their fabrication into devices. We examine WBG synthesis by using three different growth techniques: (1) sputtering in a reactive environment of nitrogen, (2) atomic layer deposition (ALD), and (3) metal organic chemical vapor deposition (MOCVD). Using advanced $\mathrm{x}$-ray imaging techniques, we follow the evolution of strain and defect behavior during the growth, processing, and cycling of test devices to isolate critical processes. Complementary multiscale computational models predict strain evolution and defect generation.

\section{MISSION RELEVANCE}

WBG semiconductors such as GaN and AIN have enormous potential for revolutionizing the control and transmission of electrical power, but their development requires a deeper understanding of WBG physics and chemistry. Thus, a microscopic understanding of the synthesis and processing of WBG materials will directly benefit the DOE energy mission by enabling higher-performance devices.

\section{RESULTS AND ACCOMPLISHMENTS}

In FY 2014, we developed computational tools to simulate the growth of GaN epitaxial thin films by MOCVD and $A L D$, and performed preliminary $x$-ray measurement and imaging of defect generation in WBG semiconductor thin films. Building on these results in FY 2015, we continued to develop the experimental and computational techniques and use them to develop multi-scale models of the synthesis of WBG semiconductor thin films.

Localized growth measurements are a key capability needed to address the epitaxial growth of WBG semiconductors on three-dimensional (3D) vertical device structures. Over the past year, we achieved this capability by installing a nitride MOCVD system for $x$-ray scattering and imaging studies at beamline 12-ID-D of the Advanced Photon Source (APS). To fully utilize this new instrument, we are completing the development of a new coherent $x$-ray imaging technique that will resolve individual dislocations in WBG crystals at 3D spatial resolutions of $<10$ nanometers $(\mathrm{nm})$, thus enabling multiscale visualization of structure and strain fields. We measured coherent nanodiffraction data (Figure 1) that will be used to reconstruct 3D images of internal strain and defects within nanoscale islands that AIGaN initially forms on single-crystal sapphire. This new imaging techniquesparse-angle Bragg ptychography-will be deployed for in-situ visualization of heterogeneous WBG materials.
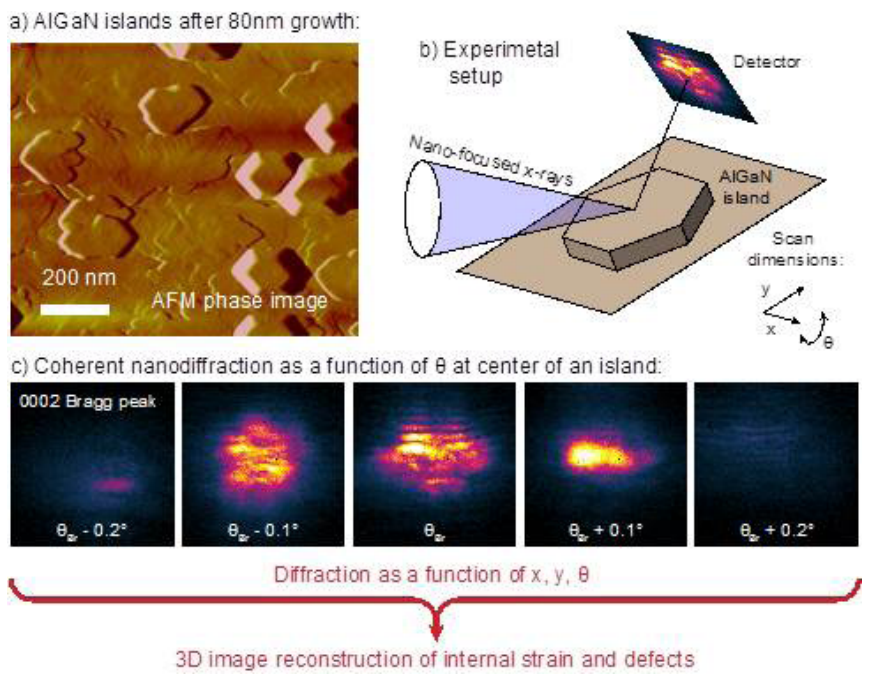

Figure 1. We are developing visualization tools to examine the complex strain and defect distributions in the nanoscale AIGaN islands (a) that form during growth on sapphire. With a 40-nm focused x-ray nanoprobe (b), we collected coherent Bragg diffraction patterns from several islands and their surrounding film. These large data sets (c) will be used to reconstruct the island shape and strain with $<10-\mathrm{nm}$ resolution.

A key process in the fabrication of WBG device structure is relaxation of the strain associated with epitaxy of materials with different lattice constants. We have used in-situ x-ray scattering to study the growth of AIN nucleation layers via reactive magnetron sputtering and followed the evolution of AIN films. Our results indicate that the quality and properties of the films depend heavily not only on the growth conditions, but also on the initial growth surface. These results are being used to guide the development of predictive models of the growth of AIN on the (0001) plane of $\mathrm{Al}_{2} \mathrm{O}_{3}$ substrates. As the film thickness increases, so does the stress due to lattice mismatch, which drives structural transitions. From first principles calculations, we found that the interface energy quickly approaches the bulk value with increasing number of layers. This result allowed us to formulate a model that predicts the limits of stability for an epitaxial film. Ongoing density functional theory calculations are exploring interfaces with different lattice mismatches.

Current WBG semiconductor growth models, which typically use effective reaction probabilities, do not reproduce the growth of defects and $3 D$ nanostructures. Therefore, our focus has been on the development of atomistic simulations to understand the morphology and growth rate of WBG semiconductors for arbitrary surfaces and orientations. Using energetics obtained from classical potentials developed for these materials, we modeled thin film epitaxy and 3D crystal growth for both $\mathrm{GaN}$ and $\mathrm{SiC}$, and successfully reproduced the transition from step flow to island growth mode on c-plane surfaces. 
We also mapped out the dependence of growth rate on miscut and orientation for different growth conditions. These results will be incorporated into coarser-scale models that reach up to the feature scale. We have implemented a simple model based on a dynamic Lagrangian approach to track surface evolution during trench coating. This gives us a unique approach to infer the angular dependence of growth rate in different MOCVD processes based on trench profile data and construct a full map of the 3D coating.

\section{PROPOSED FUTURE WORK}

In FY 2016, we will work toward the following milestones:

$\square$ Provide conceptual models and simulations for growth in trenches.

$\square$ Develop computational models of growth and defect densities with input from $x$-ray imaging studies.

$\square$ Use in-situ x-ray techniques to monitor simultaneous MOCVD growth on the different faces of a GaN trench.

$\square$ Image defect generation in real time during growth.

$\square$ Compare growth results from different techniques and identify key parameters controlling properties.

$\square$ Image defect formation and breakdown in active devices.

\section{Bridging the Electronic and Atomistic Scales: Force Field Development for Reactive Interfaces from First Principles}

\section{4-161-R1}

Subramanian Sankaranarayanan, Maria Chan, Michael J. Davis, Stephen K. Gray, and Benoit Roux

\section{PROJECT DESCRIPTION}

Chemical interactions at reactive interfaces underlie much of energy capture, conversion, and storage by materials. We currently have an unprecedented level of detail in our understanding of the structural and transport characteristics of various interfaces at the electronic, atomic, and molecular levels. However, an accurate atomistic-scale description of reactive interfaces is still in its infancy. Such dynamical processes combine a multitude of interfacial reactions, transport phenomena, and microstructural evolution with the formidable subtleties of material defect chemistry and solvation dynamics at the interface. Understanding such reactive interfaces has emerged as a key materials science challenge.

While ab-initio molecular dynamics (AIMD) simulations are becoming more feasible, there is still a substantial gulf between time and length scales accessible to AIMD versus force fields (FFs). AIMD simulations involve hundreds of atoms and trajectories of picoseconds, while FF simulations encompass billions of atoms and several microsecond trajectories. Ultimately, we need a new generation of classical AIMD simulations employing reactive FFs. Thus, the goal of this project is to develop new, first-principles-based, more accurate, and more robust inter-atomic potentials for accurate simulations of dynamical processes at reactive interfaces.

\section{MISSION RELEVANCE}

We envision our effort to become an important part of DOE's Materials Genome initiative. These themes are also of interest to agencies beyond DOE, including defense research agencies and companies such as Exxon-Mobil, DE Shaw, Chevron, and Intel. We will establish strong ties with other focus groups within Argonne and external groups to engage in larger-scale efforts focused on FF development. Success in this program has the potential to change the landscape for the role and impact of simulation on the design and synthesis of new materials, including those for energy applications and new chemistries.

\section{RESULTS AND ACCOMPLISHMENTS}

During FY 2014 and 2015, we established an FF fitting methodology that allows for development of new $a b$-initio based FFs for classical simulations of materials and interfaces. Specifically, we developed new reactive ab-initio FFs for a broad range of materials: metal clusters, oxides, and soft-matter organic systems. We also developed a new class of flexible potentials that overcome the limitations imposed by the predefined functional forms used in classical AIMD simulations. Specifically, we have introduced a new hybrid FF to capture dimensionality effects and global minimum structures in metal clusters. Our new FF-termed hybrid bond order potential (HyBOP) accurately predicts structures of gold nanoclusters from two atoms up to bulk (Figure 1). We also developed a genetic algorithm code for improved sampling to aid in the generation of an extensive $a b$-initio training set for potential fitting. For planar clusters, such as 13-atom gold (Au), we identified a new global minimum structure that has not been reported previously. We also developed a new variable charge potential model for evaluating the structural stability 
of iridium oxygen (Ir-O) polymorphs (Figure 2), which are widely used as photocatalysts. We also embarked on development of a more robust Drude polarizable model for organic molecules in solvents. Evolutionary optimization was used to reparameterize existing FF for six molecules, including water, benzene, ethane, butane, methanol, and ethanol. We also extended HyBOP to other transition metals and mixed covalent-metallic systems, such as cobalt/carbon (Co/C). We compared various local optimization schemes, such as Levenberg-Marquardt (L-M); Simplex; derivative free optimization such as ORBIT and POUNDERS (with Stefan Wild of Argonne's Mathematics and Computer Science [MCS] Division); and local search algorithms with global optimization methods, including multi-start L-M and genetic algorithms. We also tested the advantages of using a multi-objective scheme to remove ambiguities caused by the selection of weights in the single objective optimization.

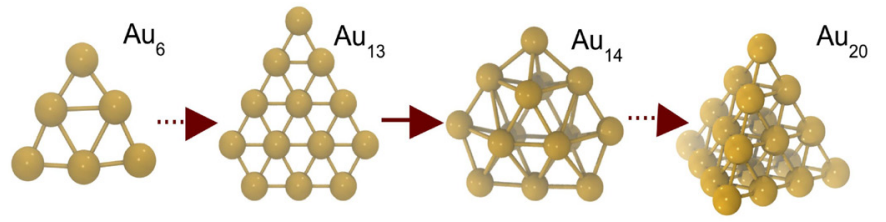

Figure 1. New HyBOP method successfully describes the diverse geometries of gold nanoclusters. The global minimum energy configurations for various cluster sizes predicted by HyBOP are shown above. HyBOP is the first empirical force field which successfully captures the planar to globular transition (from $\mathrm{Au}_{13}$ to $\mathrm{Au}_{14}$ ) in gold nanoclusters. A pyramidal structure for $A_{20}$ is also successfully predicted by HyBOP.

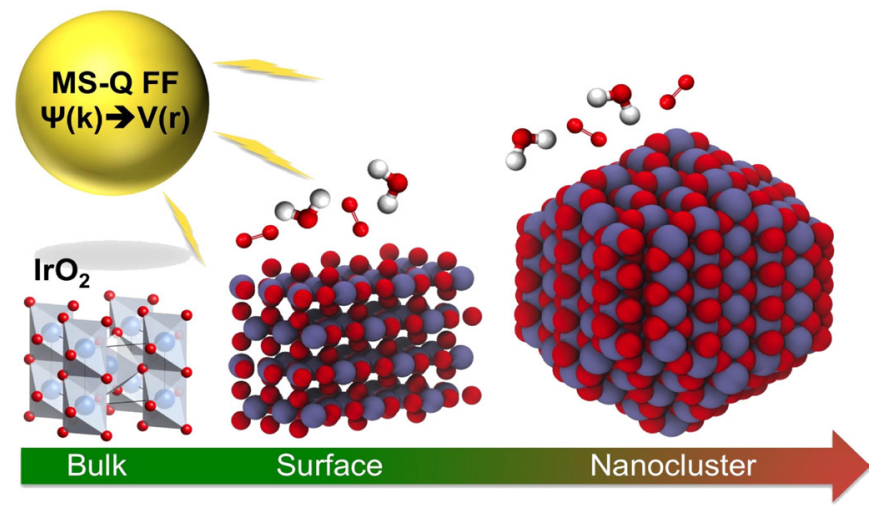

Figure 2. Multi-objective approach enables development of first empirical reactive $\mathrm{FF}$ model for $\mathrm{IrO}_{\mathrm{x}}$. A first principles-based force-field for the $\mathrm{Ir}-\mathrm{O}$ system was developed by parametrizing a Morse potential coupled with a variable charge equilibration method (QEq) (MS-Q FF). MS-Q predicts with reasonable accuracy the structural and thermodynamic properties of bulk, surfaces as well as nanoclusters. Our MS-Q FF should enable large-scale atomistic modeling of Ir-O systems and therefore be useful for gaining atomistic insights into structure, catalytic, and dynamic properties of $\mathrm{IrO}_{2}$ nanoclusters, nanoparticles, and phases of relevance to a range of energy applications.

\section{PROPOSED FUTURE WORK}

During FY 2016, continued development of HyBOP will involve several steps: (1) generation and manipulation of extensive fitting data sets through electronic structure calculations, (2) defining functional forms, (3) formulating highly optimized fitting procedures, (4) employing dualHamiltonian optimization to leverage FF with more accurate approaches, and (5) implementing these algorithms on high-performance computers. We will focus on developing a flexible functional form consisting of a linear combination of independent basis sets and will employ state-of-the-art machine learning and regression techniques such as compressive sensing, evolutionary techniques such as particle swarm, and differential evolution to derive optimal parameter sets for complex systems such as Li/Si. Finally, we will develop a computational workflow tool for FF fitting using a parallel scripting language.

\section{Defect-Localized Spins in Semiconductors for Quantum Optoelectronics}

\section{4-191-R1 \\ David D. Awschalom}

\section{PROJECT DESCRIPTION}

The purpose of this project is to create robust electronic spins that can be used as quantum bits (qubits) in gallium nitride ( $\mathrm{GaN})$. These spins will be bound to crystalline point defects that are generated in GaN through damage or ion implantation. Therefore, they will be only a few atoms large in scale, making them suitable for use in a number of emerging applications in quantum information processing, quantum communication, and quantum sensing.

Gallium nitride has already proven to be an extremely versatile semiconductor in the microelectronics industry. We would like to leverage the unique capabilities of GaN materials growth and device design to develop a new generation of quantum-enabled electronics. To do this, we must create and discover robust quantum states suitable for integration into GaN-based device structures. Defect-bound spin qubits may be ideal for this purpose, as they have been shown to exhibit exceptionally robust quantum properties in other wide-bandgap systems such as diamond and silicon carbide.

\section{MISSION RELEVANCE}

This project is relevant to DOE's basic science mission. Electronic access to the quantum-mechanical phase space of a spin qubit would enable a new class of quantum-coherent optoelectronic devices in which 
traditional microelectronic interfaces are used to control extended quantum modes throughout a given structure. These fundamental material studies may establish a powerful device paradigm in which the quantum state of a material can be seamlessly interconverted between electronic, magnetic, optical, and vibrational degrees of freedom via the quantized spin of a single point defect. Such capabilities could have profound implications for energy-efficient electronics, high-speed computing (quantum information processing), secure encrypted communication, and basic sensor technologies.

\section{RESULTS AND ACCOMPLISHMENTS}

In FY 2015, we successfully created and characterized quantum spin states in GaN and aluminum nitride (AIN) when engineered with ion implantation. While seeking to precisely study these defects, we constructed a magneto-optical spectroscopy apparatus operating from the ultraviolet to infrared regions of the spectrum.

Further Characterization of Spin Properties. With a new equipment setup-including a closed-cycle cryostat with optical access and magnet goniometer allowing precise placement of a permanent magnet relative to the defects inside a sample, we began to quantitatively characterize a level anti-crossing (LAC) dip observed in the magnetoluminescence of the defect and its relationship to field angle and temperature. The dip is sharpest when the magnet angle is aligned along the c-axis of the $\mathrm{GaN}$ crystal, which is also the primary axis of the defect. This behavior is similar to what has been observed in the diamond nitrogen-vacancy center as well as the silicon carbide divacancy, suggesting optically-active spin states.

We also began to quantitatively characterize optically detected magnetic resonance (ODMR) in this system as a function of several experimental parameters. At cryogenic temperatures (5-20 K), we observed several interesting ODMR resonances (Figure 1). Like the LAC observed in magnetoluminescence, these ODMR features are indicative of a spin-selective optical process that may include spin polarization on some level within the defect. The first ODMR feature is a large and broad resonance that is observed to increase in frequency with magnetic field with a g-factor of approximately 2 . This finding suggests that the spin lifetime associated with this feature is in the nanoseconds timescale, which is shorter than what has been observed for defect spins in diamond and SiC. This result might indicate that the spin interacts significantly with the surrounding nuclear spin bath. Every atom in GaN has a non-zero nuclear spin, whereas in contrast, the nuclear spin density of diamond and $\mathrm{SiC}$ is only a few percentage points. Spin-mixing in the ground state at this magnetic field reduces the contrast of the excited state ODMR. Further exploration is needed to determine whether a similar effect is being observed here in GaN.

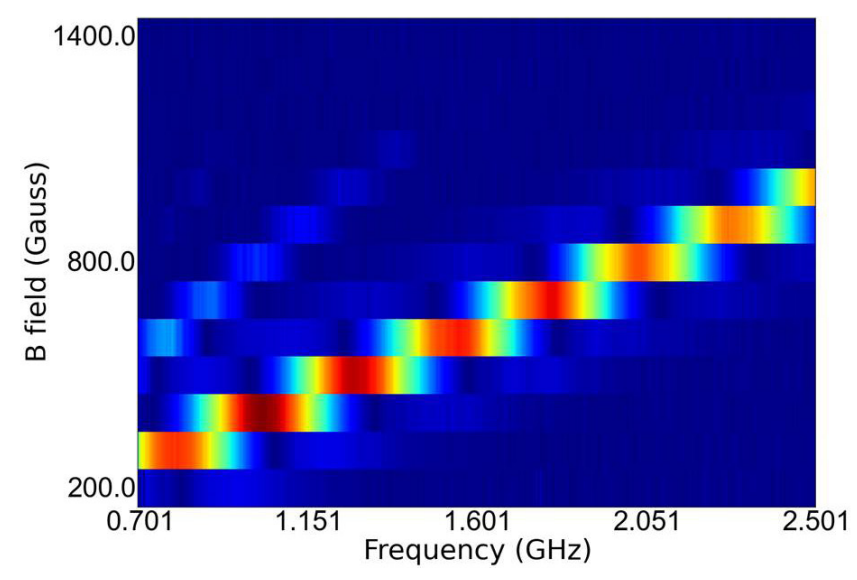

Figure 1. Optically Detected Magnetic Resonance (ODMR) vs. Magnetic Field. ODMR is measured as a function of magnetic field magnitude. The magnetic field is oriented along the $c$-axis of the sample. A microwave driving field is generated perpendicular to this direction along the sample plane. This driving field geometry can be used to drive spins with an out-of-plane component. Two resonances are observed at frequencies $f$ and $f / 2$, suggesting that the lighter feature is attributable to a two-photon transition.

In addition to the large ODMR peak, a second shorter ODMR peak can be observed at lower frequencies when the magnetic field is slightly misaligned away from the $c$-axis. It is also fairly broad and appears to increase in frequency with magnetic field but at half the rate. Because of these features, we believe that this peak is a double-quantum resonance.

Initial Exploration of Defect Optical Structure. Our FY 2014 work showed that defect photoluminescence in these electron-irradiated samples could be enhanced significantly by using a dual-excitation scheme in which UV and IR light was used simultaneously to excite the sample. In addition, the defect luminescence at $0.88 \mathrm{eV}$ was known to exhibit two zero phonon lines (ZPLs) roughly $3 \mathrm{meV}$ apart. These properties are distinct from what has been observed in the diamond nitrogen vacancy center, as well as the silicon carbide divacancy, which suggests that these GaN-based defect spins differ somehow in their electronic structure and optical dynamics. To study these aspects in more detail, we performed a series of optical excitation experiments in which defect luminescence was monitored as a function of various excitation conditions.

As an initial experiment, the defect luminescence spectrum was monitored as a function of excitation wavelength. Several interesting observations were then made, based on the data shown in Figure 2. First, while the phonon sideband increases marginally when the monochromator is tuned from red to blue 
(400 nm), a sudden and marked increase occurs at $\sim 375 \mathrm{~nm}$. This threshold behavior is also observed in the higher-energy ZPL at 1,412 nm, with the line being largest between $375 \mathrm{~nm}$ and $355 \mathrm{~nm}$ (the bandgap energy of GaN). Notably, this line is difficult to observe at longer wavelengths, whereas the magnitude of its lower-energy counterpart at $1,418 \mathrm{~nm}$ remains roughly similar across the full visible-to-UV tuning range of the experiment. Last, a new ZPL feature at 1,399 $\mathrm{nm}$ was observed in these measurements that does not appear to have been noticed or discussed in the literature previously.

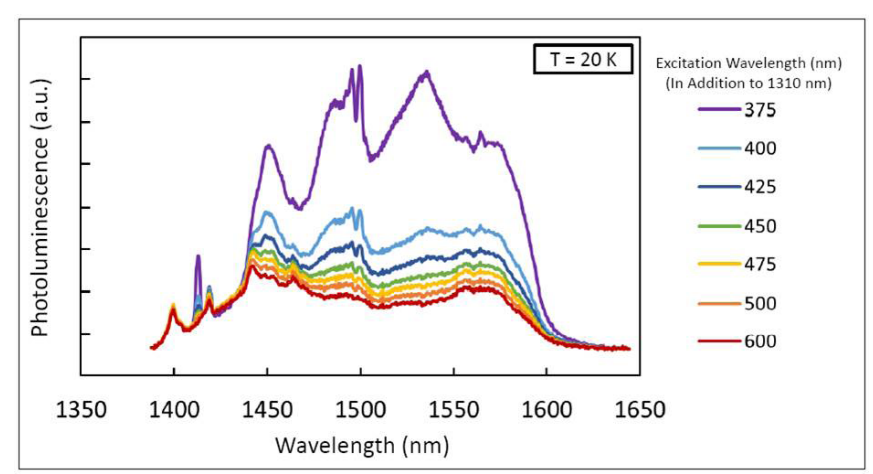

Figure 2. Wavelength Dependence of Defect Photoluminescence. Defect photoluminescence is measured as a function of excitation wavelength. A clear threshold behavior is observed at $\sim 375 \mathrm{~nm}$ in the phonon sideband and the higher-energy ZPL at 1,412 $\mathrm{nm}$. However, little wavelength dependence is observed for the feature at $1,399 \mathrm{~nm}$, which appears to be a third ZPL that has never been noticed previously.

To further explore the relationship between these three lines, we performed a series of photoluminescence excitation (PLE) experiments in which the luminescence intensity of the phonon sideband was monitored while resonantly exciting the defect ensemble using a narrow-line tunable laser. We confirmed that the new line at 1,399 $\mathrm{nm}$ was indeed a physically meaningful feature of the luminescence spectrum and not simply an artifact of our measurements. Second, we were able to gather data suggesting that all three ZPL features originate from the same defect species rather than multiple distinct species that happen to have similar optical emission energies. This data came in the form of several photobleaching experiments in which defect emission was greatly reduced by turning off the UV laser while resonantly exciting the defects at one of the three ZPL wavelengths. Last, we attempted several ensemble hole-burning experiments to test whether any substructure in the optical excitation pathway could be observed. However, we were unable to generate any hole or anti-hole features in these initial experiments.

\section{PROPOSED FUTURE WORK}

Several experimental pathways exist for FY 2016. First, we will attempt to modulate the spin or orbital state of these defects by using strain or the piezoelectric properties of the sample. A major motivation for our GaN and AIN explorations is to identify promising defect spin species for precisely this purpose. Ultimately, we would like to demonstrate coherent quantum control over a localized spin state using mechanical degrees of freedom. We can attempt initial experiments along these lines by designing an fabricating a sample mount that enables mechanical forces to be applied to the defect spins in situ.

As a continued effort for FY 2016, we would like to further our understanding of the electronic structure of these defects, especially with regard to the photo and spin dynamics of this system. Time-resolved optical experiments would help in this regard. And while the short spin lifetimes of these defects make time-resolved studies challenging, efforts to improve the spin lifetime through fine-tuning of the sample preparation recipe (e.g., by reducing the electron irradiation dose) may also be worthwhile.

Finally, it would be worthwhile to evaluate whether defect systems in GaN or AIN are promising targets for optical coherent control. In particular, recent theoretical and computational work to identify vacancy impurity complexes in AIN with properties similar to the diamond $\mathrm{NV}$ offers some promising opportunities for further exploration.

\section{Computational Spectroscopy of Heterogeneous Interfaces}

\section{4-192-R1}

\section{Giulia Galli}

\section{PROJECT DESCRIPTION}

This project aims to develop and apply large-scale quantum simulation methods to model, at the microscopic scale, the physical and chemical processes involved in photo-electrochemical (PEC) energy conversion. The main focus is on processes occurring at the interface between solid photo-electrodes and simple electrolytes (e.g., water with dissolved salts). Although primarily applied to PEC energy conversion, the results of this project are also relevant to other problems of interest in renewable energy applications, including electrical energy storage and solarto-thermal energy conversion.

This project encompasses ab-initio molecular dynamics (MD) simulations to obtain atomic trajectories and compute ensemble averages of thermodynamic properties, and the development and use of firstprinciples methods to obtain vibrational and electronic 
spectra of heterogeneous interfaces. These methods are applied to oxides and semiconducting electrodes interfaced with simple aqueous solutions, with two main goals: (1) to provide knowledge and computational tools to interpret a large body of ongoing experiments on fuel production from water; and (2) to establish design rules to predict Earth-abundant, nontoxic oxides and semiconductors with interfacial properties optimally suited to oxidize and reduce water.

\section{MISSION RELEVANCE}

The project aligns well with DOE's mission in the development of renewable energy sources. Within Argonne, beneficiaries include the Center for Nanoscale Materials (CNM), the Advanced Photon Source (APS), and the Materials Design Laboratory; this project is also well aligned with several ongoing activities in the Joint Center for Energy Storage Research (JCESR).

\section{RESULTS AND ACCOMPLISHMENTS}

In FY 2014, we focused on developing a computational strategy to compute, from first principles, band edge positions of semiconductors and insulators interfaced with liquid water and with respect to vacuum and water redox potentials.

We also completed work on functionalized silicon (Si) surfaces used as p-type photoelectrodes for water splitting. In particular, we combined ab-initio MD simulations of structural properties with many-body perturbation theory calculations of electronic energy levels. The computational approach employed in this work explicitly includes the effect of the interaction of liquid water with the solid surface, including structural rearrangements occurring at the interface and finite temperature effects.

Two main results were obtained in FY 2015:

$\square$ We developed a new computational technique to compute surface-sensitive sum frequency generation (SFG) spectra that will be applied in the future to study heterogeneous interfaces: In particular, the method is based on density functional theory and the use of maximally localized Wannier functions to compute the response to electric fields, and it includes the effect of electric field gradients at surfaces. In addition, it includes quadrupole contributions to SFG spectra, thus enabling the verification of the dipole approximation, whose validity determines the surface specificity of SFG spectroscopy. We computed the SFG spectra of ice Ih basal surfaces and identified which spectral components are affected by bulk contributions. Our results are in good agreement with experiments at low temperature.

$\square$ We studied interfaces between photoabsorbers and catalysts, in particular the ability to extract charges. The design of optimal interfaces between photoelectrodes and catalysts is a key challenge in building photoelectrochemical cells to split water. Iridium dioxide $\left(\mathrm{IrO}_{2}\right)$ is an efficient catalyst for oxygen evolution, is stable in acidic conditions, and hence is a good candidate to be interfaced with photoanodes. Using first-principles quantum mechanical calculations, we investigated the structural and electronic properties of tungsten trioxide $\left(\mathrm{WO}_{3}\right)$ surfaces interfaced with an $\mathrm{IrO}_{2}$ thin film (see Figure 1). We built a microscopic model of the interface that exhibits a formation energy lower than the surface energy of the most stable $\mathrm{IrO}_{2}$ surface, in spite of a large lattice mismatch, and which has no impurity states pinning the Fermi level. We found that upon full coverage of $\mathrm{WO}_{3}$ by $\mathrm{IrO}_{2}$, the two oxides form undesirable Ohmic contacts. However, our calculations predicted that if both oxides are partially exposed to a water solvent, the relative position of the absorber conduction band and the catalyst Fermi level favors charge transfer to the catalyst and hence water splitting. We propose that for oxide photoelectrodes interfaced with $\mathrm{IrO}_{2}$, it is advantageous to form rough interfaces with the catalyst, for example, by depositing nanoparticles instead of sharp interfaces with thin films.

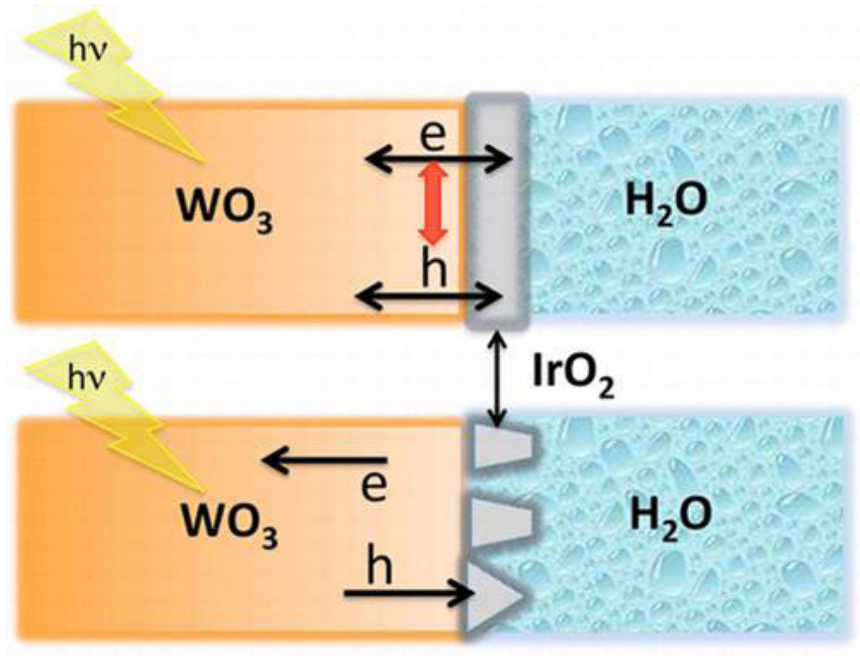

Figure 1. Schematic representation of different catalysts' morphology on wo and their influence on charge extraction. 


\section{PROPOSED FUTURE WORK}

The main ongoing work concerns the further implementation and use of GW approximation techniques for surface and solution calculations using the WEST code (http://www.west-code.org/). Reports on preliminary results are being prepared or submitted.

\section{Framework for Integrating Multi-Modal Imaging of Materials for Energy Storage}

\author{
2015-144-NO \\ Doga Gursoy, Charudatta Phatak, Lynn Trahey, \\ and Xianghui Xiao
}

\section{PROJECT DESCRIPTION}

Lithium-ion batteries have had a remarkable effect on the adoption of portable electronic devices. Improving batteries requires understanding the interaction of many materials at multiple length scales, ranging from micro (structure of various components, transport of ions and charges) to nano (electronic interactions, local charge accumulation). To address this challenge, we are investigating battery materials using a combination of electron microscopy and x-ray microscopy. Our goal is to integrate the data from each modality by using the computational imaging tools that will be developed within this project. We will develop improved computational methods based on probability theory for faster and reliable analysis, reconstruction, and correlation of multi-dimensional micro- and nanoscale datasets. This approach will allow us to understand the nano- to microscale evolution of change in the material as a function of electrochemical cycling. Results of this research will help create pathways for breakthroughs in design of battery electrodes.

\section{MISSION RELEVANCE}

This research will assist the energy community, as well as the vast general user communities (e.g., materials science and engineering), which require robust multi-modal characterizations. The development of standardized data analysis tools will also benefit researchers at a range of DOE facilities (Advanced Photon Source, Electron Microscopy Center, Center for Nanoscale Materials) and thus will be useful to the wider scientific community. The outcome of this research will have direct impact on reducing the data acquisition and quantification time and will lead to a holistic understanding of fundamental processes, leading to faster improvements and innovations in materials design for future energy technology. This research will directly advance the goals of the Integrated Imaging Initiative at Argonne, while strongly supporting the overall DOE mission of innovative and improved research on energy materials as well as the missions of several DOE BES Energy Frontier Research Centers and the Joint Center for Energy Storage Research.

\section{RESULTS AND ACCOMPLISHMENTS}

Preliminary experiments were conducted to obtain data using current instrumentation to study the structural changes in uncycled and cycled lithium- and manganese-rich - nickel manganese cobalt cathode particles. We obtained experimental data and performed reconstructions from transmission $\mathrm{x}$-ray microscopy (TXM), bright-field scanning transmission electron microscopy (STEM), and high-angle annular dark-field STEM from the same cycled particle. Currently, both $\mathrm{x}$-ray and electron imaging-based modalities reveal structural information, such as the presence of porosity at different resolutions and for different volumes of the particle. We developed data registration workflows for $\mathrm{x}$-ray and electron tomography datasets and obtained preliminary 3D visualization of the agglomerate from the reconstruction of tomography datasets. We are now exploring whether learning-based approaches can be used in improving the resolution of TXM from STEM data. We are also working to improve and automate the registration of images obtained at different projection angles (or tilt-series) as well as across x-ray and electron imaging modalities using machine learning methods such as sparse dictionary learning and artificial neural networks.

\section{PROPOSED FUTURE WORK}

We will build the $x$-ray microscope to initially characterize the illumination beam with a standard sample and then perform a tomographic scan of a battery sample. The phase-contrast images can be treated with single-distance phase retrieval algorithms, and the contribution of the illumination beam can be removed with the pre-characterized illumination wave. This approach enables one to obtain the 3D morphological structure of a large volume of battery electrode at high temporal resolution in minutes. On the basis of the 3D tomographic structure, a region of interest (ROI) can be identified and moved to the $x$-ray beam focal position. A 3D diffraction mapping of the ROI can provide crystallographic information from this volume. Because of the achromatic $x$-ray focusing system, the variation of incident x-ray energy does not require a change in the configuration of the optics. By carefully choosing a ROI on the outermost 
regions of an electrode, it is possible to utilize $x$-ray fluorescence and scattering measurements from a thin layer of the electrode that can provide valuable information about the solid-electrolyte interface in an operando battery unit.

\section{Integrated Imaging, Modeling, and Analysis of Ultrafast Energy Transport in Nanomaterials}

\author{
2015-149-NO
}

Tom Peterka, Nicola Ferrier, Ross J. Harder, Sven Leyffer, Ian McNulty, Todd Munson, Subramanian Sankaranarayanan, and Haidan Wen

\section{PROJECT DESCRIPTION}

Integrating ultrafast imaging with molecular dynamics (MD) modeling and data analysis and visualization can provide crucial insights for energy research. The temporal behavior of externally stimulated materials beyond equilibrium can lead to breakthroughs in controlling, for example, heat dissipation of next-generation semiconductors, conversion of waste heat into electricity in thermoelectric (TE) materials, and electrochemical processes across liquid-solid interfaces in water purification. These diverse applications all transport energy through phonons (sound waves that carry heat) in a time-evolving crystal lattice. We are researching an integrated approach to predict, image, and analyze phonon dynamics that can be applied to externally stimulated systems. The project consists of three main components: modeling, analysis, and imaging.

\section{MISSION RELEVANCE}

Lattice vibrations in individual nanoparticles affect phase transitions, bond softening/hardening, ferroelectricity, solid/liquid interfaces, heat dissipation, phononic local structure, phase front propagation, and spectrometry. Understanding such phenomena can enable energy applications such as photocatalysis, photonics, thermoelectrics, semiconductor design, groundwater photo-remediation, and heat transfer in battery interfaces, all critical to DOE's mission to design new materials for energy. The novel integration of model-guided imaging and image-guided modeling allows iterative feedback between the two processes that ultimately enables improved utilization of valuable resources at the Advanced Photon Source (APS) and Argonne Leadership Computing Facility. Modeling information such as the sample temperature and image resolution allows researchers to conduct measurements that are scientifically significant. Likewise, the veracity of simulated results, crucial to the outcome of the experiments, is improved by timely analysis of reconstructed experimental images. In the context of the APS upgrade for transformational sciences, this proposal addresses the need for high-speed, high-volume data processing for novel time-resolved imaging; and it aligns with other strategic directions in hard $\mathrm{x}$-ray sciences and advanced computing.

\section{RESULTS AND ACCOMPLISHMENTS}

We executed MD simulations and analyzed the MD models to compute trajectories of phonons, and we used these MD trajectories to generate strain fields. Such data analysis is the "glue" between forward modeling and reverse image reconstruction. We developed advanced graphics processing unit methods for rapid, near-real-time reconstruction of ptychographic diffraction patterns and for feature detection and tracking in materials. We applied these methods to reconstruct three-dimensional (3D) models from coherent diffraction images (CDI). To complement the imaging experiments and gain further insights into the mechanistic sequence of steps that leads to the onset of strain in a gold-ascorbic acid system, we performed MD simulations using the ReaxFF force-field. These simulations allow for dynamic charge transfer and are suitable for modeling chemically reactive systems. The model test system comprises a gold slab with 200 ascorbic acid molecules dispersed in 10,000 water molecules. Our preliminary analysis indicated a total of $\sim 90$ reactions occurring in the adsorption pathway in the short time frame of 20 picoseconds (ps). Many of these are fast, reversible reactions associated with the rapid dissociation and recombination of ascorbic acid. To identify the atomistic mechanism by which strain is induced, we adapted the Molfract analysis tool in LAMMPS (Large-scale Atomic/Molecular Massively Parallel Simulator) to obtain chemical reaction pathways using gold-ascorbic acid as a representative test system. We developed detailed post-processing of the simulation trajectories in order to understand the correlation between the size of the gold nanocrystal and the time scales for fluctuation of ascorbic acid-induced strain in the gold lattice (Figure 1). Meanwhile, at beamline 34-ID-C, we conducted experiments involving the same reaction, the reduction of ascorbic acid on a nanocrystalline gold catalyst. The coherent imaging (Figure 2) was performed on gold nanocrystals ( 200 nanometers) before, during, and after exposure to 1 molar ascorbic acid. The crystals were observed to exhibit distortions of their lattice near edges of the top and bottom oriented facets as a function of time in the acid solution, which agreed with our simulations. 


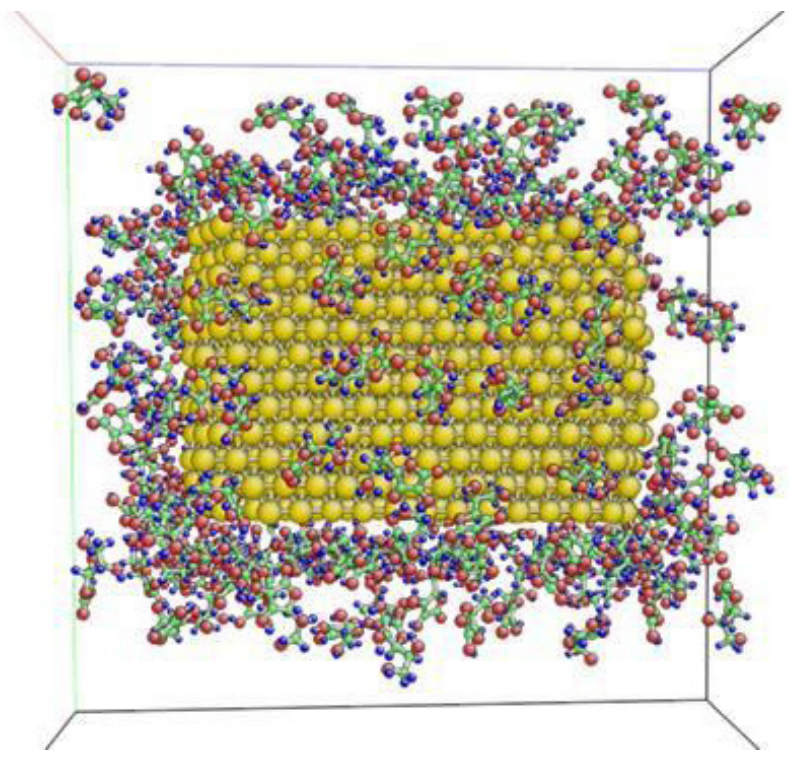

Figure 1. Modeling the atomistic mechanism leading to strain using reactive MD.

\section{PROPOSED FUTURE WORK}

We will continue to model the gold-ascorbic system using ReaxFF and post-process the MD trajectories to derive various structural and dynamical correlation functions (pair correlation functions, structure factors, etc.) to compare/complement the experiments. Because the ascorbic acid reduction is exothermic and might lead to localized heating, we will also carry out non-equilibrium MD simulations to study phonon transport across solid-liquid interfaces such as in the gold-ascorbic acid system. We will subsequently extend the non-equilibrium MD simulations to study phonon transport across solid interfaces such as in TE multilayered materials to complement the imaging experiments. We will carry out coherent imaging of phonon modes excited in a multilayer structure via ultrafast laser pumping. Multilayer structures are particularly promising for TE materials, as the interfaces between different lattices in the layers act as scattering centers for phonons striking them, leading to phononic band gaps in the materials. This behavior is highly desired in TE materials, as the goal is to transfer maximal energy into the electrons and not into the lattice in the form of phonons. The experiments are proposed for Sector 7 of the APS, as this sector is equipped with the necessary laser and $x$-ray diffraction instrumentation.

\section{$200 \mathrm{~nm} \times 320 \mathrm{~nm} \times 195 \mathrm{~nm}$}

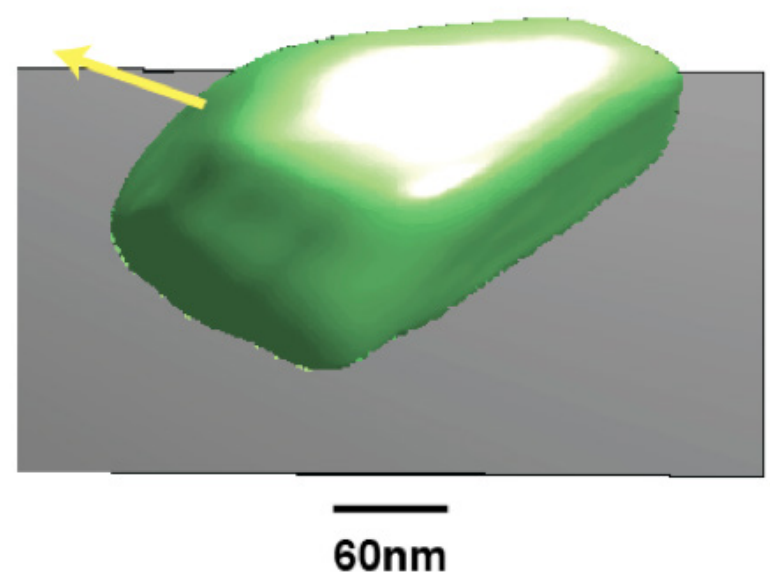

Acid $\Delta \mathrm{t}=20 \mathrm{~m}$

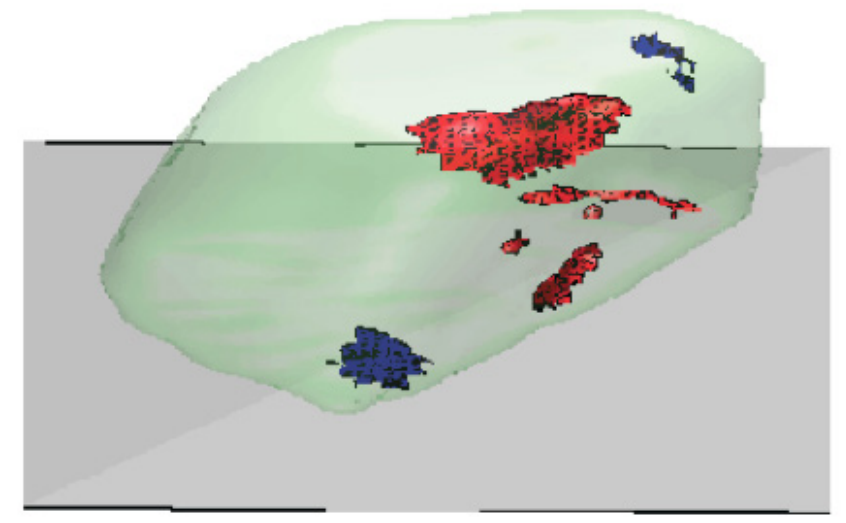

Figure 2. Imaging gold nanoparticles in ascorbic acid using coherent x-rays: Top: Initial crystal surface. Bottom: After $\mathbf{2 0}$ minutes in ascorbic acid, positive and negative strain in red and blue, respectively, at the corners of the crystal match model predictions.

\section{In-situ Co-Analysis of Atomic and Electronic Structural Evolution for Materials Synthesis}

\section{5-152-NO}

\section{Hawoong Hong, Jessica McChesney, and Peter Zapol}

\section{PROJECT DESCRIPTION}

Multi-faceted efforts are essential in materials discovery. The project goal is to combine two synchrotron-based diagnostics to determine atomic structures and electronic states for new materials. The initial step is to employ theoretical/computational techniques to predict new energy-related functional materials and their atomic/ 
electronic structures. New materials and interfaces are then synthesized in the form of thin films. The fabrication is monitored and guided by real-time $x$-ray diffraction utilizing hard x-rays at the Advanced Photon Source (APS). In-situ $x$-ray scattering provides significant advantages over conventional electron diffraction observation during thin-film growth. It can provide picometer-scale positional information and accurate compositional estimation (at the $10^{-2}$ (evel). Electronic structures of the fabricated thin films are to be measured with angle-resolved photoemission spectroscopy (ARPES). The structural and electronic information obtained is to be fed back to the theoretical/ computational efforts to discover new materials. The beamline at APS Sector 33 has a thin-film deposition system on a diffractometer (Figure 1). The high brightness of the APS allows us to follow structural evolution of thin films at the atomic level, with sub-angstrom precision. The deposition system is under ultra-high vacuum (UHV) and can grow various forms of complex functional oxides, topological insulators, superconductors, and materials with other exotic properties. The system can handle several different evaporation sources (most of the Group 4d transition metals: lanthanum, strontium, palladium, lead, bismuth, silver, titanium, tellurium, and selenium, etc.) at the same time and has an ozone source for oxide films. Deposition sources utilizing metal organic chemical vapor phase deposition (MOCVD) are being added. The system is quite flexible, and the base pressure of $1 \times 10^{-10}$ torr can be achieved even after prolonged high-pressure operation for oxide synthesis. High-energy $x$-rays at the APS enable in-situ real-time structural analysis during film synthesis. The capability of oxide film fabrication via molecular beam epitaxy (MBE) is unique among hard $x$-ray synchrotron facilities worldwide. In addition, resonant $x$-ray scattering utilizing tunable $x$-ray energies provides element-specific information. In this project, the ARPES technique will be used for electronic structure measurements. This way, the research team can correlate the structural evolution with electronic states. APS Sector 29 (the intermediate energy x-ray beamline, 250-2500 eV) has a state-of-the-art Scienta R4000 electron analyzer. A helium ultraviolet lamp (photon energies of 21.2 and 41.8 electron volts) will be added to the Scienta chamber. We can utilize this helium lamp/ Scienta system in both on- and off-line modes.

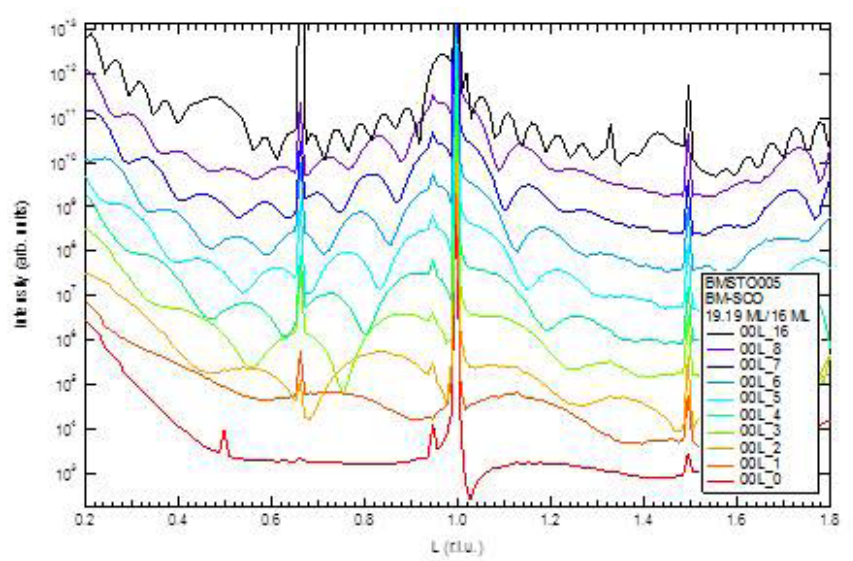

Figure 1. X-ray diffraction [intensity vs. scattering vector (in reciprocal lattice units)]: (OOL) scans during the film deposition of $\mathbf{1 9 . 2}$ monolayers (ML) of brownmillerite-structure-type $\mathrm{SrCoO}_{2.5}$. The film fabrication and measurements were performed at APS Sector 33.

\section{MISSION RELEVANCE}

This project has implications across a number of DOE Basic Energy Sciences programs, including Synthesis and Processing Science, Experimental and Theoretical Condensed Matter Physics, and Solar Photochemistry. Our multimodal in-situ diagnostic techniques will play significant roles in developing solar energy harvesting material and in discovering new material systems with energy band gaps matching the solar spectrum. All of these efforts will require precise atomic structural determination and detection of electronic states. Our co-analysis concept can also speed up the new efforts involving materials beyond perovskite oxides, such as organic intercalation in layered-transition-metal dichalcogenides, organometallic trihalide perovskites, or oxynitrides. Our efforts can address three of the DOE Grand Challenges, namely, \#1-“How do we control materials processes at the level of electrons?"), \#2-“How do we design and perfect atom- and energy-efficient synthesis of revolutionary new forms of matter with tailored properties?”) and \#3-“How do remarkable properties of matter emerge from complex correlations of the atomic or electronic constituents and how can we control these properties?"). Existing thin-film deposition capabilities (e.g., oxide MBE, pulsed laser deposition, atomic layer deposition, and MOCVD) will enable our establishing a strong research base. 


\section{RESULTS AND ACCOMPLISHMENTS}

A UHV sample transportation method between multiple chambers located at three different APS beamlines (in Sectors 33, 4, and 29) has been established. Researchers can perform three different synchrotron-radiation-based measurements without degrading the sample films by keeping them in a UHV "suitcase" (Figure 2). Thin-film samples have been fabricated in the Sector 33 oxide-MBE chamber and characterized with in-situ x-ray diffraction. Then the samples were transferred, under vacuum, to the Sector 4 x-ray photo-electron spectroscopy (XPS) chamber, which has a port for sample transport from the suitcase, and successful XPS measurements were made. The Sector 29 ARPES chamber is also designed to accommodate the UHV suitcase; the ARPES measurement is waiting for the completion of the Sector 29 ARPES branch. These techniques will reveal atomic structures and electronic/chemical states of new materials created in the form of thin films.

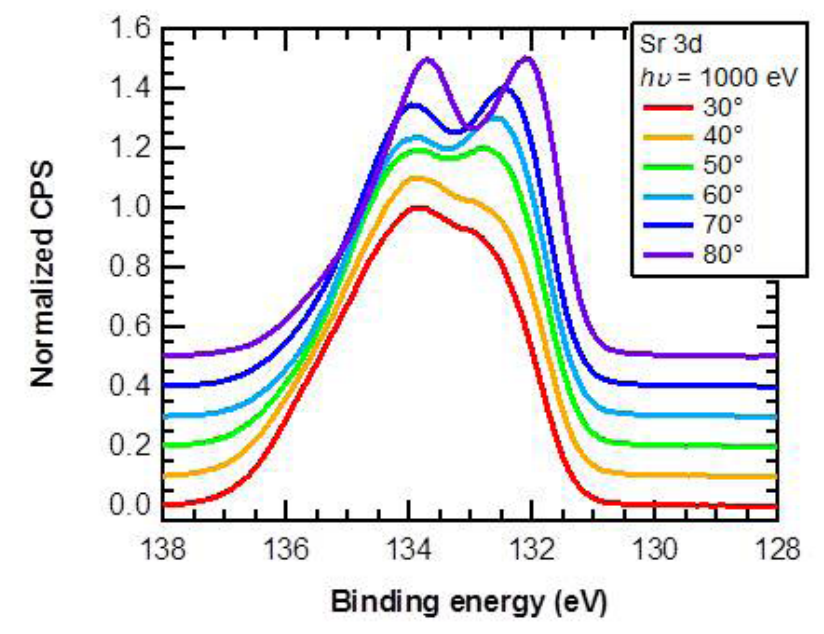

Figure 2. XPS data from the final sample shown in Figure 1. The sample was transferred to APS Sector 4 in the UHV suitcase. The smaller take-off angle confers more surface sensitivity. [CPS=counts per second]

\section{Integrated Imaging to Understand and Advance Photocatalysis}

\author{
2015-154-NO
}

Jeffrey R. Guest, Zhonghou Cai, Maria Chan, Yuzi Liu, lan McNulty, and Tijana Rajh

\section{PROJECT DESCRIPTION}

The photocatalytic conversion of carbon dioxide $\left(\mathrm{CO}_{2}\right)$ to liquid fuels has the dual advantages of carbon recycling for global climate change mitigation and solar energy capture for renewable energy development.
Directly harnessing sunlight to convert $\mathrm{CO}_{2}$ and water to energy-dense products (formic acid, formaldehyde, and methanol) allows easy collection, storage, and transport. However, the obstacles in photocatalytic $\mathrm{CO}_{2}$ reduction are great because the process involves many proton-coupled electron transfer reactions, posing several fundamental challenges in electrochemistry, photochemistry, and semiconductor physics.

Fundamental understanding of active sites in catalytic and photocatalytic materials and their role in charge distribution, charge transport, and efficiency of catalytic reactions trails that of the technological development of materials for energy. The objective of this project is to simultaneously (1) advance the understanding of elementary processes involved in $\mathrm{CO}_{2}$ reduction to liquid fuel and spatial and kinetic control of the active sites and (2) develop integrated imaging and visualization approaches. These aspects are intertwined because understanding these complex processes requires a multimodal approach in which atomic-level understanding of how active sites are involved in electron transfer processes will be gained using local atomic-scale probes coupled with optical methods, while the knowledge of how electronic states are correlated with their mobility and longevity during electrochemical activation or photoactivation will be explored using atomic- and nanometer-scale dynamic structure probes through electron and $\mathrm{x}$-ray techniques. We are developing our integrated imaging approach on promising next-generation materials (e.g., copper(I) oxide $\left[\mathrm{Cu}_{2} \mathrm{O}\right]$ ) that may hold advantages for photocatalytic processes. The major tasks of this project include developing a cross-platform sample holder (for electron microscopy, $\mathrm{x}$-ray fluorescence microscopy, and scanning probe microscopy) for multimodal imaging studies on a single system, and corresponding computational modeling.

\section{MISSION RELEVANCE}

This project supports the DOE mission in use-inspired fundamental science and photocatalysis. This problem presents an opportunity and an ideal testbed to develop the experimental, theoretical and analytic methods for attacking a complex problem that spans orders of magnitude in length and time scales and requires the complementary modalities of various (x-ray, electron, scanning probe, optical) microscopy and spectroscopy platforms. The project bridges Argonne's existing expertise in these areas, leveraging it not only to enhance the control and understanding of photocatalytic $\mathrm{CO}_{2}$ conversion, but also to enable multimodal capabilities for a broader materials design, synthesis, characterization, and modeling program. Beyond fundamental science alone, advancing $\mathrm{CO}_{2}$ conversion technology supports DOE's environmental quality mission as well. 


\section{RESULTS AND ACCOMPLISHMENTS}

For our first cross-platform experiments, we targeted large $\mathrm{Cu}_{2} \mathrm{O}$ nanoparticles with a variety of well-defined facets that we can interrogate in parallel through our various experimental modalities. We successfully synthesized $\mathrm{Cu}_{2} \mathrm{O}$ nanoparticles with well-defined facets of different shapes and surface terminations (surfactants such as sodium dodecyl sulfate or SDS), using multiple techniques. The observed crystal shapes were primarily cubeand truncated concave octahedral-shaped (Figure 1). Figures 2a and 2c show transmission electron microscopy (TEM) images of two different shapes of the $\mathrm{Cu}_{2} \mathrm{O}$ nanocrystals. The primary facets in Figure 2a were found to be (100), with corresponding nanobeam diffraction patterns (Figure 2b). A corresponding atomic model of a (100) facet of $\mathrm{Cu}_{2} \mathrm{O}$ is shown to the left of Figure 2a. In comparison, the primary facets of Figure $2 c$ were found to be (110), with corresponding nanobeam diffraction patterns (Figure 2d). A corresponding atomic model of a (110) facet of $\mathrm{Cu}_{2} \mathrm{O}$ is shown to the left of Figure 2c. This structural information and computational atomic modeling are important for investigating the dependence of $\mathrm{CO}_{2}$ reduction to methanol on facet termination, using in-situ scanning X-ray fluorescence microscopy (SXFM), and for connection with ultrahigh-vacuum (UHV) scanning tunneling microscopy (STM) measurements.

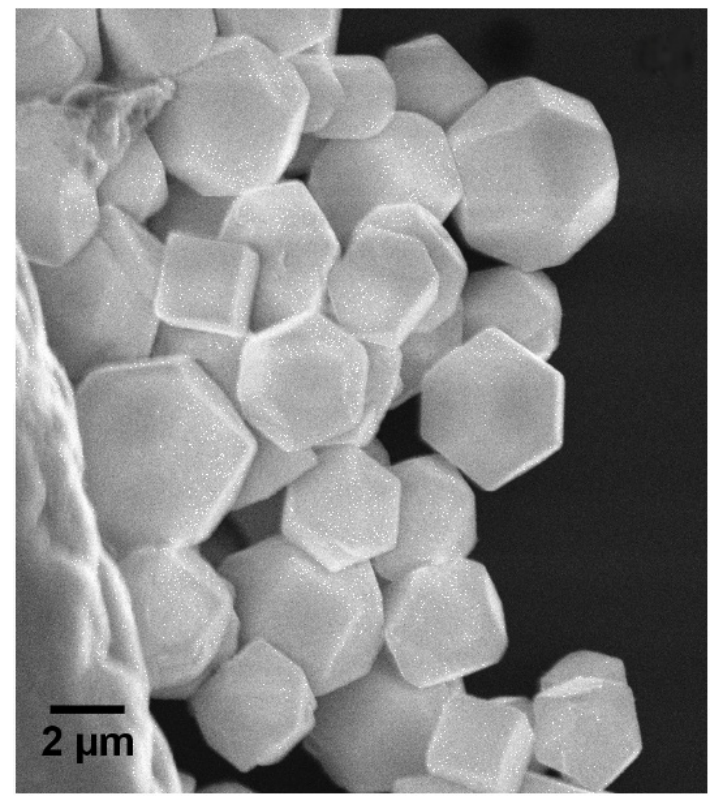

Figure 1. Scanning electron microscopy image of $\mathrm{Cu}_{2} \mathrm{O}$ nanoparticles.

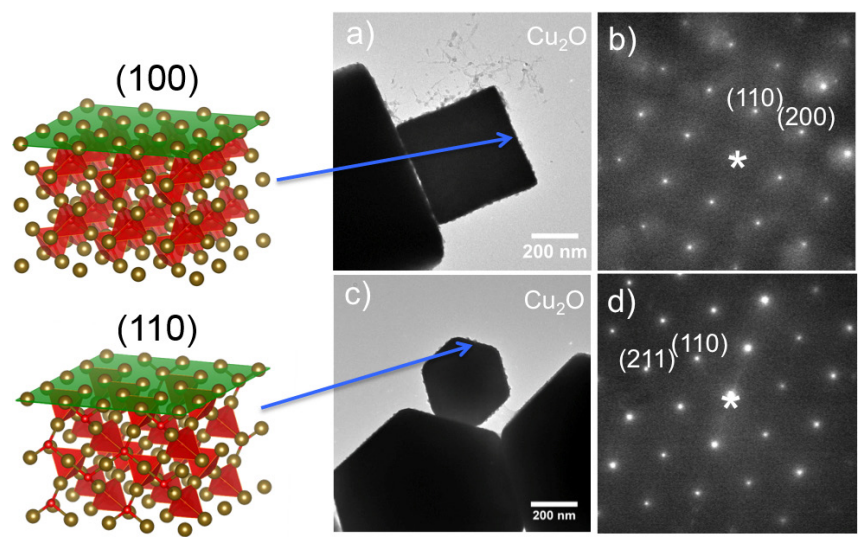

Figure 2. (a) TEM image of cube- shaped $\mathrm{Cu}_{2} \mathrm{O}$; (b) nanobeam diffraction pattern of (a); (c) TEM image of truncated concave octahedral $\mathrm{Cu}_{2} \mathrm{O}$; (d) nanobeam diffraction pattern of (c).

The structure of defects in as-synthesized $\mathrm{Cu}_{2} \mathrm{O}$ nanoparticles with SDS ligands was studied using electron paramagnetic resonance (EPR). It was found that SDS, while stabilizing the growth of nanoparticles by complexing $\mathrm{Cu}$ atoms on the nanoparticle surface, also leaches $\mathrm{Cu}^{2+}$ ions from nanoparticles into the solution. Both for particles with and without SDS ligands, EPR measurements showed a distinct change in the spectrum associated with $\mathrm{Cu}$ atoms after exposing the sample to $\mathrm{CO}_{2}$, suggesting the importance of surface-bound $\mathrm{Cu}(\mathrm{II})$ in binding $\mathrm{CO}_{2}$ to $\mathrm{Cu}_{2} \mathrm{O}$.

In order to interpret the experimental data and interface it with atomistic models, we performed first-principles density functional theory modeling of $\mathrm{Cu}_{2} \mathrm{O}$. The goal of this work is to simulate images and spectroscopic signals that would be observed on pristine surfaces, surfaces with adsorbates, surfaces with molecular modifications, and surfaces coated with adlayer(s). We investigated the (100) and (110) surface facets of $\mathrm{Cu}_{2} \mathrm{O}$, including off-stoichiometric and defective surfaces, and compared their stability under different chemical environments. We also calculated the adsorption energies and configurations of $\mathrm{CO}_{2}$ and carbon monoxide (CO) adsorbates, and determined the change in oxidation state of surface $\mathrm{Cu}$ atoms due to adsorption.

\section{PROPOSED FUTURE WORK}

In order to extend these studies to SXFM and UHV STM measurements on the same sample region or nanostructure, we have been developing a cross-platform sample holder; this device will allow us to bring these complementary modalities to bear on the (ideally) identical system. Furthermore, to explore photocatalysis in operando, we have designed the holder to perform these measurements under optical illumination and (in the case of TEM and SXFM) under gas flow. Future 
experimental work will center on exploring the same particles under SXFM and with UHV STM, and utilizing analytical techniques to develop correlations between these modalities to discern the underlying physics associated with the photocatalytic behavior of these systems. Future computational work involves modeling x-ray fluorescence spectra, electron energy loss spectra, and STM signals on the same underlying system undergoing surface chemical reactions.

\section{Coherent X-ray Studies of Materials Synthesis and Dynamics}

\section{5-167-NO}

\section{G. Brian Stephenson}

\section{PROJECT DESCRIPTION}

New tools are on the horizon that can revolutionize the science of materials synthesis. Advances in accelerator technology now being implemented will produce thousand-fold increases in the brightness of hard x-ray sources, enabling in-situ coherent x-ray studies of synthesis mechanisms down to the atomic scale with the needed sub-nanosecond time resolution. The ultimate payoff and success of major DOE investments in these new sources depends upon developing and applying these novel coherent $x$-ray imaging and dynamics techniques to critical problems, such as materials synthesis. In parallel, orders-of-magnitude increases in computing power are enabling ab-initio simulations, not just of ground-state materials structure and properties, but also of the competing chemical reactions and materials kinetics that occur during synthesis. Both coherent x-ray techniques and atomic-scale modeling enable us to see beyond the average behavior of a fluctuating system and reveal the microscopic arrangements, correlations, and dynamics.

This project focuses on understanding materials synthesis and processing mechanisms by developing new techniques to observe and model the atomic-scale mechanisms; for example, using more complex space/time correlations that go beyond standard pair correlations. Synthesis processes and materials studied (e.g., growth of nitrides by chemical vapor deposition) will be chosen to illustrate fundamental materials synthesis issues (e.g., control of surface morphology, formation of defects, creation of metastable phases, and impurity incorporation) that are broadly applicable and relevant to critical energy applications. The combination of in-situ observation of structure and dynamics during synthesis with advanced computational studies will provide the insight needed to re-write the textbooks on materials synthesis and processing.

Coherent $x$-ray techniques provide qualitatively new information regarding the structure and dynamics of the defects and disorder that mediate crystal growth and phase transformations. Coherent techniques are sensitive to the exact arrangement of nanoscale structure, rather than just spatially averaged quantities (such as defect density). This project will focus on advancing the capabilities of x-ray photon correlation spectroscopy (XPCS), because its space and time resolutions are well suited to studies of synthesis dynamics.

The standard XPCS method determines the time spectrum of fluctuations in the speckle intensity at each location $q$ in reciprocal space, which gives the dynamics of the pair correlations as a function of $q$. This is very powerful, revealing the dynamics of rearrangements in equilibrium systems that are not observable in the diffuse scattering measured with an incoherent beam. However, it should be possible to go well beyond current XPCS analysis. In principle, analysis of intensity correlations between two or more q's in the speckle pattern obtained with a coherent beam can reveal spatial correlations of a higher order than pair correlations, which are inaccessible in conventional incoherent $x$-ray analysis. The analysis of these higher-order correlations is still in its infancy. Furthermore, non-equilibrium processes, such as phase transformations, can have non-steady behavior that is best characterized by two-time correlation functions. In addition, recent work indicates that correlations between two q's and two times will allow separation of diffusive rearrangements and deformations.

Measurement and analysis of these complex space/time correlations during materials synthesis will be explored in this project. Initial experiments will be carried out at the Advanced Photon Source (APS) on a new instrument being commissioned at beamline 12-ID-D. Experiments will be designed to demonstrate what will be possible with much higher coherent flux in the future, that is, with the APS Upgrade.

Multi-length- and time-scale simulations of atomic-scale dynamics during synthesis will be developed to guide the design and analysis of the experimental studies. These will be key to interpreting the new observations and give breakthroughs in scientific understanding. These models will allow prediction of the complex space/time atomic-scale correlations that will be observed for the first time during synthesis using coherent $x$-ray methods. 


\section{MISSION RELEVANCE}

A critical mission of the National Laboratories is to make breakthroughs in discovering the new materials and processes needed to address the global challenges in energy, environment, health, and security. Not only do we need to understand the proper arrangement of atoms in materials and nanostructures that gives the desired functionality, but also to learn how to synthesize and stabilize these arrangements. Rather than a trial-and-error approach, developing the science underlying materials synthesis promises the most extensive and sustainable progress. This project will further the DOE Basic Energy Sciences mission to design, discover, and synthesize new materials through atomic-scale control. It addresses the Grand Challenge, "How do we design and perfect atom- and energy-efficient synthesis of revolutionary new forms of matter with tailored properties?" The results will contribute to the scientific cases for high-brightness $x$-ray facilities such as the APS Upgrade.

\section{RESULTS AND ACCOMPLISHMENTS}

During FY 2015, we commissioned a new diffractometer capable of coherent $x$-ray measurements, as well as a new system for in-situ materials growth, at APS beamline 12-ID-D. The coherence properties of beamline 12-ID-D were characterized in December 2014. We found that the coherent flux could be improved by more than an order of magnitude by removing unnecessary unpolished beryllium $(\mathrm{Be})$ windows and/ or replacing them with coherence-preserving windows. These modifications were completed by September 2015, in collaboration with APS. Initial surface scattering tests were made with the new growth chamber in April and July 2015. The chamber and diffractometer performed as desired. Simulations of gallium nitride (GaN) growth began in February using 3D Kinetic Monte Carlo methods. This approach allows simulations using the correct crystal structure geometry of atomic sites, which is critical for understanding the atomic-scale growth mechanisms on different crystallographic orientations, for example, step-edge attachment during island growth.

Initial results of this work appear, without individual attribution, in "Early Science at the Upgraded Advanced Photon Source," section 2.3.C. "Understanding Structure \& Dynamics During Materials Synthesis." Find a link to the report at https://www1.aps.anl.gov/APS-Upgrade.

\section{PROPOSED FUTURE WORK}

In FY 2016, we will demonstrate new coherent x-ray methods combining experiment and simulation to characterize atomic-scale mechanisms of materials synthesis. Simulation results now being collected will be analyzed in advance to predict the most interesting parameters to vary in experiments (e.g., temperature, growth rate, and crystal orientation) to observe effects in two-time and higher-order correlation functions.

\section{The Computational Design of New Functional Materials from Complex Transition Metal Oxides}

\section{5-168-NO}

\section{Hyowon Park}

\section{PROJECT DESCRIPTION}

We will pursue the theoretical study of novel electronic and structural phases of complex oxide materials in which the strong correlation of electrons plays an important role. Strongly correlated oxides exhibit novel electronic behaviors intimately coupled to their spin, charge, orbital, and lattice degrees of freedom. We will adopt the state-of-the-art ab-initio method-that is, density functional theory plus dynamical mean field theory (DFT+DMFT). The main objectives are (1) to calculate various spectroscopic quantities of complex oxides in both bulk and heterostructured forms and (2) to develop a code for computing interatomic forces within DFT+DMFT in order to investigate the structural properties and energetics of oxides. The output of DFT+DMFT calculations will be directly compared with experimental measurements, including photoemission, x-ray scattering, x-ray absorption, and neutron scattering spectra. Ultimately, our project can lead to the computational design of functional oxide materials or devices with a desired functionality.

\section{MISSION RELEVANCE}

This project is relevant to the mission of DOE Basic Energy Sciences. It addresses the fundamental understanding of the relationship among charge, spin, orbital, and lattice degrees of freedom. Ultimately, the knowledge of electronic correlations among different degrees of freedom will provide the ability to control and design new functional materials. For example, novel electronic phases and chemical reactions occurring at the interfaces or surfaces can be used for new electronic devices or energy materials (such as batteries). Our project is also relevant to programs at other federal agencies, including the National Science Foundation and the Defense Advanced Research Projects Agency. 


\section{RESULTS AND ACCOMPLISHMENTS}

During FY 2015, we studied electronic and structural properties of strained oxide thin films and superlattices by using DFT+DMFT. The lanthanum-nickel-oxide, $\mathrm{LaNiO}_{3}$ is an interesting test case since its electronic properties and correlation effects are strongly associated with the structural lattice distortions. The lattice distortions include the deformation, tilting, and rotations of $\mathrm{NiO}_{6}$ octahedra under strains. First, we quantified the electronic correlation in films of $\mathrm{LaNiO}_{3}$ by computing the momentum resolved spectral function and the mass enhancement of a correlated electron compared to a bare electron in a strained thin film and compared those to angle-resolved photoemission spectroscopy (ARPES) experimental data. The electron mass enhancement due to the interaction with other electrons is a key measure of correlation strength. We found that DFT+DMFT can accurately describe the correlation strength of strained $\mathrm{LaNiO}_{3}$ films compared to ARPES experiment, producing a quantitatively consistent band bottom energy and mass enhancement of $\mathrm{LaNiO}_{3}$. We also found that the treatment of realistic $\mathrm{NiO}_{6}$ octahedral rotations under strain is crucial for producing the quantitatively accurate correlations in transition metal oxides.

Second, we quantified orbital polarization of strained $\mathrm{LaNiO}_{3} / \mathrm{LaAlO}_{3}$ superlattices consisting of four layers of nominally metallic $\mathrm{NiO}_{2}$ and four layers of insulating $\mathrm{AlO}_{2}$ separated by LaO layers. We showed that the overall dependence of orbital polarization on strain is qualitatively consistent with recent $\mathrm{x}$-ray absorption and resonant reflectometry measurements. The strain effect results in the $\mathrm{NiO}_{6}$ octrahedral deformation, and the orbital polarization is directly affected due to the structural change. Moreover, we discovered that there are interesting differences, depending on the sign of strain. Tensile strain produces orbital polarization similar to that calculated for the strained bulk and observed in experiment, while compressive strain produces a larger dependence of orbital polarization on Ni layers, and even the inner Ni layer exhibits different polarization from the bulk. The quantum confinement effect was found to be as important as the strain effect and stronger for tensile strain.

\section{PROPOSED FUTURE WORK}

For future work, we will focus on an efficient implementation of computational codes for calculating atomic forces within DFT+DMFT and apply it to the study of structural and electronic properties of strongly correlated oxides. The atomic force calculation will be implemented based on an efficient plane-wave DFT code; by doing so, the study of rather large supercells (including the interface of heterostructures or the surface of thin films) will also be possible. The stringent test of the DFT+DMFT force calculation will be the study of structural properties on the surface or the interface of strained $\mathrm{LaNiO}_{3}$ thin films. The atomic structure of a $\mathrm{LaNiO}_{3}$ thin film is extremely difficult to determine experimentally, and the electronic structure is closely related to the atomic structure. We will also study the structural and electronic properties of $\mathrm{LaCoO}_{3}$, in which the origin of the spin-state transition and its relation to the structural change are not yet clearly understood.

\section{Magnetic Phases in Highly Oxidized, Low-Dimensional Oxides}

\section{5-175-NO}

\section{John F. Mitchell and Nathaniel Schreiber}

\section{PROJECT DESCRIPTION}

We are carrying out a discovery crystal synthesis project that leverages the high oxygen fugacity achievable in a recently installed high-pressure floating zone (FZ) image furnace to stabilize new materials. We will follow a control strategy of using this high partial pressure of oxygen $\left(\mathrm{pO}_{2}\right)$ to grow stoichiometric phases that are known at ambient pressure to either develop anion vacancies or decompose upon heating as a result of a high targeted metal oxidation state. Such high oxidation state materials are an open area of opportunity because of the difficulty of growing specimens, but they offer views on the role of $\mathrm{O} 2 p$ hole states in the electronic structure and the variation of magnetic and orbital ordering.

The material system in question, $\mathrm{La}_{2-2 x} \mathrm{Sr}_{1+2 x} \mathrm{Mn}_{2} \mathrm{O}_{7}$, is one that we have previously characterized using polycrystalline specimens, but the lack of single-crystal specimens has prevented a complete and definitive study of the unique intrinsic magnetic phenomena. In particular, a region of the phase diagram near $x=0.7$ anomalously shows no magnetic long-range order. We have developed a framework for understanding this based on short-range orbital order "seeding" short-range magnetic order that would be largely invisible to the neutron powder diffraction studies to date. The objective of the present work is to create these heretofore "ungrowable" crystals and explore magnetism and phase competition unique to the low-dimensional electronic structure using a combination of $x$-ray and neutron scattering tools. 


\section{MISSION RELEVANCE}

This is a fundamental science project. The work directly supports DOE's Office of Basic Energy Sciences missions in discovery science, particularly grand challenges in control of matter at the level of electrons, understanding and harnessing correlated systems, and synthesis of new functional compounds. The beneficiaries will be the wide base of condensed matter scientists studying transition metal oxides, particularly those who have worked on correlated electron manganites (a very large fraction of the community).

\section{RESULTS AND ACCOMPLISHMENTS}

The project started in mid-July 2015. The main activity during this time was to familiarize with single-crystal growth using the floating-zone method. In this regard, by mid-August, we grew a crystal of $\mathrm{LaCoO}_{3}$, which we considered a "simple" example but suitable for use. The specimen has been sent to a collaborator at Karlsruhe Institute of Technology for phonon measurements. The remainder of the fiscal year was focused on preparing starting materials for the growth of $\mathrm{La}_{2-2 x} \mathrm{Sr}_{1+2 x} \mathrm{Mn}_{2} \mathrm{O}_{7}$ and performing some preliminary experiments.

We found quickly that extreme pressures of oxygen were actually counterproductive, leading to many second phases and ultimately none of the target compound. We thus began a systematic investigation at lower $\mathrm{pO}_{2}$ to optimize phase stability prior to crystal growth. As a result of this set of experiments, we have found that the desired phase crystallizes when $\mathrm{pO}_{2}$ is in the range 6 to 10 bar. With small specimens, we were able to show uniform magnetization, and preliminary single crystal $x$-ray diffraction on small pieces showed clean, well-defined structure with the expected lattice structure. We are encouraged that growth of suitably large specimens is feasible. We also submitted a proposal to the Spallation Neutron Source (SNS) to do the magnetic studies, anticipating that we will indeed succeed with these specimens. Reviews of the proposal are pending at this writing.

\section{PROPOSED FUTURE WORK}

During FY 2016, we will work to optimize the crystal growth. Our target is to reach a 250-500 milligram specimen, which is the size needed by the SNS instrument for magnetic diffuse scattering. When we achieve this, we will prepare other samples at slightly higher and lower doping levels to bracket the region of missing long-range magnetic order.
In conjunction with the magnetic diffraction studies, we intend to propose $\mathrm{x}$-ray experiments at the Advanced Photon Source designed to explore the charge channel, particularly looking for distinct signs of either long- or short-range order of the mixed-valent $\mathrm{Mn}^{3+}-\mathrm{Mn}^{4+}$ ions. This kind of phenomenon is common in related manganese oxides and has been observed in the target material and lower values of the Sr excess dopant level, $x$.

\section{Towards lonotronics: First- Principles Strategies for Coupling Electronic and Ionic Properties in Complex Oxides}

\section{5-178-NO}

James Rondinelli

\section{PROJECT DESCRIPTION}

This project aims to deliver control strategies over sine materia (the absence of matter), e.g., vacant cation or anion sites, in a designed fashion to establish a materials platform for an ionic-based electronic device. Anion-deficient $\mathrm{ABO}_{3-\delta}$ oxides based on the perovskite structure are the materials platform for investigation; they could be the ideal channel materials for an ionics-based field-effect transistor (FET). Although they have been studied previously experimentally, the main focus has been on exploring polymorphic crystallography based on redox chemistry rather than phase stability or electronic properties. The polymorphic flexibility of these materials is the focus of this project, which will be used to evaluate the suitability for FETs.

\section{MISSION RELEVANCE}

The project is relevant to the scientific discovery and innovation mission of DOE. The ability to manipulate ion concentration in an ultrathin oxide film via electric fields represents an exciting new operational platform for solid-state devices because of the sensitive coupling between stoichiometry and functional properties. The main effort of the project will be to develop an understanding of suitable pathways to manipulating the electronic structure of complex oxides through engineered static and dynamic control of the anion structure. The incorporation of novel materials into advanced nanoelectronics has implications for the development of superior computing technology and reduced power consumption while forging pathways to new state variables beyond temperature or voltage, which would represent a "game-changing" advance in computing. 


\section{RESULTS AND ACCOMPLISHMENTS}

In FY 2015, the focus was on understanding the polymorphic stability of $\mathrm{Sr}_{2} \mathrm{Fe}_{2} \mathrm{O}_{5}$ (i.e., the $\mathrm{ABO}_{3-\delta}$ with $\delta=0.5$ ) in bulk and under epitaxial strain. The structure consists of alternating layers of $\mathrm{FeO}_{6}$ octahedra and $\mathrm{FeO}_{4}$ tetrahedra, viz., ordered arrangements of oxygen vacancies. Electronic structure calculations based on density functional theory (DFT) were performed to disentangle the complex interactions in $\mathrm{Sr}_{2} \mathrm{Fe}_{2} \mathrm{O}_{5}$, relating the stability of the equilibrium (strain-free) and thin-film structures to both previously identified and new descriptors. The simulations indicate that cation size and intralayer separation of the tetrahedral chains provide key contributions to the preferred ground state. Interestingly, the bulk ground-state structure is retained in the ferrates over a range of strain values; however, a change in the orientation of the tetrahedral chains (i.e., a perpendicular orientation of the vacancies relative to the substrate) is stabilized in the compressive region. The structural stability under strain is largely governed by maximizing the intraplane separation of the dipoles generated from rotations of the $\mathrm{FeO}_{4}$ tetrahedra. Understanding the change in atomic structure is important because it directs the electronic band gap in these materials. Thus the atomistic understanding established by the DFT calculations provides key insight that may be used to design the electronic properties of thin-film materials in thin-film $\mathrm{Sr}_{2} \mathrm{Fe}_{2} \mathrm{O}_{5}$ and related compounds.

\section{PROPOSED FUTURE WORK}

In FY 2016, the focus will be on formulating a quantitative theory of anion-deficient structural (polymorphic) stability based on the previous results, which will be required to reversibly move ions and induce electronic transitions. This will be achieved by (i) enumerating symmetries and structure types available to the tiling of two polyhedral units followed by (ii) performing ab-initio DFT calculations on $\mathrm{SrFeO}_{3-\delta}$ and $\mathrm{SrMnO}_{3-\delta}$, which are anticipated to result from the tiling of the anion-deficient polyhedral motifs.

\section{Development of a Cryogenic Correlative Confocal Light Microscope ( $\left.C^{3} L M\right)$ for Integrated Imaging}

\author{
2015-185-NO \\ Amanda Petford Long and Chris Jacobsen
}

\section{PROJECT DESCRIPTION}

This project seeks to develop a cryogenic confocal light microscope $\left(C^{3} L M\right)$ for correlative studies of soft and hard materials under vacuum. The microscope will be configured with an in-vacuum microscope objective (that is maintained at room temperature) and an out-of-vacuum confocal microscope head. A cryo-robot will be used for sample exchange. Once developed, the microscope will be applied to studies of soft and biological materials and will provide much-needed microscopic capabilities for the cryo-analysis of these materials. By using the same cryogenic sample handling system as is used for scanning $\mathrm{x}$-ray ptychography and fluorescence microscopy at the Advanced Photon Source at Argonne, it will allow us to combine visible light fluorescence and emission microscopy with x-ray microscopy studies of the same specimen. Visible light fluorescence provides information about molecules with chemically specific binding affinities, while $x$-ray ptychography provides structural views down to a resolution of 20 nanometers, and x-ray fluorescence offers a means to image trace metal concentrations independent of specific chemical binding affinities.

\section{MISSION RELEVANCE}

This instrument is relevant to DOE's mission in science by enabling studies of soft materials, quantum devices, and biological and environmental science specimens. The microscope will contribute to Argonne's emerging efforts in brain connectomics (an area to be supported by DOE's Offices of Biology and Environmental Research [BER], Basic Energy Sciences [BES], and Advanced Scientific Computing Research [ASCR]) and to a bioimaging program aimed at exploring microbial communities in soil (an area supported by BER). The instrument will also contribute to the APS upgrade project (supported by BES) by permitting exploration of the effects of $x$-ray irradiation on samples under various cryogenic conditions. 


\section{RESULTS AND ACCOMPLISHMENTS}

The project was awarded in September 2015. During September, we developed a design concept and evaluated commercial components that could be used in the design. We then ordered and received the confocal microscope and in-vacuum micropositioning system, along with some associated hardware. The confocal microscope is expected to provide a transverse spatial resolution of better than 0.3 micrometers, with sensitive detection of visible light fluorescence from a selected depth plane with a resolution of better than 3 micrometers. It should allow acquisition of a three-dimensional image stack in less than 10 minutes.

\section{PROPOSED FUTURE WORK}

In FY 2016, we wish first to obtain the components for the cryo-robot design from Zeiss, and then develop the system using this design. Following full development of the $C^{3} L M$ instrument, we will use it for initial studies in two areas. One will involve looking at synapses in hippocampal neuron cultures; the goal will be to understand the relationship between total zinc as measured by x-ray fluorescence and visible light confocal fluorescence images of zinc bound to molecular labels (there is reason to believe that tight molecular binding of zinc to enzymes such as superoxide dismutase can make zinc invisible to molecular fluorophores). Another use will involve carrying out basic studies of radiation damage in polymers such as those used in organic photovoltaics; by seeing the degree to which visible fluorescence dyes are deactivated or even removed (via mass loss) from these polymers as a function of radiation dose at cryogenic temperatures, we can better understand the ultimate resolution that we can reach in x-ray imaging studies of these materials and how that relates to the range of exciton transport at the boundaries of immiscible polymers. 


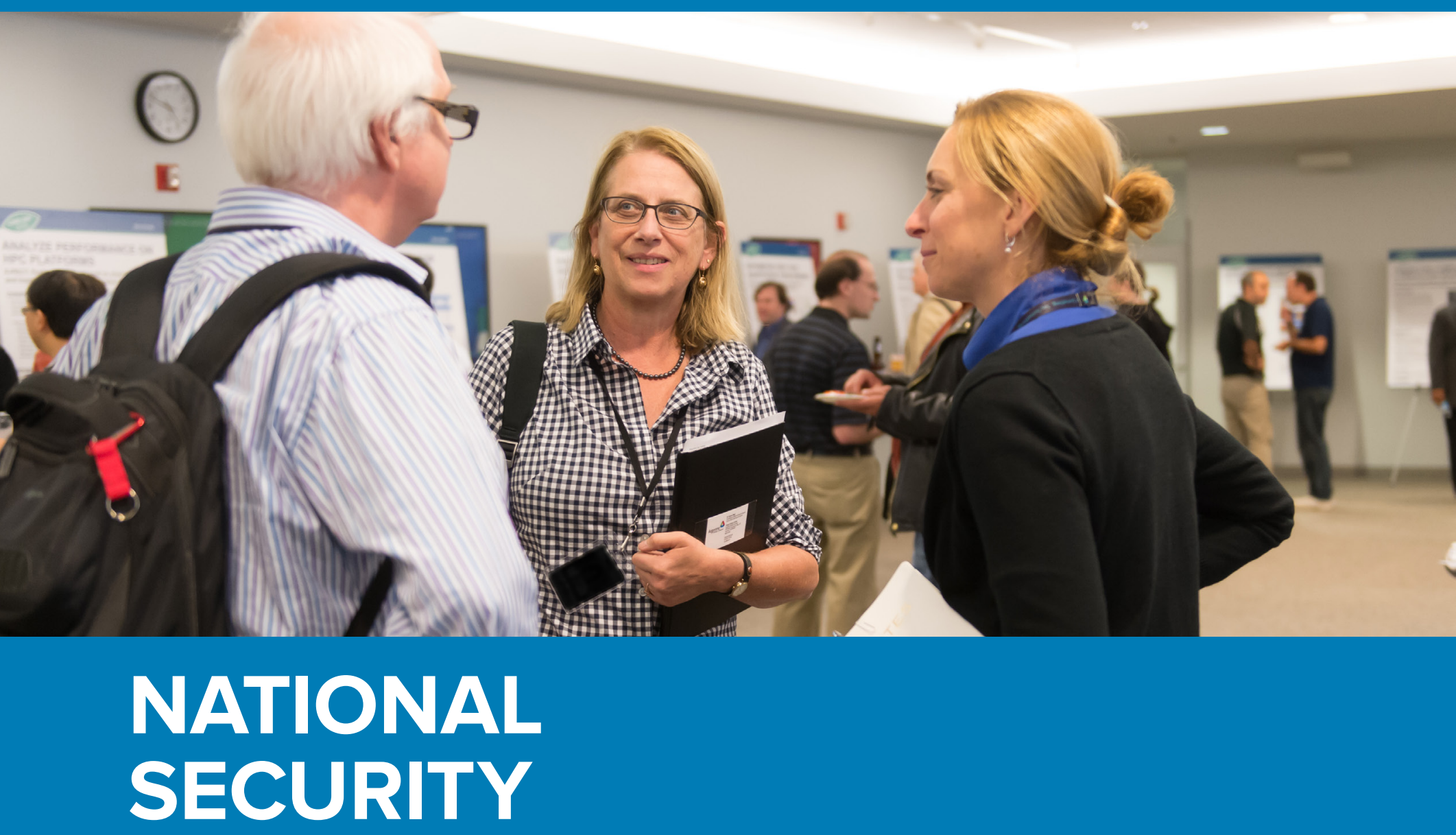




\section{Unconventional Signatures for Characterizing Culture Conditions}

\section{3-171-R2}

Daniel Schabacker, Lucy Stols, and Rosemarie Wilton

\section{PROJECT DESCRIPTION}

Microbial forensics is the scientific discipline dedicated to analyzing evidence from a bioterrorist attack, bio-crime, or inadvertent release of a microorganism or toxin for the purpose of identifying the perpetrator (i.e., for attribution). An important facet of microbial forensics is the analysis of variations (whether cellular or molecular) among strains and among preparation methods, with the goal of inferring the origin of a particular isolate or forensic sample. At present, microbial forensics is dominated by analysis of DNA. While DNA analysis provides a wealth of information, identifying the methods used to culture and prepare the biological threat agent (BTA)-information that can be critical for forensic analysis-requires a different data set. That information is often resident in the matrix associated with the BTA and in the molecular composition (in particular, the proteome) of the BTA itself. Therefore, while DNA analysis is a critical tool, it is not adequate as the sole forensic methodology.

Delivering an agent requires preparing it to remain effective when outside of its optimal growing conditions. Exposure to environmental stresses, such as temperature, ultraviolet radiation, and drying, can reduce the agent's activity. Some pathogens, like the anthrax bacterium, can encapsulate themselves into a hardy, long-lasting spore not easily susceptible to those conditions. Other agents require further processing that minimizes damage and allows the BTA to retain its activity when dispersed.

The inherent need to stabilize and protect BTAs from inactivation during storage, transport, and dissemination results in a state of suspended animation or metabolic inactivity. While in this state, the molecular signatures indicative of growth conditions and preparation methods are preserved, thus providing an ideal opportunity for forensic analysis.

In this project, we are developing a novel forensic methodology that is especially suited to BTA attribution. Our approach quantitatively analyzes the molecular composition (mainly the proteome) of BTAs, which varies with culture conditions and preparation methods. As a result, our methodology can provide detailed information regarding the methods used to culture, purify, and store the BTA. Our approach consists of four stages: $\square$ Lysis of the BTA;

$\square$ Automated, high-resolution fractionation of the extract by chromatofocusing and reversed-phase highperformance liquid chromatography;

$\square$ Adsorption of the protein(s) in each fraction to a spot on a protein "chip" by using a conventional robotic system; and

$\square$ Interrogation of the types and levels of proteins on the chip by applying nonspecific protein dyes, specific antibodies, and lectins (to detect glycoproteins), followed by quantitation of binding using a commercially available chip-reader. The resulting information-rich data set can be rapidly and quantitatively analyzed by using existing software.

Our method requires a minimum amount of sample, can be implemented by using off-the-shelf technology, and is relatively insensitive to environmental contamination.

\section{MISSION RELEVANCE}

This project supports the national security mission of DOE to reduce the global danger from weapons of mass destruction by enhancing the capability to attribute a BTA to individuals or organizations. This approach goes beyond current microbial forensics methods that identify the BTA strain; it provides information on how a BTA was cultured and prepared for dissemination, providing insight into the operator's expertise and resources and enhancing attribution. The Interagency Microbial Forensics Advisory Board, led by the Federal Bureau of Investigation (FBI) and White House Office of Science and Technology Policy, has identified our technical approach as a viable solution for identifying culture conditions and preparation methods used in BTA production. Our approach is specific, relatively insensitive to interferents, high-throughput, and inexpensive, and it provides a depth of characterization unavailable with any other known method.

In addition, high-resolution characterization of protein expression resulting from varying culture conditions and sample preparation methods would be valuable in cancer research, where identifying these changes would facilitate more precise biomarker discovery by removing uninformative changes due to variations in laboratory technique. Stakeholders who may be sponsors and customers include the FBI Laboratory; Defense Intelligence Agency, Chemical, Biological, Radiological, Nuclear Explosives Technical Collection Office; National Bioforensic Analysis Center; U.S. Department of Homeland Security Science and Technology Directorate; and the Defense Threat Reduction Agency. 


\section{RESULTS AND ACCOMPLISHMENTS}

In FY 2013, we confirmed that spent media from E. coli had adequate protein complexity in both autoclaved and untreated forms for use as a discriminator. Results were analyzed by using the newly developed ProFAST (PROtein Fraction Array Statistics Tool) software (software copyright number ANL-SF-13-015).

In FY 2014, we focused on expanding growth conditions to obtain the data sets required to confirm the ability to discern growth conditions. Broadening the number of media types, particularly by matrixing media selection such that a media component (e.g., agar) or condition (e.g., growth at $27^{\circ} \mathrm{C}$ ) was shared by at least two media types, provided more resolution power with more conditions for the same number of experimental samples than a series of binary comparisons would provide.

ProFAST was significantly expanded and optimized to include Quantile Normalization, Cluster, Heatmap, Principal Component Analysis, M2 Statistics, and Prediction Analysis for Microarrays in R analysis. Additional statistical tools such as analysis of variance tools (ANOVA), Artificial Neural Networks, Bayesian Networks, and Support Vector Machine (SVM), were evaluated. These statistical tools were used to classify data, by using whole-proteome signatures (all fractions) or a subset of fractions.

Four growth conditions were analyzed by using ANOVA (Adjusted Bonferroni correction, 10,000 permutations, $\mathrm{pVal}<0.05)$ to identify fractions significant by media type. We identified 79 out of 960 fractions as significant by media type. These 79 fractions are displayed on the $x$-axis in Figure 1. The various growth media are seen on the $y$-axis near the right side of the figure. Looking closely, one can see that all four growth media were successfully clustered using the 79 prioritized fractions.

An SVM model was built and successfully classified unknown growth media by using the 79 data points chosen by ANOVA as significant. The SVM model was validated by introducing random noise to the data and re-checking SVM prediction accuracy. The model was determined to be highly resistant to the addition of noise and able to assign a confidence to an unknown classification.

In FY 2015, we focused on the development of a JAVA version of ProFAST, expansion of media compositions, validation of the classification methodology, and validation of fraction prioritization.

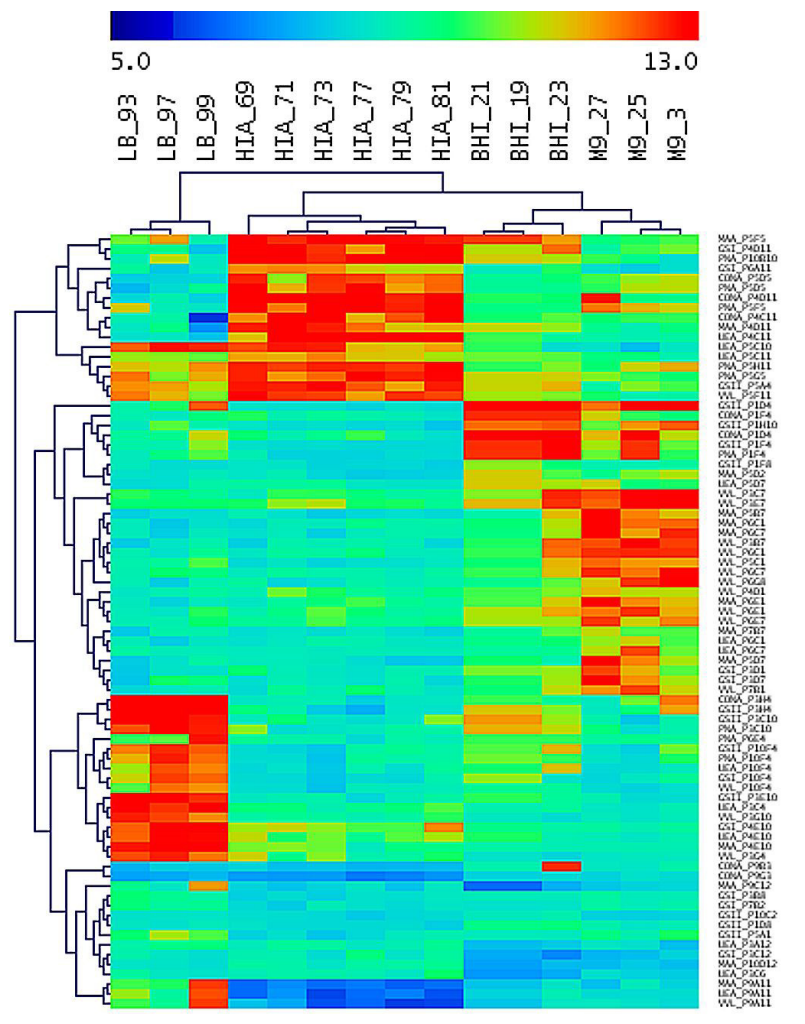

Figure 1. Hierarchical Cluster: Cluster (average linkage, Euclidian distance) for 79 fractions. Data were normalized and log2-transformed. Signal is identified as significant by media according to ANOVA. Media compositions (3-LB, 6-HIA, 3-BHI, 3-M9) are on the y-axis; ANOVA prioritized fractions are on the $\mathrm{x}$-axis.

Technical milestones achieved include the following:

$\square$ ProFAST software. To address the limitations of the Matlab version, ProFAST was ported to a Java implementation to provide a cross-platform (Windows, OSX, and Linux) and open source application that is freely available to researchers under an open source software license. The Java version:

- Has a much smaller file size (Matlab is several hundred MB, Java is $\sim 500 \mathrm{~KB})$.

- Runs faster - complete loading, processing, and result generation for several thousand GenePix Results (GPR) files typically take less than a minute on a standard desktop computer.

- Is user-friendly - The Project tree can be saved and reloaded so that entire GPR hierarchy, class, and plate settings are preserved for further analysis.

- Is flexible - The tree structure is editable and may be arranged dynamically, which allows the user to add additional GPRs or delete existing files in the tree.

- Is efficient - Results files can be exported to Excel and easily processed in other programs or continuing analysis in ProFAST. 
$\square$ Additional signatures were added to the reference library. Complete analysis of growth conditions provided by the National Biodefense Analysis and Countermeasures Center (NBACC) and Northwestern University were added to the database.

$\square$ Validated classification and fraction prioritization methodology. Statistical tools were evaluated with the expanded signature database for the ability to discern components of growth conditions while providing a statistical certainty. Using a Leave-One-Out validation scheme, SVM predicted incubation time and media source with high accuracy (F-scores 1.0 and 0.97, respectively). Predictions were highly robust to the addition of random noise, with strong accuracy, even with the addition of noise equal to five times the standard deviation of array feature signal intensities (average $\mathrm{F}$-scores $\mathbf{0 . 9 4}$ for incubation time and 0.88 for media source). Even after the addition of random noise equal to nine times the feature standard deviation, the SVM performed better than chance ( $p$-value $1.2 \times 10^{-6}$ for incubation time and $p$-value $2.8 \times 10^{-5}$ for media source).

These results constitute a powerful proof-of-principle, outlining a combined proteomics and computational approach for the accurate prediction of fermentation conditions of biological agents from proteins.

\section{Ratiometric Semiconductor Nanocrystal-Based Sensors for Threat Reduction Applications}

\section{3-173-R2}

Richard D. Schaller, Elena A. Rozhkova, Daniel Schabacker, Elena V. Shevchenko, and Rosemarie Wilton

\section{PROJECT DESCRIPTION}

This project focuses on the development of low-cost, easy-to-use ratiometric fluorescence resonance energy transfer (FRET) -based sensors with applications in chem-bio-rad-nuclear (CBRN) threat reduction scenarios. Our objective has been to produce a sensor capable of rapidly and sensitively detecting the presence of such species as botulism toxin or uranium ions with high specificity. Our approach entails causing association or dissociation of a FRET donor (D) and acceptor (A), each of which is highly fluorescent but not significantly overlapping spectrally at emission wavelengths, upon exposure to analyte. Because FRET efficiency is highly sensitive to $D$-to-A distance, the ratio of $D$ and $A$ spectrally resolved emission will change sensitively with small amounts of analyte binding. Relative $D$ and $A$ emission maxima intensities then can be correlated with lab-based assays to directly read out analyte absence/presence and a calibrated analyte concentration. Semiconductor quantum dot (QD) FRET donors are targeted due to optical tunability and high optical stability.

\section{MISSION RELEVANCE}

Our project is relevant to the DOE mission in national security. Accurate, reliable sensors are crucial to a wide array of national security efforts. Ideally, one would like a non-expert to be capable of sensitively, reliably, and persistently detecting a wide assortment of threat agents ranging from biological to nuclear in the field. In addition to facility inspectors, such ability would bolster the effectiveness of individuals in the field and overall anti-terrorism efforts. While lab-based methods can sensitively detect numerous threat agents, most require specialist operators or otherwise lack fieldability. Our efforts target a sensor platform that is field compatible, simple to operate and interpret, compatible with sensitivity to multiple threat agents, and robust against minor system degradation. The technology pursued has the potential to yield compact, hand-held sensors that rapidly facilitate the identification and quantification of threat agents.

\section{RESULTS AND ACCOMPLISHMENTS}

In FY 2015, we demonstrated a sensitive ratiometric FRET scheme (see Figure 1) that implemented highly photostable semiconductor QD energy donors and chromophore conjugation to compact, single-chain variable antibody fragments (scFvs) to yield a fast, fieldable, easy-to-use homogeneous assay sensor for botulinum neurotoxin (BoNT) with a 20- to 40-picomoles/ liter (pM/L) detection limit (which is a non-lab-based method record), toxin quantification over three orders of magnitude (adjustable dynamic range), sensitivity in the presence of frequently confounding interferents, and sensing times as fast as 5 minutes (see Figure 2). This result was a culmination of developments that we began working on in FY 2013 and continued through FY 2014 that involved development and expression of scFv components, modes of functionalization of semiconductor nanocrystals for biofunctionalization, and protocols for performing assays. Through a combination of mutations, we achieved stabilized scFv denaturation temperatures of more than 60 degrees Celsius $\left({ }^{\circ} \mathrm{C}\right)$, which further bolsters fieldability and increases the shelf life of isolated scFv components. We further adapted the assay into a microarray format that offers persistent monitoring, reuse, 
and spatially resolved multiplexing. The approach could be adapted with furthur work so as to yield sensitivity to a range of other biological threat agents in a fast, easy-to-use multiplex sensor.
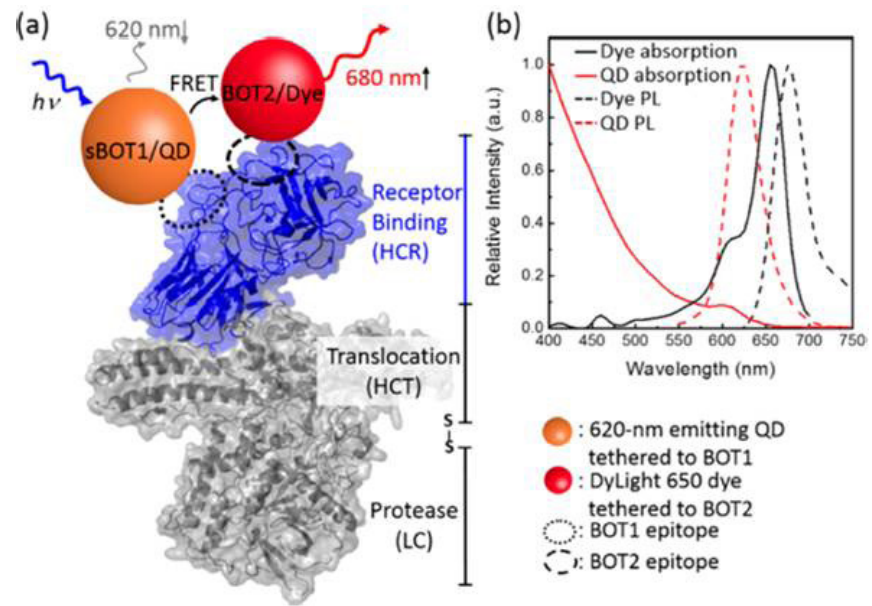

Wavelength $(\mathrm{nm})$

Figure 1. Ratiometric FRET-based BoNT sensor components. (a) Ribbon/ space-fill diagram of BoNT/A1 (Protein Data Bank entry no. 3BTA). Dashed lines show approximate HCR antigen binding locations for labeled scFvs sBOT1 and BOT2. (b) Optical properties of the QD donor and Dylight 650 acceptor.

(a)
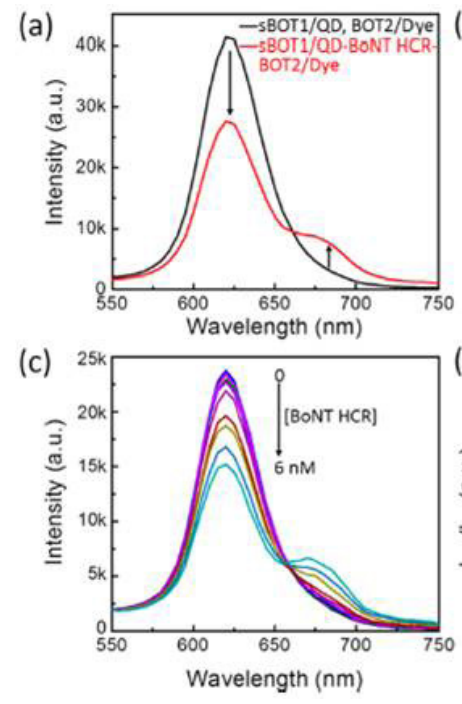

Figure 2. (a) Photoluminescence (PL) spectra of a solution containing both SBOT1/QD and BOT2/dye before (black line) and after (red line) addition of BoNT HCR. (b) Ratio of acceptor/donor PL intensity as a function of time following BoNT HCR addition. (c) PL spectra vs BoNT HCR relay assay sensitivity with IDye/IQD plotted in (d), as a function of BoNT HCR. The LOD of the FRET-based BoNT sensing platform was found to be $31 \mathrm{pM}$.

Key developments this year included (1) reduction of limit-of-detection (LOD) for BoNT to just 20 to 40 pM and (2) demonstration of detection of intact holotoxin, including (3) in the presence of interferents. At the beginning of the year, our homogeneous assay achieved $1.2 \mathrm{nM}$, which was important in that our homogeneous assay format was still far more rapid than competing technologies. However, lower LOD is desirable because of the high toxicity of BoNT (nanogram/kilogram [ng/kg] lethality in animals). By altering the concentrations of the chromophore-conjugated scFvs, we were able to reduce our LOD by a factor of 50 and still retain the rapid readout. Further, for safety reasons, our detection work of BoNT to this point had made use of the isolated heavy chain region (HCR) of the toxin, which lacks toxicity as this portion of the protein lacks the enzymatic activity of the light chain (LC). Use of the isolated HCR was critical to the successful development of BOT1 and BOT2, since lack of toxicity permitted examination in BSL1 (biosafety level 1) facilities.

However, because scFvs were screened (in year two) to attach to the isolated HCR with high selectivity, it was possible that the two developed scFvs might attach to regions of the mutated $\mathrm{HCR}$ that are not present on the wild-type holotoxin, or that the structure of the HCR might itself differ sufficiently that BOT1 and BOT2 would not attach with high affinity to intact toxin. As such, we sought approval this year to perform homogeneous assays on intact holotoxin containing the HCR and LC. Appropriate approvals were obtained, and we moved our experiments into a BSL2 facility for intact toxin and toxin-complex assays. These measurements were successfully performed.

Similarly, we examined the intact toxin and complex in the presence of interferents successfully. Our developed sensing scheme is insensitive to non-specific binding with interferents, because close proximity of the donor and acceptor chromophores is required for ratiometric FRET signals to be impacted. Non-specific binding offers little chance for our two scFv components to bind with high proximity, as they do in the presence of the HCR of BoNT.

\section{Uranium and Plutonium Detection by Plasmonic Graphene-Based Nanosensors}

\section{3-178-R2 \\ Michael Kaminski, Carol Mertz, Leonidas Ocola, and Anirudha V. Sumant}

\section{PROJECT DESCRIPTION}

Determining the presence of uranium $(U)$ and/or plutonium (Pu) after a release of nuclear material is an important task. Unfortunately, for a release in the field, that represents an acute gap in detection capabilities. Low-energy gamma-ray spectroscopy can be used to assay clean samples emitting little background radiation; however, a significant $\mathrm{x}$-ray and gamma-ray background renders 
such techniques useless. This work seeks to develop a new hand-held analytical instrument that identifies $U$ and $\mathrm{Pu}$ isotopes in a liquid and possibly a solid state. The basis for the device is the graphene nanosensor, which, according to theoretical calculations, is able to detect elemental $U$ and Pu by characteristic changes in the plasmonic properties of graphene sheets. The size of the graphene sensor sheets is in the micrometer range $(\sim 1 \times 1 \mu \mathrm{m})$, thus enabling the sensor device to be entirely hand-held and compatible with minimal sample size, while requiring minimal battery power.

Our project comprises two primary approaches. The first is to conduct computational studies of the basic interaction of graphene with actinide ions in solutions. A basic interaction model will allow us to predict graphene structures and solution chemistry that maximize the sensitivity of the device and minimize potential interferences. The second is the experimental fabrication and testing of graphene for integration into an experimental device.

The milestones are summarized below and are consistent with our original seven-goal plan.

$\square$ Goal 3 (FY 2015): Continue to develop more complex simulations for real molecular systems.

$\square$ Goal 6 (FY 2015): Optimize the sensing mechanism and device integration.

$\square$ Goal 7 (FY 2015): Perform preliminary experiments on sensor response to $\mathrm{U}$ and $\mathrm{Pu}$ using liquid samples.

\section{MISSION RELEVANCE}

Our project addresses DOE's mission in nuclear security. The need for hand-held devices for the determination of $U$ and Pu was identified by the Program Needs Joint Working Group of the American Association for the Advancement of Science with world-leading co-authors from Lawrence Livermore and Los Alamos National Laboratories. Specifically, they described a priority need for a portable system to provide rapid indication of the presence of $U$ and $\mathrm{Pu}$ in post-detonation debris and an accurate and precise measurement of isotopic ratios. The International Atomic Energy Agency has expressed similar interest in developing a portable system to screen samples as part of its nonproliferation and verification activities. Intelligence agencies have expressed an interest in obtaining small, field-robust units for remote or stand-off detection of undeclared materials. The U.S. Environmental Protection Agency has expressed similar interest in a system that meets its demands for analysis of radionuclides in waters. Our sensor may be able to meet these needs.

\section{RESULTS AND ACCOMPLISHMENTS}

In FY 2013, we tested the performance of graphene for electrical detection of a model f-element europium (III) nitrate. Graphene fabricated by using chemical vapor deposition on copper foil was transferred to a silicon wafer by using various methods, and two-electrode configurations were microfabricated on top of graphene, with different geometries and distances between electrodes. One configuration corresponded to a graphene ribbon positioned between two metal electrodes, and one corresponded to a gapped graphene. We then measured the graphene electrical response to europium (III) nitrate solution. We reported a change in the graphene device conductance of up to an order of magnitude upon exposure to $\sim 0.1 \mathrm{mM}$ europium nitrate water solution. We continued with fabrication and characterization studies in FY 2014 and tested the electrical response of working sensors (different configurations) under a variety of solution conditions.

In FY 2015, we sought to improve the fabrication process to produce nanoribbons with reproducible voltage-current response curves so that these could be tested in controlled experiments with various salt solutions. It appears that we are introducing defects into the graphene during the fabrication process. We think the descumming process is the source of the defects. We spent the majority of the effort trying to develop an improved method to descum the various electrode designs. Many designs and their voltage-current response curves were developed, representing numerous fabrication trials. The defect rate remained high (25-75\%). We achieved Goal 3, development of more sophisticated modeling capabilities, by working with Prof. Jorge Seminario of Texas A\&M University. We failed to achieve Goal 6, optimization of the sensing mechanism, because of problems associated with defects. A further investment of resources would be needed to fully explore the ultimate feasibility of the detection schemes envisioned here. 


\section{Crime on the Urban Edge: Simulating the Interface between Transnational and Local Crime}

\author{
2014-194-R1 \\ Pamela Sydelko, Ignacio Martinez-Moyano, \\ and Michael North
}

\section{PROJECT DESCRIPTION}

Ultimately, this research will provide a systems framework that promotes better understanding of systems complexity. It will also support multi-stakeholder intervention planning that takes into account that for every intervention action, there are adaptive behavior changes in the system that emerge from new intervention pathways.

\section{MISSION RELEVANCE}

This project is relevant to DOE's research in energy planning, policy, and the economy. We are developing an overarching systemic intervention framework that includes systemic problem structuring methods (PSMs) for use on complex "wicked" problems. These PSMs contribute soft systems capabilities that can be used by multiple stakeholders to define and systemically map and visualize problems in any domain, and would be especially pertinent to energy planning and energy security applications. Under the systemic intervention framework, we are designing soft systems to work side by side with hard systems approaches such as system dynamics modeling or agent-based modeling. The hard system model we developed during this project is a genetic algorithm for automatically generating system dynamics models that represent potential system adaptations. This systemic intervention framework can be applied to any class of complex problems where initial interventions (e.g., a purposeful action to create change) create second- and third-order effects. Many energy-planning problems come from this class because consumer behavior, economic behavior, environmental impacts, and technological changes can all be highly complex and adaptive.

\section{RESULTS AND ACCOMPLISHMENTS}

Work began on this project in late fiscal year (FY) 2014. The FY 2015 effort focused on the development of a systemic intervention approach to support the use of multiple methods in the design of interagency meta-organizational structures for addressing wicked problems. Specifically, the viable system model (VSM), systemic PSMs, and boundary critique will continue to be used in a mixed-method approach to structure wicked problems collaboratively and to design an interagency meta-organizational structure capable of addressing them:

$\square$ Systemic Problem Structuring Methods: Transnational organized crime (TOC) and domestic urban crime gangs (DUGs) are wicked problems with an extraordinary amount of interdependency; they also have many stakeholders who have varying opinions and perceptions about the problems. Intervention requires bringing these stakeholders together to collaboratively and holistically define and structure the wicked problem. The choice of systemic PSM is related to its ability to provide for robust participatory problem formulation. The use of outside "experts" to define wicked problems could alienate multiple agency stakeholders who have their own strongly held values and perceptions about the problem. For the CUE project, we conducted PSMs with three individual stakeholders in FY 2014. An example of one of those system maps is shown in Figure 1.

$\square$ Anticipatory Systems: An anticipatory systems model called AnticipatoRy Complex Adaptive Network Extrapolation (ARCANE) may be introduced into the systemic intervention. ARCANE is a genetic algorithm system for automatically generating system dynamics models that represent potential system adaptations.

For ARCANE, a genetic algorithm was formulated to automatically generate system dynamics models that differ in both their parameters and their equations while maintaining dimensional consistency. Two demonstration scenarios, one small and one large, were created to test the genetic algorithm formulation's ability to generate new system dynamics models. Statistically significant results were found in both scenarios. Our method for using genetic algorithms to automatically generate candidate anticipatory system dynamics models can find dimensionally consistent models that produce better fitness function values than baseline models, providing, in turn, a new way to use systems dynamics modeling to explore possible future configurations of complex adaptive networks. 


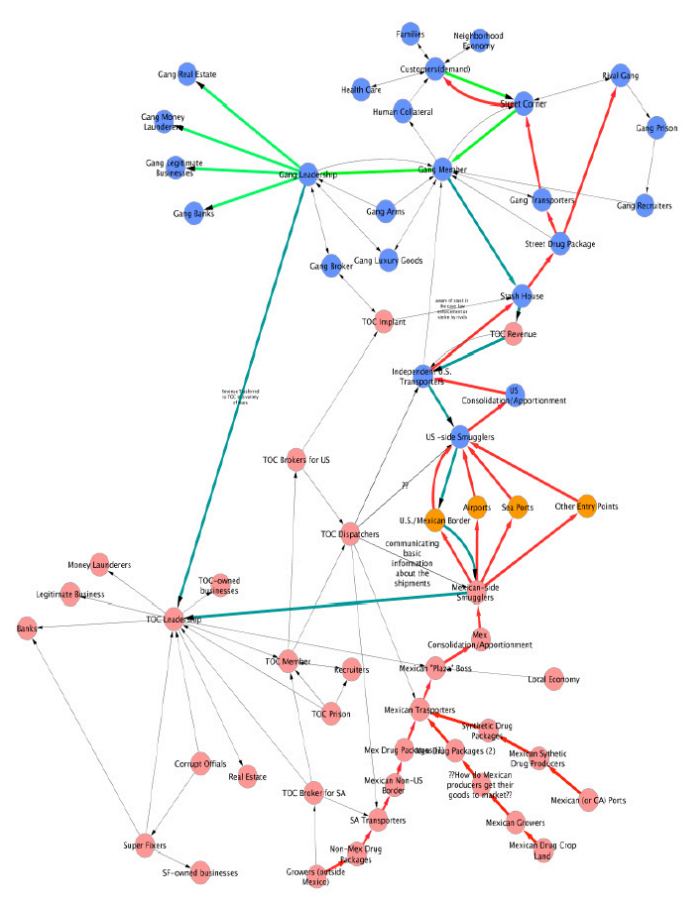

Figure 1. Cytoscape System Map for Interview \#3.

$\square$ Three-dimensional (3D) Printing for Systems Visualization: Understanding and communicating the structure of complex abstractions is known to be extremely difficult. One solution is to use people's natural ability to work manually with 3D structures in the physical world: 3D printing makes this practical today.

For our project, we use a multistep, free, and open-source software-focused process to produce 3D printouts of complex systems and networks. The workflow is shown in Figure 2. A custom script was created to help automate the workflow. First, the system data are created using the free and open-source Cytoscape computational biology environment. Second, the system data are rendered in 3D using a special 3D version of Cytoscape. Third, the system is stored in an extensible markup language $(\mathrm{XML})$ version of a graph modeling language (GML) format called XGML. Fourth, the $X G M L$ is compiled into the solid computer-aided design (SCAD) file format. The SCAD file is then converted into the stereolithography or standard tessellation language (STL) file format via the free and open-source OpenSCAD application. The STL file can then be loaded into common 3D printer drivers to determine the required temporary overhang supports and then print. Temporary overhang supports are needed for long horizontal spans without intermediate support. They are manually removed once printing is complete.
The last panel in Figure 2 shows a 3D printout of the two-dimensional (2D) influence diagram in the first panel. Early discussions with a variety of stakeholders indicate that the use of 3D printouts dramatically improves their ability to understand the networks being studied, at least for networks small enough to print. The use of 3D printouts also seems to substantially increase their level of engagement.

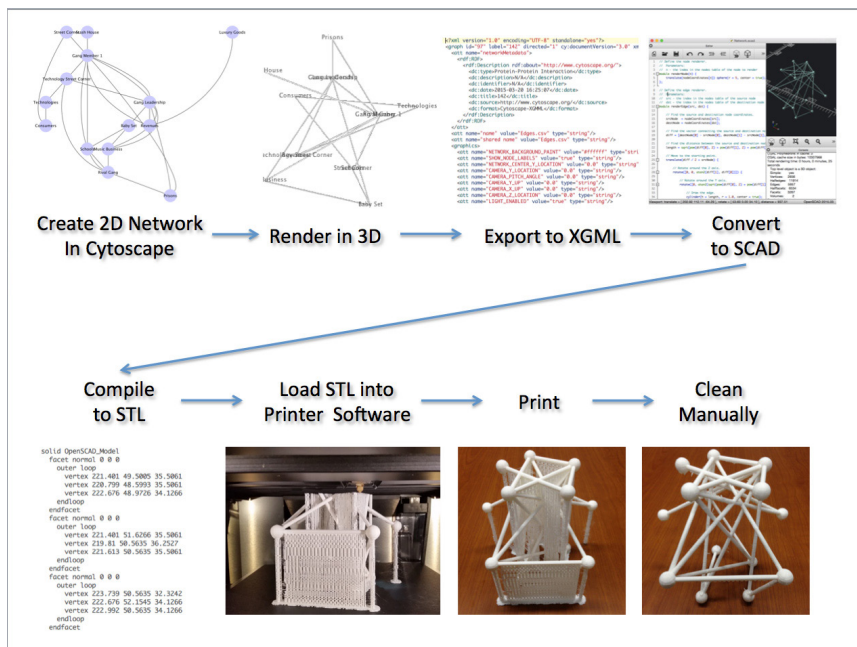

Figure 2. 3D Printing Workflow for System Visualization.

\section{PROPOSED FUTURE WORK}

Our work in FY 2016 will entail the following:

$\square$ Data Collection: Collection of data through individual stakeholder problem structuring interviews (described above) will continue, with an expected eight to ten more interviews being scheduled. The system maps generated from these interviews will be entered into Cytoscape. The whole collection of stakeholder system maps will then be compiled into a single common map. Advanced systems visualization techniques will be further developed to understand and explore complex system data.

$\square$ Group Systemic Problem Structuring: We will hold a facilitated group problem structuring meeting to finalize the common view of TOC and DUG systems. Facilitating collaborative problem structuring will no doubt reveal conflicts and power struggles between agencies.

Clearly, wicked problems are challenging to delineate. If the values of all stakeholders are not accounted for, the interagency collaboration will suffer. Boundary critique offers a method for exploring issues of marginalization and unveiling any value-laden ramifications of making boundary decisions. Boundary critique will be a key method used at the group problem structuring meeting. 
$\square$ ARCANE Implementation: ARCANE will be ported to a free and open-source big data platform such as the Apache Hadoop stack with Apache Spark. A full version will be developed using the common view system map and information from interviews.

$\square$ Multi-agency Meta-organization Design/ARCANE Demonstration: A second group stakeholder meeting will be held in late summer to facilitate design of a meta-organization for interagency collaboration toward this wicked problem using the VSM. The VSM is used as the primary framework for designing a viable meta-organizational structure. Governments typically organize task forces to "solve" problems in a reductionist and linear manner (which may sometimes work for complicated or tame problems). However, designing the interagency structure to deal with highly adaptive wicked problems requires a strong coupling between the organization and its environment (the wicked problem). The VSM is a powerful instrument for diagnosing and designing flexible and adaptable organizations by weaving the environment with operations so that real-time adjustments can be made. The VSM offers a better approach for interagency meta-organizational design than other, more hierarchical, approaches because it supports autonomous agencies while still providing a structure for collaboration, shared resources, and flow of information.

This meeting will also include the demonstration of ARCANE. Stakeholders will be able to test various intervention strategies and explore the adaptation outcomes ARCANE produces.

$\square$ Evaluation: We will develop and implement an evaluation process for evaluating (1) problem structuring methods, (2) the VSM intervention, and (3) the ARCANE anticipatory modeling. 


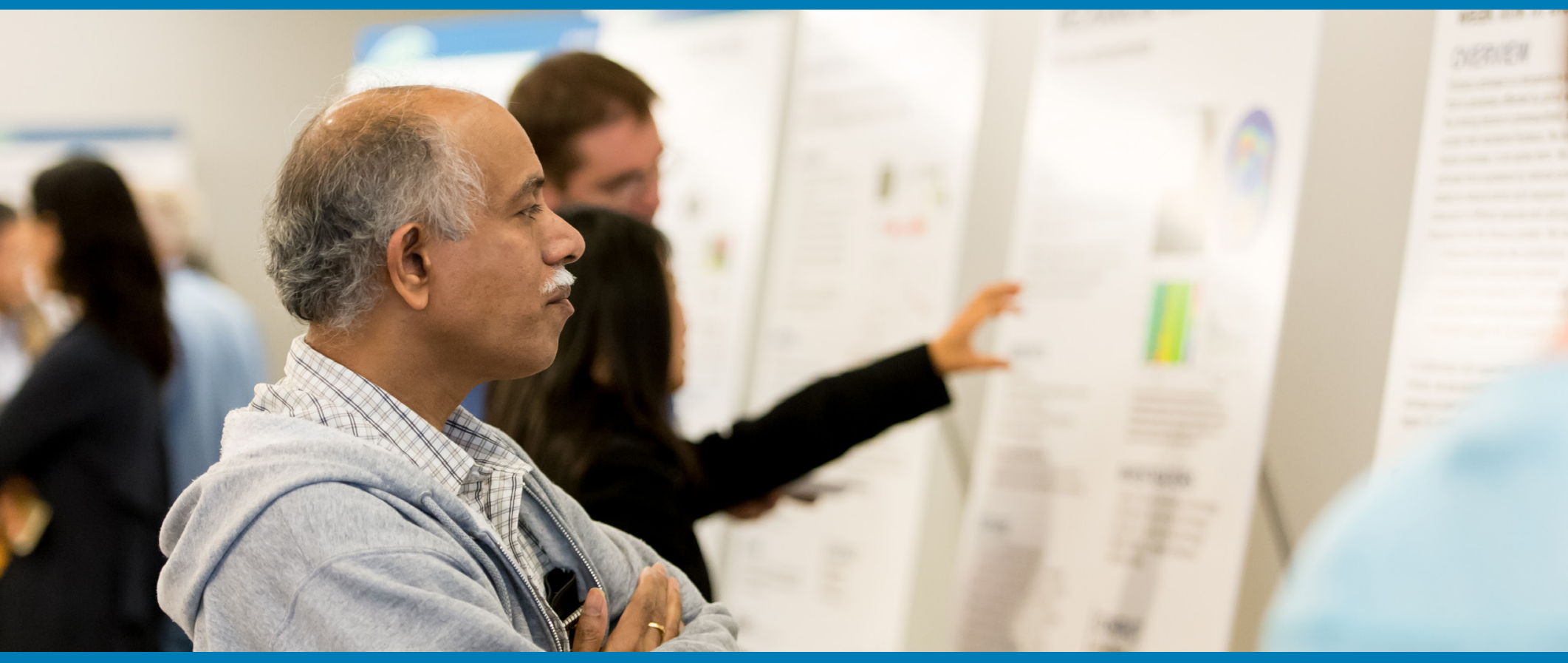

\section{NUCLEAR ENERGY AND SECURITY}


Nanolaminate Materials for Extreme Environments: A Demonstration of Argonne Capabilities for Design, Synthesis, and Accelerated Testing of Radiation-Tolerant Nuclear Engineering Materials

\author{
2013-152-R2
}

Abdellatif Yacout, Jonathan D. Almer, Meimei Li,
Michael J. Pellin, and Marius Stan

PROJECT DESCRIPTION

Argonne has a long history of proposing, developing, and testing new materials for nuclear reactors. By combining the capabilities of high-energy MeV/nucleon (mega-electron-volt/nucleon) heavy-ion irradiation at the Argonne Tandem Linear Accelerator System (ATLAS), high-energy $x$-ray analysis at the Advanced Photon Source (APS), and multiscale modeling and simulations through the Argonne Leadership Computing Facility (ALCF), this work will make available a new nuclear materials research capability. This capability can accelerate materials development for the extreme nuclear reactor environment, thereby enabling the testing of materials at radiation doses of hundreds of displacements per atom and reducing the required testing time from years (in the reactor) to days (in this study). While this approach is not expected to be a replacement for in-reactor neutron irradiations done in support of reactor licensing activities, it can shorten the development cycle of new or improved nuclear materials by limiting in-reactor testing to those candidates that show the most promise.

This project will make a "first" demonstration of the power of this concept by examining the stability of nuclear materials, including a class of nanomaterialsnanolaminates-under simulated extreme conditions, to provide insight into the damage mechanisms of neutrons and the roles of fission fragments at high doses.

\section{MISSION RELEVANCE}

This project is relevant to DOE's missions in science and energy. Within the scope of this work is the development of an understanding of the characteristics of nanolaminates and the characteristics of other materials that promote material stability in extreme environments, such as those found in a nuclear reactor. This understanding will be achieved through the synergistic use of Argonne's user facilities that will demonstrate the capabilities of these facilities to rapidly accelerate materials screening for extreme environments. The demonstration will begin to develop a case for an in-situ irradiation effects capability through the use of similar tools (the proposed extreme materials [XMAT] beamline at the APS). It will also support the case for the DOE's Office of Nuclear Energy's (NE's) investment in the proposed XMAT beamline by demonstrating its technical viability.

\section{RESULTS AND ACCOMPLISHMENTS}

In FY 2013 and FY 2014, we achieved significant progress in using Argonne's facilities to demonstrate the objectives of this project. In addition, innovations were achieved in materials testing and characterization in a high-temperature steam environment, thermal analysis modeling, nanolaminate synthesis and characterization, and lower-scale modeling and simulations. Activities at Argonne's facilities included MeV/nucleon heavy-ion irradiations (at ATLAS) of nuclear cladding materials (including nanolaminate-coated materials) and nuclear fuel materials, as well as the characterization of those materials at the APS and at the Intermediate Voltage Electron Microscope (IVEM). Sample characterizations were performed at APS beamlines 1, 10, and 34 using various characterization techniques (extended x-ray absorption fine structure [EXAFS], small- and wide-angle x-ray scattering [SAXS, WAXS], and diffraction tomography). Modeling and simulation activities included density functional theory (DFT) simulation of cerium (IV) oxide $\left(\mathrm{CeO}_{2}\right)$, which is a promising material as a non-radioactive surrogate for studying nuclear fuel containing plutonium (IV) oxide $\left(\mathrm{PuO}_{2}\right)$.

In FY 2014, more activities were performed at ATLAS, APS, and IVEM facilities. We irradiated different fuel and structural materials using high-energy ions (xenon $[\mathrm{Xe}]$ and iron [Fe] ions) at ATLAS. Very high radiation damage and high burn-up levels were achieved in the structural and fuel materials, respectively. The irradiated materials were then studied at the APS. In addition to the synchrotron techniques used in FY 2013, more advanced techniques were utilized in FY 2014. These included in-situ micro-tomography at APS Sector 2 and nano-tomography at APS Sector 32.

Further progress was achieved in FY 2015, including the following activities and accomplishments.

\footnotetext{
$\square$ High-energy ion irradiations at the ATLAS. In FY 2015, two high-energy ion irradiation experiments were conducted on various nuclear materials, including uranium oxide $\left(\mathrm{UO}_{2}\right)$ and uranium-molybdenum (U-Mo) fuels, silicon carbide (SiC), advanced ferritic steels, and Zircaloy. Two ion sources were used: 55-MeV Fe
} 
ions and 80-MeV Xe ions. To achieve the desired dose and/or radiation damage levels at specific radiation temperatures, an irradiation chamber with two different sample stages was designed, fabricated, and installed at ATLAS. The first stage, with exceptional heat-transfer capability, was built for the dispersion of U-Mo fuel (U-Mo alloy fuel particles dispersed in an Al based matrix) to achieve a relatively low sample temperature during irradiations. The second stage was equipped with a ceramic heater that connected to the temperature controller for closed-loop temperature control. The irradiation chamber will be installed permanently at the ATLAS and it will be ready to use when additional beam time is allocated.

$\square$ Application of high-energy synchrotron wide- and small-angle scattering to analyze in-pile irradiated high-burnup U-Mo dispersion fuels. U-7wt\%Mo samples used in this experiment were irradiated at the Advanced Test Reactor (ATR) at Idaho National Laboratory (INL) in experiment RERTR-7. The experiment was a joint Argonne/INL experiment in support of the highly enriched uranium (HEU) minimization effort sponsored by DOE's National Nuclear Security Administration (NNSA). Samples at three different burnup levels were prepared at INL and transported to Argonne for synchrotron characterizations and further ion-irradiations (to even higher burnup levels). These samples were studied first at Sector 1 at the APS with high-energy WAXS and SAXS, as shown in Figure 1. A fission gas bubble superlattice was observed to form at the intermediate burnup. The superlattice constant was determined to be 11.7 nanometers $(\mathrm{nm})$ and $12.1 \mathrm{~nm}$ by WAXS and SAXS, respectively. Grain sub-division takes place throughout the irradiation and causes the collapse of the superlattice at high burnup. The bubble superlattice expands the lattice constant and acts as a strong sink for radiation induced defects. The modified Williamson-Hall analysis was utilized to interpret the broadening of diffraction peaks so that the dislocation density in these samples was deduced. The evolution of dislocation loops was found to be suppressed until the bubble superlattice collapsed.

$\square$ Application of high-energy synchrotron wide- and small-angle scattering to analyze high-energy ion irradiated U-Mo dispersion fuels. Different types of metallic fuel samples were ion irradiated at the ATLAS and then machined into specimens of $\sim 10 \times 10 \times 10$ micrometers $^{3}\left(\mu \mathrm{m}^{3}\right)$ by using a focused ion beam (FIB). The energy of the synchrotron $x$-rays was adjusted to $\sim 62 \mathrm{keV}$ in the experiment. The $\mathrm{x}$-ray beam was focused to $\sim 2 \mu \mathrm{m}$ (vertical) by $20 / 40 \mu \mathrm{m}$ (horizontal). This focused $\mathrm{x}$-ray beam was axially scanned from the top to the bottom of the specimens. A few microstructural developments that were found in the in-pile neutron irradiated dispersion fuel were observed in the ion-irradiated samples. For example, the U-Mo $\gamma$ phase was stabilized during both neutron and heavy-ion irradiations (Figure 1); bubble structures produced by fission fragments in the reactor were simulated by Xe implantation in the ATLAS; both neutron and ion irradiations promote the formation of the uranium aluminide $\left(\mathrm{UAl}_{3}\right)$ phase due to the radiation-induced inter-diffusion of $U$ and $A$ latoms in the fuel. With the focused $x$-ray beam, all of the regions of interest, including the interaction layer of the $\mathrm{UAl}_{3}$ near the surface and the Xe bubble concentration region, were characterized by the $x$-ray scan. The results of the WAXS demonstrated the utility of combining high-energy ion irradiation and high-energy $\mathrm{x}$-ray radiation for research on nuclear fuel materials.
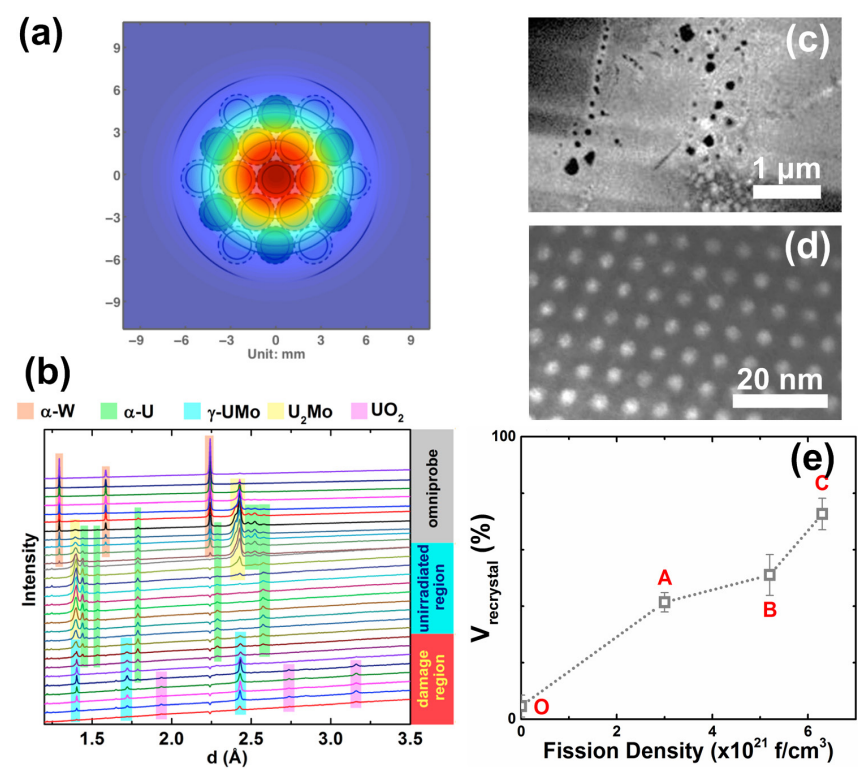

Figure 1. Key APS and ATLAS experiments in FY 2015. (a) Schematic of a multiple sample holder for heavy-ion irradiations at ATLAS, (b) Synchrotron x-ray diffraction patterns of a U-Mo dispersion fuel irradiated at ATLAS, showing in-depth phase development induced by heavy-ion irradiation, (c) Scanning electron microscope image showing the Xe bubble structures induced by Xe implantation at ATLAS, (d) Transmission electron microscope image showing the fission gas bubble superlattice induced by neutron irradiation at ATR, and (e) Volume fraction evolution of recrystallized U-Mo grains, derived from analysis of the WAXS data.

$\square$ Synchrotron radiation study of nano-grained $\mathrm{UO}_{2+x}$. Several methodology studies essential to the success of in-situ measurements were completed. The experimental setup for a high-temperature annealing experiment was configured at APS Sector 1-ID. Three nano-grained $\mathrm{UO}_{2+x}$ samples, sintered under different conditions, were characterized with high-energy synchrotron x-rays. The stoichiometry of each sample was determined from the measured lattice 
constants. The dislocation density and grain size of the nano-grained $\mathrm{UO}_{2}$ samples were determined using both conventional and modified Williamson-Hall methods.

$\square$ Application of x-ray micro-diffraction, x-ray nano-tomography, and $x$-ray absorption fine structure to analyze high-energy ion-irradiated fuel and structural materials. Two micro-diffraction experiments were performed at APS Sector 34 to analyze the ion-irradiated $\mathrm{U}-\mathrm{Mo}$ and $\mathrm{UO}_{2}$ samples. Through the experiments, the capability of x-ray Laue diffraction to probe a specific ion-irradiated region was demonstrated. X-ray nano-tomography experiments were performed at APS Sector 32 to study the Xe bubble distribution in irradiated U-Mo samples. Finally, an x-ray absorption fine structure experiment was conducted at APS Sector 10 to analyze ion-irradiated $\mathrm{UO}_{2}, \mathrm{U}-\mathrm{Mo}$, and HT9 steels. The changes in coordination number (the total number of neighbors of an atom in a crystal) of each material after ion-irradiation were observed.

$\square$ Modeling and simulation. We performed a number of atomic scale simulations of fission fragments (Xe gas) in nuclear fuel that support investigations of the impact of defect and damage formation on microstructure evolution during ion irradiation of nuclear materials. A molecular dynamics (MD) study of diffusion coefficients of intrinsic defects and fission gas in U-Mo alloy was performed. The predicted diffusivities of vacancies, interstitials, and Xe gas are used as input parameters for meso-scale phase field simulation studies. A molecular dynamics study of the formation of a Xe gas bubble superlattice in Mo and U-Mo alloy based on thermodynamics was also performed where it was found that the formation of a bubble superlattice is due to the Xe diffusion along certain preferred directions. Other investigations included the multiscale simulation of grain growth in the U-Mo alloy as a result of the annealing and multiscale simulation of the intragranular and intergranular fission gas bubble evolution in U-Mo as a result of irradiation, where a van der Waals equation of state (vdW EOS) for Xe gas was implemented into the phase-field model to yield a more accurate model of gas pressure in the over-pressurized bubbles.

$\square$ Considerations of in-situ characterization of irradiated materials. Beyond the scoping studies of heavy-ion irradiation and synchrotron x-ray radiation to study radiation damage in nuclear materials, this project has demonstrated that there is a need to establish the capability of performing simultaneous irradiation and characterization. First of all, the sample condition during heavy-ion irradiation is combined with temperature and radiation exposures. Although the sample can be rapidly cooled after ion-irradiation, the sample condition after irradiation cannot completely represent the sample condition during the irradiation. More important, the kinetics of the material's microstructural development cannot be observed solely by characterizing the post-irradiation materials. Furthermore, radioactive materials transported from ATLAS or ATR to APS have shown that there is a risk of sample damage by vibration, oxidation, and potential human error during sample transport. All of these problems can be avoided if irradiation and characterization are collocated.

\section{Development of a Computational Fluid Dynamics Multiphase Boiling Capability to Predict the Critical Heat Flux in Nuclear Reactor Fuel Assemblies}

\section{4-177-R1}

Adrian Tentner, Oana Marin, Elia Merzari, Aleksandr Obabko, Ananias Tomboulides, and Prasad Vegendla

\section{PROJECT DESCRIPTION}

The project will further develop and expand the extended boiling framework (EBF) two-phase (2P) flow modeling methodology previously developed by Argonne and will implement it in Nek5000, a high-performance, highly scalable, open-source computational fluid dynamics (CFD) code developed at Argonne. The goal of the project is to develop a new 2P version of the Nek5000 code, named NEK-2P, to simulate the two phase flow and heat transfer phenomena that occur in a light-water-reactor (LWR) fuel bundle under various operating conditions, with special emphasis on the prediction of critical heat flux (CHF). Boiling flows are subject to the phenomenon known as "boiling crisis," where heat transfer between the cladding and coolant deteriorates and the cladding surface temperature rises sharply. The avoidance of CHF conditions is essential for the safe functioning of LWRs.

\section{MISSION RELEVANCE}

Multi-phase flow and boiling are of fundamental importance in nuclear reactors used for energy generation, the vast majority of which are cooled by water. This work is therefore supportive of DOE's energy mission. The assessment of the fuel assembly behavior under 2P flow conditions is of particular importance, because many design and safety criteria depend on 
it. The U.S. Nuclear Regulatory Commission requires vendors to evaluate the CHF phenomenon accurately in their nuclear reactor designs and to demonstrate that the "boiling crisis" can be avoided. The EBF 2P code developed by the project will help reduce the number of costly hardware experiments required to develop new reactor designs.

\section{RESULTS AND ACCOMPLISHMENTS}

During FY 2014, the research and development effort was focused on (1) evaluation and extension of the EBF models in the context of the existing CFD code STAR-CD, with the goal of exploring and improving the capability of the EBF models to predict the occurrence of CHF under boiling flow conditions similar to those encountered in boiling water reactors, and (2) development of a homogeneous $2 \mathrm{P}$ variable density solver for the Nek5000 code, which can be used for the simulation of $2 \mathrm{P}$ boiling flows in heated channels.

During FY 2015, the research and development effort was focused on numerical and physical $2 \mathrm{P}$ modeling and simulations of boiling. Numerical 2P modeling comprised two separate efforts: (1) continued development of the 2P Navier-Stokes solver based on a drift-flux model (2P-DF) and (2) implementation of the 2P, two-velocity Navier-Stokes solver (2P-2V) in Nek5000. Completion of the 2P-DF solver in Nek5000 was required for the boiling simulations described below.

For the phenomenological $2 \mathrm{P}$ modeling, we continued implementing the EBF 2P models in the 2P-DF Nek5000 solver. The EBF models and the Nek5000 2P-DF were evaluated through analyses of several subcooled boiling flow experiments. The vapor fraction distribution calculated with 2P-DF in an axial cross-section of three experiments is shown in Figure 1. The calculated results were shown to be in reasonably good agreement with the experimental data measured by Bartolomei et al., as illustrated in Figure 2.

Finally, we explored the simulation of boiling phenomena using the mesoscale Lattice Boltzmann Method (LBM), with the goal of refining the boiling models used in the CFD simulation. The LBM simulations focused on the modeling of vapor bubble formation and growth on a heated wall in contact with a subcooled flowing liquid, bubble departure, and subsequent coalescence in the subcooled liquid flow. The LBM phenomenological information was used to evaluate and refine the EBF 2P models.
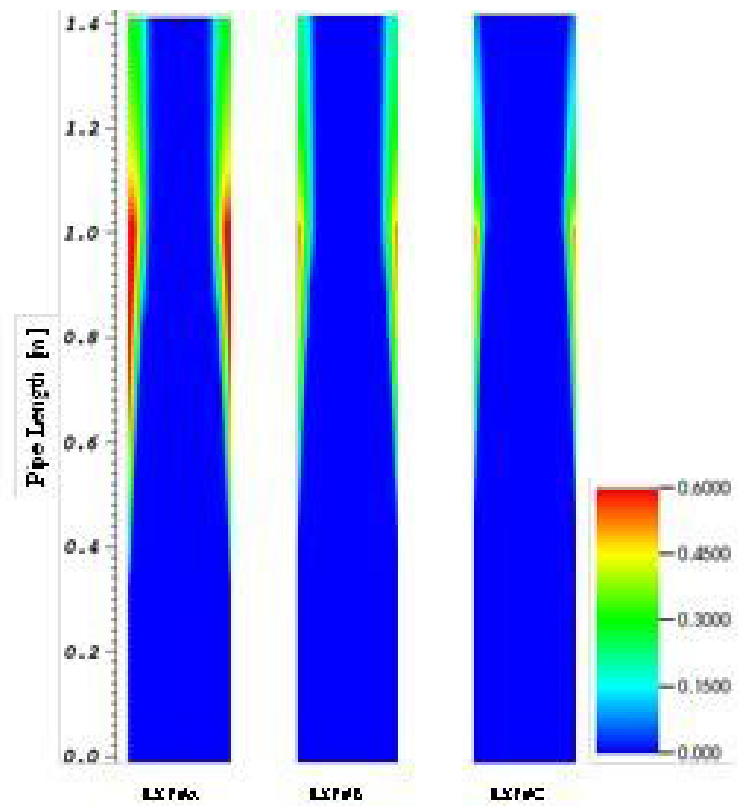

Figure 1. Vapor fraction distribution calculated with the NEK-2P drift-flux model in an axial cross-section of three experiments. Vapor is generated near the heated wall (red), while the bulk of the fluid remains subcooled and free of vapor (blue).

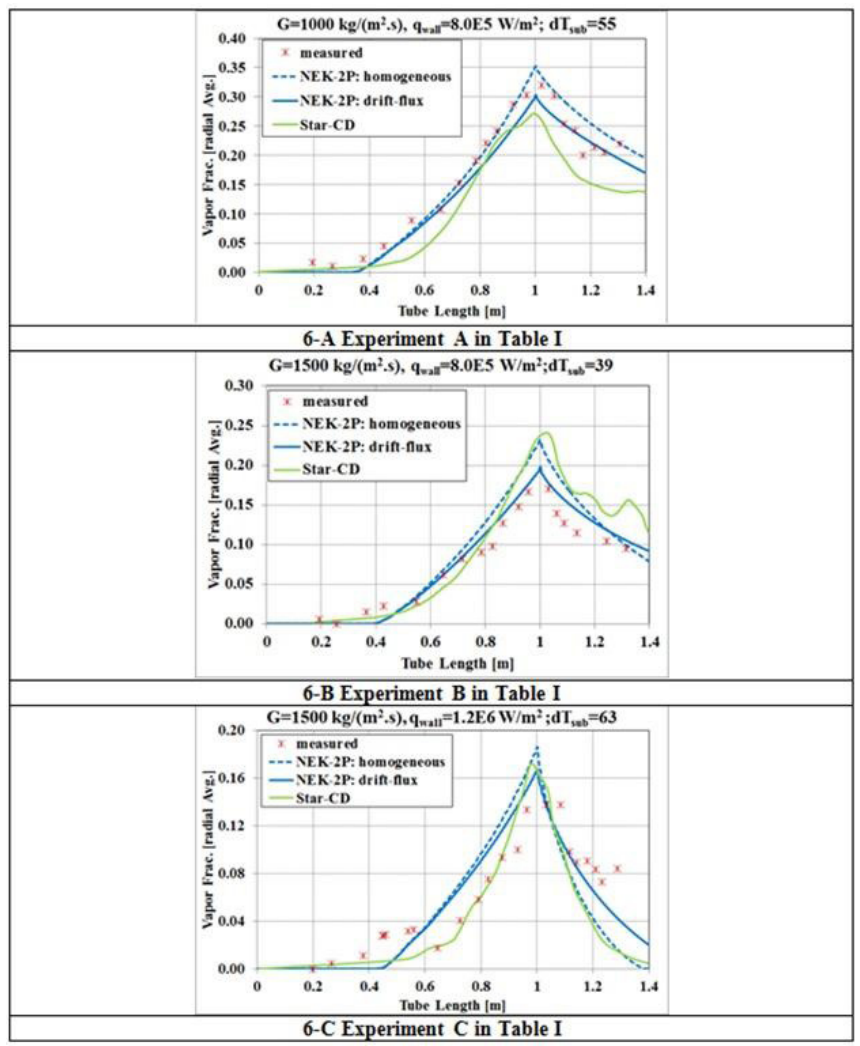

Figure 2. Radially averaged vapor fraction profiles along the length of the pipe for the same three experiments. The measured and calculated average void fraction increases along the 1.0-m heated section of the pipe, then begins to decrease due to condensation in the remaining $0.4-\mathrm{m}$ unheated section. Measured data (x) are from G.G. Bartolomei, G.N. Batashov, V.G. Brantov, et al. Teplomassoobmen-IV, Vol. 5. Minsk. ITMO AN BSSR Press, Vol. 5, pp. 38 (1980). 


\section{PROPOSED FUTURE WORK}

We have identified three tasks that will continue in the third year:

$\square$ Numerical Modeling. We will continue development of the spectral-element 2P Navier-Stokes solver with a focus on the implementation of the $2 \mathrm{P}-2 \mathrm{~V}$ model.

$\square$ Physical Modeling. The EBF 2P model will be implemented in Nek5000: (1) the EBF models initially implemented in the Nek5000 2P-DF solver will be expanded and implemented in the Nek5000 2P-2V solver and (2) the EBF models will be evaluated for their ability to capture CHF phenomena.

$\square$ Testing and Validation. We will run large-scale simulations of boiling and CHF processes for which experimental data are available in the open literature: (1) multi-phase boiling flow in a simple experimental geometry (e.g., heated pipe flow) and (2) multi-phase boiling flow in a realistic or simplified rod-bundle geometry.

\section{Economic and Technical Aspects of Nuclear Energy Competitiveness in the Current U.S. Deregulated Electricity Markets}

\section{5-129-NO}

Francesco Ganda, Audun Botterud, Fernando de Sisternes, Roberto Ponciroli, Richard Vilim, and Zhi Zhou

\section{PROJECT DESCRIPTION}

The objective of this project is to investigate a wide range of technology, policy, and market issues, with the goal of identifying those options that have the greatest potential to restore the economic competitiveness of nuclear power plants (NPPs) in the United States in the mid- to long term. Nuclear power in general, and existing nuclear reactors in particular, are pivotal to achieving the electricity-based carbon emissions reduction goals for 2030 established by the U.S. Environmental Protection Agency in its recently released Clean Power Plan. Yet the economic viability of nuclear energy is increasingly challenged in our nation's restructured electricity markets, as evidenced by some recent nuclear plant closures and discussion about further closings for economic reasons (e.g., "Exelon's six nuclear plants in Illinois have failed to turn a profit over the last five years," Chicago Tribune, March 9, 2014, and "Response to the Illinois General Assembly Concerning House Resolution 1146, Potential
Nuclear Power Plant Closings in Illinois," January 5, 2015, prepared by the Illinois Commerce Commission, Illinois Power Agency, Illinois Environmental Protection Agency, and Illinois Department of Commerce and Economic Opportunity). Whereas there are also nuclear construction projects currently ongoing in the United States (e.g., four combined AP1000 units [pressurized water reactor NPPs being built by Westinghouse Electric Co.] at Vogtle [in Georgia] and VC Summer [in South Carolina]), these are all areas with traditional vertically integrated utility regulation. Two of the existing issues are connected with the abundance of inexpensive natural gas and the increased number of incentive programs that promote the out-of-market deployment of renewable energy capacity with virtually zero marginal operating cost. These factors tend to reduce electricity market prices, impeding the cost recovery of the capital investment in NPPs. Understanding how existing and future NPPs can adapt to this situation, from both an economic and a technical perspective, is the focus of this work.

\section{MISSION RELEVANCE}

Understanding how existing and future nuclear technologies can adapt to evolving market conditions is essential to ensuring the economic viability of this zero-carbon-emissions technology, and is therefore relevant to the DOE's energy security and environmental quality missions.

\section{RESULTS AND ACCOMPLISHMENTS}

In the first year of this project, we completed a literature review and the establishment of a framework for the model development, including the methodology to estimate the resource variability and the reserve requirements in systems with increasing shares of wind and solar resources. We also started to explore the extent to which existing NPPs have been affected by low natural gas prices and low-price events caused by excess supply of renewable generation (Figure 1). We found that while the main economic challenge at present is caused by current low natural gas prices, the exposure of NPPs to zero or negative prices likely will gain importance in the long run, once renewables represent a more significant share of the energy mix. 


\section{Historical Negative Prices}

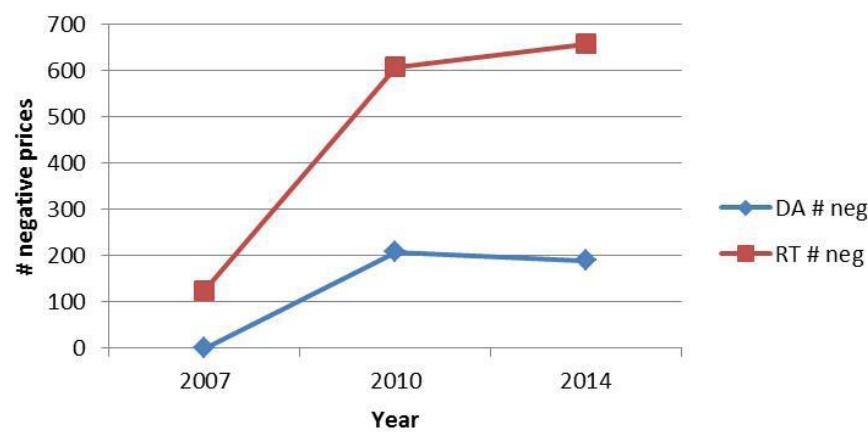

Figure 1. Number of negative prices (in which power companies pay to provide power to the grid) in day-ahead (DA) and real-time (RT) electricity markets at the location of the Quad Cities nuclear power plant in Illinois. The data are taken from the PJM electricity market (www.pjm.com).

While load-following operations have been practiced extensively in France and Germany, very little experience exists in the United States. This "flexible" operating mode will gain importance as the number of zero-price events increases with greater penetration of renewable energy sources into the energy mix. We set out to understand the economic, regulatory, and technical implications of operating nuclear reactors in flexible mode. The following were identified as among the most important technical issues associated with flexible operations and load following:

$\square$ Moderator density reactivity feedback;

$\square$ Fuel temperature reactivity feedback (Doppler effect);

$\square$ Redistribution of the core power profile following the power excursion and the associated control-rod adjustments;

$\square$ Fission product poisoning (by ${ }^{135} \mathrm{Xe}$ and ${ }^{149} \mathrm{Sm}$ isotopes of xenon and samarium);

$\square$ Reduced maneuverability with nuclear fuel burnup;

$\square$ Accelerated aging of components due to thermal fatigue (observed to be limited if the power ramp speeds are within certain limits); and

$\square$ Fuel performance in load-following mode (the experience in France and Germany does not show increased fuel failure due to daily load-cycling and extended low-power operations, provided ramp speeds are within certain limits).

In the short run, operating existing reactors flexibly can reduce their exposure to zero- or negative-price events, since NPPs can reduce their output in situations with surplus renewable generation. Moreover, from a system perspective, flexibility has increasing value with more wind and solar power in the electric grid, and flexible NPPs can contribute toward this increased value. Initial results from modeling nuclear flexibility in a unit commitment/economic dispatch (UC/ED) model indicate significant benefits to NPP owners as well as to the system as a whole.

Using the Investment Model for Renewable Electricity Systems (http://web.mit.edu/ceepr/www/publications/ workingpapers/2013-016r.pdf), a generation capacity expansion model with unit commitment constraints, we also analyzed the impact of operating nuclear reactors flexibly on the optimal generation mix, on electricity prices, and on curtailment of renewables. We found that operating nuclear reactors in flexible mode also adds value to the system (albeit small) in the long run, by reducing the electricity price and reducing renewable curtailment. Figure 2 shows an example of how the nuclear capacity is dispatched in the system in the presence of renewable generation when it is allowed to operate flexibly.

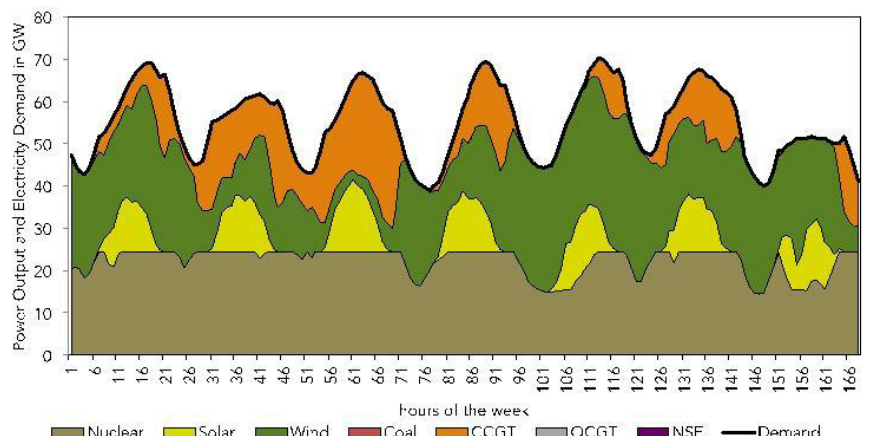

Figure 2. Hourly dispatch by technology for one week in a test system with nuclear power plants operating in flexible mode. Frequent reductions in the power level of nuclear plants are observed, meaning that the flexible operation capability of nuclear capacity adds value to the system (CCGT: combined cycle gas turbine; OCGT: open cycle gas turbine; NSE: non-served energy).

We are also working on a more detailed representation of the phenomena occurring in the reactor when it is operated in flexible mode. Among the constraints governing the reactor operation, it is important to accurately model the reactivity defect due to xenon poisoning. Since this fission product is a strong neutron absorber, high concentrations might limit NPPs' operational flexibility. The xenon concentration is a function of the time-dependent power level at which the reactor is operated. In particular, as a result of a power drop, the xenon concentration initially increases and afterwards decreases because of radioactive decay until it reaches a lower value at equilibrium. However, particularly close to the end of the nuclear fuel reloading cycle, the initial peak might not be balanced by the externally induced reactivity (i.e., the operation of the control rods and the dilution of the neutron-absorbing boric acid might not be sufficient). A dedicated constraint was initially defined to represent this effect. In particular, 
we introduced constraints in the UC/ED model to ensure that the system cannot perform any power ramp-up for a certain amount of time after a ramp-down. This initial representation was improved: from the time-dependent evolution of the neutron multiplication factor, it is possible to derive the instantaneous value of the externally induced reactivity, which can be used by the operator. Therefore, by comparing the control rod capabilities with the reactivity defect due to xenon poisoning at each time-step, one can establish whether the power ramp-up decision can be taken or not. This criterion for the operation of the NPP is going to be implemented in the model.

\section{PROPOSED FUTURE WORK}

In the next steps, we will complete the novel algorithms for improved representation of nuclear energy in generation expansion, scheduling, and dispatch for the power system. Moreover, we will use these algorithms to complete the analysis of nuclear flexibility benefits and then investigate a wider set of issues affecting the competitiveness of nuclear power in U.S. electricity markets.

\section{Nuclear Materials under Extreme Conditions}

\section{5-136-NO \\ Mark Williamson, Chris Benmore, and Rick Weber}

\section{PROJECT DESCRIPTION}

The goal of this project, which builds upon capabilities in high-energy $x$-ray characterization and ultrahigh-temperature containerless melting techniques, is to develop a dedicated station for the experimental evaluation of nuclear materials under extreme conditions. The goal is to investigate the properties of nuclear materials under well-controlled conditions and determine fundamental data important to nuclear energy systems.

\section{MISSION RELEVANCE}

Events at the Fukushima Daiichi nuclear power station in 2011 emphasized the need for experimentally derived data on the behavior of nuclear materials under extreme conditions. Loss of electrical power to the reactor site resulted in loss of coolant flow to the reactor core, which resulted in the fuel melting and interacting with cladding and supporting hardware to form a multiphase material called corium. Coupled to the formation of corium was the generation of hydrogen that resulted in explosions that severely damaged the reactor containment structure. Consequent renewed interest in reactor safety assessments arose, including severe-accident experimentation and modeling and simulation, as well as in research and development on so-called accident-tolerant fuels. Also, these events underscored the need for a more thorough understanding of the high-temperature behavior of irradiated fuel and its interaction with cladding and structural materials in a reactor system. These data needs are paramount in designing and testing advanced fuels and reactor materials, as well as in validating nuclear system codes (e.g., fuels, materials, severe accidents).

\section{RESULTS AND ACCOMPLISHMENTS}

A first-of-a-kind experiment demonstrating the feasibility of the approach resulted in the experimental determination of the structure of hot crystalline and molten uranium dioxide. A new state-of-the-art instrument for containerless, high-temperature $x$-ray interrogation of hot crystalline solid and liquid materials at very high temperatures was designed, fabricated, and used in experiments during the first year of the study. The containerless environment allows investigation of high-purity solids and liquids at temperatures up to approximately 3600 kelvins (K). It contains functionality to equilibrate the samples with mixtures of gases to enable the study of materials either under the oxidizing environments that may be encountered in severe reactor accident scenarios or the reducing environments experienced by nuclear fuels contained in metallic cladding. In addition to avoiding container reactions, aerodynamic levitation enables supercooling of liquids (by avoiding extrinsic nucleation of the solid phase). We demonstrated the functionality of the system through a series of experiments conducted at the APS that resulted in x-ray measurements of hot crystalline and molten uranium dioxide, zirconium dioxide, and solid solutions of uranium dioxide and zirconium dioxide, each of which is relevant to current and advanced nuclear energy systems. Analysis of the high-quality synchrotron scattering data obtained from the recent experiment is ongoing but preliminary pair distribution function results indicate that the $\mathrm{U}-\mathrm{O}$ coordination changes from $\mathrm{UO}_{8}$ to a mixture of $\mathrm{UO}_{6}$ and $\mathrm{UO}_{7}$, which suggests the polyhedra are not linked rigidly and leads to a dynamic, reactive melt.

\section{PROPOSED FUTURE WORK}

Experiments conducted with the instrument developed during the first year identified the need for several modifications to the sample chamber and control software to improve system functionality in areas such as mass flow 
control of oxidizing and reducing cover gas, and enhance data quality. These modifications will be completed and tested during the second year of the project. Specifically, the performance of the modified apparatus will be evaluated through a series of experiments with urania-zirconia- iron oxide samples relevant to severe-accident analysis. Data for the materials will be collected as a function of temperature and oxidizing atmosphere. In addition to yielding technically significant results, the work will demonstrate the full utility of the platform.

\section{Understanding Embrittlement in Cast Austenitic Stainless Steels and Stainless Steel Welds}

\section{5-145-NO}

Yiren Chen, Jonathan D. Almer, Meimei Li, and Ken Natesan

\section{PROJECT DESCRIPTION}

Cast austenitic stainless steels (CASSs) and stainless steel (SS) welds are structural materials used for components at the primary pressure boundaries and core internals of light water reactors (LWRs). Consisting of a dual-phase microstructure of delta ferrite and austenite, CASS alloys and SS welds are vulnerable to thermal aging embrittlement and neutron irradiation damage. The service performance of CASS alloys and SS welds are of concern after long-term service exposure to reactor core environments. Although the effects of thermal aging and neutron irradiation have been studied separately, it is difficult to predict whether these two degradation mechanisms will interact with each other during long-term service, leading to unexpected failures of reactor core components.

This research project is built upon the long history of Argonne's LWR materials programs and core capabilities of hard $\mathrm{x}$-ray science and advanced characterization tools. In this project, we will investigate the embrittlement mechanisms of CASS alloys and SS welds subjected to both neutron irradiation and thermal aging. Using advanced microstructural characterization techniques and $x$-ray in-situ mechanical testing, we will study (1) the combined effect of thermal aging and neutron irradiation on the microstructural evolution and (2) the deformation behavior of the dual-phase microstructure responsible for the embrittlement. Through this study, we will answer fundamental questions regarding the degradation of LWR core components and provide a scientific basis for assessing and managing the embrittlement of CASS alloys and SS welds under the combination of neutron irradiation and thermal aging.

\section{MISSION RELEVANCE}

Nuclear power generates about $20 \%$ of U.S. electric power without generating carbon emissions. This critical role of nuclear energy must be maintained for long-term operations to meet the nation's clean energy goal. The objective of the current project is well aligned with the DOE's Light Water Reactor Sustainability Program, which is aiming to develop technical solutions to ensure safe and economical operations of existing nuclear power plants. The current project will also benefit the mission of the U.S. Nuclear Regulatory Commission to ensure nuclear safety of current LWRs and other nuclear systems. As the life extension of current LWRs is under consideration, a better understanding of the combined effect of thermal aging and neutron irradiation on the deformation behavior of duplex microstructures will be crucial for assessing the integrity and service lifetime of reactor components. The results of this study can help develop an effective aging management strategy, leading to significant economic benefits and adequate safety margins.

\section{RESULTS AND ACCOMPLISHMENTS}

A recently developed in-situ mechanical testing system for activated materials at the Advanced Photon Source (APS) offers a new opportunity to study the plastic deformation of dual-phase microstructures under aging and neutron irradiation with high-energy synchrotron $x$-rays. The synchrotron-based characterization techniques are being used, along with high-resolution transmission electron microscopy (TEM) and atom probe tomography (APT), to reveal subtle and fine-scale microstructural and microchemical changes resulting from thermal aging and neutron irradiation.

In FY 2015, we completed the TEM characterization of unirradiated samples and select low-dose neutron-irradiated specimens using the Intermediate Voltage Electron Microscope-Tandem at Argonne. Figure 1 shows the precipitate microstructures resulting from thermal aging and neutron irradiation. These precipitates were identified as G-phase particles, a nickel-rich intermetallic silicide known to be a precursor of the $\alpha / \alpha^{\prime}$ phase separation in the iron-chromium-nickel (Fe-Cr-Ni) alloy system. It is evident that the neutron irradiation conducted in this study enabled and facilitated the precipitation or phase separation processes that would otherwise occur too slowly to be visible at the irradiation temperature. Quantitative analysis of the 
TEM data clearly showed that neutron irradiation and thermal aging are related, not independent, degradation processes. Thus, the combined effect of thermal aging and neutron irradiation must be evaluated for CASS alloys and SS welds.

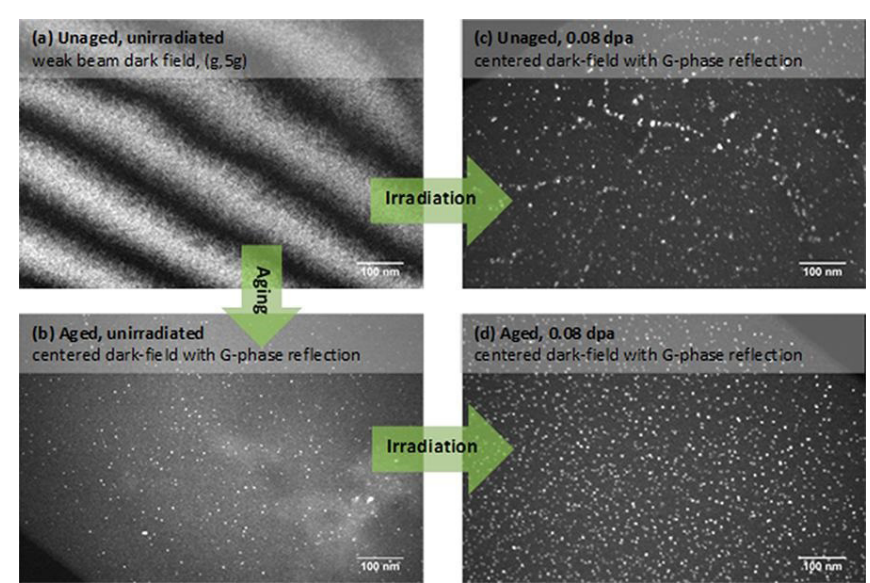

Figure 1. Dark-field TEM images formed with the G-phase reflection showing precipitate microstructure in the delta ferrite of CF-8, a CASS alloy with normal carbon content, in the as-cast specimen (a), after thermal aging (b), after neutron irradiation (c), and after thermal aging plus neutron irradiation (d).

We also performed an APT analysis in FY 2015 on a pair of unirradiated CASS specimens (unaged and aged) at the Center for Nanophase Materials Sciences, Oak Ridge National Laboratory. In contrast to the TEM observation, in which G-phase precipitates were visible only in aged specimens, the APT revealed nanoscale nickel-silicon (Ni-Si) clusters in both unaged and aged specimens. These nanoscale solute clusters are embryos of G-phase precipitates and have not been fully developed into crystallographically distinct regions. The role of these solute clusters under neutron irradiation will be explored in the future. The APT data also revealed $\alpha / \alpha$ ' phase separation that is difficult to identify with TEM. Using the chromium distribution results, we showed that the extent of phase separation was elevated considerably by thermal aging.

Synchrotron-based x-ray diffraction and scattering techniques will be used extensively for straining experiments to understand the combined effect of thermal aging and neutron irradiation on embrittlement. Experiments conducted at APS beamline 1-ID-E explored the different methodologies of revealing fine precipitates and constituent phases and their effects on plastic deformation. Selected CASS specimens in the forms of TEM disks and small coupons were scanned with a monoenergetic beam of 86 kilo-electron volts (keV) and a beam size of $30 \times 4$ microns $(\mu \mathrm{m})$. Wide-angle $x$-ray scattering and three-dimensional (3D) high-energy $x$-ray diffraction microscopy (HEDM) data were recorded.
Figure 2 shows an example of HEDM measurement of an unaged CF-3 specimen, which provides a wealth of grain-scale information including grain orientation, center of mass, grain size, and stress-state of each individual grain within the examined volume. The coarse solidification microstructure yielded a heavily "textured" pattern, and the orientations of ferrite and austenite grains appear to be correlated. In combination with upcoming in-situ strain experiments, the load partitioning between ferrite and austenite and the stress state in individual grains can be obtained to understand the deformation behavior of CASS and SS welds. Baseline mechanical tests were also conducted in FY 2015 to obtain tensile properties and prepare for in-situ x-ray straining tests. The aged specimens showed consistently higher yield and tensile stresses than their unaged counterparts.

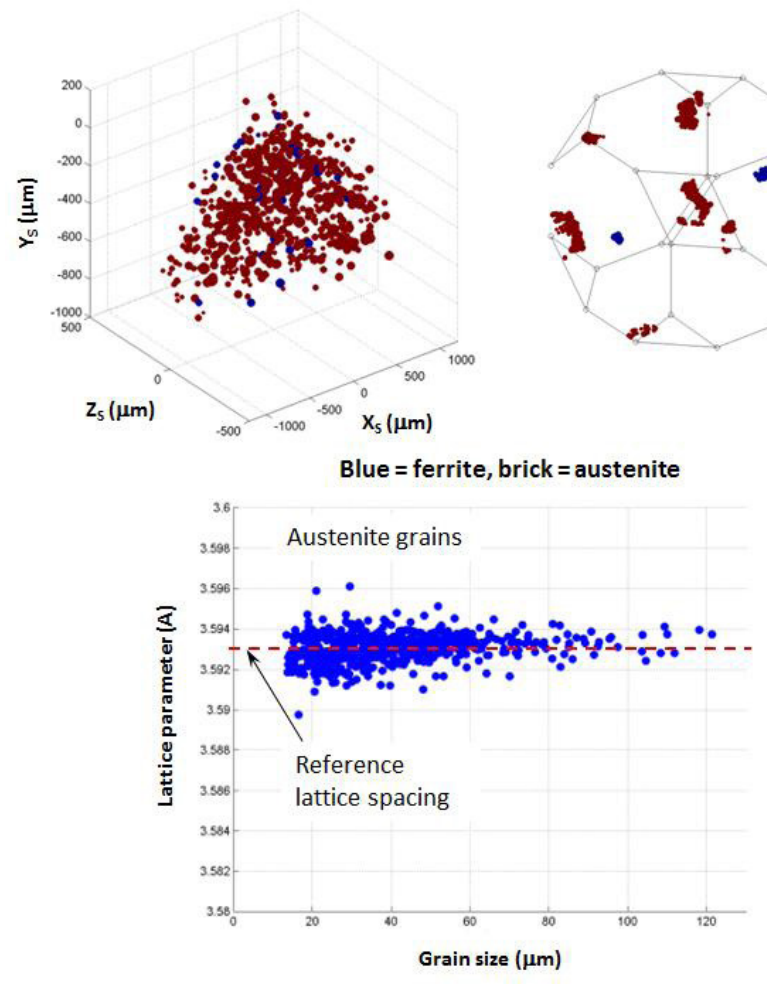

Figure 2. A wealth of grain-scale information derived from a 3D high-energy x-ray diffraction microscopy measurement on an unaged CF-3 specimen, a CASS alloy with low carbon content. The top-left and -right figures are a center-of-mass map and a spatial orientation map, respectively, of the austenite and ferrite grains. The bottom figure shows the variation of lattice constant as a function of austenite grain size.

\section{PROPOSED FUTURE WORK}

In FY 2016, we will carry out TEM characterization on high-dose neutron-irradiated specimens and investigate the dose effects on the microstructural evolution. We will also perform APT analyses on neutron-irradiated specimens and focus on the nanoscale solute clusters and phase separation that are invisible under TEM. The in-situ 
straining tests on aged and unaged tensile specimens will be initiated in FY 2016 to understand the deformation behavior of the dual-phase microstructure and to investigate the effects of precipitate and phase separation on the plastic deformation. 


\section{Enabling Sodium-Ion Batteries for Grid Storage}

\section{4-185-R1 \\ Christopher Johnson, Joel Blauwkamp, Arturo Gutierrez, and Eungje Lee}

\section{PROJECT DESCRIPTION}

The technological development of new high-performance, low-cost batteries is a key driver for the deployment of advanced large-scale electrical energy storage systems that deliver electricity to the grid and maintain reliable power flow. Sodium-ion batteries (SIBS) that operate at ambient temperature would be a serious contender for this application, if the energy density could be improved. The search for electrode materials that possess desirable properties, such as low cost and low toxicity, long cycle life, fast charge and discharge rates, and high energy density and that are easily synthesized will foster the rollout of an environmentally sustainable battery technology using earth-abundant and recyclable elements.

In this project, we will synthesize new materials for both electrodes (cathodes and anodes), characterize the chemistries and structures, and operate the electrodes in a SIB. Using the information so gained, we will optimize the SIB and continue seeking to identify new systems and emerging technologies, such as the use of nanoscience, in making improvements for high-power applications. Nanoparticles are useful in SIBs, because the diffusion path length is very small for the electrochemical reaction. This allows for high power in the battery because the current rate is synchronized with the diffusion speed of the sodium cations within the host intercalation electrode. This effect leads to very low impedance in the battery. According to our calculations, in order to achieve targeted grid storage requirements, the anodes and cathodes must meet specific capacity values of 600 milliampere-hours/gram (mAh/g) and 200 mAh/g, respectively, at $\sim 3.5$ volts $(\mathrm{V})$ in order to generate at least 350 watt-hours/kilogram (Wh/kg) in energy density. After full-cell optimization and fabrication, the SIB technology will: (a) have a specific energy of $350 \mathrm{Wh} / \mathrm{kg}$ and an energy density of 750 watt-hours/liter (Wh/L), (b) be compact and lightweight, (c) be economical and scalable, (d) have exceptional cycle life $(-1,000$ cycles) and calendar life ( 15 years), and (e) exhibit fast recharge in SIB full pouch-type cells. In addition, the total cost will be no more than $\$ 100 /$ kilowatt-hour ( $\mathrm{kWh}$ ) to meet the DOE's grid storage objective. Currently, this project is at the discovery stage; we are synthesizing and characterizing new battery materials to operate as state-of-the-art functional electrodes, with a focus on the energy density performance objectives laid out above.

\section{MISSION RELEVANCE}

The project is relevant to the DOE's missions in energy security and the environment. Grid storage topics fall in the domain of the DOE Office of Electricity. SIBs are drawing interest as an energy storage option to satisfy backup power needs for stationary grid power networks. The energy security and reliability of the grid are paramount in the United States. The needs for new materials-based technologies for this area are outlined in the DOE report "Energy Storage Program Planning Document” (February 2011). Our strategy is based on the potential of our technology in achieving an optimal balance between battery performance and cost. A grid storage system will require large-capacity batteries for which material production cost and raw material expense must be minimally apportioned. SIBs may offer a good, low-cost option, provided the correct electrode and electrolyte chemistry for this application can be identified and deployed. Highly synergistic and mutual benefits between this project and other electrochemical energy storage programs in DOE Energy Efficiency and Renewable Energy (EERE) and Advanced Battery Research could be expected, because the core technologies comprising the SIB system complement the battery system activities (non-SIB research) in the Joint Center for Energy Storage Research and at other national laboratories pursuing SIB technologies.

\section{RESULTS AND ACCOMPLISHMENTS}

In FY 2014, we focused on cathode research. The first demonstration of an intergrowth layered structure consisting of P2- and 03-type stacked layers within a crystal domain was reported and published. This novel layered intergrowth $\mathrm{Na}_{1-x} \mathrm{Li}_{x} \mathrm{Ni}_{0.5} \mathrm{Mn}_{0.5} \mathrm{O}_{2}$ cathode showed fast charge-discharge performance. The good electrochemical properties were attributed to the synergistic effect of an intergrowth structure that results from the direct incorporation of $\mathrm{Li}$ in the matrix. This finding highlights the importance of multiphase intergrowths with orientation relationships that can attain the stability and performance for future optimized SIBs.

Sodium-iron oxides were discussed in last year's report as the material that we had targeted in this project, going forward. Recognizing the impact that Mössbauer spectroscopy can have on understanding iron chemistry, we first reported Mössbauer spectroscopic measurements on our systems. As a result, we are collecting important characterization data and evaluating new materials that 
we have synthesized in the lab for this project. Mössbauer spectroscopy reveals the coordination geometry of the iron atom and its redox character (i.e., oxidation state), knowledge of both being critical in the design of the host intercalation material. However, these iron-based layered oxides still show low cycle performance, and the redox of the unusual $\mathrm{Fe}^{3+} / \mathrm{Fe}^{4+}$ couple needs to be better understood. In this past fiscal year, we analyzed the data and found only quasi-reversibility of the layered $\alpha-\mathrm{NaFeO}_{2}$ cathode in SIBs. A NaFeO 2 powder sample that has the O3-type layered structure (the most common oxygen $A B C$ sub-lattice stacking, with octahedral interstitials filled) was synthesized via a solid-state synthesis method. The changes in Fe oxidation states and crystallographic structures were examined during the electrochemical sodium cycling of the $\mathrm{NaFeO}_{2}$ electrodes. Ex-situ Mössbauer spectroscopy analysis revealed the chemical instability of $\mathrm{Fe}^{4+}$ in a battery cell environment-more than $20 \%$ of the $\mathrm{Fe}^{4+}$ species that was generated in the de-sodiated $\mathrm{Na}_{1-x} \mathrm{FeO}_{2}$ electrode was spontaneously reduced back to $\mathrm{Fe}^{3+}$ states during open-circuit storage of the charged cell. In-situ synchrotron x-ray diffraction further observed the nonequilibrium phase transition behavior of the $\mathrm{NaFeO}_{2}$ cathode. A new layered phase, denoted as $\mathrm{O}$ " 3 , or a $2^{\text {nd }} \mathrm{O} 3$ structure with a monoclinic distortion, was observed in the course of sodium deintercalation, and its asymmetric structural behavior during cycling was confirmed. These findings explain the quasi-reversibility of $\alpha-\mathrm{NaFeO}_{2}$ in the sodium cell and provide guidance for the future development of iron-based cathode materials for SIBs.

Turning to anodes, we screened a number of carbonaceous materials and found that a spherical carbon that is about 0.3 micrometers in diameter (Figure 1) gives at least 1,000 reversible charge-discharge cycles. Microporous spherical carbon particles were synthesized by surfactant-assisted emulsion polymerization of a low-cost industrial chemical, furfuryl alcohol, followed by pyrolysis of the resultant polymer in an argon atmosphere. The synthesized carbon particles were evaluated as a potential anode electrode material for SIBs. A highly reversible capacity of $253 \mathrm{mAh} / \mathrm{g}$ (at a $20 \mathrm{~mA} / \mathrm{g}$ rate) was achieved, and a high rate of $200 \mathrm{~mA} / \mathrm{g}(1 \mathrm{~h}$ charge and discharge times) yielded $155 \mathrm{mAh} / \mathrm{g}$ over a long cycle life of 1,000 cycles (Figure 2) with 99\% coulombic efficiency. Results from solid-state ${ }^{23} \mathrm{Na}$ magic angle spinning (MAS) nuclear magnetic resonance (NMR) measurements suggest a storage mechanism whereby sodium (de) insertion occurs simultaneously within both internal particle graphene interlayer regions and particle surface nanopores. No correlation in voltage profiles with the nature of the sodium site in the carbon is observed, which is a new finding for the sodium-ion battery field.

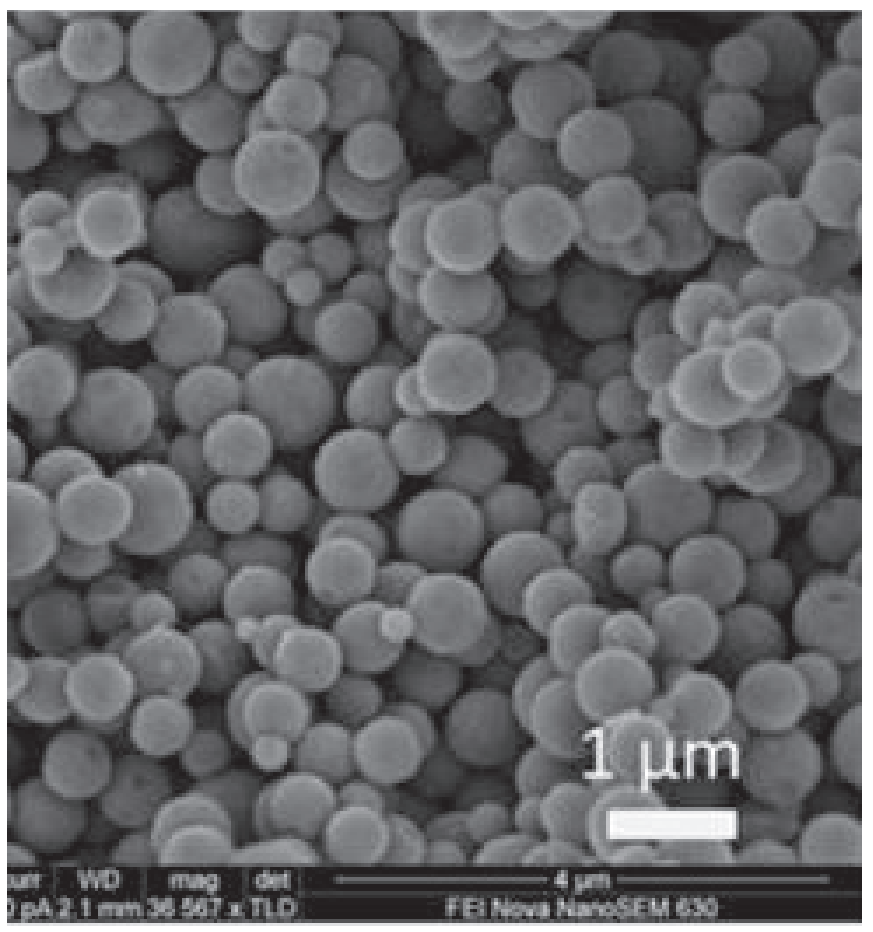

Figure 1. A scanning electron micrograph of the spherical carbon used in the long-life $\mathrm{Na} / \mathrm{C}$ cell.

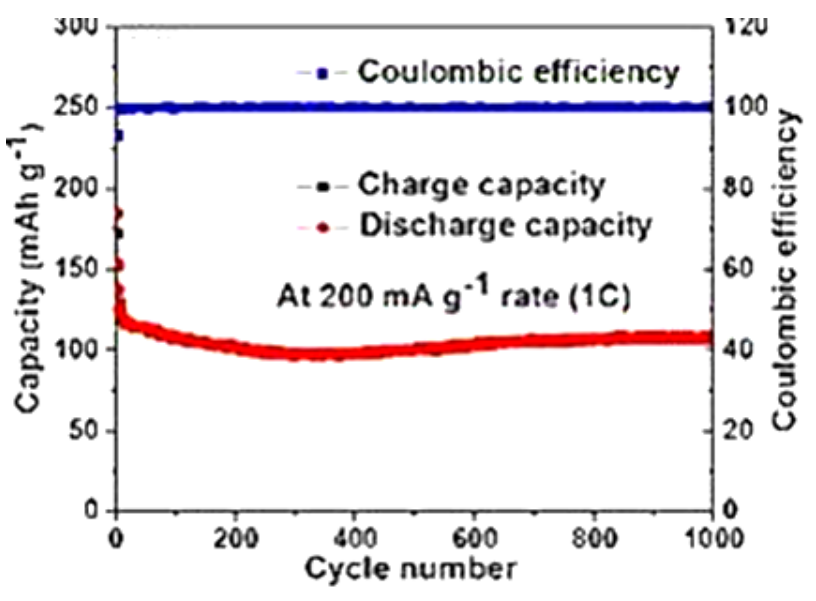

Figure 2. Capacity and coulombic efficiency of a $\mathrm{Na} / \mathrm{C}$ cell using the 0.3-micrometer carbon sphere particles as the anode plotted versus cycle number. The electrolyte is carbonate-based, and the salt is $\mathrm{NaPF}_{6}$. The $1 \mathrm{C}$ label within the figure refers to $\mathrm{a} 1 \mathrm{~h}$ charge time and a $1 \mathrm{~h}$ discharge time.

\section{PROPOSED FUTURE WORK}

New materials research drives the discovery of the best electrodes that yield superior performance. In FY 2016, we will concentrate on continuing the synthesis of new SIB electrode materials. With regard to cathodes, we plan to continue on two fronts: (1) continue our evaluation of the effect of $\mathrm{Li}$ incorporation in sodium layered transition metal oxides and (2) incorporate Fe in the layered oxides. For example, some preliminary data shows that the composition $\mathrm{Na}_{x} \mathrm{Li}_{1-x} \mathrm{Fe}_{0.5} \mathrm{Mn}_{0.5} \mathrm{O}_{2}$ yields $140 \mathrm{mAh} / \mathrm{g}$, 
which indicates active iron in the composition. We strive to conduct further Mössbauer measurements and are planning to conduct in-operando battery studies.

For carbon anodes, we will vary the closed and open pore ratios in the graphene to both understand the nature of the intercalated sodium site in the materials and to increase the battery energy density. To do this, we will change the heating time and temperatures during the pyrolysis of the polymer precursors.

Lastly, we plan to conduct low-temperature and high-temperature cycling studies of full cells to see if the operation temperature window is superior to that of Li-ion batteries. Certainly these comparisons are valid, particularly since Li-ion batteries are on the roadmap to grid storage batteries, but SIBs may be better suited to energy storage locations having extreme temperature environments. Also, safety studies of the sodium battery electrodes in charge states will be done in order to determine if there is improved (lower) thermal reactivity than with Li-ion batteries. Once the full-cell chemistry is determined, we will produce and evaluate larger pouch cells to check the durability of the battery. We plan to discharge the cell to the zero energy state, let it sit in a shorted state for weeks, and then revive it upon charging to see if it cycles normally. The pouch cell-formatted batteries are an important test vehicle for accelerating-rate calorimetry (ARC) studies within our department. ARC is a method for evaluating the thermal runaway behavior of batteries. The pouch cells can be fabricated by the Argonne battery department Cell Analysis, Manufacturing, and Prototype (CAMP) facility, sponsored by DOE EERE. Pouch full cells feature larger capacities up to 300-400 mAh and are useful for demonstrating energy density, safety, and lifetime to the battery community.

\section{Conversion of $\mathrm{C}_{2}$ and $\mathrm{C}_{3}$ Paraffins into Liquid-Phase Products}

\section{5-174-NO}

Christopher L. Marshall

\section{PROJECT DESCRIPTION}

In this project, we will create tandem catalysts for converting gas-phase molecules directly into higher-value liquids. This project couples two successes with atomic layer deposition (ALD) synthesis at Argonne: (1) ALD overcoating of metallic catalysts, and (2) high-precision synthesis of controlled acidic functions to create a tandem catalyst that will convert light gases directly into $C_{4}$ to $C_{6}$ liquids. The two reactions will be coupled together as shown below.

$$
\begin{aligned}
& \mathrm{C}_{2} \mathrm{H}_{6}+1 / 2 \mathrm{O}_{2} \rightarrow \mathrm{C}_{2} \mathrm{H}_{4}+\mathrm{H}_{2} \mathrm{O} \\
& 2 \mathrm{C}_{2} \mathrm{H}_{4} \rightarrow \mathrm{C}_{4} \mathrm{H}_{8}
\end{aligned}
$$

Reaction 1 takes place at the bottom of a pore over the catalytic metal nanoparticle and will selectively convert ethane and propane to ethylene and propylene, respectively, with little or no coking. After leaving the surface of the metal nanoparticle, the olefin will encounter acidic sites on the sides of the pore 1 to 2 nanometers $(\mathrm{nm})$ away from the metal surface. The acidic site will protonate the olefin, thus creating a carbenium ion. This carbenium ion will react with a second olefin forming an olefinic dimer, the desired reaction product.

ALD overcoating will be used to stabilize the catalytic metal nanoparticles and to boost the yield of heavier olefins. By designing a bimetallic catalyst composed of a primary alumina coating and a secondary silica coating, an acidic site will be created inside the catalytic pore capable of polymerizing olefins. The catalysts' uniform and restricted pore dimensions will limit olefin oligomerization to $\mathrm{C}_{4}$ to $\mathrm{C}_{6}$ olefins. The modular design of the catalyst will allow the system to have high transportability and can thus be used to monetize stranded and remote resources that are not easily accessible to large commercial plants, which currently must be built adjacent to vast gas fields.

\section{MISSION RELEVANCE}

The importance of our project to DOE's energy mission is confirmed by an excerpt from the web site of the Office of Basic Energy Sciences (BES): Catalytic transformations impact a large range of DOE mission areas. Particular emphasis is placed on catalysis relevant to the conversion and use of fossil and renewable energy resources, such as the conversion of crude petroleum and biomass into clean burning fuels and materials. Catalysts are used in fuel cells and batteries as well as photocells. They are essential for energy-efficient routes for the production of basic chemical feedstocks and value-added chemicals, as well as for minimizing the production of unwanted products.

The catalysts developed from this project will benefit the natural gas industry in the United States where much of the gas being processed in Pennsylvania, Texas, and North Dakota is termed "wet gas," that is, gas containing up to $20 \% \mathrm{C}_{2}$ and $\mathrm{C}_{3}$ paraffins. Because it is difficult to separate these heavier components from the methane, much of this gas is simply flared, leading to high $\mathrm{CO}_{2}$ 
emissions. An effective catalyst could be used at a wellhead to separate the heavier components from the methane. This project is therefore relevant to DOE's environmental quality mission as well.

\section{RESULTS AND ACCOMPLISHMENTS}

This project began in mid-April 2015. In the first 3 months, we ordered chemicals, completed training on the integrated ALD synthesis-catalysis (I-ALD-CAT) tool, and tested the feasibility of reproducing previous results on overcoating of palladium (Pd) catalysts. Initial studies of Pd coated on alumina powder (low surface area "spherical alumina") showed the catalyst to be very nonselective for oxidative dehydrogenation of ethane, producing more than $90 \% \mathrm{CO}_{2}$ and $\mathrm{H}_{2} \mathrm{O}$ and very little ethylene. In the next 2 months of FY 2015, overcoating this Pd with a monolayer of alumina (from ALD of trimethyl aluminum [TMA]) was tested for oxidative dehydrogenation of propane. Initial results showed low activity prior to calcination due to loss in the metal surface area. Total activity continues to decrease and is eliminated after 20 cycles of TMA deposition. At this point, it is assumed that all Pd sites are covered, and that there are effectively no accessible Pd sites for catalysis. Calcination of the alumina overcoating resulted in resumption of catalytic activity, but at a lower rate than found with the initial uncoated material. The catalyst does show improved selectivity to the olefin (propylene), which is consistent with previous work conducted in a more traditional ALD reactor. Because the catalyst contains no Brønsted acidity, it was not expected to demonstrate either coupling or hydroxylation.

Additional FY 2015 activities were dedicated to incorporating Brønsted acidity into the support and looking for coupling (to form dimers, trimers, etc.). The I-ALD-CAT unit has shown that it will speed the studies of reaction and compositional space compared to traditional methods. We also included adding 5 to 10 monolayers of silica followed by 1 to 2 monolayers of alumina to create Brønsted acidity sites of varying distances from the Pd oxidative dehydrogenation (ODH) site. The gas chromatograph has been set up for analyzing not only the cracked products ( $C_{2}$ and $C_{3}$ olefins), but also the polymeric and even hydroxylated products from those olefins. We anticipate that the distance from the dehydrogenation site to the Brønsted acid site will be the controlling factor for the tandem catalysis. As these sites grow farther apart, the less effective bifunctional routes will dominate, and selectivity to higher boiling components will decrease.

\section{PROPOSED FUTURE WORK}

The primary technical challenges to resolve in the coming fiscal year (FY 2016) will be the proper synthesis of the two catalytic sites in terms of distance, reactivity, and selectivity, and scale-up of the ALD process. Such coupling is unique in the catalyst literature. The research risk addressed is how to chemically link these two catalytic sites. Work will concentrate on the effect of the ratio between the amount of noble metal and the amount of overcoating applied in each of the two steps. Too little overcoating will not stabilize the noble metal. Too much, and the metal will be completely buried under the substrate. This ratio will also be dependent on the size of the noble metal particle placed on the support. Following optimization of these two parameters, work will focus on controlling the nature of the acidic site. It is known that mixing silica with alumina will result in a Brønsted acid site; however, other combinations can also provide acidity that could be more effective in promoting the desired coupling reactions.

\section{Integration of Multiple Infrastructure Dependencies and Interdependencies into Infrastructure Hazard Analysis}

\section{5-177-NO}

\section{Megan Clifford and Mike McElfresh}

\section{PROJECT DESCRIPTION}

The modeling of infrastructure dependencies and interdependencies is often handled by using a linear approach (i.e., outputs of one model are inputs to another model), which limits the evaluation of the impacts between even two different infrastructure systems. To address this gap, we focused on automating and integrating existing energy system modeling tools (i.e., EPfast and NGfast, for electric power and natural gas systems, respectively) to create an Infrastructure Forecaster. NGfast is a linear model that uses a progressive forward natural gas pipeline ownership identification and flow quantification process to track lost flow volumes due to pipeline breaks. EPfast explores the possibility of uncontrolled islanding caused by successive (or cascading) steady-state electric power line overloads. Such overloads are initially triggered by a major, non-reclosable, line-to-line fault or simply by a de-energization of a major line due to a seismic event or other natural causes. This Infrastructure Forecaster tool will be used to anticipate cascading failures and support the analysis of infrastructure security and resilience. The conceptual process is shown in Figure 1. 


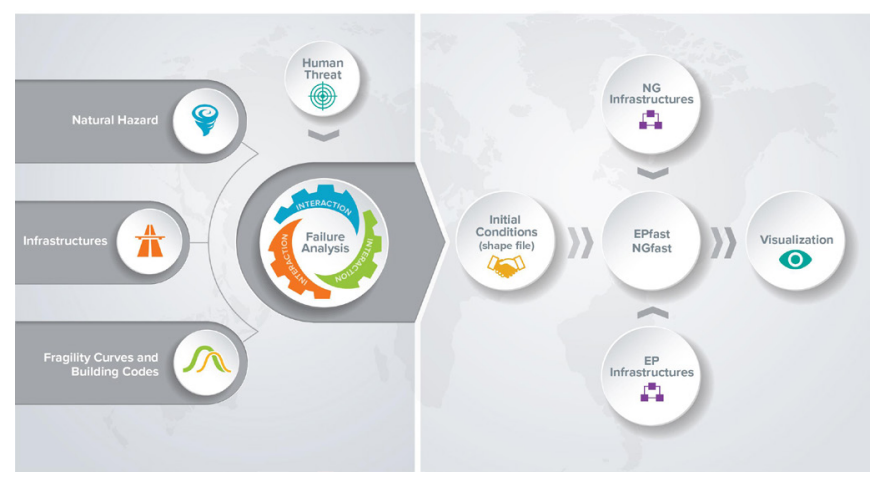

Figure 1. Argonne's Infrastructure Forecaster process.

To begin developing the Infrastructure Forecaster, the project focused on three main objectives:

$\square$ Automate the steps needed for defining the initial degradation conditions and simulating the cascading failures of energy systems,

$\square$ Integrate the dependencies and interdependencies existing between electricity and natural gas distribution systems, and

$\square$ Propose a flexible computing architecture for supporting the integration of other simulation tools and the use of different databases.

\section{MISSION RELEVANCE}

Infrastructure dependencies and interdependencies create complexities that mask many systemic risks. As a result, an impact to a single node or link-the proverbial "single point of failure" that is often hidden deep within these interconnected systems-can result in significant economic and physical damage on a city-wide, regional, national, or international scale. Accordingly, the DOE's 2015 Quadrennial Energy Review includes a recommendation calling for an analytical framework and a tool to assess the resilience and security of energy infrastructures. The results of this project are relevant to this recommendation.

\section{RESULTS AND ACCOMPLISHMENTS}

The results of this project include automation of the failure analysis, which characterizes initial conditions (i.e., state of energy infrastructures) used as inputs for the cascading failure analysis. The automation algorithm was written in Python, version 2.7. The algorithm automatically integrates the geospatial distribution of the infrastructure asset(s), the geospatial distribution of the hazard(s), and the failure criteria of each asset. For each asset, the infrastructure type and location are matched with the hazard intensity and failure criteria. The output includes the geospatial distribution of the original infrastructure asset modified with a binary "outage" variable, which equals 1 for asset failure and 0 otherwise.

Another key result was the cascading failure analysis, which involved automating the integration of EPfast and NGfast capabilities, which were used independently prior to this project. To automate the integration, we used Data Centric Modeling/Simulation (DCMS), a platform-independent, device-independent, paradigm-shifting framework for model interactions. DCMS keeps the current models intact, with little to no code changes, while still allowing them to be connected to other models. With these updates in place, the initial conditions from the automated failure analysis were input into the updated and integrated versions of EPfast and NGfast. The two programs are iterated until the results converge. We also improved NGfast's numerical stability and solution time by reformulating its optimization model with additional variables and differentiable functions, enabling numerical stability in a nonlinear optimization solver. Also, we implemented optimization problems for EPfast and NGfast by using the JuMP modeling package in Julia, an open-source, extensible, portable, high-level programming language. Julia is faster than other script languages such as MATLAB, Python, and R, but uses easily accessible and similar syntax.

Finally, we applied the developed methods to a hypothetical 64-bus electric grid set on the coast of Louisiana. We used wind and storm surge data from Hurricane Ivan, a Category 5 hurricane that caused widespread damage in the Caribbean and United States in the 2004 Atlantic hurricane season. The automated failure analysis determined that failure occurs in one electric power bus, one electric transmission line, and five natural gas processing plants. Then, we increased the vulnerability of the infrastructure assets using a $20 \%$ increase in wind gusts. As expected, the increase in wind speed resulted in a dramatic increase in the number of failures in the same selection of assets. We used the initial conditions as inputs for the next steps in the program, which iterated through the newly integrated EPfast and NGfast until the results converged. The final output visually depicted the outage area and islanding effects.

With this effort, it is now possible to rapidly test the impact of hypothetical scenarios, establish detailed quantification of the uncertainties attendant to any predicted impact, study ensembles of scenarios, execute searches through infrastructure configurations to probe potential weaknesses, and provide analyses to guide infrastructure planning strategies. 


\section{PROPOSED FUTURE WORK}

We will undertake several activities in FY 2016, including:

$\square$ Verify the outputs of the automated process by comparing them to the results of manual failure analyses conducted by experienced infrastructure analysts.

$\square$ Advance the automation of hazards analyses.

$\square$ Develop reliable and flexible interfaces to a variety of data sources and create a flexible scheme for representing probabilistic descriptions of infrastructural responses to stresses induced by the hazards.

$\square$ Convert $\mathrm{C \#}$ and Python code to $\mathrm{C}++$ to achieve a uniform code base and to become platform-independent and enable future scalability.

$\square$ Expand the Infrastructure Forecaster to evaluate the inclusion of telecommunications and/or transportation.

$\square$ Address data accessibility and usability.

$\square$ Enhance visualization of the interactions among infrastructures to identify the areas affected by the degradation and the cascading failures resulting from the initial event.

\section{Fine Resolution Reconstruction of Large Volumes of a Brain}

\section{5-181-NO}

Narayanan Kasthuri

\section{PROJECT DESCRIPTION}

This project proposes to explore research in both electron and high-energy $\mathrm{x}$-ray microscopy for imaging the fine structure of the brain. There is a current push to develop approaches to map all the neurons and connections at the nanometer $(\mathrm{nm})$ scale in a large volume of a brain to accomplishing the same for entire brains ("connectomics"). The current approach is to use serial section electron microscopy (EM). This research project will continue those investigations along with determining how synchrotron-based, high energy x-ray microscopy of the same brains can complement and extend the capabilities for connectomics. The Advanced Photon Source (APS), one of the brightest and most coherent $x$-ray sources, is therefore ideal for such experiments. Technical objectives for this project include producing: (1) a map of an entire mouse brain at sub-micron resolution ( $100 \mathrm{~nm}$ voxels), where the goal is to map all the cells in a brain, their long-distance projections, and the blood vessels that supply their energy and remove their waste; (2) a map of a "functional module" of a single brain at nanometer scale ( $10 \mathrm{~nm} /$ voxel over a cubic millimeter $\left.\left[\mathrm{mm}^{3}\right]\right)$, where the goal is to find every neuronal connection in that volume; and (3) the creation of both maps in the same brain.

The long-term goal of this project is to provide a "wiring diagram" for brains that will significantly improve our computational models of brain processing and eventually lead to better algorithms for real-world objectives (e.g., face recognition, novel object detection in scenes, etc.) based on how brains process similar information. Similar data will eventually be collected from pathological brains (i.e., autistic or schizophrenic brains) to determine whether "mis-wiring" of neurons contributes to the pathological behavior of those diseases; ultimately, they will serve as targets for therapy. In the more intermediate term, successful pursuit of the project will yield insights into how brains develop and age. Finally, mapping the brain at nanometer resolution will necessitate dealing with large amounts (hundreds to thousands of terabytes' worth) of data and developing a new range of algorithms to analyze and manage such large datasets.

\section{MISSION RELEVANCE}

DOE now is taking a more active role in the broader BRAIN (Brain Research through Advancing Innovative Neurotechnologies) Initiative, a mandate of the Federal Government. This project reflects that role and also fits within the broader DOE mission of supporting large-scale science with new hardware and computing tools.

\section{RESULTS AND ACCOMPLISHMENTS}

We have already demonstrated that we can use the APS to create micron-scale maps of brains where every neuron and blood vessel can be mapped at fast rates amenable to the automatic segmentation analyses of our collaborators. We have demonstrated that the brains imaged by the APS can still be subsequently imaged with automated serial EM, and we are preparing these results for publication.

\section{PROPOSED FUTURE WORK}

In fiscal years 2016 and 2017, we will primarily evaluate the ability of $x$-ray mapping to provide sub-micron resolution maps of entire mouse brains. We will determine whether we can prepare samples of brains that are consistent with both x-ray mapping and subsequent serial EM, and we will work on $x$-ray-specific stains to increase the contrast and imaging speed of x-ray microscopy. In parallel in years 2016 and 2017, we will evaluate whether $\mathrm{x}$-ray mapping can provide nanometer reconstructions 
of smaller volumes of brains in order to identify synaptic connections. We will create mathematical simulations of $x$-ray interactions with stained and embedded brains to determine whether entire brains can be penetrated with x-rays at nanometer resolution or whether we can "thick-slice" brains (at tens of microns in thickness) and then serially image these thick slices and reconstruct neuronal wires over entire brains. We will optimize staining and sample preparation for this approach, as well. By the end of the project, from the lessons learned from previous years, we will be in a position to start imaging large mammalian brains at sub-micron resolution to make mesoscale maps of brains compatible with subsequent serial EM and/or reconstructions of all the neurons and their connections in smaller brains (e.g., the larval zebrafish).

A specific breakdown of our plan of experimentation includes:

$\square$ Determining the optimal size and staining for embedded brains for x-ray microtomography and projection microscopy. We will explore both the thickness of brain samples (ranging from $100 \mu \mathrm{m}$ thick to $2 \mathrm{~mm}$ thick) and the amount of heavy metal (e.g., increasing concentrations or incubation times of osmium) used to stain a brain with subsequent embedding in standard electron microscopy plastic. For each of our samples, we will evaluate different beam conditions (e.g., energy, coherence, etc.) to determine whether x-rays can penetrate these embedded samples with good signal quality.

$\square$ We will investigate a range of embedding media from standard polymers used for electron microscopy to more exotic plastics with different refractiveness and scattering properties for $x$-rays. We will choose a slice thickness and stained protocol from \#1 to investigate a variety of plastics, and we will quantify signal-noise for each of the embedding conditions. In all cases, we will automatically cut serial ultra-thin sections from these embedded samples and ensure that we can cut reliably over long stretches (thousands of sections at 30-nm thickness) and that we see little beam damage from the scanning electron microscope.

$\square$ We will collaborate with the Electron Microscopy Center at Argonne to develop a way, using a focused ion beam, to extract smaller sub-volume samples from a bigger embedded brain. These small-volume samples (about $15 \mu \mathrm{m}^{3}$ ) should be more amenable to nano-x-ray imaging including nano-transmission $\mathrm{x}$-ray microscopy and ptychography. After imaging with nano-x-rays, we will then serial section the same blocks and analyze the same brain material using electron microscopy. Images from the resulting datasets ( $\mathrm{x}$-ray and EM of the same samples) will be analyzed to compare signal-noise ratios, speed of acquisition, and algorithmic tracing of neural processes for both EM and x-ray microscopy.

Points \#1 and \#2 will be in service of our first objective of making a map of an entire mouse brain at submicron resolution and for our third objective of combining such mesoscale maps with EM for nanoscale maps. Point \#3 outlines how we will start thinking about using $x$-rays for the second objective stated in the introduction: creating a nanometer map of a 1-cubic-millimeter $\left(\mathrm{mm}^{3}\right)$ area of a brain. 


\section{PERFORMANCE METRICS}

The LDRD Program Office collects statistical data on current and completed LDRD projects. The data cover various items such as publications (see appendices to this report), inventions (see appendix to this report), follow-on sponsorship, and the enhancement of staff by post-doctoral appointees and new hires. Although these data are of little value on a project-by-project basis, in the aggregate, they do provide a picture of overall program productivity. Summaries of the most recently collected data are provided here in Tables 1 and 2. The first table displays project outcomes realized only during FY 2015 but derived from projects active in any year or years from FY 2012 forward.

The data in Table 1 are subdivided by LDRD Program component. For the same prior and currently active projects, the second table displays those metrics for which cumulative results are monitored, with no restriction as to when the accomplishments occurred.

Table 1. Aggregate FY 2015 Outcomes for Recently Completed and Current Projects

\begin{tabular}{|c|c|c|c|c|c|c|}
\hline $\begin{array}{l}\text { Most Recent } \\
\text { Project Year }\end{array}$ & $\begin{array}{l}\text { Number of } \\
\text { Refereed } \\
\text { Publications }\end{array}$ & $\begin{array}{l}\text { Number of } \\
\text { Students/ } \\
\text { Postdocs } \\
\text { Supported }\end{array}$ & $\begin{array}{c}\text { Number of } \\
\text { New Staff } \\
\text { Hires }\end{array}$ & $\begin{array}{l}\text { Number of } \\
\text { Non-Publication } \\
\text { Copyrights }\end{array}$ & $\begin{array}{l}\text { Number of } \\
\text { Invention } \\
\text { Disclosures }\end{array}$ & $\begin{array}{l}\text { Number } \\
\text { of Patents } \\
\text { Issued/ } \\
\text { Pending }\end{array}$ \\
\hline \multicolumn{7}{|l|}{ FY 2012} \\
\hline Competitive Grants & 3 & 0 & 0 & 0 & 0 & 4 \\
\hline Strategic Initiative & 21 & 0 & 2 & 0 & 6 & 8 \\
\hline \multicolumn{7}{|l|}{ FY 2013} \\
\hline Competitive Grants & 4 & 0 & 0 & 0 & 0 & 4 \\
\hline Strategic Initiative & 22 & 0 & 6 & 0 & 0 & 1 \\
\hline \multicolumn{7}{|l|}{ FY 2014} \\
\hline Competitive Grants & 5 & 0 & 0 & 0 & 3 & 6 \\
\hline Strategic Initiative & 8 & 0 & 4 & 0 & 0 & 0 \\
\hline \multicolumn{7}{|l|}{ FY 2015} \\
\hline Competitive Grants & 23 & 34 & 1 & 0 & 3 & 3 \\
\hline Strategic Initiative* & 90 & 138 & 17 & 1 & 23 & 5 \\
\hline $\begin{array}{l}\text { Grand Totals: } \\
248 \text { Projects }\end{array}$ & 176 & 172 & 30 & 1 & 35 & 31 \\
\hline
\end{tabular}

* Data for the Director's Grand Challenge component is included in the Strategic Initiative data. 
Table 2. Aggregate Outcomes for All Years of Recently Completed and Current Projects (2012-2015)

\begin{tabular}{|c|c|c|c|c|}
\hline $\begin{array}{l}\text { Most Recent } \\
\text { Project Year }\end{array}$ & $\begin{array}{c}\text { Number of } \\
\text { Projects } \\
\text { Receiving } \\
\text { Follow-on Funds }\end{array}$ & $\begin{array}{l}\text { Number of } \\
\text { Proposals } \\
\text { Submitted to } \\
\text { Sponsors }\end{array}$ & $\begin{array}{l}\text { Number of } \\
\text { Intellectual } \\
\text { Property } \\
\text { Events }\end{array}$ & $\begin{array}{c}\text { Number of } \\
\text { External } \\
\text { Reviews }\end{array}$ \\
\hline & & & & $20 \mathrm{DOE}$ \\
\hline \multirow[t]{4}{*}{ FY 2012} & 16 & 138 & 98 & 7 Other \\
\hline & & & & 47 UofC \\
\hline & & & & 74 Total \\
\hline & & & & $10 \mathrm{DOE}$ \\
\hline \multirow[t]{4}{*}{ FY 2013} & 31 & 177 & 49 & 9 Other \\
\hline & & & & 83 UofC \\
\hline & & & & 102 Total \\
\hline & & & & $6 \mathrm{DOE}$ \\
\hline \multirow[t]{4}{*}{ FY 2014} & 12 & 79 & 37 & 2 Other \\
\hline & & & & 20 UofC \\
\hline & & & & 28 Total \\
\hline & & & & $14 \mathrm{DOE}$ \\
\hline \multirow[t]{4}{*}{ FY 2015} & 24 & 158 & 66 & 6 Other \\
\hline & & & & 70 UofC \\
\hline & & & & 90 Total \\
\hline & & & & $50 \mathrm{DOE}$ \\
\hline \multirow[t]{3}{*}{ Totals: 248 Projects } & 83 & 552 & 250 & 24 Other \\
\hline & & & & 220 UofC \\
\hline & & & & 294 Total \\
\hline
\end{tabular}




\section{LDRD PROJECT CHARACTERISTICS}

\section{MISSION RELEVANCE}

Evaluating the relevance of LDRD projects with respect to DOE mission areas is of utmost importance during the review and selection process. All LDRD projects have demonstrable ties to one or more of the science, energy, environment, and national security missions of the U.S. Department of Energy (DOE), and many are also relevant to the missions of other federal agencies that sponsor work at Argonne. Figure 1 represents the number of FY 2015 LDRD projects supporting one or more of the four DOE mission areas.

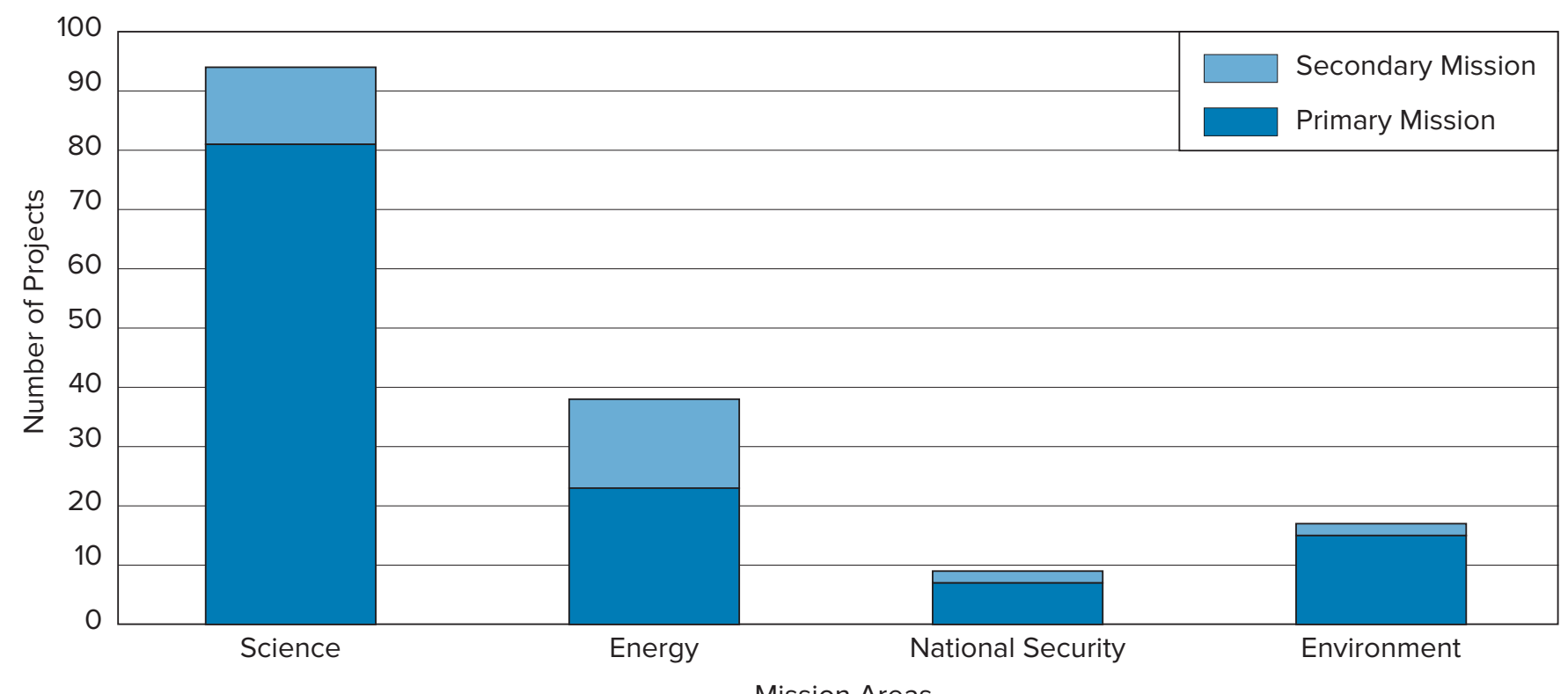

Figure 1. Number of LDRD Projects Supporting the DOE Mission Areas

\section{LEVEL OF FUNDING}

Figure 2 depicts the funding distribution for the 126 projects funded in FY 2015. About 58\% of the projects were in the $\$ 101 \mathrm{~K}$ to $\$ 300 \mathrm{~K}$ range, with a little more than $17 \%$ receiving $\$ 100 \mathrm{~K}$ or less. About $21 \%$ of the projects received between $\$ 301 \mathrm{~K}$ and $\$ 500 \mathrm{~K}$, while only 3\% of projects were in the range of $\$ 501 \mathrm{~K}$ to $\$ 1 \mathrm{M}$. Less than $1 \%$ of projects received more than $\$ 1 \mathrm{M}$. The average funding level of projects in FY 2015 was approximately $\$ 246 \mathrm{~K}$.

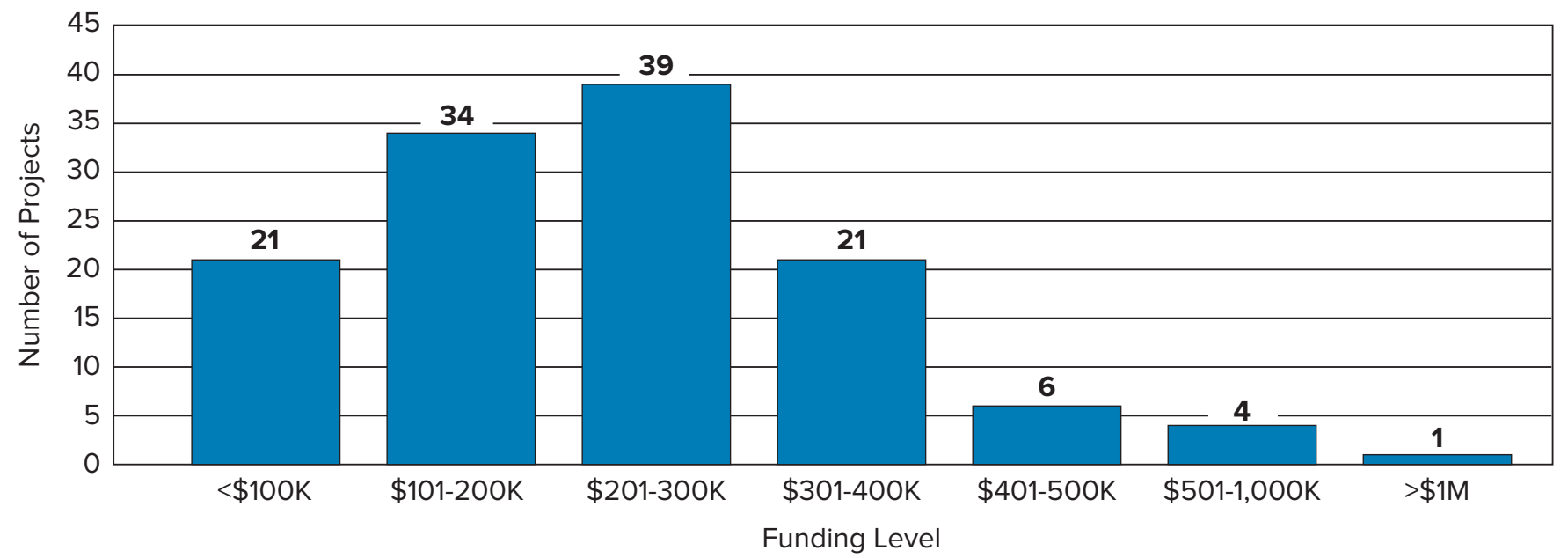

Figure 2. Number of Projects and Levels of Funding 


\section{FY 2016 LDRD PROGRAM UPDATE}

DOE approved Argonne's FY 2016 LDRD Plan and program expenditures not to exceed $\$ 38$.0M. A list of projects begun so far in FY 2016, by component/focus area and title, is provided at the end of this section.

For FY 2016, we have begun implementing changes to the structure of the program components. We have renamed components: the Strategic Initiative component has been renamed "LDRD Prime," and the Director's Competitive Grants component has been renamed "LDRD Innovate." The focus of each component remains the same. All new LDRD Innovate proposal submissions will be restricted to a two-year maximum duration with funding not to exceed $\$ 250 \mathrm{~K}$ per year. LDRD Prime proposals will not be subject to the same restrictions; however, investigators will be strongly encouraged to submit new proposals that do not exceed a two-year duration: exceptions for three-year projects will be granted if a sufficiently strong justification is provided. Also, for FY 2016, we will no longer invest in the Director's Grand Challenge component. We have instead created a new "LDRD Swift" component. LDRD Swift projects will be no more than one year in duration

\section{LDRD PROJECTS BEGUN IN FY 2016}

\section{LDRD INNOVATE}

$\square$ A Missing Protein in the Bacterial Methylmercury Pathway

$\square$ A Novel Gas-Filled Microchannel Plate (GFMCP) X-ray Polarimetry Imager

$\square$ A Theory of Out-of-Equilibrium Phase Transitions

$\square$ Efficient Droplet-Based Environmental Mechanical Energy Harvesting Through Reverse Electrowetting

$\square$ GO-IN-EM - Genetic algorithm Optimization of INterface structure from Electron Microscopy

$\square$ Images from Inner Space: Exposing Quantum Mechanics within Nucleons and Nuclei

$\square$ Nano-mechanical Delivery of Biomolecules into Live Bacterial Cells

$\square$ Ordered Core-shell Nanostructure for Transverse Thermoelectric Applications

$\square$ Perovskite Halide-based IntermediateBand Solar Cells

$\square$ Real-time Monitoring of Material Structure Evolution in Additive Manufacturing Processes

$\square$ Spin Vortex-based Non-volatile Superconducting Memory with funding not to exceed \$150K. LDRD Swift projects will focus more on the applied sciences and target a specific near-term follow-on funding opportunity. LDRD Swift will also include proof-of-concept projects for early career researchers. Finally, beginning in FY 2016, the Argonne Fellowship program is incorporated into LDRD as the LDRD Named Fellows component. The Fellowship program has and will continue to attract highly talented postdoctoral scientists and engineers to Argonne.

In FY 2016, we will continue to refine our use of electronic tools to communicate with investigators and reviewers and to collect and document performance information on LDRD projects. Based on user feedback and other forms of input, we are improving our web-based applications and creating new tools as necessary. One such example is a modification to our online proposal submission system to accept concept papers, that is, brief accounts of ideas for new projects. That enhancement allows easier processing of submissions, linking concept papers to full proposals, if invited, and gives users an online tool to check the status of their submissions.

$\square$ Structure and Dynamics of Chiral Molecules and Radicals

$\square$ Top Down Fabrication of Large Area Monolayers of 2D Materials

\section{LDRD NAMED FELLOWS}

$\square$ Charge Transport in Nanostructured Materials from $a b$-initio Simulations

$\square$ Coherent X-ray Investigations of Defect Dynamics in Next-Generation Nanostructured Materials

$\square$ Core-shell Nanowire Magnetic/Ferroelectric Multiferroic Heterostructure for Voltage Tunable RF Devices

$\square$ In-situ Polarized Spectroscopy of Optically Transparent TRGO-Polymer Solar Cells

$\square$ Investigation of Solid-Liquid Interfaces in Energy Materials Interfacing Multi-scale Modeling with Experimental Characterization

$\square$ The Search for Weyl Semimetals

$\square$ Ultrafast Spectroscopy of Nanometer-scale Heterojunctions Fabricated by Self-assembly

$\square$ Understanding and Controlling Charge, Spin, Pseudospins and Lattice Degrees of Freedom in Layered Transition Metal Dichalcogenides

$\square$ Understanding the Structure of Matter 


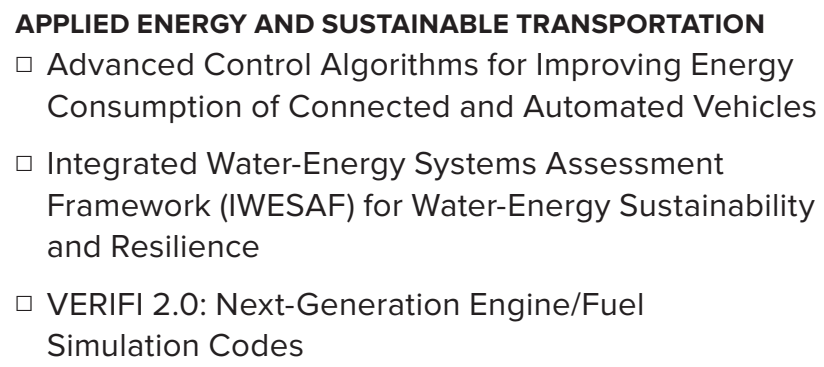

\section{HARD X-RAY SCIENCES}

$\square$ A Conveyer Belt of Nanoliter to Picoliter Droplets for Hard X-ray Pump-probe Experiments

\section{MATERIALS AND MOLECULES TO MANUFACTURING}

$\square$ Managing Emission and Thermal Absorption

$\square$ Supported Single-site Catalysts for Selective Alkane Oxidation

\section{NATIONAL AND GLOBAL SECURITY}

$\square$ A Novel Interferometric Terahertz Phase Imager (THzPI) for National Security Applications

$\square$ Fabrication and Testing of a Borosilicate Microchannel Plate Thermal Neutron Detector with Optimized Geometry

$\square$ New ${ }^{6}$ Li Rich Semiconductors for Neutron Detection

$\square$ Understanding the Resiliency of Interdependent Infrastructures Using Multi-scale Agent-based Simulation

\section{NEXT GENERATION COMPUTING}

$\square$ Data-driven Multiscale Coupled Urban Systems Modeling

$\square$ Event-based Monte Carlo Transport Methods for Next-Generation Node Architectures

$\square$ Re-form: Leveraging FPGA Reconfigurability and Floating-point Capabilities for Next-generation Computing Systems

\section{NUCLEAR ENERGY SCIENCE AND TECHNOLOGY}

$\square$ Additive Manufacturing for Nuclear Energy Applications

$\square$ Development of Analysis Methods for Non-Destructive Evaluation of Concrete Degradation in Light Water Reactors

$\square$ Developing a Program for the Production of Medical Isotopes using the Argonne Electron LINAC

\section{UNIVERSE AS THE LAB (ULAB)}

$\square$ Cross-Correlating Ground-based Optical Surveys with the Cosmic Microwave Background

$\square$ Data-Intensive Computing and the Cosmic Frontier

$\square$ Detectors for CMB experiments

$\square$ Hydrodynamic Simulations with HACC on Next Generation Supercomputers

$\square$ Superconducting Materials for Using the Universe As Our Lab

$\square$ Synthetic Sky Catalogs for Space Based Cosmology Missions

\section{OTHER NOVEL R\&D}

$\square$ Development of a Pre-Conceptual Design of a New Tracking System for CLAS12 Detector

$\square$ Highly Anisotropic Magnetic Materials for Microwave Applications

$\square$ Hybrid Silicon Nanolasers

$\square$ New Thin Film Oxide, Chalcogenide and Oxy-chalcogenide Materials Discovery

$\square$ Plasmonic Grating-Launched Nanoscale Light Source for Optical Spectroscopy on Atomically Resolved Systems 


\section{APPENDICES}




\section{INTELLECTUAL PROPERTY EVENTS OCCURING IN FY 2015}

(As a Result of LDRD Projects and Subsequent Related Sponsored Research) Invention Disclosures

\section{5-147}

PATENT APPLICATION

Rajh, T., N. Dimitrijevic, E. Rozhkova, B. Grybowski, H. Fry, and T. Koritarov. "Use of Metal Oxide Nanoparticles for Cancer.” Patent application filed April 2015. [ANL-IN-10-079] [Also see 2012-181.]

\section{6-075}

\section{PATENT GRANTED}

Pellin, M., H.-H. Wang, K. Byrum, H. Frisch, S. Lee, and J. Elam. “Micro-channel Plate Detector.” Patent No. 9,139,905 granted September 2015. [ANL-IN-09-017]

\section{6-149}

\section{INVENTION DISCLOSURE}

Elam, J., S. Darling, Y.-C. Tseng, and Q. Peng. "Sequential Infiltration Synthesis for Advanced Lithography." Invention report disclosed March 2015. [ANL-IN-10-106B]

\section{PATENT APPLICATION}

Elam, J., S. Darling, Y.-C. Tseng, and Q. Peng. "Sequential Infiltration Synthesis for Advanced Lithography." Patent Application No. 14/645,162 filed March 2015. [ANL-IN-10-106B]

\section{PATENTS GRANTED}

Elam, J., A. Martinson, and M. Pellin. "Atomic Layer Deposition of Metal Sulfide Thin Films Using Non-halogenated Precursors.” Patent No. 9,040,113 granted May 2015. [ANL-IN-08-085]

Elam, J., and A. Yanguas-Gil. "Doping Control in ALD by Surface Functionalization." Patent No. 8,951,615 granted February 2015. [ANL-IN-10-028]

Elam, J., S. Darling, Y.-C. Tseng, and Q. Peng. "Sequential Infiltration Synthesis for Advanced Lithography." Patent No. 8,980,418 granted March 2015. [ANL-IN-10-106B]

\section{7-044}

\section{INVENTION DISCLOSURE}

Liu, D.-J., G. Boenaga, and S. Yuan. "Electrocatalysts Using Porous Polymers and Method of Preparation.” Invention report disclosed March 2015. [ANL-IN-09-109B] [Also see 2010-158.]

\section{PATENT APPLICATION}

Liu, D.-J., G. Boenaga, and S. Yuan. "Electrocatalysts Using Porous Polymers and Method of Preparation." Patent Application No. 14/659,330 filed March 2015. [ANL-IN-09-109B] [Also see 2010-158.]

\section{PATENT GRANTED}

Liu, D.-J., and S. Yuan. "Electrocatalysts Using Porous Polymers and Method of Preparation." Patent No. 9,012,344 granted April 2015. [ANL-IN-09-109] [Also see 2010-158.] 


\section{8-195}

\section{INVENTION DISCLOSURE}

Lopez, O., G. Wiederrecht, D. Gosztola, and D. Mancini. "Microelectromechanical (MEMS) Manipulators for Control of Nanoparticle Coupling Interactions." Invention report disclosed December 2014. [ANL-IN-09-050B]

\section{PATENT APPLICATION}

Lopez, O., G. Wiederrecht, D. Gosztola, and D. Mancini. "Microelectromechanical (MEMS) Manipulators for Control of Nanoparticle Coupling Interactions." Patent application filed December 2014. [ANL-IN-09-050B]

\section{PATENT GRANTED}

Lopez, D., D. Mancini, G. Wiederrecht, and D. Gosztola. "Microelectromechanical (MEMS) Manipulators for Control of Nanoparticle Coupling Interactions." Patent No. 8,922,094 granted December 2014. [ANL-IN-09-050]

\section{9-115}

\section{PATENT APPLICATION}

Li, J., and Y. Liu. "Method for Fluidizing and Coating Ultrafine Particles, System for Coating Ultrafine Particles." Patent Application No. 14/581,154 filed December 2014. [ANL-IN-11-048B]

\section{PATENT GRANTED}

Li, J., and Y. Liu. "Method for Fluidizing and Coating Ultrafine Particles, System for Coating Ultrafine Particles." Patent No. 8,936,831 granted January 2015. [ANL-IN-09-048]

\section{0-074}

\section{PATENT GRANTED}

Chapman, K., B. Shyam, K. Wiaderek, O. Borkiewicz, P. Chupas, and C. Kurtz. "Transmission-Geometry

Electromechanical Cell for In Situ Scattering and Spectroscopy Investigations." Patent No. 9,022,652 granted May 2015. [ANL-IN-12-028]

\section{0-117}

\section{PATENT GRANTED}

Wang, J., D. Mukhopadhyay, I.-W. Jung, G. Shenoy, D. Walko, and O.D. Lopez. "Method for Spatially Modulating X-ray Pulses Using MEMS-based X-ray Optics." Patent No. 8,976,933 granted March 2015. [ANL-IN-11-021]

\section{0-138}

\section{PATENT GRANTED}

Islam, Z., R. Das, and R. Weinstein. "Dual Stage Trapped-Flux Magnet Cryostat for Measurements at High Magnetic Fields.” Patent No. 9,007,058 granted April 2015. [ANL-IN-11-075]

\section{0-158}

\section{INVENTION DISCLOSURE}

Liu, D.-J., G. Goenaga, and S. Yuan. "Electrocatalysts Using Porous Polymers and Method of Preparation." Invention report disclosed March 2015. [ANL-IN-09-109B] [Also see 2007-044.]

\section{PATENT APPLICATIONS}

Liu, D.-J. "Alkaline Fuel Cell Non-Platinum Group Metal Electrocatalysts Using Metal Organic Framework Materials and Method of Preparation." Patent Application No. 14/634,581 filed February 2015. [ANL-IN-14-079] [Also see 2014-051.]

Liu, D.-J., G. Boenaga, and S. Yuan. "Electrocatalysts Using Porous Polymers and Method of Preparation." Patent Application No. 14/659,330 filed March 2015. [ANL-IN-09-109B] [Also see 2007-044.] 


\section{PATENT GRANTED}

Liu, D.-J., and S. Yuan. "Electrocatalysts Using Porous Polymers and Method of Preparation." Patent No. 9,012,344 granted April 2015. [ANL-IN-09-109] [Also see 2007-044.]

\section{0-185}

\section{INVENTION DISCLOSURE}

Amine, K., L. Curtiss, J. Lu, K.C. Lau, Z. Zhang, Y.-K. Sun. “LI-Air Batteries Having Ether-based Electrolytes.” Invention report disclosed February 2015. [ANL-IN-11-129B]

\section{PATENT APPLICATION}

Amine, K., L. Curtiss, J. Lu, K.C. Lau, Z. Zhang, Y.-K. Sun. "LI-Air Batteries Having Ether-based Electrolytes.” Patent Application No.14/633,408 filed February 2015. [ANL-IN-11-129B]

\section{PATENTS GRANTED}

Amine, K., and Z. Chen. "Lithium Air Cells Using Polyethylene Oxide Based Non-Aqueous Electrolytes." Patent No. 9,130,241 granted September 2015. [ANL-IN-07-060B]

Amine, K., L. Curtiss, Y.-K. Sun, Z. Zhang, K.C. Lau, and J. Lu. "Li-Air Batteries Having Ether-based Electrolytes." Patent No. 8,968,941 granted March 2015. [ANL-IN-11-129]

Amine, K., P. Du, J. Lu, Y. Lei, and J. Elam. "Coating of Porous Carbon for Improving the Efficiency of Li-Air Battery." Patent No. 9,005,816 granted April 2015. [ANL-IN-12-058]

\section{0-191}

\section{INVENTION DISCLOSURES}

Krumdick, G., A. Kiricova, and T. Dzwiniel. "Advanced Wetting Process for Battery Cell Production." Invention disclosure filed February 2015. [ANL-IN-15-007]

Krumdick, G., T. Dzwiniel, and K. Pupek. "Process for the Production of High Voltage Electrolyte Solvents for Li-lon Batteries." Invention disclosure filed February 2015. [ANL-IN-15-015]

Krumdick, G., T. Dzwiniel, and K. Pupek. “Fluorinated Electrolyte Solvent.” Invention disclosure filed March 2015. [ANL-IN-15-018]

Krumdick, G., T. Dzwiniel, and K. Pupek. “Supramolecular Binder for Li-Ion Battery Si Anode.” Invention disclosure filed June 2015. [ANL-IN-15-059]

Krumdick, G., and A. Kiricova. "Advanced Wet Coating Process." Invention disclosure filed August 2015. [ANL-IN-15-094]

\section{PATENTS GRANTED}

Krumdick, G., K. Pupek, and T. Dzwiniel. "Process for Producing Redox Shuttles.” Patent No. 8,921,611 granted December 2014. [ANL-IN-039]

Krumdick, G., K. Pupek, and T. Dzwiniel. “Method for Producing Redox Shuttles.” Patent No. 8,969,625 granted March 2015. [ANL-IN-11-105]

\section{0-194}

\section{PATENT APPLICATION}

Campos Ortega, E., and R. Ware. "Method and System for Icing Condition Detection." Patent application filed December 2014. [ANL-IN-14-051] 


\section{0-203}

\section{PATENT APPLICATION}

Jansen, A., F. Brushett, and J. Vaughey. "Quinoxaline and Its Derivatives as Redox Species for Aqueous Flow Batteries." Patent application filed February 2015. [ANL-IN-12-125]

\section{1-012}

\section{PATENT GRANTED}

Lewellen, J., D. Walters, J. Noonan, and M. Virgo. “Electron Linac for Medical Isotope Production.” Patent No. 9,129,714 granted September 2015. [ANL-IN-10-001]

\section{1-116}

\section{PATENTS GRANTED}

Johnson, C., and S.-H. Kang. “Electrode Materials for Rechargeable Battery.” Patent No. 9,126,844 granted September 2015. [ANL-IN-10-031B]

Johnson, C., Y. Kim, and E. Lee. “Sodium Chalcogenide Electrodes for Sodium Batteries." Patent No. 8,906,542 granted December 2014. [ANL-IN-12-082]

\section{1-193}

\section{PATENT APPLICATION}

Vajda, S. "Subnanometer to Nanometer Pt-based Mono and Multicomponent CO Oxidation Catalyst." Patent application filed September 2015. [ANL-IN-14-039]

\section{2-012}

\section{INVENTION DISCLOSURE}

Abouimrane, A., and K. Amine. "Electrode Materials for Rechargeable Batteries." Invention disclosure filed March 2015. $[\mathrm{ANL}-\mathrm{IN}-10-110 \mathrm{~B}]$

\section{PATENT APPLICATION}

Abouimrane, A., and K. Amine. "Electrode Materials for Rechargeable Batteries." Patent Application No. 14/639,917 filed March 2015. [ANL-IN-10-110B]

\section{PATENT GRANTED}

Abouimrane, A., and K. Amine. "Electrode Materials for Rechargeable Batteries." Patent No. 9,005,808 granted April 2015. [ANL-IN-10-110]

\section{2-072}

\section{INVENTION DISCLOSURE}

Sumant, A., D. Berman, and A. Erdemir. "Graphene as a Protective Coating and Best Lubricant for Electrical Contacts." Invention report disclosed October 2014. [ANL-IN-14-105]

\section{PATENT APPLICATION}

Sumant, A., D. Berman, J. Choi, and A. Erdemir. "Superlubricating Graphene and Graphene Oxide Films." Patent Application No. 14/415,499 filed January 2015. [ANL-IN-11-056]

\section{2-087}

\section{PATENT APPLICATION}

Timofeeva, E., J. Kastoudas, D. Singh, C. Segre, and J. Terry. "Radiolysis Mediated Charging of Nanoelectrofuels from Different Sources of Radiation.” Patent application filed January 2015. [ANL-IN-14-036] 


\section{2-181}

\section{PATENT APPLICATION}

Rajh, T., N. Dimitrijevic, E. Rozhkova, B. Grybowski, H. Fry, and T. Koritarov. "Use of Metal Oxide Nanoparticles for Cancer." Patent application filed April 2015. [ANL-IN-10-079] [Also see 2005-147.]

\section{3-096}

\section{INVENTION DISCLOSURE}

Sumant, A., and R. Gulotty. "Direct Synthesis of Reduced Graphene Oxide Films on Dielectric Substrates.” Invention report disclosed January 2015. [ANL-IN-14-110]

\section{PATENT APPLICATION}

Sumant, A., and R. Gulotty. "Direct Synthesis of Reduced Graphene Oxide Films on Dielectric Substrates." Patent application filed May 2015. [ANL-IN-14-110]

\section{3-116}

\section{INVENTION DISCLOSURE}

Pellin, M., A. Yacout, S. Bhattacharya, and D. Sedimann. "Intermetallic Formation from High Enthalpy Coatings." Invention disclosure filed December 2014. [ANL-IN-14-093] [Also see 2013-152.]

\section{PATENT APPLICATION}

Pellin, M., A. Yacout, S. Bhattacharya, and D. Sedimann. "Intermetallic Formation from High Enthalpy Coatings." Patent application filed April 2015. [ANL-IN-14-093] [Also see 2013-152.]

\section{3-152}

\section{INVENTION DISCLOSURE}

Pellin, M., A. Yacout, S. Bhattacharya, and D. Sedimann. "Intermetallic Formation from High Enthalpy Coatings." Invention disclosure filed December 2014. [ANL-IN-14-093] [Also see 2013-116.]

\section{PATENT APPLICATION}

Pellin, M., A. Yacout, S. Bhattacharya, and D. Sedimann. "Intermetallic Formation from High Enthalpy Coatings." Patent application filed April 2015. [ANL-IN-14-093] [Also see 2013-116.]

\section{3-154}

\section{INVENTION DISCLOSURES}

Elam, J., J. Libera, A. Mane, and A. Yanguas-Gil. "Chemical Vapor Processing for Additive Manufacturing." Invention disclosure filed October 2014. [ANL-IN-14-074] [Also see 2015-151.]

Libera, J., and J. Elam. “A Batch Reader for Industrial Scale Atomic Layer Deposition onto High Surface Area Powder.” Invention report disclosed January 2015. [ANL-IN-15-001]

Libera, J., A. Yanguas-Gil, A. Mane, and J. Elam. "Refractory Solar Selective Coatings." Invention disclosure filed March 2015. [ANL-IN-15-016] [Also see 2015-151.]

Elam, J., T. Seidel, and A. Mane. "Silicon Doping Source Films by ALD Deposition.” Invention disclosure filed August 2015. [ANL-IN-15-087]

\section{PATENT APPLICATION}

Elam, J., A. Mane, and T. Seidel. "Silicon Doping Source Films by ALD Deposition.” Patent application filed June 2015. [ANL-IN-15-087] 


\section{3-156}

\section{INVENTION DISCLOSURE}

Brotzman, R., E. Rabe, M. Urgun Demirtas, X. Chen, and P. Ignacio de Leon. "Magnetic Nanotube Composite Membranes." Invention report disclosed April 2015. [ANL-IN-15-030]

\section{3-208}

\section{INVENTION DISCLOSURE}

Murphy-Olson, D., S. Boisvert, and R. Aydelott. "Betterclouds." Invention disclosure filed September 2015.

[ANL-IN-15-073]

\section{4-004}

\section{INVENTION DISCLOSURE}

Ellis, R. "Magnetically Enhanced Phase Separation for Solvent Extraction." Invention report disclosed February 2015.

[ANL-IN-15-014]

\section{PATENT APPLICATION}

Ellis, R. "Magnetically Enhanced Phase Separation for Solvent Extraction." Patent application filed July 2015.

[ANL-IN-15-014]

\section{4-051}

\section{PATENT APPLICATION}

Liu, D.-J. "Alkaline Fuel Cell Non-Platinum Group Metal Electrocatalysts Using Metal Organic Framework Materials and Method of Preparation." Patent Application No. 14/634,581 filed February 2015. [ANL-IN-14-079] [Also see 2010-158.]

\section{4-137}

\section{INVENTION DISCLOSURE}

Welp, U., I. Kesgin, C. Doose, M. Kasa, and Y. Ivanyushenkov. "A New Method for Continuous Winding of REBCO Coated Conductor Magnets with No Resistive Joints.” Invention report disclosed May 2015. [ANL-IN-15-054]

\section{4-160}

\section{NON-PUBLICATION COPYRIGHT}

Beckman, P., R. Sankaran, and C. Catlett. “WAGGLE Software.” Software copyright issued August 2015. [ANL-SF-15-099]

\section{4-169}

\section{INVENTION DISCLOSURES}

Chen, X., and Z. Zhou. "Tunable Broadband Microwave Absorber." Invention report disclosed October 2014.

[ANL-IN-14-097]

Chen, X., and Z. Zhou. "RF Energy Harvesting with Magnetodielectric Composite Substrate." Invention report disclosed October 2014. [ANL-IN-14-098]

Chen, X., and R. Stoddard. "Image Processing Tool for Automatic Feature Recognition and Quantification." Invention report disclosed October 2014. [ANL-IN-14-107]

Chen, X., Z. Zhou, G. Grocke, and V. Vajda. "Integrated System for High Aspect Ratio Nanofiber Production." Invention report disclosed November 2014. [ANL-IN-14-109]

Chen, X. "Field Controlled Direct-write Electrospinning." Invention report disclosed October 2014. [ANL-IN-14-111] 


\section{PATENT APPLICATIONS}

Chen, X., Z. Zhou, G. Grocke, and V. Vajda. "Integrated System for High Aspect Ratio Nanofiber Production." Patent application filed March 2015. [ANL-IN-14-109]

Chen, X., and R. Stoddard. "Image Processing Tool for Automatic Feature Recognition and Quantification." Patent application filed May 2015. [ANL-IN-14-107]

Chen, X. "Field Controlled Direct-write Electrospinning." Patent application filed July 2015. [ANL-IN-14-111]

\section{4-174}

\section{INVENTION DISCLOSURE}

Heald, M. “Freckles-Scheme for Labeling Everything." Invention disclosure filed August 2015. [ANL-IN-15-091]

\section{4-185}

\section{INVENTION DISCLOSURE}

Johnson, C., and M. Peer. "Carbonaceous Negative Electrodes for Sodium Batteries." Invention disclosure filed July 2015. [ANL-IN-15-078]

\section{4-187}

\section{INVENTION DISCLOSURE}

Darling, S., J. Libera, and J. Elam. "Hydrophilic, Visible-Light-Active Conformal Coatings for Membranes." Invention report disclosed August 2015. [ANL-IN-15-102]

\section{5-121}

\section{INVENTION DISCLOSURE}

Li, J., R. Muehleisen, P. Wang, and Y. Sun. "Continuous Flow Synthesis of $\mathrm{VO}_{2}$ Nanoparticles or Nanorods by Using a Capillary-Based Microreactor.” Invention disclosure filed May 2015. [ANL-IN-15-033]

\section{5-132}

\section{INVENTION DISCLOSURE}

Krumdick, G., and O. Kahvencioglu-Feridun. "Pressurized Taylor Vortex Reactor with Capillary Pressure Let Down Apparatus." Invention disclosure filed June 2015. [ANL-IN-15-082]

\section{5-151}

\section{INVENTION DISCLOSURES}

Elam, J., and A. Mane. "Atomic Layer Writer (ALW)." Invention disclosure filed April 2015. [ANL-IN-15-031]

Elam, J., J. Libera, A. Mane, and A. Yanguas-Gil. "Chemical Vapor Processing for Additive Manufacturing." Invention disclosure filed October 2014. [ANL-IN-14-074] [Also see 2013-154.]

Libera, J., A. Yanguas-Gil, A. Mane, and J. Elam. "Refractory Solar Selective Coatings." Invention disclosure filed March 2015. [ANL-IN-15-016] [Also see 2013-154.]

\section{5-153}

\section{INVENTION DISCLOSURE}

Preissner, C., D. Cine, C. Jacobsen, J. Maser, B. Lai, C. Roehrig, O. Schmidt, and S. Vogt. "Ultra-high Stability Long-vertical Travel Stage.” Invention disclosure filed September 2015. [ANL-IN-15-072] 
2015-161

\section{INVENTION DISCLOSURES}

Conley, R., and D. Windt. "Dynamic Aperture for Three-Dimensional Control of Thin-Film Deposition and Ion-Beam Erosion." Invention disclosure filed August 2015. [ANL-IN-15-022]

Conley, R., S. Izzo, L. Assoufid, M. Erdmann, J. Qian, and E. Kasman. "Method for Precision Surface Measurement in Vacuum." Invention disclosure filed April 2015. [ANL-IN-15-024]

\section{PATENT APPLICATION}

Conley, R., and D. Windt. "Dynamic Aperture for Three-Dimensional Control of Thin-Film Deposition and Ion-Beam Erosion." Patent application filed August 2015. [ANL-IN-15-022] 


\section{PUBLICATIONS AND PRESENTATIONS I}

(Derived from LDRD Projects Active in Fiscal Year 2015)

\section{DIRECTOR'S COMPETITIVE GRANTS}

\section{3-016-R3}

\section{REFEREED PUBLICATIONS}

Bai, L., P. Hyde, Y.S. Gui, C.-M. Hu, V. Vlaminck, J.E. Pearson, S.D. Bader and A. Hoffmann (2013). "Spin Pumping in the Nonlinear Dynamics Regime Analyzed Using a Symmetry-Based Method.” Physical Review Letters 111(217602).

Hoffmann, A. (2013). “Spin Hall Effects in Metals.” IEEE Transactions on Magnetics 49(10): 5172-5193.

Houchen, C., L. Peng, Z. Wei, L. Tao, A. Hoffmann, D. Longjiang and W. Mingzhong (2014). "Nanometer-Thick Yttrium Iron Garnet Films With Extremely Low Damping." IEEE Magnetics Letters 5: 1-4.

Jungfleisch, M.B., W. Zhang, W. Jiang and A. Hoffmann (2016). "New Pathways Towards Efficient Metallic Spin Hall Spintronics." SPIN 05(03): 1530005. (Also see 2014-018.)

Li, S., W. Zhang, J. Ding, J.E. Pearson, V. Novosad and A. Hoffmann. "Epitaxial Patterning of Nanometer-thick $\mathrm{Y}_{3} \mathrm{Fe}_{5} \mathrm{O}_{12}$ Films with Low Magnetic Damping." Nanoscale. (To be published.)

Liu, T., H.C. Chang, V. Vlaminck, Y.Y. Sun, M. Kabatek, A. Hoffmann, L.J. Deng and M.Z. Wu (2014). "Ferromagnetic Resonance of Sputtered Yttrium Iron Garnet Nanometer Films.” Journal of Applied Physics 115(17).

Schultheiss, H., J.E. Pearson, S.D. Bader and A. Hoffmann (2012). "Thermoelectric Detection of Spin Waves." Physical Review Letters 109(23): 237204.

Sklenar, J., W. Zhang, M.B. Jungfleisch, W. Jiang, H. Chang, J.E. Pearson, M. Wu, J.B. Ketterson and A. Hoffmann (2016). "Driving and Detecting Ferromagnetic Resonance in Insulators with the Spin Hall Effect." Physical Review Letters 92(17): 174406. (Also see 2014-018.)

Vlaminck, V., H. Schultheiss, J.E. Pearson, F.Y. Fradin, S.D. Bader and A. Hoffmann (2013). "Mapping Microwave Field Distributions via the Spin Hall Effect." Applied Physics Letters 102(19): 252406.

Wu, S.M., F.Y. Fradin, J. Hoffman, A. Hoffmann and A. Bhattacharya (2015). "Spin Seebeck Devices Using Local On-chip Heating." Journal of Applied Physics 117(17).

Zhang, W., M.B. Jungfleisch, F. Freimuth, W. Jiang, J. Sklenar, J.E. Pearson, J.B. Ketterson, Y. Mokrousov and A. Hoffmann (2016). "All-electrical Manipulation of Magnetization Dynamics in a Ferromagnet by Antiferromagnets with Anisotropic Spin Hall Effects.” Physical Review B: Condensed Matter 92(14).

Zhang, W., M.B. Jungfleisch, W.J. Jiang, Y.H. Liu, J.E. Pearson, S.G.E. te Velthuis, A. Hoffmann, F. Freimuth and Y. Mokrousov (2015). "Reduced Spin Hall Effects from Magnetic Proximity." Physical Review B: Condensed Matter 91(11): 115316.

Zhang, W., M.B. Jungfleisch, W. Jiang, J.E. Pearson, A. Hoffmann, F. Freimuth and Y. Mokrousov (2015). "Spin Hall Effects in Metallic Antiferromagnets." Physical Review Letters 113(19): 196602.

Zhang, W., M.B. Jungfleisch, W. Jiang, J.E. Pearson and A. Hoffmann (2015). "Spin Pumping and Inverse Rashba-Edelstein Effect in NiFe/Ag/Bi and NiFe/Ag/Sb.” Journal of Applied Physics 117(17): 17C727-717C730. 
Zhang, W., M.B. Jungfleisch, W. Jiang, J. Sklenar, F.Y. Fradin, J.E. Pearson, J.B. Ketterson and A. Hoffmann (2015). "Spin Pumping and Inverse Spin Hall Effects-Insights for Future Spin-orbitronics." Journal of Applied Physics 117(17): $172610-172615$.

Zhang, W., V. Vlaminck, J.E. Pearson, R. Divan, S.D. Bader and A. Hoffmann (2014). "Determination of Pt Spin Diffusion Length by Spin-Pumping and Spin Hall Effect." Applied Physics Letters 103(24): 242414.

\section{NON-REFEREED PUBLICATIONS}

Hoffmann, A., H. Schultheiss, S. Vogt, S. Jain, F.Y. Fradin, S. Bader, Z. Wang, Y. Sun, Y.-T. Song, M. Wu, T. Sebastian and B. Hillebrands (2014). "Electric Control and Detection of Spin Waves." Concepts in Spintronics, Santa Barbara, CA, September 30-October 4, 2013. (Also see 2014-018.)

Hoffmann, A., W. Zhang, V. Vlaminck, J.E. Pearson, S.D. Bader, R. Divan, L. Bai, P. Hyde, Y. Gui and C.-M. Hu (2014). "Insight About Spin Hall Effects from Spin Pumping." SPIE NanoScience+Engineering, San Diego, CA, August 17-24, 2014. (Also see 2014-018.)

Sola, A., M. Kuepferling, V. Basso, S. Zullino, E. Olivetti, M. Pasquale, T. Kikkawa, K. Uchida, E. Saitoh, D. Meier, T. Kuschel, G. Reiss, H. Jin, S. Boona, S. Watzman, J. Heremans, W. Zhang, M. B. Jungfleisch, J.E. Pearson, A. Hoffmann and H.W. Schumacher (2014). "Round Robin Comparison of the Spin-seebeck Coefficient: Results and Outlook." Spin Caloritronics VI, Isree, Germany, July 14-18, 2014. (Also see 2014-018.)

Zhang, W., V. Vlaminck, J.E. Pearson, R. Divan, S.D. Bader and A. Hoffmann (2014). "Determination of the Spin Diffusion Length via Spin Pumping and Spin Hall Effects." American Physical Society March Meeting, Denver, CO, March 3-7, 2014. (Also see 2014-018.)

Zhang, W., V. Vlaminck, J.E. Pearson, R. Divan, S.D. Bader and A. Hoffmann (2014). "Quantifying Spin Diffusion Lengths from Spin Pumping and Spin Hall Effects." Intermag 2014, Dresden, Germany, May 4-8, 2014. (Also see 2014-018.)

\section{PRESENTATIONS}

Chang, H., P. Li, W. Zhang, T. Liu, A. Hoffmann, L. Deng and M. Wu (2015). "Nanometer-Thick Yttrium Iron Garnet Films with Extremely Low Damping." 59th Annual Magnetism and Magnetic Materials Conference, Honolulu, HI, November 3-7, 2014.

Hoffmann, A., W. Zhang, V. Vlaminck, M.B. Jungfleisch, W. Jiang, J.E. Pearson, S.D. Bader, R. Divan, L. Bai and P. Hyde (2015). "Insights About Spin Hall Effects from Spin Pumping." 59th Annual Magnetism and Magnetic Materials Conference, Honolulu, HI, November 3-7, 2014.

Hoffmann, A. (2015). "Magnetism at the Mesoscale: Harnessing Competing Interactions." University of California, Davis, CA, February 12, 2015. (Also see 2014-018 and 2014-054.)

Hoffmann, A. (2015). "Magnetism at the Mesoscale: Harnessing Competing Interactions." Fudan University, Shanghai, China, January 30, 2015. (Also see 2014-018 and 2014-054.)

Hoffmann, A. (2015). "Magnetism at the Mesoscale: Harnessing Competing Interactions." University of California-San Diego, La Jolla, CA, June 4, 2015. (Also see 2014-018 and 2014-054.)

Hoffmann, A. (2015). "Magnetism at the Mesoscale: Harnessing Competing Interactions." University of California, Riverside, CA, June 5, 2015. (Also see 2014-018 and 2014-054.)

Hoffmann, A. (2015). "Magnetism at the Mesoscale: Harnessing Competing Interactions." Nanjing University, Nanjing, China, May 20, 2015. (Also see 2014-018 and 2014-054.)

Hoffmann, A. (2015). "Magnetism at the Mesoscale: Harnessing Competing Interactions." 2015 American Vacuum Society Prairie Chapter Symposium, South Bend, IN, September 10, 2015. (Also see 2014-018 and 2014-054.)

Hoffmann, A. (2015). "Magnetism at the Mesoscale: Harnessing Competing Interactions." California State University, Long Beach, CA, September 16, 2015. (Also see 2014-018 and 2014-054.) 
Hoffmann, A., W. Zhang, J. Sklenar, M.B. Jungfleisch, W. Jiang, H. Chang, B. Hsu, F.Y. Fradin, Y. Liu and J.E. Pearson (2015). "Magnetization Dynamics Driven by Spin Hall Effects." International Colloquium on Magnetic Films and Surfaces, Cracow, Poland, July 12-17, 2015. (Also see 2014-018.)

Hoffmann, A., W. Zhang, M.B. Jungfleisch, W. Jiang, Y. Liu, J.E. Pearson, S.G.E. te Velthuis, F. Freimuth and Y. Mokrousov (2015). "Spin Hall Effects in Magnetically Ordered Materials." Recent Trends in Nanomagnetism, Spintronics, and their Application, San Sebastian, Spain, June 30-July 3, 2015. (Also see 2014-054.)

Hoffmann, A. (2015). "Spin Hall Effects in Metals.” 2015 Spinlcur Summer School, Braga, Portugal, September 7-11, 2015. (Also see 2014-054.)

Hoffmann, A. (2015). "Spin Hall Effects: From Spin Current Detection to Manipulation of Skyrmions." SLAC National Accelerator Laboratory, Menlo Park, CA, December 5, 2014. (Also see 2014-054.)

Hoffmann, A. (2015). "Spin Hall Effects: From Spin Current Detection to Manipulation of Skyrmions." New York University, New York, NY, June 2, 2015.

Hoffmann, A. (2015). "Spin Hall Effects: From Spin Current Detection to Manipulation of Skyrmions." Bar-llan University, Ramat Gan, Israel, May 7, 2015. (Also see 2014-054.)

Hoffmann, A. (2014). "Connecting Spin Waves to Charge Currents." International Workshop on Nanoscale Spectroscopy and Nanotechnology 8, Chicago, IL, July 28-31, 2014. (Also see 2014-018.)

Hoffmann, A. (2014). "Connecting Spin Waves to Charge Currents." Ohio State University, Columbus, OH, November 21, 2013. (Also see 2014-018.)

Hoffmann, A. (2014). "Magnetization Dynamics in Nanometer-thick Yttrium Iron Garnet Films." Workshop on Oxide Materials: Nanoscale Spectroscopy and Nanotechnology, Cali, Columbia, September 15-19, 2014. (Also see 2014-018.)

Hoffmann, A. (2014). "Reduction of Spin Hall Effects Due to Magnetic Proximity." Spin Caloritronics VI, Isree, Germany, July 14-18, 2014. (Also see 2014-018.)

Jungfleisch, M.B., W. Zhang, W. Jiang, F.Y. Fradin, J.E. Pearson and A. Hoffmann (2015). “DC Voltage Generation in a Pure Permalloy Layer Under Ferromagnetic Resonance." 59th Annual Magnetism and Magnetic Materials Conference, Honolulu, HI, November 3-7, 2014. (Also see 2013-018.)

Schultheiss, H., J.E. Pearson, S.D. Bader and A. Hoffmann (2013). "Thermoelectric Detection of Spin Waves." 12th Joint Magnetism and Magnetic Materials (MMM)/Intermag Conference, Chicago, IL, January 14-18, 2013.

Sklenar, J., J.B. Ketterson, M.B. Jungfleisch, W. Zhang, W. Jiang, J.E. Pearson and A. Hoffmann (2015). "DC Rectification of Microwaves in Yttrium Iron Garnet/Pt/Py Trilayers." American Physical Society March Meeting, San Antonio, TX, March 2-6, 2015.

Zhang, W., M.B. Jungfleisch, W. Jiang, J.E. Pearson and A. Hoffmann (2015). "Spin Hall Effect in Mn-based Metallic Antiferromagnets." 59th Annual Magnetism and Magnetic Materials Conference, Honolulu, HI, November 3-7, 2014.

Zhang, W., B. Jungfleisch, W. Jiang, J. Pearson, A. Hoffmann, F. Freimuth and Y. Mokrousov (2015). "Spin Hall Effects in CuAu-I-type Metallic Antiferromagnets." American Physical Society March Meeting 2015, San Antonio, TX, March 2-6, 2015.

2013-022-R2

REFEREED PUBLICATION

Burgo, T.A. and A. Erdemir (2014). "Bipolar Tribocharging Signal During Friction Force Fluctuations at Metal-insulator Interfaces." Angewandte Chemie 53(45): 12101-12105. 


\section{PRESENTATIONS}

De Lima Burgo, T., A. Erdemir, O. Eryilmaz and J.G. Ramirez Gonzalez (2014). "Tribocurrent-like Friction Force at a Metal-Insulator Interfaces." 2014 Annual Meeting of the Electrostatistics Society of America, South Bend, IN, June 17-19, 2014.

Eryilmaz, O., G. Ramirez and A. Erdemir (2015). "Tribological Performance of Diamond Like Carbon and Nitride Based Coatings Against PTFE Based Composite Seal Materials in Oil Free Methane Environment." 42nd International Conference on Metallurgical Coatings and Thin Films (ICMCTF-2015), San Diego, CA, April 20-24, 2015.

Ramirez, G., O.L. Eryilmaz and A. Erdemir (2016). "Cupronickel-based Nanostructured Coatings with Exceptional Tribological Properties.” 2015 STLE Tribology Frontiers Conference, Denver, CO, October 25-27, 2015.

Ramirez, G., O. Eryilmaz and A. Erdemir (2016). "Friction and Wear of Carbon Films Derived from Natural Gas.” Annual Postdoctoral Research and Career Symposium, Argonne National Laboratory, Argonne, IL, October 8, 2015.

Ramirez, G., O.L. Eryilmaz, A. Erdemir, Y. Liao, S.E. Rodil, R. Mirabal and O. Depablos-Rivera (2016). “Tribology of Nanocomposite Coatings in Methane Environment." VIII International Conference on Surfaces, Materials and Vacuums, Puebla, Mexico, October 21-25, 2015.

Ramirez, G., O.L. Eryilmaz, R. Mirabal, O. Depablos-Rivera, S.E. Rodil and A. Erdemir (2015). "Development and Tribological Characterization of Ni-doped Vanadium Nitride Coatings." 42nd International Conference on Metallurgical Coatings and Thin Films (ICMCTF-2015), San Diego, CA, April 20-24, 2015.

Ramirez, G., A. Erdemir, O.L. Eryilmaz, O. Depablos-Rivera, R. Mirabal and S.E. Rodil (2015). “Tribological Behavior of $\mathrm{Ni}$-doped Vanadium Nitride Coatings in Methane Environment." 70th Society of Tribologists and Lubrication Engineers (STLE) Annual Meeting and Exhibition, Dallas, TX, May 17-21, 2015.

\section{3-035-R2}

\section{REFEREED PUBLICATION}

Pelton, M., D. Chakraborty, E. Malachosky, P. Guyot-Sionnest and J.E. Sader (2014). “Viscoelastic Flows in Simple Liquids Generated by Vibrating Nanostructures." Physical Review Letters 111: 244502.

\section{PRESENTATION}

Ahmed, A., A. Klinkova, D. Chakraborty, E. Kumacheva, J. Sader and J. Guest (2015). “Acoustic Vibrations of Complex Bimetallic Nanostructures.” 2015 American Physical Society March Meeting, San Antonio, TX, March 2-6, 2015.

\section{3-036-R2 \\ REFEREED PUBLICATIONS}

Chen, F., Y. Zhu, S. Liu, Y. Qi, H.Y. Hwang, N.C. Brandt, J. Lu, F. Quirin, H. Enquist, P.E. Zalden, T. Hu, J. Goodfellow, M.C. Hoffmann, D. Zhu, H.T. Lemke, J. Glownia, M.C. Chollet, A. Damodaran, J. Park, Z. Cai, I. Jung, M.J. Highland, D.A. Walko, J.W. Freeland, P.G. Evans, A. Vailionis, J. Larsson, K. Nelson, A.M. Rappe, K. Sokolowski-Tinten, L. Martin, H. Wen and A. Lindenberg. "Terahertz Electric-field-driven lonic Response in Ferroelectrics." Nature Photonics.

(To be published.)

Park, J., Q. Zhang, P. Chen, M. Cosgriff, J.A. Tilka, C. Adamo, D.G. Schlom, H. Wen, Y. Zhu and P.G. Evans (2015). "Spatially Confined Low-power Optically Pumped Ultrafast Synchrotron X-ray Nanodiffraction." Review of Scientific Instruments 86(8): 083904.

Zhu, Y., Z. Cai, P. Chen, Q. Zhang, M.J. Highland, I.W. Jung, D.A. Walko, E.M. Dufresne, J. Jaewoo, M.G. Samant, S.S.P. Parkin, J.W. Freeland, P.G. Evans and H. Wen "Mesoscopic Structural Phase Progression in Photo-excited VO Revealed by Time-resolved X-ray Diffraction Microscopy." Nature Communications. (To be published.)

\section{PRESENTATIONS}

Wen, H. (2016). "Spatiotemporal Imaging of Heterogeneities in Correlated Materials." Probing Structure and Dynamics of Quantum Materials via X-ray Scattering at LCLS Workshop, SSRL/LCLS User Meeting, October 9, 2015. 
Wen, H. (2015). “Spatiotemporally Resolved X-ray Imaging." DOE-BES 'X-ray Scattering Principal Investigators' Meeting, Washington, D.C., November 5-7, 2014.

Wen, H. (2013). "Correlation of Structural and Electronic Dynamics in Complex Oxides." Symposium of Complex Metal Oxides and Unconventional Interfaces, CINT User Conference, Santa Fe, NM, September 24-26, 2013.

Zhu, Y., Z. Cai, I.W. Jung, M. Highland, H. Wen, F. Chen, P.E. Zalden, A. Lindenberg, J. Park, P.G. Evans, A. Damodaran and L. Martin (2015). "Metamaterial-assisted High-field THz Pulse Induced Structural Dynamics in $\mathrm{BaTiO}_{3}$." Optical Terahertz Science and Technology Conference, San Diego, CA, March 8-13, 2015.

Zhu, Y., Q. Zhang, P. Chen, Z. Cai, D.A. Walko, E.M. Dufresne, M. Highland, I.W. Jung, J.-U. Thiele, E.E. Fullerton, C. Adamo, D.G. Schlom, J. Wang, J.W. Freeland, P.G. Evans and H. Wen (2014). "Transient Grating-induced Phase Inhomogeneity in FeRh Studied by Time-resolved Hard X-ray Nanodiffraction." American Physical Society (APS) March Meeting, Denver, CO, March 3-7, 2014.

\section{3-063-R2}

\section{REFEREED PUBLICATIONS}

Choi, Y.-Y., S. Tong, S.P. Ducharme, A. Roelofs and S. Hong. "Charge Collection Kinetics on Ferroelectric Polymer Surface Using Charge Gradient Microscopy.” ACS Nano. (To be published.)

Choi, Y.-Y., P. Sharma, C. Phatak, D.J. Gosztola, Y. Liu, J. Lee, B. Lee, J. Li, A. Gruverman, S.P. Ducharme and S. Hong (2015). "Enhancement of Local Piezoresponse in Polymer Ferroelectrics via Nanoscale Control of Microstructure." ACS Nano 9(2): 1809-1819.

Choi, Y.Y., T.G. Yun, N. Qaiser, H. Paik, H.S. Roh, J. Hong, S. Hong, S.M. Han and K. No (2015). “Vertically Aligned P(VDF-TrFE) Core-shell Structures on Flexible Pillar Arrays." Scientific Reports 5: 10728.

Kim, D., H.S. Roh, Y. Kim, K. No and S. Hong (2015). "Selective Current Collecting Design for Spring-type Energy Harvesters." RSC Advances 5(14): 10662-10666.

Kim, D., S. Hong, J. Hong, Y. Choi, J. Kim, M. Park, T. Sung and K. No (2013). "Fabrication of Vertically Aligned Ferroelectric Polyvinylidene Fluoride Mesoscale Rod Arrays." Journal of Applied Polymer Science 130(6): 3842-3848.

Kim, D., S. Hong, D. Li, H.S. Roh, G. Ahn, J. Kim, M. Park, J. Hong, T.H. Sung and K. No (2013). "A Spring-type Piezoelectric Energy Harvester." RSC Advances 3(10): 3194-3198.

Kim, S., S. Hong, Y.Y. Choi and K. No (2013). "Effect of Nucleation Time on Bending Response of lonic Polymer Metal Composite Actuators." Electrochimica Acta 108: 547-553.

Kim, Y., S. Hong, S. Oh, Y.-Y. Choi, H. Choi and K. No (2015). "Influence of Alkaline Treatment on the Ferroelectric Properties of Poly(vinylidene fluoride trifluoroethylene) Films." Electronic Materials Letters 11(4): 586-591.

Li, D., S. Hong, S. Gu, Y. Choi, S. Nakhmanson, O. Heinonen, D. Karpeev and K. No (2014). “Polymer Piezoelectric Energy Harvesters for Low Wind Speed.” Applied Physics Letters 104(1): 012902-012904.

Paik, H., Y.-Y. Choi, S. Hong and K. No (2015). "Effect of Ag Nanoparticle Concentration on the Electrical and Ferroelectric Properties of Ag/P(VDF-TrFE) Composite Films.” Scientific Reports 5: 13209.

\section{PRESENTATIONS}

Chang, S., S.K. Selvaraj, Y.-Y. Choi, S. Hong, S.M. Nakhmanson and C.G. Takoudis (2014). "Atomic Layer Deposition of Tin-titanate as a Potential Ferroelectric Material." 14th International Conference on Atomic Layer Deposition, Kyoto, Japan, June 15-18, 2014.

Choi, Y.-Y., P. Sharma, C. Phatak, D.J. Gosztola, Y. Liu, J. Lee, B. Lee, J. Li, A. Gruverman, S.P. Ducharme and S. Hong (2015). "Enhancement of Local Piezoresponse in Polymer Ferroelectrics via Nanoscale Control of Microstructure." Electronic Materials and Applications 2015 Conference (EMA 2015), Orlando, FL, January 21-23, 2015. 
Choi, Y.-Y., P. Sharma, C. Phatak, D.J. Gosztola, Y. Liu, J. Lee, B. Lee, J. Li, A. Gruverman, S.P. Ducharme and S. Hong (2015). "Mechanical Annealing of Ferroelectric Polymers at the Nanoscale." International Workshop on Topological Structures in Ferroic Materials, Sydney, Australia, May 19-21, 2015.

Choi, Y., P. Sharma, C. Phatak, D.J. Gosztola, Y. Liu, J. Lee, J. Li, A. Gruverman, S.P. Ducharme and S. Hong (2014). "Enhancement of Local Piezoresponse in Polymer Ferroelectrics via Nanoscale Control of Microstructure." International Workshop on Nanoscale Spectroscopy and Nanotechnology, Chicago, IL, July 28-31, 2014.

Heinonen, O., D. Karpeyev, S. Gu, S.M. Nakhmanson, B. Lee and S. Hong (2013). "Real Space Formulation for Coupled Elastic Problems." Purdue University, West Lafayette, IN, April 15-16, 2013.

Hong, S. (2016). "Mechanical Annealing at the Nanoscale: Integrated Imaging." Argonne National Laboratory-Purdue Integrated Imaging Workshop, Argonne National Laboratory, October 26-27, 2015.

Hong, S. (2015). "Functional Hybrid Materials Design Based on Nanoscale Visualization and Manipulation."

3rd International Symposium on Hybrid Materials and Processing, Busan, South Korea, November 10-13, 2014.

Hong, S. (2015). "Functional Materials Design Based on Nanoscale Visualization and Manipulation." University of Texas, Dallas, TX, November 21, 2014.

Hong, S. (2015). "Functional Materials Design Based on Nanoscale Visualization and Manipulation." University of Texas at Dallas, Richardson, TX, March 13, 2015.

Hong, S. (2015). "Functional Materials Design Based on Nanoscale Visualization and Manipulation." South Dakota School of Mines and Technology, Rapid City, SD, April 9, 2015.

Hong, S., Y. Choi, S. Tong, C.M. Phatak and A.K. Roelofs (2014). "Functional Materials Design Based on Nanoscale Visualization Using Atomic Force Microscopy." 2014 Piezoresponse Force Microscopy and Nanoscale Phenomena in Polar Materials, Ekaterinburg, Russia, July 14-17, 2014.

Hong, S., D. Kim, Y.Y. Choi, H.S. Roh and K. No (2014). "Proof of Principle Experiments for Piezoelectric Energy Harvesters." International Conference on Small Science 2013, Las Vegas, NV, December 15-18, 2013.

Hong, S. (2014). "Visualization and Manipulation of Electric Polarization and Charges Using Atomic Force Microscopy." 2014 Joint IEEE International Symposium on the Applications of Ferroelectric, International Workshop on Acoustic Transduction Materials and Devices \& Workshop on Piezoresponse Force Microscopy (ISAF/IWATMD/PFM), State College, PA, May 12-16, 2014.

Hong, S. (2014). "Visualization and Manipulation of Polarization and Screen Charges Using Atomic Force Microscopy." Ecole Polytechnique Federale de Lausanne, Lausanne, Switzerland, June 6, 2014.

Hong, S. (2013). "Visualization and Manipulation of Electric Polarization and Charges using Atomic Force Microscopy." IEEE-Ultrasonics, Ferroelectrics, Frequency Control Society (UFFCS), European Frequence and Time Forum (EFTF), and PFM Symposium, Prague, Czech Republic, July 21-26, 2013.

Kim, D., S. Hong, D. Li, H. Roh and K. No (2013). "Spring-type Piezoelectric Energy Harvester." The 7th World Congress on Biomimetics, Artificial Muscles and Nano-Bio (BAMN2013), Jeju Island, South Korea, August 26-30, 2013.

Kim, Y., S. Hong, Y. Choi, J. Ryu and K. No (2013). "Characterization of Poly(Vinylidene Fluoride-trifluoroethylene) Films Fabricated by Electrophoretic Deposition." The 7th World Congress on Biomimetics, Artificial Muscles and Nano-Bio (BAMN2013), Jeju Island, South Korea, August 26-30, 2013. 


\section{3-070-R2}

\section{PRESENTATIONS}

O’Brien, S.L., M.D. Whiteside, D.J. Sholto-Douglas, A. Dohnalkova, D.M. Durall, G. Doga, M. Jones, L. Kovarik, B. Lai, C. Roehrig, S.Z. Sullivan, S. Vogt and K.M. Kemner (2016). "Identifying Microbial Habitats in Soil Using Quantum Dots and X-ray Fluorescence Microtomography." 7th Annual Argonne Soil Metagenomics Meeting, Lisle, IL, October 21-23, 2015.

O’Brien, S.L. (2015). “The Microbial Habitat in Soil.” University of Chicago, Chicago, IL, October 7, 2014.

O’Brien, S.L. (2015). "Quantum Dots: A Novel Tool for Studying Soil Microbial Ecology." Oak Ridge National Laboratory, Oak Ridge, TN, May 18, 2015.

O'Brien, S.L., M.D. Whiteside, D.J. Sholto-Douglas, D.A. Antonopoulos, M.I. Boyanov, D.M. Durall, M. Jones, B. Lai, E.J. O'Loughlin and K.M. Kemner (2014). "Imaging Nutrient Uptake in Bacterial Biofilms Using Quantum Dots." 99th Ecological Society of America Annual Meeting, Sacramento, CA, August 10-15, 2014.

O’Brien, S.L., M.D. Whiteside, D.J. Sholto-Douglas, D.A. Antonopoulos, M.I. Boyanov, D.M. Durall, M. Jones, B. Lai, E.J. O'Loughlin and K.M. Kemner (2014). "A Novel Nanoparticle Approach for Imaging Soil Bacteria." Midwest Geobiology Symposium, Chicago, IL, September 27, 2014.

O’Brien, S.L., M.D. Whiteside, D.J. Sholto-Douglas, D.A. Antonopoulos, M.I. Boyanov, D.M. Durall, M. Jones, B. Lai, E.J. O'Loughlin and K.M. Kemner (2014). "A Novel Nanoparticle Approach for Imaging Soil Bacteria." Synchrotron Environmental Science VI, Argonne National Laboratory, September 11-12, 2014.

O’Brien, S. (2013). “Connecting Microbial Ecology with Soil Biogeochemistry." University of British Columbia, Okanagan, British Columbia, Canada, September 11, 2013.

\section{3-080-R2}

\section{REFEREED PUBLICATIONS}

Benson, B.A., P.A.R. Ade, Z. Ahmed, S.W. Allen, K. Arnold, J.E. Austermann, A.N. Bender, L.E. Bleem, J.E. Carlstrom, C.L. Chang, H.M. Cho, S.T. Ciocys, J.F. Cliche, T.M. Crawford, A. Cukierman, T. de Haan, M.A. Dobbs, D. Dutcher, W. Everett, A. Gilbert, N.W. Halverson, D. Hanson, N.L. Harrington, K. Hattori, J.W. Henning, G.C. Hilton, G.P. Holder, W.L. Holzapfel, K.D. Irwin, R. Keisler, L. Knox, D. Kubik, C.L. Kuo, A.T. Lee, E.M. Leitch, D. Li, M. McDonald, S.S. Meyer, J. Montgomery, M. Myers, T. Natoli, H. Nguyen, V. Novosad, S. Padin, Z. Pan, J. Pearson, C.L. Reichardt, J.E. Ruhl, B.R. Saliwanchik, G. Simard, G. Smecher, J.T. Sayre, E. Shirokoff, A.A. Stark, K. Story, A. Suzuki, K.L. Thompson, C. Tucker, K. Vanderlinde, J.D. Vieira, A. Vikhlinin, G. Wang, V. Yefremenko and K.W. Yoon (2014). "SPT-3G: A Next-Generation Cosmic Microwave Background Polarization Experiment on the South Pole Telescope." Proceedings of the SPIE-The International Society for Optical Engineering, Montreal, Quebec, Canada, July 23, 2014, 9153(Millimeter, Submillimeter, and Far-infrared Detectors and Instrumentation for Astronomy VII). (Also see 2013-219.)

Chang, C., P. Ade, Z. Ahmed, S. Allen, K. Arnold, J. Austermann, A. Bender, B. Benson, L. Bleem, J. Carlstrom, H.-m. Cho, S. Ciocys, J.-F. Cliche, T. Crawford, A. Cukierman, T. de Haan, M. Dobbs, D. Dutcher, W. Everett, A. Gilbert, N. Halverson, D. Hanson, N. Harrington, K. Hattori, J. Henning, G. Hilton, G. Holder, W. Holzapfel, K. Irwin and R. Keisler (2015). "Low Loss Superconducting Microstrip Development at Argonne National Laboratory." IEEE Transactions on Applied Superconductivity PP(99): 1-1.

\section{3-100-R2}

\section{REFEREED PUBLICATIONS}

Martinson, A.B.F. " $V x \ln _{(2-x)} S_{3}$ Intermediate Band Absorbers Deposited by Atomic Layer Deposition." (To be published.)

McCarthy, R.F., R.D. Schaller, D.J. Gosztola, G.P. Wiederrecht and A.B. Martinson (2015). "Photoexcited Carrier Dynamics of $\operatorname{In}_{2} \mathrm{~S}_{3}$ Thin Films." Journal of Physical Chemistry Letters 6(13): 2554-2561. 
McCarthy, R.F., M.S. Weimer, A.S. Hock and A.B.F. Martinson (2014). "Chemical and Spatial Control of Substitutional Intermediate Band Materials: Toward the Atomic Layer Deposition of $V_{0.25} \operatorname{In}_{1.75} S_{3}$." Proceedings of the 40th IEEE Photovoltaic Specialists Conference, Denver, CO, June 8-13, 2014: 250-253.

McCarthy, R.F., M.S. Weimer, J.D. Emery, A.S. Hock and A.B.F. Martinson (2014). "Oxygen-free Atomic Layer Deposition of Indium Sulfide." ACS Applied Materials \& Interfaces 6(15): 12137-12145.

\section{NON-REFEREED PUBLICATIONS}

McCarthy, R.F., R.D. Schaller and A.B.F. Martinson (2015). "Physical and Optoelectronic Properties of $V_{x} I_{y} S_{z}$ Intermediate Band Semiconductors Deposited by Atomic Layer Deposition." 42nd IEEE Photovoltaic Specialists Conference (PVSC), New Orleans, LA, June 14-19, 2015.

\section{PRESENTATIONS}

McCarthy, R.F., R.D. Schaller and A.B.F. Martinson (2015). "Physical and Optoelectronic Properties of VxInySIntermediate Band Semiconductors Deposited by Atomic Layer Deposition." 42nd IEEE Photovoltaic Specialists Conference (PVSC), New Orleans, LA, June 14-19, 2015.

McCarthy, R.F., M.S. Weimer, A.S. Hock and A.B.F. Martinson (2015). "Spatial and Chemical Control of Substitutional Intermediate Band Materials: Toward the Atomic Layer Deposition of $V_{0.25} \operatorname{In}_{1.75} S_{3}$." Argonne National Laboratory Postdoctoral Research and Career Symposium, Argonne, IL, October 23, 2014.

McCarthy, R.F., M.S. Weimer, M.J. Pellin, A.S. Hock and A.B.F. Martinson (2014). "Atomic Layer Deposition of $\operatorname{In}_{2} \mathrm{~S}_{3}$ Via a Novel Indium Amidinate Precursor." Argonne National Laboratory Postdoctoral Research and Career Symposium, Argonne, IL, October 10, 2013.

McCarthy, R.F., M.S. Weimer, A.S. Hock and A.B.F. Martinson (2014). "Chemical and Spatial Control of Substitutional Intermediate Band Materials: Toward the Atomic Layer Deposition of $V_{0.25} \operatorname{In}_{1.75} S_{3}$." 40th IEEE Photovoltaic Specialists Conference (PVSC), Denver, CO, June 8-13, 2014.

\section{3-116-R2}

\section{REFEREED PUBLICATIONS}

Mo, K., D. Yun, Y. Miao, X. Liu, M. Pellin, J. Almer, J.-S. Park, J.F. Stubbins, S. Zhu and A. Yacout (2016). "Investigation of High-Energy lon-Irradiated MA957 Using Synchrotron Radiation under in situ Tension." Materials 9(1): 15.

(Also see 2013-152.)

Mohamed, W., D. Yun, K. Mo, M.J. Pellin, M.C. Billone, J. Almer and A.M. Yacout (2015). "Depth Profile of Oxide Volume Fractions of Zircaloy-2 in High-temperature Steam: An in situ Synchrotron Radiation Study." Journal of Nuclear Materials 454(1-3): 192-199. (Also see 2013-152.)

Mo, K., D. Yun, W. Mohamed, M. Pellin, J. Almer and A.M. Yacout (2014). "Synchrotron Radiation Study on Steam Oxidation Behavior of Zircaloy-2 with Advanced Coatings." Proceedings of the Nuclear Fuels and Structural Materials for the Next Generation Nuclear Reactors (NFSM) 2014 American Nuclear Society Annual Meeting, Reno, NV, June 15-19, 2014.

Pellin, M.J., A.M. Yacout, K. Mo, J. Almer, S. Bhattacharya, W. Mohamed, D. Seidman, B. Ye, D. Yun, R. Xu and S. Zhu. "MeV per Nucleon Ion Irradiation of Nuclear Materials with High Energy Synchrotron X-ray Characterization." Journal of Nuclear Materials. (To be published.) (Also see 2013-152.)

Pellin, M.J., A.M. Yacout, K. Mo, J. Almer, S. Bhattacharya, W. Mohamed, D. Seidman, B. Ye, D. Yun, R. Xu and S. Zhu. "MeV per Nucleon Ion Irradiation of Nuclear Materials with High Energy Synchrotron X-ray Characterization." Journal of Nuclear Materials. (To be published.) (Also see 2013-152.)

Yacout, A.M., M. Pellin and M.C. Billone (2013). "Development and Testing of Nanolaminate Coatings for Conventional LWR Cladding." Proceedings of the LWR Fuel Performance Meeting/Top Fuel 2013, Charlotte, NC, September 15-19, 2013: 847. (Also see 2013-152.) 


\section{NON-REFEREED PUBLICATION}

Yun, D., W. Mohamed, B. Ye, M. Kirk, P. Baldo and A. Yacout (2013). "Preliminary Assessment of Microstructural Evolution of U-10Mo Thin Specimen Annealed at Typical Fast Reactor Temperature Regime." 2014 American Nuclear Society Annual Meeting, Atlanta, GA, June 16-20, 2013.

\section{PRESENTATIONS}

Mo, K., D. Yun, Y. Miao, W. Mohamed, M.J. Pellin, J. Almer, J. Park, J.F. Stubbins and A.M. Yacout (2015). “Microstructural Characterization of High-energy Ion Irradiated HT9 \& MA 957." NuMat Conference 2014, Clearwater, FL, October 27-30, 2014.

Yacout, A., M. Mendelsohn, D. Yun and W. Mohamed (2014). "Multilayer ALD Coating of Light Water Reactor Zirconium Alloy Cladding Materials.” Multilayers'13, Madrid, Spain, October 1-4, 2013. (Also see 2013-152.)

Yun, D., K. Mo, W. Mohamed, M.J. Pellin and A.M. Yacout (2015). “Irradiation Behavior Study of U-Mo/Al Dispersion Fuel with High Energy Xe.” NuMat Conference 2014, Clearwater, FL, October 27-30, 2014. (Also see 2013-152.)

Yun, D., K. Mo, W. Mohamed, M.J. Pellin and A.M. Yacout (2015). “Modeling Beam Heating and Sample Temperature by High Energy Heavy Ion Irradiation, and Comparison with Experimental Measurements." NuMat Conference 2014, Clearwater, FL, October 27-30, 2014. (Also see 2013-152.)

Yun, D., J. Wright, J. Terry, K. Mo, R. Xu, W. Mohamed, B. Yei, K. Logan, M. Pellin and A. Yacout (2015). "Study of Xe Ion Beam Irradiated Mo Single Crystal by Synchrotron Extended X-ray Absorption Fine Structure and Microdiffraction." NuMat Conference 2014, Clearwater, FL, October 27-30, 2014. (Also see 2013-152.)

\section{4-018-R1}

\section{REFEREED PUBLICATIONS}

Jungfleisch, M.B., W. Zhang, W. Jiang, J. Sklenar, J.E. Pearson, J.B. Ketterson and A. Hoffmann. "All-electrical Detection of Spin Dynamics in Magnetic Antidot Lattices by the Inverse Spin Hall Effect." Applied Physics Letters. (To be published.)

Jungfleisch, M.B., W. Zhang, J. Sklenar, W. Jiang, J.E. Pearson, J.B. Ketterson and A. Hoffmann. "Interface-driven Spin-torque Ferromagnetic Resonance by Rashba Coupling at the Interface Between Non-magnetic Materials." Physical Review Letters. (To be published.)

Jungfleisch, M.B., W. Zhang, J. Sklenar, J. Ding, W. Jiang, H. Chang, F.Y. Fradin, J.E. Pearson, J.B. Ketterson, V. Novosad, M. Wu and A. Hoffmann. "Large Spin-wave Bullet in a Ferrimagnetic Insulator Driven by Spin Hall Effect." Physical Review Letters. (To be published.)

Jungfleisch, M.B., W. Zhang, W. Jiang and A. Hoffmann (2016). "New Pathways Towards Efficient Metallic Spin Hall Spintronics." SPIN 05(03): 1530005. (Also see 2013-016.)

Jungfleisch, M.B., W. Zhang, W. Jiang, H. Chang, J. Sklenar, S.M. Wu, J.E. Pearson, A. Bhattacharya, J.B. Ketterson, M. Wu and A. Hoffmann (2015). "Spin Waves in Micro-structured Yttrium Iron Garnet Nanometer-thick Films." Journal of Applied Physics 117(17): 17D128-117D131.

Sklenar, J., W. Zhang, M.B. Jungfleisch, W. Jiang, H. Chang, J.E. Pearson, M. Wu, J.B. Ketterson and A. Hoffmann (2016). “Driving and Detecting Ferromagnetic Resonance in Insulators with the Spin Hall Effect." Physical Review Letters 92(17): 174406. (Also see 2013-016.)

\section{NON-REFEREED PUBLICATIONS}

Hoffmann, A., H. Schultheiss, S. Vogt, S. Jain, F. Y. Fradin, S. Bader, Z. Wang, Y. Sun, Y.-T. Song, M. Wu, T. Sebastian and B. Hillebrands (2014). "Electric Control and Detection of Spin Waves." Concepts in Spintronics, Santa Barbara, CA, September 30-October 4, 2013. (Also see 2013-016.) 
Hoffmann, A., W. Zhang, V. Vlaminck, J.E. Pearson, S.D. Bader, R. Divan, L. Bai, P. Hyde, Y. Gui and C.-M. Hu (2014). "Insight About Spin Hall Effects from Spin Pumping." SPIE NanoScience+Engineering, San Diego, CA, August 17-24, 2014. (Also see 2013-016.)

Sola, A., M. Kueoferling, V. Basso, S. Zullino, E. Olivetti, M. Pasquale, T. Kikkawa, K. Uchida, E. Saitoh, D. Meier, T. Kuschel, G. Reiss, H. Jin, S. Boona, S. Watzman, J. Heremans, W. Zhang, M. B. Jungfleisch, J.E. Pearson, A. Hoffmann and H.W. Schumacher (2014). "Round Robin Comparison of the Spin-Seebeck Coefficient: Results and Outlook." Spin Caloritronics VI, Isree, Germany, July 14-18, 2014. (Also see 2013-016.)

Zhang, W., V. Vlaminck, J.E. Pearson, R. Divan, S.D. Bader and A. Hoffmann (2014). "Determination of the Spin Diffusion Length via Spin Pumping and Spin Hall Effects." American Physical Society March Meeting, Denver, CO, March 3-7, 2014. (Also see 2013-016.)

Zhang, W., V. Vlaminck, J.E. Pearson, R. Divan, S.D. Bader and A. Hoffmann (2014). "Quantifying Spin Diffusion Lengths from Spin Pumping and Spin Hall Effects." Intermag 2014, Dresden, Germany, May 4-8, 2014. (Also see 2013-016.)

\section{PRESENTATIONS}

Heinonen, O. (2014). "Resonant Dynamics of Topological Magnetic Structures." American Physical Society March Meeting, Denver, CO, March 3-7, 2014.

Hoffmann, A., W. Zhang, J. Sklenar, M.B. Jungfleisch, W. Jiang, H. Chang, B. Hsu, F.Y. Fradin, Y. Hoffmann, A. (2015). "Spin Hall Effects: From Spin Current Detection to Manipulation of Skyrmions." University of Illinois, Chicago, IL, October 30, 2014. (Also see 2014-054.)

Hoffmann, A. (2015). "Electric Manipulation of Spin Textures." 20th International Conference on Magnetism, Barcelona, Spain, July 5-10, 2015. (Also see 2014-054.)

Hoffmann, A. (2015). "Electric Manipulation of Spin Textures." Conference on Magnetism and Its Applications, Shanghai, China, January 31-February 2, 2015. (Also see 2014-054.)

Hoffmann, A. (2015). “Electric Manipulation of Spin Textures." Centro Brasileiro de Pesquisas Fisicas (CBPF), Rio de Janeiro, Brazil, October 30, 2014. (Also see 2014-054.)

Hoffmann, A. (2015). “Electric Manipulation of Spin Textures." Northwestern University, Evanston, IL, October 16, 2014. (Also see 2014-054.)

Hoffmann, A. (2015). "Magnetism at the Mesoscale: Harnessing Competing Interactions." 2015 American Vacuum Society Prairie Chapter Symposium, South Bend, IN, September 10, 2015. (Also see 2013-016 and 2014-054.)

Hoffmann, A. (2015). "Magnetism at the Mesoscale: Harnessing Competing Interactions." California State University, Long Beach, CA, September 16, 2015. (Also see 2013-016 and 2014-054.)

Hoffmann, A. (2015). "Magnetism at the Mesoscale: Harnessing Competing Interactions." Nanjing University, Nanjing, China, May 20, 2015. (Also see 2013-016 and 2014-054.)

Hoffmann, A. (2015). "Magnetism at the Mesoscale: Harnessing Competing Interactions." University of California, Riverside, CA, June 5, 2015. (Also see 2013-016 and 2014-054.)

Hoffmann, A. (2015). "Magnetism at the Mesoscale: Harnessing Competing Interactions." University of California-San Diego, La Jolla, CA, June 4, 2015. (Also see 2013-016 and 2014-054.)

Hoffmann, A. (2015). "Magnetism at the Mesoscale: Harnessing Competing Interactions." Fudan University, Shanghai, China, January 30, 2015. (Also see 2013-016 and 2014-054.)

Hoffmann, A. (2015). "Magnetism at the Mesoscale: Harnessing Competing Interactions." University of California, Davis, CA, February 12, 2015. (Also see 2013-016 and 2014-054.) 
Hoffmann, A. (2014). "Connecting Spin Waves to Charge Currents." International Workshop on Nanoscale Spectroscopy and Nanotechnology 8, Chicago, IL, July 28-31, 2014. (Also see 2013-016.)

Hoffmann, A. (2014). "Connecting Spin Waves to Charge Currents." Ohio State University, Columbus, OH, November 21, 2013. (Also see 2013-016.)

Hoffmann, A. (2014). "Magnetization Dynamics in Nanometer-thick Yttrium Iron Garnet Films." Workshop on Oxide Materials: Nanoscale Spectroscopy and Nanotechnology, Cali, Columbia, September 15-19, 2014. (Also see 2013-016.)

Hoffmann, A. (2014). "Reduction of Spin Hall Effects due to Magnetic Proximity." Spin Caloritronics VI, Isree, Germany, July 14-18, 2014. (Also see 2013-016.)

Jungfleisch, M.B., W. Zhang, W. Jiang, F.Y. Fradin, J.E. Pearson and A. Hoffmann (2015). "DC Voltage Generation in a Pure Permalloy Layer Under Ferromagnetic Resonance." 59th Annual Magnetism and Magnetic Materials Conference, Honolulu, HI, November 3-7, 2014.

Jungfleisch, M.B., W. Zhang, J. Sklenar, W. Jiang, J. Ding, H. Chang, F.Y. Fradin, J.B. Ketterson, M. Wu and A. Hoffmann (2015). "Excitation and Detection of Magnetization Dynamics in Insulators by Spin Hall Effects." IEEE Magnetics Society, Santa Clara Valley Chapter, San Jose, CA, May 23, 2015.

Jungfleisch, M.B., W. Zhang, W. Jiang, S.M. Wu, J.E. Pearson, A. Bhattacharya, A. Hoffmann, J. Sklenar, J.B. Ketterson, H. Chang and M. Wu (2015). "Spin Dynamics in Patterned Nanometer-thick Yttrium Iron Garnet Films." American Physical Society March Meeting 2015, San Antonio, TX, March 2-6, 2015.

Jungfleisch, M.B. (2015). "Spin Pumping and Inverse Spin Hall Effect in Metallic Ferromagnet Structures." Technische Universität Kaiserslautern, Kaiserslautern, Germany, June 11, 2014.

Jungfleisch, M.B., W. Zhang, W. Jiang, J. Ding, H. Chang, F.Y. Fradin, J.E. Pearson, J.B. Ketterson, V. Novosad, M. Wu and A. Hoffmann (2015). "Spin-torque Driven Magnetization Dynamics by Interface- and Bulk Spin-orbital Phenomena." Technische Universität Kaiserslautern, Kaiserslautern, Germany, August 18, 2015.

Jungfleisch, M.B., W. Zhang, W. Jiang, H. Chang, J.E. Pearson, M. Wu and A. Hoffmann (2015). "Spin Waves in Micro-structured Yttrium Iron Garnet Thin Film." 59th Annual Magnetism and Magnetic Materials Conference, Honolulu, HI, November, 3-7, 2014.

Liu Y. and J.E. Pearson (2015). "Magnetization Dynamics Driven by Spin Hall Effects." International Colloquium on Magnetic Films and Surfaces, Cracow, Poland, July 12-17, 2015. (Also see 2013-016.)

Schultheiss, H., A. Hoffmann, K. Vogt, F. Fradin, J.E. Pearson, T. Sebastian, B. Hillebrands and S.D. Bader (2015). "Propagation, Steering and Detection of Spin Waves for Magnonic Applications." Intermag 2015, Beijing, China, May 11-15, 2015.

Sklenar, J., W. Zhang, M.B. Jungfleisch, W. Jiang, H. Saglam, S. Grudichak, J.E. Pearson, J.B. Ketterson and A. Hoffmann (2015). "Spin-torque Ferromagnetic Resonance Studies in Out-of-plane Magnetization Configurations." American Physical Society March Meeting, San Antonio, TX, March 2-6, 2015.

Vogt, K., F. Fradin, J.E. Pearson, T. Sebastian, S.D. Bader, B. Hillebrands, A. Hoffmann and H. Schultheiss (2015). "Realization of Spin-wave Multiplexer." 59th Annual Magnetism and Magnetic Materials Conference, Honolulu, HI, November 3-7, 2014.

2014-019-R1

REFEREED PUBLICATIONS

Flender, S., L. Bleem, H. Finkel, S. Habib, K. Heitmann and G. Holder. "Simulations of the Pairwise Kinematic Sunyaev-Zeldovich Signal.” Astrophysical Journal. (To be published.) 
Van Engelen, A., S. Bhattacharya, N. Sehgal, G.P. Holder, O. Zahn and D. Nagai (2014). "CMB Lensing Power Spectrum Biases from Galaxies and Clusters Using High-Angular Resolution Temperature Maps.” Astrophysical Journal 786(1): 13.

\section{4-023-R1}

\section{NON-REFEREED PUBLICATION}

Dharmapalan, R., A. Mane, K. Byrum, M. Demarteau, J. Elam, E. May, R. Wagner, D. Walters, L. Xia, J. Xie and H. Zhao (2015). "MCP-based Photodetectors for Cryogenic Applications." Light Detection in Noble Elements International Conference (LIDINE 2015), Albany, NY, August 28-30, 2015.

\section{PRESENTATIONS}

Dharmapalan, R. and J.M. Paley (2014). "LArLArPD: Large Area Picosecond Photodetector (LAPPD) in Liquid Argon (LAr)." Liquid Argon TPC R\&D Workshop (LArTPC14), Fermi National Accelerator Laboratory, Batavia, IL, July 8-9, 2014.

Wagner, B. and R. Dharmapalan (2015). “MCP-based Photodetectors for Cryogenic Applications.” Light Detection in Noble Elements International Conference, Albany, NY, August 28-30, 2015.

\section{4-046-R1}

\section{REFEREED PUBLICATION}

Hadt, R.G., L. Li, S. Yao, W. Lo, Z. Cai, Q. Wu, B. Pandit, L.X. Chen and L. Yu. "Photocatalysts Based on Cobalt-chelating Conjugated Polymers for Hydrogen Evolution from Water.” Nature Chemistry. (To be published.)

\section{4-051-R1}

\section{REFEREED PUBLICATIONS}

Barkholtz, H.M., L.N. Chong, Z.B. Kaiser, T. Xu and D.J. Liu (2015). "Highly Active Non-PGM Catalysts Prepared from Metal Organic Frameworks." Catalysts 5(2): 955-965.

Shui, J., C. Chen, L.R. Grabstanowicz, D. Zhao and D.-J. Liu (2015). "Highly Efficient Non-precious Metal Catalyst Prepared with Metal-Organic Framework in a Continuous Carbon Nanofibrous Network." Proceedings of the National Academy of Sciences of the United States of America 112(10629-10634).

Zhao, D., J.L. Shui, L.R. Grabstanowicz, C. Chen, S.M. Commet, T. Xu, J. Lu and D.J. Liu (2014). "Highly Efficient Non-precious Metal Electrocatalysts Prepared from One-pot Synthesized Zeolitic Imidazolate Frameworks (ZIFs)." Advanced Materials 26(7): 1093-1097.

\section{PRESENTATIONS}

Barkholtz, H.M., Z.B. Kaiser and D.-J. Liu (2015). "Highly Active Non-PGM Catalysts Prepared from Metal-Organic Frameworks." 227th Meeting of the Electrochemical Society, Chicago, IL, May 24-28, 2015.

Liu, D.-J. (2015). “MOFs and POPs (and more...) - Rationally Designed Porous Precursors for Synthesis of Highly Efficient Non-PGM Catalyst." Electrocatalysis and Fuel Cell Discussion, La Grande Motte, France, September 13-16, 2015.

Liu, D.-J. (2015). "Novel Non-PGM Catalysts from Rationally Designed 3-D Precursors." 5th European PEFC and H2 Forum, Lucerne Switzerland, June 30-July 3, 2015.

Liu, D.-J. (2015). "Recent Advancements in Non-precious Metal Fuel Cell Catalysts Prepared by Rationally Designed Porous Materials." XIV International Congress of the Mexican Hydrogen Society, Cancun, Mexico, October 2, 2014.

Liu, D.-J. (2015). “Recent Developments in New Materials \& Characterization Techniques for Energy Storage and Conversion." University of Illinois at Chicago, Chicago, IL, February 26, 2015. (Also see 2015-091.)

Liu, D.-J. (2015). "Recent Developments in New Materials and Characterization Techniques for Energy Storage and Conversion.” University of Nebraska, Lincoln, NE, October 20, 2014. (Also see 2015-091.) 
Liu, D.-J. (2014). "Improving Non-PGM Catalyst Performance through Rationally Designed PEMFC Cathode Architectures.” 2014 Gordon Research Conference for Fuel Cell, Bryant University, Smithfield, RI, August 3-8, 2014.

Liu, D.-J. (2014). "Oxygen Reduction Reactions in Fuel Cell \& Li-Air Battery: Insights of New Catalyst Design \& Catalytic Mechanism." Peking University, Beijing, China, September 23, 2014.

Zhao, D., J.-L. Shui, L.R. Grabstanowicz and D.-J. Liu (2015). "A Versatile Preparation of Highly Active ZIF-based Non-PGM Catalysts through Solid State Synthesis." 226th Meeting of the Electrochemical Society, Cancun, Mexico, October 5-10, 2014.

\section{4-054-R1}

\section{REFEREED PUBLICATIONS}

Jiang, W., P. Upadhyaya, W. Zhang, G. Yu, M.B. Jungfleisch, F.Y. Fradin, J.E. Pearson, Y. Tserkovnyak, K.L. Wang, O. Heinonen, S.G. te Velthuis and A. Hoffmann (2015). "Magnetism: Blowing Magnetic Skyrmion Bubbles." Science 349(6245): 283-286.

\section{NON-REFEREED PUBLICATIONS}

Hoffmann, A., W. Jiang, P. Upadhyaya, Q. Yang, G. Yu, W. Zhang, M.B. Jungfleisch, F.Y. Fradin, J. E. Pearson, Z. Wang, Y. Fan, O. Heinonen and S.G.E. te Velthuis (2015). "Electric Manipulation of Skyrmions in Metals and Insulators." 2015 IEEE International Magnetics Conference (Intermag), Beijing, China, May 11-15, 2015.

Hoffmann, A. (2015). "Magnetism at the Mesoscale: Harnessing Competing Interactions." American Vacuum Society Prairie Chapter Symposium, South Bend, IN, September 10, 2015. (Also see 2013-016 and 2014-018.)

Jiang, W., P. Upadhyaya, Q. Yang, G. Yu, Z. Wang, J. Tang, K. Wong, M. Akyol, L.-T. Chang, M. Lang, S.G.E. te Velthuis and A. Hoffmann (2015). "Manipulation of Skyrmion Bubbles in a Magnetic Insulator via Pure Spin Current." 59th Annual Magnetism and Magnetic Materials Conference, Honolulu, Hawaii, November 3-7, 2014.

\section{PRESENTATIONS}

Heinonen, O., W. Jiang, S.G.E. te Velthuis and A. Hoffmann (2015). "Micromagnetic Modeling of Skyrmion Injection in an Inhomogeneous Geometry." 2015 American Physical Society March Meeting, San Antonio, TX, March 2-6, 2015.

Hoffmann, A., W. Jiang, P. Upadhyaya, W. Zhang, G. Yu, M.B. Jungfleisch, F.Y. Fradin, J.E. Pearson, Y. Tserkovnyak, K.L. Wang, O. Heinonen and S.G.E. te Velthuis (2015). "Blowing Magnetic Skyrmion Bubbles." Recent Advances in Spintrontics, Safed, Israel, May 10-14, 2015.

Hoffmann, A. (2015). "Electric Manipulation of Spin Textures." Centro Brasileiro de Pesquisas Fisicas (CBPF), Rio de Janiero, Brazil, November 20, 2014. (Also see 2014-018.)

Hoffmann, A. (2015). "Electric Manipulation of Spin Textures." Conference on Magnetism and its Applications, Shanghai, China, January 31-February 2, 2015. (Also see 2014-018.)

Hoffmann, A. (2015). “Electric Manipulation of Spin Textures." Northwestern University, Evanston, IL, October 16, 2014. (Also see 2014-018.)

Hoffmann, A. (2015). "Electric Manipulation of Spin Textures." 20th International Conference on Magnetism, Barcelona, Spain, July 5-10, 2015. (Also see 2014-018.)

Hoffmann, A. (2015). "Magnetism at the Mesoscale: Harnessing Competing Interactions." California State University, Long Beach, CA, September 16, 2015. (Also see 2013-016 and 2014-018.)

Hoffmann, A. (2015). "Magnetism at the Mesoscale: Harnessing Competing Interactions." Fudan University, Shanghai, China, January 30, 2015. (Also see 2013-016 and 2014-018.)

Hoffmann, A. (2015). "Magnetism at the Mesoscale: Harnessing Competing Interactions." Nanjing University, Nanjing, China, May 20, 2015. (Also see 2013-016 and 2014-018.) 
Hoffmann, A. (2015). "Magnetism at the Mesoscale: Harnessing Competing Interactions." University of California, Davis, CA, February 12, 2015. (Also see 2013-016 and 2014-018.)

Hoffmann, A. (2015). "Magnetism at the Mesoscale: Harnessing Competing Interactions." University of California, Riverside, CA, June 5, 2015.

Hoffmann, A. (2015). "Magnetism at the Mesoscale: Harnessing Competing Interactions." University of California, San Diego, CA, June 4, 2015. (Also see 2013-016 and 2014-018.)

Hoffmann, A., W. Zhang, M.B. Jungfleisch, W. Jiang, Y. Liu, J.E. Pearson, S.G.E. te Velthuis, F. Freimuth and Y. Mokrousov (2015). "Spin Hall Effects in Magnetically Ordered Materials." International Workshop on Recent Trends in Nanomagnetism, Spintronics and their Applications (RTNSA), Gipuzkoa, Spain, June 1-3, 2015. (Also see 2013-016.)

Hoffmann, A. (2015). "Spin Hall Effects in Metals." 2015 Spinlcur Summer School, Braga, Portugal, September 7-11, 2015. (Also see 2013-016.)

Hoffmann, A. (2015). "Spin Hall Effects: From Spin Current Detection to Manipulation of Skyrmions." Bar-llan University, Ramat Gan, Israel, May 7, 2015. (Also see 2013-016.)

Hoffmann, A. (2015). "Spin Hall Effects: From Spin Current Detection to Manipulation of Skyrmions." New York University, New York, NY, June 2, 2015.

Hoffmann, A. (2015). "Spin Hall Effects: From Spin Current Detection to Manipulation of Skyrmions." SLAC National Accelerator Laboratory, Menlo Park, CA, December 5, 2014. (Also see 2013-016.)

Hoffmann, A. (2015). "Spin Hall Effects: From Spin Current Detection to Manipulation of Skyrmions." University of Illinois, Chicago, IL, October 30, 2014. (Also see 2014-018.)

Hoffmann, A. (2014). "Magnetic Skyrmions in Metals and Insulators." Workshop on Oxide Materials: Nanoscale Spectroscopy and Nanotechnology, Cali, Colombia, September 15-19, 2014.

Jiang, W. (2015). "Electrical Creation and Manipulation of Magnetic Skyrmoin Bubbles." 2015 American Physical Society March Meeting, San Antonio, TX, March 2-6, 2015.

\section{4-077-R1}

\section{NON-REFEREED PUBLICATIONS}

Lee, J., X.-M. S. Lin, A.R. and S. Narayanan (2016). "Shear Stress Induced Microstructure in Concentrated Silica Dispersions." 87th Society of Rheology Annual Meeting, Baltimore, MD, October 11-15, 2015.

Lee, J., X.-M. Lin, A.R. Sandy and S. Narayanan (2015). "Dynamics of Concentrated Silica Suspension Under Oscillatory Shear Studied by SAXS and XPCS." American Physical Society March Meeting 2015, San Antonio, TX, March 2-6, 2015.

Lee, J., S. Narayanan, X.-M. Lin and A.R. Sandy (2015). "Rheological Behavior of Silica Nanoparticles Measured by Small Angle X-ray Scattering." 2014 Society of Rheology (SOR) Annual Meeting, Philadelphia, PA, October 5-9, 2014.

\section{PRESENTATION}

Lee, J., X.-M. Lin, S. Narayanan and A.R. Sandy (2015). "Structure and Dynamics of Concentrated Silica Suspension Under Oscillatory Shear Studied by SAXS and XPCS." 89th ACS Colloid and Surface Science Symposium,

Pittsburgh, PA, June 15-17, 2015.

\section{4-081-R1}

\section{NON-REFEREED PUBLICATION}

Kim, J., C. Park, H. Hong, T. Kim, S. Kim, C.B. Bahn, J.D. Hoffman, A. Bhattacharya, J.H. Kim and S. Hong (2015).

"Pb-effects on $\mathrm{NiO}$ and $\mathrm{Pb}$-contained Solution Interface with High-resolution X-ray Reflectivity." 17th International Conference on Environmental Degradation of Materials in Nuclear Power Systems-Water Reactors, Ottawa, Ontario, Canada, August 9-13, 2015. 


\section{PRESENTATIONS}

Hong, S., Y.-Y. Choi and J.J. Kim (2015). “Design of Model Experiments for Stress-induced Nanoscale Phenomena." School of Mechanical Engineering, Pusan National University, Busan, Korea, November 11, 2014.

Kim, J., C. Park, H. Hong, T. Kim, S. Kim, C.B. Bahn, J.D. Hoffman, A. Bhattacharya, J.H. Kim and S. Hong (2015). "Pb-effects on $\mathrm{NiO}$ and $\mathrm{Pb}$-contained Solution Interface with High-resolution X-ray Reflectivity." 17th International Conference on Environmental Degradation of Materials in Nuclear Power Systems-Water Reactors, Ottawa, Ontario, Canada, August 9-13, 2015.

Park, C., J. Kim, C.B. Bahn, H. Hong, T.-H. Kim, S.-H. Kim, B. Hou, S. Hong and J.-H. Kim (2015). "Synchrotron X-ray Reflectivity Study of $\mathrm{Ni}$-oxide-passive-layer-and-water Interface and Yttrium-stabilized-zirconia-and-water Interface." 227th Electrochemical Society Meeting, Chicago, IL, May 24-28, 2015.

\section{4-084-R1}

\section{PRESENTATIONS}

Feng, Y. (2015). “Understanding the Aerosol Interactions with Warm Clouds." 26th General Assembly of the International Union of Geodesy and Geophysics (IUGG), Prague, Czech Republic, June 22-July 2, 2015.

Feng, Y., A. Ito, B. Lai, A. Lango and E. Ingall (2014). "Bio-availability of Iron Inputs to the Ocean Driven by Aerosol Chemistry in Dust.” 2014 Climate Modeling Principal Investigator (PI) Meeting, Potomac, MD, May 12-15, 2014.

Feng, Y., A. Lango, B. Lai, E. Ingall and Y. Gao (2014). "Characterization of Dust Deposition and Mineralogy Export to the Southern Ocean." Workshop on Clouds, Aerosols, Radiation and Air-Sea Interface of the Southern Ocean: Establishing Directions for Future Research, University of Washington, Seattle, WA, March 18-19, 2014.

Longo, A.F., Y. Feng and B. Lai (2015). "Key Factors Controlling the Solubility of Iron in Saharan Dust." Association for the Sciences of Limnology and Oceanography (ASLO) 2015 Aquatic Sciences Meeting, Granada, Spain,

February 22-27, 2015.

\section{4-095-R1}

\section{REFEREED PUBLICATION}

DeGottardi, W. (2014). “Topological Insulating Phases of Non-Abelian Anyonic Chains.” Physical Review B 90(7): 075129.

\section{PRESENTATIONS}

DeGottardi, W. (2015). "Topological Insulating Phases of Non-Abelian Anyonic Chains." Advances in Topological Phases of Matter in Crystalline Solids and Cold Atom Systems, Hong Kong, China, June 28-July 3, 2015.

DeGottardi, W. (2015). “Topological Insulating Phases of Non-Abelian Anyonic Chains.” Gordon Conference, Hong Kong, China, August 1-2, 2015.

\section{4-108-R1 \\ REFEREED PUBLICATION}

Michalska, K., A.D. Steen, G. Chhor, M. Endres, A.T. Webber, J. Bird, K.G. Lloyd and A. Joachimiak (2015). "New Aminopeptidase from "Microbial Dark Matter" Archaeon." The Journal of the Federation of American Societies of Experimental Biology 29(9): 4071-4079.

\section{PRESENTATIONS}

Michalska, K., A. Steen, G. Chhor, M. Endres, A.T. Webber, J. Bird, K. Lloyd and A. Joachimiak (2016). "Microbial Degradation of Biopolymers-Structural Biology Insights." University of Tennessee, Knoxville, TN, October 19, 2015.

Michalska, K., A. Steen, G. Chhor, K.M. Fayman, M. Endres, G. Babnigg, K. Lloyd, R. Jedrzejczak and A. Joachimiak (2014). “Discovery of Proteins from 'Microbial Dark Matter'.” NIH Structural Biology Horizons Workshop, Bethesda, MD, December 9-10, 2013. 
Michalska, K., A. Steen, G. Chhor, K.M. Fayman, M. Endres, G. Babnigg, K. Lloyd, R. Jedrzejczak and A. Joachimiak (2014). "Structural Genomics of Sedimentary Archaea in Postgenomic Era." DOE Joint Genome Institute 9th Annual Genomics of Energy \& Environment Meeting, Walnut Creek, CA, March 18-20, 2014.

Michalska, K., A. Steen, G. Chhor, K. Fayman, M. Endres, G. Babnigg, K. Lloyd, R. Jedrzejczak and A. Joachimiak (2014). "Structure and Specificity of Novel Aminopeptidase from Marine Sediment Archaea." 23rd Congress and General Assembly of the International Union of Crystallography, Montreal, Quebec, Canada, August 5-13, 2014.

Steen, A., K. Michalska, G. Chhor, M. Endres, J. Vazin, K. Lloyd, S.W. Wilhelm and A. Joachimiak (2014). "Strategies to Assess the Biochemical Properties of Extracellular Hydrolases in Aquatic Environments." Goldschmidt 2014 Conference, Sacramento, CA, June 8-13, 2014.

\section{5-078-NO}

\section{REFEREED PUBLICATION}

Benseman, T.M., A.E. Koshelev, V. Vlasko-Vlasov, Y. Hao, W.K. Kwok, U. Welp, C. Keiser, B. Gross, M. Lange, D. Kölle, R. Kleiner, H. Minami, C. Watanabe and K. Kadowaki (2015). "Current Filamentation in Large $\mathrm{Bi}_{2} \mathrm{Sr}_{2} \mathrm{CaCu}_{2} \mathrm{O}_{8+\delta} \mathrm{Mesa}$ Devices Observed via Luminescent and Scanning Laser Thermal Microscopy." Physical Review Applied 3(4).

\section{PRESENTATIONS}

Benseman, T.M., A.E. Koshelev, V. Vlasko-Vlasov, Y. Hao, W.-K. Kwok, U. Welp, C. Keiser, B. Gross, M. Lange, D. Koelle, R. Kleiner and K. Kadowaki (2015). "Comparison of Luminescent and Scanning Laser Thermal Micro-imaging of Self-heating in $\mathrm{Bi}_{2} \mathrm{Sr}_{2} \mathrm{CaCu}_{2} \mathrm{O}_{8+\delta}$ Mesa THz Sources." American Physical Society March Meeting, San Antonio, TX, March 2-6, 2015.

Benseman, T.M., A.E. Koshelev, V. Vlasko-Vlasov, Y. Hao, U. Welp, W.-K. Kwok, B. Gross, M. Lange, D. Koelle, R. Kleiner and K. Kadowaki (2015). "Terahertz Radiation from $\mathrm{Bi}_{2} \mathrm{Sr}_{2} \mathrm{CaCu}_{2} \mathrm{O}_{8+\delta}$ Interlayer Josephson Junctions: Progress and Future Strategies." AMN-7: Advanced Materials and Nanotechnology Conference, Nelson, New Zealand, February 8-12, 2015.

Benseman, T.M., A.E. Koshelev, V. Vlasko-Vlasov, Y. Hao, U. Welp, W.-K. Kwok, B. Gross, M. Lange, D. Koelle, R. Kleiner and K. Kadowaki (2015). "Terahertz Radiation from $\mathrm{Bi}_{2} \mathrm{Sr}_{2} \mathrm{CaCu}_{2} \mathrm{O}_{8+\delta}$ Interlayer Josephson Junctions: Progress and Future Strategies." City of University of NY-Queens College Colloquium, New York, NY, February 25, 2015.

Hao, Y., T.M. Benseman, A.E. Koshelev, V. Vlasko-Vlasov, W.-K. Kwok, U. Welp, C. Keiser, B. Gross, M. Lange, D. Koelle, R. Kleiner and K. Kadowaki (2015). "The Stability of Current Filaments in $\mathrm{Bi}_{2} \mathrm{Sr}_{2} \mathrm{CaCu}_{2} \mathrm{O}_{8+\delta}$ Observed Via Luminescent Thermal Microscopy." American Physical Society March Meeting, San Antonio, TX, March 2-6, 2015.

Welp, U., T. Benseman, Y. Hao, A.E. Koshelev, V. Vlasko-Vlasov, W.-K. Kwok, H. Minami, C. Watanabe, K. Kadowaki, B. Gross, M. Lange, D. Koelle and R. Kleiner (2015). "Current Filamentation in Large $\mathrm{Bi}_{2} \mathrm{Sr}_{2} \mathrm{CaCu}_{2} \mathrm{O}_{8+\delta}$ Mesas Observed by Luminescent and Scanning Laser Thermal Microscopy.” Plasma 2014, Kyoto, Japan, November 30-December 3, 2014.

2015-091-NO

REFEREED PUBLICATION

Chong, L., X. Zeng, W. Ding, D.J. Liu and J. Zou (2015). "NaBH ${ }_{4}$ in "Graphene Wrapper:" Significantly Enhanced Hydrogen Storage Capacity and Regenerability through Nanoencapsulation.” Advanced Materials 27(34): 5070-5074.

\section{PRESENTATIONS}

Liu, D.-J. (2015). "Recent Developments in New Materials and Characterization Techniques for Energy Storage and Conversion." University of Illinois at Chicago, Chicago, IL, February 26, 2015. (Also see 2014-051.)

Liu, D.-J. (2015). "Recent Developments in New Materials and Characterization Techniques for Energy Storage and Conversion.” University of Nebraska, Lincoln, NE, October 20, 2014. (Also see 2014-051.) 


\section{5-096-NO}

\section{REFEREED PUBLICATIONS}

Benmore, C.J., L.B. Skinner, B. Lee, J.R. Weber, J.B. Parise and M.A. Williamson. "Topological Ordering in Liquid $\mathrm{UO}_{2}$." Journal of Physics: Condensed Matter. (To be published.) (Also see 2015-136.)

Skinner, L.B., C.J. Benmore, J.R. Weber, M.A. Williamson, A.J. Tamalonis, A.S. Hebden, T. Wiencek, O.L.G. Alderman, M. Guthrie, L. Leibowitz and J.B. Parise (2015). "Molten Uranium Dioxide Structure and Dynamics.” Science 346(6212): 984-987. (Also see 2015-136.)

\section{DATA-DRIVEN SCIENCE GRAND CHALLENGE}

\section{3-165-R2}

\section{REFEREED PUBLICATIONS}

Chen, J., L. Wang and M. Anitescu. "A Parallel Tree Code for Computing Matrix-Vector Products with the Matern Kernel." ACM Transactions on Mathematical Software. (To be published.)

Habib, S., A. Pope, H. Finkel, N. Frontiere, K. Heitmann, D. Daniel, P. Fasel, V. Morozov, G. Zagaris, T. Peterka, V. Vishwanath, Z. Lukic, S. Sehrish and W.-K. Liao (2016). "HACC: Simulating Sky Surveys on State-of-the-Art Supercomputing Architectures.” New Astronomy 42: 49-65.

Heitmann, K., E. Lawrence, J. Kwan, S. Habib and D. Higdon (2014). “The Coyote Universe Extended: Precision Emulation of the Matter Power Spectrum.” Astrophysical Journal 780(1): 111.

Heitmann, K., S. Habib, H. Finkel, N. Frontiere, A. Pope, V. Morozov, S. Rangel, E. Kovacs, J. Kwan, N. Li, S. Rizzi, J. Insley, V. Vishwanath, T. Peterka, D. Daniel, P. Fasel and G. Zagaris (2014). "Large-Scale Simulations of Sky Surveys." Computing in Science \& Engineering 16(5): 14-23.

Kwan, J., S. Bhattacharya, K. Heitmann and S. Habib (2013). “Cosmic Emulation: The Concentration-Mass Relation for Wcdm Universes." Astrophysical Journal 768(2): 123.

Sunayama, T., N. Padmanabhan, K. Heitmann, S. Habib and E. Rangel. "Efficient Construction of Mock Catalogs for Baryon Acoustic Oscillation Surveys." Journal of Cosmology and Astroparticle Physics. (To be published.)

\section{NON-REFEREED PUBLICATIONS}

Bicer, T., D. Gürsoy, R. Kettimuthu, F. De Carlo, G. Agrawal and I.T. Foster (2015). "Rapid Tomographic Image Reconstruction via Large-Scale Parallelization.” Euro-Par 2015: Parallel Processing-Proceedings of the 21st International Conference on Parallel and Distributed Computing, Vienna, Austria, August 24-28, 2015.

Foster, I. (2014). “Networking Materials Data.” 2014 International Advanced Research Workshop on High Performance Computing: From Clouds and Big Data to Exascale and Beyond, Centraro, Italy, July-11, 2014.

Malik, T., K. Chard and I. Foster (2014). "Benchmarking Cloud-based Tagging Services.” 6th International Workshop on Cloud Data Management, 30th IEEE International Conference on Data Engineering, Chicago, IL, March 31, 2014.

Wozniak, J.M., K. Chard, B. Blaiszik, R. Osborn, M. Wilde and I. Foster (2016). "Big Data Remote Access Interfaces for Light Source Science." 2nd IEEE/ACM International Symposium on Big Data Computing, Limassol, Cyprus, December 7-10, 2015.

Wozniak, J.M., T.G. Armstrong, K.C. Maheshwari, D.S. Katz, M. Wilde and I.T. Foster (2016). "Interlanguage Parallel Scripting for Distributed Memory Scientific Computing." 10th Workshop on Workflows in Support of Large-Scale Science, Austin, TX, November 15, 2015.

Wozniak, J.M., T.G. Armstrong, S.J. Krieder, K. Maheshwari, M. Wilde and I.T. Foster (2014). "Mega Python: Scalable Interlanguage Scripting for Scientific Computing." Super Computer Conference (SC13), Denver, CO, November 18-22, 2013. 
Wozniak, J.M., T.G. Armstrong, D.S. Katz, M. Wilde and I.T. Foster (2014). “Toward Computational Experiment Management via Multi-language Applications." 2014 ASCR Workshop on Software Productivity for Extreme-Scale Science, Rockville, MD, January 13-14, 2014.

\section{PRESENTATIONS}

Almer, J., S. Stock and R. Suter (2014). "Studies of Complex Materials using High-Energy X-rays." Diamond Light Source, Oxfordshire, England, May 29, 2014.

Blaiszik, B., K. Chard, R. Anathakrishnan, S. Tuecke and I. Foster (2015). "Globus Data Publication Services." US-UK Workshop on Materials Theory and Simulation, Argonne National Laboratory, Argonne, IL, January 26, 2015.

Blaiszik, B., K. Chard, S. Tuecke and I. Foster (2015). "Globus: Research Data Management-as-a-Service." Opportunities in Materials Informatics (OMI), University of Wisconsin-Madison, Madison, WI, February 9, 2015.

Blaiszik, B., K. Chard, H. Pruyne, R. Anathakrishnan, S. Tuecke and I. Foster (2014). "Globus Scientific Data Services Current and Future." Materials Genome Initiative Materials Data Workshop, Air Force Research Laboratory, Dayton, OH, July 15, 2014.

Foster, I. (2014). "An Architecture for a National Data Service." 1st National Data Service Consortium Workshop, Boulder, CO, Jun 12-13, 2014.

Foster, I. (2014). "Networking Materials Data." Workshop on Building an Integrated MGI Accelerator Network, Atlanta, GA, June 2014.

Foster, I. (2014). "Publication Services for Materials." 2014 Seminar at the PRISMS Center, University of Michigan, Ann Arbor, MI, June 24, 2014.

Habib, S. (2014). "Computing the Universe: How to Stuff a Supercomputer Inside a Laptop." Carnegie Mellon University, Pittsburgh, PA, April 2014.

Habib, S. (2014). "HACCing the Universe on the BG/Q." 20th Annual ScicomP 2014 BG Consortium Meeting, Chicago, IL, May 27-30, 2014.

Habib, S. (2014). “Thoughts on Next-Generation Computing and LSST DESC.” LLST DESC Dark Energy Science Collaboration Meeting, Philadelphia, PA, June 2014.

Heitmann, K. (2014). "Cosmological Simulations for Large-Scale Sky Surveys." Oak Ridge Leadership Computing Facility (OLCF) Users Meeting, Oak Ridge, TN, July 22-24, 2014.

Krogstad, M., R. Osborn, S. Rosenkranz, K. Taddei, J. Allred and O. Chmaissem (2015). "Single Crystal Diffusion X-ray Scattering Using Continuous Rotation." American Physical Society March Meeting, San Antonio, TX, March 2-6, 2015.

Osborn, R. and S. Rosenkranz (2015). “Advances in Single Crystal Diffuse Scattering." 21st National Synchrotron Radiation Research Center User’s Meeting, Hsinchu, Taiwan, September 9-10, 2015.

Osborn, R., P. Zapol, A. Ngo, S. Rosenkranz, J. Allred, O. Chmaissem, M. Krogstad, J. Morris, K. Taddei, D. Bugaris, G. Jennings, P. Chaupas, X. Zhang, I. Foster, M. Wilde, J. Wozniak, M. Anitescu, K. Petra, R. Ananthakrishnan, B. Blaiszik, K. Chard and B. Allen (2015). "Big Data: Beyond the Workflow." 2015 Workshop on Grand Challenges for Neutrons and Supercomputing, Argonne, IL, March 30-31, 2015.

Osborn, R., P. Zapol, A. Ngo, S. Rosenkranz, J. Allred, O. Chmaissem, M. Krogstad, J. Morris, K. Taddei, D. Bugaris, G. Jennings, P. Chaupas, X. Zhang, I. Foster, M. Wilde, J. Wozniak, M. Anitescu, K. Petra, R. Ananthakrishnan, B. Blaiszik, K. Chard and B. Allen (2015). "Single Crystal Diffuse Scattering: Beyond the Workflow." APS/CNM User's Meeting, Argonne, IL, May 11-14, 2015.

Rosenkranz, S. (2014). "Quantitative Analysis of the Diffuse Scattering from a Bilayer Manganite." American Physical Society March Meeting 2014, Denver, CO, March 3-7, 2014. 
Wozniak, J.M. (2014). "Parallel Scripting for Beamline Science: Connecting Big Data and HPC.” 2014 BES Facilities Computing Working Group Technical Meeting, Berkeley, CA, February 20, 2014.

Wozniak, J.M. (2014). "Studies in Big Data and HPC from X-ray Crystallography." 1st Workshop of the INRIA-IIlinois-ANL-BSC Joint Laboratory on Extreme Scale Computing, Sophia Antipolis, France, June 9-11, 2014.

\section{ADVANCED COMPUTING}

\section{3-199-R2}

\section{REFEREED PUBLICATIONS}

Romero, N.A., R.J. Harrison, G. Beylkin, F.A. Bischoff, J. Calvin, G. Fann, J. Fosso-Tande, D. Galindo, J. Hammond, R. Hartman-Baker, J.C. Hill, J. Jia, J.S. Kottmann, M.-J.Y. Ou, L.E. Ratcliff, M.G. Reuter, A.C. Richie-Halford, H. Sekino, W.A. Shelton, B.E. Sundahl, W.S. Thornton, E.F. Valeev, A. Vazquez Mayagoitia, N. Vence and Y. Yokoi. "MADNESS: A Multiresolution, Adaptive Numerical Environment for Scientific Simulation." SIAM Journal on Scientific and Statistical Computing. (To be published.)

Vázquez-Mayagoitia, A., J.R. Hammond, R.J. Harrison and W.S. Thornton (2015). "Quantum Chemistry Methods with Multiwavelet Bases on Massive Parallel Computers." Annual Reports in Computational Chemistry. R.A. Wheeler, Elsevier B.V. 10: Chapter 1.

\section{PRESENTATIONS}

Ratcliff, L.E., A. Vazquez Mayagoitia and N.A. Romero (2015). "Massively Parallel Density Functional Theory with Multiwavelets." Psi-k 2015 Conference, San Sebastian, Spain, September 6-12, 2015.

Vazquez Mayagoitia, A. (2015). "Tenors of Dynamic (Hyper) Polarizabilities from TDHF/TDDFT Schemes with Multiresolution Multiwavelet Bases.” 15th International Congress of Quantum Chemistry, Beijing, China, June 8-13, 2015.

Vázquez-Mayagoitia, A. and J.R. Hammond (2013). "DFT and TD-DFT Using Multiresolution Multiwavelet Basis Sets on Petaflop Supercomputers." 15th International Conference on Density Functional Theory and Its Applications (DFT 2013), Durham University, Durham, United Kingdom, September 9-13, 2013.

\section{3-206-R2}

\section{REFEREED PUBLICATION}

Cattlet, C. and R. Ghiani (2015). “Big Data for Social Good.” Big Data Journal Special Issue 3(1): 1-2.

Catlett, C., I. Foster, T. Malik, B.J. Goldstein, J. Giuffrida, Y. Shao, A. Panella, D. Eder, E. van Zanten, R. Mitchum and S. Thaler (2015). "Plenario: An Open Data Discovery and Exploration Platform for Urban Science." Digest of Papers. IEEE Symposium on Mass Storage Systems 37(4): 27-42.

\section{PRESENTATIONS}

Catlett, C. (2015). "Big Data and New Institutions." Bringing Social Science Back In: The Big Data Revolution and Urban Theory, Harvard University, Cambridge, MA, December 2014.

Catlett, C. (2015). “Computation, Information, Embedded Systems, and the Future of Cities." The International Conference for High Performance Computing, Networking, Storage and Analysis (SC14), New Orleans, LA, November 16-21, 2014.

Catlett, C. (2015). “Computation, Information, Embedded Systems, and the Future of Cities." Acting Locally, Understanding Globally: Scaling Up Community Collected Data in Developing Cities Workshop, Santa Fe, NM, November 19-21, 2014.

Catlett, C. (2015). "Creating Integrated Computation and Mathematical Capabilities to Transform the Design, Planning, and Operation of Cities to Achieve Sustainability and Resilience." URBAN IMPRINT: The Art and Science Shaping our Cities, Chicago, IL, November 14, 2014. 
Catlett, C. (2015). "Data and Cities." Workshop of the U.S.-U.K. Trans-Atlantic Platform Digital Scholarship Workshop, Washington, D.C., January 2015.

Catlett, C. (2015). "Data and the City." Workshop on Instrumented Cities, Future Cities Catapult, London, UK, March 2015.

Catlett, C. (2015). "Instrumenting The (Urban) Environment." Exploring Points of Connection Workshop, Chicago, IL, July 1, 2015.

Catlett, C. (2015). "Instrumenting the City." Greater Chicago Area Systems Research (GCASR) Workshop, Chicago, IL, April 27, 2015.

Catlett, C. (2015). “Instrumenting the City.” Northwestern University Science Café, Evanston, IL, February 2015.

Catlett, C. (2015). "Instrumenting the City." Action Canada, University of Chicago Harris School of Public Policy, Chicago, IL, May 2015.

Catlett, C. (2015). “Instrumenting the City." University of Chicago, Chicago, IL, May 2015.

Catlett, C. (2015). "Open Data and Instrumenting Cities.” New York Scientific Data Summit, New York, NY, August 2, 2015.

Catlett, C. (2015). "Open Data and Instrumenting Cities." Monash Undergraduate Research Projects Abroad (MURPA), University of Queensland, St. Lucia, Australia, August 2015.

Catlett, C. (2015). "Open Data and Instrumenting Cities." Monash Undergraduate Research Projects Abroad (MURPA), Monash University, Clayton, Australia, August 2015.

Catlett, C. (2015). “Urban Center for Computation and Data." National Science Foundation, Arlington, VA, June 2015.

Catlett, C. (2015). “Urban Sciences: Sensing and Data.” University of Chicago, Chicago, IL, May 2015.

Catlett, C. (2015). “Urban Systems Science and Engineering.” DOE Big Ideas Summit, Washington, D.C., April 2015.

Catlett, C. (2015). “Urban Systems Science and Engineering." DOE Big Ideas Workshop National Renewable Energy Laboratory (NREL), Golden, CO, February 2015.

Catlett, C. (2015). "Urban Systems Science and Engineering." National Research Council Study, Chattanooga, TN, July 2015.

Catlett, C. (2014). "The City of Chicago Technology Plan." Chicago Architecture Foundation, Chicago, IL, June 4, 2014.

Catlett, C. (2014). "Computation and Data-Enabled Urban Design and Operation." Illinois Institute of Technology, Chicago, IL, October 23, 2014.

Catlett, C. (2014). "Computational Science and Urban Policy." 20th ACM SIGKDD Conference on Knowledge Discovery and Data Mining, New York, NY, August 24-27, 2014.

Catlett, C. (2014). "Computational Science and Urban Policy." 2014 Coalition for Academic Scientific Computation Fall Meeting, Arlington, VA, September 17-19, 2014.

Catlett, C. (2014). "Computational Urban Sciences." NCSA Blue Waters Symposium for Petascale Science and Beyond, Champaign, IL, May 12-15, 2014.

Catlett, C. (2014). "Creating Integrated Computational and Mathematical Capabilities to Transform the Design, Planning, and Operation of Cities to Achieve Sustainability and Resilience." University of Illinois, Urbana-Champaign, IL, November 4, 2013. 
Catlett, C. (2014). "Open Data, Big Computation, and a New Era for Urban Research." American Association for the Advancement of Science Annual Meeting (AAAS 2014), Chicago, IL, February 7-13, 2014.

Catlett, C. (2014). "Rapid Urbanization in Developing Economies." Committee on National Statistics, Washington, D.C., February 6, 2014.

Catlett, C. (2014). "Science for Cities." 7th World Urban Forum: Urban Equity in Development-Cities for Life, Medellin, Columbia, April 5-11, 2014.

Catlett, C. (2014). "Toward an "Urban Science." Argonne Training Program on Extreme-Scale Computing, St. Charles, IL, August 3-15, 2014.

Catlett, C. (2014). "Toward an "Urban Science." Science and Big Data to Improve Urban Planning, Design, and Policy, Washington, D.C., July 21, 2014.

Catlett, C. (2014). “Urban Data Sciences.” 2014 Big Data and Health, Chicago, IL, May 9, 2014.

Catlett, C. (2014). "URBAN IMPRINT: The Art and Science Shaping Our Cities." Alumni Club of the Bay Area Webcast, San Francisco, CA, May 14, 2014.

Catlett, C. (2014). "Urban Science." International Advanced Research Workshop on High Performance Computing: From Clouds and Big Data to Exascale and Beyond, Centraro, Italy, July 7-11, 2014.

Catlett, C. (2013). "Big Data and Urban Social Sciences." ORDCamp, Chicago, IL, January 26, 2013.

Catlett, C. (2013). "Computation Urban Sciences: Emerging Opportunities." 2nd ACM SIGKDD International Workshop on Urban Computing (UrbComp 2013), ACM-KDD, Chicago, IL, August 11, 2013.

Catlett, C. (2013). "Computational Opportunities in Urban Sciences." IEEE eScience Conference, Chicago, IL, October 3, 2012.

Catlett, C. (2013). "Data Complexity in the Sciences: The Computation Institute." 2013 National Big Data Week, Chicago, IL, April 24, 2013.

Catlett, C. (2013). "Emerging Opportunities in Urban Sciences." University of Illinois at Urbana-Champaign, Urbana, IL, December 10, 2012.

Catlett, C. (2013). "Interdisciplinary Urban Sciences." Kickoff Workshop of Urban Sciences Research Coordination Network, Chicago, IL, February 15, 2013.

Catlett, C. (2013). "Opportunities for Data and Computational Sciences in Present and Future Cities." NCSA 2nd Annual CHANGES Workshop, Chicago, IL, September 10, 2013.

Catlett, C. (2013). "Urban Center for Computation and Data." 2013 Workshop: How Far Can "Big Data" Take Us Towards Understanding Cities?, Santa Fe, NM, September 20, 2013.

Catlett, C. (2013). "Urban Center for Computation and Data: Opportunities for Computational Urban Sciences." Extreme Scale Computing School, Chicago, IL, August 8, 2013.

Catlett, C. (2013). “Urban Sciences and Computer Science.” University of Chicago, Chicago, IL, January 15, 2013.

Catlett, C. (2013). “Urban Sciences in Chicago.” Mayor's Innovation Project, Chicago, IL, August 15, 2013.

Catlett, C. (2013). "Urban Sciences in Chicago." Exploratory Workshop on Energy Policy and Computational Urban Sciences, 8th IEEE Conference on eScience, The University of Chicago Beijing Center, Beijing, China, January 30, 2013.

Catlett, C. (2013). "Urban Sciences in Chicago." Chicago Harris Public Policy Lecture Series, University Club of Chicago, Chicago, IL, September 18, 2013. 
Catlett, C. (2013). "Urban Security and Risk Management." 14th International Latin American Smart Cities Conference, Quito, Ecuador, September 26, 2013.

Catlett, C. (2013). “Urban Sensing in Chicago.” 2013 Workshop on Urban Sensing, Argonne, IL, August 27, 2013.

Catlett, C. (2013). "Urban Social Sciences and Mathematics." Argonne Fermilab Annual Collaboration Workshop, Chicago, IL, October 7, 2012.

\section{3-208-R2}

\section{REFEREED PUBLICATIONS}

Devoid, S., N. Desai and L. Hochstein (2014). "Poncho: Enabling Smart Administration of Full Private Clouds."

Proceedings of the 27th Large Installation System Administration (LISA) Conference, Washington, D.C.,

November 3-8, 2013, USENIX Association: 17-26.

Gerlach, W., W. Tang, K. Keegan, T. Harrison, A. Wilke, J. Bischof, M. D'Souza, S. Devoid, D. Murphy-Olson, N. Desai and F. Meyer (2015). "Skyport-Container-Based Execution Environment Management for Multi-Cloud Scientific Workflows." DataCloud 2014: Proceedings of the 5th International Workshop on Data-Intensive Computing in the Clouds (SC14), New Orleans, LA, November 21, 2014, IEEE Press: 25-32.

\section{3-212-R2}

\section{REFEREED PUBLICATIONS}

Arsenault, L.F., A. Lopez-Bezanilla, O.A. von Lilienfeld and A.J. Millis (2015). "Machine Learning for Many-body Physics: The Case of the Anderson Impurity Model." Physical Review B 90(15).

Bereau, T., D. Andrienko and O.A. von Lilienfeld (2015). "Transferable Atomic Multipole Machine Learning Models for Small Organic Molecules.” Journal of Chemical Theory and Computation 11(7): 3225-3233.

Faber, F., A. Lindmaa, O. Anatole von Lilienfeld and R. Armiento (2015). "Crystal Structure Representations for Machine Learning Models of Formation Energies.” International Journal of Quantum Chemistry 115(16): 1094-1101.

Hansen, K., F. Biegler, R. Ramakrishnan, W. Pronobis, O. Anatole von Lilienfeld, K.-R. Muller and A. Tkatchenko (2015). "Machine Learning Predications of Molecular Properties: Accurate Many-Body Potentials and Nonlocality in Chemical Space." Journal of Physical Chemistry Letters 6(12): 2326-2331.

Lopez-Bezanilla, A. and O.A. von Lilienfeld (2014). "Modeling Electronic Quantum Transport with Machine Learning." Physical Review B 89(23).

Montavon, G., K. Hansen, S. Fazli, M. Rupp, F. Biegler, A. Ziehe, A. Tkatchenko, O.A. von Lilienfeld and K.-R. Müller (2013). "Learning Invariant Representations of Molecules for Atomization Energy Prediction." Proceedings of the Advances in Neural Information Processing Systems (NIPS 2012), Lake Tahoe, NV, December 3-6, 2012, 25: 449-457.

Montavon, G., M. Rupp, V. Gobre, A. Vazquez-Mayagoitia, K. Hansen, A. Tkatchenko, K.R. Muller and O.A. von Lilienfeld (2013). "Machine Learning of Molecular Electronic Properties in Chemical Compound Space." New Journal of Physics 15.

Ramakrishnan, R., O. Anatole von Lilienfeld, P.O. Dral and M. Rupp (2015). "Big Data meets Quantum Chemistry Approximations: The $\Delta$-Machine Learning Approach." Journal of Chemical Theory and Computation 11(5): 2087-2096.

Ramakrishnan, R., P.O. Dral, M. Rupp and O.A. von Lilienfeld (2014). "Quantum Chemistry Structures and Properties of 134 Kilo Molecules." Scientific Data 1: 140022.

von Lilienfeld, O.A. (2013). "First Principles View on Chemical Compound Space: Gaining Rigorous Atomistic Control of Molecular Properties." International Journal of Quantum Chemistry 113(12): 1676-1689. 


\section{PRESENTATIONS}

Vazquez Mayagoitia, A. (2015). "Multiscale Methods with Machine Learning Approaches: Production and Analysis of Large Datasets with HPC." UCLA-IPAM Machine Learning for Many-Particle Systems Workshop, Los Angeles, CA, February 23-27, 2015.

von Lilienfeld, O.A. (2015). "Alchemy and Machine Learning Methods for The Sampling of Chemical Space from First Principles." 10th Congress of the World Association of Theoretical and Computational Chemists (WATOC 2014), Santiago, Chile, October 5-10, 2014.

\section{3-213-R2}

\section{REFEREED PUBLICATIONS}

Balaprakash, P., L.A. Bautista Gomez, M.-S. Bouguerra, S.M. Wild, F. Cappello and P.D. Hovland (2015). "Analysis of the Tradeoffs Between Energy and Run Time for Multilevel Checkpointing." Proceedings of the 5th International Workshop on High Performance Computing Systems. Performance Modeling, Benchmarking, and Simulation (PMBS 2014), New Orleans, LA, November 16-21, 2014, Springer International Publishing 8966(Lecture Notes in Computer Science): 249-263.

Balaprakash, P., Y. Alekseev, S. Mickelson, S. Leyffer, R. Jacob and A. Craig (2015). "Machine-Learning-Based Load Balancing for Community Ice Code Component in CESM." Proceedings of the 11th International Conference on High Performance Computing for Computational Science (VECPAR 2014), Eugene, OR, June 30-July 3, 2014, Springer International Publishing 8969(Revised Selected Papers): 79-91.

Boyer, M., J. Meng and K. Kumaran (2013). "Improving GPU Performance Prediction with Data Transfer Modeling." Proceedings of the 3rd International Workshop on Accelerators and Hybrid Exascale Systems (AsHES), Boston, MA, May 20, 2013: 1097-1106.

Bui, H., P. Malakar, V. Vishwanath, T.S. Munson, E.S. Jung, A.E. Johnson, M.E. Papka and J. Leigh (2016). “Improving Communication Throughput by Multipath Load Balancing on Blue Gene/Q." Proceedings of the 22nd IEEE International Conference on High-Performance Computing, Bengaluru, India, December 16-19, 2015.

Guo, J., J. Meng, Q. Yi, V. Morozov and K. Kumaran (2014). “Analytically Modeling Application Execution for Software-Hardware Co-design "Proceedings of the 2014 IEEE 28th International Parallel \& Distributed Processing Symposium (IPDPS), Phoenix, AZ, May 19-23, 2014, IEEE: 468-477.

Joohwan, L., J. Meng and H. Kim (2014). "SESH Framework: A Space Exploration Framework for GPU Application and Hardware Codesign." Proceedings of the 4th International Workshop on Performance Modeling, Benchmarking and Simulation of High Performance Computer Systems (PMBS13) and Supercomputing Conference (SC), Denver, CO, November 17-22, 2013.

Maheshwari, K., E.-S. Jung, J. Meng, V. Morozov, V. Vishwanath and R. Kettimuthu (2016). "Workflow Performance Improvement Using Model-based Scheduling Over Multiple Clusters and Clouds." Future Generation Computer Systems 54: $206-218$.

Malakar, P., V. Vishwanath, T. Munson, C. Knight, M. Hereld, S. Leyffer and M.E. Papka (2016). "Optimal Scheduling of in situ Analysis for Large-scale Scientific Simulations." Proceedings of the 28th IEEE/ACM International Conference for High Performance Computing, Networking, Storage and Analysis (SC 2015), Article 52, Austin, TX, November 15-20, 2015.

Malakar, P. and V. Vishwanath (2015). "Hierarchical Read-write Optimizations for Scientific Applications with Multi-variable Structured Datasets." Proceedings of the 12th Annual IFIP International Conference on Network and Parallel Computing (NPC), New York, NY, September 17-19, 2015.

Meng, J., T. Uram, V.A. Morozov, V. Vishwanath and K. Kumaran (2015). "Modeling Cooperative Threads to Project GPU Performance for Adaptive Parallelism." Proceedings of the IPDPS 16th IEEE International Workshop on Parallel and Distributed Scientific and Engineering Computing (PDSEC15), Hyderabad, India, May 25-29, 2015. 
Meng, J., X. Wu, V.A. Morozov, V. Vishwanath, K. Kumaran, V. Taylor and C.W. Lee (2014). "SKOPE: A Framework for Modeling and Exploring Workload Behavior." Proceedings of the 11th Association for Computer Machinery (ACM) Conference on Computing Frontiers (CF), Cagliari, Italy, May 20-22, 2014, ACM New York, NY (Article 6).

Meng, J., V. Morozov, V. Vishwanath and K. Kumaran (2013). "Dataflow-Driven GPU Performance Projection for Multi-Kernel Transformations." Proceedings of the International Conference for High Performance Computing, Networking, Storage, and Analysis (SC 2012), Salt Lake City, UT, November 10-16, 2012, (82).

Morozov, V., J. Meng, V. Vishwanath, K. Kumaran and M.E. Papka (2013). “Characterization and Understanding Machine-Specific Interconnect." Proceedings of the 12th International Conference on Parallel Computing Technologies (PaCT 2013), St. Petersburg, Russia, September 30-October 4, 2013, Lecture Notes in Computer Science 7979(2013): 90-104.

Morozov, V., V. Vishwanath, K. Kumaran, J. Meng and M.E. Papka (2013). "Early Experience on the Blue Gene/Q Supercomputing System." Proceedings of the 27th IEEE International Parallel \& Distributed Processing Symposium (IPDPS 2013), Boston, MA, May 20-24, 2013: 1229-1240.

Nelson, T., A. Rivera, P. Balaprakash, M. Hall, P.D. Hovland, E. Jessup and B.R. Norris (2015). “Generating Efficient Tensor Contractions for GPUs." Proceedings of the 44th International Conference on Parallel Processing (ICPP 2015), Beijing,

China, September 1-4, 2015: 969.

Panwar, L.S., A.M. Aji, J. Meng, P. Balaji and W. Feng (2014). "Online Performance Projection for Clusters with Heterogeneous GPUs." Proceedings of the IEEE International Conference on Parallel and Distributed Systems (ICPADS), Seoul, South Korea, December 15-18, 2013.

\section{NON-REFEREED PUBLICATIONS}

Balaprakash, P., V. Morozov, R. Kettimuthu and I. Foster. "Improving Data Transfer Throughput with Direct Search Optimization." 30th IEEE International Parallel and Distributed Processing Symposium (IPDPS 2016), Chicago, IL, May 23-27, 2016. (To be published.)

Bui, H., R. Jacob, P. Malakar, V. Vishwanath, A.E. Johnson, M.E. Papka and J. Leigh (2015). "Multipath Load Balancing for M $\times$ N Communication Patterns on the Blue Gene/Q Supercomputer Interconnection Network." 1st IEEE International Workshop on High-Performance Interconnection Networks Towards the Exascale and Big-Data Era, Chicago, IL, September 8, 2015.

\section{PRESENTATION}

Zhang, Y., P. Balaprakash, J. Meng, V. Morozov, S. Parker and K. Kumaran (2015). "Raexplore: Enabling Rapid, Automated Architecture Exploration for Full Applications." ACM/IEEE Conference on Supercomputing (SC14), New Orleans, LA, November 16-21, 2014.

\section{4-167-R1}

\section{PRESENTATIONS}

Drewniak, B., F. Chen, R. Jacob and C. Catlett (2014). "Urban Landscapes and Climate Change: Workshop Report." 11th Symposium on the Urban Environment, American Meteorological Society 94th Annual Meeting, Atlanta, GA, February 2-6, 2014.

Jacob, R. (2015). "Continuous Sensing of the Urban Boundary Layer." Air \& Waste Management Association: Lake Michigan State Section 2014 Air Quality Management Conference, Chicago, IL, November 12, 2014.

Jacob, R. (2015). "Standard Onboard Sensor Control and Compute for UAVs." International Society for Atmospheric Research using Remotely-piloted Aircraft (ISARRA 2015), Norman, OK, May 20-22, 2015.

Sankaran, R., P. Beckman, C. Catlett, R. Jacob and K. Keahey (2015). "Waggle: A Framework for Intelligent Attentive Sensing and Actuation." American Geophysical Union (AGU) Fall Meeting, San Francisco, CA, December 17, 2014. 


\section{4-174-R1}

\section{PRESENTATIONS}

Ferrier, N. (2015). "Image-based Methods for 3D Structure." Institute for Molecular Engineering Seminar, Chicago, IL, June 2015.

Ferrier, N. (2014). “Image-based Metrology.” Imaging Workshop, Taipei, Taiwan, May 2014.

\section{4-181-R1}

\section{REFEREED PUBLICATIONS}

Bautista Gomez, L.A. and F. Cappello (2014). "Detecting Silent Data Corruption Through Data Dynamic Monitoring for Scientific Applications." Proceedings of the 19th ACM SIGPLAN Symposium on Principals and Practice of Parallel Programing (ACM PPoPP 2014), Orlando, FL, February 15-19, 2014, ACM, New York, NY: 381-382.

Cappello, F., A. Geist, W. Gropp, S. Kale, B. Kramer and M. Snir (2014). “Toward Exascale Resilience: 2014 Update.” Supercomputing Frontiers and Innovations 1(1): 5-8.

Snir, M., R.W. Wisniewski, J.A. Abraham, S.V. Adve, S. Bagchi, P. Balaji, J. Belak, P. Bose, F. Cappello, B. Carlson, A.A. Chien, P. Coteus, N.A. Debardeleben, P. Diniz, C. Engelmann, M. Erez, S. Fazzari, A. Geist, R. Gupta, F. Johnson, S. Krishnamoorthy, S. Leyffer, D. Liberty, S. Mitra, T. Munson, R. Schreiber, J. Stearley and E.V. Hensbergen (2014). "Addressing Failures in Exascale Computing." International Journal of High Performance Computing Applications 28(2): $129-173$.

\section{NON-REFEREED PUBLICATIONS}

Balaprakash, P., L.A. Bautista Gomez, M.S. Bouguerra, S.M. Wild, F. Cappello and P.D. Hovland (2014).

"Energy-Performance Tradeoffs in Multilevel Checkpoint Strategies." IEEE International Conference on Cluster Computing (CLUSTER), Madrid, Spain, September 22-26, 2014.

Bautista-Gomez, L. and F. Cappello (2015). "Detecting and Correcting Data Corruption in Stencil Applications through Multivariate Interpolation." 1st International Workshop on Fault Tolerant Systems (FTS 2015) at IEEE Cluster 2015, Chicago, IL, September 8-11, 2015.

Bautista-Gomez, L. and F. Cappello (2015). "Detecting Silent Data Corruption for Extreme-Scale MPI Applications." 2015 EuroMPI (Message Passing Interface) Meeting, Bordeaux, France, September 21-23, 2015.

Bautista-Gomez, L. and F. Cappello (2015). "Exploiting Spatial Smoothness in HPC Applications to Detect Silent Data Corruption." 17th IEEE International Conference on High Performance Computing and Communications, New York, NY, August 24-26, 2015.

Bautista Gomez, L.A., F. Cappello, L. Carro, N. DeBardeleben, B. Fang, S. Gurumurthi, S. Keckler, K. Pattabiraman, P. Rech and M. Sonza Reorda (2014). "GPGPUs: How to Combine High Computational Power with High Reliability." Design, Automation \& Test in Europe (DATE '14), Dresden, Germany, March 24-28, 2014.

Berrocal, E., L. Bautista-Gomez, S. Di, Z. Lan and F. Cappello (2014). "Lightweight Silent Data Corruption Detection Based on Runtime Data Analysis for HPC Applications." IEEE International Parallel \& Distributed Processing Symposium, Phoenix, AZ, May 19-23, 2014.

Di, S. and F. Cappello. "Fast Error-bounded Lossy HPC Data Compression with SZ." 30th IEEE International Parallel and Distributed Processing Symposium (IPDPS 2016), Chicago, IL, May 23-27, 2016. (To be published.)

Di, S., E. Berrocal and F. Cappello (2015). "An Efficient Silent Data Corruption Detection Method with Error-Feedback Control and Even Sampling for HPC Applications." 15th IEEE/ACM International Symposium on Cluster, Cloud and Grid Computing (IEEE/ACM CCGrid 2015), Shenzhen, Guangdong, China, May 4-7, 2015.

Di, S., L. Bautista-Gomez and F. Cappello (2015). "Optimization of Multi-Level Checkpoint Model with Uncertain Execution Scales." ACM/IEEE International Conference for High Performance Computing, Networking, Storage and Analysis (SC14), New Orleans, LA, November 16-21, 2014. 
Di, S., M.S. Bouguerra, L. Bautista-Gomez and F. Cappello (2014). "Optimization Multi-Level Checkpoint Model for Large Scale HPC Applications.” IEEE International Parallel \& Distributed Processing Symposium (IPDPS'14), Phoenix, AZ, May 19-23, 2014.

Gainaru, A., M.S. Bouguerra, F. Cappello, M. Snir and W. Kramer (2015). "Navigating the Blue Waters: Online Failure Prediction in the Petascale Era." 29th IEEE International Parallel \& Distributed Processing Symposium, Hyderabad, India, May 25-29, 2015.

Gainaru, A., G. Aupy, A. Benoit, F. Cappello, Y. Robert and M. Snir (2015). "Scheduling the I/O of HPC Applications Under Congestion.” 2015 IEEE International Parallel and Distributed Processing Symposium (IPDPS), Hyperabad, India, May 25-29, 2015.

Martsinkevich, T., T. Ropars and F. Cappello (2015). "Addressing the Last Roadblock for Message Logging in HPC: Alleviating the Memory Requirement Using Dedicated Resources." 21st International European Conference on Parallel and Distributed Computing (Euro-Par) 2015-8th Workshop on Resiliency in High Performance Computing in Clusters, Clouds and Grids, Vienna, Austria, August 24-28, 2015.

Martsinkevich, T., O. Subasi, O. Unsal, F. Cappello and J. Labarta (2015). "Fault-tolerant Protocol for Hybrid Task-parallel Message-passing Applications." 1st International Workshop on Fault Tolerant Systems (FTS 2015) at IEEE Cluster 2015, Chicago, IL, September 8-11, 2015.

Subasi, O., S. Di, L. Bautista-Gomez, P. Balaprakash, O. Unsal, J. Labarta, A. Cristal and F. Cappello. "Spatial Support Vector Regression to Detect Silent Errors in the Exascale Era." 16th IEEE/ACM International Symposium on Cluster, Cloud and Grid (CCGRID 2016) Cartagena, Columbia, May 16-19, 2016. (To be published.)

\section{PRESENTATIONS}

Bautista-Gomez, L. (2016). "Opportunities for Online Data Analytics in HPC." 4th Joint-Laboratory on Extreme Scale Computing Workshop, Bonn, Germany, December 2-4, 2015.

Bautista-Gomez, L. (2015). “Analytic Based Corruption Detection.” 3rd Joint-Laboratory for Extreme-Scale Computing Workshop, Barcelona, Spain, June 29-July 1, 2015.

Bautista-Gomez, L. (2015). "Detecting Silent Data Corruption for Extreme-Scale Application through Data Mining." 2nd Joint-Laboratory on Extreme Scale Computing Workshop, Chicago, IL, November 24-26, 2014.

Cappello, F. (2016). "Trust in Results of Numerical Simulation: The New Challenging Scientific Problem in Reliability." Conference on Data Analysis: CODA 2016, Santa Fe, NM, March 2-4, 2016.

Cappello, F. (2015). "Let's Forget About "Fault Tolerance" and "Resilience" for HPC; Trusting is the New Challenging Scientific Problem in Reliability." 1st International Workshop on Fault Tolerant Systems (FTS 2015), Chicago, IL, September 8, 2015.

Di, S., E. Berrocal, L. Bautista-Gomez, K. Heisey, R. Gupta and F. Cappello (2015). "Towards Effective Detection of Silent Data Corruptions for HPC Applications." International Conference for High Performance Computing, Networking, Storage and Analysis (SC14), New Orleans, LA, November 16-21, 2014.

\section{4-182-R1}

\section{REFEREED PUBLICATIONS}

Foster, I., R. Ananthakrishnan, B. Blaiszik, K. Chard, R. Osborn, S. Tuecke, M. Wilde and J. Wozniak (In press).

"Networking Materials Data: Accelerating Discovery at Experimental Facilities." Advances in Parallel Computing. L. G. a. G. Joubert. Amsterdam, Netherlands, IOS Press Ebooks. 26: Big Data and High Performance Computing: $117-132$.

Wozniak, J.M., K. Chard, B. Blaiszik, R. Osborn, M. Wilde and I. Foster (2015). "Big Data Staging with MPI-IO for Interactive X-ray Science." Proceedings of the 2014 IEEE/ACM International Symposium on Big Data Computing (BDC 2014), London, United Kingdom, December 8-9, 2014, IEEE Computer Society: 26-34. 


\section{5-169-NO}

\section{NON-REFEREED PUBLICATIONS}

Collier, N., J. Ozik and C.M. Macal (2015). "Large-Scale Agent-based Modeling with Repast HPC: A Case Study in Parallelizing an Agent-based Model." 3rd Workshop on Parallel and Distributed Agent-Based Simulations (PADABS) at the Workshop of Euro-Par 2015, Vienna, Austria, August 24-28, 2015.

Ozik, J., N.T. Collier and J.M. Wozniak (2015). "Many Resident Task Computing in Support of Dynamic Ensemble Computations." 8th Workshop on Many-Task Computing on Clouds, Grids, and Supercomputers (MTAGS 2015), Austin, TX, November 15, 2015.

\section{PRESENTATIONS}

Collier, N.T. and J. Ozik (2015). "High Performance Computing and Computational Modeling: Repast HPC." University College London Big Data Institute, London, England, April 30, 2015.

Macal, C.C. (2015). “Agent-Based Modeling of Ebola Spread in a Large Urban Ara.” INFORMS Healthcare Conference, Nashville, TN, July 29-31, 2015.

\section{APPLIED ENERGY AND SUSTAINABLE TRANSPORTATION}

\section{3-148-R2}

REFEREED PUBLICATIONS

Bai, S., M.J. Davis and R.T. Skodje (2016). "The Sum over Histories Representation for Kinetic Sensitivity Analysis: How Chemical Pathways Change when Reaction Rate Coefficients are Varied.” The Journal of Physical Chemistry A 119(45): 11039-11052.

Bai, S., D. Zhou, M.J. Davis and R.T. Skodje (2015). “Sum over Histories Representation for Chemical Kinetics.” Journal of Physical Chemistry Letters 6(1): 183-188.

Hawkes, E.R., Y. Pei, S. Kook and S. Som (2014). "An Analysis of the Structure of an n-Dodecane Spray Flame Using PDF Modelling." Proceedings of the Australian Combustion Symposium, the University of Western Australia, Australia, November 6-8, 2013.

Knox, K.R., A.M.M. Abeykoon, H. Zheng, W.G. Yin, A.M. Tsvelik, J.F. Mitchell, S.J.L. Billinge and E.S. Bozin (2014). "Local Structural Evidence for Strong Electronic Correlations in Spinel $\mathrm{LiRH}_{2} \mathrm{O}_{4}$ " Physical Review B 88(17).

Kodavasal, J., C. Kolodziej, S. Ciatti and S. Som (2015). “CFD Simulation of Gasoline Compression Ignition.” Proceedings of the 2014 American Society of Mechanical Engineers (ASME) Internal Combustion Engine Division Fall Technical Conference, Columbus, IN, October 19-22, 2014, ICEF2014-5591(V002T06A008): 15 pages.

Kodavasal, J., C.P. Kolodziej, S.A. Ciatti and S. Som (2015). "A Numerical Study on the Effects of Injection Parameters and Swirl on Gasoline Compression Ignition at Idle-Load Conditions." Proceedings of the 5th Annual Meeting of the Saudi Arabian Section of the Combustion Institute, Riyadh, Saudi Arabia, May 3-4, 2015.

Kodavasal, J., C.P. Kolodziej, S.A. Ciatti and S. Som (2015). “Computational Fluid Dynamics Simulation of Gasoline Compression Ignition.” Journal of Energy Resources Technology 137(3): 032212 (032213 pages).

Kundu, P., Y.J. Pei, M.J. Wang, R. Mandhapati and S. Som (2015). "Evaluation of Turbulence-Chemistry Interaction Under Diesel Engine Conditions with Multi-flamelet RIF Model." Atomization and Sprays 24(9): 779-800.

Luo, Z.Y., S. Som, S.M. Sarathy, M. Plomer, W.J. Pitz, D.E. Longman and T.F. Lu (2014). "Development and Validation of an n-Dodecane Skeletal Mechanism Forspray Combustion Applications." Combustion Theory and Modelling 18(2): 187-203.

Payri, R., J.P. Viera, Y. Pei and S. Som (2015). “Experimental and Numerical Study of lift-off Length and Ignition Delay of a Two-component Diesel Surrogate.” Fuel 158: 957-967. 
Pei, Y., S. Som, E. Pomraning, P.K. Senecal, S.A. Skeen, J. Manin and L.M. Pickett (2016). "Large Eddy Simulation of a Reacting Spray Flame with Multiple Realizations Under Compression Ignition Engine Conditions." Combustion and Flame 162(12): 4442-4455.

Pei, Y., B. Hu and S. Som (2016). "Large Eddy Simulation of an n-Dodecane Spray Flame Under Different Ambient Oxygen Conditions." Proceedings of the 2015 ASME Internal Combustion Engine Division Fall Technical Conference, Houston, TX, November 8-11, 2015.

Pei, Y., M.J. Davis, L.M. Pickett and S. Som (2015). "Engine Combustion Network (ECN): Global Sensitivity Analysis of Spray A for Different Combustion Vessels." Combustion and Flame 162: 2337-2347.

Pei, Y., M. Mehl, W. Liu, T. Lu, W.J. Pitz and S. Som (2015). "A Multi-Component Blend as a Diesel Fuel Surrogate for Compression Ignition Engine Applications." Journal of Engineering for Gas Turbines and Power 137(11): 111502.

Pei, Y., W. Liu, M. Mehl, T. Lu, W.J. Pitz and S. Som (2015). "A Multi-Component Blend as a Diesel Fuel Surrogate for Compression Ignition Engine Applications." Proceedings of the 2014 American Society of Mechanical Engineers (ASME) Internal Combustion Engine Division Fall Technical Conference, Columbus, IN, October 19-22, 2014, ICEF2014-5625(V002T06A012): 11 pages.

Pei, Y., R. Shan, S. Som, M.J. Davis, D.E. Longman and T. Lu (2014). "Global Sensitivity Analysis of a Diesel Engine Simulation with Multi-Target Functions." Proceedings of the SAE 2014 International World Congress, Detroit, MI, April 8-10, 2014, SAE Technical Paper: 2014-2001-1117.

Raju, M., M. Wang, M. Dai, S. Quan, P.K. Senecal, S. Som, M. McNenly and D.L. Flowers (2013). “Towards Accommodating Comprehensive Chemical Reaction Mechanisms in Practical Internal Combustion Engine Simulations." Proceedings of the 8th U.S. National Combustion Meeting Organized by the Western States Section of the Combustion Institute, University of Utah, Salt Lake City, UT, May 19-22, 2013, 3: 2020-2025.

Senecal, P.K., E. Pomraning, K.J. Richards and S. Som (2013). "Grid-convergent Spray Models for Internal Combustion Engine CFD Simulations.” Journal of Energy Resources Technology 136(1): 012204.

Senecal, P.K., E. Pomraning, K.J. Richards and S. Som (2013). "Grid-convergent Spray Models for Internal Combustion Engine CFD Cimulations." Proceedings of the American Society of Mechanical Engineers (ASME) 2012 Internal Combustion Engine Division Fall Technical Conference, Vancouver, BC, Canada, September 23-26, 2012.

Som, S., W. Liu, D.D.Y. Zhou, G.M. Magnotti, R. Sivaramakrishnan, D.E. Longman, R.T. Skodje and M.J. Davis (2013). "Quantum Tunneling Affects Engine Performance." Journal of Physical Chemistry Letters 4(12): 2021-2025.

Wang, M., M. Raju, E. Pomraning, P. Kundu., Y. Pei and S. Som (2015). "Comparison of Representative Interactive Flamelet and Detailed Chemistry Based Combustion Models for Internal Combustion Engines." Proceedings of the 2014 American Society of Mechanical Engineers (ASME) Internal Combustion Engine Division Fall Technical Conference, Columbus, IN, October 19-22, 2014, ICEF2014-5522(V002T06A003): 13 pages.

Wang, Z., W. Liu, S. Som and D.E. Longman (2014). "Comparison of Different Chemical Kinetic Models for Biodiesel Combustion." Proceedings of the American Society of Mechanical Engineers (ASME) 2013 Internal Combustion Engine Division Fall Technical Conference (ICEF2013), Dearborn, MI, October 13-16, 2013, ICEF2013-19094(V002T02A004): 11 pages.

Wang, Z., W. Liu, S. Som and D.E. Longman (2013). "Comparison of Different Chemical Kinetic Models for Biodiesel Combustion." Proceedings of the 8th U.S. National Combustion Meeting Organized by the Western States Section of the Combustion Institute, University of Utah, Salt Lake City, UT, May 19-22, 2013, Curran Associates, Inc. 1: 556-566.

Xue, Q., S. Som, P.K. Senecal and E. Pomraning (2014). "Large Eddy Simulation of Fuel Spray Under Non-Reacting IC Engine Conditions." Atomization and Sprays 23(10): 925-955. 


\section{NON-REFEREED PUBLICATIONS}

Kodavasal, J., K. Harms, P. Srivastava, S. Som, S. Quan, K. Richards and M. Garcia (2016). "Development of a Stiffness-based Chemistry Load Balancing Scheme, and Optimization of I/O and Communication, to Enable Massively Parallel High-fidelity Internal Combustion Engine Simulations." 2015 ASME Internal Combustion Engine Division Fall Technical Conference, Houston, TX, November 8-11, 2015.

Kolodziej, C.P., J. Kodavasal, S. Som, J.P.P. Delhom, S.A. Ciatti and N.S. Shidore (2015). "Achieving Stable Engine Operation of Gasoline Compression Ignition Using 87 AKI Gasoline Down to Idle." SAE 2015 World Congress and Exhibition, Detroit, MI, April 14, 2015.

Kundu, P., Y. Pei and S. Som (2014). "Combustion Modeling under Diesel Engine Conditions with Multi-Flamelet RIF Model." Spring Technical Meeting of the Central States Section of the Combustion Institute, Tulsa, OK, March 16-18, 2014.

Liu, W., S. Som, D.Y. Zhou, R. Sivaramakrishnan, D.E. Longman, R.T. Skodje and M.J. Davis (2013). "The Role of Individual Rate Coefficients in the Performance of Compression Ignition Engine Models." 8th U.S. National Combustion Meeting Organized by the Western States Section of the Combustion Institute, University of Utah, Salt Lake City, UT, May 19-21, 2013.

Pei, Y., P. Kundu, G.M. Goldin and S. Som (2015). "Large Eddy Simulation of an n-dodecane Spray Flame under Diesel Engine Conditions." 9th U.S. National Combustion Meeting, Cincinnati, OH, May 17-20, 2015.

Pei, Y., E.R. Hawkes, S. Kook, G.M. Goldin and S. Som (2014). "An Analysis of the Structure of Spray A flame using TPDF Modelling." Spring Technical Meeting of the Central States Section of the Combustion Institute, Tulsa, OK, March 16-18, 2014.

Pei, Y., W. Liu, M. Mehl, S. Som, T. Lu and W.J. Pitz (2014). "A Multi-Component Blend as a Diesel Fuel Surrogate for Compression Ignition Engine Applications." Spring Technical Meeting of the Central States Section of the Combustion Institute, Tulsa, OK, March 16-18, 2014.

Tong, Y., Y. Pei, B. Zhong, S. Som and T. Lu (2015). "A Hybrid Mechanism for n-Dodecane Combustion with Optimized Low-Temperature Chemistry." 9th U.S. National Combustion Meeting, Cincinnati, OH, May 17-20, 2015.

\section{PRESENTATIONS}

Kodavasal, J., C.P. Kolodziej, S.A. Ciatti and S. Som (2015). "The Effects of Injection Pressure and Inclusion Angle on Gasoline Compression Ignition at Low-load and Idling Conditions." SAE 2015 World Congress and Exhibition, Detroit, Ml, April 21-23, 2015.

Som, S. (2013). "Simulation of Compression Ignition Engines with Detailed Chemistry and Spray Models." The American Association for the Advancement of Science, Boston, MA, February 14-18, 2013.

Som, S. (2013). "Towards Predictive Simulations of the Internal Combustion Engine." University of Connecticut, Storr, CT, July 11, 2013.

\section{3-154-R2}

\section{REFEREED PUBLICATIONS}

Yanguas-Gil, A., J.A. Libera and J.W. Elam (2015). "Multiscale Simulations of ALD in Cross Flow Reactors." ECS Transactions 64(9): 63-71.

Yanguas-Gil, A. and J.W. Elam (2014). "Analytic Expressions for Atomic Layer Deposition: Coverage, Throughput, and Materials Utilization in Cross-flow, Particle Coating, and Spatial Atomic Layer Deposition." Journal of Vacuum Science \& Technology A: Vacuum, Surfaces, and Films 32(3): 031504.

\section{PRESENTATION}

Yanguas-Gil, A. (2016). "Growth Under the Influence of Chemistry: The Emergence of Microstructure and Metastable Phases Far From Equilibrium." American Physical Society Fall 2015 Prairie Section Meeting, South Bend, IN, November 19-21, 2015. 


\section{3-156-R2}

\section{REFEREED PUBLICATION}

Ignacio-de Leon, P.A., X. Chen, E.J. Rabe, M. Urgun-Demirtas, M. Puga, Z. Zhou and R.W. Brotzman. "Magnetic Iron Cobalt Oxide Nanotube Composite Membranes." Journal of Membrane Science. (To be published.)

\section{PRESENTATION}

Ignacio-de Leon, P., E. Rabe, M. Urgun-Demirtas, R.W. Brotzman and X. Chen (2015). "Magnetic Nanotube Composite Membranes." 25th North American Membrane Society Annual Meeting, Boston, MA, May 30-June 3, 2015.

\section{4-120-R1}

\section{REFEREED PUBLICATIONS}

Bessac, J., E. Constantinescu and M. Anitescu. "Stochastic Simulation of Predictive Space-Time Scenarios of Wind Speed Using Observations and Physical Models." Annals of Applied Statistics. (To be published.)

de Sisternes, F.J., J.D. Jenkins and A. Botterud. "The Value of Energy Storage in Deep Decarbonization of the Electricity Sector." Applied Energy. (To be published.)

Li, N., C. Uckun, E. Constantinescu, J. Birge, K. Hedman and A. Botterud. "Flexible Operation of Batteries in Power System Scheduling with Renewable Energy." IEEE Transactions on Sustainable Energy. (To be published.)

\section{PRESENTATIONS}

Botterud, A. (2015). “Energy Storage for Grid Integration of Renewable Energy." Next Generation Batteries 2015, San Diego, CA, April 22, 2015.

Botterud, A., C. Uckun, P. Thimmapuram, K. Gallagher, E. Constantinescu and J.R. Birge (2015). "Grid Level Energy Storage for Integration of Renewable Energy." The Institute for Operations Research and the Management Sciences (INFORMS) Annual Meeting, San Francisco, CA, November 9-12, 2014.

Botterud, A. (2015). "Integration of Renewable Energy, Possible Solutions, the Role of Storage." MITei Symposium: Storage, Renewables and the Evolution of the Grid, Cambridge, MA, May 1, 2015.

Li, N., K. Hedman, A. Botterud, C. Uckun and J.R. Birge (2015). "Economic Assessment of Energy Storage in Systems with High Renewable Penetration." The Institute for Operations Research and the Management Sciences (INFORMS) Annual Meeting, San Francisco, CA, November 9-12, 2014.

Botterud, A. (2014). “Energy Storage for the Power Grid: A U.S. Perspective.” Workshop on Energy Storage: From Technologies to Grids and Electricity Markets, Porto, Portugal, July 4, 2014.

\section{4-169-R1}

\section{REFEREED PUBLICATION}

Zhang, Y.J., M. Liu, B. Peng, Z.Y. Zhou, X. Chen, S.M. Yang, Z.D. Jiang, J. Zhang, W. Ren and Z.G. Ye (2016). “Controlled Phase and Tunable Magnetism in Ordered Iron Oxide Nanotube Arrays Prepared by Atomic Layer Deposition." Scientific Reports 6.

\section{PRESENTATIONS}

Chen, X. (2016). "Functional Highly Anisotropic Nanostructures." University of Denver, Denver, CO, October 16, 2015.

Chen, X. (2016). "Functional Highly Anisotropic Nanostructures." Johns Hopkins University, Baltimore, MD, April 20, 2015.

Chen, X. (2016). "High Anisotropic Magnetic Nanostructures for Microwave Applications." University of Colorado, Boulder, CO, October 15, 2015.

Chen, X. (2015). "Fast Design Cycles and Concept Validation in a Multi-Discipline Laboratory." COMSOL Midwest Meeting, Columbus, OH, July 22, 2015. 
Chen, X. (2015). "Functional Highly Anisotropic Nanostructures." Naval Research Laboratory, Washington, D.C., April 21, 2015.

Chen, X. (2015). “Functional Highly Anisotropic Nanostructures.” University of Arizona, Tucson, AZ, May 26, 2015.

Chen, X. (2015). "Functional Nanostructures by Electrospinning and Atomic Layer Deposition." Northeastern University, Boston, MA, October 10, 2014.

2015-132-NO

\section{PRESENTATIONS}

Kahvecioglu Feridun, O., Y. Shin and G.K. Krumdick (2016). "Synthesis of a Layered-layered Cathode Material Using a Taylor Vortex Reactor." Annual Postdoctoral Research and Career Symposium, Argonne, IL, October 8, 2015.

Kahvecioglu Feridun, O., Y. Shin and G.K. Krumdick (2015). "Continuous Production of a Layered-layered Cathode Material using a Taylor Vortex Reactor." Advanced Automotive and Industrial Stationary Battery Conference, Detroit, MI, June 15-19, 2015.

Kahvecioglu Feridun, O., Y. Shin and G.K. Krumdick (2015). " $\mathrm{Li}_{1.14} \mathrm{Mn}_{0.53} \mathrm{Ni}_{0.28} \mathrm{Co}_{0.19} \mathrm{Oy}$ Layered-spinel Material for LIB Prepared by Using Taylor Vortex Reactor.” 227th Electro Chemical Society Meeting, Chicago, IL, May 25-29, 2015.

\section{5-151-NO}

\section{REFEREED PUBLICATIONS}

Babar, S., A.U. Mane, A. Yanguas-Gil, R. Haasch and J.W. Elam. "Bandgap Tailoring of W: $\mathrm{Al}_{2} \mathrm{O}_{3}$ Nanocomposite Thin Films Prepared by Atomic Layer Deposition." Chemistry of Materials. (To be published.)

Biswas, M., J.A. Libera, S.B. Darling and J.W. Elam (2015). "Kinetics for the Sequential Infiltration Synthesis of Alumina in Poly(methyl methacrylate): An Infrared Spectroscopic Study." Journal of Physical Chemistry C 119(26): 14585-14592.

\section{5-159-NO}

\section{REFEREED PUBLICATIONS}

Campos, E. and J. Wang (2016). "Numerical Simulation and Analysis of the April 2013 Chicago Floods." Journal of Hydrology 531(Part 2): 454-474.

Qiu, F., J. Wang, C. Chen and T. Jianzhong (2015). “Optimal Black Start Resource Allocation.” IEEE Transactions on Power Systems PP(99): 1-2.

\section{BIOLOGY AND ENVIRONMENT}

\section{2-205-R3}

\section{REFEREED PUBLICATIONS}

Fan, Z., J.C. Neff and N.P. Hanan (2015). "Modeling the Pulsed Soil Respiration Measured from an African Savanna Ecosystem." Agricultural and Forest Meteorology 200: 282-292.

Fan, Z. and C. Liang (2015). "Significance of Microbial Asynchronous Anabolism to Soil Carbon Dynamics Driven by Litter Inputs.” Scientific Reports 5: 9575.

Fan, Z.S., J.C. Neff, M.P. Waldrop, A.P. Ballantyne and M.R. Turetsky (2015). "Transport of Oxygen in Soil Pore-water Systems: Implications for Modeling Emissions of Carbon Dioxide and Methane from Peatlands." Biogeochemistry 121(3): 455-470.

Fan, Z., J.D. Jastrow, C. Liang, R. Matamala and R.M. Miller (2014). "Priming Effects in Boreal Black Spruce Forest Soils: Quantitative Evaluation and Sensitivity Analysis." PLOS ONE 8(10): e77880. 
Mishra, U., J.D. Jastrow, R. Matamala, G. Hugelius, C.D. Koven, J.W. Harden, C.L. Ping, G.J. Michaelson, Z. Fan, R.M. Miller, A.D. McGuire, C. Tarnocai, P. Kuhry, W.J. Riley, K. Schaefer, E.A. G. Schuur, M.T. Jorgenson and L.D. Hinzman (2013). "Empirical Estimates to Reduce Modeling Uncertainties of Soil Organic Carbon in Permafrost Regions: A Review of Recent Progress and Remaining Challenges." Environmental Research Letters 8(3).

\section{PRESENTATIONS}

Fan, Z., A.D. McGuire, M. Turetsky, J.W. Harden, J.M. Waddington and E. Kane (2013). "Modeling the Response of Boreal Peatlands to Climate Change Using Peatland-DOS-TEM." Terrestrial Ecosystem Model Workshop, Fairbanks, AK, June 17-21, 2013.

Fan, Z. (2013). "Priming Effects in Northern Boreal Forest Soils: A Quantitative Evaluation." 4th NACP All-Investigators Meeting, Albuquerque, NM, February 4-7, 2013.

Fan, Z. and J.D. Jastrow (2013). "Priming Effects in Northern Boreal Forest Soils: A Quantitative Evaluation." American Geophysical Union Annual Meeting, San Francisco, CA, December 3-7, 2012.

Fan, Z. (2013). "Response of High-Latitude Peatlands to Climate Change-Improving Model Predictive Capabilities." University of Chicago, Chicago, IL, February 14, 2013.

Jastrow, J., R. Matamala, K. McFarlane, Z. Fan, R. Porras, M. Torn, T. Guilderson and P. Hanson (2014). "Climatic and edaphic effects on root- and leaf-litter carbon inputs to temperate forest soils." 2014 TES SBR PI Meeting, Potomac, MD, June 6-7, 2014.

Mishra, U., J.D. Jastrow, R. Matamala Paradeda, Z. Fan, B.A. Drewniak, W. Riley and J. Krummel (2014). “Spatial Representation of Soil Properties in Earth System Models." 20th World Congress of Soil Science, Jeju, South Korea, June 8-13, 2014. (Also see 2012-206.)

\section{2-206-R3}

\section{REFEREED PUBLICATIONS}

Drewniak, B.A., U. Mishra, J. Song, J. Prell and V.R. Kotamarthi (2014). "Modeling the Impact of Agricultural Land Use and Management on U.S. Carbon Budgets." Biogeosciences Discussions 11(9): 13675-13698.

Hugelius, G., J. Strauss, S. Zubrzycki, J.W. Harden, E.A.G. Schuur, C.L. Ping, L. Schirrmeister, G. Grosse, G.J. Michaelson, C.D. Koven, J.A. O’Donnell, B. Elberling, U. Mishra, P. Camill, Z. Yu, J. Palmtag and P. Kuhry (2014). “Improved Estimates Show Large Circumpolar Stocks of Permafrost Carbon While Quantifying Substantial Uncertainty Ranges and Identifying Remaining Data Gaps." Biogeosciences Discussions 11(3): 4771-4822.

Jiang, Y., A. Rocha, E. Rastetter, G. Shaver, U. Mishra, Q. Zhuang and B. Kwiatkowski (2015). "C-N-P Interactions Control Climate Driven Changes in Regional Patterns of C Storage on the North Slope of Alaska." Landscape Ecology 31: 195-213.

Mishra, U. and W. Riley (2015). "Scaling Impacts on Environmental Controls and Spatial Heterogeneity of Soil Organic Carbon Stocks." Biogeosciences 12(13): 3993-4004.

Mishra, U. and W.J. Riley (2014). "Active-Layer Thickness across Alaska: Comparing Observation-Based Estimates with CMIP5 Earth System Model Predictions." Soil Science Society of America Journal 78: 848-902.

Mishra, U., J.D. Jastrow, R. Matamala, G. Hugelius, C.D. Koven, J.W. Harden, C.L. Ping, G.J. Michaelson, Z. Fan, R.M. Miller, A.D. McGuire, C. Tarnocai, P. Kuhry, W.J. Riley, K. Schaefer, E.A. G. Schuur, M.T. Jorgenson and L.D. Hinzman (2013). "Empirical Estimates to Reduce Modeling Uncertainties of Soil Organic Carbon in Permafrost Regions: A Review of Recent Progress and Remaining Challenges." Environmental Research Letters 8(3).

Reitsma, K.D., B. Dunn, U. Mishra, S.A. Clay, T. DeSutter and D.E. Clay (2015). “Land-Use Change Impact on Soil Sustainability in a Climate and Vegetation Transition Zone." Agronomy Journal 107(6): 2363-2372. 


\section{PRESENTATIONS}

Drewniak, B. and U. Mishra (2014). "Modeling Agriculture Impacts on Soil Organic Carbon under Different Management Practices with the Community Land Model." American Geophysical Union Fall Meeting, San Francisco, CA, December 9-13, 2013.

Drewniak, B.A. and U. Mishra (2014). "Soil Organic Carbon Response to Cultivation in the Community Land Model." RCN FORECAST Workshop, Breckenridge, CO, June 11-14, 2014.

Mishra, U., Z. Fan, J.D. Jastrow, R.M. Matamala and W. Vitharana (2016). "Capturing Spatial Heterogeneity of Soil Organic Carbon Under Changing Climate." 2015 American Geophysical Union Fall Meeting, San Francisco, CA, December 14-18, 2015.

Mishra, U., Z. Fan and B.A. Drewniak (2016). “Informing Climate Change Studies Using Soil Survey Information.” 2015 Soil Science Society of America International Annual Meeting, Minneapolis, MN, November 15-18, 2015.

Mishra, U., J.D. Jastrow, R.M. Matamala and Z. Fan (2015). "ANL TES SFA: Capturing the Spatial Heterogeneity of Soil Organic Carbon Stocks Using Soil-forming Factors.” DOE-TES/SBR Joint Investigators Meeting, Potomac, MD, April 28-29, 2015.

Mishra, U., K.E. LaGory, J. Krummel, J.D. Jastrow and R. Matamala Paradeda (2014). "Benchmarking Earth System Models: A New Domain for Soil Scientists.” USDA-Natural Resource Conservation Services, Lincoln, NE, March 18, 2014.

Mishra, U., J.D. Jastrow, R.M. Matamala, K.E. LaGory and J.R. Krummel (2014). "Environmental Controls and Spatial Representation of Soil Properties Across Alaska: Comparison Between Geospatial and CMIP5 Earth System Models." Korea Polar Research Institute, Seoul, South Korea, June 16, 2014.

Mishra, U., W. Riley, M. Torn, S. Ogle and B. Drewniak (2015). "Predicting Land Use and Climate Change Impacts on Soil Organic Carbon at Regional Scales." Soil Science Society of America Annual Meeting, Long Beach, CA,

November 5, 2014.

Mishra, U., J.D. Jastrow, R. Matamala Paradeda, Z. Fan, B.A. Drewniak, W. Riley and J. Krummel (2014). "Spatial Representation of Soil Properties in Earth System Models." 20th World Congress of Soil Science, Jeju, South Korea, June 8-13, 2014. (Also see 2012-205.)

Mishra, U., J.D. Jastrow, R. Matamala, G. Hugelius, C.L. Ping and G. Michaelson (2014). "Spatial Variability of Surface Organic Horizon Thickness across Alaska." American Geophysical Union Fall Meeting, San Francisco, CA, December 9-13, 2013.

Mishra, U., W.J. Riley and C.D. Koven (2014). "Topographic Controls, Spatial Heterogeneity, and Prediction Accuracies of SOC Stocks across Geospatial and Earth System Models." Soil Science Society of America Meeting, Tampa, FL, November 3-6, 2013.

Mishra, U., W.J. Riley and C.D. Koven (2013). "Assessment of Spatial Heterogeneity, Environmental Controls, and Prediction Accuracy of Soil Organic Carbon Stocks across Geospatial and Earth Systems Models." American Geophysical Union Fall Meeting, San Francisco, CA, December 3-7, 2012.

\section{3-177-R2}

\section{PRESENTATIONS}

O'Loughlin, E.J., M.F. Sladek, D.A. Antonopoulos, T.M. Flynn, J.C. Koval, C.L. Marshall and K.M. Kemner (2016). "Effects of Soluble Electron Shuttles on Microbial Fe(III) Reduction and Methanogenesis in Wetland Sediment Microcosms." 7th Annual Argonne Soil Metagenomics Meeting, Lisle, IL, October 21-23, 2015.

O'Loughlin, E.J., M.F. Sladek, D.A. Antonopoulos, T.M. Flynn, J.C. Koval, C.L. Marshall and K.M. Kemner (2016). "Effects of Soluble Electron Shuttles on Microbial Fe(III) Reduction and Methanogenesis in Wetland Sediment Microcosms." 2015 Midwest Geobiology Symposium, Bloomington, IN, October 9-10, 2015. 
O'Loughlin, E.J., T.M. Flynn, J.C. Koval, S.M. Owens, K. Arend and D.A. Antonopoulos (2015). "Effects of 2-chloro-6-methylpyridine Concentration on Microbial Community Development During Aerobic Methane Oxidation." 6th Annual Argonne National Laboratory Soil Metagenomics Meeting, St. Charles, IL, October 1-3, 2014.

O'Loughlin, E.J., T.M. Flynn, J. Koval, S. Owens, K. Arend and D.A. Antonopoulos (2014). "Effects of 2-chloro-6-methylpyridine Concentration on Microbial Community Development During Aerobic Methane Oxidation.” 114th General Meeting, American Society of Microbiology, Boston, MA, May 17-20, 2014.

O’Loughlin, E.J., D.A. Antonopoulos, K.M. Kemner, J. Koval, S.L. O’Brien and S. Owens (2014). “Identifying Novel Pathways for Anaerobic Microbial Oxidation of Methane." 5th Annual Argonne National Laboratory Soil Metagenomics Meeting, Bloomington, IL, October 2-4, 2013.

\section{3-194-R2}

\section{REFEREED PUBLICATIONS}

Cruz-Morales, P., C.E. Martínez-Guerrero, M.A. Morales-Escalante, L. Yáñez-Guerra, J.F. Kopp, J. Feldmann, H.E. Ramos-Aboites and F. Barona-Gómez. "Recapitulation of the Evolution of Biosynthetic Gene Clusters Reveals Hidden Chemical Diversity on Bacterial Genomes." bioRxiv. (To be published.)

Jeffryes, J.G., R.L. Colastani, M. Elbadawi-Sidhu, T. Kind, T.D. Niehaus, L.J. Broadbelt, A.D. Hanson, O. Fiehn, K.E. Tyo and C.S. Henry (2015). "MINEs: Open Access Databases of Computationally Predicted Enzyme Promiscuity Products for Untargeted Metabolomics." Journal of Cheminformatics 7(1): 44.

Noda-García, L., A.L. Juárez-Vázquez, M.C. Ávila-Arcos, E.A. Verduzco-Castro, G. Montero-Morán, P. Gaytán, M. Carrillo-Tripp and F. Barona-Gómez (2015). "Insights into the Evolution of Enzyme Substrate Promiscuity after the Discovery of $(\beta a) 8$ Isomerase Evolutionary Intermediates from a Diverse Metagenome." BMC Evolutionary Biology 15: 107.

Verduzco-Castro, E.A., K. Michalska, M. Endres, A.L. Juárez-Vazquez, L. Noda-García, C.L. Chang, C.S. Henry, G. Babnigg, A. Joachimiak and F. Barona-Gómez. "Co-occurrence of Analogous Enzymes Determines Evolution of a Novel (Ba)8-Isomerase Sub-family After Non-conserved Mutations in Flexible Loop." Journal of Biological Chemistry. (To be published.)

\section{4-141-R1}

\section{PRESENTATIONS}

Flynn, T.M., J.C. Koval, S.M. Greenwald, S.M. Owens, K.M. Kemner and D.A. Antonopoulos (2015). "Community Level Physiological Profiling of Diverse Environments Reveals Functional and Taxonomic Diversity within Aerobic, Single Carbon-source Enrichments." 115th American Society of Microbiology General Meeting (ASM 2015), New Orleans, LA, May 30-June 2, 2015.

Flynn, T.M., J.C. Koval, S.M. Moormann, S.M. Owens, S.L. O'Brien, S. Alvarez-Clare, E.J. O’Loughlin, K.M. Kemner and D.A. Antonopoulos (2015). "Community Level Physiological Profiling of Diverse Soil Environments Reveals Functional and Taxonomic Diversity Within Aerobic, Single-carbon Source Enrichments." 6th Annual Argonne National Laboratory Soil Metagenomics Meeting, St. Charles, IL, October 1-3, 2014.

Flynn, T.M., J.C. Koval, S.M. Moormann, S.M. Owens, S.L. O'Brien, S. Alvarez-Clare, E.J. O'Loughlin, K.M. Kemner and D.A. Antonopoulos (2014). "Community Level Physiological Profiling of Diverse Environments Reveals Functional and Taxonomic Diversity Within Aerobic, Single Carbon-Source Enrichments." 2014 Ecological Society of America Annual Meeting, Sacramento, CA, August 10-15, 2014.

\section{4-145-R1}

\section{REFEREED PUBLICATIONS}

Dishaw, L.J., J. Flores-Torres, S. Lax, K. Gemayel, B. Leigh, D. Melillo, M.G. Mueller, L. Natale, I. Zucchetti, R. De Santis, M.R. Pinto, G.W. Litman and J.A. Gilbert (2014). "The Gut of Geographically Disparate Ciona intestinalis Harbors a Core Microbiota." PLoS ONE 9(4): e93386. 
Gibbons, S.M., N.M. Scott, E. Jones, A. Bearquiver, F. Blackwolf, W. Roundstone, J. Hooker, R. Madsen, M. Coleman and J.A. Gilbert (2014). “Human and Environmental Impacts on River Sediment Microbial Communities." PLoS ONE 9(5): e97435.

Gilbert, J.A. (2015). “Science is Innate!” Genome Biology 15(477).

Gilbert, J.A., A. Kumar, J.F. Dames, A. Gupta, S. Sharma and P. Ahmad (2014). "Current Developments in Arbuscular Mycorrhizal Fungi Research and Its Role in Salinity Stress Alleviation: A Biotechnological Perspective." Critical Reviews in Biotechnology: 1-14.

Gilbert, J.A., J. Jansson and R. Knight (2014). "The Earth Microbiome Project: Successes and Aspirations.” BMC Biology 12(69).

Gilbert, J.A., O.U. Mason, N.M. Scott, A. Gonzalez, A. Robbins-Pianka, J. Baelum, J. Kimbrel, N. Bouskill, E. Prestat and S. Borglin (2014). "Metagenomics Reveals Sediment Microbial Community Response to Deepwater Horizon Oil Spill." ISME Journal 8: 1464-1475.

Gilbert, J.A., D. Van Der Lelie and I. Zarraonaindia Martinez (2014). "Microbial terroir for Wine Grapes." Proceedings of the National Academy of Sciences of the United States of America 111(1): 5-6.

Groer, M.W., A.A. Luciano, L.J. Dishaw, T.L. Ashmeade, E. Miller and J.A. Gilbert (2015). "Development of the Preterm Infant Gut Microbiome: A Research Priority." Microbiome 2(38).

Hamada, Y., J.A. Gilbert, P.E. Larsen and M.J. Norgaard (2014). "Toward Linking Aboveground Vegetation Properties and Soil Microbial Communities Using Remote Sensing.” Photogrammetric Engineering and Remote Sensing 80(4): 311-321.

Handley, K.M., E.J. O’Loughlin, W.L. Trimble, K.A. Skinner, J.A. Gilbert, N. Desai, E.M. Glass, T. Paczian, A. Wilke, D.A. Antonopoulos, K.M. Kemner and F. Meyer (2015). "The Complete Genome Sequence for Putative H2- and S- Oxidizer Candidatus Sulfuricuvum sp., Assembled de novo from an Aquifer-derived Metagenome." Environmental Microbiology 16(11): 2443-3462.

Handley, K.M., K.C. Wrighton, C.S. Miller, M.J. Wilkins, J.A. Gilbert, R. Kantor, B.C. Thomas, K.H. Williams, P.E. Long and J.F. Banfield (2014). "Disturbed Subsurface Microbial Communities Follow Equivalent Trajectories Despite Different Structural Starting Points.” Environmental Microbiology: DOI: 10.1111/1462-2920.12467.

Kyrpides, N.C., P. Hugenholtz, J. Eisen, T. Woyke, M. Goeker, C.T. Parker, R. Amann, B.J. Beck, P.S.G. Chain, J. Chun, R.R. Colwell, A. Danchin, P. Dawyndt, T. Dedeurwaerdere, E.F. DeLong, J.C. Detter, P. De Vos, T.J. Donohue, X.-Z. Dong, D.S. Ehrlich, C. Fraser, R. Gibbs, J.A. Gilbert, P. Gilna, F.O. Glöckner, J.K. Jansson, J.D. Keasling, R. Knight, D. Labeda, A. Lapidus, J.-S. Lee, W.-J. Li, J. MA, V. Markowitz, E.R.B. Moore, M. Morrison, F. Meyer, K.E. Nelson, M. Ohkuma, C.A. Ouzounis, N. Pace, J. Parkhill, N. Qin, R. Rossello-Mora, J. Sikorski, D. Smith, M. Sogin, R. Stevens, U. Stingl, K.-i. Suzuki, D. Taylor, H.M. Tiedje, B. Tindall, M. Wagner, G. Weinstock, J. Weissenbach, O. White, J. Wang, L. Zhang, Y.-G. Zhou, D. Feld, W.B. Whitman, G.M. Garrity and H.-P. Klenk (2014). "Genomic Encyclopedia of Bacteria and Archaea: Sequencing a Myriad of Type Strains." PLoS ONE 12(8): e1001920.

LaBelle, E.V., C.W. Marshall, J.A. Gilbert and H.D. May (2015). "Influence of Acidic pH on Hydrogen and Acetate Production by an Electrosynthetic Microbiome." PLOS ONE 9(10): e109935.

Lax, S., D.P. Smith, J. Hampton-Marcell, S.M. Owens, K.M. Handley, N.M. Scott, S.M. Gibbons, P. Larsen, B.D. Shogan, S. Weiss, J.L. Metcalf, L.K. Ursell, Y. Vazquez-Baeza, W. Van Treuren, N.A. Hasan, M.K. Gibson, R. Colwell, G. Gantas, R. Knight and J.A. Gilbert (2014). "Longitudinal Analysis of Microbial Interaction Between Humans and the Indoor Environment.” Science 345(6200): 1048-1052.

Nielsen, S.F., T. Minchin, S. Kimber, L. van Zwieten, J.A. Gilbert, P. Munroe, S. Joseph and T. Thomas (2014). "Comparative Analysis of the Microbial Communities in Agricultural Soil Amended with Enhanced Biochars or Traditional Fertilizers." Agriculture, Ecosystems \& Environment 191: 73-82. 
Parfey, L.W., W. Walters, C.L. Lauber, J.C. Clemente, D. Berg-Lyons, C. Teiling, C. Kodira, M. Mohiuddin, J. Brunelle, M. Driscoll, N. Fierer, J.A. Gilbert and R. Knight (2014). "Communities of Microbial Eukaryotes in the Mammalian Gut Within the Context of Environmental Eukaryotic Diversity." Frontiers in Microbiology 5(298).

Peer, X., J.A. Gilbert and G.C. An (2014). "Examining the Microbial Ecological Dynamics of Clostridium Difficile Infections and the Efficacy of Fecal Microbiome Transplant (FMT) using an Agent-based Model." Journal of Surgical Research 186(2): 689.

Pfister, C.A., J.A. Gilbert and S.M. Gibbons (2015). "The Role of Macrobiota in Structuring Microbial Communities Along Rocky Shores." PeerJ 2(631).

Piombino, P., A. Genovese, S. Esposito, L. Moio, P.P. Cutolo, A. Chambery, V. Severino, E. Moneta, D.P. Smith, S. Owens, J.A. Gilbert and D. Ercolini (2014). "Saliva from Obese Individuals Suppresses the Release of Aroma Compounds from Wine." PLOS ONE 9(1): e85611.

Rideout, J.R., Y. He, J.A. Navas-Molina, W.A. Walters, L.K. Ursell, S.M. Gibbons, J. Chase, D. McDonald, A. Gonzalez, A. Robbins-Pianka, J.C. Clemente, J.A. Gilbert, S.M. Huse, H.W. Zhou, R. Knight and J.G. Caporaso (2014). "Subsampled Open-reference Clustering Creates Consistent, Comprehensive OTU Definitions and Scales to Billions of Sequences." PeerJ 2(e545).

Rubin, B.R., J.G. Sanders, J. Hampton-Marcell, S.M. Owens, J.A. Gilbert and C.S. Moreau (2014). "DNA Extraction Protocols Cause Differences in 16S rRNA Amplicon Sequencing Efficiency but not in Community Profile Composition or Structure." MicrobiologyOpen 3(6): 910-921.

Shade, A., S.E. Jones, G. Caporasoc, J. Handelsman, R. Knight, N. Fierer and J.A. Gilbert (2014). "Conditionally Rare Taxa Disproportionately Contribute to Temporal Changes in Microbial Diversity." mBio 5(4): e01371-01314.

Shogan, B.D., D.P. Smith, S. Christley, J.A. Gilbert, O. Zaborina and J.C. Alverdy (2014). "Intestinal Anastomotic Injury Alters Spatially Defined Microbiome Composition and Function." Microbiome 2(35).

Winston, M., J. Hampton-Marcell, I. Zarraonaindia Martinez, S. Owens, C.S. Moreau, J.A. Gilbert, J. Hartsel and S.M. Gibbons (2014). "Understanding Cultivar-Specificity and Soil Determinants of the Cannabis Microbiome." PLoS ONE 9(6): e99641.

Xiong, J., H. Sun, F. Peng, H. Zhang, X. Xue, S.M. Gibbons, J.A. Gilbert and H. Chu (2014). "Characterizing Changes in Soil Bacterial Community Structure in Response to Short-term Warming." FEMS Microbiology Reviews 89(2): 281-292.

Zaborin, A., D. Smith, K. Garfield, J. Quensen, S. Baddr, M. Kade, M. Tirrell, J. Tiedje, J.A. Gilbert, O. Zaborina and J.C. Alverdya (2014). "Membership and Behavior of Ultra-Low-Diversity Pathogen Communities Present in the Gut of Humans During Prolonged Critical Illness." mBio 5(5): e01361-01314.

\section{PRESENTATIONS}

Marshall, C.W. and J.A. Gilbert (2016). "Microbial Ecology of Floodplains and Electrosynthesis Systems." US-China Biodiversity Workshop, Raleigh, NC, October 16-18, 2015.

Marshall, C., S.L. O’Brien, K.M. Kemner, E.J. O'Loughlin, P. Weisenhorn, N.R. Gottel, S. Alvarez Clare, A. Best, T.M. Flynn and J.A. Gilbert (2016). "Microbial Ecology of Restored Floodplains." 7th Annual Argonne Soil Metagenomics Meeting, Argonne, IL, October 21-23, 2015.

Marshall, C., S.L. O’Brien, K.M. Kemner, E.J. O’Loughlin, N.R. Gottel, S. Alvarez Clare, A. Best, T.M. Flynn and J.A. Gilbert (2015). "Microbial Ecology of Floodplains." 115th American Society for Microbiology General Meeting, New Orleans, LA, May 30-June 2, 2015. 


\section{4-183-R1}

\section{REFEREED PUBLICATIONS}

Albrecht, B.A., M. Fang and V.P. Ghate (2015). "Exploring Stratocumulus Cloud-Top Entrainment Processes and Parameterizations by Using Doppler Cloud Radar Observations." Journal of the Atmospheric Sciences(2015): e-View.

Ghate, V.P., M.A. Miller, B.A. Albrecht and C.W. Fairall (2015). "Thermodynamic and Radiative Structure of Stratocumulus-topped Boundary Layers." Journal of the Atmospheric Sciences 72(1): 430-451.

Fang, M., B.A. Albrecht, V.P. Ghate and P. Kollias (2014). "Turbulence in Continental Stratocumulus, Part I: External Forcings and Turbulence Structures.” Boundary-Layer Meteorology 150(3): 341-360.

Fang, M., B.A. Albrecht, V.P. Ghate and P. Kollias (2014). "Turbulence in Continental Stratocumulus, Part II: Eddy Dissipation Rates and Large-Eddy Coherent Structures.” Boundary-Layer Meteorology 150(3): 361-380.

\section{PRESENTATION}

Schwartz, M.C. and V.P. Ghate (2015). "Stratocumulus-to-Cumulus Cloud Transition: A Case Study from the MAGIC Field Campaign.” Atmospheric System Research (ASR) Science Team Meeting, Vienna, VA, March 16-20, 2015.

\section{4-187-R1}

\section{REFEREED PUBLICATIONS}

Biswas, M., J.A. Libera, S.B. Darling and J.W. Elam (2015). "New Insight into the Mechanism of Sequential Infiltration Synthesis from Infrared Spectroscopy." Chemistry of Materials 26(21): 6135-6141.

Lee, A., J.W. Elam and S.B. Darling (2015). "Membrane Materials for Water Purification: Design, Development and Application." Environmental Science: Water Research \& Technology Advance Article: DOI:10.1039/C1035EW00159E.

\section{4-188-R1}

\section{REFEREED PUBLICATIONS}

Buizert, C., D. Baggenstos, W. Jiang, R. Purtschert, V.V. Petrenko, Z.T. Lu, P. Mueller, T. Kuhl, J. Lee, J.P. Severinghaus and E.J. Brook (2014). "Radiometric ${ }^{81} \mathrm{Kr}$ Dating Identifies 120,000-year-old Ice at Taylor Glacier, Antarctica." Proceedings of the National Academy of Sciences of the United States of America, May 13, 2014, 111(19): 6876-6881.

Jiang, W., K. Bailey, Z.T. Lu, P. Mueller, T.P. O'Connor and R. Purtschert (2014). "Ion Current as a Precise Measure of the Loading Rate of a Magneto-optical Trap." Optics Letters 39(2): 409-412.

Sturchio, N.C., K.L. Kuhlman, R. Yokochi, P.C. Probst, W. Jiang, Z.T. Lu, P. Mueller and G.M. Yang (2014). “Krypton-81 in Groundwater of the Culebra Dolomite Near the Waste Isolation Pilot Plant, New Mexico." Journal of Contaminant Hydrology 160: 12-20.

Zappala, J.C., K. Bailey, Z.T. Lu, T.P. O’Connor and W. Jiang (2014). “Efficient Generation of Optical Sidebands at GHz With a High-power Tapered Amplifier." Review of Scientific Instruments 85(4): 046104.

\section{NON-REFEREED PUBLICATION}

Lu, Z.-T., W. Jiang, J.C. Zappala, K. Bailey, P. Mueller and T.P. O'Connor (2015). “" Kr: A New Tracer of Old Groundwater Flow." International Symposium on Isotope Hydrology: Revisiting Foundations and Exploring Frontiers, Vienna, Austria, May 11-15, 2015.

\section{PRESENTATIONS}

Jiang, W., J.C. Zappala, K. Bailey, Z.-T. Lu, P. Mueller and T.P. O'Connor (2015). "Radiokrypton Dating with Atom Trap Trace Analysis." 46th Annual Meeting of the American Physical Society Division of Atomic, Molecular, and Optical Physics, Columbus, OH, June 8-12, 2015.

Zappala, J.C., W. Jiang, K. Bailey, Z.-T. Lu, P. Mueller and T.P. O'Connor (2016). "Laboratory for Radiokrypton Dating." American Geophysical Union 2016 Fall Meeting, San Francisco, December 14-18, 2015. 
Zappala, J.C., W. Jiang, Z.-T. Lu, K. Bailey, P. Mueller and T.P. O'Connor (2015). "Radio-Krypton Dating with Atom Trap Trace Analysis." 7th International Conference on Laser Probing, East Lansing, MI, June 7-11, 2015.

\section{4-189-R1}

\section{NON-REFEREED PUBLICATIONS}

Badalov, S., N. Wardrip, M. Dsouza, Y. Oren, M. Urgun-Demirtas, S.W. Snyder, J.A. Gilbert and C. Arnusch (2016). "2-D and 3-D Printing Assisted Fabrication and Modification of UF/NF/RO Membranes." Israeli Polymer and Plastics Society 44th Meeting, Jerusalem, Israel, December 13, 2015.

Urgun-Demirtas, M., C.J. Arnusch, J.A. Gilbert, S.W. Snyder, J. Dittrich, P. Ignacio-de Leon and K.M. Handley (Accepted). "A Systematic Tool for Development of More Robust 'Smart' Membranes and Energy Efficient Water Treatment Processes." Water and Energy 2015: Opportunities for Energy and Resource Recovery in the Changing World, Washington, D.C., June 8-10, 2015.

\section{PRESENTATIONS}

Badalov, S., N. Wardrip, M. Dsouza, Y. Oren, M. Urgun-Demirtas, S.W. Snyder, J.A. Gilbert and C. Arnusch (2016). "2-D and 3-D Printing Assisted Fabrication and Modification of UF/NF/RO Membranes for Water Treatment." Water Technology and Environmental Control Conference (WATEC 2015), Tel Aviv, Israel, October 13-14, 2015.

Wardrip, N., S. Badalov, E. Matzkin, Y. Oren, M. Urgun-Demirtas, S.W. Snyder, J.A. Gilbert and C. Arnusch (2015). "2-D and 3-D Printing Assisted Fabrication and Modification of UF/NF/RO Membranes." Euromembrane (EMC 2015), Aachen, Germany, September 6-10, 2015.

Wardrip, N., M. Urgun-Demirtas, S. Snyder, J.A. Gilbert and C. Arnusch (2015). "3-D Printing Assisted Modification of Ultrafiltration Membranes Towards Novel Patterned Anti Fouling Coatings." 2nd International Conference on Desalination using Membrane Technology, Singapore, July 26-29, 2015.

\section{4-193-R1 \\ REFEREED PUBLICATIONS}

Collow, A.B., V.P. Ghate, M.A. Miller and L.C. Trabachino (2015). "A One-Year Study of the Diurnal Cycle of Meteorology, Clouds, and Radiation in the West African Sahel Region." Quarterly Journal of the Royal Meteorological Society.

Ghate, V.P., M.A. Miller and P. Zhu (2016). "Differences Between Nonprecipitating Tropical and Trade Wind Marine Shallow Cumuli." American Meteorological Society Journal Monthly Weather Review 2015(e-View).

Wood, R., M. Wyant, C.S. Bretherton, J. Rémillard, P. Kollias, J. Fletcher, J. Stemmler, S. deSzoeke, S. Yuter, M. Miller, D. Mechem, G. Tselioudis, C. Chiu, J. Mann, E. O'Connor, R. Hogan, X. Dong, M. Miller, V. Ghate, A. Jefferson, Q. Min, P. Minnis, R. Palinkonda, B. Albrecht, E. Luke, C. Hannay and Y. Lin (2014). "Clouds, Aerosol, and Precipitation in the Marine Boundary Layer: An ARM Mobile Facility Deployment." Bulletin of the American Meteorological Society e-View: 140617093021002.

\section{PRESENTATIONS}

Ghate, V.P., P. Kollias and R.L. Coulter (2015). "Climatology of Boundary Layer Depth at the ARM SGP Central Facility." 2015 Atmospheric Radiation Measurement (ARM)/Atmospheric System Research (ASR) Joint User Facility and Principal Investigator Meeting, Vienna, VA, April 16-20, 2015.

Ghate, V.P., M.A. Miller and P. Zhu (2015). "Similarities and Differences between Tropical and Trade Wind Cumulus Topped Marine Boundary Layers.” Jet Propulsion Laboratory Earth Science Seminar, California Institute of Technology, March 27, 2015.

Ghate, V.P. and M.A. Miller (2014). "Effects of Clouds on Cross-atmospheric Radiative Flux Divergence: Case-studies in Different Cloud Conditions." American Geophysical Union (AGU) Fall Meeting, San Francisco, CA, December 9-13, 2013.

Ghate, V.P., M.A. Miller and B.A. Albrecht (2014). "On the Dynamics and Radiation of Cumulus Topped Marine Boundary Layers." 4th Atmospheric System Research (ASR) Science Team Meeting, Potomac, MD, March 10-13, 2014. 


\section{5-124-NO}

\section{PRESENTATIONS}

Botterud, A., F. Ganda and F.J. De Sisternes (2015). "Economic and Technical Aspects of Nuclear Energy in Electricity Markets with Renewables." Low-Carbon Energy Economy Workshop, Cambridge, MA, May 26-27, 2015. (Also see 2015-129.)

Botterud, A., F. Ganda and F.J. De Sisternes (2015). "Economic and Technical Aspects of Nuclear Energy in Electricity Markets with Renewables." U.S.-Japan Workshop on Compatibility of Nuclear and Renewables with Grid Stability, Economics and Deregulation, Cambridge, MA, June 18, 2015.

\section{5-170-NO}

\section{PRESENTATIONS}

Negri, M.C. and H. Ssegane (2015). "Biomimetic Approaches for Water Smart Landscape." University of Chicago Institute for Molecular Engineering (IME) Collaborative Water Research Conference, Chicago, IL, August 17, 2015.

Negri, M.C. and H. Ssegane (2015). "Efficient Green Roofs." The Array of Things Workshop, Chicago, IL, September 3, 2015.

\section{HARD X-RAY SCIENCES}

\section{3-111-R2}

\section{REFEREED PUBLICATIONS}

Holzbauer, J.P. and A. Nassiri (2015). "Thermal Study of a Cryogen-less $\mathrm{MgB}_{2}$ Cavity." Nuclear Instruments \& Methods in Physics Research Section A-Accelerators Spectrometers Detectors and Associated Equipment 767: 407-414.

Nassiri, A., R. Kustom and T. Proslier (2013). "Cryogen-Free RF System Studies Using Cryocooler-cooled MgB 2 Coated Copper RF Cavities." Proceedings of the 16th International Conference on RF Superconductivity (SRF 2013), Paris, France, September. 23-27, 2013.

\section{PRESENTATION}

Nassiri, A., R. Kustom and T. Proslier (2015). "Large Area Superconducting Magnesium Diboride Films on Copper Substrates for SRF Applications Using HPCVD.” International Conference on RF Superconductivity (SRF 2015), Whistler, British Columbia, Canada, September 13-18, 2015.

\section{3-168-R2}

\section{REFEREED PUBLICATIONS}

De Carlo, F., D. Gursoy, F. Marone, M. Rivers, D.Y. Parkinson, F. Khan, N. Schwarz, D.J. Vine, S. Vogt, S.-C. Gleber, S. Narayanan, M. Newville, T. Lanzirotti, Y. Sun, Y.P. Hong and C. Jacobsen (2015). "Scientific Data Exchange: A Schema for HDF5-Based Storage of Raw and Analyzed Data.” Journal of Synchrotron Radiation 21(6): 1224-1230.

Gursoy, D., F. De Carlo, X. Xiao and F. Jacobsen (2014). “TomoPy: A Framework for the Analysis of Synchrotron Tomographic Data.” Journal of Synchrotron Radiation 21(5): 1188-1193.

Hong, Y.P., S.C. Gleber, T.V. O’Halloran, E.L. Que, R. Bleher, S. Vogt, T.K. Woodruff and C. Jacobsen (2014). “Alignment of Low-dose X-ray Fluorescence Tomography Images Using Differential Phase Contrast." Journal of Synchrotron Radiation 21(Pt 1): 229-234.

Lerotic, M., R. Mak, S. Wirick, F. Meirer and C. Jacobsen (2014). "MANTiS: A Program for the Analysis of X-ray Spectromicroscopy Data.” Journal of Synchrotron Radiation 21(Pt 5): 1206-1212.

Mak, R., M. Lerotic, H. Fleckenstein, S. Vogt, S.M. Wild, S. Leyffer, Y. Sheynkin and C. Jacobsen (2015). "Non-negative Matrix Analysis for Effective Feature Extraction in X-ray Spectromicroscopy." Royal Society of Chemistry Faraday Discussions 171: 357-371. 
Wang, S., J. Ward, S. Leyffer, S.M. Wild, C. Jacobsen and S. Vogt (2013). "Unsupervised Cell Identification on Multidimensional X-ray Fluorescence Datasets." Proceedings of the Journal of Synchrotron Radiation (ACM SIGGRAPH 2013) International Conference on Computer Graphics and Interactive Techniques, Anaheim, CA, July 21-25, 2013, ACM, New York, NY 88.

\section{NON-REFEREED PUBLICATIONS}

Gursoy, D., F. De Carlo, X. Xiao and C. Jacobsen (2014). "TomoPy: A Framework for the Analysis of Synchrotron Tomographic Data.” 2014 SPIE Optics \& Photonics: Developments in X-ray Tomography, San Diego, CA, August 17-21, 2014.

Maser, J., B. Lai, T. Buonassisi, Z. Cai, S. Chen, L. Finney, S.C. Gleber, R. Harder, C. Jacobsen, W. Liu, C. Murray, C. Preissner, C. Roehrig, V. Rose, D. Shu, D. Vine and S. Vogt (2013). "A Next-generation in situ Nanoprobe Beamline for the Advanced Photon Source." SPIE 2013: Optics \& Photonics, San Diego, CA, August 25-29, 2013.

\section{PRESENTATIONS}

Jacobsen, C.J. (2014). "Big Data at the Advanced Photon Source: Some Order Out of Chaos, and Getting Quick Pictures.” Research Data Alliance 4th Plenary Meeting, Amsterdam, The Netherlands, September 22-24, 2014.

Jacobsen, C.J. (2014). "The Bionanoprobe at the Advanced Photon Source at Argonne." 2nd 108-SXM Workshop on Scanning X-ray Microscopy, Diamond Light Source, Oxfordshire, United Kingdom, March 25, 2014.

Jacobsen, C.J. (2014). "The Cold, Hard Truth About Cells: Cryo X-ray Microscopy at the Advanced Photon Source." MAX-IV Laboratory, Lund University, Sweden, September 26, 2014.

Jacobsen, C. (2014). "Cryo Microscopy: Advantages, Challenges and Opportunities." Canadian Light Source, Saskatchewan, Canada, January 14, 2014.

Jacobsen, C.J. (2014). "Data and Network Needs at the Advanced Photon Source." DOE ESNet Networking Requirements Review, Washington, D.C., September 10, 2014.

Jacobsen, C.J., A. Kastengren and K. Fezzaa (2014). "Imaging at Fast Times: APS Experience and Speculations." Workshop on Scientific Opportunities Using High Repetition Rate X-ray Sources with 1-10 ps Bunch Length, SLAC National Accelerator Laboratory, Menlo Park, CA, October 1-4, 2013.

Jacobsen, C.J. (2014). "Oscillators, X-rays and Global Carbon: Adventures in X-ray Nanofocusing." Colloquium at the University of Illinois Chicago, Chicago, IL, April 2, 2014.

Jacobsen, C. (2014). "Radiation Damage Limits to X-ray Imaging, and Their (Partial) Mitigation." Workshop on Radiation Effects at X-ray Doses from MegaGrays and PetaGrays and Above-the Impact for Science at NSLS-II, NSLS/CFN User Meeting, Brookhaven National Laboratory, Upton, NY, May 21, 2014.

Jacobsen, C.J. (2014). "Scientific Data Exchange: A Flexible HDF5 Schema for Data and Analysis." PaNData ODI Open Workshop (POOW14), Amsterdam, The Netherlands, September 25, 2014.

Jacobsen, C.J. (2014). "Soft X-ray Microscopy and Spectroscopy with Zone Plates." Higher European Research Course for Users of Large Experimental Systems (HERCULES), Grenoble, France, March 14, 2014.

Jacobsen, C. (2014). “X-ray Microscopy.” X-ray and Neutron School, Argonne/Oak Ridge, June 16, 2014.

Jacobsen, C. (2014). "X-ray Nanofocusing and the MBA Lattice Upgrade." APS/CNM/EMS User Meeting, Advanced Photon Source, Argonne National Laboratory, Argonne, IL, May 13, 2014.

Jacobsen, C. (2014). "X-rays Part 2: Comparison with Electrons, Resolution and Damage, Cryo Ptychography." Workshop on Physical, Engineering and Biological Limits to Brain Measurement, National Science Foundation (NSF), May 31, 2014.

Jacobsen, C.J. (2013). "Cold and in Color: Towards Combining Ptychography with Fluorescence in a Cryogenic Microscope.” Real and Reciprocal Space X-ray Imaging, Newport Pagnell, United Kingdom, February 13-14, 2013. 
Jacobsen, C.J. (2013). “Oscillators, X-rays and Global Carbon: Adventures in X-ray Nanofocusing." Kansas State University, Manhattan, KS, October 22, 2012.

Jacobsen, C.J. (2013). "Simplicity from Complexity: Pattern Recognition Techniques Applied to Soft X-ray Spectromicroscopy." Canadian Light Source 16th Annual Users' Meeting and Related Workshops, Saskatoon, Saskatchewan, Canada, May 2-3, 2013.

Jacobsen, C.J. (2013). "Vignettes on the History of X-rays and X-ray Microscopy." Xradia, Inc. Workshop, Concord, CA, February 22, 2013.

Jacobsen, C.J. (2013). "X-ray Imaging." 15th National School on Neutron and X-ray Scattering, Argonne/Oak Ridge School on X-ray and Neutron Science, Argonne, IL, August 10-24, 2013.

Jacobsen, C.J. (2013). "X-ray Imaging and Microscopy: Methods, Applications, and Detectors." 15th International Workshop on Radiation Imaging Detectors, Paris, France, June 23-27, 2013.

Jacobsen, C.J. (2013). "X-ray Microscopy." Higher European Research Course for Users of Large Experimental Systems (HERCULES), Grenoble, France, February 24-March 27, 2013.

Jacobsen, C.J. (2013). "X-ray Microscopy: Combining Imaging and Spectroscopy." RACIRI Summer School on X-ray Science Between Germany, Russia, and Sweden, St. Petersburg, Russia, August 17-25, 2013.

Jacobsen, C.J. (2013). "X-ray Radiation Damage: Limitations and Solutions.” Xradia, Inc. Workshop, Concord, CA, February 22, 2013.

\section{4-134-R1}

\section{PRESENTATIONS}

Cha, W., S. Hruszkewycz, M. Highland, R. Sichel-Tissot, P. Fuoss, R. Harder, J. Maser and W. Liu (2015).

"Three-dimensional Bragg Coherent Diffractive Imaging Using Polychromatic X-rays." 144th TMS Annual Meeting and Exhibition, Orlando, FL, March 15-19, 2015.

Cha, W., S. Hruszkewycz, R. Sichel-Tissot, M.J. Highland, R. Harder, W. Liu, J. Maser and P. Fuoss (2014). "Three-dimensional Bragg Coherent Diffraction Imaging Using Polychromatic X-rays." International Workshop of Phase Retrieval and Coherent Scattering, Evanston, IL, September 2-5, 2014.

\section{4-137-R1}

\section{REFEREED PUBLICATIONS}

Kesgin, I., M. Kasa, C. Doose, Y. Ivanyushenkov, Y. Zhang, P. Brownsey, D. Hazelton and U. Welp. “Feasibility and Electromagnetic Analysis of a REBCO Superconducting Undulator." Superconductor Science \& Technology. (To be published.)

Kesgin, I., C.L. Doose, M.T. Kasa, Y. Ivanyushenkov and U. Welp (2016). "Design of a REBCO Thin Film Superconducting Undulator." Proceedings of the International Cryogenic Materials Conference Series: Advances in Cryogenic Engineering Materials, Tucson, AZ, June 28-July 2, 2015, 101: 912953.

\section{NON-REFEREED PUBLICATION}

Kesgin, I., C. Doose, M. Kasa, Y. Ivanyushenkov and U. Welp. "High-temperature Superconducting REBCO Conductors for Undulators in X-ray Sources." 2016 Materials Research Society Spring Meeting and Exhibit, Phoenix, AZ, March 28-April 1, 2016. (To be published.)

\section{PRESENTATIONS}

Kesgin, I., C.L. Doose, M.T. Kasa, Y. Ivanyushenkov and U. Welp (2015). "Design of a REBCO HTS Superconducting Undulator." 2015 Cryogenic Engineering Conference and International Cryogenic Materials Conference, June 28-July 2, 2015. 
Kesgin, I., Y. Ivanyushenkov, U. Welp, C.L. Doose and M. Kasa (2015). "Design of a Short Period 2G-HTS Base Undulator." 6th International Particle Accelerator Conference (IPAC 2015), Richmond, VA, May 3-8, 2015.

\section{4-175-R1}

\section{REFEREED PUBLICATION}

Murray, T.D., A. Lyubimov, C.M. Ogata, H. Vo, M. Uervirojinangkoorn, A. Brunger and J. Berger (2015).

"A High-transparency, Micro-patternable Chip for X-ray Diffraction Analysis of Microcrystals Under Native Growth Conditions." Acta Crystallographica Section D-Biological Crystallography 71(Pt 10): 1987-1997.

\section{4-184-R1}

\section{REFEREED PUBLICATION}

Shiroyanagi, Y., C. Doose, J. Fuerst, K. Harkay, Q. Hasse, Y. Ivanyushenkov and M. Kasa (2014). "Thermal Analysis of a Superconducting Undulator." Proceedings of the Applied Superconductivity Conference (ASC 2014), Charlotte, NC, August 10-15, 2014.

\section{PRESENTATION}

Ivanyushenkov, Y., C. Doose, J. Fuerst, K. Harkay, Q. Hasse, M. Kasa, D. Skiadopoulos, E.M. Trakhtenberg, Y. Shiroyanagi and E. Gluskin (2015). "Development and Performance of 1.1-M Long Superconducting Undulator at the Advanced Photon Source." 6th International Particle Accelerator Conference (IPAC'15), Richmond, VA, May 3-8, 2015.

\section{5-150-NO}

\section{REFEREED PUBLICATIONS}

Dufresne, E.M., S. Narayanan, A.R. Sandy, D.M. Kline, Q.T. Zhang, E.C. Landahl and S. Ross (2016). "Pushing X-ray Photon Correlation Spectroscopy Beyond the Continuous Frame Rate Limit." Optics Express 24(1): 355-364.

Rumaiz, A.K., D.P. Siddons, G. Deptuch, P. Maj, A.J. Kuczewski, G.A. Carini, S. Narayanan, E.M. Dufresne, A. Sandy, R. Bradford, A. Fluerasu and M.D. Sutton (2016). "First Experimental Feasibility Study of VIPIC: A Custom-made Detector for X-ray Speckle Measurements." Journal of Synchrotron Radiation 23(2).

Zhang, Q., E.M. Dufresne, P. Grybos, P. Kmon, P.G. Maj, S. Narayanan, G.W. Deptuch, R. Szcygiel and A. Sandy. "Submillisecond X-ray Photon Correlation Spectroscopy from Pixel-array-detector with Fast Dual Gating and No Readout Deadtime." Journal of Synchrotron Radiation. (To be published.)

\section{5-153-NO}

\section{PRESENTATION}

Preissner, C., S. Sullivan, C. Jacobsen, J. Maser, B. Lai and C. Roehrig (2015). “The VelociProbe Instrument Design.” NSLS-II Hard X-ray Nanoprobe Team, Upton, NY, September 9, 2015.

\section{5-161-NO}

\section{REFEREED PUBLICATION}

Windt, D. and R. Conley (2015). "Two-dimensional Differential Deposition: Figure Correction of Thin-shell Mirror Substrates for X-ray Astronomy." Proceedings of the SPIE, Optics for EUV, X-ray, and Gamma-Ray Astronomy VII, San Diego, CA, August 9, 2015, 9603: 96031H.

\section{5-182-NO}

\section{PRESENTATIONS}

$\mathrm{Hu}, \mathrm{M}$. (2015). "Pressure Induced Amorphization in Snl 4 ." International Conference on the Applications of the Mössbauer Effect (ICAME), Hamburg, Germany, September 13-18, 2015.

Leu, B.M. (2015). "High-energy Resolution Inelastic X-ray Scattering at Beamline 30-ID, Advanced Photon Source." 8th North American Mössbauer Symposium, Boston, MA, January 8-9, 2015. 
Perez, D. and B.M. Leu (2015). "Vibrational and Superconducting Properties of ${ }^{119}$ Sn Nanowires." Superconductivity on the Verge, Leiden, The Netherlands, July 27-31, 2015.

\section{MATERIALS FOR ENERGY}

\section{2-209-R3}

\section{REFEREED PUBLICATION}

Welland, M.J., K.C. Lau, P.C. Redfern, L. Liang, D. Zhai, D. Wolf and L.A. Curtiss (2016). "An Atomistically Informed Mesoscale Model for Growth and Coarsening During Discharge in Lithium-oxygen Batteries." Journal of Chemical Physics 143(22): 224113.

\section{3-184-R2}

\section{REFEREED PUBLICATIONS}

Armas-Pérez, J.C., A. Londono-Hurtado, O. Guzmán, J.P. Hernández-Ortiz and J.J. de Pablo (2015). "Theoretically Informed Monte Carlo Simulation of Liquid Crystals by Sampling of Alignment-tensor Fields." Journal of Chemical Physics 143(4): 044107.

Buchanan, L.E., E.B. Dunkelberger, H.Q. Tran, P.-N. Cheng, C.C. Chiu, P. Cao, D.P. Raleigh, J.J. de Pablo, J.S. Nowick and M.T. Zanni (2014). "Mechanism of IAPP Amyloid Fibril Formation Involves an Intermediate with a Transient $\beta$-sheet." Proceedings of the National Academy of Sciences of the United States of America, November 26, 2013, 110(19285-19290).

Chiu, C.-c. and J.J. de Pablo (2015). “Fibrillar Dimer Formation of Islet Amyloid Polypeptides.” AIP Advances 5(9): 092501.

Chiu, C.C., S. Singh and J.J. de Pablo (2013). "Effect of Proline Mutations on the Monomer Conformations of Amylin." Biophysical Journal 105(5): 1227-1235.

Fluitt, A.M. and J.J. de Pablo (2015). "An Analysis of Biomolecular Force Fields for Simulations of Polyglutamine in Solution.” Biophysical Journal 109(5): 1009-1018.

Freeman, G.S., D.M. Hinckley, J.P. Lequieu, J.K. Whitmer and J.J. de Pablo (2015). "Coarse-grained Modeling of DNA Curvature." Journal of Chemical Physics 141(16): 165103.

Freeman, G.S., D.M. Hinckley, J.P. Lequieu, J.K. Whitmer and J.J. de Pablo (2015). "DNA Shape Dominates Sequence Affinity in Nucleosome Formation." Physical Review Letters 113(16): 168101.

Hinckley, D.M. and J.J. de Pablo (2016). "Coarse-grained lons for Nucleic Acid Modeling." Journal of Chemical Theory Computation 11(11): 5436-5446.

Hinckley, D.M., J.P. Lequieu and J.J. de Pablo (2014). "Coarse-grained Modeling of DNA Oligonucleotide Hybridization: Length, Sequence, and Salt Effects.” Journal of Chemical Physics 141(3): 035102.

Hinckley, D.M., G.S. Freeman, J.K. Whitmer and J.J. de Pablo (2014). "An Experimentally-Informed Coarse-Grained 3-Site-Per-Nucleotide Model of DNA: Structure, Thermodynamics, and Dynamics of Hybridization." Journal of Chemical Physics 139(14): 144903.

Hoffmann, K., M. McGovern, C.C. Chiu and J.J. de Pablo (2015). "Secondary Structure of Rat and Human Amylin Across Force Fields." PLOS One 10(7): e0134091.

Hur, S.M., V. Thapar, A. Ramirez-Hernandez, G. Khaira, T. Segal-Peretz, P.A. Rincon-Delgadillo, W. Li, M. Muller, P.F. Nealey and J.J. de Pablo (2016). "Molecular Pathways for Defect Annihilation in Directed Self-assembly." Proceedings of the National Academy of Sciences (PNAS) 112(46): 14144-14149.

Hur, S.M., G.S. Khaira, A. Ramirez-Hernandez, M. Mueller, P.F. Nealey and J.J. de Pablo (2015). "Simulation of Defect Reduction in Block Copolymer Thin Films by Solvent Annealing." ACS Macro Letters 4(1): 11-15. 
Joshi, A.A., J.K. Whitmer, O. Guzman, N. Abbott and J.J. de Pablo (2014). "Measuring Liquid Crystal Elastic Constants with Free Energy Perturbations." Soft Matter 10(6): 882-893.

Li, W., P.F. Nealey, J.J. de Pablo and M. Müller (2015). "Defect Removal in the Course of Directed Self-Assembly is Facilitated in the Vicinity of the Order-Disorder Transition." Physical Review Letters 113(16).

Liu, C.C., A. Ramirez-Hernandez, E. Han, G.S. W. Craig, Y. Tada, H. Yoshida, H.M. Kang, S.X. Ji, P. Gopalan, J.J. de Pablo and P.F. Nealey (2013). "Chemical Patterns for Directed Self-assembly of Lamellae-Forming Block Copolymers with Density Multiplication of Features." Macromolecules 46(4): 1415-1424.

Martinez-Gonzalez, J.A., Y. Zhou, M. Rahimi, E. Bukusoglu, N.L. Abbott and J.J. de Pablo (2016). "Blue-phase Liquid Crystal Droplets." Proceedings of the National Academy of Sciences (PNAS) 112(43): 13195-13200.

Onses, M.S., A. Ramirez-Hernandez, S.M. Hur, E. Sutanto, L. Williamson, A.G. Alleyne, P.F. Nealey, J.J. de Pablo and J.A. Rogers (2014). "Block Copolymer Assembly on Nanoscale Patterns of Polymer Brushes Formed by Electrohydrodynamic Jet Printing." ACS Nano 8(7): 6606-6613.

Qin, J., G.S. Khaira, Y.R. Su, G.P. Garner, M. Miskin, H.M. Jaeger and J.J. de Pablo (2014). “Evolutionary Pattern Design for Copolymer Directed Self-assembly." Soft Matter 9(48): 11467-11472.

Qin, J., D. Priftis, R. Farina, S.L. Perry, L. Leon, J. Whitmer, K. Hoffmann, M. Tirrell and J.J. de Pablo (2014). "Interfacial Tension of Polyelectrolyte Complex Coacervate Phases." ACS Macro Letters 3(6): 565-568.

Rahimi, M., T.F. Roberts, J.C. Armas-Perez, X.G. Wang, E. Bukusoglu, N.L. Abbott and J.J. de Pablo (2015). "Nanoparticle Self-assembly at the Interface of Liquid Crystal Droplets." Proceedings of the National Academy of Sciences (PNAS) 112(17): 5297-5302.

Ramirez-Hernandez, A., H.S. Suh, P.F. Nealey and J.J. de Pablo (2014). "Control of Directed Self-assembly in Block Copolymers by Polymeric Top Coats." Macromolecules 47(10): 3520-3527.

Ramirez-Hernandez, A., F.A. Detcheverry, B.L. Peters, V.C. Chappa, K.S. Schweizer, M. Muller and J.J. de Pablo (2013). "Dynamical Simulations of Coarse Grain Polymeric Systems: Rouse and Entangled Dynamics." Macromolecules 46(15): 6287-6299.

Ramirez-Hernandez, A., M. Muller and J.J. de Pablo (2013). "Theoretically Informed Entangled Polymer Simulations: Linear and Non-linear Rheology of Melts." Soft Matter 9(6): 2030-2036.

Whitmer, J.K., C.C. Chiu, A.A. Joshi and J.J. de Pablo (2015). "Basis Function Sampling: A New Paradigm for Material Property Computations." Physical Review Letters 113: 190602.

Whitmer, J.K., A.A. Joshi, R.J. Carlton, N.L. Abbott and J.J. de Pablo (2015). "Surface Adsorption in Nonpolarizable Atomic Models." Journal of Chemical Theory and Computation 10(12): 5616-5624.

Whitmer, J.K., X.G. Wang, F. Mondiot, D.S. Miller, N.L. Abbott and J.J. de Pablo (2014). "Nematic-Field-Driven Positioning of Particles in Liquid Crystal Droplets." Physical Review Letters 111(22): 227801.

Whitmer, J.K., A.A. Joshi, T.F. Roberts and J.J. de Pablo (2013). "Liquid-Crystal Mediated Nanoparticle Interactions and Gel Formation.” Journal of Chemical Physics 138(19): 194903.

2013-202-R1

REFEREED PUBLICATIONS

Jorn, R., R. Kumar, D.P. Abraham and G.A. Voth (2013). "Atomistic Modeling of the Electrode-Electrolyte Interface in Li-lon Energy Storage Systems: Electrolyte Structuring.” Journal of Physical Chemistry C 117(8): 3747-3761.

Ottochian, A., G. Dezanneau, C. Gilles, P. Raiteri, C. Knight and J.D. Gale (2014). "Influence of Isotropic and Biaxial Strain on Proton Conduction in Y-doped $\mathrm{BaZrO}_{3}$ : A Reactive Molecular Dynamics Study." Journal of Materials Chemistry A 2(9): 3127-3133. 
PUBLICATIONS AND PRESENTATIONS I

Sode, O. and G.A. Voth (2015). "Electron Transfer Activation of a Second Water Channel for Proton Transport in [FeFe]-Hydrogenase." Journal of Chemical Physics 141(22): D527.

\section{PRESENTATIONS}

Knight, C. (2014). "Computationally Efficient Reactive Models Derived from Condensed Phase ab Initio Simulations." Louisiana State University, Baton Rouge, LA, July 17, 2014.

Knight, C.J. (2014). "Computationally Efficient Reactive Models Derived from Condensed Phase ab Initio Simulations." University of Kansas, Lawrence, KS, December 12, 2013.

Knight, C.J., G.E. Lindberg, S. Tse, A.M. Herring, T.W. Witten and G.A. Voth (2014). "Progress Towards a Fundamental Understanding of Charge Transport Mechanisms in Fuel Cell Membranes." 7th Bishop's Lodge Workshop: Materials for Energy Conversion, Santa Fe, NM, November 3-5, 2013.

\section{3-216-R2}

\section{REFEREED PUBLICATIONS}

Doxastakis, M., H.S. Suh, X. Chen, P.A.R. Delgadillo, L. Wan, L. Williamson, Z. Jiang, J. Strzalka, J. Wang, W. Chen, N. Ferrier, A. Ramirez-Hernandez, J.J. de Pablo, R. Gronheid and P. Nealey (2015). "Grazing-incidence Small Angle X-ray Scattering Studies of Nanoscale Polymer Gratings." Proceedings of the SPIE Advanced Lithography: Metrology, Inspection, and Process Control for Microlithography XXIX, San Jose, CA, March 19, 2015, 9424(1N): 7.

Segal-Peretz, T., J. Winterstein, M. Doxastakis, A. Ramirez-Hernandez, M. Biswas, J. Ren, H.S. Suh, S.B. Darling, J.A. Liddle, J.W. Elam, J.J. de Pablo, N.J. Zaluzec and P.F. Nealey (2015). "Characterizing the Three-Dimensional Structure of Block Copolymers via Sequential Infiltration Synthesis and Scanning Transmission Electron Tomography." ACS Nano 9(5): 5333-5347.

\section{3-219-R2}

\section{REFEREED PUBLICATIONS}

Benson, B.A., P.A.R. Ade, Z. Ahmed, S.W. Allen, K. Arnold, J.E. Austermann, A.N. Bender, L.E. Bleem, J.E. Carlstrom, C.L. Chang, H.M. Cho, S.T. Ciocys, J.F. Cliche, T.M. Crawford, A. Cukierman, T. de Haan, M.A. Dobbs, D. Dutcher, W. Everett, A. Gilbert, N.W. Halverson, D. Hanson, N.L. Harrington, K. Hattori, J.W. Henning, G.C. Hilton, G.P. Holder, W.L. Holzapfel, K.D. Irwin, R. Keisler, L. Knox, D. Kubik, C.L. Kuo, A.T. Lee, E.M. Leitch, D. Li, M. McDonald, S.S. Meyer, J. Montgomery, M. Myers, T. Natoli, H. Nguyen, V. Novosad, S. Padin, Z. Pan, J. Pearson, C.L. Reichardt, J.E. Ruhl, B.R. Saliwanchik, G. Simard, G. Smecher, J.T. Sayre, E. Shirokoff, A.A. Stark, K. Story, A. Suzuki, K.L. Thompson, C. Tucker, K. Vanderlinde, J.D. Vieira, A. Vikhlinin, G. Wang, V. Yefremenko and K.W. Yoon (2014). "SPT-3G: A Next-Generation Cosmic Microwave Background Polarization Experiment on the South Pole Telescope." Proceedings of the SPIE-The International Society for Optical Engineering, Montreal, Quebec, Canada, July 23, 2014, 9153(Millimeter, Submillimeter, and Far-infrared Detectors and Instrumentation for Astronomy VII). (Also see 2013-080.)

Chang, C., P. Ade, Z. Ahmed, S. Allen, K. Arnold, J. Austermann, A. Bender, B. Benson, L. Bleem, J. Carlstrom, H.-m. Cho, S. Ciocys, J.-F. Cliche, T. Crawford, A. Cukierman, T. de Haan, M. Dobbs, D. Dutcher, W. Everett, A. Gilbert, N. Halverson, D. Hanson, N. Harrington, K. Hattori, J. Henning, G. Hilton, G. Holder, W. Holzapfel, K. Irwin and R. Keisler (2015). "Low Loss Superconducting Microstrip Development at Argonne National Laboratory." IEEE Transactions on Applied Superconductivity PP(99): 1-1. (Also see 2013-080.)

Posada, C.M., P.A.R. Ade, Z. Ahmed, K. Arnold, J.E. Austermann, A.N. Bender, L.E. Bleem, B.A. Benson, K. Byrum, J.E. Carlstrom, C.L. Chang, H.M. Cho, S.T. Ciocys, J.F. Cliche, T.M. Crawford, A. Cukierman, D. Czaplewski, J. Ding, R. Divan, T. de Haan, M.A. Dobbs, D. Dutcher, W. Everett, A. Gilbert, N.W. Halverson, N.L. Harrington, K. Hattori, J.W. Henning, G.C. Hilton, W.L. Holzapfel, J. Hubmayr, K.D. Irwin, O. Jeong, R. Keisler, D. Kubik, C.L. Kuo, A.T. Lee, E.M. Leitch, S. Lendinez, S. S. Meyer, C.S. Miller, J. Montgomery, M. Myers, A. Nadolski, T. Natoli, H. Nguyen, V. Novosad, S. Padin, Z. Pan, J. Pearson, J.E. Ruhl, B.R. Saliwanchik, G. Smecher, J.T. Sayre, E. Shirokoff, L. Stan, A.A. Stark, J. Sobrin, K. Story, A. Suzuki, K.L. Thompson, C. Tucker, K. Vanderlinde, J.D. Vieira, G. Wang, N. Whitehorn, V. Yefremenko, K.W. Yoon and K.E. Ziegler (2015). "Fabrication of Large Dual-polarized Multichroic TES Bolometer Arrays for CMB Measurements with the SPT-3G Camera." Superconductor Science \& Technology 28(9): 094002. 
Wang, G., C.L. Chang, V. Yefremenko, V. Novosad, J. Pearson, R. Divan and J.E. Carlstrom (2015). "Mo/Au Bilayer TES Resistive Transition Engineering “IEEE Transactions on Applied Superconductivity-ACS 2014 Special Issue PP(99): 1-1.

\section{4-121-R1}

\section{REFEREED PUBLICATIONS}

Gao, M.-R., M.K.Y. Chan and Y. Sun (2015). "Edge-terminated Molybdenum Disulfide with a 9.4 A Interlayer Spacing for Electrochemical Hydrogen Production.” Nature Communications 6: 7493.

Liu, Q., M.-R. Gao, Y. Liu, J.S. Okasinski, Y. Ren and Y. Sun (2016). "Quantifying the Nucleation and Growth Kinetics of Microwave Nanochemistry Enabled by in situ High-Energy X-ray Scattering.” Nano Letters(Article ASAP): DOI: 10.1021/ acs.nanolett.1025b04541.

Liu, Q., Z. Li, J.S. Okasinski, Y. Ren and Y. Sun (2015). “In situ High-energy Synchrotron X-ray Diffraction Revealing Precipitation Reaction Kinetics of Silver Ions with Mixed Halide Ions." Journal of Materials Chemistry C: Materials for Optical and Electronic Devices 3(28): 7492-7498.

\section{PRESENTATIONS}

Sun, Y. (2015). “Discovery Enabled by in situ Synchrotron X-ray Techniques.” 51st Annual Technical Meeting, Society of Engineering Science (SES 2014), West Lafayette, IN, October 1-3, 2014.

Sun, Y., S. Peng, Z. Li, Y. Ren, J.D. Almer and J.S. Okasinski (2014). "In situ Study on the Growth and Transformation of Colloidal Noble Metal Nanocrystals.” 2014 Gordon Research Conference on Noble Metal Nanoparticles, South Hadley, MA, June 15-20, 2014.

\section{4-128-R1}

\section{REFEREED PUBLICATION}

Zhong, X., I. Rungger, P. Zapol and O. Heinonen (2015). "Electronic and Magnetic Properties of $\mathrm{Ti}_{4} \mathrm{O}_{7} \mathrm{Predicted} \mathrm{by}$ Self-interaction-corrected Density Functional Theory." Physical Review B: Condensed Matter and Materials Physics 91: 115143.

\section{NON-REFEREED PUBLICATION}

Maheshwari, K.C., J.M. Wozniak, T. Armstrong, D.S. Katz, T.A. Binkowski, X. Zhong, O. Heinonen, D. Karpeyev and M. Wilde (2015). "Porting Ordinary Applications to Blue Gene/Q Supercomputers." 2015 IEEE 11th International Conference on e-Science, Munich, Germany, August 31-September 4, 2015.

\section{PRESENTATIONS}

Heinonen, O. (2015). “Coupling Molecular Dynamics and First-principle Electronic Structure Modeling of Disordered Heterostructures." International Workshop on High Performance and Parallel Computing for Materials Defects and Multiphase Flows, National University of Singapore, Singapore, February 9-13, 2015.

Heinonen, O. (2015). “Length Scale Bridges." Los Alamos National Laboratory, Los Alamos, NM, April 23, 2015.

Heinonen, O. (2015). “Length Scale Bridges.” University of Chicago Computation Institute, Chicago, IL, October 22, 2014.

Zapol, P., D. Karpeyev, K.C. Maheshwari, X. Zhong, B. Narayanan, S. Sankaranarayanan, M. Wilde and O. Heinonen (2015). "Coupled Molecular-dynamics and First-principle Transport Calculations of Metal/Oxide/Metal Heterostructures." American Physical Society March Meeting, San Antonio, TX, March 2-6, 2015. (Also see 2014-161.)

Zhong, X., I. Rungger, P. Zapol and O. Heinonen (2015). "Self-interaction Corrected Electronic Structure of $\mathrm{Ti}_{4} \mathrm{O}_{7}, \mathrm{TiO}_{2}$ and $\mathrm{Ti}_{2} \mathrm{O}_{3}$ " American Physical Society March Meeting, San Antonio, TX, March 2-5, 2015. 


\section{4-133-R1}

\section{REFEREED PUBLICATIONS}

Klug, J.A., C.J. Alvarez, N.G. Becker, C.M. Schleputz, A. Petford-Long and T. Proslier. "Epitaxial Atomic Layer Deposition of $\mathrm{MgO}$ on a-Al $\mathrm{O}_{3}(0001)$." Applied Physics Letters. (To be published.)

Klug, J.A., C. Schleputz, A. Petford-Long, M.J. Pellin, N.G. Becker, P. Zapol, D.C. Ford and T. Proslier. "Second Phase Nucleation During Epitaxial Atomic Layer Deposition of Molybdenum Nitride on Sapphire." Physical Review B: Condensed Matter and Materials Physics. (To be published.)

Klug, J.A., M.S. Weimer, J.D. Emery, A. Yanguas-Gil, S. Seifert, C.M. Schleputz, A.B.F. Martinson, J.W. Elam, A.S. Hock and T. Proslier (2016). "A Modular Reactor Design for in situ Synchrotron X-ray Investigation of Atomic Layer Deposition Processes." Review of Scientific Instruments 86(11): 113901.

Groll, N.R., J.A. Klug, C.Y. Cao, S. Altin, H. Claus, N.G. Becker, J.F. Zasadzinski, M.J. Pellin and T. Proslier (2014). "Tunneling Spectroscopy of Superconducting MoN and NbTiN Grown by Atomic Layer Deposition." Applied Physics Letters 104(9): 092602.

Klug, J.A., N.G. Becker, N.R. Groll, C. Cao, M.S. Weimer, M.J. Pellin, J. Zasadzinski and T. Proslier (2014). “Heteroepitaxy of Group IV-VI Nitrides by Atomic Layer Deposition." Applied Physics Letters 103(21): 211602.

Yanguas-Gil, A., T. Proslier, J.A. Klug, M.S. Weimer, J.W. Elam, N.G. Becker and A.S. Hock. "Structure and Stability of Amorphous Doped $\mathrm{HfO}_{2}$ Thin Films and Nanolaminates." Applied Physics Letters. (To be published.)

\section{PRESENTATIONS}

Klug, J. (2015). "Atomic Layer Deposition of Nanoscale Superconductors." Illinois Institute of Technology, Chicago, IL, November 20, 2014.

Weimer, M., J. Klug, J. Emery, A. Yanguas-Gil, C. Schleputz, M. Pellin, J. Elam, A. Hock and T. Proslier (2015). "Atomic Layer Deposition of Doped Films Studied by in situ Synchrotron Techniques with a Mobile and Modular Reactor." APS/CNM Users Meeting, Argonne, IL, May 11, 2015.

\section{4-139-R1}

\section{REFEREED PUBLICATION}

Keceli, M., H. Zhang, P. Zapol, D.A. Dixon and A.F. Wagner (2016). "Shift-and-Invert Parallel Spectral Transformation Eigensolver: Massively parallel performance for Density-Functional Based Tight-Binding." Journal of Computational Chemistry 37(4): 448-459.

\section{PRESENTATIONS}

Dixon, D.A. (2015). "Computational Studies of Heavy Element Clusters and Molecular Halides." Southeast Theoretical Chemistry Association Annual Meeting (SETCA 2015), Orlando, FL, May 14-16, 2015.

Keceli, M., H. Zhang, P. Zapol, D.A. Dixon and A.F. Wagner (2015). "Exploiting Sparsity to Enable Petascale Applications in Material Science and Quantum Chemistry." 249th American Chemical Society National Meeting Denver, CO, March 22-26, 2015.

Keceli, M., H. Zhang, P. Zapol, D.A. Dixon and A.F. Wagner (2015). "A Scalable Sparse Eigensolver for Petascale Applications.” American Physical Society March Meeting, San Antonio, TX, March 2-6, 2015.

Wagner, A., P. Zapol, M. Keceli and H. Zhang (2014). "The SIPs Eigensolver for Petascale Applications." 46th Midwest Theoretical Chemistry Conference, Northwestern University, Evanston, IL, June 15-17, 2014. 


\section{4-151-R1}

\section{REFEREED PUBLICATION}

Yanguas-Gil, A. and J.W. Elam. "Exact Solution of Kinetic Monte Carlo Simulations: Application to Particle-surface Interaction During Materials Growth." Physical Review E: Statistical Physics, Plasmas, Fluids, and Related Interdisciplinary Topics. (To be published.)

\section{PRESENTATIONS}

Fuoss, P. (2015). "In situ Studies of GaN Growth on Polar and Non-polar Substrates." DOE EERE Solid State Lighting R\&D Workshop, San Francisco, CA, January 27-29, 2015.

Fuoss, P. (2015). "In situ X-ray Studies of Thin Film Crystal Growth." Gordon Research Conference on Crystal Growth and Self-assembly, Bitteford, ME, June 28-July 3, 2015.

Fuoss, P., J. Eastman, M. Highland, M. Hopper, S. Hruszkewycz, B. Ingram, B. Stephenson and C. Thompson (2015). "Millimeters to Nanometers: The Evolution of Real-time in situ X-ray Measurements." Materials Research Society Spring Meeting, San Francisco, CA, April 6-10, 2015.

Fuoss, P., M. Highland, S. Hruszkewycz, P. Zapol, B. Stephenson and C. Thompson (2015). "Synchrotron X-ray Studies of the Synthesis and Processing of Wide Bandgap Semiconductors." DOE EERE/BES Solid State Lighting Roundtable, Washington, D.C., October 7, 2014.

Ju, G., M.J. Highland, C. Thompson, J.A. Eastman, G.B. Stephenson and P.H. Fuoss (2015). "Diffractometer and Growth System for in situ Coherent X-ray Studies of Epitaxy." 20th American Conference on Crystal Growth and Epitaxy (ACCGE-20), Big Sky, MT, August 2-7, 2015.

Sichel-Tissot, R., S. Hruszkewycz, M. Highland, W. Cha, R.J. Holt, S.R. Lee, D.D. Koleske and P.H. Fuoss (2014). "X-ray Probes of Local Composition and Structure Near Defects in InGaN Multi-quantum Wells." International Workshop of Phase Retrieval and Coherent Scattering, Evanston, IL, September 2-5, 2014.

Yanguas-Gil, A. (2015). "First-order Kinetic Model for SiC Epitaxy: Miscut, Microstructure, Polytypes and Kinetic Wulff Constructions." Electronic Materials Conference, Columbus, OH, June 24-26, 2015.

Yanguas-Gil, A. and P. Zapol (2015). "Impact of Microstructure and Defects on Surface Reactivity during Wide Bandgap Semiconductor Epitaxy.” 227th Electrochemical Society Meeting, Chicago, IL, May 24-28, 2015.

Yanguas-Gil, A. (2015). "The Role of Surface Kinetics on Defect Generation and Propagation During Epitaxy of WBG Semiconductors." American Physical Society March Meeting, San Antonio, TX, March 2-6, 2015.

Yanguas-Gil, A. (2014). "The Role of Surface Kinetics on Surface Morphology and Defect Evolution during WBG Semiconductor Epitaxy." Workshop on Defects in Wide Band Gaps Semiconductors, University of Maryland, College Park, MD, September 23, 2014.

\section{4-161-R1}

\section{REFEREED PUBLICATIONS}

Das, S., M.K. Bera, S. Tong, B. Narayanan, G.K. Kamath, A. Mane, M.R. Antonio, S.K.R.S. Sankaranarayanan and A.K. Roelofs. "A Self-Limiting Electro-Ablation Technique for the Top-Down Synthesis of Wafer Scale Monolayers of 2D Materials." ACS Nano. (To be published.)

Deshmukh, S., G. Kamath and S.K.R.S. Sankaranarayanan (2014). "Effect of Nanoscale Confinement on Freezing of Modified Water at Room Temperature and Ambient Pressure." ChemPhysChem 15(8): 1632-1642.

Kamath, G.K., B. Narayanan and S.K.R.S. Sankaranarayanan (2015). “Atomistic Origin of Superior Performance of Ionic Liquid Electrolytes for Al-ion Batteries.” Physical Chemistry Chemical Physics 16(38): 20387-20391.

Sen, F.G., A. Kinaci, B. Narayanan, S.K. Gray, M.J. Davis, S.K.R.S. Sankaranarayanan and M.K.Y. Chan (2015). "Towards Accurate Prediction of Catalytic Activity in $\mathrm{IrO}_{2}$ Nanoclusters via First Principles-based Variable Charge Force Field." Journal of Materials Chemistry A 3(37): 18970-18982. 
Sumant, A.V., D. Berman, S. Deshmukh, B. Narayanan, S.K.R.S. Sankaranarayanan, Z. Yan, A.A. Balandin, A. Zinovev and D. Rosenmann. "Metal Induced Transformation of Diamond into Single Domain Graphene on Wafer Scale in Seconds." Nature Communications. (To be published.)

\section{PRESENTATIONS}

Kinaci, A., B. Narayanan, M.J. Davis, S. Gray, S. Sankaranarayanan and M. Chan (2015). "Evolutionary Algorithm Search for Global Minimum Structures of Au Nano-Clusters." Materials Research Society Fall Meeting, Boston, MA, November 30-December 5, 2014.

Kinaci, A., B. Narayanan, M. Davis, S. Gray, S. Sankaranarayanan and M. Chan (2015). "Evolutionary Algorithm Search for Global Minimum Structures of Au Nano-Clusters.” CNM/APS Users Meeting, Argonne, IL, May 11-14, 2015.

Narayanan, B., F.G. Sen, A. Kinaci, M.J. Davis, S.K. Gray, Z.-G. Mei, M.K.Y. Chan and S.K.R.S. Sankaranarayanan (2016). "Evolutionary Strategy for Developing Interatomic Potentials to Bridge the Electronic and Atomistic Length Scales." Materials Research Society Fall Meeting, Boston, MA, November 29-December 4, 2015.

Narayanan, B., S.A. Deshmukh, G. Kamath, E.V. Shevchenko and S.K.R.S. Sankaranarayanan (2016). "Role of Ligand Dynamics in Structural Stability and Pressure Behavior of Supercrystals Self-assembled from Crystalline Nanoparticles." Materials Research Society Fall Meeting, Boston, MA, November 29-December 4, 2015.

Narayanan, B., S.A. Deshmukh, S. Ramanathan and S.K.R.S. Sankaranarayanan (2015). "Atomistic Insights into the Interaction of Copper Oxide Surfaces with Chloride Ions in Aqueous Media." 227th Electrochemical Society Meeting, Chicago, IL, May 24-28, 2015.

Narayanan, B., A. Kinaci, F.G. Sen, M.J. Davis, S.K. Gray, M.K.Y. Chan and S.K.R.S. Sankaranarayanan (2015). "Bond Order Potential to Capture Size-dependent Dimensionality Effects in Au Clusters." CNM/APS Users Meeting Argonne, IL, May 11-14, 2015.

Narayanan, B., A. Kinaci, M. Davis, M. Chan, S. Sankaranarayanan and S. Gray (2015). "Development of Novel Force Field for Gold Nanoclusters.” Materials Science and Technology Conference, Pittsburgh, PA, October 12-16, 2014.

Narayanan, B., A. Kinaci, F.G. Sen, M.J. Davis, S. Gray, M. Chan and S.K.R.S. Sankaranarayanan (2015). "A Novel Empirical Force Field to Capture Size-dependent Dimensionality Effects in Au Nanoclusters." 227th Electrochemical Society Meeting, Chicago, IL, May 24-28, 2015.

Narayanan, B., A. Kinaci, F.G. Sen, M.J. Davis, S.K. Gray, M.K.Y. Chan and S.K.R.S. Sankaranarayanan (2015). "Size-dependent Dimensionality Effects and Structures in Au Nanoclusters Using a Novel Empirical Force Field." Materials Research Society Spring Meeting, San Francisco, CA, April 6-10, 2015.

Narayanan, B., A. Kinaci, M.J. Davis, M.K. Chan, S. Sankaranarayanan and S.K. Gray (2014). "Development of Force Field for Reactive Interfaces from First Principles." Center for Nanoscale Materials Users Meeting, Argonne National Laboratory, Argonne, IL, May 12-15, 2014.

Sen, F.G., A. Kinaci, B. Narayanan, M.J. Davis, S.K. Gray, S.K.R.S. Sankaranarayanan and M.K.Y. Chan (2016). "Atomistic

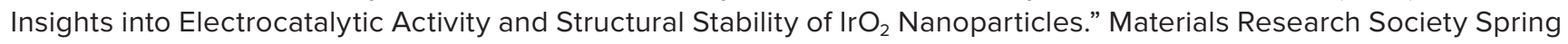
Meeting, Phoenix, AZ, March 28-April 1, 2016.

Sen, F.G., B. Narayanan, A. Kinaci, M.J. Davis, S.K. Gray, S.K.R.S. Sankaranarayanan and M.K.Y. Chan (2016). "A Comparative Study of Optimization Methods for Force Field Fitting." Materials Research Society Fall Meeting, Boston, MA, November 29-December 4, 2015.

Sen, F.G., A. Kinaci, B. Narayanan, M J. Davis, S.K. Gray, S.K.R.S. Sankaranarayanan and M.K.Y. Chan (2015). "Development of a Variable Charge Force-field to Gain Atomistic Insights into $\mathrm{IrO}_{2}$ Surfaces and Nanoclusters." Materials Research Society Spring Meeting, San Francisco, CA, April 6-10, 2015. 
Sen, F., B. Narayanan, A. Kinaci, S. Gray, M. Davis, S. Sankaranarayanan and M. Chan (2015). "Force Field Development from First Principles for Materials Design." American Physical Society March Meeting, San Antonio, TX, March 2-6, 2015.

Sen, F.G., A. Kinaci, B. Narayanan, M.J. Davis, S.K. Gray, S.K.R.S. Sankaranarayanan and M.K.Y. Chan (2015). "IrO $\mathrm{r}_{2}$ Surface and Nanostructure Stability from First Principles and Variable Charge Force Field Calculations." 227th Electrochemical Society Meeting, Chicago, IL, May 24-28, 2015.

Sen, F.G., B. Narayanan, A. Kinaci, M.J. Davis, S.K. Gray, S.K.R.S. Sankaranarayanan and M.K.Y. Chan (2015). "IrO Surface and Nanostructure Stability from first Principles and Variable Charge Force Field Calculations." CNM/APS Users Meeting Argonne, IL, May 11-14, 2015.

Zapol, P., D. Karpeyev, K.C. Maheshwari, X. Zhong, B. Narayanan, S. Sankaranarayanan, M. Wilde and O. Heinonen (2015). "Coupled Molecular-dynamics and First-principle Transport Calculations of Metal/Oxide/Metal Heterostructures." American Physical Society March Meeting, San Antonio, TX, March 2-6, 2015. (Also see 2014-128.)

\section{4-191-R1}

\section{REFEREED PUBLICATION}

Koehl, W.F., H. Seo, G. Galli and D.D. Awschalom (2016). “Designing Defect Spins for Wafer-scale Quantum Technologies." MRS Bulletin 40(12): 1146-1153.

\section{4-192-R1 \\ REFEREED PUBLICATIONS}

Pham, T.A., D. Lee, E. Schwegler and G. Galli (2015). "Interfacial Effects on the Band Edges of Functionalized Si Surfaces in Liquid Water." Journal of the American Chemical Society 136(49): 17071-17077.

Ping, Y., W.A. Goddard and G.A. Galli (2015). "Energetics and Solvation Effects at the Photoanode/Catalyst Interface: Ohmic Contact Versus Schottky Barrier." Journal of American Chemical Society 137(16): 5264-5267.

Wan, Q. and G. Galli (2015). "First-principles Framework to Compute Sum-frequency Generation Vibrational Spectra of Semiconductors and Insulators." Physical Review Letters 115(24): 246404.

\section{PRESENTATIONS}

Galli, G.A. (2016). "Aqueous Interfaces: Insights From First Principles Calculations." Materials Research Society Fall Meeting (2015 MRS), Boston, MA, November 29-December 4, 2015.

Galli, G. (2016). "Predicting the Properties of Interfaces Between Electrolytes and Electrodes: Condensed Matter Physics Meets Electrochemistry." 228th ECS Meeting, Phoenix, AZ, October 11-15, 2015.

Galli, G.A. (2015). "First Principles Spectroscopy of Heterogeneous Systems: GW and Hybrid Functionals." 250th American Chemical Society National Meeting, Boston, MA, August 16-20, 2015.

Galli, G. (2015). "Materials Discovery and Scientific Design by Computation: What Does It Take?" Psi-K Conference, San Sebastien, Spain, September 6-10, 2015.

Galli, G. (2015). “Predicting Complex Materials from First Principles.” Gordon Research Conference: Nanomaterials for Applications in Energy Technology, Ventura, CA, February 22-27, 2015.

Galli, G. (2014). "Electronic Properties of Aqueous Interfaces: Coupled ab initio Molecular Dynamics and GW Calculations." International Center for Materials Research (ICMR) Workshop on Ab-initio Description of Charged Systems and Solid/Liquid Interfaces for Semiconductors and Electrochemistry, University of California, Santa Barbara, CA, July 7-11, 2014.

Galli, G. (2014). "Heterogeneous Interfaces: Insights From First Principles Calculations." American Conference on Theoretical Chemistry (ACTC) 2014, Telluride, CO, July 21-24, 2014. 
Galli, G. (2014). "Light Absorbers for Photo-electrochemical Energy Conversion: First Principles Calculations." 248th American Chemical Society (ACS) National Meeting, San Francisco, CA, August 10-14, 2014.

Galli, G. (2014). "Photo-excitations in Nanostructured Semiconductors." American Physical Society (APS) March Meeting, Denver, CO, March 3-7, 2014.

Galli, G. (2014). "Predicting Materials for Water Splitting: Condensed Matter Physics Meets Electrochemistry." 248th American Chemical Society (ACS) National Meeting, Presidential Symposium Photocatalytic Conversion of Water to Hydrogen and Oxygen, San Francisco, CA, August 10-14, 2014.

Galli, G. (2014). "Water at Surfaces: Insights from First Principles Calculations." Water and Aqueous Solutions Gordon Research Conference, Holderness, NH, July 27-August 1, 2014.

\section{5-144-NO}

\section{REFEREED PUBLICATION}

Phatak, C. and D. Gursoy (2015). "Iterative Reconstruction of Magnetic Induction using Lorentz Transmission Electron Tomography." Ultramicroscopy 150: 54-64.

\section{NON-REFEREED PUBLICATION}

Phatak, C., D. Gursoy, E.B. Gulsoy, L. Trahey and V. De Andrade (2016). "Integrated Multimodal Imaging of Cathodes for Lithium-Ion Battery.” 145th Annual TMS Meeting (TMS 2016), Nashville, TN, February 14-18, 2016.

\section{PRESENTATIONS}

Gürsoy, D. (2015). "Compressive Sensing and Its Potential in Nanoimaging." Joint NSRC Workshop 2015: Big, Deep, and Smart Data Analytics in Materials Imaging, Oak Ridge, TN, June 8-10, 2015.

Gürsoy, D. (2015). "Current and Future Data Analysis Trends for Synchrotron Imaging." 23rd International Congress on X-ray Optics and Microanalysis, Upton, NY, September 14-18, 2015.

Phatak, C., X. Yang, D. Gursoy, E.B. Gulsoy, L. Trahey, V. De Andrade, Q. Liu and X. Xiao (2016). "MIMES: Multimodal Imaging of Materials for Energy Storage.” 145th TMS Annual Meeting, Nashville, TN, February 14-18, 2016.

Yang, X., D. Gürsoy, C. Phatak, F. De Carlo and V.J. De Andrade. "Enhancing Structural Resolution of Lithium-ion Battery Particles by Multimodal Analysis of TXM and STEM Datasets." 3D Materials Science (3DMS), St. Charles, IL, July 10-13, 2016. (To be presented.)

Yang, X., D. Gursoy, C. Phatak, V. De Andrade, E.B. Gulsoy and F. De Carlo. "Learning from Scanning Transmission Electron Microscopy to Enhance Transmission X-ray Microscopy: How we can merge STEM and TXM datasets?” Microscopy and Microanalysis Meeting (M\&M 2016), Columbus, OH, July 24-28, 2016. (To be presented.)

Yang, X., B. Gulsoy, V.J. De Andrade, F. De Carlo, C. Phatak and D. Gürsoy (2016). "Multimodal Imaging for Lithium-ion Battery Particles." Lorentz Workshop on Quantitative 3D X-ray Imaging: From Tomographic Images to Metrics, Leiden Netherlands, January 11-15, 2016.

\section{5-149-NO}

\section{REFEREED PUBLICATIONS}

Deng, J., Y.S.G. Nashed, S. Chen, N.W. Phillips, T. Peterka, R. Ross, S. Vogt, C. Jacobsen and D.J. Vine (2015). "Continuous Motion Scan Ptychography: Characterization for Increased Speed in Coherent X-ray Imaging." Optics Express 23(5): 5438-5451.

Li, Y., Z. Jiang, X.-M. Lin, H. Wen, D.A. Walko, S.A. Deshmukh, R. Subbaraman, S.K.R.S. Sankaranarayanan, S. Gray and P. Ho (2015). "Femto-second Laser Pulse Driven Melting in Gold Nanorod Aqueous Colloidal Suspension: Identification of a Transition from Stretched to Exponential Kinetics." Nature Scientific Reports 5(8146). 


\section{5-154-NO}

\section{REFEREED PUBLICATIONS}

Cai, Z., S. Cai, W. Yang and M. Tang (2015). "Development of Differential Analysis Techniques for Multivariate Imaging Data Analysis." Proceedings of the SPIE X-ray Nanoimaging II Conference, San Diego, CA, August 2015.

Wu, Y.A., L. Li, Z. Li, A. Kinaci, M.K. Chan, Y. Sun, J.R. Guest, I. McNulty, T. Rajh and Y. Liu. "Visualizing Redox Dynamics of a Single $\mathrm{Ag} / \mathrm{AgCl}$ Heterogeneous Nanocatalyst at Atomic Resolution.” ACS Nano. (To be published.)

\section{PRESENTATIONS}

Li, L., M.K. Chan, L. Luo, J. Ciston, W. Saidi, E.A. Stach, J. Yang and G. Zhou (2015). "First-Principles Study on the Epitaxial Growth of $\mathrm{Cu}_{2} \mathrm{O}$ Thin Film and Catalytic Reactivity of $\mathrm{Cu}_{2} \mathrm{O}$ Surfaces." 27th Annual Workshop on Recent Developments in Electronic Structure Theory, Seattle, WA, June 21-24, 2015.

McNulty, I. (2015). “Argonne Integrated Imaging Initiative: the Sum is Greater Than the Parts.” Big, Deep, and Smart Data Analytics in Materials Imaging Workshop, Oak Ridge, TN, June 2015.

\section{5-168-NO REFEREED PUBLICATIONS}

Kim, C., H. Park and C.A. Marianetti. "New Class of Planar Ferroelectric Mott Insulators via First Principles Design.” Physical Review B. (To be published.)

Kim, C., H. Park and C.A. Marianetti (2016). “New Class of Planar Ferroelectric Mott Insulators via First Principles Design." Physical Review B 92(23).

Nowadnick, E.A., J.P. Ruf, H. Park, P.D.C. King, D.G. Schlom, K.M. Shen and A.J. Millis (2016). "Quantifying Electronic Correlation Strength in a Complex Oxide: A Combined DMFT and ARPES Study of $\mathrm{LaNiO}_{3}$." Physical Review B 92(24): 245109.

Upton, M.H., Y. Choi, H. Park, J. Liu, D. Meyers, J. Tchakhalian, S. Middey and J.-W. Kim (2015). "Novel Electronic Behavior Facilitating the $\mathrm{NdNiO}_{3}$ Metal-insulator Transition.” Physical Review Letters 115: 036401.

\section{NATIONAL SECURITY}

\section{3-171-R2}

\section{PRESENTATION}

Schabacker, D. and A. Driks (2014). "Enhanced Molecular Attribution Through Proteomic Signatures (EMAPS)." International Microbial Forensics Symposium, Ottawa, Ontario, Canada, February 11-12, 2014.

\section{3-173-R2}

\section{REFEREED PUBLICATION}

Lee, J., M.B. Brennan, R. Wilton, C.E. Rowland, E.A. Rozhkova, S. Forrester, D.C. Hannah, J.N. Carlson, E.V. Shevchenko, D.S. Schabacker and R.D. Schaller (2015). "Fast, Ratiometric FRET from Quantum Dot Conjugated Stabilized Single Chain Variable Fragments for Quantitative Botulinum Neurotoxin Sensing." Nano Letters 15(10): 7161-7167.

\section{PRESENTATIONS}

Schaller, R.D. (2015). "Colloidal Semiconductor Nanocrystals for Sensing and Optoelectronic Applications."

6th International Conference on Metamaterials, Photonic Crystals and Plasmonics, New York, NY, August 4-7, 2015.

Schaller, R.D. (2015). "Colloidal Semiconductor Nanocrystals for Sensing and Optoelectronic Applications." 227th Electrochemical Society Meeting: State of the Art Program on Compound Semiconductors, Chicago, IL, May 24-28, 2015. 
Schaller, R. (2015). "Quantum Confined Semiconductor Nanocrystals: Elevated Temperature Performance and Sensing Application." 6th International Conference on Metamaterials, Photonic Crystals and Plasmonics (META 2015), New York, NY, August 4-7, 2015.

Schaller, R.D. (2014). "Ratiometric Sensing of Bioweapon and Nuclear Threat Agents." National Counterproliferation Center Lab Days, The MITRE Corporation, McLean, VA, April 9, 2014.

Schaller, R. D. (2013). "Probing Energy Migration in Semiconductor Nanocrystals for Sensing and Energy Applications." Telluride Science Research Center, Telluride, CO, June 27, 2013.

\section{3-178-R2}

\section{REFEREED PUBLICATION}

Bobadilla, A.D., L.E. Ocola, A.V. Sumant, M. Kaminski, N. Kumar and J.M. Seminario (2015). "Europium Effect on the Electron Transport in Graphene Ribbons.” Journal of Physical Chemistry C 119(39): 22486-22495.

Mertz, C.J., M.D. Kaminski, I.A. Shkrob, M. Kalensky, V.S. Sullivan and Y. Tsai (2015). “Development of a Field-based Separator for the Rapid Identification of Uranium and Plutonium." Journal of Radioanalytical and Nuclear Chemistry 305(1): 199-205.

\section{PRESENTATIONS}

Bobadilla, A., N. Kumar, A. Sumant, L. Ocola, C. Mertz, G. Sandi, M. Kaminski and J. Seminario (2014). "Detection of Uranium and Plutonium by Graphene-Based Nanosensors." American Vacuum Society 60th International Symposium, Long Beach, CA, October 27-November 1, 2013.

Bobadilla, A., L. Ocola, A. Sumant, J.M. Seminario and M. Kaminski (2014). "Electrochemical Response of Graphene Ribbon to Eu (III)." APS/CNM/EMC Users Meeting 2014, Argonne, IL, May 12-15, 2014.

Bobadilla, A., L. Ocola, A. Sumant, J.M. Seminario and M. Kaminski (2014). "Graphene Based Electrochemical Detection of Europium (III)." New Diamond and Nano Carbons Conference, Chicago, IL, May 25-29, 2014.

Bobadilla, A., L. Ocola, A. Sumant, J.M. Seminario and M. Kaminski (2014). “Electrochemical Response of Graphene Ribbon to Eu (III)." Texas A\&M, College Station, TX, June 5, 2014.

Bobadilla, A., G. Sandi, L. Ocola, A. Sumant, J. Seminario, M. Kaminski and C. Mertz (2013). "Uranium and Plutonium Detection by Plasmonic Graphene-Based Nanosensors." APS/CNM/EMC Users Meeting, Argonne, IL, May 6-9, 2013.

\section{4-194-R1}

\section{REFEREED PUBLICATIONS}

North, M.J., P. Sydelko and I. Martinez-Moyano. "Anticipatory Complex Adaptive Network Extrapolation." Complex Adaptive Systems Modeling. (To be published.)

North, M.J., P. Sydelko and I. Martinez-Moyano (2016). "Applying 3D Printing and Genetic Algorithm-Generated Anticipatory System Dynamics Models to a Homeland Security Challenge." Proceedings of the 2015 Winter Simulation Conference, Huntington Beach, CA, December 6-9, 2015: 2511-2522.

North, M.J., P. Sydelko and I. Martinez-Moyano (2015). "Structurally Evolving System Dynamics Models Using Genetic Algorithms." Proceedings of the 33rd International Conference of the System Dynamics Society, Cambridge, MA, July 19-23, 2015. 


\section{NUCLEAR ENERGY AND SECURITY}

2013-152-R2

\section{REFEREED PUBLICATIONS}

Kun, M., Z. Zhou, Z. Miao, Y. Yun, D. Tung, H.M. Zhang, G. Chen, W. Almer and J.F. Stubbins (2014). "Synchrotron Study on Load Partitioning between Ferrite/Martensite and Nanoparticles of a 9Cr ODS Steel." Proceedings of the 16th International Conference on Fusion Reactor Materials (ICFRM-16), Beijing, China, October 20-26, 2013, 455(1-3): 376-381.

Lin, J.-L., K. Mo, D. Yun, Y. Miao, X. Liu, H. Zhao, D.T. Hoelzer, J.-S. Park, J. Almer, G. Zhang, Z. Zhou, J.F. Stubbins and A.M. Yacout. "In situ Synchrotron Tensile Investigations on 14 YWT, MA957, and 9-Cr ODS Alloys." Journal of Nuclear Materials. (To be published.)

Mei, Z.-G., L. Liang, Y.S. Kim, T. Wiencek, E. O’Hare, A.M. Yacout, G. Hofman and M. Anitescu. "Grain Growth in U-Mo Alloy: A Combined First-principles and Phase Field Study." Journal of Nuclear Materials. (To be published.)

Miao, Y., K. Mo, B. Ye, L. Jamison, Z.-G. G. Mei, Jian, B. Miller, J. Madden, J.-S. Park, J. Almer, S. Bhattacharya, Y.S. Kim, G.L. Hofman and A.M. Yacout (2016). "High-Energy Synchrotron Study of In-Pile-Irradiated U-Mo Fuels." Scripta Materialia 114: 146-150.

Miao, Y., K. Mo, B. Ye, L. Jamison, Z. Mei, Y.S. Kim, G.L. Hofman, A.M. Yacout, J.S. Park, J. Almer, J. Gan, B. Miller, J. Madden and S. Bhattacharya (2016). "High-Energy Synchrotron Study of In-Pile-Irradiated U-Mo Fuels." 36th International Meeting on Reduced Enrichment for Research and Test Reactors (RERTR 2015), Seoul, South Korea, October 11-14, 2015.

Mo, K., D. Yun, Y. Miao, X. Liu, M. Pellin, J. Almer, C. Park, J.F. Stubbins, S. Zhu and A. Yacout (2016). “Investigation of High-Energy Ion-Irradiated MA957 Using Synchrotron Radiation under In situ Tension." Materials 9(15): 1-11.

(Also see 2013-116.)

Mohamed, W., D. Yun, K. Mo, M. Pellin, M. Billone, J. Almer and A.M. Yacout (2015). "Depth Profile of Oxide Volume Fractions of Zircaloy-2 in High-temperature Steam: An in situ Synchrotron Radiation Study." Journal of Nuclear Materials 454(1-3): 192-199. (Also see 2013-116.)

Pellin, M.J., A.M. Yacout, K. Mo, J. Almer, S. Bhattacharya, W. Mohamed, D. Seidman, B. Ye, D. Yun, R. Xu and S. Zhu. "MeV per Nucleon Ion Irradiation of Nuclear Materials with High Energy Synchrotron X-ray Characterization." Journal of Nuclear Materials. (To be published.) (Also see 2013-116.)

Yacout, A.M., M. Pellin and M.C. Billone (2013). "Development and Testing of Nanolaminate Coatings for Conventional LWR Cladding." Proceedings of the LWR Fuel Performance Meeting/Top Fuel 2013, Charlotte, NC, September 15-19, 2013: 847. (Also see 2013-116.)

Ye, B., S. Bhattacharya, K. Mo, D. Yun, W. Mohamed, M. Pellin, J. Fortner, Y.S. Kim, G.L. Hofman, A.M. Yacout, T. Wiencek, S. Van den Berghe and A. Leenaers (2015). "Irradiation Behavior Study of U-Mo/AL Dispersion Fuel with High Energy Xe." Journal of Nuclear Materials 464: 236-244.

Yun, D., Y. Miao, R. Xu, Z. Mei, K. Mo, W. Mohamed, B. Ye, M.J. Pellin and A.M. Yacout. "Characterization of High Energy Xe Ion Irradiation Effects in Single Crystal Molybdenum with Depth-resolved Synchrotron Microbeam Diffraction." Journal of Nuclear Materials. (To be published.)

Yun, D., K. Mo, W. Mohamed, B. Ye, M.A. Kirk, P. Baldo, R.Q. Xu and A.M. Yacout (2016). "In situ TEM and Synchrotron Characterization of U-10Mo Thin Specimen Annealed at the Fast Reactor Temperature Regime." Materials Characterization 110: 208-214.

Yun, D., W. Mohamed, B. Ye, M. Kirk, P. Baldo and A. Yacout (2013). "Preliminary Assessment of Microstructural Evolution of U-10Mo Thin Specimen Annealed at Typical Fast Reactor Temperature Regime." Proceedings of the American Nuclear Society (ANS) Annual Meeting, Atlanta, GA, June 12-16, 2013: 373. 


\section{NON-REFEREED PUBLICATIONS}

Mei, Z.G., M. Stan and A.M. Yacout (2014). “Thermophysical Properties of Uranium Dioxide by First-Principles.” 2014 American Nuclear Society (ANS) Annual Meeting and Nuclear Fuels \& Structural Materials for Next Generation Nuclear Reactors (NFSM), Reno, NV, June 15-19, 2014.

Mo, K., Y. Miao, Z. Zhou, D. Yun, X. Liu, J. Almer and J.F. Stubbins (2014). "Nanoparticles Loading Behavior Before and After Matrix Necking: An in situ Synchrotron Radiation Study in a 9Cr ODS Alloy." 2014 American Nuclear Society (ANS) Annual Meeting and Nuclear Fuels \& Structural Materials for Next Generation Nuclear Reactors (NFSM), Reno, NV, June 15-19, 2014.

Mo, K., D. Yun, W. Mohamed, M. Pellin, J. Almer and A.M. Yacout (2014). "Synchrotron Radiation Study on Steam Oxidation Behavior of Zircaloy-2 with Advanced Coatings." 2014 American Nuclear Society (ANS) Annual Meeting and Nuclear Fuels \& Structural Materials for Next Generation Nuclear Reactors (NFSM), Reno, NV, June 15-19, 2014.

Yun, D., K. Mo, R. Xu, W. Mohamed, B. Ye, M.J. Pellin and A.M. Yacout (2014). "Characterization of High Energy Xe Ion Damage in U-10Mo Metallic Alloy Fuel with Depth Resolved Synchrotron Microbeam Diffraction." 2014 American Nuclear Society (ANS) Annual Meeting and Nuclear Fuels \& Structural Materials for Next Generation Nuclear Reactors (NFSM), Reno, NV, June 15-19, 2014.

Yun, D., K. Mo, R. Xu, W. Mohamed, B. Ye, M.J. Pellin and A.M. Yacout (2014). "Characterization of High Energy Xe Ion Irradiation Effects in Single Crystal Molybdenum with Depth Resolved Synchrotron Microbeam Diffraction." 2014 American Nuclear Society (ANS) Annual Meeting and Nuclear Fuels \& Structural Materials for Next Generation Nuclear Reactors (NFSM), Reno, NV, June 15-19, 2014.

\section{PRESENTATIONS}

Bhattacharya, S., B. Ye, K. Mo, W. Mohamed, L. Jamison, G. Hofman, Y.S. Kim, M. Pellin, A. Yacout, S. Van den Berghe, A. Leenaers and D.K. Seidman (2016). "A Comparative Post lon-irradiation Study of ALD and PVD Coated ZrN U-7wt\%Mo Dispersion Fuel Microplates." 36th International Meeting on Reduced Enrichment for Research and Test Reactors (RERTR 2015), Seoul, South Korea, October 11-14, 2015.

Mei, Z.-G., L. Liang, Y.S. Kim, T. Wiencek, G. Hofman, M. Anitescu and A.M. Yacout (2016). "Grain Growth and Bubble Evolution in U-Mo Alloy by Multiscale Simulations." 36th International Meeting on Reduced Enrichment for Research and Test Reactors (RERTR 2015), Seoul, South Korea, October 11-14, 2015.

Mei, Z.G., A. Yacout, Y.S. Kim, G. Hofman and M. Stan (2015). "A First-principles Study of ZrN as Diffusion Barrier." The Nuclear Materials Conference (NuMat 2014), Clearwater Beach, FL, October 27-30, 2014.

Mei, Z.G., M. Stan, A. Yacout and J. Yang (2015). “Thermal Transport in Uranium Dioxide by Atomic Simulations." The Nuclear Materials Conference (NuMat 2014), Clearwater, FL, October 27-30, 2014.

Mei, Z.G., M. Stan, A.M. Yacout and J. Yang (2014). "Atomistic Study of the Effects of Point Defects and Xe Atoms on the Thermal Conductivity of $\mathrm{UO}_{2}$." 143rd Annual TMS 2014 Meeting, San Diego, CA, February 16-20, 2014.

Mei, Z.G. and M. Stan (2014). "First-principles Study of Phase Stability and Thermodynamics of Uranium Nitride." Materials Science \& Technology 2013 Conference (MS\&T 2013), Montreal, Quebec, Canada, October 27-31, 2013.

Miao, Y., K. Mo, L. Jamison, B. Ye, J. Gan, B. Miller, J.S. Park, J. Almer, J. Madden, S. Bhattacharya, Z. Mei, Y.S. Kim, G.L. Hofman and A.M. Yacout (2016). "High-Energy Synchrotron Study of In-Pile Irradiated U-Mo Fuels." 36th International Meeting on Reduced Enrichment for Research and Test Reactors (RERTR 2015), Seoul, South Korea, October 11-14, 2015.

Stan, M., D. Yun, Z. Mei, A. Yacout, B. Mihaila and S. Hu (2013). "Microstructural Heterogeneity and Thermal Transport." Pacific Rim International Conference on Advanced Materials Processing (PRICM-8), Waikoloa, HI, August 4-9, 2013.

Yacout, A., M. Mendelsohn, D. Yun and W. Mohamed (2014). "Multilayer ALD Coating of Light Water Reactor Zirconium Alloy Cladding Materials.” Multilayers'13, Madrid, Spain, October 1-4, 2013. (Also see 2013-116.) 
Ye, B., S. Bhattacharya, K. Mo, D. Yun, M. Pellin, J. Fortner, Y.S. Kim, G.L. Hofman, A.M. Yacout, T. Wiencek, S. Van den Berghe and A. Leenaers (2015). "Irradiation Behavior Study of U-Mo/Al Dispersion Fuel with High Energy Xe." The Nuclear Materials Conference (NuMat 2014), Clearwater Beach, FL, October 27-30, 2014. (Also see 2013-116.)

Ye, B., Y.S. Kim, G. Hofman, A. Yacout, S. Bhattcharya, K. Mo, D. Yun, W. Mohamed, M. Pellin and J. Fortner (2015). "Xe Irradiation on ZrN-Coated U-Mo/AI Dispersion Fuel." International Meeting on Reduced Enrichment for Research and Test Reactors (RERTR 2014), Vienna, Austria, October 12-15, 2014.

Ye, B., S. Bhattacharya, K. Mo, D. Yun, W. Mohamed, M. Pellin, J. Fortner, Y.S. Kim, G. Hofman and A. Yacout (2014). "80 MeV Xe Irradiation on U-Mo/Al Dispersion Fuel." 19th International Conference on Ion Beam Modification of Materials, Leuven, Belgium, September 14-19, 2014.

Yun, D., K. Mo, W. Mohamed, M.J. Pellin and A.M. Yacout (2015). "Modeling Beam Heating and Sample Temperature by High Energy Heavy Ion Irradiation, and Comparison with Experimental Measurements." The Nuclear Materials Conference (NuMat 2014), Clearwater Beach, FL, October 27-30, 2014. (Also see 2013-116.)

Yun, D., J. Wright, J. Terry, K. Mo, R. Xu, W. Mohamed, B. Yei, K. Logan, M. Pellin and A. Yacout (2015). "Study of Xe lon Beam Irradiated Mo Single Crystal by Synchrotron Extended X-ray Absorption Fine Structure and Microdiffraction." The Nuclear Materials Conference (NuMat 2014), Clearwater Beach, FL, October 27-30, 2014. (Also see 2013-116.)

\section{4-177-R1}

\section{REFEREED PUBLICATIONS}

Tentner, A.M., P. Vegendla, A. Obabko, A. Tomboulides, P. Fischer, O. Marin and E. Merzari (2015). "Modeling of Two-Phase Flow in a BWR Fuel Assembly Using a Highly-Scalable Code." Proceedings of the 16th International Topical Meeting on Nuclear Reactor Thermal Hydraulics (NURETH-16) Chicago, IL, August 30-September 4, 2015.

Tentner, A., E. Merzari and P. Vagendla (2014). "Computational Fluid Dynamics Modeling of Two-Phase Boiling Flow and Critical Heat Flux." Proceedings of the 22nd International Conference on Nuclear Engineering ICONE22, Prague, Czech Republic, July 7-11, 2014, ASME Proceedings 4(ICONE22-30844): V004T010A037.

\section{5-129-NO}

\section{PRESENTATION}

Botterud, A., F. Ganda and F.J. De Sisternes (2015). "Economic and Technical Aspects of Nuclear Energy in Electricity Markets with Renewables." U.S.-Japan Workshop on Compatibility of Nuclear and Renewables with Grid Stability, Economics and Deregulation, Massachusetts Institute of Technology, Cambridge, MA, June 18, 2015. (Also see 2015-124.)

\section{5-136-NO}

\section{REFEREED PUBLICATIONS}

Benmore, C.J., L.B. Skinner, B. Lee, J.R. Weber, J.B. Parise and M.A. Williamson. "Topological Ordering in Liquid $\cup_{2}$." Journal of Physics: Condensed Matter. (To be published.) (Also see 2015-096.)

Skinner, L.B., C.J. Benmore, J.R. Weber, M.A. Williamson, A.J. Tamalonis, A.S. Hebden, T. Wiencek, O.L.G. Alderman, M. Guthrie, L. Leibowitz and J.B. Parise (2015). "Molten Uranium Dioxide Structure and Dynamics." Science 346(6212): 984-987. (Also see 2015-096.)

\section{5-145-NO}

\section{REFEREED PUBLICATION}

Chen, Y., B. Alexandreanu, W.Y. Chen, K. Natesan, Z. Li, Y. Yang and A.S. Rao (2016). "Cracking Behavior of Thermally Aged and Irradiated CF-8 Cast Austenitic Stainless Steel.” Journal of Nuclear Materials 466: 560-568. 


\section{NON-REFEREED PUBLICATION}

Chen, Y., B. Alexandreanu, W. Chen, Z. Li, Y. Yang, K. Natesan and A. Rao (2015). "Crack Growth Rate and Fracture Toughness J-R Curve Tests on Irradiated Cast Austenitic Stainless Steels." 17th International Conference on Environmental Degradation of Materials in Nuclear Power Systems-Water Reactors, Ottawa, Ontario, Canada, August 9-13, 2015.

\section{OTHER NOVEL R\&D}

\section{4-185-R1}

\section{REFEREED PUBLICATIONS}

Karan, N.K., M.D. Slater, F. Dogan, D. Kim, C.S. Johnson and M. Balasubramanian (2014). "Operando Structural Characterization of the Lithium-Substituted Layered Sodium-Ion Cathode Material P2- $\mathrm{Na}_{0.85} \mathrm{Li}_{0.17} \mathrm{Ni}_{0.21} \mathrm{Mn}_{0.64} \mathrm{O}_{2}$ by $\mathrm{X}-\mathrm{ray}$ Absorption Spectroscopy." Journal of the Electrochemical Society 161(6): A1107-1115.

Lee, E., S. Sahgong, C.S. Johnson and Y. Kim (2015). “Comparative Electrochemical Sodium Insertion/Extraction Behavior in Layered $\mathrm{Na}_{x} \mathrm{VS}_{2}$ and $\mathrm{Na}_{x} \mathrm{TiS}_{2}$." Electrochimica Acta 143: 272-277.

Lee, E., D.E. Brown, E.E. Alp, Y. Ren, J. Lu, J.-J. Woo and C.S. Johnson (2015). "New Insights into the Performance Degradation of Fe-based Layered Oxides in Sodium-Ion Batteries: Instability of $\mathrm{Fe}^{3+} / \mathrm{Fe}^{4+} \mathrm{Redox}$ in a- $\mathrm{NaFeO}_{2}$." Chemistry of Materials 27(19): 6755-6764.

Lee, E., J. Lu, Y. Ren, X.Y. Luo, X.Y. Zhang, J.G. Wen, D. Miller, A. DeWahl, S. Hackney, B. Key, D. Kim, M.D. Slater and C.S. Johnson (2014). “Layered P2/O3 Intergrowth Cathode: Toward High Power Na-Ion Batteries." Advanced Energy Materials 4(17).

Zhou, D., M. Slater, D. Kim, E. Lee, J. Jorne and C.S. Johnson (2014). “SnSb Carbon Composite Anode in a SnSb_C/ $\mathrm{NaNi}_{1} / \mathrm{Mn}_{1} /{ }_{3} \mathrm{Fe}_{1} /_{3} \mathrm{O}_{2} \mathrm{Na}$-Ion Battery." ECS Transactions: Battery Chemistries Beyond Lithium Ion 58(12): 59-64.

\section{PRESENTATIONS}

Johnson, C.S., E. Lee, D. Zhou and M. Slater (2015). “Na-ion Battery Technologies: Present Status and Update.” 226th Electrochemical Society (ECS) Meeting, Cancun, Mexico, October 4-10, 2014.

Johnson, C.S. (2014). “Emergence of Na-Ion Battery Technologies.” 248th American Chemical Society Meeting, San Francisco, CA, August 10, 2014.

Johnson, C.S. (2014). "Na-ion Batteries: A New Energy Storage Focus." International Society of Electrochemistry Society (ISE) Meeting, Lausanne, Switzerland, August 30, 2014.

Lee, E., D. Zhou, M. Slater and C.S. Johnson (2015). “Energy Storage using Sodium-ion Batteries (SIB)." Beyond Li-ion Batteries Conference III, Oak Ridge, TN, June 2-4, 2015.

Lee, E. (2014). “Layered P2/O3 Intergrowth Cathode: Toward High Capacity and High Power Na-Ion Batteries.” 17thvInternational Meeting on Lithium Batteries (IMLB) Meeting, Como, Italy, June 10-14, 2014.

Senguttuvan, P., A. Gutierrez, S.-D. Han, S. Tepacevic, C.K. Lin, S. Lapidus, Y. Ren, A.K. Burrell and C.S. Johnson (2015). "NaCoPO ${ }_{4}$ Cathodes for Sodium Batteries and Nanostructured Bi-layered $\mathrm{V}_{2} \mathrm{O}_{5}$ for Multivalent Battery Applications." Beyond Li-ion Batteries Conference VIII, Oak Ridge, TN, June 2-4, 2015.

\section{5-174-NO}

\section{PRESENTATIONS}

Marshall, C.L. (2016). "Enhancing the Stability/Performance of Catalysts via Atomic Layer Deposition." University of Kansas, Lawrence, KS, November 13, 2015.

Marshall, C.L. (2015). “Atomic Layer Deposition Overcoating: Tuning Catalyst Selectivity for Biomass Conversion.” North American Catalysis, Pittsburgh, PA, June 14-19, 2015. 


\section{PUBLICATIONS AND PRESENTATIONS II}

\section{(Arising from LDRD projects completed prior to FY 2015 but produced and/or delivered during FY 2015)}

\section{0-043 \\ REFEREED PUBLICATION}

Drake, G., M. Garcia-Scivres, A. Paramonov, R. Stanek, and D. Underwood (2015). "Fiber-optic Links Based on Silicon Photonics for High-speed Readout of Trackers." Journal of Instrumentation 9.

\section{0-117}

\section{REFEREED PUBLICATION}

Mukhopadhyay, D., D.A. Walko, I.W. Jung, C.P. Schwartz, J. Wang, D. Lopez, and G.K. Shenoy (2015). "X-ray Photonic Microsystems for Manipulation of Synchrotron Light." Nature Communications 6: 7057.

\section{0-119}

\section{PRESENTATIONS}

Matamala, R., J. Jastrow, Z. Fan, U. Mishra, C. Liang, F. Calderon, G.J. Michaelson, and C.-L. Ping (2015). “ANL Terrestrial Ecosystem Science SFA: Characterizing Organic Matter Quality and Lability of Alaskan Soils Using Mid Infrared Spectroscopy." Environmental System Science PI Meeting, Potomac, MD, April 28-29, 2015.

Matamala, R., J. Jastrow, F. Calderon, C. Liang, R. Miller, C.-L. Ping, G. Michaelson, and S. Hofmann (2015). "Characterizing Soil Organic Matter Degradation Levels in Permafrost-affected Soils Using Infrared Spectroscopy." Fall Meeting of the American Geophysical Union, San Francisco, CA, December 15-19, 2015.

\section{0-158}

\section{REFEREED PUBLICATION}

Shui, J., C. Chen, L.R. Grabstanowicz, D. Zhao, and D.-J. Liu (2015). "Highly Efficient Non-precious Metal Catalyst Prepared with Metal-Organic Framework in a Continuous Carbon Nanofibrous Network." Proceedings of the National Academy of Sciences of the United States of America, August 25, 2015, 112(10629-106-34).

\section{PRESENTATIONS}

Liu, D.-J. (2015). "New Material Chemistries and Structural Investigations for Next-Generation Energy Storage and Conversion Applications." University of Illinois at Chicago, Chicago, IL, February 26, 2015.

Liu, D.-J. (2015). "Recent Developments in New Materials \& Characterization Techniques for Energy Storage and Conversion." University of Nebraska, Lincoln, NE, October 20, 2014.

\section{0-183}

\section{REFEREED PUBLICATIONS}

Chen, S., T. Paunesku, Y. Yuan, Q. Jin, L. Finney, B. Hornberger, C. Flachenecker, B. Lai, K. Brister, C. Jacobsen, G. Woloschak, and S. Vogt (2015). "The Bionanoprobe: Synchrotron-based Hard X-ray Fluorescence Microscopy for 2D/3D Trace Elements Mapping." Microscopy Today 23(03): 26-29. 
Chen, S., J. Deng, D. Vine, Y. Nashed, Q. Jin, T. Peterka, C. Jacobsen, and S. Vogt (2015). "Simultaneous X-ray Nano-Ptychography and Fluorescence Microscopy at the Bionanoprobe." Proceedings of the SPIE 9592, X-Ray Nanoimaging: Instruments and Methods II, San Diego, CA, September 18, 2015, 9592.

Deng, J., D.J. Vine, S. Chen, Y.S.G. Nashed, T. Peterka, R. Ross, S. Vogt, and C. Jacobsen (2015). "Opportunities and Limitations for Combined Fly-scan Ptychography and Fluorescence Microscopy." Proceedings of the SPIE 9592, X-Ray Nanoimaging: Instruments and Methods II, San Diego, CA, September 18, 2015, 95920U. (Also see 2010-193.)

Deng, J., D.J. Vine, S. Chen, Y.S.G. Nashed, Q. Jin, N.W. Phillips, T. Peterka, R. Ross, S. Vogt, and C.J. Jacobsen (2015). "Simultaneous Cryo X-ray Ptychographic and Fluorescence Microscopy of Green Algae." Proceedings of the National Academy of Sciences of the United States of America 112(8): 2314-2319.

(Also see 2010-193.)

Hong, Y.P., S. Chen, and C. Jacobsen (2015). "A New Workflow for X-ray Fluorescence Tomography: MAPStoTomoPy." Proceedings of the SPIE 9592, X-Ray Nanoimaging: Instruments and Methods II, San Diego, CA, September 18, 2015, 95920W. (Also see 2010-193.)

Jin, Q., S. Vogt, B. Lai, S. Chen, L. Finney, S.-C. Gleber, J. Ward, J. Deng, R. Mak, N. Moonier, and C. Jacobsen (2015). "Ultraviolet Germicidal Irradiation and Its Effects on Elemental Distributions in Mouse Embryonic Fibroblast Cells in X-ray Fluorescence Microanalysis.” PLoS One 10(2): e0117437. (Also see 2010-193.)

Punshon, T., S. Chen, L. Finney, L. Howard, B.P. Jackson, M.R. Karagas, and K. Ornvold (2015). "High-resolution Elemental Mapping of Human Placental Chorionic Villi Using Synchrotron X-ray Fluorescence Spectroscopy." Analytical and Bioanalytical Chemistry 407(22): 6839-6850.

Que, E.L., R. Bleher, F.E. Duncan, B.Y. Kong, S.C. Gleber, S. Vogt, S. Chen, S.A. Garwin, A.R. Bayer, V.P. Dravid, T.K. Woodruff, and T.V. O'Halloran (2015). "Quantitative Mapping of Zinc Fluxes in the Mammalian Egg Reveals the Origin of Fertilization-induced Zinc Sparks." Nature Chemistry 7(2): 130-139.

\section{0-188}

\section{REFEREED PUBLICATION}

Tepavcevic, S., Y. Liu, D. Zhou, B. Lai, J. Maser, X. Zuo, H. Chan, P. Kral, C.S. Johnson, V. Stamenkovic, N.M. Markovic, and T. Rajh (2015). "Nanostructured Layered Cathode for Rechargeable Mg-lon Batteries." ACS Nano 9(8): 8194-8205.

\section{PRESENTATION}

Tepavcevic, S., Y. Liu, B. Lai, J. Maser, V. Stamenkovic, C. Johnson, N. Markovic, and T. Rajh (2015). "Nanostructured Layered Cathode For Rechargeable Mg-ion Batteries." Materials Research Society Spring Meeting (MRS 2015), San Francisco, CA, April 6-10, 2015.

\section{0-193}

\section{REFEREED PUBLICATIONS}

De Carlo, F., D. Gursoy, F. Marone, M. Rivers, D.Y. Parkinson, F. Khan, N. Schwarz, D.J. Vine, S. Vogt, S.-C. Gleber, S. Narayanan, M. Newville, T. Lanzirotti, Y. Sun, Y.P. Hong, and C. Jacobsen (2015). "Scientific Data Exchange: a Schema for HDF5-Based Storage of Raw and Analyzed Data." Journal of Synchrotron Radiation 21(6): $1224-1230$.

Deng, J., Y. Nashed, S. Chen, N. Phillips, T. Peterka, R. Ross, S. Vogt, C. Jacobsen, and D. Vine (2015). "Continuous Motion Scan Ptychography: Characterization for Increased Speed in Coherent X-ray Imaging." Optics Express 23(5): $5438-5451$. 
Deng, J., D. Vine, S. Chen, Y. Nashed, T. Peterka, R. Ross, S. Vogt, and C. Jacobsen (2015). "Opportunities and Limitations for Combined Fly-scan Ptychography and Fluorescence Microscopy." Proceedings of the SPIE 9592, X-ray Nanoimaging: Instruments and Methods II, San Diego, CA, September 18, 2015, 95920U. (Also see 2010-183.)

Deng, J., D.J. Vine, S. Chen, Y.S.G. Nashed, Q. Jin, N.W. Phillips, T. Peterka, R. Ross, S. Vogt, and C. Jacobsen (2015). "Simultaneous Cryo X-ray Ptychographic and Fluorescence Microscopy of Green Algae." Proceedings of the National Academy of Sciences of the United States of America (PNAS), February 24, 2015, 112(8): 2314-2319. (Also see 2010-183.)

Hong, Y.P., S. Chen, and C. Jacobsen (2015). "A New Workflow for X-ray Fluorescence Tomography: MAPStoTomoPy." Proceedings of the SPIE 9592, X-ray Nanoimaging: Instruments and Methods II, San Diego, CA, September 18, 2015, 95920W. (Also see 2010-183.)

Jin, Q., S. Vogt, B. Lai, S. Chen, L. Finney, S.-C. Gleber, J. Ward, J. Deng, R. Mak, N. Moonier, and C. Jacobsen (2015). "Ultraviolet Germicidal Irradiation and Its Effects on Elemental Distributions in Mouse Embryonic Fibroblast Cells in X-ray Fluorescence Microanalysis." PLoS One 10(2): e0117437. (Also see 2010-183.)

Mak, R., M. Lerotic, H. Fleckenstein, S. Vogt, S.M. Wild, S. Leyffer, Y. Sheynkin, and C. Jacobsen (2015). “Non-Negative Matrix Analysis for Effective Feature Extraction in X-Ray Spectromicroscopy." Royal Society of Chemistry Faraday Discussions 171: 357-371.

Nashed, Y.S.G., D.J. Vine, T. Peterka, J. Deng, R. Ross, and C. Jacobsen (2015). "Parallel Ptychographic Reconstruction." Optics Express 22(26): 32082-32097.

Sun, Y., S.-C. Gleber, C. Jacobsen, J. Kirz, and S. Vogt (2015). “Optimizing Detector Geometry for Trace Element Mapping by X-ray Fluorescence." Ultramicroscopy 152: 44-56.

\section{0-195}

\section{REFEREED PUBLICATIONS}

Gilbert, J.A., and N.M. Scott (2015). "Introduction to Genetic, Genomic, and System Analyses for Communities." Hydrocarbon and Lipid Microbiology Protocols: Cultivation. T.J. McGenity, K.N. Timmis, and B. Nogales Fernandez (eds.), Totowa, NJ, Springer Protocol Handbooks.

Hampton-Marcell, J.T., A. Frazier, S.M. Moormann, S.M. Owens, and J.A. Gilbert (2015). "Preparation and Analysis of Metatranscriptomic Libraries in Petroleum Hydrocarbon Microbe Systems." Hydrocarbon and Lipid Microbiology Protocols: Cultivation. T.J. McGenity, K.N. Timmis, and B. Nogales Fernandez, Totowa, NJ, Springer Protocol Handbooks.

\section{0-197}

\section{PRESENTATIONS}

Mei, Z.G., A. Yacout, Y.S. Kim, G. Hofman, and M. Stan (2015). "A First-principles Study of ZrN as Diffusion Barrier." Nuclear Materials Conference 2014, Clearwater, FL, October 27-30, 2014.

Mei, Z.G., M. Stan, A. Yacout, and J. Yang (2015). “Thermal Transport in Uranium Dioxide by Atomic Simulations." Nuclear Materials Conference 2014, Clearwater, FL, October 27-30, 2014.

\section{1-005}

\section{REFEREED PUBLICATION}

Shen, B., M. Leroux, Y.L. Wang, X. Luo, V.K. Vlasko-Vlasov, A.E. Koshelev, Z.L. Xiao, U. Welp, W.K. Kwok, M.P. Smylie, A. Snezhko, and V. Metlushko (2015). "Critical Fields and Vortex Pinning in Overdoped $\mathrm{Ba}_{0.2} \mathrm{~K}_{0.8} \mathrm{Fe}_{2} \mathrm{As}_{2}$." Physical Review B 91(17): 45121-45128. 


\section{1-012}

\section{REFEREED PUBLICATIONS}

Benmore, C.J. (2015). "Advanced X-Ray Analytical Methods to Understand Structure, Properties, and Risk." Discovering and Developing Molecules with Optimal Drug-Like Properties. A.C. Templeton, S.R. Byrn, R.J. Haskell, and T.E. Prisinzano (eds.), Springer New York. 15: 263-283.

Mou, Q., C.J. Benmore, and J.L. Yarger (2015). "X-ray Intermolecular Structure Factor (XISF) - Separation of Intra- and Inter-molecular Interactions from Total X-ray Scattering Data." Journal of Applied Crystallography 48(3): 950-952.

\section{1-023}

\section{REFEREED PUBLICATIONS}

Fabbris, G., J. Lim, L.S.I. Veiga, D. Haskel, and J.S. Schilling (2015). "Electronic and Structural Ground State of Heavy Alkali Metals at High Pressure." Physical Review B: Condensed Matter 91(8).

Mardegan, J., G. Fabbris, L.S.I. Veiga, S. Francoual, J. Strempfer, D. Haskel, R.A. Ribeiro, M.A. Avila, and C. Giles (2015). "Magnetic Properties of $\mathrm{GdT}_{2} \mathrm{Zn}_{2} \mathrm{O}$ ( $\mathrm{T}=\mathrm{Fe}, \mathrm{Co}$ ) Investigated by X-ray Diffraction and Spectroscopy." Physical Review B: Condensed Matter 93: 024421.

Morrow, R., J.Q. Yan, M.A. McGuire, J.W. Freeland, D. Haskel, and P.M. Woodward (2015). "Effects of Chemical Pressure on the Magnetic Ground States of the Osmate Double Perovskites $\mathrm{SrCaCoOsO}_{6}$ and $\mathrm{Ca}_{2} \mathrm{CoO}_{5} \mathrm{O}_{6}$." Physical Review B: Condensed Matter 92(9): 094435.

\section{PRESENTATION}

Haskel, D. (2015). "X-ray Spectroscopy and High-pressure Tuning of the Spin-orbit Coupled Ground State of Iridate (5d) Oxides." 2014 Fall Condensed Matter Physics Seminar, Notre Dame University, South Bend, IN, October 9, 2014.

\section{1-027}

\section{REFEREED PUBLICATION}

Weinstein, M., A. Heifetz, and R. Klann (2015). "Detection of Nuclear Sources in Search Survey Using Dynamic Quantum Clustering of Gamma-ray Spectral Data." The European Physical Journal Plus 129(11): 239.

\section{1-038}

\section{REFEREED PUBLICATION}

Jenkins, J., J. Dinan, P. Balaji, T. Peterka, N.F. Samatova, and R. Thakur (2014). "Processing MPI Derived Datatypes on Noncontiguous GPU-Resident Data." IEEE Transactions on Parallel and Distributed Systems 25(10): 2627-2637.

\section{1-052}

\section{REFEREED PUBLICATION}

Lu, Z., D.G. Streets, E. Winijkul, F. Yan, Y. Chen, T.C. Bond, Y. Feng, M.K. Dubey, S. Liu, J.P. Pinto, and G.R. Carmichael (2015). "Light Absorption Properties and Radiative Effects of Primary Organic Aerosol Emissions." Environmental Science and Technology 49(8): 4868-4877.

\section{1-063}

\section{REFEREED PUBLICATION}

Park, J.S., X. Zhang, H. Sharma, P. Kenesei, D. Hoelzer, M.M. Li, and J. Almer (2015). "High-energy Synchrotron X-ray Techniques for Studying Irradiated Materials." Journal of Materials Research 30(9): 1380-1391. 


\section{PRESENTATIONS}

Park, J.S., P. Kenesei, H. Sharma, A. Mashayekhi, J. Okasinski, J. Almer, E. Benda, F. Westferro, X. Zhang, M. Li, Y. Chen, R. Pokharel, D. Brown, and B. Clausen (2015). "High-energy X-ray Techniques for Studying Nuclear Energy Relevant Materials." 2015 APS Users Meeting, Lemont, IL, May 11-14, 2015.

Zhang, X., C. Xu, M. Li, J.S. Park, P. Kenesei, J. Almer, K. Mo, C. Tomchik, J. Stubbins, and J. Gan (2015). "Characterization of Neutron-irradiated HT-UPS Steel by High-energy X-ray Diffraction Microscopy." 144th TMS Annual Meeting and Exhibition, Orlando, FL, March 15-19, 2015.

\section{1-067}

\section{REFEREED PUBLICATION}

Adams, B.W., A.U. Mane, J.W. Elam, R. Obaid, M. Wetstein, and M. Chollet (2015). "Towards a Microchannel-based X-ray Detector with Two-dimensional Spatial and Time Resolution and High Dynamic Range." Journal of Synchrotron Radiation 22(5): 1202-1206.

\section{1-109}

\section{REFEREED PUBLICATION}

Cattlet, C., and R. Ghiani (2015). “Big Data for Social Good.” Big Data Journal Special Issue 3(1): 1-2. (Also see 2011-214.)

\section{PRESENTATIONS}

Catlett, C. (2015). "Big Data and New Institutions.” Bringing Social Science Back In: The Big Data Revolution and Urban Theory, Harvard University, Cambridge, MA, December 2014. (Also see 2011-214.)

Catlett, C. (2015). "Computation, Information, Embedded Systems, and the Future of Cities." The International Conference for High Performance Computing, Networking, Storage and Analysis (SC14), New Orleans, LA, November 16-21, 2014. (Also see 2011-214.)

Catlett, C. (2015). "Computation, Information, Embedded Systems, and the Future of Cities." Acting Locally, Understanding Globally: Scaling Up Community Collected Data in Developing Cities Workshop, Santa Fe, NM, November 19-21, 2014. (Also see 2011-214.)

Catlett, C. (2015). "Data and Cities." Workshop of the U.S.-U.K. Trans-Atlantic Platform Digital Scholarship Workshop, Washington, D.C., January 2015. (Also see 2011-214.)

Catlett, C. (2015). "Data and the City." Workshop on Instrumented Cities, Future Cities Catapult, London, UK, March 2015. (Also see 2011-214.)

Catlett, C. (2015). “Instrumenting The (Urban) Environment." Exploring Points of Connection Workshop, Chicago, IL, July 1, 2015. (Also see 2011-214.)

Catlett, C. (2015). "Instrumenting the City." Greater Chicago Area Systems Research (GCASR) Workshop, Chicago, IL, April 27, 2015. (Also see 2011-214.)

Catlett, C. (2015). “Instrumenting the City.” Northwestern University Science Café, Evanston, IL, February 2015. (Also see 2011-214.)

Catlett, C. (2015). "Instrumenting the City.” Action Canada, University of Chicago Harris School of Public Policy, Chicago, IL, May 2015. (Also see 2011-214.)

Catlett, C. (2015). “Instrumenting the City.” University of Chicago, Chicago, IL, May 2015. (Also see 2011-214.)

Catlett, C. (2015). “Open Data and Instrumenting Cities.” New York Scientific Data Summit, New York, NY, August 2, 2015. (Also see 2011-214.)

For information regarding projects listed here, see previous years' annual reports 
Catlett, C. (2015). "Open Data and Instrumenting Cities." Monash Undergraduate Research Projects Abroad (MURPA), University of Queensland, St. Lucia, Australia, August 2015. (Also see 2011-214.)

Catlett, C. (2015). "Open Data and Instrumenting Cities." Monash Undergraduate Research Projects Abroad (MURPA), Monash University, Clayton, Australia, August 2015. (Also see 2011-214.)

Catlett, C. (2015). “Urban Center for Computation and Data." National Science Foundation, Arlington, VA, June 2015. (Also see 2011-214.)

Catlett, C. (2015). “Urban Sciences: Sensing and Data.” University of Chicago, Chicago, IL, May 2015. (Also see 2011-214.)

Catlett, C. (2015). “Urban Systems Science and Engineering.” DOE Big Ideas Summit, Washington, D.C., April 2015.

(Also see 2011-214.)

Catlett, C. (2015). "Urban Systems Science and Engineering." DOE Big Ideas Workshop National Renewable Energy Laboratory (NREL), Golden, CO, February 2015. (Also see 2011-214.)

Catlett, C. (2015). "Urban Systems Science and Engineering." National Research Council Study, Chattanooga, TN, July 2015. (Also see 2011-214.)

\section{1-121}

\section{REFEREED PUBLICATION}

Goldberg, N., S. Leyffer, and I. Safro (2015). "Optimal Response to Epidemics and Cyber Attacks in Networks." Networks 66(2): 145-158.

\section{1-123}

\section{REFEREED PUBLICATION}

Kwon, S.G., G. Krylova, P.J. Phillips, R.F. Klie, S. Chattopadhyay, T. Shibata, E.E. Bunel, Y. Liu, V.B. Prakapenka, B. Lee, and E.V. Shevchenko (2015). "Heterogeneous Nucleation and Shape Transformation of Multicomponent Metallic Nanostructures.” Nature Materials 14(2): 215-223.

\section{1-134}

\section{REFEREED PUBLICATION}

Kalaji, A., and L. Soderholm (2015). "Aqueous Hafnium Sulfate Chemistry: Structures of Crystalline Precipates." Inorganic Chemistry 53(20): 11252-11260.

\section{1-147}

\section{PRESENTATIONS}

Novosad, V. (2015). “Geometrically Confined Spin Vortices.” University of South Florida, Tampa, FL, March $27,2015$.

Novosad, V. (2015). “Top-Down Synthesis and Applications of Magneto-Responsive Nanomaterials.” 2015 Materials Research Society Sprint Meeting and Exhibit, San Francisco, CA, April 6-10, 2015.

\section{1-149}

\section{PRESENTATION}

Vogt, S. (2015). “Next Generation Data Exploration for X-ray Microscopy: Intelligence in Data Analysis." The Big Data in X-Ray Microscopy Workshop, Melbourne, Australia, October 27, 2014. 


\section{1-153}

\section{REFEREED PUBLICATION}

De Lurgio, P., Z. Djurcic, G. Drake, R. Hashemian, A. Kreps, M. Oberling, T. Pearson, and H. Sahoo (2015). "A Prototype of Wireless Power and Data Acquisition System for Large Detectors." Nuclear Instruments and Methods in Physics Research Section A: Accelerators, Spectrometers, Detectors and Associated Equipment 785(11): 99-104.

\section{1-170}

\section{REFEREED PUBLICATION}

Pelliccia, D., R. Vaz, I. Svalbe, K.S. Morgan, S. Marathe, X. Xiao, L. Assoufid, R.A. Anderson, J. Topczewski, and R.J. Bryson-Richardson (2015). "Comparison of Different Numerical Treatments for X-ray Phase Tomography of Soft Tissue from Differential Phase Projections.” Physics in Medicine and Biology 60(8): 3065-3080.

\section{PRESENTATION}

Assoufid, L., S. Marathe, E. Benda, X. Shi, A. T. Macrander, S. Stoupin, M.J. Wojcik, K. Lang, and K. Goetze (2015). "Development and Implementation of a Portable Grating Interferometer System as a Standard Tool for Testing Optics at the APS Beamline 1-BM.” International Workshop on X-ray Mirror Design, Fabrication and Metrology (IWXM 2015), Berkeley, CA, July 14-16, 2015.

\section{1-196}

\section{PRESENTATION}

Owens, S.M., W.L. Trimble, S.L. O’Brien, S.M. Greenwald, D.A. Antonopoulos, and F. Meyer (2015). "Exploring Clonal Populations of Verrucomicrobia from Grassland Soil Metagenomes Using Moleculo." Multi-omics for Microbiome Conference, Kennewick, WA, September 14-16, 2015.

\section{1-198}

\section{PRESENTATIONS}

Jellinek, J. (2015). "Analysis of Structural and Dynamical Complexities in Finite Homogeneous and Heterogeneous Systems." R.S. Berry Tribute Symposium, Telluride, CO, June 22-27, 2015.

Jellinek, J. (2015). "Analysis of Structural and Dynamical Complexities in Finite Homogeneous and Heterogeneous Systems.” The Fritz Haber Institute, Berlin, Germany, July 16, 2015.

Jellinek, J. (2015). "Analysis of Structural and Dynamical Complexities in Finite Homogeneous and Heterogeneous Systems." 7th International Symposium on Atomic Cluster Collisions (ISACC 2015), Madrid, Spain, July 17-21, 2015.

Jellinek, J. (2015). "Analysis of Structural and Dynamical Complexities in Homogeneous and Heterogeneous Finite Systems." Karlsruhe Institute of Technology, Karlsruhe, Germany, July 28, 2015.

Jellinek, J. (2015). "Computational Analysis and Characterization of Structural and Dynamical Complexities in Finite Heterogeneous Systems.” 11th International Conference on Computational Methods in Science and Engineering (ICCMSE 2015), Athens, Greece, March 19-24, 2015.

Jellinek, J. (2015). “Dynamics-based Solution to the Problem of Anharmonic Densities of States." Second International Conference on Anharmonicity in Medium-sized Molecules and Cluster (AMOC 2015), Madrid, Spain, April 26-30, 2015.

Jellinek, J. (2015). "Solving the Problem of Anharmonic Densities of States." The Fritz Haber Institute, Berlin, Germany, July 2, 2015. 


\section{1-199}

\section{REFEREED PUBLICATIONS}

Frazer, L., K.B. Chang, K.R. Poeppelmeier, and J.B. Ketterson (2015). "Cupric Oxide Inclusions in Cuprous Oxide Crystals Grown by the Floating Zone Method." Science and Technology of Advanced Materials 16(3).

Frazer, L., E.J. Lenferink, K.B. Chang, K.R. Poeppelmeier, N.P. Stern, and J.B. Ketterson (2015). "Evaluation of Defects in Cuprous Oxide Through Exciton Luminescence Imaging." Journal of Luminescence 159: 294-302.

\section{1-204}

\section{PRESENTATION}

Grimmer, J. (2015). “A Revolver Undulator for Sector 35 (DCS) - Two Undulators in One!” APS User Operations Meeting, Argonne, IL, October 29, 2014.

\section{1-207}

\section{REFEREED PUBLICATION}

Wang, J.B., K. Byrum, M. Demarteau, J. Elam, A. Mane, E. May, R. Wagner, D. Walters, L. Xia, J.Q. Xie, and H.Y. Zhao (2015). "Development and Testing of Cost-effective, $6 \mathrm{~cm} \times 6 \mathrm{~cm}$ MCP-based Photodetectors for Fast Timing Applications." Nuclear Instruments \& Methods in Physics Research Section a-Accelerators Spectrometers Detectors and Associated Equipment 804: 84-93.

\section{1-214}

\section{REFEREED PUBLICATION}

Cattlet, C., and R. Ghiani (2015). "Big Data for Social Good." Big Data Journal Special Issue 3(1): 1-2. (Also see 2011-109.)

\section{PRESENTATIONS}

Catlett, C. (2015). "Big Data and New Institutions." Bringing Social Science Back In: The Big Data Revolution and Urban Theory, Harvard University, Cambridge, MA, December 2014. (Also see 2011-109.)

Catlett, C. (2015). "Computation, Information, Embedded Systems, and the Future of Cities." The International Conference for High Performance Computing, Networking, Storage and Analysis (SC14),

New Orleans, LA, November 16-21, 2014. (Also see 2011-109.)

Catlett, C. (2015). "Computation, Information, Embedded Systems, and the Future of Cities." Acting Locally, Understanding Globally: Scaling Up Community Collected Data in Developing Cities Workshop, Santa Fe, NM, November 19-21, 2014. (Also see 2011-109.)

Catlett, C. (2015). "Data and Cities." Workshop of the U.S.-U.K. Trans-Atlantic Platform Digital Scholarship Workshop, Washington, D.C., January 2015. (Also see 2011-109.)

Catlett, C. (2015). "Data and the City." Workshop on Instrumented Cities, Future Cities Catapult, London, UK, March 2015. (Also see 2011-109.)

Catlett, C. (2015). "Instrumenting The (Urban) Environment." Exploring Points of Connection Workshop, Chicago, IL, July 1, 2015. (Also see 2011-109.)

Catlett, C. (2015). "Instrumenting the City." Greater Chicago Area Systems Research (GCASR) Workshop, Chicago, IL, April 27, 2015. (Also see 2011-109.)

Catlett, C. (2015). “Instrumenting the City.” Northwestern University Science Café, Evanston, IL, February 2015. (Also see 2011-109.)

Catlett, C. (2015). "Instrumenting the City." Action Canada, University of Chicago Harris School of Public Policy, Chicago, IL, May 2015. (Also see 2011-109.)

For information regarding projects listed here, see previous years' annual reports 
Catlett, C. (2015). “Instrumenting the City." University of Chicago, Chicago, IL, May 2015. (Also see 2011-109.)

Catlett, C. (2015). “Open Data and Instrumenting Cities." New York Scientific Data Summit, New York, NY, August 2, 2015. (Also see 2011-109.)

Catlett, C. (2015). "Open Data and Instrumenting Cities." Monash Undergraduate Research Projects Abroad (MURPA), University of Queensland, St. Lucia, Australia, August 2015. (Also see 2011-109.)

Catlett, C. (2015). "Open Data and Instrumenting Cities." Monash Undergraduate Research Projects Abroad (MURPA), Monash University, Clayton, Australia, August 2015. (Also see 2011-109.)

Catlett, C. (2015). “Urban Center for Computation and Data." National Science Foundation, Arlington, VA, June 2015. (Also see 2011-109.)

Catlett, C. (2015). “Urban Sciences: Sensing and Data." University of Chicago, Chicago, IL, May 2015.

(Also see 2011-109.)

Catlett, C. (2015). “Urban Systems Science and Engineering.” DOE Big Ideas Summit, Washington, D.C., April 2015.

(Also see 2011-109.)

Catlett, C. (2015). “Urban Systems Science and Engineering." DOE Big Ideas Workshop National Renewable Energy Laboratory (NREL), Golden, CO, February 2015. (Also see 2011-109.)

Catlett, C. (2015). "Urban Systems Science and Engineering." National Research Council Study, Chattanooga, TN, July 2015. (Also see 2011-109.)

\section{1-215}

\section{REFEREED PUBLICATION}

Collins, J.T., J. Nudell, G. Navrotski, Z. Liu, and P. Den Hartog (2015). "Results from Studies of ThermomechanicallyInduced Fatigue in GlidCop ${ }^{\circledR}$." Proceedings of the Mechanical Engineering Design of Synchrotron Radiation Equipment and Instrumentation Conference (MEDSI2014), Melbourne, Australia, October 20-24, 2014: 7.

\section{PRESENTATION}

Collins, J., J. Nudell, G. Navrotski, Z. Liu, and P. Den Hartog (2015). "Results from Studies of ThermomechanicallyInduced Fatigue in GlipCop ${ }^{\circledast}$." Mechanical Engineering Design of Synchrotron Radiation Equipment and Instrumentation Conference (MEDSI2014), Melbourne, Australia, October 20-24, 2014.

\section{1-216}

\section{REFEREED PUBLICATION}

Feng, Y., V.R. Kotamarthi, R. Coulter, C. Zhao, and M.P. Cadeddu (2015). "Radiative and Thermodynamic Responses to Aerosol Extinction Profiles During the Pre-monsoon Month over South Asia." Atmospheric Chemistry and Physics Discussions 15(15): 16901-16943.

\section{2-015}

\section{REFEREED PUBLICATION}

Welland, M.J., D. Karpeyev, D.T. O'Connor, and O. Heinonen (2015). "Miscibility Gap Closure, Interface Morphology, and Phase Microstructure of 3D $\mathrm{Li}_{x} \mathrm{FePO}_{4}$ Nanoparticles from Surface Wetting and Coherency Strain." ACS Nano 9(10): 9757-9771.

\section{PRESENTATION}

Welland, M.J., D. O'Connor, O. Heinonen, P. Voorhees, and D. Wolf (2015). "Simulation of $\mathrm{LiFePO}_{4} \mathrm{Nanoparticle}$ Microstructure with a Coupled Phase-field, Elastomechanics and Surface Tension Model." 144th TMS Annual Meeting and Exhibition, Orlando, FL, March 15-19, 2015.

For information regarding projects listed here, see previous years' annual reports 


\section{2-052}

\section{REFEREED PUBLICATION}

Liu, G. (2015). "Advances in Theoretical Understanding of Photon Upconversion in Rare-earth Activated Nanophosphors." Chemical Society Reviews 44(6): 1635-1652.

\section{2-072}

\section{REFEREED PUBLICATIONS}

Berman, D., S.A. Deshmukh, S.K.R.S. Sankaranarayanan, A. Erdemir, and A.V. Sumant (2015). "Extraordinary Macroscale Wear Resistance of One Atom Thick Graphene Layer." Advanced Functional Materials 24(42): 6640-6646.

Berman, D., A. Erdemir, and A.V. Sumant (2015). "Graphene as a Protective Coating and Superior Lubricant for Electrical Contacts." Applied Physics Letters 105(23): 231907.

Berman, D., S.A. Deshmukh, S.K.R.S. Sankaranarayanan, A. Erdemir, and A.V. Sumant (2015). "Macroscale Superlubricity Enabled by Graphene Nanoscroll Formation." Science 348(6239): 1118-1122.

Berman, D., A. Erdemir, A.V. Zinovev, and A.V. Sumant (2015). “Nanoscale Friction Properties of Graphene and Graphene Oxide." Diamond and Related Materials 54: 91-96.

\section{2-074}

\section{REFEREED PUBLICATION}

Fan, Z., and C. Liang (2015). "Significance of Microbial Asynchronous Anabolism to Soil Carbon Dynamics Driven by Litter Inputs.” Scientific Reports 5(9575).

\section{PRESENTATIONS}

Matamala, R., J. Jastrow, Z. Fan, U. Mishra, C. Liang, F. Calderon, G.J. Michaelson, and C.-L. Ping (2015). “ANL Terrestrial Ecosystem Science SFA: Characterizing Organic Matter Quality and Lability of Alaskan Soils Using Mid Infrared Spectroscopy." Environmental System Science PI Meeting, Potomac, MD, April 28-29, 2015.

Matamala, R., J. Jastrow, F. Calderon, C. Liang, R. Miller, C.-L. Ping, G. Michaelson, and S. Hofmann (2015). "Characterizing Soil Organic Matter Degradation Levels in Permafrost-affected Soils Using Infrared Spectroscopy." Fall Meeting of the American Geophysical Union, San Francisco, CA, December 15-19, 2015.

\section{2-082}

\section{NON-REFEREED PUBLICATION}

Zounmevo, J.A., S. Perarnau, K. Iskra, K. Yoshii, R. Gioiosa, B.C. Van Essen, M.B. Gokhale, and E.A. Leon (2015). "A Container-Based Approach to OS Specialization for Exascale Computing." 2015 IEEE International Conference on Cloud Engineering (IC2E), Tempe, AZ, March 9-13, 2015.

\section{PRESENTATION}

Zounmevo, J.A., K. Iskra, K. Yoshii, R. Gioiosa, B.C. Van Essen, M.B. Gokhale, and E.A. Leon (2015).

"A Single-Kernel Approach to OS Specialization and Node Resource Partitioning for Exascale Computing." 11th Usenix Symposium on Operating System Design and Implementation, Broomfield, CO, October 6-10, 2014.

\section{2-087}

\section{REFEREED PUBLICATION}

Pelliccione, C.J., E.V. Timofeeva, J.P. Katsoudas, and C.U. Segre (2015). "Note: Sample Chamber for in situ X-ray Absorption Spectroscopy Studies of Battery Materials." Review of Scientific Instruments 85(12): 126108. 


\section{2-114}

\section{REFEREED PUBLICATION}

Otten, M., R.A. Shah, N.F. Scherer, M. Min, M. Pelton, and S.K. Gray (2015). “Entanglement of Two, Three and Four Plasmonically Coupled Quantum Dots." Physical Review B 92(12).

\section{PRESENTATIONS}

Min, M.S. (2015). "Highly Tuned Hybrid MPI/OpenACC Implementation with GPUDirect Communication for Electromagnetic and Uid Solvers Based on High Order Spectral Element Discretization." 8th International Congress on Industrial Applied Mathematics (ICIAM 2015), Beijing, China, August 10-14, 2015.

Min, M.S. (2015). "Highly Tuned Hybrid MPI/OpenACC Implementation with GPUDirect Communication for Electromagnetics Solvers Based on Spectral Element Discretizations." 9th International Congress on Industrial and Applied Mathematics, Beijing, China, August 10-14, 2015.

Min, M.S. (2015). “Highly Tuned MPI/OpenACC Implementation and Performance Analysis.” Nek5000 Users Meeting, Argonne, IL, July 14, 2015.

Min, M.S. (2015). “High-order Methods for High-performance Simulations." 8th International Congress on Industrial Applied Mathematics (ICIAM 2015), Beijing, China, August 10-14, 2015.

Min, M.S. (2015). "Hybrid MPI/OpenACC Implementation for a High-order Electromagnetics Solver on GPUDirect Communication." Porting Apps to Titan: Results from the Inaugural GPU Hackathon at GPU Technology Conference, Silicon Valley, CA, March 17-20, 2015.

Min, M.S. (2015). "Scalable Algorithms for Large Scale Quantum-mechanical Density Matrix Calculations for a Nanoparticle System Interacting with Multi-state Multiple Quantum Dots." 8th International Congress on Industrial Applied Mathematics (ICIAM 2015), Beijing, China, August 10-14, 2015.

Min, M.S. (2015). "Scaling Limits for Transport Simulations Based on High-order Spectral Element Discretizations." 2nd International Conference in Applied Mathematics and Scientific Computing, Qingdao, China, August 16-18, 2015.

\section{2-181}

\section{PRESENTATION}

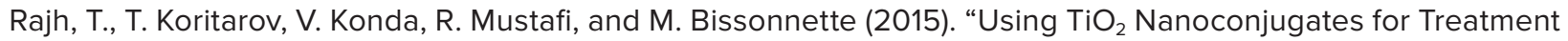
of Cancer Cells." 19th International Conference on Semiconductor Photocatalysis and Solar Energy Conversion, San Diego, CA, November 16-20, 2014.

\section{2-203}

\section{REFEREED PUBLICATIONS}

Fridlyand, A., S.S. Goldsborough, and K. Brezinsky (2015). "Chemical Kinetic Influences of Alkyl Chain Structure on the High Pressure and Temperature Oxidation of a Representative Unsaturated Biodiesel: Methyl Nonenoate." Journal of Physical Chemistry A 119(28): 7559-7577.

Grogan, K.P., S.S. Goldsborough, and M. Ihme (2015). “Ignition Regimes in Rapid Compression Machines.” Combustion and Flame 162(8): 3071-3080.

\section{NON-REFEREED PUBLICATION}

Grogan, K.P., S.S. Goldsborough, and M. Ihme (2015). “Mild Ignition Phenomena in Rapid Compression Machines.” 25th International Colloquium on the Dynamics of Explosions and Reactive Systems, Leeds, UK, August 2-7, 2015. 


\section{2-208}

\section{REFEREED PUBLICATIONS}

Arribas, A.P., F. Shang, M. Krishnamurthy, and K. Shenai (2015). "A Simple and Accurate Circuit Simulation Model for SiC Power MOSFET." IEEE Transactions on Electron Devices 62(2): 449-457.

Pozo Arribas, A., M. Krishnamurthy, and K. Shenai (2015). "Accurate Estimation of Switching Losses in SiC Power MOSFET's." ECS Transactions 64(7): 283-287.

Pozo Arribas, A., M. Krishnamurthy, and K. Shenai (2015). "Future Prospects of Wide Bandgap (WBG) Semiconductor Power Switching Devices." IEEE Transactions on Electron Devices 62(2): 248-257.

Shenai, K., and A. Chattopadhyay (2015). “Optimization of High-Voltage Wide Bandgap Semiconductor Power Diodes.” IEEE Transactions on Electron Devices 62(2): 359-365.

\section{3-129}

REFEREED PUBLICATION

Chun, S.H., J.W. Kim, J. Kim, H. Zheng, C.C. Stoumpos, C.D. Malliakas, J.F. Mitchell, K. Mehlawat, Y. Singh, Y. Choi, T. Gog, A. Al-Zein, M.M. Sala, M. Krisch, J. Chaloupka, G. Jackeli, G. Khaliullin, and B.J. Kim (2015). "Direct Evidence for Dominant Bond Directional Interactions in a Honeycomb Lattice Iridate $\mathrm{Na}_{2} \mathrm{IrO}_{3}$." Nature Physics 11(6): 462-466.

\section{3-218}

\section{REFEREED PUBLICATION}

Bergerson, J., R.T. Muehleisen, W.B. Rodda, J.A. Auld, L.B. Guzowski, J. Ozik, and N. Collier (2015). “Designing Future Cities: LakeSIM Integrated Design Tool for Assessing Short and Long Term Impacts of Urban Scale Conceptual Designs." ISOCARP Review 11. 


\section{AUTHOR INDEX}

Ahluwalia, Rajesh K.

Alatas, Ahmet

Alexeev, Yuri

Allcock, William

Almer, Jonathan D.

Almer, Jonathan D.

Almer, Jonathan D.

Almer, Jonathan D.

Alp, E. Ercan

Alp, E. Ercan

Ananthakrishnan, Rachana

Anitescu, Mihai

Antonopoulos, Dionysios A.

Antonopoulos, Dionysios A.

Antonopoulos, Dionysios A.

Antonopoulos, Dionysios A.

Assoufid, Lahsen

Auld, Joshua

Awschalom, David D.

Aydelott, Ryan

Babnigg, Gyorgy

Babnigg, Gyorgy

Babnigg, Gyorgy

Beckman, Peter

Benmore, Chris

Benmore, Chris

Berry, Matt

Bessac, Julie

Bhattacharya, Suman

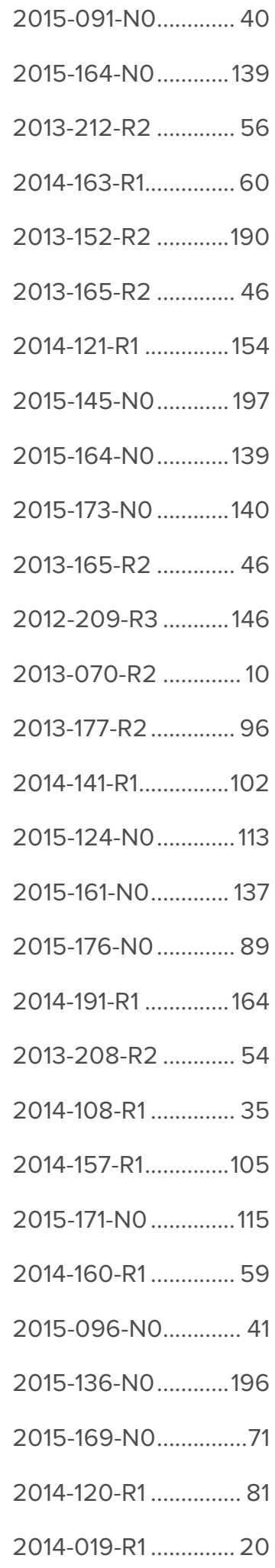

Billone, Michael

Binkowski, Andrew

Blaiszik, Ben

Blaiszik, Benjamin

Blauwkamp, Joel

Botterud, Audun

Botterud, Audun

Boyanov, Maxim

Bradford, Robert

Bromberek, David

Brombosz, Scott M.

Byrum, Karen

Cai, Zhonghou

Cai, Zhonghou

Campos Ortega, Edwin

Cappello, Franck

Carlstrom, John

Carrera, Julie

Carter, Jason

Castiglioni, Andrew

Catlett, Charlie

Catlett, Charlie

Chan, Maria

Chan, Maria

Chang, Clarence

Chang, Clarence

Chapman, Karena

Chard, Kyle

Chard, Kyle

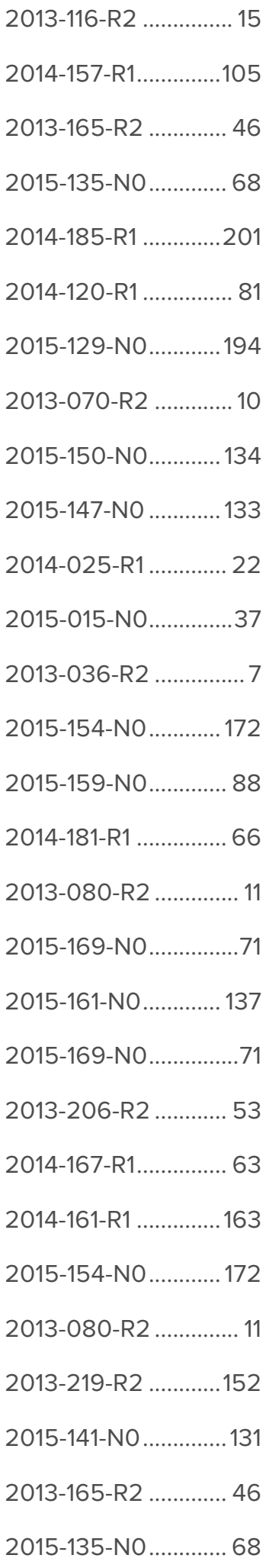


AUTHOR INDEX

\begin{tabular}{|c|c|c|c|}
\hline Chen, Chen & 2015-159-NO............ 88 & Desai, Narayan & 2013-208-R2 ........... 54 \\
\hline Chen, Lin X. & 2013-165-R2 …........ 46 & DiChiara, Anthony & 2015-150-NO........... 134 \\
\hline Chen, Lin X. & 2014-046-R1 ............. 23 & Dixon, David & 2014-139-R1..............160 \\
\hline Chen, Xing & 2013-156-R2 _.............79 & Domagala, Paul & 2015-135-NO ............... 68 \\
\hline Chen, Xing & 2014-169-R1 .............. 83 & Doose, Charles & 2014-184-R1_.............130 \\
\hline Chen, Xing & 2015-157-NO .............. 86 & Drewniak, Beth & 2014-167-R1_.............. 63 \\
\hline Chen, Yiren & 2015-145-NO ............. 197 & Drewniak, Beth & 2015-124-NO ..............113 \\
\hline Chen, Zonghai & 2014-121-R1 ..............154 & Dufresne, Eric & 2015-150-NO............ 134 \\
\hline Choi, YoonYoung & 2013-063-R2 …...........9 & Eastman, Jeffrey A. & 2014-151-R1 ................161 \\
\hline Chupas, Peter & 2013-165-R2 ….......... 46 & Elam, Jeffrey W. & 2013-154-R2 .............. 77 \\
\hline Chupas, Peter & 2015-141-NO ............... 131 & Elam, Jeffrey W. & 2014-133-R1..............159 \\
\hline Ciatti, Stephen & 2015-157-NO ............. 86 & Elam, Jeffrey W. & 2014-151-R1 ...............161 \\
\hline Clifford, Megan & 2015-177-NO ...........204 & Elam, Jeffrey W. & 2014-187-R1_............108 \\
\hline Collart, Frank & 2015-171-NO ...............115 & Elam, Jeffrey W. & 2015-151-NO .............. 85 \\
\hline Collier, Nick & 2015-169-N0 ................71 & Ellis, Ross J. & 2014-004-R1 .................17 \\
\hline Conley, Raymond & 2015-161-N0 .............. 137 & Erdemir, Ali & 2013-022-R2 ............... 4 \\
\hline Constantinescu, Emil M. & 2013-165-R2 ….......... 46 & Erdmann, Mark & 2015-161-NO .............. 137 \\
\hline Constantinescu, Emil M. & 2014-120-R1 ….......... 81 & Eryilmaz, Osman & 2013-022-R2 …........... 4 \\
\hline Cook, David & 2014-132-R1...............101 & Fan, Zhaosheng & 2012-205-R3 ............ 93 \\
\hline Curtiss, Larry A. & 2012-209-R3 ….......146 & Fan, Zhaosheng & 2015-124-NO ..............113 \\
\hline Darling, Seth B. & 2014-187-R1_.............108 & Fang, Lei & 2014-095-R1 ...............34 \\
\hline Darling, Seth B. & 2015-151-NO.............. 85 & Feng, Yan & 2014-084-R1 ............. 32 \\
\hline Davis, Michael J. & 2013-148-R2 …...........76 & Feng, Yejun & 2015-184-NO ............. 144 \\
\hline Davis, Michael J. & 2014-161-R1 ..............163 & Fernandez-Gonzalez, Liliana & 2015-170-NO ............. 114 \\
\hline De Carlo, Francesco & 2013-168-R2 ............123 & Ferrier, Nicola & 2014-132-R1_..............101 \\
\hline de Pablo, Juan J. & 2013-184-R2 ............ 147 & Ferrier, Nicola & 2014-160-R1 ............. 59 \\
\hline de Pablo, Juan J. & 2014-166-R1 ............. 62 & Ferrier, Nicola & 2014-174-R1_............. 64 \\
\hline de Pablo, Juan J. & 2015-172-N0 ...............73 & Ferrier, Nicola & 2015-149-NO ............169 \\
\hline de Sisternes, Fernando & $2014-120-R 1$............... 81 & Finkel, Hal & 2013-165-R2 …......... 46 \\
\hline de Sisternes, Fernando & 2015-129-NO .............194 & Finkel, Hal & 2014-019-R1 ............... 20 \\
\hline Demarteau, Marcel & 2015-015-N0..............37 & Finney, Lydia & 2015-183-NO ............ 143 \\
\hline
\end{tabular}


Flender, Samuel

Flynn, Theodore M.

Flynn, Theodore M.

Flynn, Theodore M.

Fong, Dillon D.

Foster, Ian T.

Foster, Ian T.

Foster, Ian T.

Foster, Ian T.

Frontiere, Nicholas

Fuerst, Joel

Fuoss, Paul H.

Fuoss, Paul H.

Fuoss, Paul H.

Gallagher, Kevin

Galli, Giulia

Ganda, Francesco

Garcia Martinez, Marta

Gerlach, Wolfgang

Ghate, Virendra P.

Ghate, Virendra P.

Gilbert, Jack A.

Gilbert, Jack A.

Gilbert, Jack A.

Gilbert, Jack A.

Gluskin, Efim

Goel, Aditya

Goetze, Kurt

Gog, Thomas

Goldsborough, S. Scott

Goodenough, Lisa

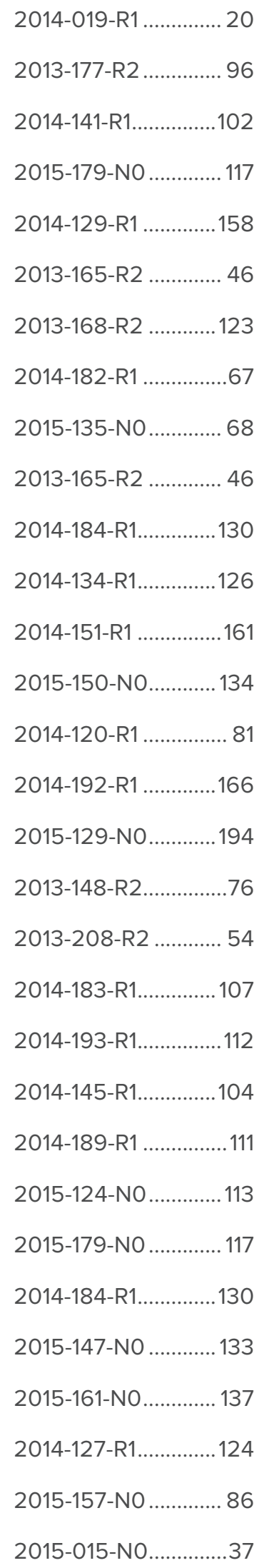

Gray, Stephen K.

Gregar, Joseph

Gros, Marie-Francoise

Guest, Jeffrey R.

Guest, Jeffrey R.

Gursoy, Doga

Gutierrez, Arturo

Gutierrez, Ben

Guzowski, Leah

Habib, Salman

Halder, Gregory

Hamada, Yuki

Hamada, Yuki

Hamada, Yuki

Harder, Ross J.

Harder, Ross J.

Harder, Ross J.

Hasse, Quentin

Heald, Steve

Heinonen, Olle

Heinonen, Olle

Heinonen, Olle

Heinonen, Olle

Heitmann, Katrin

Heitmann, Katrin

Henry, Christopher

Henry, Christopher

Henry, Christopher

Hereld, Mark

Highland, Matthew J.

Highland, Matthew J.

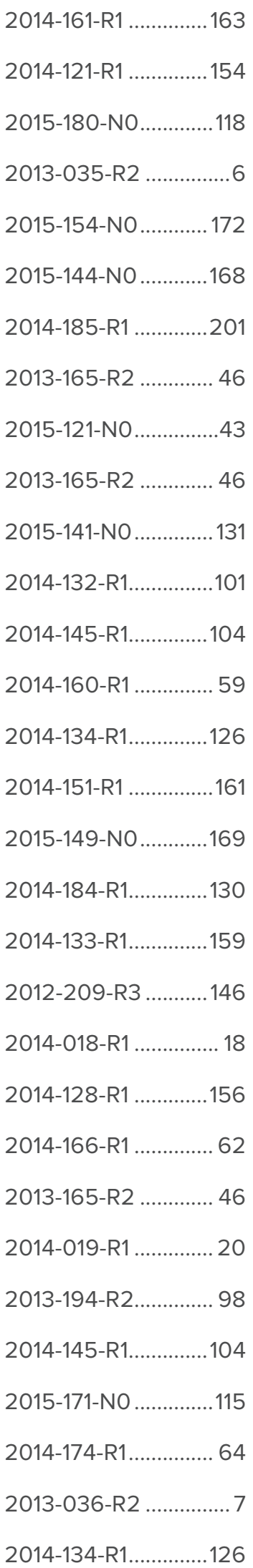


AUTHOR INDEX

\begin{tabular}{|c|c|c|}
\hline Highland, Matthew J. & 2014-151-R1 ................161 & Joachimiak, Andrzej \\
\hline Ho Kim, Jung & 2014-127-R1.............124 & Joachimiak, Andrzej \\
\hline Hock, Adam & 2014-133-R1.............159 & Joachimiak, Andrzej \\
\hline Hoffmann, Axel & 2013-016-R2 …............3 & Johnson, Christopher \\
\hline Hoffmann, Axel & 2014-018-R1 ................ 18 & Jung, II Woong \\
\hline Hoffmann, Axel & 2014-054-R1 ..............27 & Kahvecioglu Feridun, Ozgenur \\
\hline Holt, Martin V. & 2014-151-R1 ................161 & Kaminski, Michael \\
\hline Hong, Hawoong & 2014-081-R1 ................30 & Karbowski, Dominik \\
\hline Hong, Hawoong & 2015-152-NO .............170 & Karpeyev, Dmitry \\
\hline Hong, Seungbum & 2013-063-R2 _............... & Karpeyev, Dmitry \\
\hline Hong, Seungbum & 2014-081-R1 ................30 & Karpeyev, Dmitry \\
\hline Horan, Douglas & 2015-147-NO .............133 & Kasa, Matthew \\
\hline Hruszkewycz, Stephan O. & 2014-134-R1.............126 & Kastengren, Alan \\
\hline Hruszkewycz, Stephan O. & 2014-151-R1 ................. 161 & Kasthuri, Narayanan \\
\hline Hu, Michael & 2015-164-NO ..............139 & Kavicky, James \\
\hline Hu, Michael & 2015-173-NO .............140 & Keahey, Katarzyna \\
\hline Huang, Xianrong & 2014-127-R1..............124 & Keceli, Murat \\
\hline Ignacio-de Leon, Patricia & 2013-156-R2 …...........79 & Kemner, Kenneth M. \\
\hline Insley, Joe & 2015-169-NO ................71 & Kemner, Kenneth M. \\
\hline Ivanyushenkov, Yury & 2014-137-R1 ............... 127 & Kemner, Kenneth M. \\
\hline Ivanyushenkov, Yury & 2014-184-R1_.............130 & Kemner, Kenneth M. \\
\hline Jacob, Robert & 2014-167-R1_.............. 63 & Kemner, Kenneth $\mathrm{M}$. \\
\hline Jacobsen, Chris & 2013-168-R2 ............123 & Kettimuthu, Raj \\
\hline Jacobsen, Chris & 2015-153-NO ............136 & Kim, Jongjin \\
\hline Jacobsen, Chris & 2015-185-NO ............178 & Kissick, David \\
\hline Jedrzejczak, Robert & 2014-108-R1 ............... 35 & Klug, Jeffrey \\
\hline Jedrzejczak, Robert & 2014-157-R1_.............105 & Knight, Christopher J. \\
\hline Jennings, Guy & 2013-165-R2 .............. 46 & Koshelev, Alexei \\
\hline Jennings, Guy & 2015-141-NO ...............131 & Kotamarthi, Rao \\
\hline Jiang, Wei & 2014-188-R1 ...............110 & Kotamarthi, Rao \\
\hline Jiang, Zhang & 2015-150-NO.............134 & Kotamarthi, Rao \\
\hline
\end{tabular}

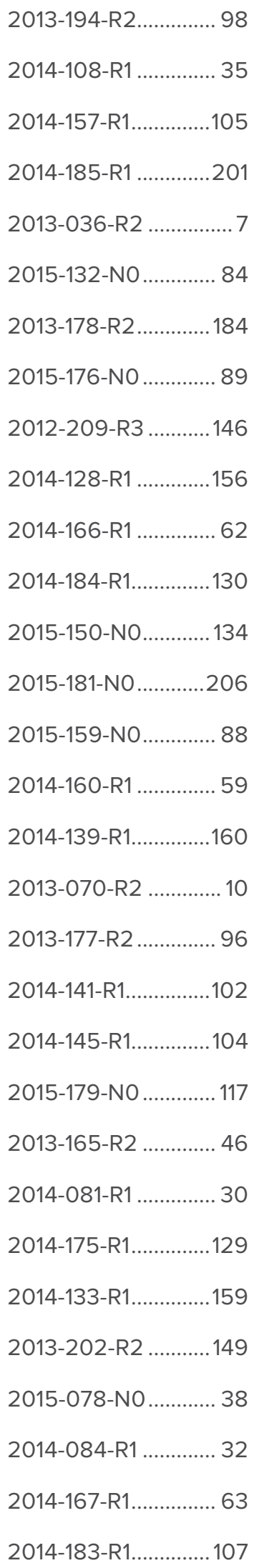


AUTHOR INDEX

\begin{tabular}{|c|c|c|c|}
\hline Kotamarthi, Rao & 2014-193-R1................112 & Liu, Di-Jia & 2015-091-NO............. 40 \\
\hline Kovacs, Eve & 2013-165-R2 ….......... 46 & Liu, Wenjun & 2014-134-R1_............126 \\
\hline Krumdick, Gregory & 2015-132-N0 ............. 84 & Liu, Yuzi & 2015-154-NO ............. 172 \\
\hline Krummel, John & 2012-206-R3 …......... 94 & Longman, Douglas E. & 2013-148-R2 ................ \\
\hline Krummel, John & 2014-183-R1...............107 & Lu, Zheng-Tian & 2014-188-R1 ..............110 \\
\hline Krummel, John & 2014-193-R1................112 & Macal, Charles & 2015-169-NO ................71 \\
\hline Kumaran, Kalyan & 2013-213-R2 .............. 58 & Madden, Timothy & 2015-150-NO............. 134 \\
\hline Kurtz, Charles & 2015-141-NO ............... 131 & Madduri, Ravi & 2013-165-R2 ............ 46 \\
\hline Kustom, Robert & 2013-111-R2 …..........122 & Magill, Stephen & 2015-015-N0...............37 \\
\hline Kwok, Wai-Kwong & 2014-025-R1 ............. 22 & Malik, Tanu & 2013-165-R2 …......... 46 \\
\hline Kwok, Wai-Kwong & 2014-095-R1 ...............34 & Mane, Anil & 2013-154-R2............... 77 \\
\hline Lai, Barry & 2014-084-R1 ............. 32 & Marin, Oana & 2014-177-R1 ..............192 \\
\hline Lai, Barry & 2015-153-NO .............136 & Marshall, Christopher L. & 2014-145-R1..............104 \\
\hline Laible, Phillip & 2015-157-NO ............. 86 & Marshall, Christopher L. & 2015-174-NO ...........203 \\
\hline Larsen, Peter & 2015-124-NO .............. 113 & Martinez-Moyano, Ignacio & 2014-194-R1.............186 \\
\hline Lee, Eungje & 2014-185-R1 .............201 & Martinson, Alex B.F. & 2013-100-R2 ............... 13 \\
\hline Lee, Jonghun & 2014-077-R1 .............. 28 & Maser, Jörg & 2014-134-R1..............126 \\
\hline Leu, Bogdan & 2015-182-NO .............142 & Maser, Jörg & 2015-153-NO ............136 \\
\hline Leyffer, Sven & 2013-168-R2 …........123 & Matamala, Roser & 2014-132-R1...............101 \\
\hline Leyffer, Sven & 2013-206-R2 ............ 53 & Matveev, Konstantin & 2014-095-R1 ...............34 \\
\hline Leyffer, Sven & 2015-149-NO ............169 & McChesney, Jessica & 2015-152-NO ............170 \\
\hline Leyffer, Sven & 2015-159-NO ............. 88 & McElfresh, Mike & 2015-177-NO ...........204 \\
\hline Li, Jie & 2015-121-NO ................43 & McNulty, Ian & 2015-149-NO .............169 \\
\hline Li, Meimei & 2013-152-R2 .............190 & McNulty, Ian & 2015-154-NO ............ 172 \\
\hline Li, Meimei & 2015-145-NO ............. 197 & Mertz, Carol & 2013-178-R2 .............184 \\
\hline Li, Meimei & 2015-150-NO............134 & Merzari, Elia & 2014-177-R1 ..............192 \\
\hline Libera, Joseph & 2013-154-R2 ...............77 & Meyer, Folker & 2014-141-R1..............102 \\
\hline Lin, Xiao-Min & 2013-035-R2 ................ 6 & Miceli, Antonino & 2015-150-NO............ 134 \\
\hline Lin, Xiao-Min & 2014-077-R1 .............. 28 & Michalska, Karolina & 2014-108-R1 .............. 35 \\
\hline Lin, Xiao-Min & 2015-150-NO.............134 & Michalska, Karolina & 2014-157-R1...............105 \\
\hline Liu, Di-Jia & 2014-051-R1 .............. 25 & Miller, Jeffery & 2014-051-R1 .............. 25 \\
\hline
\end{tabular}




\begin{tabular}{|c|c|c|c|}
\hline Mishra, Umakant & 2012-206-R3 ............ 94 & O’Loughlin, Edward J. & 2015-179-NO ............... 117 \\
\hline Mitchell, John F. & 2015-175-NO ............. 176 & Obabko, Aleksandr & 2014-177-R1 ...............192 \\
\hline Mooney, Tim & 2015-161-NO .............. 137 & Ocola, Leonidas & 2013-178-R2 ............. 184 \\
\hline Mootz, Joseph & 2014-108-R1 ............... 35 & Ogata, Craig M. & 2014-175-R1..............129 \\
\hline Morozov, Vitali & 2013-213-R2 ............. 58 & Okasinski, John & 2014-121-R1 ...............154 \\
\hline Muehleisen, Ralph & 2015-121-N0 ...............43 & Osborn, Ray & 2013-165-R2 ….......... 46 \\
\hline Mueller, Peter & 2014-188-R1 ................110 & Overbeek, Ross & 2013-194-R2 2.............. 98 \\
\hline Munson, Todd & 2013-168-R2 ............123 & Ozik, Jonathan & 2015-169-NO ................71 \\
\hline Munson, Todd & 2015-149-N0 .............169 & Paley, Jonathan M. & 2014-023-R1 ............... 21 \\
\hline Murphy, John T. & 2015-169-NO...............71 & Park, Hyowon & 2015-168-NO ............. 175 \\
\hline Murphy-Olson, Daniel & 2013-208-R2 ........... 54 & Pellin, Michael J. & 2013-100-R2 .............. 13 \\
\hline Narayanan, Suresh & 2013-168-R2 ...........123 & Pellin, Michael J. & 2013-116-R2 …........... 15 \\
\hline Narayanan, Suresh & 2014-077-R1 ............... 28 & Pellin, Michael J. & 2013-152-R2 ............190 \\
\hline Narayanan, Suresh & 2015-150-NO.............134 & Pellin, Michael J. & 2014-133-R1..............159 \\
\hline Nassiri, Alireza & 2013-111-R2 ..............122 & Pelton, Matthew & 2013-035-R2 …............6 \\
\hline Nassiri, Alireza & 2015-147-NO .............. 133 & Peterka, Tom & 2013-168-R2 ….........123 \\
\hline Natesan, Ken & 2015-145-NO ............. 197 & Peterka, Tom & 2015-149-NO .............169 \\
\hline Nealey, Paul F. & 2013-216-R2 ..............151 & Petford Long, Amanda & 2015-185-NO ............. 178 \\
\hline Nealey, Paul F. & 2015-150-NO............ 134 & Phatak, Charudatta & 2013-063-R2 …...........9 \\
\hline Negri, M. Cristina & 2015-170-NO .............. 114 & Phatak, Charudatta & 2015-144-NO .............168 \\
\hline Nocek, Boguslaw & 2014-157-R1..............105 & Phillips, Carolyn & 2015-172-NO ..............73 \\
\hline Noirot, Philippe & 2015-171-NO ...............115 & Poeppelmeier, Kenneth & 2015-141-NO ................131 \\
\hline North, Michael & 2014-194-R1...............186 & Pokkuluri, P. Raj & 2013-013-R2 …............. \\
\hline Novosad, Valentyn & 2013-080-R2 .............. 11 & Ponciroli, Roberto & 2015-129-NO.............194 \\
\hline Novosad, Valentyn & 2013-219-R2 ...........152 & Pope, Adrian & 2013-165-R2 ............. 46 \\
\hline O’Brien, Sarah & 2013-070-R2 .............. 10 & Preissner, Curt & 2015-153-NO .............136 \\
\hline O’Brien, Sarah & 2014-145-R1.............104 & Proslier, Thomas & 2013-080-R2 .............. 11 \\
\hline O’Loughlin, Edward J. & 2013-070-R2 .............. 10 & Proslier, Thomas & 2014-133-R1..............159 \\
\hline O’Loughlin, Edward J. & 2013-177-R2 ….......... 96 & Qian, Jun & 2015-161-NO ............. 137 \\
\hline O’Loughlin, Edward J. & 2014-141-R1...............102 & Qiu, Feng & 2015-159-NO ............. 88 \\
\hline O’Loughlin, Edward J. & 2014-145-R1...............104 & Rajh, Tijana & 2015-135-NO .............. 68 \\
\hline
\end{tabular}


AUTHOR INDEX

\begin{tabular}{|c|c|c|c|}
\hline Rajh, Tijana & 2015-154-NO ............. 172 & Sharma, Hemant & 2013-165-R2 …......... 46 \\
\hline Ramirez, Giovanni & 2013-022-R2 …............ 4 & Shevchenko, Elena V. & 2013-173-R2 .............183 \\
\hline Rangel, Esteban & 2013-165-R2 ............. 46 & Shi, Bing & 2015-161-NO ............. 137 \\
\hline Rask, Eric & 2015-176-NO .............. 89 & Shi, Xianbo & 2015-141-NO ...............131 \\
\hline Ratcliff, Laura E. & 2013-199-R2 …......... 52 & Shin, Youngho & 2015-132-N0 ............. 84 \\
\hline Ren, Yang & 2014-121-R1 ...............154 & Shiroyanagi, Yuko & 2014-184-R1...............130 \\
\hline Roehrig, Chris & 2015-153-NO .............136 & Sivaramakrishnan, Raghu & 2013-148-R2 ...............76 \\
\hline Romero, Nichols A. & 2013-199-R2 …......... 52 & Sivaramakrishnan, Raghu & 2015-157-NO ............. 86 \\
\hline Romero, Nichols A. & 2014-128-R1 .............156 & Smith, Barry F. & 2015-139-NO ...............70 \\
\hline Rondinelli, James & 2015-178-NO ............. 177 & Snyder, Seth W. & 2014-189-R1 ................111 \\
\hline Rosenberg, Richard & 2014-129-R1 .............158 & Sokolov, Vadim & 2015-169-NO ...............71 \\
\hline Rosenblum, Emily & 2015-169-NO ................71 & Sokolov, Vadim & 2015-176-NO .............. 89 \\
\hline Rosenkranz, Stephan & 2013-165-R2 ….......... 46 & Som, Sibendu & 2013-148-R2 ...............76 \\
\hline Rousseau, Aymeric & 2015-176-NO ............. 89 & Ssegane, Herbert & 2015-170-NO ..............114 \\
\hline Roux, Benoit & 2014-161-R1 ...............163 & Stacey, Craig & 2014-163-R1.............. 60 \\
\hline Rozhkova, Elena A. & 2013-173-R2 .............. 183 & Stan, Marius & 2012-209-R3 ............146 \\
\hline Sandy, Alec & 2014-077-R1 .............. 28 & Stan, Marius & 2013-152-R2 …........190 \\
\hline Sandy, Alec & 2015-150-NO.............134 & Stephenson, G. Brian & 2015-167-NO ............ 174 \\
\hline Sankaran, Rajesh & 2014-160-R1 .............. 59 & Stols, Lucy & 2013-171-R2 ...............181 \\
\hline Sankaran, Rajesh & 2014-167-R1............... 63 & Strzalka, Joseph & 2015-150-NO............ 134 \\
\hline Sankaranarayanan, Subramanian & 2014-161-R1 ..............163 & Sullivan, Shane & 2015-153-NO ............136 \\
\hline Sankaranarayanan, Subramanian & 2015-149-NO ............169 & Sumant, Anirudha V. & 2013-178-R2 .............184 \\
\hline Schabacker, Daniel & 2013-171-R2 ............... 181 & Sun, Yugang & 2014-121-R1 .............154 \\
\hline Schabacker, Daniel & 2013-173-R2 .............183 & Sun, Yugang & 2015-121-NO ...............43 \\
\hline Schaller, Richard D. & 2013-173-R2 ............. 183 & Sydelko, Pamela & 2014-194-R1..............186 \\
\hline Schiffer, Marianne & 2013-013-R2 …............. 2 & Talaga, Richard & 2015-015-NO...............37 \\
\hline Schlepuetz, Christian & 2014-133-R1..............159 & te Velthuis, Suzanne G.E. & 2014-054-R1 .............27 \\
\hline Schreiber, Nathaniel & 2015-175-NO ............. 176 & Tentner, Adrian & 2014-177-R1 ...............192 \\
\hline Schwarz, Nicholas & 2013-168-R2 …........123 & Thimmapuram, Prakash & 2014-120-R1 ............... 81 \\
\hline Schwarz, Nicholas & 2015-150-NO.............134 & Tiede, David & 2013-165-R2 …......... 46 \\
\hline Seaver, Samuel & 2013-194-R2 2.............. 98 & Tischler, Jonathan Z. & 2014-129-R1 .............158 \\
\hline
\end{tabular}


AUTHOR INDEX

\begin{tabular}{|c|c|c|c|}
\hline Toellner, Thomas S. & 2015-164-NO .............139 & Wang, Jianhui & 2015-159-NO ............. 88 \\
\hline Tomboulides, Ananias & 2014-177-R1 ................192 & Wang, Jin & 2015-150-NO ............. 134 \\
\hline Trahey, Lynn & 2015-144-NO .............168 & Weber, Rick & 2015-136-NO .............196 \\
\hline Trail, Jessica & 2015-169-NO ................71 & Weimer, Matt & 2014-133-R1..............159 \\
\hline Trakhtenberg, Emil & 2014-184-R1...............130 & Weizeorick, John & 2015-150-NO............134 \\
\hline Tranter, Robert & 2015-157-NO ............. 86 & Welland, Michael & 2012-209-R3 ...........146 \\
\hline Tse, Olivia & 2015-170-NO .............. 114 & Welp, Ulrich & 2014-137-R1_.............. 127 \\
\hline Tuecke, Steven & 2014-182-R1 ...............67 & Wen, Haidan & 2013-036-R2 ............... 7 \\
\hline Uckun, Canan & 2014-120-R1 ............... 81 & Wen, Haidan & 2015-149-NO .............169 \\
\hline Uram, Tom & 2013-165-R2 ….......... 46 & Wen, Haidan & 2015-150-NO............134 \\
\hline Urgun-Demirtas, Meltem & 2013-156-R2 …...........79 & Wild, Stefan & 2013-168-R2 ............123 \\
\hline Urgun-Demirtas, Meltem & 2014-189-R1 ................111 & Wilde, Michael & 2013-165-R2 ............. 46 \\
\hline van Gemmeren, Peter & 2013-165-R2 ….......... 46 & Williamson, Mark & 2015-096-NO.............. 41 \\
\hline Vázquez-Mayagoitia, Álvaro A. & 2013-199-R2 …......... 52 & Williamson, Mark & 2015-136-NO .............196 \\
\hline Vázquez-Mayagoitia, Álvaro A. & 2013-212-R2 ............. 56 & Wilton, Rosemarie & 2013-013-R2 ................ 2 \\
\hline Vázquez-Mayagoitia, Álvaro A. & 2014-139-R1...............160 & Wilton, Rosemarie & 2013-171-R2 ................181 \\
\hline Vegendla, Prasad & 2014-177-R1 ...............192 & Wilton, Rosemarie & 2013-173-R2 ..............183 \\
\hline Vilim, Richard & 2015-129-NO .............194 & Winans, Randall & 2015-151-NO .............. 85 \\
\hline Vishwanath, Venkatram & 2013-165-R2 …......... 46 & Winkler, Linda & 2014-163-R1.............. 60 \\
\hline Vishwanath, Venkatram & 2013-213-R2 ............. 58 & Wolf, Dieter & 2012-209-R3 ...........146 \\
\hline Vlasko-Vlasov, Vitalii & 2015-078-NO ............. 38 & Wozniak, Justin & 2013-165-R2 …......... 46 \\
\hline Vogt, Stefan & 2013-168-R2 …........123 & Xia, Fangfang & 2013-194-R2 .............. 98 \\
\hline Vogt, Stefan & 2014-084-R1 ............. 32 & Xiao, Xianghui & 2015-144-NO .............168 \\
\hline Vogt, Stefan & 2015-153-NO .............136 & Yacout, Abdellatif & 2013-116-R2 ............... 15 \\
\hline von Lilienfeld, Anatole & 2013-212-R2 ............. 56 & Yacout, Abdellatif & 2013-152-R2 ............190 \\
\hline Voth, Gregory A. & 2013-202-R2 …........149 & Yanguas-Gil, Angel & 2013-154-R2 ...............77 \\
\hline Wagner, Albert & 2014-139-R1..............160 & Yanguas-Gil, Angel & 2014-133-R1.............159 \\
\hline Waldschmidt, Geoff & 2015-147-NO .............133 & Yanguas-Gil, Angel & 2014-151-R1 ................161 \\
\hline Walko, Donald & 2015-150-NO............. 134 & Yefremenko, Volodymyr & 2013-080-R2 .............. 11 \\
\hline Wang, Gensheng & 2013-080-R2 ............... 11 & Yefremenko, Volodymyr & 2013-219-R2 ............152 \\
\hline Wang, Gensheng & 2013-219-R2 ............152 & You, Hoydoo & 2015-150-NO............ 134 \\
\hline
\end{tabular}


Zapol, Peter

Zapol, Peter

Zapol, Peter

Zapol, Peter

Zapol, Peter

Zhang, Junchao

Zhang, Yao

Zhao, Jiyong

Zhou, Zhi

Zhou, Ziyao

Zumpf, Colleen
2013-165-R2 …......... 46

2014-128-R1 ..............156

2014-139-R1..............160

2014-151-R1 ................ 161

2015-152-NO ..............170

2015-139-NO ...............70

2013-213-R2 ............. 58

2015-164-NO ..............139

2015-129-NO ............. 194

2014-169-R1 .............. 83

2015-170-NO ............... 114 


\section{ACKNOWLEDGMENTS}

Argonne's LDRD Program Office comprises the Associate Chief Scientist for LDRD, Dr. Kawtar Hafidi; LDRD Program Manager, Rebecca R. Rank; and Administrative Specialist, Marianne I. Lohr-Dietz. Those of us in the program's management are grateful for the critical support received throughout the year from the individuals listed below. Special appreciation is due to the many experts who perform the research and provide their project reports; to the technical and administrative management of Argonne's programmatic divisions, within which the research and development are pursued; to the many members of the several peer-review panels that evaluate LDRD proposals; and to the Laboratory's management, whose vision and understanding of the nation's technological needs help guide and refine the content of Argonne's LDRD portfolio.

$\square$ Computing and Information Systems

○ Jason Boggs

o Fred Moszur

o Ross Pallan

$\square$ Legal Department

o Mark Hilliard

o Debbie Krakowski

$\square$ Office of the Chief Financial Officer

- Richard Crowley

o Luba Zholents

$\square$ Office of the Director

○ Elton N. Kaufmann

$\square$ Communications, Education, and Public Affairs

- Kevin A. Brown (Lead Project Manager)

- Andrea Manning (Project Manager and Lead Editor)

- Michele Nelson (Lead, Design and Layout)

- Linda Conlin (Lead, Document Production)

- Janet Barrett (CD Production)

- Victor Comello (Editor)

o Pat Hollopeter (Editor)

- Katherine Obmascik (Editor)

- Bryan Schmidt (Editor)

- Carolyn Steele (Editor)

- Elizabeth Stefanski (Editor) 


\section{ABOUT ARGONNE NATIONAL LABORATORY}

Argonne is a U.S. Department of Energy Laboratory managed by UChicago Argonne, LLC, under contract DE-AC02-06CH11357. The Laboratory's main facility is located near Chicago at 9700 South Cass Avenue, Argonne, Illinois 60439, USA. For information about Argonne and its pioneering science and technology programs, visit www.anl.gov.

\section{CONTACT}

Rebecca Rank

LDRD Program Manager

Strategy and Innovation Office, Office of the Director

Phone: 630-252-6855

E-mail: rrank@anl.gov

This report was prepared as an account of work sponsored by an agency of the United States Government. Neither the United States Government nor any agency thereof, nor UChicago Argonne, LLC, nor any of their employees or officers, makes any warranty, express or implied, or assumes any legal liability or responsibility for the accuracy, completeness, or usefulness of any information, apparatus, product, or process disclosed, or represents that its use would not infringe privately owned rights. Reference herein to any specific commercial product, process, or service by trade name, trademark, manufacturer, or otherwise, does not necessarily constitute or imply its endorsement, recommendation, or favoring by the United States Government or any agency thereof. The views and opinions of document authors expressed herein do not necessarily state or reflect those of the United States Government or any agency thereof, Argonne National Laboratory, or UChicago Argonne, LLC. 